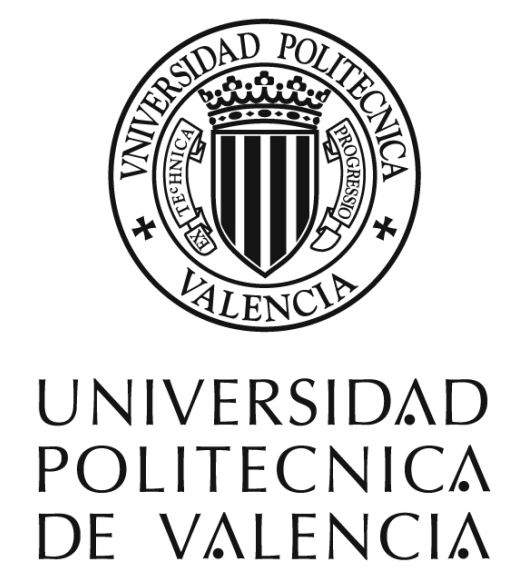

DEPARTAMENTO

DE INGENIERÍA DE LA CONSTRUCCIÓN

\title{
DISEÑO ÓPTIMO DE TABLEROS ISOSTÁTICOS DE VIGAS ARTESAS PREFABRICADAS PRETENSADAS
}




\title{
DISEÑO ÓPTIMO DE TABLEROS ISOSTÁTICOS DE VIGAS ARTESAS PREFABRICADAS PRETENSADAS
}

TESIS DOCTORAL

\author{
Presentada por \\ José Vicente Martí Albiñana
}

Dirigida por

Dr. Fernando González Vidosa

\author{
Universidad Politécnica de Valencia \\ Departamento de Ingeniería de la Construcción
}




\title{
TESIS DOCTORAL
}

Para la obtención del título de

Doctor Ingeniero de Caminos, Canales y Puertos

\section{DISEÑO ÓPTIMO DE TABLEROS ISOSTÁTICOS DE VIGAS ARTESAS PREFABRICADAS PRETENSADAS}

\author{
Presentada por \\ José Vicente Martí Albiñana \\ Dirigida por \\ Dr. Fernando González Vidosa \\ Universidad Politécnica de Valencia \\ Departamento de Ingeniería de la Construcción \\ y Proyectos de Ingeniería Civil
}

VALENCIA 2010 
"Todo era de piedras costosas, talladas a escuadra, cortadas con la sierra, por dentro y por fuera, desde los cimientos hasta las cornisas y desde fuera hasta el atrio grande.."

1 REYES 7,9

A Laura y Guillem 


\section{Agradecimientos}

Me gustaría agradecer en especial el apoyo prestado por mi compañero, mi amigo y mi director de tesis, Fernando González Vidosa, el cuál me embarcó en esta travesía que empezó hace casi cuatro años. De no ser por él, todavía estaría en el puerto de origen viendo partir los barcos.

Quiero agradecer la colaboración prestada por Julián Alcalá, siempre ha ido un paso por delante en esta singladura, me ha animado y me ha hecho ver las cosas fáciles.

Debo mostrar mi agradecimiento desinteresado de mi amigo Víctor Yepes, siempre presto a ayudarme, y que gracias a sus revisiones y consejos he podido sortear situaciones complejas.

No debo olvidarme del resto de los integrantes del grupo de investigación de procedimientos de la construcción, en especial de Francisco J. Martínez, cuya senda abierta me ha facilitado el encuentro con algunas heurísticas.

Deseo expresar mi agradecimiento a la Generalitat Valenciana por la concesión de financiación, a través del IMPIVA, -número de expediente IMIDTA/2009/777-, al proyecto de investigación realizado en colaboración con la empresa PREVALESA SL., en especial a Fernando Galán, que han permitido realizar algunos de los trabajos en los que se ha enmarcado esta tesis.

Y por último agradecer a las personas -familia, amigos y compañeros-, que aunque sin colaboración directa en el trabajo, con su apoyo, ánimo, juicio y ayuda han hecho posible que la realización de este trabajo no fuera sólo una idea, sino una realidad. De entre ellas destacar a mi esposa e hijo, y pedirles disculpas y comprensión por el tiempo que no les he podido dedicar.

Gracias. 


\section{Resumen}

MARTÍ ALBIÑANA, J.V. (2010). Diseño óptimo de tableros isostáticos de vigas artesas prefabricadas pretensadas. Tesis Doctoral. Escuela Técnica Superior de Ingenieros de Caminos, Canales y Puertos. 284 páginas. jvmartia@upv.es.

Los tableros de vigas prefabricadas de hormigón pretensado se utilizan habitualmente en todo el mundo para resolver estructuras de viaductos y de pasos superiores sobre carreteras. Son escasos los trabajos de investigación encaminados a la optimización económica de estas estructuras, donde la mayoría se han centrado en la reducción de las fuerzas de pretensado $\mathrm{y}$ han padecido de un signo considerablemente teórico, sin mejorar el aprovechamiento de los recursos que requieren, lo que ha dificultado su aplicación sobre los proyectos de ejecución. En la búsqueda bibliográfica, no se ha encontrado trabajo alguno enfocado a la optimización heurística económica de este tipo de estructuras.

Ante el hueco existente en el espectro de la investigación, el enfoque de este trabajo está basado en la aplicación de distintas técnicas de optimización económica a esta tipología. Se han elegido técnicas metaheurísticas para poder plantear el problema lo más completo posible, donde se define todo el tablero y sus armados, y se realizan las comprobaciones que marca la normativa española.

Se han analizado diferentes proyectos con esta tipología estructural, donde se han estudiado diseños de vigas artesa de distintos fabricantes, eligiendo una de ellas como modelo para realizar el presente estudio, y dejando la opción para adaptar la tipología a cualquiera de los otros diseños que se fabrican. A continuación se ha desarrollado un programa informático en lenguaje FORTRAN que incluye diferentes módulos: generación de la estructura, comprobación estructural y evaluación económica. Adicionalmente, se ha implementado un módulo que genera ficheros CAD del tablero. El estudio se realiza sobre un tablero de 12 metros de ancho, con 11 de calzada, luz entre apoyos de 35 metros, y separación entre vigas de 6 metros.

El estudio de los métodos heurísticos aplicados sobre el tablero, se realiza mediante la comparación de ocho distintos tipos de algoritmos: la estrategia de saltos múltiples aleatorios (RW), tres métodos de búsqueda local y cuatro de búsqueda poblacional. 
Los métodos de búsqueda local lo componen el método del gradiente (DLS), la cristalización simulada (SA) y la aceptación por umbrales (TA). Respecto a la búsqueda poblacional, está compuesta por tres variantes de la optimización mediante genéticos (GA1, GA2 y GA3) y por un algoritmo memético (MA). El menor coste fue obtenido por el SA con un valor de 108,008€ siendo su diferencia del $0.6 \%$ respecto al TA, del 7\% respecto al DLS y MA, y del 13\% respecto a las tres variantes de los GA. En cuanto a los tiempos de proceso para las mejores calibraciones, los valores inferiores los protagonizan el DLS con 8,122 segundos, a continuación con 18,322 segundos para el SA, seguidos por los tres GA, con valores que rondan los 21,000 segundos, los 36,233 segundos del TA, y finalmente ya muy alejados están los 129,441 segundos del MA. Los resultados han mostrado al SA como la heurística más eficaz de todas ellas.

Una vez seleccionada la mejor calibración del SA, se ha estudiado el número de reinicios necesarios para alcanzar soluciones de calidad con una probabilidad suficientemente alta, empleando un criterio de estabilización de medias y desviaciones típicas. Con ello, se recomienda un algoritmo de optimización, que combina nueve reinicios.

Se ha realizado un estudio sobre la sensibilidad del modelo a la variación del precio de los materiales. Se han tomado incrementos porcentuales en precio de las armaduras activas y pasivas del 5, 10, 15 y 20\%, haciendo lo mismo con el hormigón. Los resultados muestran una sensibilidad más acusada con la variación del precio del acero que con el del hormigón, y que el diseño del tablero se adapta para reducir su coste, aumentando el volumen del hormigón y reduciendo el peso del acero cuando se incrementa el precio de éste último, y lo contrario cuando el incremento del precio es del hormigón.

Para el estudio paramétrico del tablero se han tomado luces de 20, 25, 30, 35 y 40 metros. Se ha analizado la geometría transversal, la armadura activa, la cuantía de acero pasivo, la cuantía de hormigón y los tipos de hormigón en viga y losa. Se ha mostrado una correlación sensiblemente lineal del coste, del canto de la viga y del número de torones respecto a la luz del tablero. Los resultados obtenidos muestran secciones de espesores pequeños y cantos altos, permitiendo ahorros en el volumen de hormigón e incrementando su inercia, lo que repercute en una menor necesidad de pretensado, y una mejor calidad del hormigón, entre 40 y 50 Mpa, en viga.

Simultáneamente se ha extendido el estudio paramétrico para un hormigón reforzado con fibras de acero (HPF) en la viga, comparando los resultados con los obtenidos anteriormente. Se ha analizado el efecto que el refuerzo de las fibras produce sobre el diseño del tablero ante solicitaciones longitudinales y transversales, la variación en el número de torones, en el volumen de hormigón y en la cuantía de la armadura transversal. También se ha analizado la cuantía de fibras más adecuada, etc. Finalmente, se ha comprobado su competitividad económica respecto al hormigón convencional. 


\begin{abstract}
MARTÍ ALBIÑANA, J.V. (2010) Optimal design bridges boards of prestressed concrete precast beams. Doctoral Thesis. School of Civil Engineering. 284 pages. jvmartia@upv.es.

The boards of prestressed concrete precast beams are typically used around the world to solve structures of viaducts and overpasses on roads. Scarce research work aimed at economic optimization of these structures, where the majority have focused on the reduction of forces of prestressed and suffered a considerable theoretical sign without a better use of resources that require, which has hindered its implementation on engineering design. Bibliographic search, not found any work focused on the optimization heuristic economic of this type of structures.
\end{abstract}

Before the gap in the spectrum of research, the focus of this work has been implementing optimization techniques economic to this typology. Applied metaheuristic technical in order to define the problem as complete as possible, where it is defined all the board and its reinforcement, and checked according to the Spanish code.

Different projects with this typology, where different manufacturers U-shape beams designs have been studied have been analysed by choosing one of them as a model for the present study, and leaving the option to adapt the typology to any of the designs that are produced. Then has developed a computer program in FORTRAN language that includes different modules: generation of the structure, structural testing, and economic evaluation. In addition, there is a module that generates the board CAD files. The study is on a board of $12 \mathrm{~m}$ wide, with $11 \mathrm{~m}$ road, $35 \mathrm{~m}$ of span lengths between supports and separation between beams of $6 \mathrm{~m}$.

The study of the heuristic methods applied to the board, is performed by comparison of eight different types of algorithms: random walk (RW), three local search methods and four evolutionary algorithms. Local search methods make it up descent local search (DLS), simulated annealing (SA) and threshold accepting (TA). Regarding the evolutionary algorithms, three variants of genetic optimization (GA1, GA2 and GA3) and one memetic algorithm (MA) were used. The lowest cost solution was the SA with $108,008 €$, being $0.6 \%$ less than TA, $7 \%$ less than DLS and MA, and $13 \%$ 
less than the GA variants. As for the process times for the best calibrations, the minor values them lead the DLS with 8,122 seconds, later with 18,322 seconds for the SA, followed by three GA, with values around 21,000 seconds, them 36,233seconds of the TA, and finally already very remote they are 129,441 seconds of the MA. Results have shown the SA as better heuristics of them all.

Once selected the best calibration of the SA, we have studied the number of restart required to achieve quality solutions with a sufficiently high probability, using a stabilization of averages and standard deviations criterion. Thus designed an algorithm optimization combined nine restarts.

Conducted a study on the sensitivity of the model to the variation in the price of the materials. Percentage increases in prices of the active and passive reinforcement of 5, 10, 15 and 20\%, doing the same with the concrete has been taken. The results show a sensibility more accused with the price of the steel that with that of the concrete, and that the board design adapts to reduce its cost, increasing the volume of concrete and by reducing the weight of steel when increases the price of the latter, and vice-versa when the price increase is concrete.

For the parametric study board there have taken span lengths of 20, 25, 30, 35 and 40 meters. There have been analyzed the cross-sectional geometry, the active steel ratios, the passive steel ratios, the amount of concrete and the types of concrete in beam and slab. There has appeared a substantially linear correlation of the cost, of the beam depth and the number of strands with regard to the span lengths board. The results show small thickness sections and high depths, allowing savings in the volume of concrete and increasing its inertia, needing less active steel, and a better quality of the concrete, among 40 and $50 \mathrm{Mpa}$, in beam.

Simultaneously the parametric study for a concrete reinforced with steel fibers (HPF) in the beams has spread by comparing the results with those obtained previously. There has been analyzed the effect of the reinforcement of the fibers that produces on the design of the board with longitudinal and transverse solicitations, the variation in the number of strands, in the concrete volume, in the transverse steel ratio. Also the most suitable quantity of fibers, etc. Their economic competitiveness to conventional concrete has been verified. 


\section{Resum}

MARTÍ ALBIÑANA, J.V. (2010). Disseny òptim de taulers isostàtics de bigues pasteres prefabricades pretesades. Tesi Doctoral. Escola Tècnica Superior d'Enginyers de Camins, Canals i Ports. 284 pàgines. jvmartia@upv.es.

Els taulers de bigues prefabricades de formigó pretesat s'utilitzen habitualment en tot el món per a resoldre estructures de viaductes i de passos superiors sobre carreteres. Són escassos els treballs d'investigació encaminats a l'optimització econòmica d'estes estructures, on la majoria s'han centrat en la reducció de les forces de pretesat i han patit d'un signe considerablement teòric, sense millorar l'aprofitament dels recursos que requerixen, la qual cosa ha dificultat la seua aplicació sobre els projectes d'execució. En la busca bibliogràfica, no s'ha trobat cap treball enfocat a l'optimització heurística econòmica d'este tipus d'estructures.

Davant del buit existent en l'espectre de la investigació, l'enfocament d'este treball ha sigut aplicar tècniques d'optimització econòmica a esta tipologia. S'han aplicat tècniques metaheurístiques per a poder definir el problema el més complet possible, on es definix tot el tauler i els seus armats, i es realitzen les comprovacions de marca la normativa espanyola.

S'han analitzat diferents projectes amb esta tipologia, on s'han estudiat dissenys de bigues pastera de diferents fabricants, triant una d'elles com a model per a realitzar el present estudi, i deixant l'opció per a adaptar la tipologia a qualsevol dels dissenys que es fabriquen. A continuació s'ha desenrotllat un programa informàtic en llenguatge FORTRAN que inclou diferents mòduls: generació de l'estructura, comprovació estructural i avaluació econòmica. Addicionalment, hi ha un mòdul que genera fitxers CAD del tauler. L'estudi es realitza sobre un tauler de 12 metres d'ample, amb 11 de calçada, llum entre suports de 35 metres, i separació entre bigues de 6 metres.

L'estudi dels mètodes heurístics aplicats sobre el tauler, es realitza per mitjà de la comparació de huit distints tipus d'algoritmes: l'estratègia de bots múltiples aleatoris (RW), tres mètodes de busca local i quatre de busca poblacional. Els mètodes de busca local ho componen el mètode del gradient (DLS), la cristal-lització simulada (SA) i l'acceptació per llindars (TA). Respecte a la busca poblacional, està composta 
per tres variants de l'optimització per mitjà de genètics (GA1, GA2 i GA3) i per un algoritme memétic (MA). El menor cost va ser obtingut pel SA amb un valor de $108,008 €$ sent menor en un $0.6 \%$ respecte al TA, un $7 \%$ respecte al DLS i MA, i un $13 \%$ respecte a les tres variants dels GA. Quant als temps de procés per a les millors calibratges, els valors menors els protagonitzen el DLS amb 8,122 segons, a continuació amb 18,322 segons per al SA, seguits pels tres GA, amb valors que ronden els 21,000 segons, els 36,233segons del TA, i finalment ja molt allunyats estan els 129,441 segons del MA. Els resultats han mostrat al SA com l'heurística més eficaç de totes elles.

Una vegada seleccionada la millor calibratge del SA, s'ha estudiat el nombre de reinicis necessaris per a aconseguir solucions de qualitat amb una probabilitat prou alta, emprant un criteri d'estabilització de mitges i desviacions típiques. Amb això, s'ha dissenyat un algoritme d'optimització, que combina nou reinicis.

S'ha realitzat un estudi sobre la sensibilitat del model a la variació del preu dels materials. S'han pres increments percentuals en preu de les armadures actives i passives del 5, 10, 15 i 20\%, fent el mateix amb el formigó. Els resultats mostren una sensibilitat més acusada amb el preu de l'acer que amb el del formigó, i que el disseny del tauler s'adapta per a reduir el seu cost, augmentant el volum del formigó i reduint el pes de l'acer quan s'incrementa el preu d'este últim, i el contrari quan l'increment del preu és del formigó.

Per a l'estudi paramètric del tauler s'han pres llums de 20, 25, 30, 35 i 40 metres. S'ha analitzat la geometria transversal, l'armadura activa, la quantia d'acer passiu, la quantia de formigó i els tipus de formigó en biga i llosa. S'ha mostrat una correlació sensiblement lineal del cost, del cant de la biga i del nombre de torons respecte a la llum del tauler. Els resultats obtinguts mostren seccions de grossàries xicotetes i cantells alts, permetent estalvis en el volum de formigó i incrementant la seua inèrcia, la qual cosa repercutix en una menor necessitat pretesat, $i$ una millor qualitat del formigó, entre 40 i $50 \mathrm{Mpa}$, en biga.

Simultàniament s'ha estés l'estudi paramètric per a un formigó reforçat amb fibres d'acer (HPF) en la biga, comparant els resultats amb els obtinguts anteriorment. S'ha analitzat l'efecte del reforç de les fibres que produïx sobre el disseny del tauler davant de sol-licitacións longitudinals i transversals, la variació en el nombre de torons, en el volum de formigó, en la quantia de l'armadura transversal. També la quantia de fibres més adequada, etc. S'ha comprovat la seua competitivitat econòmica respecte al formigó convencional. 


\section{Indice general}

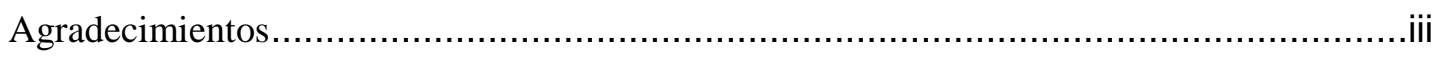

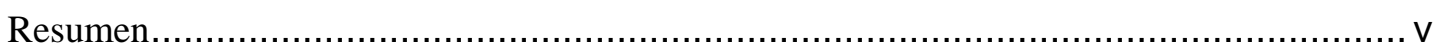

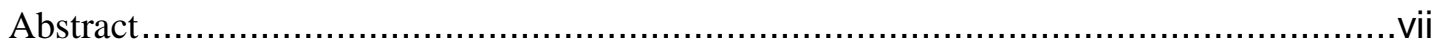

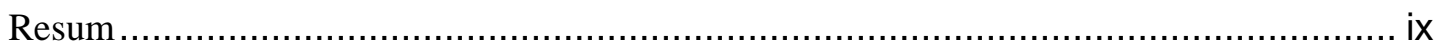

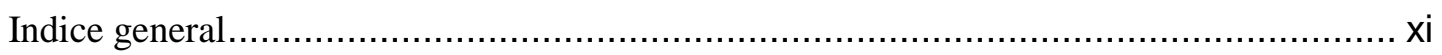

Lista de figuras .................................................................................

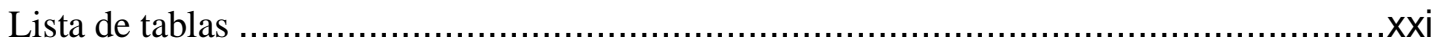

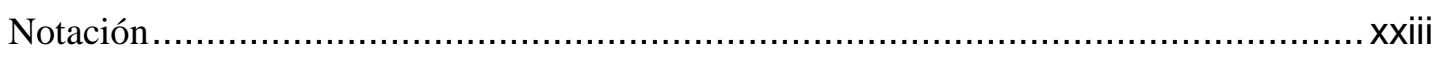

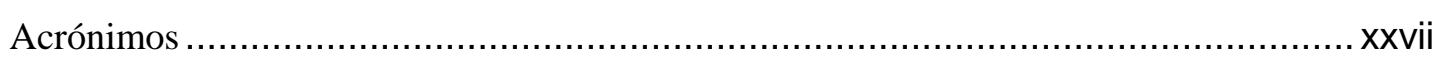

CAPÍTULO 1. INTRODUCCIÓN …........................................................... 1

1.1 Optimización de estructuras por métodos computacionales ............................. 1

1.2 Los tableros de pasos superiores de vigas artesa ............................................ 3

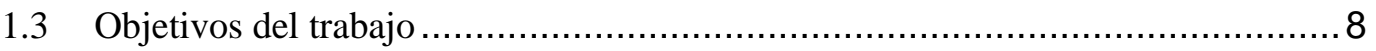

$1.4 \quad$ Naturaleza del problema de optimización .................................................... 9

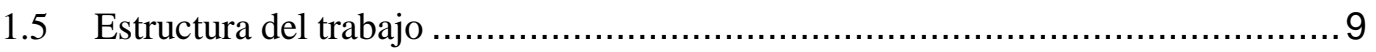

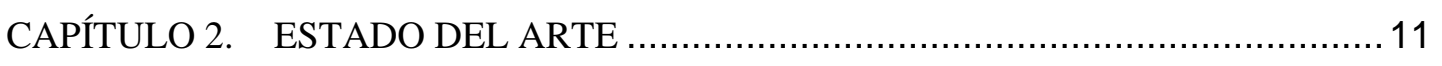

$2.1 \quad$ Antecedentes de la optimización de estructuras ...........................................11

2.1.1 Estructuras de hormigón armado ............................................. 12

2.1.2 Estructuras de hormigón pretensado....................................... 14

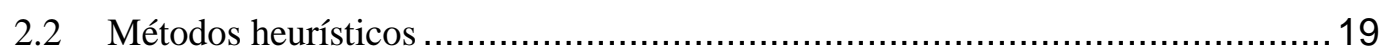

2.2.1 Estructuras de acero ........................................................... 19

2.2.2 Estructuras de hormigón ....................................................... 22

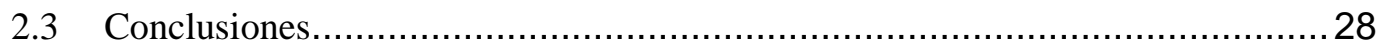

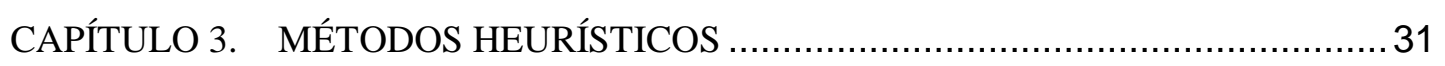

3.1 La optimización mediante técnicas heurísticas.............................................. 31 
3.1.1 El problema de la optimización condicionada ….......................... 31

3.1.2 Clasificación de las técnicas heurísticas ....................................... 33

3.2 Métodos de búsqueda secuencial por entornos .......................................... 34

3.2.1 Búsqueda por gradiente....................................................... 35

3.2.2 Cristalización simulada ...................................................... 36

3.2.3 Aceptación por umbrales................................................... 37

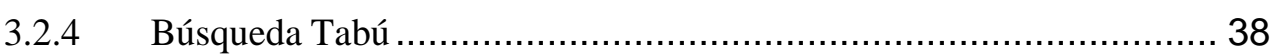

3.2.5 Algoritmo del diluvio universal............................................... 38

3.2.6 Aceptación por cercanía al líder .............................................. 38

3.2.7 Procedimientos GRASP ........................................................ 38

3.2.8 Búsqueda local iterada ....................................................... 38

3.2.9 Búsqueda en entornos variables ............................................... 39

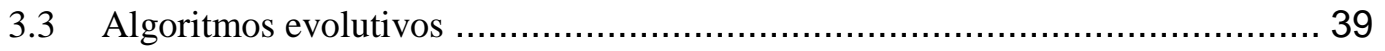

3.3.1 Colonia de Hormigas ........................................................... 39

3.3.2 Algoritmos genéticos..................................................... 39

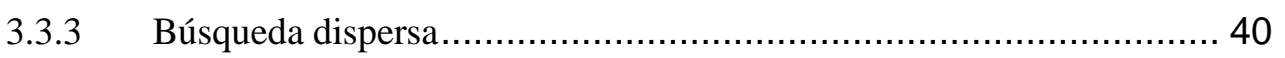

3.3.4 Algoritmos meméticos.......................................................... 40

3.3.5 Reencadenamiento de trayectorias......................................... 41

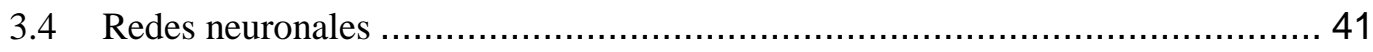

CAPÍTULO 4. DEFINICIÓN DEL PROBLEMA DE OPTIMIZACIÓN ........................ 43

4.1 Definición del problema..................................................................... 43

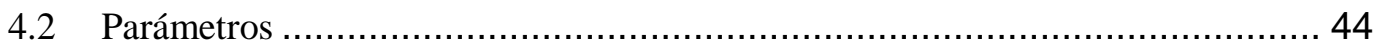

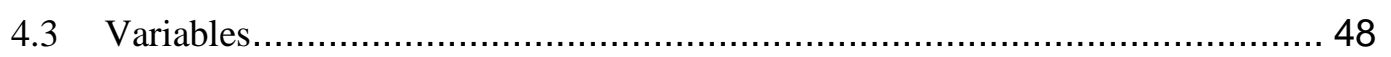

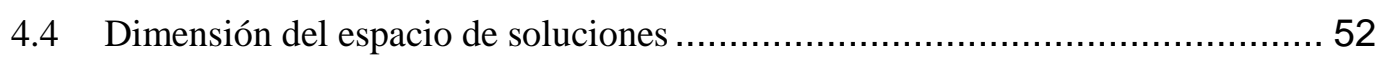

4.5 Función objetivo. Coste ................................................................... 53

4.5.1 Coste de la viga ............................................................... 53

4.5.2 Coste del transporte de la viga ............................................. 55

4.5.3 Coste de la colocación de la viga..................................................56

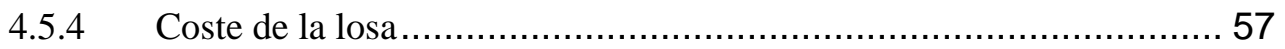

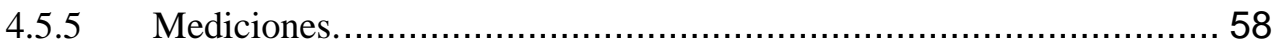

CAPÍTULO 5. COMPROBACIÓN DEL TABLERO ......................................... 59

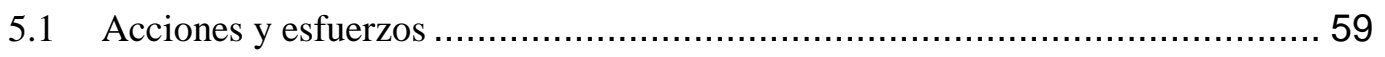

5.2 Modelización de la estructura .......................................................... 61

5.3 Envolventes de los esfuerzos ....................................................... 63

5.3.1 Obtención envolventes E.L.U. ............................................. 63

5.3.2 Obtención envolventes E.L.S................................................... 64 


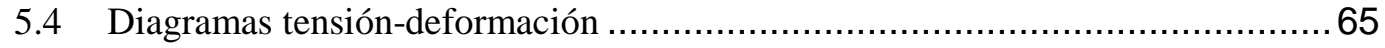

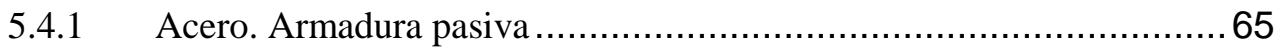

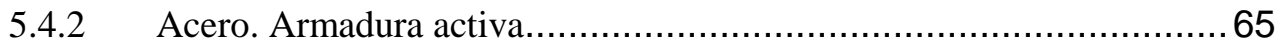

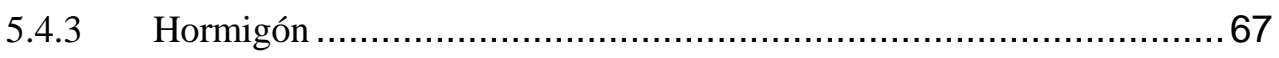

5.5 Comprobación de las secciones de hormigón .............................................. 68

5.5.1 Análisis estructural del pretensado. Armaduras pretesas ...................69

5.5.2 E.L.U. frente a solicitaciones normales......................................... 69

5.5.3 E.L.U. frente a cortante ......................................................... 72

5.5.4 E.L.U. frente a torsión....................................................... 72

5.5.5 E.L.U. disposición armaduras de cortante .................................... 73

5.5.6 E.L.U. torsión combinada con cortante....................................... 73

5.5.6 E.L.U. disposición armaduras de flexión ........................................ 73

5.5.7 E.L.U. rasantes entre ala y alma ............................................... 73

5.5.8 E.L.U. flexión transversal de la losa ............................................ 73

5.5.9 E.L.U. flexión transversal alma y ala inferior de la viga...................76

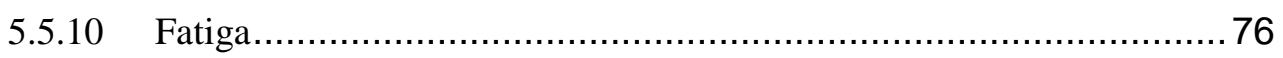

5.5.11 E.L.S. comprobación de flechas instantáneas en el tablero ..................76

5.5 .12 E.L.S. viga en planta ....................................................... 76

5.5.13 E.L.S. viga con el hormigonado de la losa ................................. 77

5.5.14 E.L.S. tablero: compresiones en el hormigón ................................ 77

5.5.15 E.L.S. tablero: aparición de fisuras ............................................ 77

5.5.16 E.L.S. comprobación de las flechas diferidas en el tablero .................77

CAPÍTULO 6. APLICACIÓN DE LOS MÉTODOS HEURÍSTICOS ...........................79

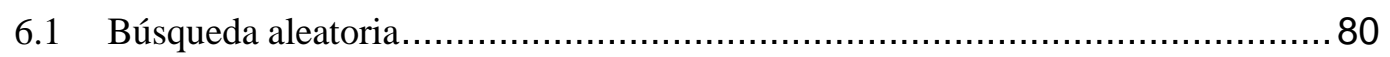

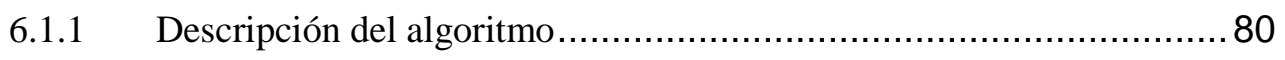

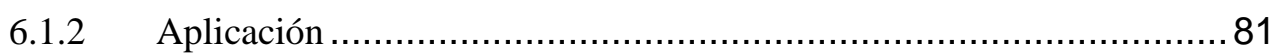

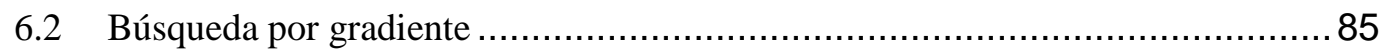

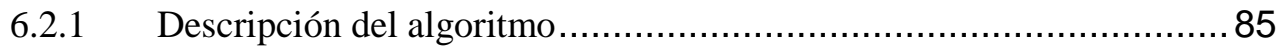

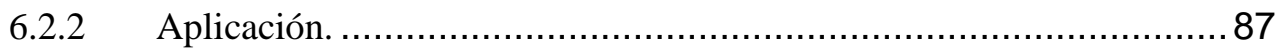

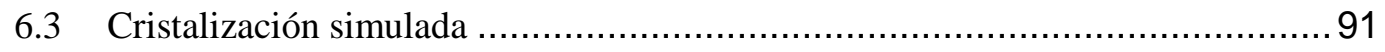

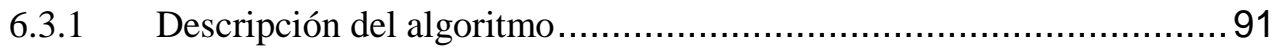

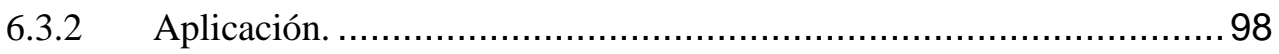

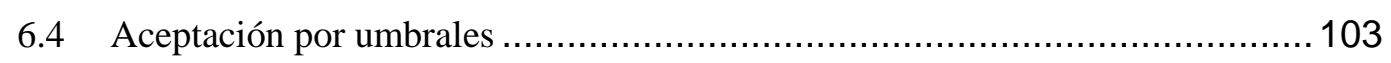

6.4.1 Descripción del algoritmo................................................. 103

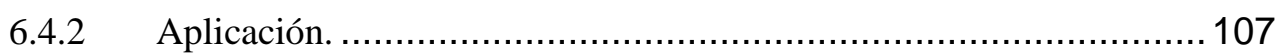

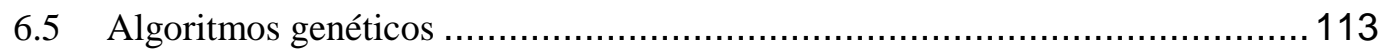


6.5.1 Descripción del algoritmo .................................................. 113

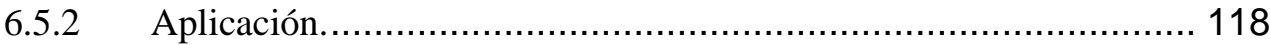

6.6 Algoritmos meméticos ................................................................. 129

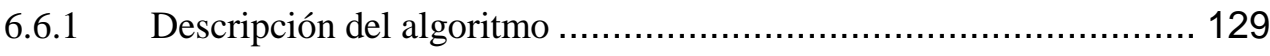

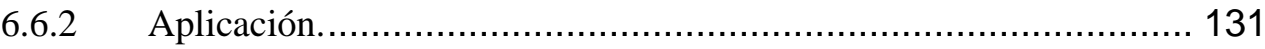

6.7 Resumen de los costes y comparación de las soluciones............................ 134

6.7.1 Resumen de los costes ...................................................... 134

6.7.2 Comparación de las soluciones ............................................... 135

6.8 Número de ejecuciones necesarias................................................... 138

6.9 Variación de precios unitarios ........................................................... 141

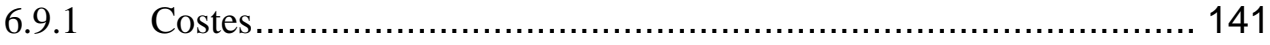

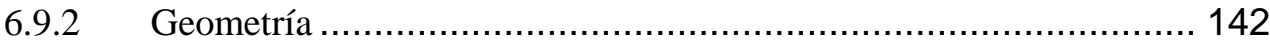

6.9.3 Cuantías de acero. ............................................................ 143

6.9.4 Cuantías de hormigón. ......................................................... 144

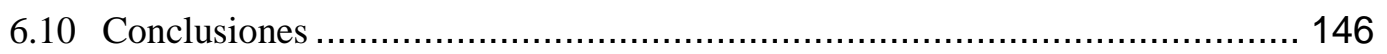

CAPÍTULO 7. ESTUDIO PARAMÉTRICO DE TABLEROS................................. 147

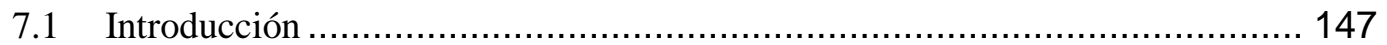

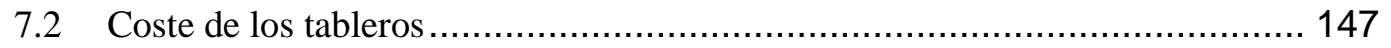

7.3 Resultados geométricos................................................................ 149

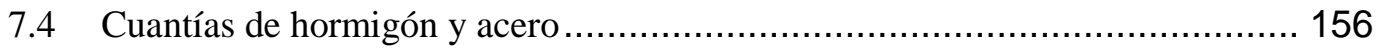

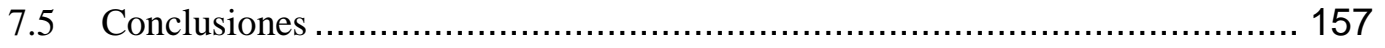

CAPÍTULO 8. APLICACIÓN A VIGAS REFORZADAS CON FIBRAS DE ACERO 159

8.1 Condicionantes sobre el modelo........................................................ 160

8.1.1 Variables........................................................................... 160

8.1.2 Coste de la viga ….......................................................... 160

8.1.3 Características mecánicas del hormigón con fibras HPF.................. 160

8.1.4 Comprobación de las secciones del tablero.................................. 162

8.2 Comparación tableros con vigas de HP vs HPF........................................ 163

8.2.1 Coste de los tableros......................................................... 163

8.2.2 Resultados geométricos ................................................... 165

8.2.3 Cuantías de hormigón y acero ................................................ 172

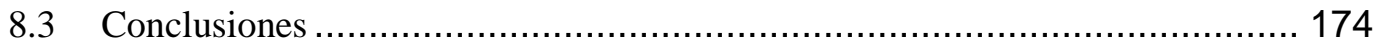

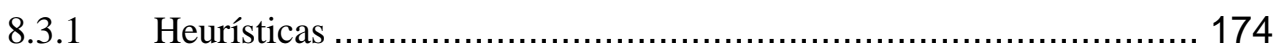

8.3.2 Hormigones con fibras..................................................... 174

CAPÍTULO 9. CONCLUSIONES Y FUTURAS LÍNEAS DE INVESTIGACIÓN ...... 175

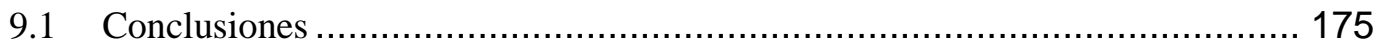


9.1.1 Estado del arte............................................................... 175

9.1.2 Optimización heurística....................................................... 176

9.1.3 Tableros con vigas artesa .................................................... 177

9.1.4 Estudio paramétrico ........................................................ 178

9.1.5 Estudio comparativo HP vs HPF ………............................... 178

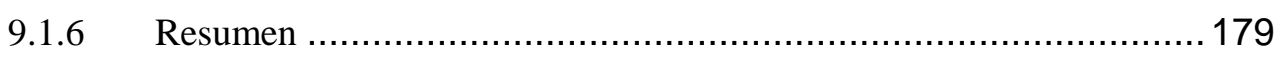

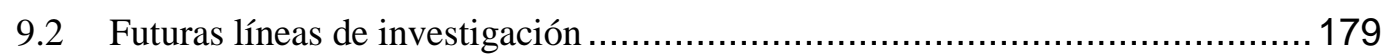

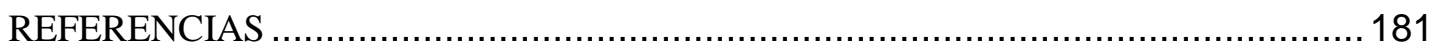

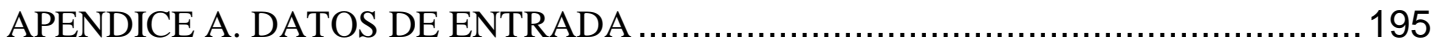

APENDICE B. RESULTADO VARIACIÓN DE PRECIOS ..................................... 199

APENDICE C. RESULTADOS DE LOS EJEMPLOS NUMÉRICOS .........................207 


\section{Lista de figuras}

Figura 1-1: Método tradicional de diseño y análisis estructural ....................................

Figura 1-2: Método de diseño empleando métodos heurísticos ..................................... 3

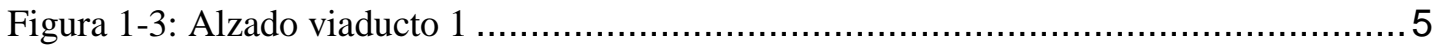

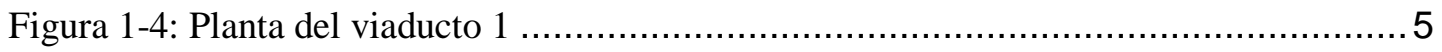

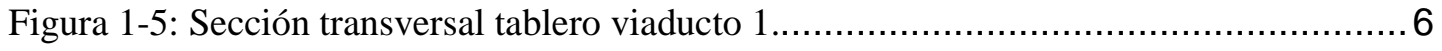

Figura 1-6: Viga artesa vano central. Modelo 180AE-345 …...................................... 6

Figura 1-7: Vigas artesa con losa. Incluye la armadura activa .................................... 7

Figura 3-1: Clasificación de las técnicas de optimización heurísticas............................. 33

Figura 3-2: Búsqueda por gradiente. Óptimos locales ............................................ 35

Figura 3-3: Búsqueda por aceptación por umbrales ................................................... 37

Figura 3-4: Red neuronal artificial con $n$ neuronas de entrada, $m$ en su capa oculta y una de

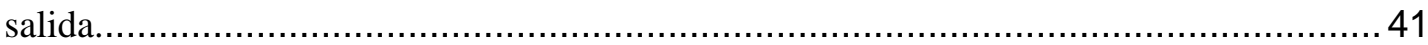

Figura 4-1: Parámetros tablero vigas artesa pretesas................................................. 47

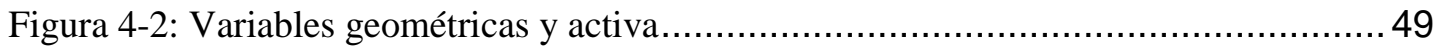

Figura 4-3: Disposición longitudinal de la armadura activa …....................................5 50

Figura 4-4: Variables armadura pasiva ……..................................................... 51

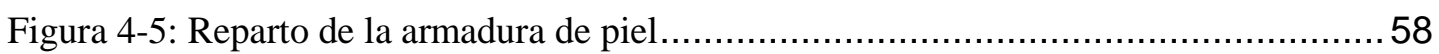

Figura 5-1: Esquema de las posiciones de la sobrecarga uniforme en distintos estados de

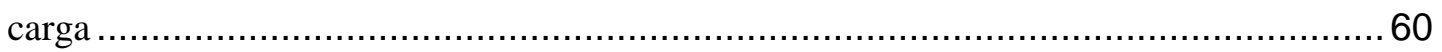

Figura 5-2: Esquema de posiciones del carro en distintos estados de carga .....................61

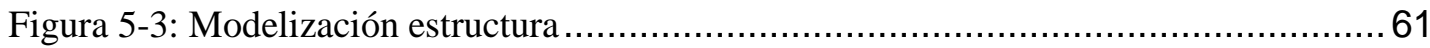

Figura 5-4: Ecuación de rigidez para un elemento longitudinal i,j ................................ 62

Figura 5-5: Ecuación de rigidez para un elemento transversal m,n .............................. 62

Figura 5-6: Diagrama tensión-deformación de cálculo armaduras pasivas. Fig 38.4 EHE-08 
Figura 5-7: Diagrama tensión-deformación característico armaduras activas. Fig 38.5 EHE08 . 66

Figura 5-8: Diagrama tensión-deformación de cálculo armaduras activas. Fig 38.7.b EHE-08 66

Figura 5-9: Diagrama tensión-deformación del hormigón en ELU. Fig 39.5.a EHE-08 ..... 67

Figura 5-10: Deformación total en una sección. Fig 42.1.2 EHE-08 ............................. 70

Figura 5-11: Dominios de deformación en una sección. Fig 42.1.3 EHE ......................... 70

Figura 5-12: Integración de secciones en ELU ....................................................... 71

Figura 5-13: Flexión en voladizo transversal I......................................................... 74

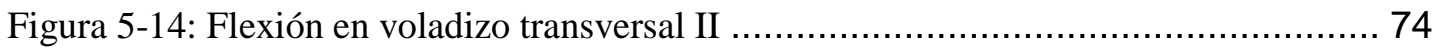

Figura 5-15: Flexión en voladizo transversal III .................................................. 74

Figura 5-16: Equilibrio de fuerzas y momentos. Figuras 39.5.b EHE-08......................... 75

Figura 5-17: Flexión transversal en losa y viga....................................................... 75

Figura 6-1: Coste de las soluciones factibles encontradas durante un RW de 25,000

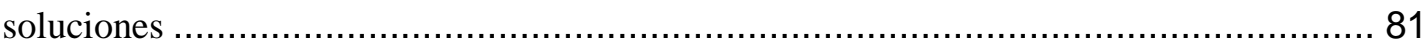

Figura 6-2: Evolución disminución del coste record durante un RW de 25000 soluciones . 82

Figura 6-3: Histograma de frecuencias de las soluciones factibles encontradas................ 83

Figura 6-4: Tablero con menor coste en un RW ................................................... 84

Figura 6-5: Diagrama de flujo de un DLS ............................................................. 86

Figura 6-6: Evolución del coste para diferentes movimientos y criterios de parada ........... 87

Figura 6-7: Evolución del coste medio para diferentes movimientos y tiempos de ejecución 89

Figura 6-8: Tablero con menor coste en un DLS .................................................. 90

Figura 6-9: Diagrama de flujo de un simulated annealing ........................................ 92

Figura 6-10: Proceso de evolución del coste y disminución de la temperatura relativo al tiempo de proceso 94

Figura 6-11: Proceso de disminución del coste relativo al número de soluciones aceptadas 94

Figura 6-12: Evolución del número de movimientos aceptados respecto al tiempo de proceso

Figura 6-13: Proceso de evolución de un annealing 96

Figura 6-14: Resultados de los valores medios de las 16 combinaciones de los parámetros del

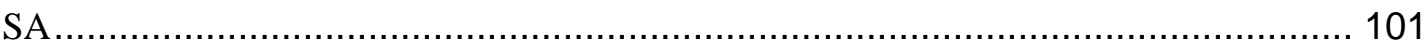

Figura 6-15: Tablero con menor coste en un SA .................................................... 102

Figura 6-16: Diagrama de flujo de un threshold accepting ........................................ 104

Figura 6-17: Proceso de evolución del coste y disminución del umbral relativo al tiempo de proceso 106 
Figura 6-18: Proceso de disminución del coste relativo al número de soluciones aceptadas 106

Figura 6-19: Proceso de evolución de un threshold. 107

Figura 6-20: Resultados de los valores medios de las 24 combinaciones de los parámetros 110

Figura 6-21: Comparativa resultados de los valores medios TA vs SA con las 24 combinaciones de los parámetros. 111

Figura 6-22: Tablero con menor coste en un TA ................................................... 112

Figura 6-23: Diagrama de flujo de GA ............................................................... 114

Figura 6-24: Diagrama terminología y ejemplo de representación de soluciones en GA... 115

Figura 6-25: Ruleta empleada en la selección de individuos ..................................... 117

Figura 6-26: Cruzamiento por un punto ….................................................... 117

Figura 6-27: Evolución de los costes medios y mínimos en un GA. (Individuos=500

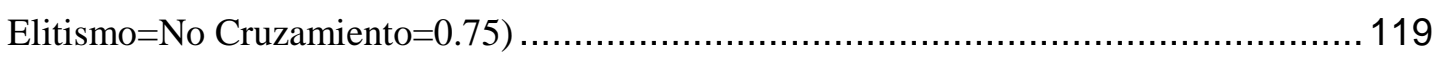

Figura 6-28: Evolución de los costes medios y mínimos en un GA. (Individuos=500

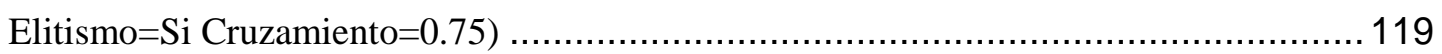

Figura 6-29: Evolución de los costes medios en el tiempo en un GA con elitismo............ 123

Figura 6-30: \% Reducción coste medio (mutación / no mutación) ................................... 126

Figura 6-31: Evolución de los costes medios y mínimos en un GA con mutación.............. 127

Figura 6-32: Tablero con menor coste en un GA.............................................. 128

Figura 6-33: Diagrama de flujo de MA ......................................................... 131

Figura 6-34: Evolución de los costes en un MA según № Cadenas Markov .................... 132

Figura 6-35: Tablero con menor coste en un MA ................................................. 133

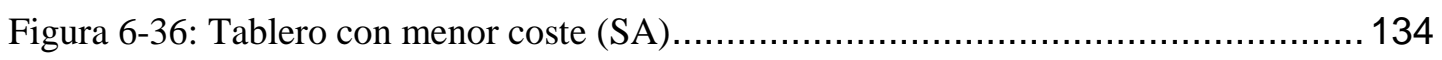

Figura 6-37: Evolución de las medias y desviaciones estándar con el número de ejecuciones 140

Figura 6-38: Variación costes medios respecto al incremento de precios acero/ hormigón 142 Figura 6-39: Cuantías acero en $\mathrm{Kg} / \mathrm{m}^{2}$ tablero sobre incremento precios acero/ hormigón 144 Figura 6-40: Cuantías hormigón en $\mathrm{m}^{3} / \mathrm{m}^{2}$ tablero sobre incremento precios acero/ hormigón 145

Figura 7-1: Coste medio y mínimo de los tableros en función de la luz......................... 148

Figura 7-2: Función del coste medio de los tableros en función de la luz........................ 148

Figura 7-3: Canto de la viga en función de la luz.................................................. 151

Figura 7-4: Espesor de la losa en función de la luz............................................... 151

Figura 7-5: Número de torones medio en función de la luz........................................ 152

Figura 7-6: Producto del canto de la viga x el número de torones en función de la luz. .... 152 
Figura 7-7: Hormigones medios en viga en función de la luz. ................................... 153

Figura 7-8: Hormigones medios en losa en función de la luz...................................... 154

Figura 7-9: Ancho medio del ala inferior en función de la luz. ................................... 155

Figura 7-10: Espesor medio del ala inferior en función de la luz................................ 155

Figura 7-11: Cuantía de hormigón por $\mathrm{m}^{2}$ de tablero.............................................. 156

Figura 7-12: Cuantía de acero pasivo por $\mathrm{m}^{2}$ de tablero............................................ 157

Figura 8-1: Diagrama tensión-deformación en tracción de cálculo del hormigón con fibras. Se corresponde con la Figura A.14.2 de la EHE-08.................................................. 162

Figura 8-2: Coste medio de los tableros en función de la luz..................................... 164

Figura 8-3: Coste mínimo de los tableros en función de la luz.................................... 164

Figura 8-4: Canto de la viga en función de la luz................................................. 167

Figura 8-5: Espesor de la losa en función de la luz................................................ 167

Figura 8-6: Número de torones medio en función de la luz. ....................................... 168

Figura 8-7: Hormigones medios en viga en función de la luz. ..................................... 169

Figura 8-8: Hormigones medios en losa en función de la luz........................................ 169

Figura 8-9: Resistencias residuales medias a tracción en función de la luz..................... 170

Figura 8-10: Ancho medio del ala inferior en función de la luz................................... 171

Figura 8-11: Espesor medio del ala inferior en función de la luz............................... 171

Figura 8-12: Cuantía de hormigón por $\mathrm{m}^{2}$ de tablero............................................ 172

Figura 8-13: Cuantía de acero pasivo por $\mathrm{m}^{2}$ de tablero.......................................... 173

Figura 0-1: Canto medio de la viga respecto al incremento de precios acero/ hormigón... 203

Figura 0-2: Espesor medio de la losa respecto al incremento de precios acero/ hormigón 203

Figura 0-3: $\mathrm{N}^{\circ}$ torones medio respecto al incremento de precios acero/ hormigón........... 203

Figura 0-4: Resistencia característica media del hormigón de la viga respecto al incremento

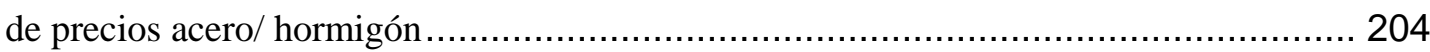

Figura 0-5: Resistencia característica media del hormigón de la losa respecto al incremento de precios acero/ hormigón 204

Figura 0-6: Ancho medio del ala inferior respecto al incremento de precios acero/ hormigón 204

Figura 0-7: Espesor medio del ala inferior respecto al incremento de precios acero/ hormigón 205

Figura 0-8: Ancho medio del ala superior respecto al incremento de precios acero/ hormigón 205

Figura 0-9: Espesor medio del ala superior respecto al incremento de precios acero/ hormigón. 205 


\section{Lista de tablas}

Tabla 4-1: Parámetros $\quad 45$

Tabla 4-2: Precios unitarios viga 53

Tabla 4-3: Coeficientes correctores de la armadura pasiva en viga. Materia prima 54

Tabla 4-4: Coeficientes correctores de la armadura pasiva en viga. Mano de obra 54

Tabla 4-5: Precios de transporte de la viga 55

Tabla 4-6: Precios de colocación de la viga 56

Tabla 4-7: Precios unitarios losa $\quad 57$

Tabla 4-8: Coeficientes correctores de la armadura pasiva en losa. Materia prima $\quad 57$

Tabla 4-9: Coeficientes correctores de la armadura pasiva en losa. Mano de obra 57

Tabla 6-1: Coste medio y tiempos para 5, 10 y $15 \%$ variables. 88

Tabla 6-2: Coste medio y tiempos para 20, 25 y $30 \%$ variables. 88

Tabla 6-3: Desviaciones (\%) del coste medio respecto al mínimo 89

Tabla 6-4: Resultados escalados. Coef enfriamiento: 0.95, Cadena Markov: $5000 \quad 97$

Tabla 6-5: Resultados con o sin reparación. Coef enfriamiento: 0.95, Cadena Markov: 1000

Tabla 6-6: Resultados del SA con las 16 combinaciones con los parámetros seleccionados 99

Tabla 6-7: Resultados medios obtenidos en SA 99

Tabla 6-8: Resultados de las 144 ejecuciones con los parámetros seleccionados en TA 108

Tabla 6-9: Resultados medios obtenidos en TA 108

Tabla 6-10: Resultados sin elitismo en GA. 100 individuos 120

Tabla 6-11: Resultados sin elitismo en GA. 250 individuos 121

Tabla 6-12: Resultados sin elitismo en GA. 500 individuos 122

Tabla 6-13: Resultados con elitismo en GA. 100 individuos 123

Tabla 6-14: Resultados con elitismo en GA. 250 individuos 124

Tabla 6-15: Resultados con elitismo en GA. 500 individuos 125

Tabla 6-16: Aplicación de "mutación” en GA 126 
Tabla 6-17: Resultados en un MA. (Indiv=500, Elit=Si, Cruz=0.5, Gen=200, Mut=4) 132

Tabla 6-18: Tabla resumen de las heurísticas estudiadas

Tabla 6-19: Resultados: Geometría, Hormigones y Activas

Tabla 6-20: Resultados: Armadura pasiva

Tabla 6-21: Resultados: Cuantías de acero

Tabla 6-22: Resultados obtenidos con 40 reinicios

Tabla 6-23: Resultados obtenidos con la variación de precios $(€)$

Tabla 6-24: Cuantías del acero en $\mathrm{Kg} / \mathrm{m}^{2}$ tablero, obtenidas con la variación de precios

Tabla 6-25: Cuantías del hormigón en $\mathrm{m}^{3} / \mathrm{m}^{2}$ tablero, obtenidas con la variación de precios

Tabla 7-1: Costes obtenidos para distintas luces.

Tabla 7-2: Resultados geométricos hormigón sin fibras HP

Tabla 7-3: Limitación cantos máximos de la viga en función de la luz

Tabla 7-4: Resultados geométricos: Canto viga, losa y $n^{\circ}$ torones

150

Tabla 7-5: Resultados hormigones: Viga y losa 153

Tabla 7-6: Resultados geométricos ala inferior: Ancho y canto

Tabla 7-7: Cuantías de hormigón y acero pasivo.

Tabla 8-1: Precios unitarios viga

Tabla 8-2: Resultados obtenidos para hormigón pretensado sin o con fibras.

Tabla 8-3: Resultados geométricos hormigón con fibras HPF

Tabla 8-4: Resultados geométricos: Canto viga, losa y n ${ }^{\circ}$ torones

Tabla 8-5: Resultados hormigones: Viga, losa, fibras

Tabla 8-6: Resultados geométricos ala inferior: Ancho y canto

Tabla 8-7: Cuantías de hormigón y acero pasivo. 


\section{Notación}

Caracteres romanos en minúsculas

a_bar ......................ancho barreras tablero

b_total ...................ancho fondo tablero

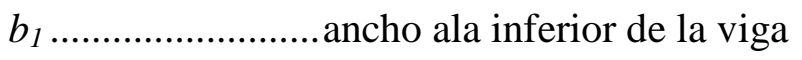

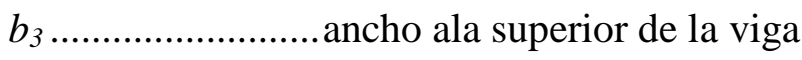

$c_{i}$........................costes unitarios

cm .........................carga muerta

d_transporte ........distancia transporte (ida)

e_pav .....................espesor del pavimento

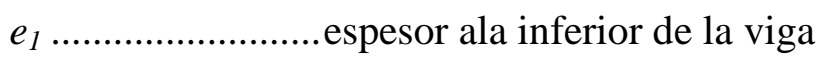

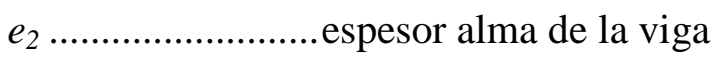

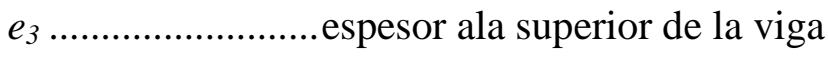

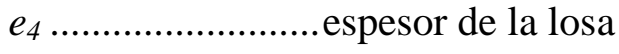

$f$..........................función objetivo

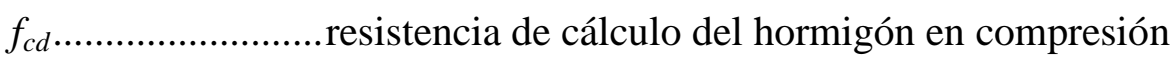

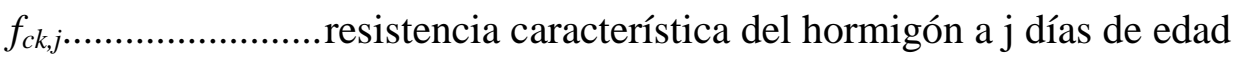

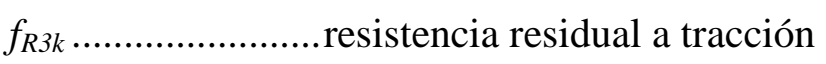

f_mon ...................factor dificultad montaje viga en obra

$g$........................número de generaciones

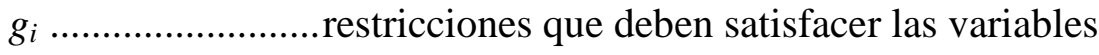

$h_{1}$.........................canto de la viga

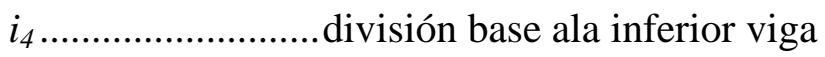

$k_{b}$.........................constante de Boltzmann

k...........................coeficiente de enfriamiento, o de reducción del umbral

$m_{i}$........................mediciones 


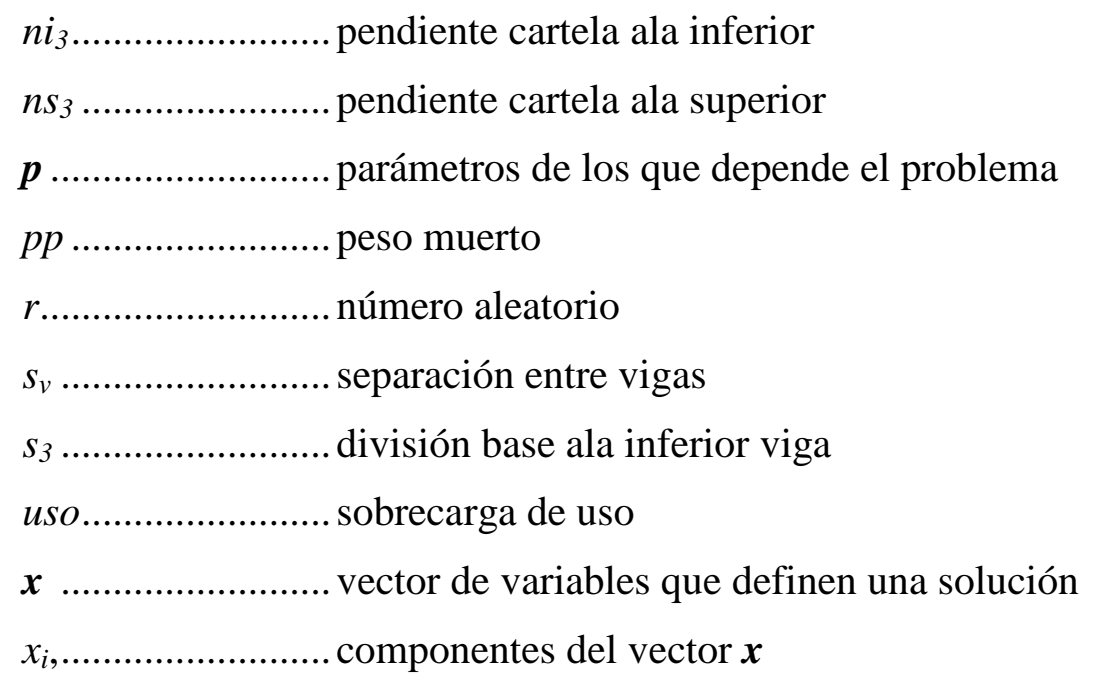

Caracteres romanos en mayúsculas

Aex ..................... Ambiente de exposición externo

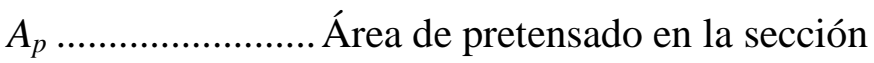

$A_{s 6} \ldots \ldots \ldots \ldots \ldots \ldots . . . . . . . . .$. Armadura longitudinal superior losa

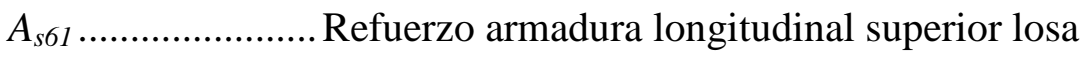

$A_{s 7}$...................... Armadura longitudinal inferior losa

$A_{s}$.......................... Área de acero traccionado en la sección

$A^{\prime}{ }_{s}$........................ Área de acero comprimido en la sección

$C_{e}$......................... coste escalado

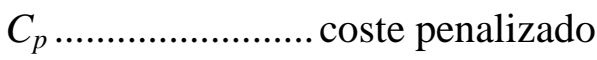

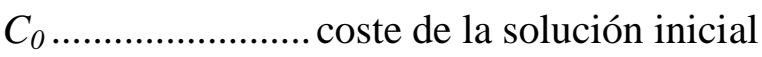

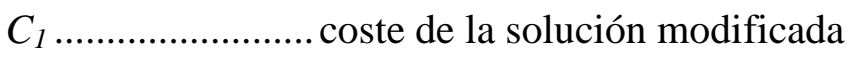

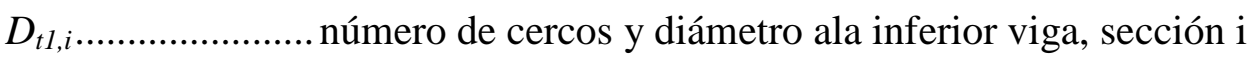

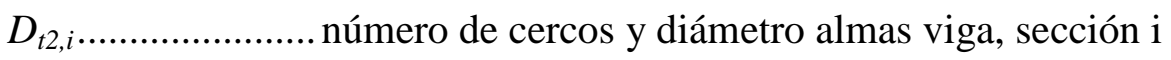

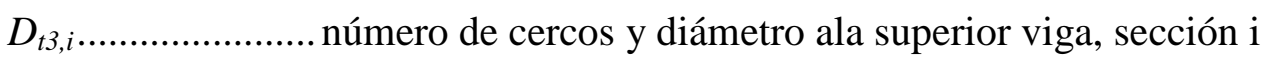

$D_{t 4} \ldots \ldots \ldots \ldots \ldots \ldots . . . . . . . . . . .$. diámetro armadura transversal losa

E........................ energía asociada a un estado

Ent .......................entrega de la viga

Esb ...................... esbeltez mínima de la viga

HA ........................ hormigón armado

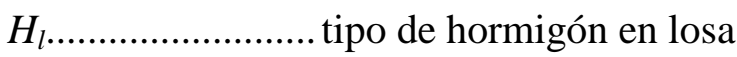

HP ....................... hormigón pretensado

$H P F$..................... hormigón pretensado reforzado con fibras de acero 
$H_{v} \ldots \ldots \ldots \ldots \ldots \ldots \ldots . . . . . . .$. tipo de hormigón en viga

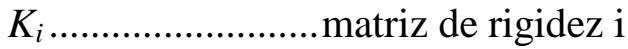

L ...........................luz

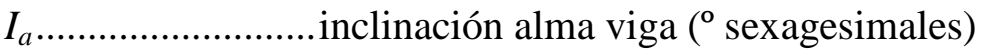

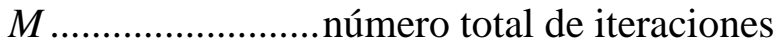

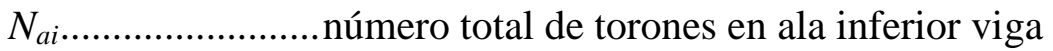

$N_{a s}$.......................número total de torones en ala superior viga

$P$.........................probabilidad

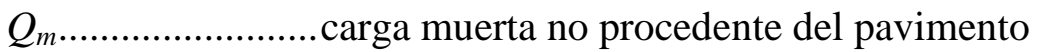

$S$.......................espacio de valores posibles que pueden tomar las variables

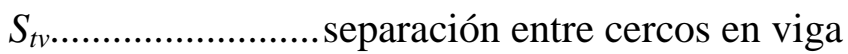

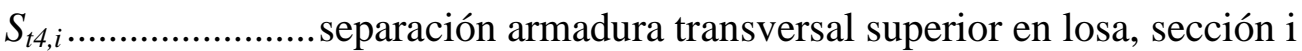

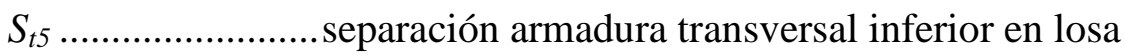

$T$...........................temperatura

U..........................umbral

$V_{2} \ldots \ldots \ldots \ldots \ldots \ldots \ldots \ldots \ldots$ número de tramos con fundas nivel 2

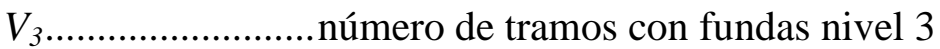

\section{$\underline{\text { Caracteres griegos }}$}

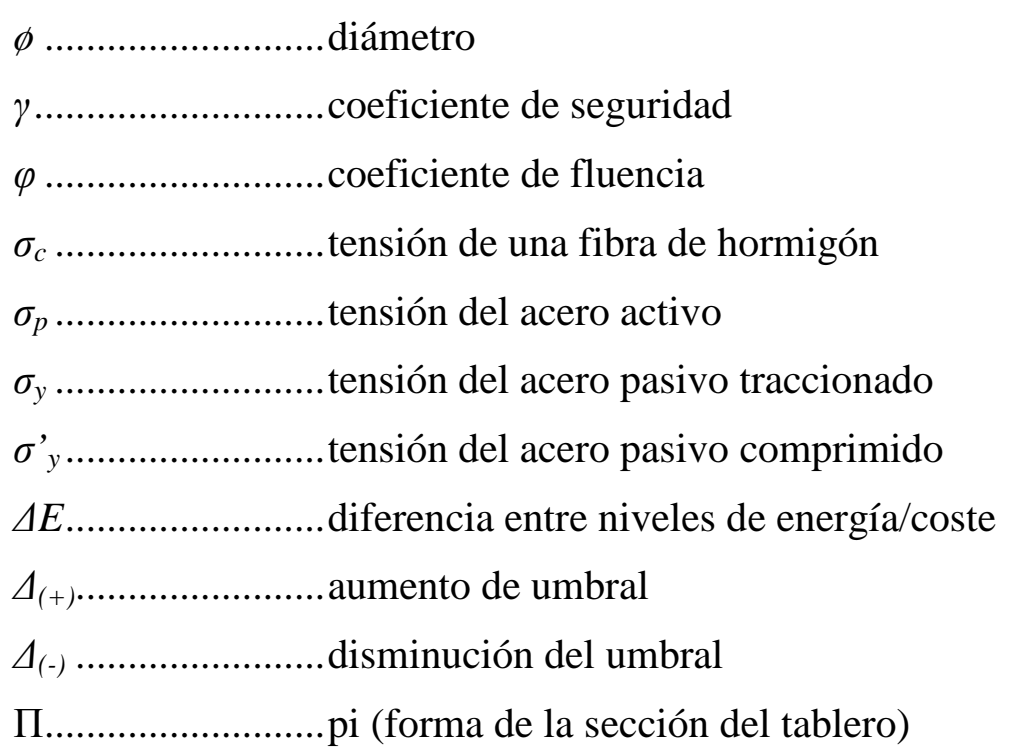




\section{Acrónimos}

ACI American Concrete Institute

$\mathrm{ACO}$ Ant Colony Optimization

ACS Ant Colony System

ADIF Administrador de Infraestructuras Ferroviarias

AISC American Institute of Steel Construction

BS8110 British Standard

CSA. Canadian Standards Association

DLS . Descent Local Search

EA Evolutionary Algorithm

ELS Estado Límite de Servicio

ELU Estado Límite Último

FB Estrategia First Best

FQD Despliegue de la función de calidad

GA Genetic Algorithm

GB .Gradiente Global Best

GEN Algoritmo Genético

GPRC Grupo de Procedimientos de Construcción GRASP Greedy Randomized Adaptive Search Procedure IA Algoritmo Inmune IABSE....... International Association for Bridge and Structural Engineering KKT Karush-Kuhn-Tucker (criterio de optimalidad)

MA Memetic Algorithm

OBA Old Bachelor Acceptance RAM Random Access Memory RNA Red Neuronal Artificial 
RW

Random Walk

SA

Simulated Annealing

SAMOSA Método de Suppapitnarm para optimiza. multiobj con SA SW Test de hipótesis de Shapiro-Wilk

TA. Threshold Accepting TOPSIS Técnica del soporte para la toma de decisiones TS Tabu Search VNS Variable Neighbourhood Search 


\section{CAPÍTULO 1. INTRODUCCIÓN}

En la práctica tradicional, el dimensionamiento de una estructura se convierte en un proceso iterativo: nace de un predimensionamiento de las dimensiones que la definen y mediante unas comprobaciones y ajustes del proyectista se llega a una solución definitiva. A continuación, se describen en este capítulo los métodos empleados en el diseño de estructuras: el tradicional y el que emplea métodos heurísticos. Además se describe el modelo a estudiar, los objetivos del trabajo y la estructura de la tesis.

\subsection{Optimización de estructuras por métodos computacionales}

El sistema tradicional usado para el diseño de estructuras de ingeniería civil está fundamentado en el conocimiento y experiencia sobre la materia de los proyectistas, que diseñan y predimensionan las distintas partes y secciones de la estructura, para a continuación, mediante la utilización de programas informáticos comprobar la bonanza del diseño inicial, y ajustar éste en los puntos críticos que puedan presentarse.

El diseño estructural tradicional empleado suele ser un proceso iterativo formado por varios pasos tal como se muestran en la Figura 1-1:

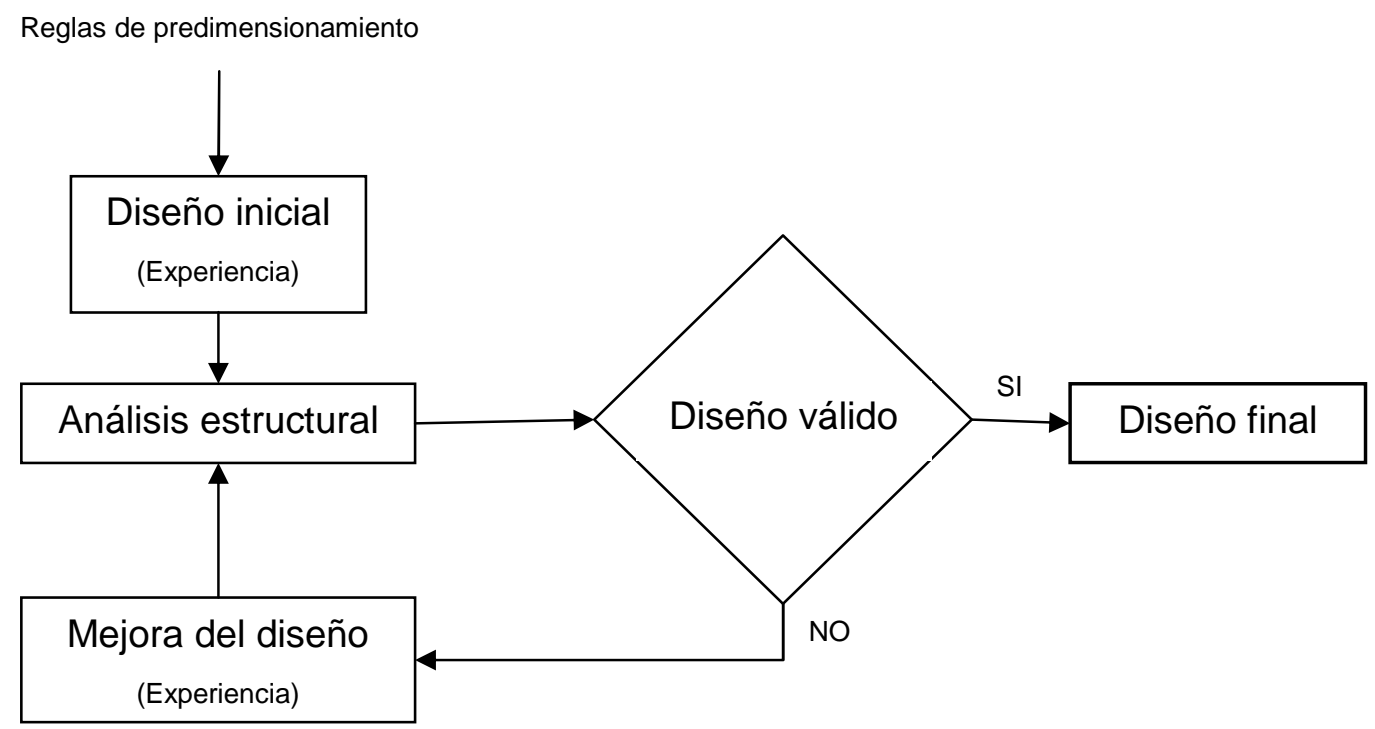

Figura 1-1: Método tradicional de diseño y análisis estructural

Este proceso parte inicialmente de un predimensionamiento en el que el proyectista establece una geometría y unos materiales de resistencia conocida, previo al cálculo 
o análisis estructural. Este diseño va recibiendo correcciones hasta que se obtiene la estructura definitiva. En este proceso iterativo es el proyectista el que aporta su experiencia en la fase de mejora del diseño. Sobre el diseño inicial se van introduciendo ajustes y correcciones, hasta obtener al final la estructura proyectada. En el análisis estructural se aplica la Normativa, condicionando la metodología del análisis, las comprobaciones a realizar y los coeficientes de seguridad mínimos para garantizar la seguridad y la funcionalidad de la obra.

Al ser los proyectistas los que aportan sus conocimientos y experiencias en la mejora del diseño, un proyecto obtendrá distintas soluciones válidas según el autor que lo haya calculado. En la mayoría de las ocasiones la solución más adecuada viene determinada principalmente por aquella que sea la más económica. Sin embargo, el proyectista tradicional no llega nunca a la estructura óptimo-económica.

La optimización de las estructuras proyectadas generalmente no se ha llevado a cabo de forma sistematizada, debido a la complejidad y lentitud que conlleva dicho proceso de prueba y error. Hay que tener en cuenta que cada estructura depende de unas condiciones de contorno, precios de los materiales, de ejecución, etc., función del sitio y de la coyuntura económica. En la práctica, las técnicas de optimización dependerán de la experiencia del proyectista. Para ello desarrollará criterios de predimensionamiento cada vez más ajustados en el proceso iterativo dentro del método tradicional, pero sin llegar a saber lo cerca que se encuentra de la solución de menor coste.

El desarrollo de la potencia de cálculo de los ordenadores personales, la aparición de diferentes técnicas de optimización entre ellas las heurísticas, y la participación de proyectistas experimentados en el desarrollo del software de la aplicación, permiten realizar de manera completamente automatizada el diseño y optimización de la estructura, sin la necesidad de intervención de un calculista experto y además, con un rendimiento superior. En el presente trabajo se desarrolla un método de diseño automatizado, con un análisis funcional que distingue dos procesos diferenciados:

- Un proceso que comprueba los estados límite, según la normativa vigente, de manera automatizada a partir de un diseño aleatorio de la estructura. La optimización requerirá realizar dicho proceso un gran número de veces, cada vez que se modifica la geometría y el resto de variables de diseño.

- Otro proceso, encargado de la optimización, desarrolla las características propias de cada heurística, y contiene dos submódulos:

a) Generador de soluciones, que realiza la variación iterativa del diseño.

b) Valoración de las soluciones, con el propósito de aceptarlas o rechazarlas.

- Adicionalmente, se desarrolla un módulo gráfico que permite obtener ficheros DFX con las secciones de la estructura. Esta representación proporciona una visión del resultado, mejorando el análisis y estudio de la heurística, y también facilita la elaboración de los planos del proyecto. 
Los procesos han sido desarrollados completamente en lenguaje FORTRAN, y las aplicaciones obtenidas han corrido sobre un procesador Intel Core 2 Duo E6850 (3.00GHz, $1333 \mathrm{MHz}, 4 \mathrm{MB}$ cache).

El diseño con técnicas de optimización se muestra en la Figura 1-2.

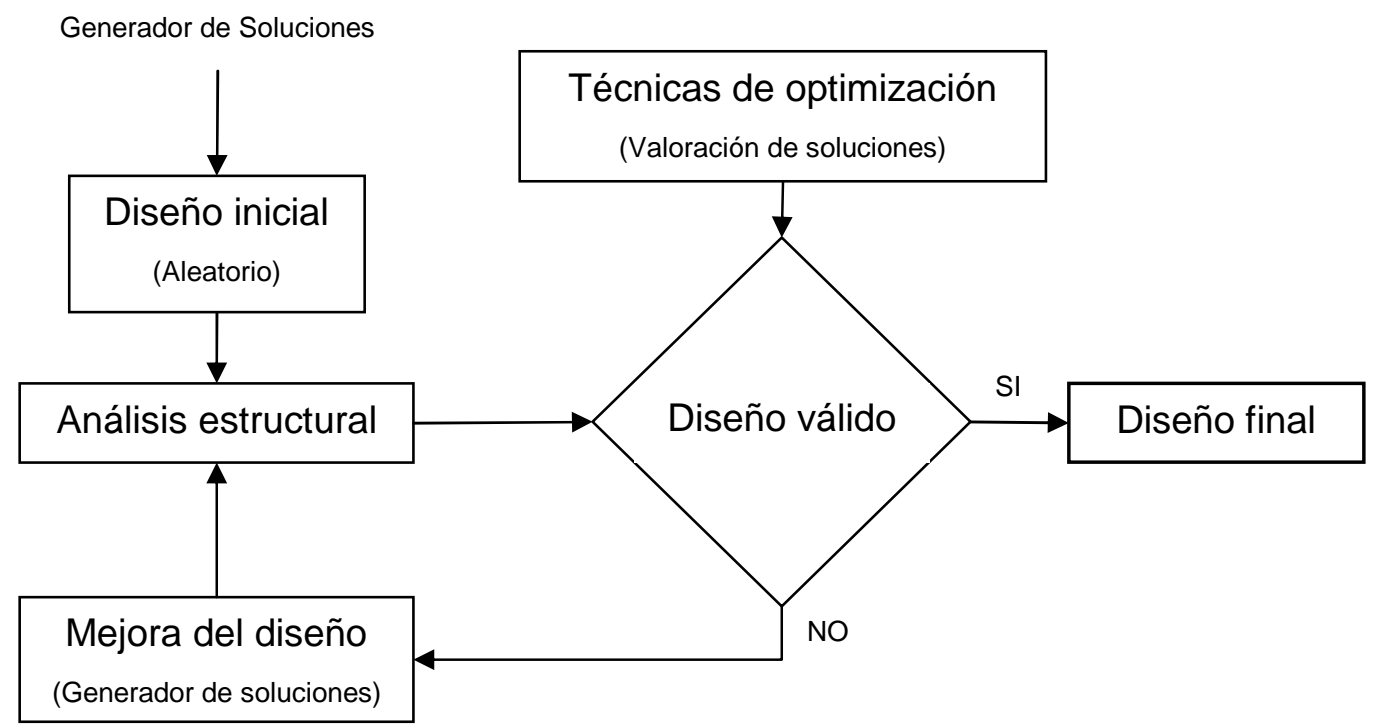

Figura 1-2: Método de diseño empleando métodos heurísticos

El presente trabajo, muestra el proceso seguido en el desarrollo de una herramienta de cálculo capaz de diseñar y optimizar una estructura de manera totalmente automatizada. No son necesarias reglas de predimensionamiento, ni una gran experiencia previa para obtener diseños económicos en un tiempo de cálculo razonable. El objetivo será encontrar la estructura más económica posible, cumpliendo la normativa, a partir de un diseño generado de modo aleatorio con algunos ajustes. Este método se convierte en una herramienta eficaz para adaptar el proceso productivo en una planta de prefabricados considerando las fluctuaciones de precios que se producen en el mercado de las materias primas, y costes de producción. El sistema permite obtener distintas soluciones optimizadas con escasa diferencia en su coste, permitiendo al ingeniero decidir el diseño más adecuado de la estructura para ser proyectada.

\subsection{Los tableros de pasos superiores de vigas artesa}

El concepto de hormigón pretensado es muy antiguo, pero no se pudo materializar en las obras de ingeniería civil mientras no se desarrollaron los hormigones y aceros de alta resistencia que, por un lado, permitían la aplicación de grandes fuerzas externas $\mathrm{y}$, por el otro, reducían las pérdidas que esas fuerzas experimentaban, como consecuencia de las deformaciones diferidas. El hormigón pretensado se aplicó en 
Europa por primera vez como técnica habitual a los puentes, al finalizar la segunda guerra mundial, y se vio impulsada por la necesidad de reconstruir numerosos puentes destruidos durante la guerra.

La tipolología que componen los pasos superiores está diferenciada por tableros de vigas prefabricadas, losas in situ, y en menor medida por tableros metálicos o mixtos. En España, está bastante repartido el uso de ambos tableros de hormigón, no así en los países más desarrollados de Europa y en los Estados Unidos, donde la mayor parte de ellos están construidos mediante tableros compuestos por vigas prefabricadas pretensadas sobre las que se ejecutan losas in situ.

El uso generalizado de los tableros ejecutados con vigas pretensadas está extendido en la construcción de puentes. El puente más largo del mundo se encuentra en Estados Unidos, y tiene 38.4 kilómetros de largo. Se le conoce como Lake Pontchartrain Causeway y fue completado en el año 1956. Se compone de dos puentes paralelos que cruzan el lago Pontchartrain, al sur de Louisiana, formados por 2,170 y 2,174 vanos iguales de $17 \mathrm{~m}$, resueltos con vigas apoyadas, y cuya longitud total es casi de $75 \mathrm{~km}$. El Puente Vasco de Gama en Lisboa, es el puente más largo de Europa y uno de los diez más largos del mundo, con sus más de diecisiete kilómetros de largo. Fue construido con motivo de la Exposición Universal de 1998 y lo componen 150 vigas prefabricadas en 75 vanos dobles formados por parejas de vigas prefabricadas de $78 \mathrm{~m}$. de luz.

Durante muchos años, incluido el comienzo del primer plan de carretera en 1984, hasta el año 1989, los tableros isostáticos de vigas prefabricadas se utilizaron con profusión para pasos superiores de la Red de Carreteras del Estado. En 1989, una Nota de Servicio de la Dirección General de Carreteras sobre pasos superiores en autovías, seguida de la O.C. 302/89T[124], derogada, limitaron significativamente el uso de vigas prefabricadas en pasos superiores, recomendando $\mathrm{u}$ obligando a disponer losas cimbradas in situ, asociando la insuficiencia estética a las vigas prefabricadas. Desde aquel momento se produjo una disminución de la aplicación de elementos prefabricados en los puentes que se hizo extensiva, no sólo a los pasos superiores, sino a todo tipo de puentes y viaductos. Se generalizó el proyecto y construcción de pasos superiores de losa in situ, cuando las soluciones prefabricadas podrían haber sido igual de válidas y, sobre todo, en general, menos costosas. Entre los aspectos negativos que se asociaban a los puentes de vigas prefabricadas se podrían citar: Su escasa valoración estética, mayores problemas de conservación y durabilidad, excesivo número de juntas en el tablero, y las contraflechas producidas por las vigas.

La O.C. 11/02[123] sobre "Criterios a tener en cuenta en el proyecto y construcción de puentes con elementos prefabricados de hormigón estructural", deroga la anterior O.C. 302/89T, e intenta rehabilitar positivamente la figura de las vigas prefabricadas para su uso en los pasos superiores de carreteras y ferrocarril. Enumera las ventajas que se producen en la mayoría de las ocasiones: su menor costo, su facilidad constructiva, la eliminación de cimbras, la mayor rapidez de construcción, la menor afección al tráfico existente en el caso de cruces con viales en servicio, la facilidad de ampliación futura (nula en el caso de puentes "in situ"), su isostaticidad y menor peso por lo que se ven menos condicionadas por las características del terreno para cimentar, etc., propiedades que deberán tenerse en cuenta en los estudios de 
tipologías y soluciones de obra nueva. Por otro lado, dicha O.C. 11/02 enumera un grupo de criterios para atenuar los aspectos negativos enumerados anteriormente: consideraciones estéticas, sobre gálibo vertical, sobre durabilidad y conservación, sobre juntas y sobre contraflechas.

La prefabricación de tableros con vigas ha experimentado una evolución importante desde sus inicios, ofreciendo soluciones de gran calidad estética y un mayor número de tipologías. En cuanto a la sección transversal, desde las propuestas iniciales constituidas por un conjunto de vigas prefabricadas doble $\mathrm{T}$ se ha pasado a la utilización de vigas artesa con forma de U, más pesadas, con gran separación entre ejes, que van de 5 ó $6 \mathrm{~m}$. a $12 \mathrm{~m}$., utilizando placas superiores prefabricadas, que constituyen un encofrado colaborante de la losa superior que se completa con un hormigonado in situ. Estas soluciones permiten eliminar completamente los cabezales sobre pila de los tableros de viga doble $\mathrm{T}$ que tanto efecto antiestético producen. Entre 10 y $12 \mathrm{~m}$. de anchura, y luces no excesivas, se pueden utilizar secciones de cajón monocelular que consiguen acabados excelentes. Además, se ofrecen soluciones de canto constante, canto variable y soluciones curvas con gran complejidad geométrica

Las vigas artesa prefabricadas se conectan mediante armadura pasiva con la losa ejecutada in situ, y forman el correspondiente tablero, descansando sobre las pilas/estribos, que transmiten las cargas, a través de la cimentación, sobre el terreno. En la Figura 1-3 y 1-4 se muestran el alzado y planta del viaducto 1 en el tramo Muro de Alcoi - Puerto de Albaida (Autopista del Mediterráneo).

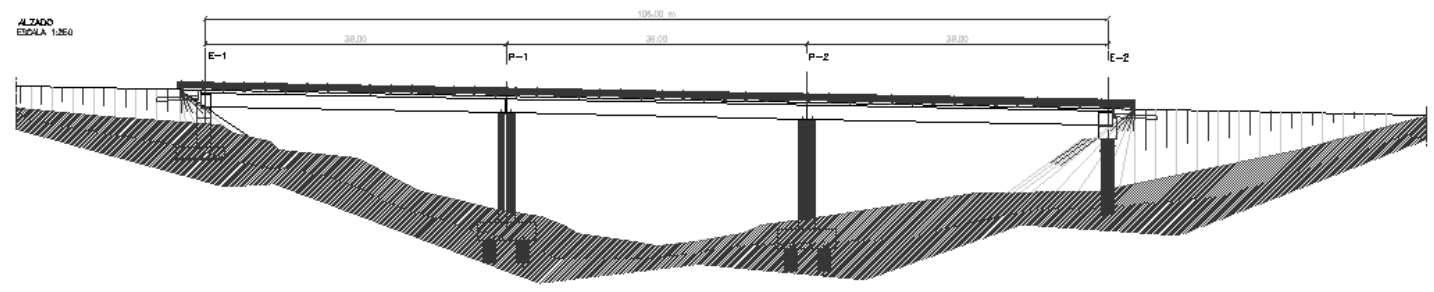

Figura 1-3: Alzado viaducto 1

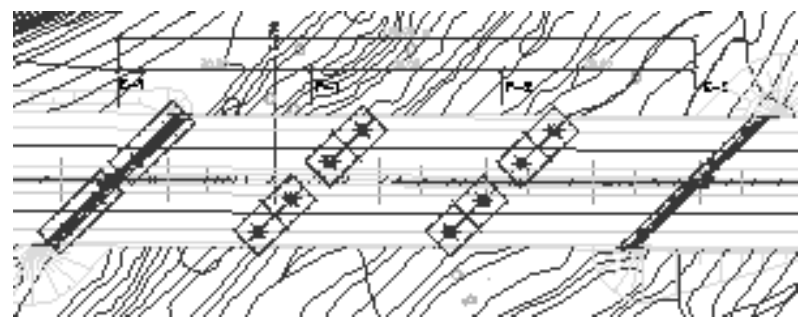

Figura 1-4: Planta del viaducto 1 
El tablero que se ha tomado como referencia en este estudio es el del vano central, con una luz entre apoyos de neopreno, de $35 \mathrm{~m}$. y unas entregas de $0.47 \mathrm{~m}$., que convierten la distancia entre las pilas de $36 \mathrm{~m}$. Cada viga se apoya sobre un neopreno. Este viaducto consta de dos puentes con tablero esviado, uno para la calzada derecha y otro para la izquierda, cada uno compuesto por tres vanos de luces 35.25-36.0035.25. Los tableros están formados por dos vigas simétricas separadas $6 \mathrm{~m}$. y una losa de sección 0.25 x 12 m. En la Figura 1-5 se define la sección transversal del tablero, con el ancho de la calzada de $11 \mathrm{~m}$. y espacio para barreras de $0.5 \mathrm{~m}$.

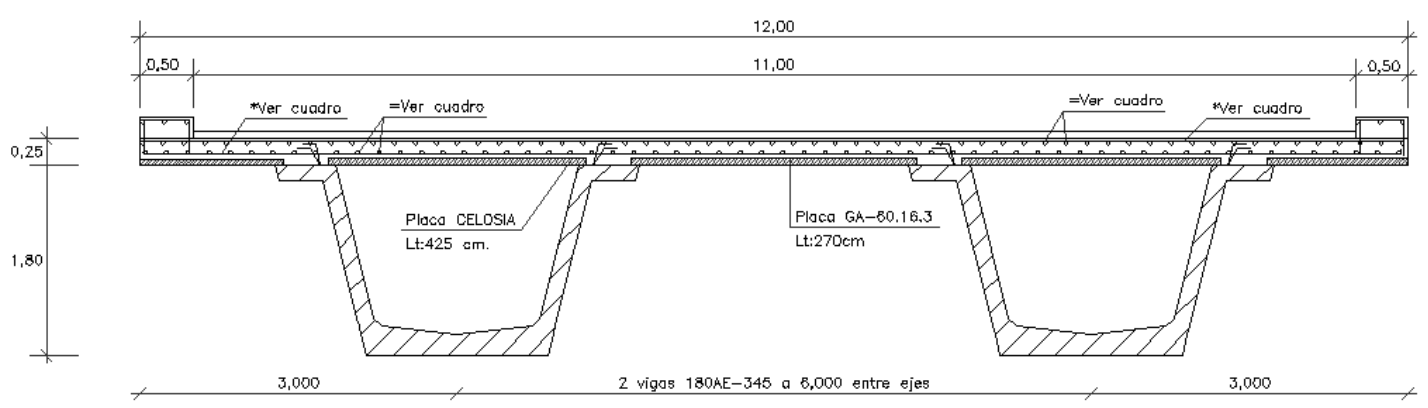

Figura 1-5: Sección transversal tablero viaducto 1.

Las vigas tienen un canto de $1.80 \mathrm{~m}$., y sus dimensiones vienen representadas en la Figura 1-6. En la misma se muestra además la armadura activa, constituida por 78 cables de acero de 0.6" distribuidos en tres niveles.

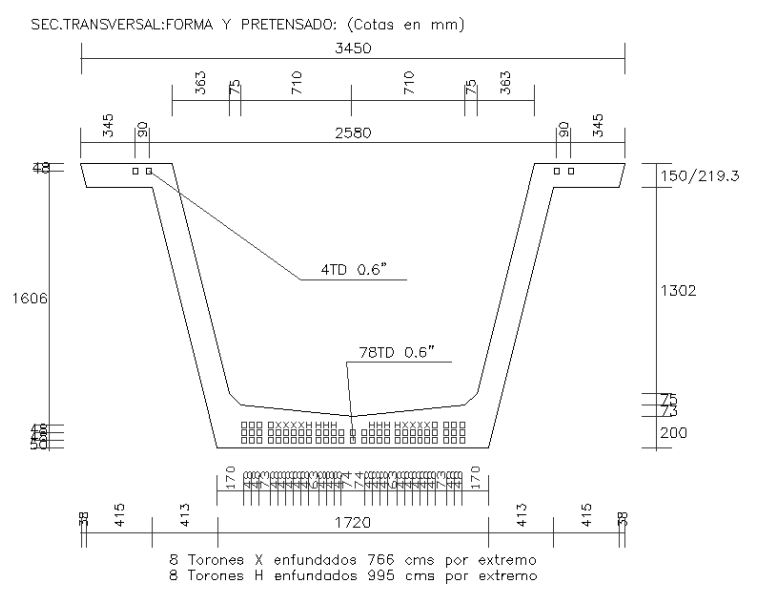

SEC.TRANSVERSAL:ARMADURA PASIVA: (Cotas en $\mathrm{mm}$ )

Armadura de piel 23 redondos D 8 (Todos los hierros no indicados)

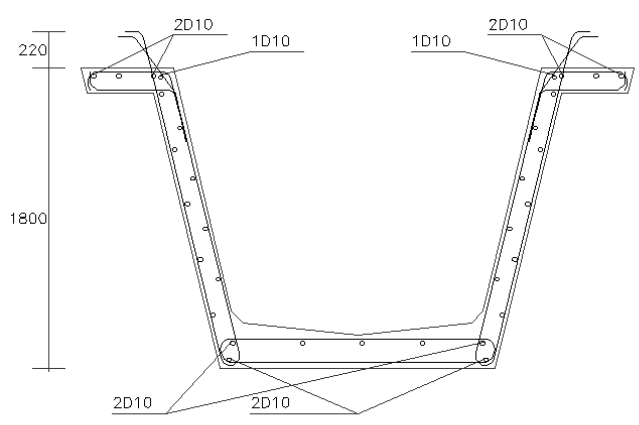

Figura 1-6: Viga artesa vano central. Modelo 180AE-345

La geometría con la que se diseñan las vigas prefabricadas artesa es muy similar, independientemente del fabricante. La artesa, básicamente está formada por un ala inferior, dos almas y dos alas superiores, cuyas secciones suelen ser delgadas para 
reducir el volumen de hormigón, y a la vez, el peso de la pieza, factor que interviene en el coste del transporte y colocación sobre los apoyos.

Los diferentes fabricantes patentan su geometría de viga artesa. Suelen diferenciarse primordialmente unas de otras en la inclinación del alma, geometría de las cartelas en alma inferior y superior, longitud del ala inferior y superior, etc. Son aspectos que no influyen decisivamente en la capacidad mecánica de la estructura. En el presente estudio, se ha tomado una inclinación de $80^{\circ}$ para las almas, y una pendiente de $3: 1$ para las cartelas (ver Apéndice A), valores que están dentro de los márgenes habituales de diseño.

Cuando se ejecuta el hormigón de la losa, la sección resistente de la viga se convierte en un cajón. El cajón en comparación de las secciones en $\prod$, formadas por dos o más vigas I más la losa, se comporta mejor ante los esfuerzos de torsión, y también, debido a su gran módulo de inercia, resiste con gran eficiencia los esfuerzos de flexión longitudinal.

La geometría de las alas superiores, que incluyen la correspondiente cartela, se tiene en cuenta principalmente en la ejecución del hormigonado de la losa, comportándose entonces como ménsula.

Las vigas pretesas se fabrican en plantas industrializadas. En ellas, se dispone de, al menos, una planta de fabricación de hormigón de alta calidad. Los moldes del encofrado, de acero, son de longitud apreciable, permitiendo la fabricación de varias vigas a la vez sobre la misma bancada. Se dispone de topes de hormigón en los extremos del molde para tensar, mediante gatos hidráulicos, los cables de acero, previo al vertido del hormigón. Cuando se endurece el hormigón, se sueltan los torones de los anclajes extremos, y los cables transmiten su presión a las vigas a través de su adherencia, teniendo como efectos visibles un acortamiento del hormigón, que se aprecia en los torones que van enfundados y sobresalen de la viga, produciendo una deformación en ella, que la eleva en el centro del vano. Las fundas se sitúan en los extremos para reducir el incremento de la tensión que se produce en el ala inferior de dichas secciones, provocado por la diferencia entre la acción del pretensado y la disminución de la solicitación a flexión que se produce cerca de los apoyos.

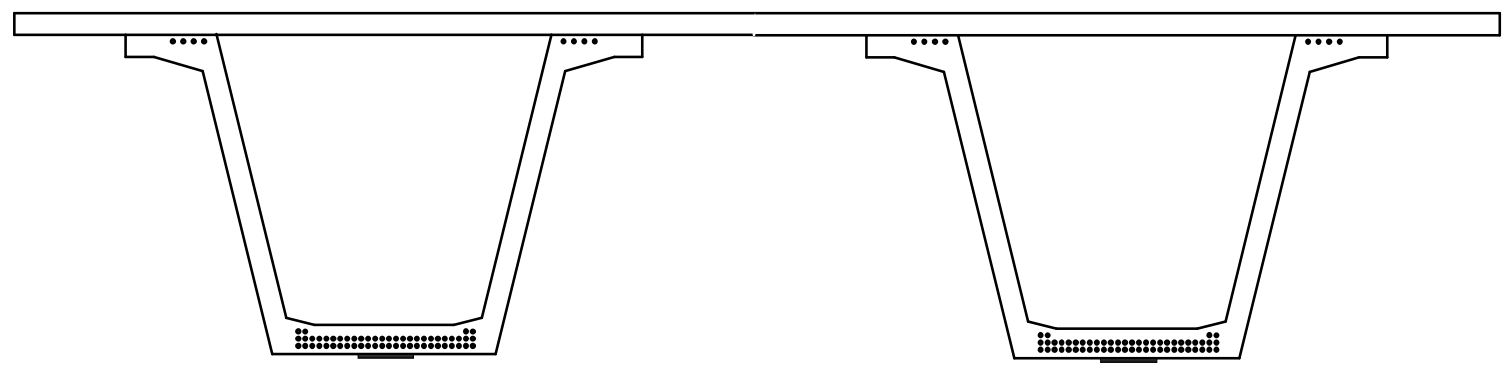

Figura 1-7: Vigas artesa con losa. Incluye la armadura activa 
En el presente estudio, se han dispuesto hasta un máximo de tres niveles de torones en el ala inferior y un nivel en el superior. El pretensado superior compensa el efecto de tracción producido en las alas de la viga en el momento de la transferencia del pretensado.

Una vez la viga está fabricada, se acopia en la planta a la espera de su transporte, que se realiza con vehículos especiales articulados hasta el lugar de colocación. Allí, mediante grúas, se sitúan sobre los apoyos de neopreno. Sobre las alas superiores de las vigas se anclan paneles prefabricados de hormigón, que ejercen la función de encofrado perdido. Posteriormente se coloca la armadura pasiva y se hormigona la losa. La viga se ve solicitada temporalmente por el peso del hormigón, deformándose nuevamente. Cuando fragua el hormigón, ya se dispone de la nueva estructura solidaria (Figura 1-7).

\subsection{Objetivos del trabajo}

Se establece como objeto de esta tesis el estudio del diseño óptimo económico automatizado aplicado al cálculo de tableros de pasos superiores constituidos con vigas artesa de hormigón pretensado. Este trabajo pretende responder a la pregunta: ¿cuál es el diseño más económico para esta tipología de puente en estos momentos?, y si aumenta/reduce el precio del acero/hormigón/colocación/.., ¿cómo queda transformado el nuevo diseño?, etc. Para la obtención del diseño se establecen dos módulos: el primero de comprobación estructural, en el que se incluirán todos los estados límite de la normativa vigente, y el segundo de optimización, de modo que se consiga la estructura de menor coste posible. Como métodos de optimización se estudian diferentes heurísticas y metaheurísticas, buscando, dentro de un conjunto de las mismas, la que mejor se adapte a nuestro problema y de mejores resultados, tanto en relación al coste medio y mínimo en función de los tiempos de ejecución, como en la dispersión que muestren los resultados.

Una vez elegida la mejor heurística, basada en criterios de robustez, costes mínimos y recursos computacionales, se realiza un estudio paramétrico de tableros para diferentes luces, obteniendo unas relaciones de cuantías, tanto en losa como en viga, de $\mathrm{m}^{3}$ de hormigón, $\mathrm{Kg}$ de acero activo y pasivo, etc., que permiten conocer mejor la estructura que se estudia. Por otro lado, modificando los dos módulos, se introduce un nuevo material para la fabricación de las vigas, el hormigón reforzado con fibras de acero (HPF), incluyendo las recientes bases de cálculo de la Instrucción del Hormigón Estructural EHE-08 [44], realizándose un estudio paramétrico similar al anterior, y permitiendo comparar ambos resultados para extraer conclusiones de ambos materiales.

Otro de los objetivos del trabajo es mostrar a los ingenieros proyectistas que la automatización del cálculo de estructuras, mediante métodos heurísticos, es una herramienta eficaz, pero sin excusar que las decisiones finales en la construcción de una estructura las deberá tomar siempre un ingeniero con experiencia y no una máquina. Además, se hace hincapié en la necesidad de la optimización estuctural, lo cual incide en un ahorro de recursos económicos y de materias primas. 
Finalmente, otro de los objetivos de esta tesis es poner a disposición de la industria del prefabricado del hormigón, una herramienta que permita adaptar sus diseños de tableros de puentes a la fluctuación de precios de un mercado globalizado, obteniendo estructuras que consuman menos recursos empresariales.

\subsection{Naturaleza del problema de optimización}

La optimización dentro del problema propuesto se ha resuelto mediante métodos aproximados, heurísticas y metaheurísticas, que pretenden minimizar los costes del tablero de vigas artesa en diferentes casos. La función objetivo que se utiliza es monoobjetivo, valorando únicamente los costes totales de cada solución calculados mediante la multiplicación de las mediciones dispuestas de cada material por el coste unitario que supone la colocación en obra de los mismos, y añadiendo los costes correspondientes al transporte y colocación de las vigas sobre los apoyos. Las restricciones que verifican todas las soluciones son las correspondientes al cumplimiento de los diferentes estados límite de servicio y últimos que indica la normativa aplicada, además de ciertas normas de construcción como pueden ser, entre otras muchas, los espesores mínimos de las paredes del alma de la viga, espesor mínimo de la losa, esbeltez mínima de la viga, etc. Como se pretende que las soluciones sean construibles, las variables que se utilizan son discretas, tratándose por tanto de un problema de optimización combinatoria.

\subsection{Estructura del trabajo}

Este trabajo se divide en nueve Capítulos. En el Capítulo I se realiza una introducción al problema planteado, la necesidad de este trabajo y los objetivos que se pretenden alcanzar. En el Capítulo II se lleva a cabo una revisión bibliográfica del estado del arte, donde se muestran los trabajos más relevantes en optimización estructural, prestando especial atención, a aquellas investigaciones que se refieren a estructuras de hormigón pretensado, y las que han aplicado alguna técnica heurística.

En el Capítulo III se tratan los elementos básicos del método de optimización, con una clasificación y descripción de diferentes métodos heurísticos y metaheurísticos, haciendo especial hincapié en los empleados en la resolución del problema. El objetivo es mostrar las principales características de cada uno de ellos, y definir los parámetros que deben calibrarse, así como el modo en que estos parámetros se pueden seleccionar en la práctica.

En el Capítulo IV, se formula el problema de optimización. Se definen las variables consideradas, así como los rangos de valores permitidos para cada una de ellas. Igualmente de enumeran los parámetros de los que depende el problema, y la composición de la función objetivo que se desea minimizar.

Una vez definido el problema, en el Capítulo $\mathrm{V}$ se describe cuales son las restricciones del mismo, y la forma en que se comprueban estas limitaciones. Para ello, se desarrolla un programa de cálculo automático de tableros, capaz de efectuar 
todas las comprobaciones que la normativa determina para este tipo de estructuras. Este programa únicamente comprueba el tablero, sin dimensionarlo.

A continuación, en el Capítulo VI se detallan los algoritmos aplicados al problema en base a las metaheurísticas descritas en el Capítulo III, realizando la calibración de los mismos y comparando los resultados obtenidos. De la colación de los distintos métodos se podrá deducir cuál es la heurística más eficaz al caso, la cuál se aplicará en los estudios paramétricos siguientes. Además, en este Capítulo se trata de cómo establecer el número de reinicios necesario para que la probabilidad de alcanzar un tablero de gran calidad sea suficientemente alta, y finalmente se estudia la sensibilidad del modelo a la variación de precios del acero y del hormigón.

En el Capítulo VII se muestra el estudio paramétrico de la estructura para distintas luces, donde se analiza la geometría, los materiales y las cuantías de los aceros y de los hormigones. En el Capítulo VIII se realiza el mismo estudio paramétrico para la utilización del hormigón con fibras de acero en la viga, comparando los resultados con los obtenidos del Capítulo anterior, y analizando la viabilidad de este material.

En el Capítulo IX se establecen las conclusiones del trabajo, algunas recomendaciones y finalmente se definen futuras líneas de investigación que puedan completar este trabajo. 


\section{CAPÍTULO 2. ESTADO DEL ARTE}

En este Capítulo se presenta un resumen de la revisión bibliográfica realizada sobre trabajos que contemplan cómo el proyecto de estructuras puede entenderse como un problema de optimización. Se muestra su evolución en el tiempo y la aplicación de los distintos métodos utilizados, tanto exactos como heurísticos. En último lugar se identifican los vacíos existentes, y se enumeran las conclusiones obtenidas.

\subsection{Antecedentes de la optimización de estructuras}

Dentro de un proceso de fabricación industrial, la optimización siempre ha tenido una importancia significativa. En la industria, los procesos son precisos y repetitivos elaborando una determinada pieza o elemento un número considerable de veces. Sin embargo, en la construcción las obras casi nunca se repiten, la localización difiere y los sistemas de ejecución son menos exactos que en la industria, debiendo desplazase cada vez el personal, la maquinaria y la propia organización, haciendo muy dificultosa la aplicación de una metodología de optimización estructural en la que se necesitan abundantes medios de cálculo. Es por ello, que solamente se empezaron a aplicar métodos de optimización a mediados del siglo $\mathrm{XX}, \mathrm{y}$ en ese caso, a estructuras simples.

La información más antigua que se tiene sobre optimización de estructuras se remonta al siglo XV, donde Leonardo da Vinci y Galileo Galilei (Grierson, en Adeli [3]) buscaban disminuir al máximo el peso de estructuras elementales de madera. En su obra póstuma, Galileo[57] publicó algunos trabajos sobre deflexión de vigas. La siguiente referencia que se encuentra fue ya en el siglo XIX con Maxwell [109] (1869) y Levy [96] (1874), y a comienzos del siglo XX con Mitchel [115] (1904). Estos autores analizaron el diseño óptimo de estructuras de arcos y cerchas metálicas para conseguir un mínimo peso, para un estado tensional dado. Estos estudios fueron pioneros en el establecimiento de una base científica para la optimización estructural, al desarrollar por primera vez una metodología capaz de proporcionar soluciones optimizadas (Navarrina [122]), aunque el diseño obtenido no fuera construible.

Klein [88] (1955) planteó que los problemas de optimización estructural deberían trazarse como problemas de optimización condicionada, a partir de lo cual comenzó a aplicarse dicha premisa, gracias a la potencia de cálculo que estaban a punto de traer los ordenadores. Schmidt [154] (1960), combina por primera vez los elementos finitos en el análisis estructural, con la programación lineal para la optimización matemática, definiéndolo como "síntesis estructural". 
Como el análisis estructural suele ser un problema no lineal de muchas variables, la aplicación del método anterior se pudo realizar a base de linealizar el problema, ya fuera mediante cambios de variable o mediante simplificaciones del mismo. Y así, comenzaron a desarrollarse y a emplearse técnicas que permitían resolver problemas no lineales, destacando los criterios de optimalidad de KKT (Karush [83], Kuhn y Tucker [91]) en su búsqueda del óptimo global. Ya en los años setenta comienzan a aplicarse los multiplicadores de Lagrange aumentados o el gradiente generalizado reducido. Desde entonces, y hasta el momento actual, han ido mejorando estas técnicas, y aumentando la dimensión de los problemas.

\subsubsection{Estructuras de hormigón armado}

Dentro del conjunto de estructuras de hormigón armado, se puede hacer un breve resumen de los autores más significativos de los últimos años que han seguido una línea de investigación sobre su optimización, mediante la aplicación de métodos exactos.

- En 1980 Moragues [118] aplicó métodos de optimización al diseño de estructuras aporticadas, siendo la función a minimizar el coste de la estructura. Estudió la forma de optimizar secciones rectangulares de hormigón armado de vigas, pilares y su composición en pórticos, dando criterios generales de predimensionamiento basados en los resultados obtenidos. La única restricción impuesta al problema era la verificación del ELU de agotamiento por solicitaciones normales, no considerando la influencia del cortante en la búsqueda de óptimos, por lo que no consideraba ni el ELU de pandeo, ni los ELS. Las estructuras estaban sometidas únicamente a fuerzas gravitatorias (verticales). El número máximo de variables consideradas fue de 7.

- En 1989 Arenas y Villegas [16] presentaron el proceso de análisis y dimensionamiento de las pilas del viaducto de Cruzul. Realizaron la optimización de las mismas mediante un proceso prueba-error. Los cálculos se realizaron teniendo en cuenta la doble no linealidad, la geométrica y la mecánica. En este trabajo representan la distribución de las fuerzas transversales y las longitudinales entre las diferentes pilas.

- En 1991 Gasch [58] optimizó la planta de un forjado reticular sometida exclusivamente a acciones verticales. La optimización era multinivel: en una primera fase optimizaba la topología (número de soportes), permaneciendo constantes las dimensiones de las secciones, y en una segunda fase, fijada la topología, optimizaba la geometría (posición de los soportes) y las dimensiones de forjados y soportes. El número máximo de variables consideradas fue de 21 .

- En 1994 Cohn y Dinovitzer [40] realizaron una revisión de los problemas de optimización resueltos hasta ese momento, dejando patente la falta de un mayor enfoque práctico de las estructuras estudiadas, de manera que las técnicas de optimización fueran una herramienta más accesible a los ingenieros, y que se resolvieran ejemplos con geometrías y cargas reales, realizando las comprobaciones de los estados límite de las mismas. Destacan la necesidad de resolver más estructuras de hormigón, dado que, el $92 \%$ de 
las resueltas eran metálicas, y de incrementar el empleo de métodos heurísticos, dado que la mayoría de los ejemplos analizados se resolvían mediante métodos exactos.

- En 1997 Balling y Yao [19] abordaron la optimización de pórticos de edificación de hormigón armado mediante tres estrategias diferentes. La primera consistía en un método exacto basado en técnicas del gradiente, considerando como variables las dimensiones del pórtico y la cuantía de armadura de flexión de las secciones críticas. La segunda consistía en un método multinivel, en primer lugar se realizaba la optimización de las dimensiones del pórtico mediante un SA y en segundo lugar, una exploración exhaustiva del espacio de soluciones para la definición de las armaduras de flexión, que se representaban mediante esquemas de armado reales. La tercera era una versión de la primera, partía de suponer que las restricciones relativas a las cuantías mínimas de armadura y al cumplimiento del ELU de agotamiento por solicitaciones normales rigen los diseños óptimos, eliminando así el armado como incógnita. Como conclusiones establecían que el tercer método es mucho más rápido que el multinivel (del orden de 280 veces) y que el método exacto (del orden de 2 veces) y que el método multicriterio proporcionaba las soluciones de menor coste, entorno a un 1.7\% respecto a las mejores del primer y tercer método. El análisis realizado no incluía la resistencia del hormigón como variable ni consideraba el cálculo de las armaduras de cortante, aunque sí realizaba ciertas comprobaciones para asegurar que la sección de hormigón era suficiente para resistir este esfuerzo.

- En 2003 Torrano [158] optimizó secciones con forma arbitraria y cualquier distribución de armado y elementos estructurales de hormigón armado. Optimiza el coste mediante un método de optimización cuadrática sucesiva. El número total de variables es de 42 , siendo todas continuas. No considera algunas disposiciones constructivas como la separación de la armadura de cortante ni verifica la fisuración. Las secciones y elementos estructurales que optimiza son: una sección cuadrada y otra en L sometidas a flexión compuesta esviada, una sección rectangular con un agujero centrado solicitada a flexión compuesta recta, una viga biapoyada de sección variable sometida a una carga vertical uniformemente repartida y una sección que representa un sistema de pantallas sometidas a flexión compuesta esviada. El método permite obtener diseños más económicos que por otros procedimientos, de una manera sencilla.

- En 2005 Rodrigues et al [147] estudiaron para un edificio de 16 alturas, sometido a acciones verticales y horizontales, la optimización de los pilares. En el estudio realizado se consideró que los pilares tenían las mismas dimensiones en una misma altura produciéndose una, dos o tres reducciones de la sección con la altura. Emplean un método multinivel: en una primera fase obtienen las dimensiones de los pilares y en una segunda el armado. No consideran como variables ni la geometría de las vigas ni la calidad del hormigón. Como restricciones consideran el desplazamiento máximo horizontal y las derivas de cada uno de los pisos, el ELU de inestabilidad de los pilares y sus cuantías máximas. No se realiza la comprobación de ELU de 
agotamiento por esfuerzo cortante ni un diseño detallado del armado de los pilares. Concluye que, disponiendo secciones de pilares diferentes en función de la planta, se puede llegar a ahorros significativos en el coste de la estructura.

\subsubsection{Estructuras de hormigón pretensado}

La optimización de las estructuras de hormigón pretensado comenzó a desarrollarse a mediados del siglo XX. Inicialmente, se buscaba el pretensado mínimo de la estructura para unas determinadas cargas y geometría de la sección. El equilibrio que establecía el pretensado se determinaba en forma de flechas (Lin [97]), o bien en forma de esfuerzos y tensiones (Brotchie [24]). A continuación se enumeran los trabajos que se ha considerado más significativos en sus aportaciones a la optimización de estas estructuras, hasta la fecha actual.

- En 1966, Torres et al. [159] plantearon el diseño económico de un tablero de puente de vigas prefabricadas pretensadas, empleando la programación lineal por niveles. En un primer nivel obtenían el número de vigas y su canto, y posteriormente la fuerza de tesado y la excentricidad de los cables. Consideraban además, el coste debido al transporte y a la colocación de las vigas sobre los apoyos.

- En 1971 Goble y Lapay [64] minimizaron el coste de vigas prefabricadas pretensadas con sección en T, empleando para ello un método de gradiente proyectado. Por primera vez se minimizaba la función objetivo del coste, que incluía los precios del hormigón, del acero activo y pasivo y del encofrado. Como conclusiones, destacan que la solución óptima no está afectada por el precio de los materiales, lo cual ha sido observado posteriormente por otros autores

- En 1972 Johnson Jr. [80] presentó un programa de cálculo automático de tableros que permitía buscar la configuración óptima de vigas y pretensado en el tablero. No incluye la función objetivo del coste, pero incorpora la comprobación de estados límites de flexión y cortante, armadura pasiva de tracción y flechas durante el tesado de la viga y con la estructura en servicio.

- En 1973 Kirsch [86] obtuvo la configuración óptima de pretensado en estructuras continuas para una determinada geometría, aplicando la programación lineal. Es posterior a otro trabajo de 1972 [87], e incluye, entre otras, la misma viga continua de dos vanos ya estudiada en aquél. Considera el trazado del cableado como una variable de decisión.

- En 1976 Naaman [121] compara, la obtención de diseños distintos de vigas biapoyadas de sección rectangular, tomando dos funciones objetivo distintas: mínimo coste y mínimo peso de vigas. Se aplican precios al hormigón, al pretensado y al encofrado. La optimización la lleva a cabo mediante una técnica de búsqueda directa. Se alcanzan resultados análogos para ambos casos, cuando el precio del metro cúbico de hormigón es del orden de 35.6 veces el precio del kilo de acero activo. En la actualidad este valor es aproximadamente de 10 . 
- El 1978 Ramamurthy [146] optimizó también losas similares a las tratadas por Naaman, pero aplicando la programación geométrica generalizada. Las variables de diseño incluyen el canto de la losa y la armadura activa y, en algunos ejemplos, la armadura pasiva de flexión si ésta es necesaria. En sus conclusiones determina la existencia de un canto por encima del cual resulta más rentable añadir armadura pasiva para conseguir la resistencia a flexión en ELU. En estos casos se mantiene el pretensado estricto para verificar solamente los ELS (tensiones, fisuración y flechas). El trabajo se completa con un estudio de la influencia de diferentes costes relativos entre el hormigón y los aceros. Los resultados obtenidos muestran una clara influencia de la relación entre los costes de los aceros activo/pasivo, en la conveniencia de emplear éste último para mejorar la resistencia a flexión en ELU. Por último, se observa cómo ni las cuantías de acero ni el coste óptimo de la losa se ven afectados por variaciones en las relaciones de costes de los materiales, en la línea sugerida por Goble y Lapay.

- En 1984 Cohn y MacRae [43], desarrollaron un programa de cálculo (OSCON) para optimizar vigas, $\mathrm{T}$ o doble $\mathrm{T}$, que además podían ser de hormigón armado, pretensado total, o pretensado parcial, y tanto con armadura pretesa como postesa. Teniendo como base una optimización económica, es posible optimizar con criterios de mínimo peso, el acero total o solamente el pretensado, anulando temporalmente los precios del hormigón o de los aceros. Se aplican las restricciones de tensión y fisuración (incluyendo la fatiga), flechas y contraflechas, aperturas de fisura, resistencia última a flexión, comprobaciones de ductilidad, y armados mínimos. Emplean para optimizar el método de direcciones conjugadas factibles. A partir de los cuatro casos que se muestran, los autores concluyen que para vigas postesadas es más económica la solución con pretensado parcial que con pretensado total, siempre que el coste unitario del acero activo respecto al del pasivo sea superior a 4.

- En 1984 Saouma y Murad [152], también estudió la optimización de vigas parcialmente pretensadas de sección doble $\mathrm{T}$ con armadura pretesa. Considera nueve variables de decisión, que incluyen la sección de hormigón, el área de armadura pasiva (superior e inferior) y el área de armadura activa. Aplicando funciones de penalización eliminan las restricciones, lo que permite aplicar el método cuasi-Newton del IMSL. Se analizan distintas longitudes de vigas, desde $6 \mathrm{~m}$ a $42 \mathrm{~m}$ de luz, tanto con fisuración permitida como impedida, llegando a la conclusión de que el pretensado parcial es ligeramente más económico que el pretensado total.

- En 1985, Jones [81] aplica por primera vez la optimización económica de vigas prefabricadas pretensadas de sección cajón. Utiliza técnicas de programación entera, que podrían interpretarse como heurísticas, ya que obtienen óptimos locales del problema. Se consideran parámetros invariables la geometría y una malla de posibles ubicaciones de los tendones, siendo las variables de decisión el tipo de hormigón, la cantidad de tendones necesarios, y la cantidad de ellos que se dispondrán oblicuos. La función objetivo contiene el precio del hormigón y el del acero activo. En cuanto a restricciones del 
problema, comprueba la flexión en ELU, las flechas, y tensiones y fisuración tanto en el momento de la transferencia como en servicio. Además considera los efectos diferidos en los límites de tensiones en el hormigón.

- En 1991 Samartín y Utrilla [151] aplicaron la programación lineal para optimizar el pretensado de losas de sección trapezoidal, propia de tableros de puentes. El objetivo es el de obtener, para la geometría dada, el pretensado mínimo (trazado y fuerza de tesado en varias familias de tendones) para las requisitos de estado límite de servicio de tensiones. Definido el trazado, queda como variables de decisión la posición de los puntos de tangencia.

- En 1992 Erbatur et al.[56], emplean el método simplex para la optimización de peso y coste de vigas de hormigón pretensado biapoyadas, aplicable a cuatro posibles secciones, sometidas a limitaciones de flexión (ELU y ELS), limitaciones de flechas en tres momentos de la vida de la viga, e incluso una comprobación de pandeo, que se justifica por la importante esbeltez de las soluciones óptimas. Las variables de decisión se reducen al ancho de la sección y la fuerza de pretensado. Los resultados muestran que las únicas restricciones que limitan el óptimo son los criterios tensionales. Realizan además un estudio de sensibilidad a los costes unitarios, del que se concluye que para vigas rectangulares con luces inferiores a $25.9 \mathrm{~m}$ el óptimo en peso y coste coinciden. Lo mismo sucede con secciones en T si la relación entre los costes del acero (activo) y hormigón es inferior a 25, y en secciones en I si este cociente es inferior a 35. La interpretación de estos cocientes es difícil, pues no se especifican las unidades a emplear en su determinación.

- En 1993 Lounis y Cohn [98], aplicaron por primera vez la optimización multiobjetivo de un tablero de vigas prefabricadas pretensadas. Señalan la importancia de esta consideración para resolver estructuras reales. Emplean algoritmos de lagrangiano con dos funciones objetivo: coste total $\mathrm{y}$ contraflecha inicial. Su estrategia consistente en ordenar por su importancia las funciones objetivo, tomando la principal como función objetivo, y estableciendo restricciones para cumplir con la otra función, que pasa a ser considerada como una nueva limitación. Los óptimos pasan a ser un conjunto de óptimos de Pareto. Se imponen restricciones en ELU y ELS.

- En 1994 Cohn y Lounis [41], presentaron una metodología para la selección de la tipología más adecuada para cada puente entre cinco posibilidades: vigas prefabricadas, cajón mono o bicelular, y losas aligeradas o macizas. Para ello proponen una optimización a tres niveles. En el primero se optimizan los componentes individuales del tablero (vigas, losa, etc.), el segundo se refiere a la configuración óptima de estos componentes en la estructura total (número de vigas, separación de almas, etc.), y el tercero se refiere a la tipología más adecuada. Emplean restricciones correspondientes a estados límites de tensiones y fisuración, fatiga, y flexión en rotura. El método admite una o varias funciones objetivo: mínimo coste, mínimo pretensado, mínimo volumen de hormigón, máxima separación entre vigas, mínimo canto para una luz dada, máxima esbeltez para luz variable, máxima luz para un canto dado o mínima flecha. Se plantea la optimización de modo multicriterio y 
multinivel. Se aplican técnicas de algoritmos de lagrangiano para la optimización del primer nivel, y a partir de este punto, técnicas de búsqueda cribada en los niveles 2 y 3 . Los resultados proporcionan valiosa información referente a los costes finales por unidad de superficie de tablero para las cinco tipologías, en función de las anchuras, número de vanos y luces en varios casos. También proporciona información relativa a cuantías de acero, disposiciones geométricas, esbelteces y estados límites activos en el óptimo. Para luces inferiores a $50 \mathrm{~m}$., los tableros con vigas I y las losas aligeradas, resultan ser los más económicos.

- En 1995, Al-Gahtani et al. [11] desarrollaron un programa de optimización de vigas continuas pretensadas (PCBDOS). Utiliza 30 variables de decisión para dos vanos. Se emplean restricciones correspondientes a estados límites de tensiones y fisuración, flexión en rotura y cuantías mínimas. La sección transversal se define como una envolvente de las diferentes formas habituales en la práctica, de tal modo que haciendo nulas algunas de sus dimensiones, esta forma puede transformarse en una viga rectangular, en $\mathrm{T}$, en I, en $\mathrm{T}$ invertida, en doble $\mathrm{T}$ o en cajón, entre otras. Obtiene resultados análogos a los obtenidos para vigas biapoyadas por Cohn y MacRae, en los que son más económicas las soluciones para pretensado parcial que para pretensado total. Además, confirma que la función objetivo debe ser el coste, y no el peso o el volumen de los materiales.

- En 1996, Aparicio et al. [15] presentaron un programa informático que obtenía diseños automatizados de puentes de carretera de hormigón pretensado. Calcula y diseña completamente toda la estructura para distintas tipologías de tableros, pilas y estribos. El programa determina en qué casos son más eficaces unas tipologías sobre otras, considerando varios tipos de puentes losa, cajón o de vigas prefabricadas I. En el ejemplo presentado, diseñan completamente un puente de 4 vanos de 60-115-115-60 m de luz a partir de los datos relativos a luces, definición de superestructura, alturas de pilas y estribos, modo de construcción y datos sísmicos. Este tipo de programas permite obtener soluciones diferentes para una estructura en particular, siendo una ayuda significativa para el ingeniero en la toma de decisión de la elección del diseño del puente más adecuado al problema.

- En 1997 Lounis et al. [99] aplicaron el lagrangiano proyectado para obtener el diseño óptimo de vigas prefabricadas pretensadas para ser empleadas indistintamente en tramos isostáticos y en continuos. Para resolver los tramos continuos, plantean un postesado en obra o con disposición de armadura pasiva. Analizan mediante un programa de optimización no-lineal, diferentes colecciones de vigas, y concluyen que las vigas con forma de bulbo en la parte inferior se adaptan mejor a los tramos continuos que las vigas en I cuasi-simétricas. Como resultado, proponen una nueva colección de vigas con bulbos, que se adaptan mejor al postesado en tramos isostáticos y en continuos.

- En 2003, Barakat et al. [22] retomaron el trabajo de 1994 de Al-Harthy y Frangopol [12]. De nuevo aplicaron la optimización combinada con el 
análisis de fiabilidad para optimizar vigas prefabricadas pretensadas doble $\mathrm{T}$, de forma que las restricciones consideradas (tensiones, flechas, momentos de agotamiento y cortante) son tratadas desde el punto de vista de la fiabilidad estructural. Establecen como variables tanto la geometría como el pretensado de las vigas. Para la optimización emplean algoritmos basados en el método de las direcciones factibles. A partir de los resultados de dieciséis casos de luces y cargas diferentes, los autores concluyen que las restricciones activas son siempre el ELS de tensiones. Igualmente observan que la esbeltez de las vigas óptimas varía entre 9 (carga pesada) y 12 (carga ligera), tomando un valor medio de 10.5 para el predimensionamiento.

- En 2004, Barakat et al. [21] continúan con el trabajo anterior, pero con optimización multiobjetivo, tomando además de la función objetivo de costes, los criterios de seguridad y límites de servicio. Permite seleccionar criterios de fiabilidad estructural, conociendo las repercusiones económicas de su decisión. Dada la no linealidad y la no convexidad del problema, consideran que el método resultaría más eficaz si se aplicaran otras técnicas de optimización, como algoritmos genéticos o cristalización simulada.

- En 2005 Sirca y Adeli [156] formulan la optimización del tablero de vigas I prefabricadas pretensadas, incluyendo el número de vigas y su pretensado. Para ello, plantean un problema mixto discreto no lineal, en cuya resolución intervienen las redes neuronales para la evaluación de los multiplicadores de Lagrange en cada iteración. Demuestran con el ejemplo presentado la aplicación práctica del método, que además contiene una excelente convergencia y robustez.

- En 2007 Srinivas y Ramanjaneyulu [157] diseñaron un procedimiento que integra algoritmos genéticos, para la optimización económica de tableros de vigas pretensadas, y una red neuronal para predecir la factibilidad de las soluciones. Los algoritmos genéticos empleados guían la búsqueda hacia el óptimo. La ventaja de este modo de proceder radica en la extraordinaria velocidad de cálculo para la obtención de la solución óptima, puesto que no calcula las restricciones del problema. Esto prueba el buen comportamiento de la aplicación de las redes neuronales en la resolución de problemas de optimización de gran tamaño.

- En 2010 S. Hernández et al. [72] diseñan un software para optimizar vigas pretesas en los tableros de puentes de carreteras. Presentan un ejemplo donde muestran la interactividad del programa. El usuario propone de un modo muy sencillo las dimensiones de las vigas y obtiene el trazado de los tendones (tres niveles) para la menor fuerza de tesado. La optimización del trazado del pretensado está formulado como un problema de formulación lineal. Proporciona salida gráfica para planos. Se puede aplicar también para tableros losa.

- Finalmente, también en 2010 H. Maleky et al [100] presentan un software que permite ayudar al equipo de ingenieros en la toma de decisiones para elegir el óptimo diseño del tipo de tablero de puentes de carreteras más adecuado al problema en estudio. En primer lugar trasladan los requerimientos del 
problema en requerimientos de diseño mediante la técnica del Despliegue de la Función de Calidad (FQD). En segundo lugar aplican la técnica del Soporte para la toma de Decisiones (TOPSIS), que permite ordenar en base al peso de los materiales el mejor diseño. Diseños que evalúa: 2 diseños de tableros de hormigón armado, 4 de hormigón postesado, 2 de hormigón pretensado, 1 de vigas postesadas, 1 de cajón en avance en voladizo y 2 mixtos con vigas metálicas en I y artesas.

\subsection{Métodos heurísticos}

En 1986 Goldberg y Samtani [66] emplearon por primera vez métodos metaheurísticos en trabajos de optimización estructural. El objeto fue una celosía articulada de 10 barras mediante algoritmos genéticos. Siguieron los trabajos de Hajela [69],-[70], Jenkins [78]-[79] o Rajeev y Krishnamoorthy [145], que aplicaron los algoritmos definidos por Goldberg (simple genetic algorithm SGA) a sencillos diseños estructurales, y problemas típicos de entramados metálicos. Se indicaba en estos trabajos, la gran ventaja derivada de la libertad de formulación que este tipo de técnicas tiene para resolver los problemas de estructuras, y la innecesaridad de la aplicación de técnicas de gradiente pero a cambio de procesar numerosas evaluaciones de las funciones.

\subsubsection{Estructuras de acero}

Se hace un breve resumen del avance de las aplicaciones heurísticas para la optimización de estructuras de acero:

- En 1991 Balling [18] (1991) aplicó por primera vez el SA (Simulated Annealing) en la optimización de estructuras a pórticos tridimensionales de acero. Las barras son cargadas, como en casos reales, con cargas debidas a la gravedad y al sismo. Dispone de una base de datos de secciones que utiliza como variables en el cálculo. Compara los resultados con los obtenidos mediante un método exacto, llegando a la conclusión de que las técnicas aproximadas pueden reducir el tiempo de computación con soluciones de calidad.

- En 1994 Adeli y Cheng [4]-[5] aplicaron GA (Genetic Algorithm) a la optimización de 4 estructuras de barras. La optimización consistió en minimizar los pesos de las estructuras, siendo el número de barras de las mismas 10, 12, 25 y 72. Investigaron diferentes formas de realizar el operador genético cruzamiento. Las estructuras se dividen en grupos de barras de manera que el número de variables disminuya, siendo 16 el empleado para la estructura de 72 barras. Concluyen que los GA son adecuados para afrontar la optimización de problemas con un gran número de barras y destacan tres características de los mismos: primera, la función objetivo empieza con varias soluciones de partida y no sólo con una, segunda, el método sólo requiere una simple función de evaluación, y tercera, la posibilidad de implementarlos en paralelo. 
- En 1994 Coello [38] aplicó GA a la optimización de estructuras metálicas de barras, en concreto, a una estructura plana formada por 10 barras y a una tridimensional formada por 25 barras. La función objetivo es el peso de la estructura, que hay que minimizar, disponiéndose de una base de datos de la que se obtienen las posibles secciones a utilizar. Con el algoritmo utilizado se obtuvieron mejores resultados que otros autores resolviendo estos mismos problemas con otros métodos.

- En 1995 Adeli y Kumar [5] presentaron un GA para la búsqueda de estructuras óptimas con gran número de variables aprovechando la adaptabilidad de los mismos a una programación en paralelo. Se dan dos propuestas diferentes: una utilizando funciones de penalización y otra aplicando el método lagrangiano. La técnica es aplicada a diferentes estructuras de barras llegando a la conclusión de la efectividad de utilizar estrategias de paralelización en GA.

- En 1999 Manoharan y Shanmuganathan [101] emplearon TS (Tabu Search), SA, GA) y un método exacto a la optimización de varias estructuras de barras. El objetivo es comparar los diferentes métodos de búsqueda, sobre la aplicación de los mismos en casos prácticos. Las conclusiones obtenidas fueron las siguientes: los tres métodos metaheurísticos llegan a soluciones buenas con tiempos razonables, TS produce soluciones de igual o mejor calidad que las otras dos metaheurísticas en tiempos menores y la aplicación del método exacto a estructuras con un gran número de barras es difícil y costosa.

- En 2002 Hasancebi y Erbatur [71] optimizaron, simultáneamente, la topología (definición de número de barras y de la posición de los nudos) y las secciones de la estructura. Aplican el SA y resuelven dos problemas tipo: una pirámide espacial y una estructura articulada plana. La función objetivo trata de minimizar el peso de la estructura. El interés del artículo radica en el intento de encontrar un método heurístico eficiente para mejorar el diseño de estructuras.

- En 2003 Ali et al. [13] desarrollaron un programa para la optimización de estructuras metálicas articuladas planas y espaciales, utilizando un programa comercial de cálculo de estructuras como módulo de comprobación. El programa constaba de un módulo en el que se realiza la optimización mediante GA y otro en el que se comprueba la estructura mediante dicho programa comercial. Las soluciones obtenidas son buenas, pero los tiempos de ejecución resultan elevados debido a los tiempos empleados en la comprobación de la estructura y en el preproceso y prosproceso de datos.

- En 2004 Camp y Bichon [25] utilizaron ACO (Ant Colony Optimization) para resolver problemas planos y espaciales de acero, típicos de la bibliografía, se comparan los resultados con los obtenidos por otros autores que aplicaron para estos problemas con GA y métodos exactos. Los resultados obtenidos muestran una alta robustez del método, proporcionando en todas las 
ejecuciones soluciones parecidas, y la alta calidad de los resultados comparándolos con GA y métodos exactos.

- En 2005 Schmidt y Thierauf [153] combinaron el TA con un algoritmo evolutivo, definiendo un algoritmo evolutivo híbrido, y lo aplicaron a la resolución de dos problemas extraídos de la bibliografía: la optimización de un pilar de acero y la de una estructura articulada de 10 barras. El algoritmo principal es evolutivo, realizando la mejora local de las soluciones aplicando TA en cada etapa. Los resultados obtenidos se comparan con los de otros autores para los mismos problemas, llegando a la conclusión de que el método muestra un comportamiento robusto con tiempos de ejecución bajos.

- En 2005 Lagaros el al. [92] aplicaron estrategias evolutivas al diseño óptimo de una estructura tipo tubo de 36 plantas. Utilizan una base de datos para dar los valores a las diversas variables de modo que la estructura sea construible, la elección de esta base debe ser adecuada al problema de modo que las variables puedan acceder a los valores necesarios. Este método da buenos resultados cuando el tamaño del programa crece.

- También en 2005 Gong et al. [67] aplicaron colonia de hormigas para optimizar estructuras de barras planas con cargas en los nodos y la estructura de una antena. Se compararon los resultados con los obtenidos mediante GA y SA llegando a la conclusión que los de colonias de hormigas eran mejores.

- En 2007 Degertekin et al. [51] aplican el TS y los GA a la optimización no lineal de estructuras de barras metálicas. La optimización consiste en minimizar el peso de las barras utilizando secciones disponibles de una base de datos. La norma empleada para el cálculo ha sido la AISC (American Institute of Steel Construction) y además de las tensiones, se tiene en cuenta las restricciones de los desplazamientos. Finalmente se comparan los resultados obtenidos con ambas heurísticas.

- En 2008 Degertekina et al. [52] plantean la optimización mediante cálculo no lineal de estructuras metálicas tridimensionales de edificación, mostrando ejemplos que alcanzan hasta 84 vigas/pilares. Aplican TS y GA.

- En 2010 Kaven y Talatahari [84] presentan una mejora en la heurística de ACO (IACO) para la optimización del diseño de pórticos planos de edificación de múltiples plantas y vanos. El algoritmo tiene dos fases, en una primera aplica colonia de hormigas para realizar una aproximación global, basada en los principios de discretización de los elementos finitos, borrando los subespacios no factibles, reduciendo así el espacio de soluciones y consecuentemente el tiempo; y en una segunda fase de búsqueda local, se obtiene un diseño final óptimo a partir de secciones tipo próximas a las secciones obtenidas en la fase anterior. Se compara este método con el ACO estándar, GA y búsqueda armónica, y se comprueba su robustez y la reducción de tiempo obtenido. 


\subsubsection{Estructuras de hormigón}

Las heurísticas aplicadas para la optimización de estructuras de hormigón están basadas, al igual que para las estructuras de acero, en métodos de búsqueda local, en poblaciones, o en una combinación de ambas. A continuación se citan algunas de las publicaciones consideradas más importantes, haciendo constar que casi todas ellas se corresponden con estructuras de hormigón armado. Las de hormigón pretensado son escasas y se muestran todas las que se han encontrado.

- En 1992 Chakrabarty [47] presentó un modelo para optimizar el coste de una viga de hormigón armado sometida a flexión.

- En 1996 Kocer y Arora [89] optimizaron la primera estructura de hormigón pretensado: postes para líneas eléctricas. Aplicaron GA y otro procedimiento denominado Ramificación y Poda.

- En 1997 Coello et al. [39] utilizaron GA para la optimización de una viga biapoyada de hormigón armado sometida a momento flector. Las variables empleadas fueron continuas. Manejaron diferentes sistemas de codificación, considerando como variables la geometría de la viga y su armadura inferior. Como conclusión, se llegó a que los GA podían ser una herramienta eficiente para problemas que requiriesen una búsqueda en un espacio de soluciones, y que un ajuste de los parámetros del algoritmo permitían llegar de manera rápida a soluciones de baja calidad, o de manera lenta a mejores soluciones.

- En 1998 Leite y Topping [94] presentaron mejoras en los algoritmos genéticos clásicos para problemas de optimización estructural, aplicando por segunda vez la técnica heurística al hormigón pretensado. Entre los ejemplos expuestos para mostrar la eficacia de este algoritmo mejorado utilizaron las vigas pretensadas continuas de tres vanos del trabajo de Cohn y Lounis [42] (1993), que resuelven para tres casos. En el primero se mantiene fija la geometría y se minimiza el acero en pretensado y armadura pasiva de flexión. En este caso se obtienen diferencias respecto al óptimo de Cohn inferiores al $1.0 \%$. En el segundo caso se liberan las dimensiones de la pieza, y permitiendo reducciones en los espesores del alma y de las alas superiores e inferiores de la viga, se obtienen mejoras del $19 \%$. Y en el tercero, añadiendo tres variables más y nuevas restricciones para el dimensionamiento completo de la viga, aumentan hasta un $21 \%$.

- En 1998 Rafiq y Southcombe [143] optimizaron mediante GA el armado de pilares sometidos a flexocompresión esviada. La geometría y el tipo de hormigón del pilar son considerados como parámetros y el armado como variable. Utilizan las especificaciones del British Standard (BS8110) satisfaciendo los requerimientos de ELU y la constructividad de las secciones. Aplicaron esfuerzos (axil y momentos en las dos direcciones) a cuatro secciones diferentes que se optimizaban, comparando los resultados obtenidos utilizando como módulo de comprobación el método simplificado de la BS8110 para la resolución de flexocompresión esviada, y un programa de ordenador que la resuelve de manera exacta mediante equilibrio de tensiones y deformaciones de la sección. Como conclusiones señalan que el 
método simplificado debe ir acompañado de otro que compruebe las secciones, especialmente en columnas con grandes secciones transversales, el método desarrollado minimiza las barras de refuerzo optimizando las secciones para los esfuerzos que la solicitan.

- En 1998 Rajeev y Krishnamoorty[144] aplicaron GA en la optimización de dos pórticos planos de hormigón armado consistentes en tres vanos y cuatro plantas, el primero de ellos, y de dos vanos y seis plantas el segundo. Destacan el uso de variables discretas con el objeto de no requerir modificaciones de la estructura obtenida a la que se vaya a construir. Tienen en cuenta aspectos de construcción como la colocación de las armaduras en los pilares y vigas. Consideran un único caso de carga y no consideran como variables la armadura de cortante ni la calidad del hormigón. Agrupa vigas iguales y pilares para reducir el número de variables. Llegan a la conclusión de que GA es una herramienta apropiada para la resolución de este tipo de problemas, haciendo especial énfasis en que la optimización debe enfocarse a estructuras construibles.

- En 2001 Ceranic et al. [35] aplicaron SA para la optimización económica de muros ménsula de hormigón armado proyectados para la retención de tierras en carreteras. Las variables consideradas son todas relativas a la geometría, incluyendo el armado. Consideran, además, estados límite debidos a la geotecnia del terreno, tales como tensiones admisibles, deslizamiento $\mathrm{y}$ vuelco. La función objetivo considera el coste del acero y del hormigón.

- En 2003 Chau y Alberman [48] diseñaron un modelo híbrido, consistente en la utilización del Abaqus para el cálculo estructural y GA para realizar la optimización, en el estudio del diseño optimizado de depósitos rectangulares de hormigón armado. Se hace un análisis optimizado del coste de la estructura considerando únicamente tres variables: canto de las losas de hormigón, diámetro de la armadura y separación entre las barras, adoptando los mismos valores para la losa de cimentación y el alzado de los depósitos. Los resultados obtenidos, permiten mostrar la idoneidad del método como herramienta a utilizar en el proyecto de estructuras.

- En 2003 Leps y Sejnoha [95] optimizaron una viga continua de dos vanos simétrica de hormigón armado, teniendo en cuenta el armado de flexión y el de cortante. El objetivo es encontrar la viga con el menor coste posible que resista los esfuerzos y cumpla las condiciones de servicio para el nivel de cargas aplicado. El algoritmo de optimización aplicado es el Augmented Simulated Annealing que combina el SA con el GA, utiliza el principio del SA pero trabajando con una población de soluciones (en vez de con una única solución) y las nuevas soluciones se obtienen a partir de las existentes mediante la aplicación de los operadores genéticos (y no mediante movimientos). La aplicación de este método la justifican ante la posibilidad de poder mejorar los resultados obtenidos utilizando únicamente GA. En el coste de la estructura no consideran el encofrado ni las cimbras, las comprobaciones se realizan para un único estado de carga y no consideran como variable el tipo de hormigón ni contemplan la posibilidad de que las 
armaduras longitudinales se adapten a los momentos flectores según las secciones, siendo este armado constante y simétrico.

- En 2003 Lee y Ahn [93] utilizaron GA, incluyendo una estrategia elitista, para el diseño optimizado de pórticos de hormigón armado. Los casos de carga utilizados son los de la normativa norteamericana (International Conference of Building Officials, 1997). El algoritmo sólo puede utilizar ciertas vigas y pilares dispuestos en una base de datos, lo que reduce el espacio de soluciones y la posibilidad de variación de la armadura en una viga o pilar a lo largo del mismo. Sólo se consideran los esfuerzos de flexión de ELU, sin considerar ni el cortante, ni los ELS de deformación y fisuración.

- En 2003 Camp et al. [26] optimizaron diversas estructuras planas de hormigón armado mediante GA. A diferencia de Lee y Ahn (2003), las flechas de las vigas se verifican a través de la imposición de un canto mínimo, y tampoco consideran, ni ELS de fisuración, ni la posibilidad de disponer varios tipos de hormigón. Disponen los pilares de una misma alineación, iguales para todas las plantas y mismo armado longitudinal para todas las vigas de una misma planta.

- En 2004 Kong [90] utilizó GA para optimizar la estructura de un edificio de hormigón armado cuya única restricción era el desplazamiento relativo entre dos plantas. La función objetivo a minimizar fue el peso del edificio. Considera como parámetros las dimensiones de las vigas y el ancho de los pilares, dejando como única variable el canto de los pilares. Sólo dimensiona la geometría de la estructura, sin tener en cuenta el armado.

- En 2004 Sahab et al. [149] presentaron un algoritmo híbrido, que consta de dos etapas, basado en GA para la optimización de estructuras de edificación con forjados tipo losa. En la primera etapa se obtiene el número óptimo de pilares mediante una búsqueda exhaustiva llevada a cabo mediante GA modificado y en la segunda se buscan las dimensiones óptimas mediante GA, discretizando las variables según el método de Hooke y Jeeves [74]. Se realiza el cálculo teniendo en cuenta el British Code BS8110 of Practice. La función objetivo considerada incluye el coste del hormigón, la mano de obra, el acero y la excavación de las cimentaciones. Sin embargo, no consideran la acción de fuerzas horizontales en la estructura, ni el tipo de hormigón como variable. Tampoco se da un armado para las losas.

- En 2004 Miyamoto et al. [116] aplicaron algoritmos inmunes (IA) y GA en la optimización de losas de hormigón armado comparando los resultados obtenidos. Los IA nacen de la idea de que en GA la diversidad de la población desaparece rápidamente en el proceso de búsqueda e intentan mantenerla para evitar que la solución converja de manera prematura. Los resultados obtenidos mediante IA son satisfactorios.

- En 2004 Carrera[112] y en 2005 Carrera et al. [32]-[33] utilizaron FB (Gradiente First Best), SA y TA (Threshold Accepting) para optimizar pórticos de hormigón armado de carretera. Los pórticos optimizados presentan cuantías de acero y esbelteces superiores a las habituales en este 
tipo de estructuras, por lo que se considera necesario realizar la comprobación a fatiga, deformaciones y vibraciones, lo que no es habitual en este tipo de estructuras. Obtienen los mejores resultados con el SA.

- En 2004 y 2006 Perea [46],[131] y en 2005, 2006 y 2007 Perea et al. [[133][135] emplearon FB, SA, TS (Tabu Search), TA, OBA (Old Bachelor Acceptance) y GA a la optimización de marcos de carreteras de hormigón armado. Los marcos optimizados tienden a altas esbelteces y armados, por lo que sería necesaria la comprobación de deformaciones, fatiga y vibraciones que no son habituales en el proyecto de esta estructura. Los mejores resultados se han conseguido con SA, TA y TS, siendo los obtenidos mediante GA de baja calidad. Aplican la metodología a un proyecto de marco realizado en Palma de Mallorca, llegando a ahorros significativos.

En 2007 Perea [132] aplicó en su Tesis Doctoral, entre otros, GA y MA (Memetic Algorithm) para la optimización de marcos de carretera de hormigón armado. En este trabajo se puede observar como los algoritmos genéticos y meméticos son especialmente indicados para una implementación en paralelo, que se beneficia de una importante reducción de los tiempos de ejecución. El mejor resultado se obtiene con los algoritmos meméticos en paralelo, empleando una parametrización del algoritmo que no hubiese sido posible ejecutar en un ordenador convencional (ver Perea et al. [138]).

En 2008 Perea et al. [136], antesala del citado de 2007 (Perea [132]), aunque publicado después, se emplean SA, TA y un algoritmo híbrido que los combina con otro de TS. Con estos algoritmos se optimizan marcos de carretera de hormigón armado, en un problema caracterizado por 44 variables de diseño, entre las que se encuentran dos tipos diferentes de hormigón (en muros y losas). De nuevo, los marcos obtenidos tienden a grandes esbelteces y fuertes armados, lo que hace necesaria la incorporación de dos estados límites que no suelen ser condicionantes en este tipo de estructuras: flechas y fatiga. El trabajo se completa con un estudio paramétrico para diferentes luces y cargas, para el cual el autor adopta un algoritmo híbrido de TA refinado con una TS. Asimismo se muestran dos aplicaciones reales (ver Perea et al. [137]), en las que se consiguieron ahorros en torno al $10 \%$ respecto a la estructura diseñada originalmente.

- En 2005 Alcalá et al. [7][8] optimizaron muros ménsula de hormigón armado mediante FB y SA. De los resultados se desprende que el SA da buenas soluciones y que los muros resultantes son más esbeltos de lo que es la práctica habitual de estas estructuras, por lo que se considera necesario la limitación de flechas en cabeza. Como continuación de este trabajo, Yepes et al. [160](2007) presentaron un estudio paramétrico de muros ménsula, dando tablas de predimensionamientos para muros con diferentes alturas de tierras y distintos terrenos. Se destaca entre las conclusiones del trabajo la poca influencia del tipo del terreno en el coste final del muro.

En 2006 [162] y en 2008 [163] Yepes et al. y, como continuación de trabajos anteriores (Alcalá [9]), aplicaron la cristalización simulada para la optimización de muros ménsula de hormigón armado. En este trabajo se 
empleaban 22 variables para caracterizar un muro, incluyendo la geometría, los armados y los tipos de acero y hormigón. La importante esbeltez a la que tendían los resultados obligó a los autores a incorporar estados límites de flechas. En sus conclusiones destacan la elección de los hormigón de la menor resistencia permitida (25 MPa), y aceros de la mayor posible (500 $\mathrm{MPa}$ ). Igualmente se obtienen muros sin armadura de cortante en todos los casos. El trabajo se completa con un estudio paramétrico que proporciona reglas útiles de predimensionamiento de muros optimizados en función de la altura, las condiciones de empujes del relleno y las condiciones de cimentación.

- En 2005 Carbonell [10][31] aplicó máximo gradiente, SA y TA a la optimización de bóvedas de hormigón armado para pasos de carretera. Obtiene los mejores resultados con SA, mejorando en un $7.60 \%$ el coste de una bóveda proyectada por un ingeniero experimentado.

En 2007, 2008 Carbonell et al. [28][30], y en 2009 Carbonell [27], en su Tesis Doctoral, aplicaron SA y TA a la optimización de bóvedas de hormigón armado para pasos de carretera. El problema tiene 21 variables entre las que se encuentran tres tipos de hormigones. Aplica procesos en paralelo para reducir tiempos de computación, y los mejores resultados se obtienen con SA. En su último trabajo realiza una parametrización de la estructura para distintas alturas de tierras y luces.

- En 2005 Sahab et al. [150] realizaron la optimización de estructuras de edificación con forjados tipo losa, transformando la estructura de tres dimensiones en una plana, mediante el uso de GA. Se trata de minimizar el coste del edificio, incluyendo los forjados, pilares y excavaciones necesarias. El proceso de optimización tiene tres etapas: en la primera se obtiene el número óptimo de pilares mediante una búsqueda exhaustiva, en la segunda, empleando un algoritmo híbrido, se realiza la optimización de las dimensiones de las columnas y espesores de las losas, y en la tercera se busca el armado óptimo para la estructura. Este método se aplica a tres edificios diferentes, comparando los resultados obtenidos con los de un diseño convencional. La norma aplicada es el British Code of Practice (BS8110). No se consideran acciones horizontales aplicadas ni diferentes tipos de hormigón. Realizan un estudio comparativo entre los costes de pilares y forjados, dividiéndolos en hormigón, acero y mano de obra.

- En 2005 Govindaraj y Ramasamy [68] optimizaron vigas de hormigón armado mediante GA utilizando las especificaciones de la norma India (Indian Standard specifications). Se tienen en cuenta, en la realización del cálculo, las condiciones de rotura, servicio, ductilidad, durabilidad y la constructividad de las soluciones. Consideran como variable el canto de la viga, suponiendo el ancho igual para los distintos vanos. La armadura de la viga no se considera como variable, sino que dadas unas dimensiones se realiza el armado óptimo a flexión, mediante la búsqueda en un catálogo de armados, y posteriormente se arma a cortante. No consideran el hormigón como variable ni tienen en cuenta la relación entre la armadura de flexión y la de cortante. Aplican los cálculos a una viga de un vano y a otras dos continuas de dos vanos, siendo 
los resultados obtenidos comparados con otros disponibles en la literatura. Concluyen que el método propuesto es adecuado para el diseño óptimo y económico de este tipo de estructuras en la práctica.

- En 2006 Rafiq et al. [142] desarrollaron una aplicación informática interactiva para la optimización de pilares sometidos a una carga axil y momentos en las dos direcciones mediante GA. La aplicación realiza, en primer lugar, una optimización del armado de la sección para los esfuerzos solicitantes mediante GA y a continuación el ingeniero proyectista puede modificar iterativamente esta configuración definiendo la zona del espacio de soluciones donde se debe centrar la búsqueda. Concluyen que aplicaciones de este tipo pueden servir de ayuda a los proyectistas y ser útiles como herramientas para conocer mejor el funcionamiento de las estructuras.

- En 2008 Martínez [105] en su Tesis Doctoral, utilizó GA, ACO, SA y TA para la optimización de pilas rectangulares huecas empleadas en viaductos, añadiendo un estudio paramétrico. También, en la misma línea, se referencian los trabajos de Martínez et al. [106][107]. Estos algoritmos se comparan entre sí, obteniendo los mejores resultados algunas variantes de los algoritmos de ACO. Dando continuidad a esta tesis, en 2010 Martínez et al. [108] presentaron un trabajo comparando el GA y TA con dos nuevas variantes del ACO, aplicando 95 variables, y recomendando para su calibración 250 hormigas y 100 etapas. La mejor solución, comparada con un diseño realizado por experto, es un $33 \%$ más económica.

- En 2005 y 2007 Payá [75][76] y en 2006 y 2008 Payá et al. [130][125][127][129] estudiaron la optimización de pórticos de edificación de hormigón armado mediante FB, SA, TA, GA y SMOSA (Método de Suppapitnarm para la Optimización Multiobjetivo mediante Cristalización Simulada). Los mejores resultados se obtienen mediante SA, heurística que utiliza para la realización de un estudio paramétrico de pórticos con diferente número de plantas y de vanos, dando las diversas características de los mismos. Realizan un estudio multiobjetivo mediante SMOSA en el que además de realizar la optimización desde el punto de vista de coste de la estructura, tienen en cuenta otros aspectos como la facilidad constructiva, la sostenibilidad ambiental (emisión de $\mathrm{CO}_{2}$ ) y un índice de seguridad global de la estructura. Como conclusiones del trabajo llegan a que el empleo de vigas planas supone un aumento considerable en el coste de las estructuras y que en el entorno de la solución optimizada, desde el punto de vista del coste económico, son posibles estructuras más fáciles de construir, más sostenibles y más seguras con incrementos de coste aceptables en la práctica. En particular, en el trabajo de Payá et al. [128] de 2009 se recogen los resultados de un algoritmo de cristalización simulada multiobjetivo para seis pórticos diferentes. En sus conclusiones estos autores destacan el importante sobrecoste que suponen las vigas planas. Por el contrario, se detecta que es posible mejorar mucho el diseño en cuanto a facilidad constructiva, sostenibilidad y seguridad, sin que ello tenga una repercusión económica importante. 
- En 2009 Martí y González [104] y Martí et al.[103] aplicaron TA y SA a la optimización económica de tableros de pasarelas de viga artesa de hormigón pretensado. Se consideran variables distintas los hormigones de la viga y de la losa, admite 4 niveles de pretensado y también fundas en los torones. Diseña la sección y arma la viga y la losa tanto longitudinalmente como transversalmente. Se tienen en cuenta los ELU y ELS de la norma española en el cálculo de las secciones. No se tiene en cuenta el coste del transporte y de la colocación de la viga. Se obtienen diseños fácilmente construibles.

- En 2009 Atabay [17] presentó la optimización de las dimensiones de muros de arriostramiento horizontal de edificios de hormigón armado. El autor pone un especial énfasis en las ventajas derivadas de emplear un método como los GA, que permiten la formulación discreta del problema. Además no necesitan la evaluación de derivadas ni integrales, lo que los simplifica notablemente.

- En 2009 Barakat y Altoubat [20], optimizaron depósitos cónicos de contención de agua. Para ello se emplearon GA, SA y una técnica conocida como Shuffled Complex Evolution. En sus conclusiones los autores proporcionan interesantes reglas de diseño para este tipo de estructuras, aunque detectan una importante sensibilidad a los precios de los materiales.

- En 2010 Alcalá [6] presentó la aplicación de las heurísticas TA, SA y OBA en la optimización de tableros de puentes losa de hormigón postesado. Se tienen en cuenta los ELU, ELS y fatiga. Diseña la sección y arma completamente el tablero continuo en los distintos vanos. Incluye el coste de la cimbra y encofrado. Compara las tres heurísticas y concluye que los resultados del TA y SA son comparables. Finalmente, realiza un estudio paramétrico para distintas luces y número de vanos en tipologías de pasos superiores de carreteras y ferrocarriles.

\subsection{Conclusiones}

Una vez revisado el estado del arte, en cuanto a la optimización de estructuras, se desprende que:

1. Dentro de la tipología de los materiales en los trabajos de optimización de estructuras, la gran mayoría han sido estructuras metálicas, siendo las soluciones resueltas con hormigón una minoría [40]. Esto es debido a la facilidad existente en la definición de las vigas metálicas (longitud y el tipo de perfil a utilizar).

2. De la optimización de estructuras de hormigón, no muchas más de 30 son de hormigón pretensado (la inmensa mayoría relativas a vigas). Ninguna de las vigas de hormigón está fabricada con refuerzos de fibras de acero (HPF). 
En cuanto a los métodos heurísticos de optimización, se desprende que:

1. La gran mayoría de las técnicas aplicadas son GA.

2. De las aplicaciones heurísticas, sólo una pequeña porción está dedicada a trabajos con estructuras de hormigón.

3. Y de estas aplicaciones heurísticas, sólo se han encontrado 5 referencias en ingeniería [89][104][94][103][6] con estructuras de hormigón pretensado: un poste de línea eléctrica, una viga continua de tres vanos, un tablero continuo de puente y dos pasarelas isostáticas de vigas artesa.

4. No se ha encontrado ningún trabajo de optimización heurística de puentes de carreteras con vigas artesa pretensadas.

Existe cierto consenso en que la variación de los precios de los materiales que componen la estructura de hormigón pretensado no afecta a la solución optimizada[64][146][56], y que para llegar a detectar esta influencia habría que recurrir a una relación de costes entre el hormigón y el acero muy superior a la que existe en la actualidad. Formando parte de esta tesis, se hace un estudio de la sensibilidad de la solución obtenida respecto a la variación de los precios.

En cuanto a la función objetivo de optimización, la mayoría de los trabajos están enfocados al coste de la estructura, aunque avanza el criterio de utilizar funciones multiobjetivo, que incluyen valoraciones medioambientales $\left(\mathrm{CO}_{2}\right)$, seguridad, mantenimiento, vida útil, etc.

Conforme aumenta la potencia de los procesadores, se tiende a eliminar simplificaciones y restricciones, aumentando las comprobaciones de la estructura y también el número de variables (incrementándose el espacio de soluciones).

El uso de aplicaciones heurísticas permite realizar estudios paramétricos de diferentes tipologías estructurales de hormigón, obteniéndose reglas de predimensionamiento que ayudan a los ingenieros proyectistas a reducir costes sin detrimento de calidad y seguridad. Se observa también, que las soluciones optimizadas tienden a reducir espesores y a aumentar cantos, reduciendo el volumen de hormigón a cambio de aumentar la inercia de la sección; ello produce aumento de las esbelteces, y posibles riesgos en pandeos localizados e incremento de flechas. 


\section{CAPÍTULO 3. MÉTODOS HEURÍSTICOS}

En este Capítulo se describe cómo tratan las técnicas heurísticas los problemas de optimización condicionada. Se describen y clasifican los distintos métodos heurísticos, incidiendo en aquellos que se aplican en este trabajo.

\subsection{La optimización mediante técnicas heurísticas}

\subsubsection{El problema de la optimización condicionada}

En la mayoría de las ocasiones, debido al elevado número de variables que participan en la resolución de algunos problemas de optimización reales, no es factible el uso de métodos de solución exacta para la obtención de soluciones óptimas. Suele ser suficiente una solución que esté relativamente cerca de la óptima, si con ello se reduce el tiempo y la complejidad del cálculo. Cuando se utilizan variables discretas, el problema a resolver se plantea sobre un conjunto finito de soluciones, asociados a un conjunto de números reales, cuya combinación trata de optimizar una función objetivo.

La optimización mediante la aplicación de técnicas heurísticas nace de la posibilidad de desarrollar un tipo de técnicas, denominadas metaheurísticas, que no dependen de las características de un problema concreto. Dada su generalidad, con ellas es posible abordar cualquier tipo de problema. Son procedimientos que buscan las soluciones óptimas, o suficientemente próximas al óptimo, mediante estrategias de búsqueda inspiradas en procesos naturales o en la inteligencia artificial. Los tiempos de cálculo de los algoritmos heurísticos, en algunas ocasiones se pueden variar por el usuario y, de esa manera, adaptarse a las circunstancias y calidad de solución requerida en las que se emplea el método. Se han aplicado diferentes métodos heurísticos en otros campos distintos a la optimización estructural, como la hidráulica (Clark et al.[37] (2006)), transporte, electricidad (De Leao [50] (1999)), etc.

Mientras que la técnica heurística consiste básicamente en la exploración del espacio de soluciones con estrategias del tipo prueba-error, pero procurando que cada prueba se realice en base a la experiencia adquirida durante el proceso, la metaheurística es una técnica diseñada para resolver problemas difíciles de optimización combinatoria, en los que los heurísticos clásicos no son efectivos. Los metaheurísticos proporcionan un marco general para crear nuevos algoritmos híbridos combinando diferentes conceptos derivados de la inteligencia artificial, la evolución biológica y la mecánica estadística. Es decir, los metaheurísticos no son métodos de optimización en sentido estricto, sino estrategias en base a las cuales es posible desarrollar algoritmos de optimización. La principal ventaja de este tipo de técnicas es que no imponen ninguna condición en el problema que deben resolver, y ello las hace muy atractivas para la optimización con unos planteamientos tremendamente prácticos. 
En el diseño automatizado del tablero isostático se pueden distinguir los siguientes elementos que se relacionan entre sí en el proceso de optimización.

- Parámetros. $\mathrm{p}=\left(\mathrm{p}_{1}, \ldots, \mathrm{p}_{\mathrm{n}}\right)$. Son magnitudes consideradas constantes y que no varían durante el proceso de optimización. Ejemplos de parámetros son los módulos de elasticidad de los materiales, coeficientes de seguridad, pendiente de las almas de las vigas, etc. Algunos de los parámetros se utilizan para limitar la geometría de la sección: ancho de la losa $(12 \mathrm{~m}$.), pendientes de las cartelas (1:3), almas $\left(80^{\circ}\right)$, etc.

- Variables de diseño. $\underline{\mathrm{x}}=\left(\mathrm{x}_{1}, \ldots, \mathrm{x}_{\mathrm{n}}\right)$. Son las variables objeto de optimización y que junto con los parámetros definen completamente la estructura. Dado que las variables utilizadas son discretas, cada posición del vector de diseño contiene un valor perteneciente a otro vector que contiene los posibles valores de una determinada característica de la estructura (por ejemplo: si $\mathrm{x}_{14}$ define la resistencia del hormigón de la viga, los valores posibles son 35, 40, 45 y 50 Mpa).

- Comprobación de la estructura. Los parámetros fijos, junto con las variables de diseño han de cumplir una serie de condiciones y de restricciones asociadas al uso o servicio que se le da a la estructura. Se refiere a restricciones geométricas (p.ej. número máximo de torones que caben en la viga), y a la comprobación de la estructura frente a los estados límites últimos y de servicio en su uso como puente de carretera. Se puede representar con la función $\mathrm{g}(\underline{\mathrm{x}}, \underline{\mathrm{p}}) \leq 0$

- Función coste (es la función objetivo). Valora el coste del tablero, compuesto por dos vigas pretensadas prefabricadas, una losa ejecutada in situ, y el trasporte y colocación de las vigas. Incluye como parámetros fijos los precios de las unidades utilizadas. Se puede representar como $C=f(\underline{x}, \underline{p})$

A partir de estas definiciones, lo que se pretende es obtener un diseño $\underline{\mathrm{x}}$ que cumpla las condiciones $\mathrm{g}(\underline{\mathrm{x}}, \underline{\mathrm{p}}) \leq 0$, y que se acerque lo más posible al mínimo de la función coste $C=f(\underline{x}, \underline{p})$.

Si $S$ es el espacio de soluciones discretas $\underline{x}$.

$F$ es el espacio de soluciones factibles (cumplen $\mathrm{g}(\underline{\mathrm{x}}, \underline{\mathrm{p})} \leq 0$ ). El espacio de soluciones $S$ se dividirá en dos regiones: factibles e infactibles, dado que no todas las posibles combinaciones $\underline{x}$ representan estructuras que cumplen las restricciones y los estados límite de servicio y últimos. El conjunto de todas las soluciones factibles es el conjunto F, por lo que F es subconjunto de $S$

El diseño optimizado ha de ser una estructura factible, y esto se expresa con la condición de que $\underline{\mathrm{x}}$ sea elemento del conjunto $\mathrm{F}$ de soluciones factibles.

El modo de obtener el coste mínimo, y de un modo sencillo, es el de evaluar la función objetivo para todos los valores posibles del espacio de soluciones $S$, pero este planteamiento es irrealizable en numerosas ocasiones, como en este caso, donde el espacio de soluciones es excesivamente amplio. En nuestro problema, tal como se 
ve en el Capítulo 4, el espacio de soluciones es aproximadamente de $1.6 \times 10^{65}$ soluciones. Si el ordenador utilizado para esta tesis tarda 0.09 segundos en comprobar una solución, tardaría del orden de $4.6 \times 10^{53}$ millones de años para resolver el problema por enumeración.

\subsubsection{Clasificación de las técnicas heurísticas}

Para evaluar la eficacia de este tipo de técnicas de optimización debe considerarse, tanto la calidad de las soluciones que son capaces de encontrar, como del tiempo empleado en su obtención, además de la robustez y flexibilidad. Es por ello, que se estudia la dualidad coste-tiempo en la comparación de ejecuciones de un algoritmo, o en la comparación entre algoritmos, y para su análisis se emplean los diagramas de óptimos de Pareto -"Se dice que una solución S1 es pareto-óptima cuando no existe otra solución $S 2$ tal que mejore en un objetivo sin empeorar en otro"-.

Existen en la actualidad numerosas técnicas de aproximación que han sido probadas con éxito en diferentes problemas de optimización. Una clasificación posible, es la propuesta por Yepes [161] (2002) mostrada en la Figura 3-1:

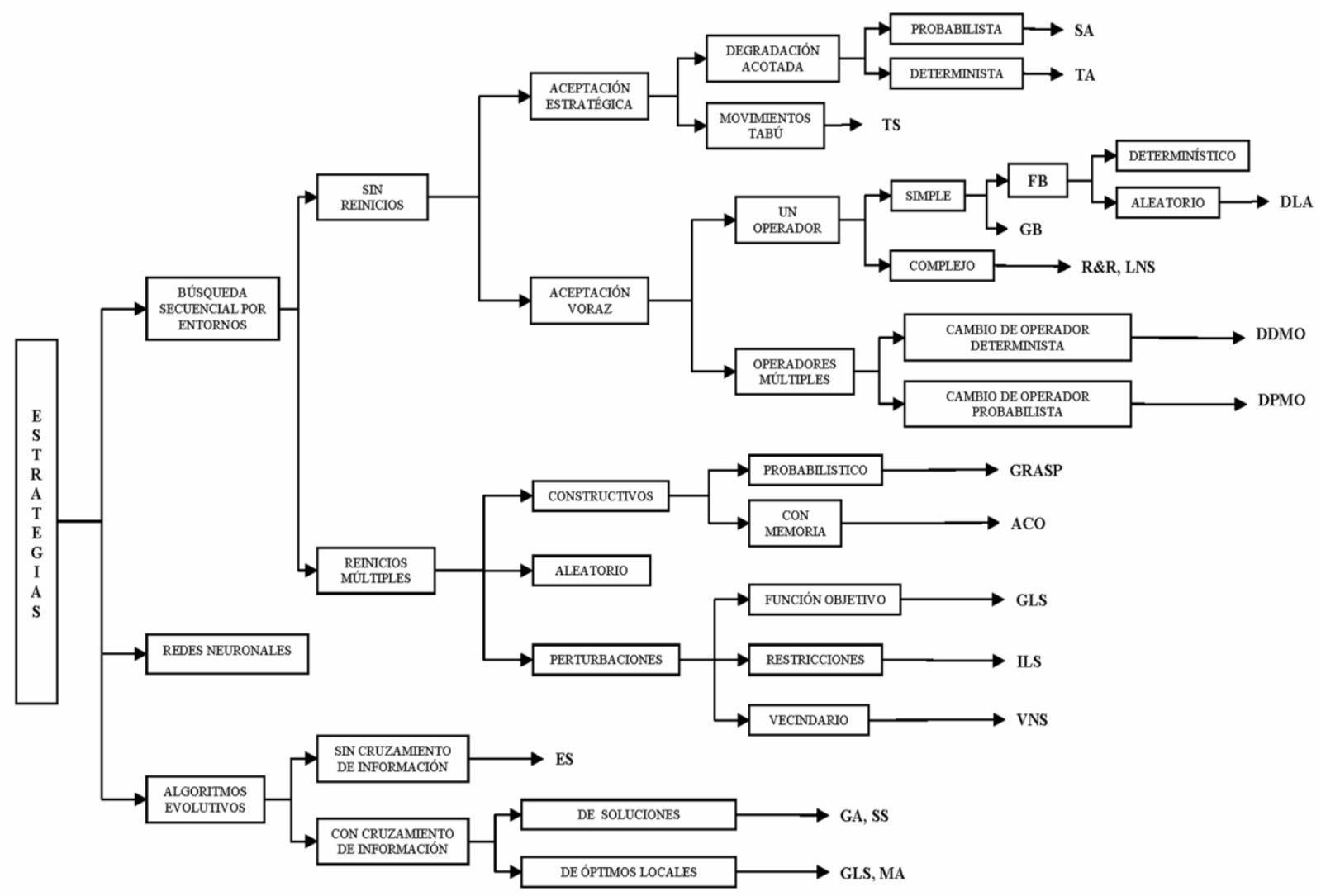

Figura 3-1: Clasificación de las técnicas de optimización heurísticas 
Las metaheurísticas se pueden subdividir en los siguientes subgrupos:

- Métodos de búsqueda secuencial por entornos

- Métodos basados en poblaciones (algoritmos evolutivos)

- Redes neuronales

El primer método estudia el espacio de soluciones mediante la exploración del entorno asociado a una solución de partida. Busca una solución que mejore a la inicial mediante movimientos discretos. Este proceso repetido varias veces puede llevar a encontrar soluciones de calidad para el problema.

Los métodos basados en poblaciones son evolutivos, partiendo de una población inicial (grupo de soluciones) trata de mejorarlas llegando a poblaciones mejores, en las que puede que destaque algún miembro de la misma, el cuál es el candidato a encontrar el óptimo.

La misión principal de las redes neuronales consiste en simular las propiedades observadas en los sistemas neuronales biológicos a través de modelos matemáticos recreados mediante mecanismos artificiales. Inicialmente, no fueron desarrolladas como técnicas de optimización sino para aplicaciones en las que no se dispone a priori de un modelo identificable pero sí de un conjunto de datos. Sin embargo se pueden emplear en la optimización de funciones.

En este trabajo se van a aplicar algoritmos de búsqueda secuencial por entornos y algoritmos evolutivos. En el problema estructural que aquí se trata no es posible conocer la factibilidad de la solución hasta que ésta ha quedado completamente definida, lo que a priori hace prever poca eficacia de algoritmos constructivos. No obstante, se van a emplear algunos principios básicos del GRASP para facilitar, al principio de cada heurística, una generación automática de soluciones.

\subsection{Métodos de búsqueda secuencial por entornos}

Resulta evidente que deben establecerse estrategias de búsqueda de óptimos que no estén basados en el azar y que no precisen la evaluación de todo el espacio de soluciones tal como se ha visto anteriormente. Se define el concepto de movimiento de una solución como la operación de modificar las variables $\underline{x}$ dentro de pequeños intervalos. Si las modificaciones son pequeñas la solución final se parecerá a la de partida, si las modificaciones fueran grandes la nueva solución no se parecería nada a la anterior, perdiendo toda la información. Definido un movimiento y una solución de partida, aquellas soluciones que pueden ser definidas a partir de aquella aplicando ese movimiento constituyen el entorno de soluciones asociado a la primera solución.

En este apartado se describen diferentes métodos de búsqueda secuencial por entornos, los cuales exploran una sucesión de entornos coligados a una solución de partida. En cada uno de ellos se define la forma en que se realiza la búsqueda, que se compone de dos elementos principales: el primero consiste en definir el 
procedimiento de selección de la nueva solución y el segundo el modo de aceptación y un apropiado criterio de parada.

\subsubsection{Búsqueda por gradiente}

El espacio de soluciones se puede recorrer mediante la exploración del entorno asociado a una solución dada. Se trata de encontrar una solución del entorno que mejore a la solución actual en su coste. Este proceso repetido sucesivas veces puede asimilarse a realizar un recorrido por el espacio de soluciones en trayectorias descendentes, es decir, gradientes negativos, debido a que buscamos minimizar la función coste. A este tipo de técnicas se les denomina búsqueda por gradiente. La solución inicial es mejorada a medida que avanza el proceso, hasta que no es posible mejorarla más. La calidad de la solución obtenida dependerá, en gran medida, de la adecuada elección de los movimientos. En este trabajo se aplica esta técnica con el objeto de estudiar los movimientos que resultan más prometedores.

Con la técnica de búsqueda por gradiente se pueden alcanzar soluciones que constituyen óptimos en el entorno de soluciones asociados a ellos, siempre que la búsqueda dure el tiempo suficiente. Se corre el riesgo que estas soluciones sean óptimos de baja calidad debido a una convergencia prematura sin que la técnica del gradiente pueda escapar de ellos. Ver Figura 3-2.

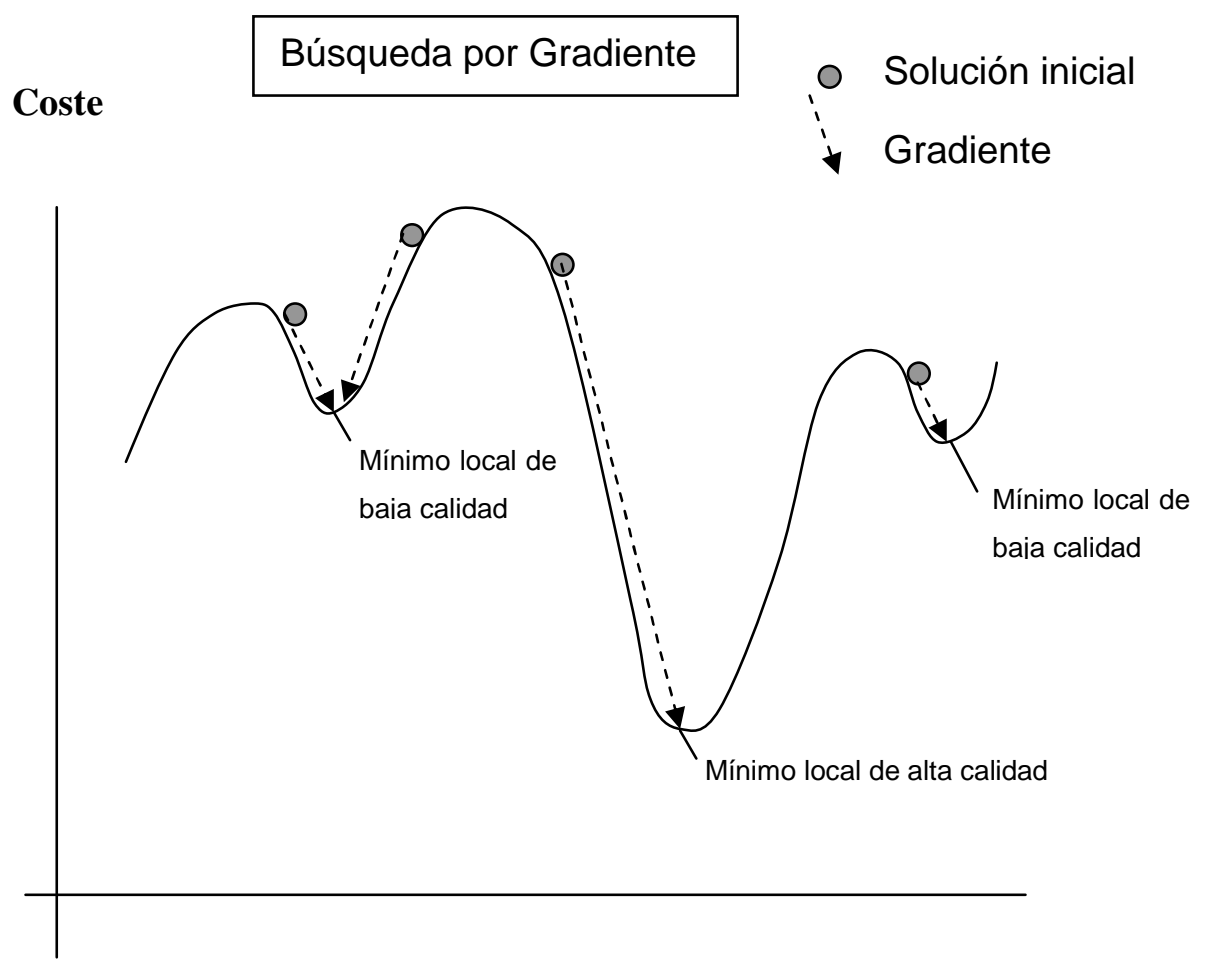

Figura 3-2: Búsqueda por gradiente. Óptimos locales 
Se pueden utilizar dos métodos para aplicar el movimiento:

- First Best (FB): Se aplica a la primera de las soluciones, sin analizar el resto del entorno.

- Global Best (GB): Se selecciona la mejor de todas las soluciones del entorno, y se aplica el movimiento.

Para resolver la convergencia prematura a soluciones de baja calidad, se han planteado diversas formas que mejoran las técnicas de gradiente, aceptando la degradación estratégica para salvar óptimos locales.

\subsubsection{Cristalización simulada}

Esta metaheurística fue propuesta inicialmente por Kirkpatrick, Gelatt y Vecchi [85] en el año 1983, y simultáneamente por Cerny [36] en 1985. "Annealing", en castellano "recocido", es el proceso químico de calentar y enfriar un material de manera controlada. Cuando un metal se calienta por encima de su punto de fundición sus propiedades cuando se enfríe dependerán, en gran medida, del proceso de enfriamiento. En el punto de fundición una gran cantidad de energía está presente en el material. Las moléculas se podrán mover libremente. Si se reduce la temperatura se reducirá la energía en el sistema y simultáneamente los movimientos del material. Si el metal se enfría lentamente se formarán cristales cuya disposición es la de energía mínima. Si se enfría demasiado rápido, las moléculas pueden llegar a estados meta-estables, sin alcanzar configuraciones adecuadas.

Metrópolis et al. (1953) [111] presentaron un algoritmo para la modelización del proceso de recocido mencionado anteriormente simulando los cambios energéticos en un sistema de partículas conforme decrece la temperatura hasta que converge a un estado estable de energía mínima. El SA es similar a la búsqueda por gradiente, pero introduce la aceptación probabilística de soluciones de peor calidad durante el proceso. Para ello acepta soluciones peores con una probabilidad $\mathrm{P}(\Delta \mathrm{E})$ dada por la expresión de Boltzmann:

$$
\mathrm{e}^{-(\Delta \mathrm{E} / \mathrm{T})}
$$

donde $\Delta \mathrm{E}$ se corresponde con la diferencia de dos niveles de energía, y que equivale al incremento del coste de la estructura, y $\mathrm{T}$ un parámetro denominado temperatura. En el algoritmo de Metrópolis se genera una perturbación aleatoria en el sistema y se calculan los cambios de energía resultantes: si hay una caída energética, el cambio se acepta automáticamente, por el contrario, si se produce un incremento energético, el cambio se acepta con una probabilidad dada por la ecuación anterior. El proceso se repite durante un número predefinido de iteraciones en series decrecientes de temperaturas, hasta que el sistema esté con la temperatura nula. El admitir soluciones peores evita la convergencia prematura a óptimos locales, lo cual favorece encontrar soluciones de mayor calidad. En este trabajo se ha aplicado la búsqueda SA.

Se puede demostrar matemáticamente que el algoritmo converge a la solución óptima si se cumplen ciertas condiciones (Aarts et al.[2](1989)). Como una de las condiciones es un enfriamiento infinitamente lento y esto corresponde a tiempo infinito la demostración no tiene ninguna importancia práctica. Esta heurística aplica 
el concepto de cadena de Markov como número de procesos aleatorios para una misma temperatura. Las cadenas de Markov aplicadas son homogéneas y con un número de ejecuciones finito, por lo que se limita el proceso de enfriamiento a un valor finito de la temperatura.

\subsubsection{Aceptación por umbrales}

Se trata de un procedimiento similar a la cristalización simulada SA y fue propuesto por Dueck y Scheuer[55] (1990) como versión parcialmente determinista de SA. La principal diferencia es el criterio con el que se aceptan soluciones modificadas. TA aceptará las nuevas soluciones si mejoran en coste la anterior o si el empeoramiento es menor que un umbral prefijado, de lo contrario se mantiene la solución actual. De esta manera en cada iteración se aceptan movimientos que mejoran la solución actual con más probabilidad que los movimientos de empeoramiento. Un parámetro de control irá disminuyendo el umbral de aceptación conforme avanza el proceso.

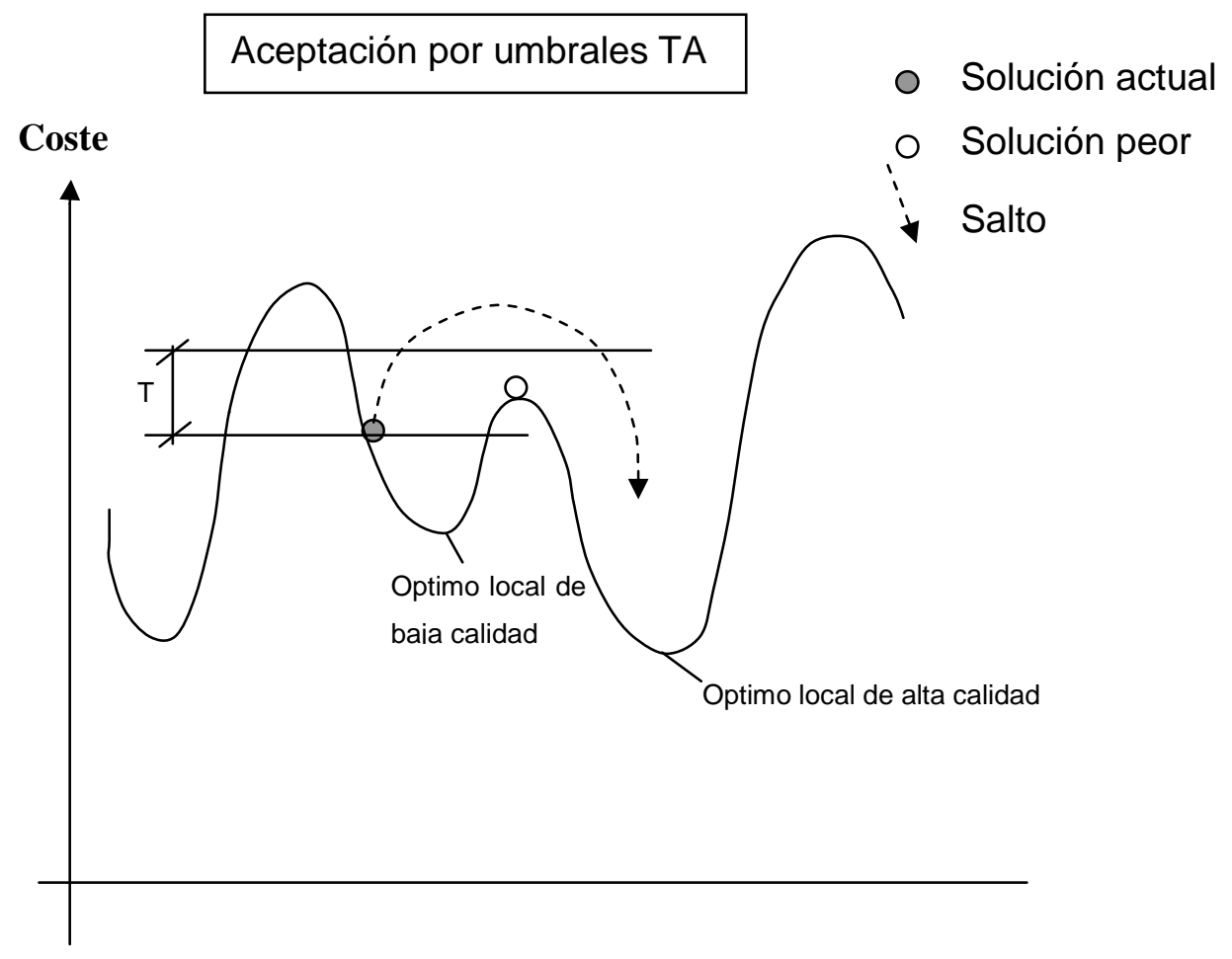

Figura 3-3: Búsqueda por aceptación por umbrales

Tanto SA y TA son métodos de optimización cuya convergencia se demuestra bajo determinadas condiciones. En el caso del TA, Althofer y Koschnick[14] demostraron que a tiempo infinito converge al óptimo global. Desde el punto de vista práctico, las demostraciones de convergencia al óptimo global, no deben sobrevalorarse porque se cumplen para tiempos de cálculo no aplicables en la realidad. En este trabajo se ha utilizado la búsqueda TA. 


\subsubsection{Búsqueda Tabú}

Introducida por Glover [59] (1983), esta estrategia se basa en una memoria que guía la búsqueda hacia zonas del espacio de soluciones aún no exploradas, evitando que la búsqueda repita soluciones visitadas previamente. Introduce dos tipos de memoria: a corto plazo y a largo plazo. A corto plazo guarda en una lista tabú las soluciones visitadas recientemente, así genera un entorno reducido que será el usual eliminando las soluciones tabú. A largo plazo, de manera que se identifiquen y mantengan aquellos atributos que inducen una cierta estructura beneficiosa para las soluciones. La memoria a largo plazo tiene dos estrategias asociadas: Intensificar, regresando a regiones ya exploradas para estudiarlas en profundidad y diversificar, visitando nuevas áreas no exploradas.

\subsubsection{Algoritmo del diluvio universal}

Se aceptarán soluciones peores siempre que éstas no superen un valor máximo (o mínimo, si se maximiza) establecido de antemano y decreciente (creciente) durante el proceso. Difiere del TA en que lo que se limita no es la diferencia de coste entre dos soluciones consecutivas, sino el coste neto de la segunda solución que en ese momento se estudia (Dueck [54], 1993).

Su analogía con un fenómeno natural es la siguiente: cuando un paisaje montañoso se va inundando, si la cota de agua asciende lo suficientemente lenta permitirá divisar y refugiarse en el punto más alto (óptimo global). Si la cota asciende muy rápida nos tendremos que refugiar en el punto más alto cercano (óptimo local) sin tener la posibilidad de bajar al valle para alcanzar un punto más alto que el de nuestro entorno. En ningún caso se podrá bajar por debajo de la cota ya alcanzada por el nivel de agua.

\subsubsection{Aceptación por cercanía al líder}

Se basa en las técnicas del TA y del GDA. A partir del coste de la mejor solución obtenida durante el proceso se acepta cualquiera que su coste no difiera más de un determinado umbral. Conforme el proceso avanza el umbral disminuirá y la mejor solución mejorará. Difiere del TA en que el umbral se valora como la diferencia de coste entre la nueva solución y la mejor obtenida durante el proceso.

\subsubsection{Procedimientos GRASP}

Propuesto por Hart and Shogan[77] (1987) combinan la idea de búsqueda aleatoria y de una búsqueda por gradiente. Se basa en la generación de soluciones mediante técnicas probabilísticas, a las que aplica después algún algoritmo de mejora local. Se consigue una muestra representativa de las mejores soluciones con una alta probabilidad de que alguna de ellas sea de gran calidad.

\subsubsection{Búsqueda local iterada}

Se basa en emplear como espacio de soluciones el de los óptimos locales. Para cada solución de partida es posible alcanzar un óptimo local empleando cualquier técnica, por ejemplo el gradiente. Una vez alcanzado el óptimo local se le aplica una perturbación que modifica la solución a otra diferente a la que le corresponde otro 
óptimo local próximo al anterior. Operando de este modo, se puede rastrear un número significativo de óptimos locales y elegir el de mayor calidad.

\subsubsection{Búsqueda en entornos variables}

Esta técnica fue propuesta, inicialmente, por Mladenovic y Hansen[117] (1997) . Se basa en la idea de que se puede salir de un óptimo local si se aplica otro operador diferente para explorar el entorno de una solución, que aquel que permitió encontrarlo. Alternando sucesivamente dos o más operadores se consiguen alcanzar óptimos de calidad.

\subsection{Algoritmos evolutivos}

Los algoritmos evolutivos están basados en poblaciones, y requieren un ajuste en la forma de valorar las mejores soluciones de cada una de las poblaciones (tamaño, criterio de cruce, de selección, etc.,). Si se sobrevaloran las mejores soluciones se puede caer en una convergencia prematura a una determinada solución y si no se valoran lo suficiente puede llevar a un desconcierto del algoritmo en la búsqueda.

\subsubsection{Colonia de Hormigas}

Es un método metaheurístico con población de soluciones. Fue inicialmente propuesto por Dorigo et al.[53] (1996). Trata de simular el comportamiento de algunas colonias de insectos que optimizan el camino de búsqueda de la comida.

Las hormigas optimizan el trabajo de la colonia mediante el intercambio de señales químicas (feromonas). En primer lugar exploran el entorno de su hormiguero de forma aleatoria. Tan pronto como un individuo encuentra una fuente de comida, evalúa su cantidad y calidad y transporta un poco al hormiguero. Durante el regreso la hormiga deja por el camino un rastro oloroso. Después de un tiempo, el rastro oloroso depositado en el camino hacia el alimento crece con el número de hormigas que pase por él, y desaparece en caso contrario. El resultado final es la consecución de rutas casi óptimas entre dos puntos. Las hormigas son cooperativas, y trabajan hacia un objetivo común.

\subsubsection{Algoritmos genéticos}

Esta técnica metaheurística fue propuesta por John Holland[73] (1975). Inspirándose en el proceso observado en la evolución natural de los seres vivos. Mediante el cruzamiento genético y la mutación, los indivíduos de una población evolucionan creando individuos mejor adaptados. Cada individuo en una población se ve afectado por el resto (compitiendo por recursos, emparejándose para procrear, huyendo de los depredadores, etc..) y también por el entorno (disponibilidad de comida, clima, etc..). Los individuos mejor adaptados son los que tienen mayores posibilidades de vivir más tiempo y reproducirse, generando una progenie con su información genética.

Los GA se inician con una población de partida teniendo soluciones de diferentes calidades (fitness). De esta población se seleccionan parejas de soluciones en función 
de sus calidades. Las soluciones generadas a partir de otras, heredarán las cualidades de ambas.

En algunos casos se aplica sobre la nueva solución un operador de mutación, que modifica ligeramente de manera aleatoria la nueva solución. De las soluciones de la población anterior y las soluciones creadas mediante combinación y mutación se seleccionan las que formarán parte de la siguiente población. En la mayoría de los casos la nueva población se selecciona de manera elitista, asegurando la supervivencia de las mejores soluciones de la población actual. Esta técnica se aplica en este trabajo.

\subsubsection{Búsqueda dispersa}

Esta técnica se debe a Glover [61][63] (1977). Es similar a los algoritmos genéticos GA, pero a diferencia de ellos sustituye el cruzamiento de soluciones por la combinación lineal, no estando fundamentado en la aleatorización sobre un conjunto relativamente grande sino en elecciones sistemáticas y estratégicas sobre un conjunto reducido. Para realizar una adecuada exploración del espacio de soluciones, se mantienen las soluciones entre padres, pero no sólo las mejores soluciones, sino también, aquellas que son más diferentes, de esta manera se evita explorar regiones donde ya se han alcanzado óptimos locales.

\subsubsection{Algoritmos meméticos}

En su libro "El gen egoísta", Dawkins[49] (1976) inventó el término meme para describir una unidad de evolución cultural humana análoga a los genes, argumentando que la replicación también ocurre en la cultura, aunque en un sentido diferente. $\mathrm{Al}$ igual que en los $\mathrm{GA}$, los algoritmos meméticos se inspiran en el proceso evolutivo, pero en este caso de la evolución cultural. Pablo Moscato[119] (1989) explica la estrategia evolutiva de los algoritmos meméticos con la metáfora de la evolución de las artes marciales. La evolución de los artes marciales se ha desarrollado en un tiempo relativamente corto comparado con la evolución biológica. $\mathrm{Su}$ mejora no ha sido un proceso aleatorio sino guiado, y sólo los grandes maestros han tenido suficiente conocimiento para mejorar un estilo existente.

En los MA se crea una población de manera aleatoria o siguiendo un procedimiento de inicialización. Cada individuo es mejorado mediante un proceso de búsqueda secuencial por entornos para obtener un óptimo local o una solución de determinada calidad. En la siguiente fase los individuos de la población interaccionan. Esta interacción se puede desarrollar de dos maneras distintas: una manera competitiva, en la que los individuos son eliminados de la población después de la competición con otro (para mantener el tamaño de la población el individuo perdedor se sustituye por un clon del individuo ganador), o de manera cooperativa, donde los componentes de diferentes individuos son intercambiados con la esperanza de que la combinación de dos componentes pueda evolucionar a soluciones mejores que las de los dos padres; en ese caso uno de los padres se reemplazaría por esta solución. El intercambio de componentes de diferentes individuos puede producir soluciones no factibles, con la necesidad de crear un procedimiento de reparación o una penalización respecto de los individuos factibles. 


\subsubsection{Reencadenamiento de trayectorias}

Esta técnica denominada Path Relinking (PR), se debe también a Glover[63][62] (1998). En ella, se sustituye la combinación lineal por trayectorias que conectan dos soluciones padre. Se fundamenta en que entre dos soluciones buenas existen soluciones que puedan ser mejores. Consiste en realizar el proceso de transformación de una solución en otra, con la esperanza de encontrar alguna intermedia que sea mejor.

\subsection{Redes neuronales}

Las redes de neuronas artificiales (RNA) son un paradigma de aprendizaje y procesamiento automático inspirado en la forma en que funciona el sistema nervioso de los animales. Se trata de un sistema de interconexión de neuronas en una red que colabora para producir un estímulo de salida. En la Figura 3-4 se muestra el esquema de una red neuronal.

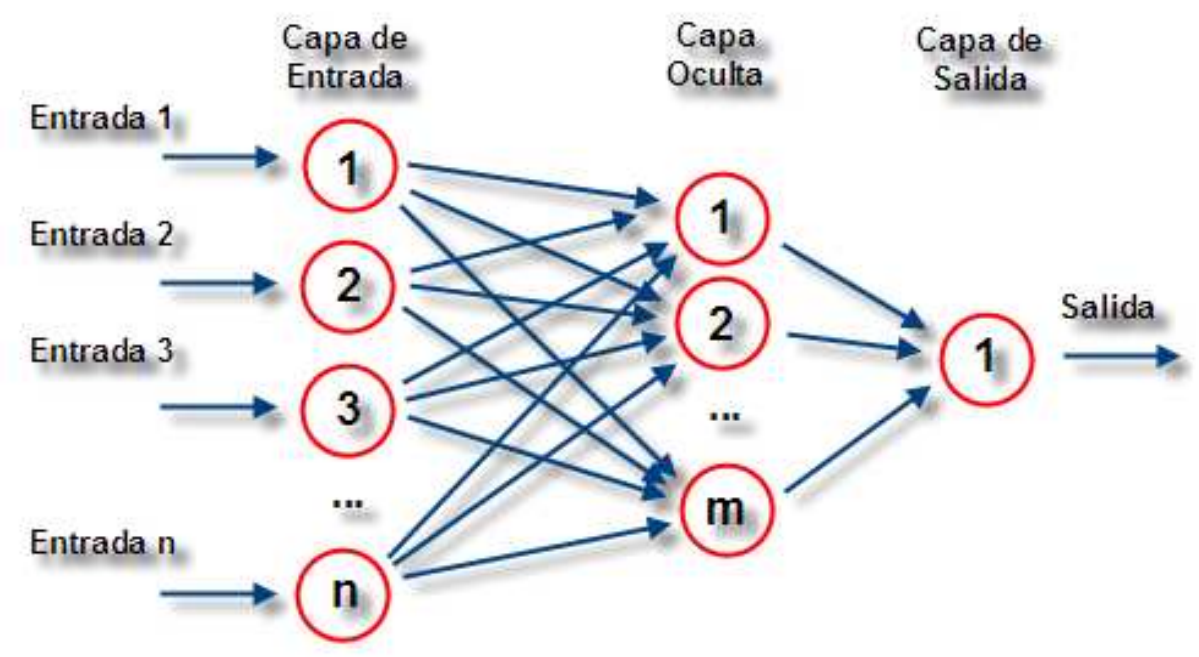

Figura 3-4: Red neuronal artificial con $n$ neuronas de entrada, $m$ en su capa oculta y una de salida.

A partir de un conjunto de datos de entrada importante, el objetivo será conseguir que la red aprenda mediante un adiestramiento las propiedades deseadas. Las características de las RNA las hacen apropiadas para aplicaciones en las que no se dispone, a priori, de un modelo identificable que pueda ser programado, pero se dispone de un conjunto de ejemplos de entrada. También han sido utilizadas en la aplicación a diversos problemas de optimización. Desde el punto de vista de las aplicaciones prácticas, la ventaja de las RNA reside en el proceso paralelo, adaptativo y no lineal de las mismas, siendo capaces de crear un modelo a partir de un número de datos suficiente, razón por la cual se han empleado con éxito en un gran número de especialidades. 


\section{CAPÍTULO 4. DEFINICIÓN DEL PROBLEMA DE OPTIMIZACIÓN}

En este Capítulo se describen las variables y los parámetros que delimitan el problema de optimización. Se muestra como se define la geometría de la sección y las armaduras activas y pasivas, y se determina el método para la obtención del coste de la estructura.

\subsection{Definición del problema}

La sección del tablero se compone de dos vigas artesa pretesas prefabricadas, y de la losa de hormigón que se ejecuta in situ sobre las vigas. Para la evaluación del coste del tablero, será necesario definirlo mediante un grupo de variables; y además, dependerá de una serie de parámetros que tienen un determinado valor y son inalterables durante todo el proceso de optimización. La función objetivo es el Coste (C), definido por la siguiente expresión:

$C=f\left(\mathrm{x}_{1}, x_{2}, \ldots x_{n} ; \mathrm{p}_{1}, p_{2}, \ldots p_{m}\right)$

Sujeta a las restricciones del problema (definidas en el Capítulo 5):

$$
g\left(\mathrm{x}_{1}, x_{2}, \ldots x_{n} ; \mathrm{p}_{1}, p_{2}, \ldots p_{m}\right) \leq 0
$$

Tal como se han definido anteriormente las expresiones:

- Parámetro. Dato $\left(\mathrm{p}_{\mathrm{i}}\right)$ que, en una familia de elementos, sirve para identificar cada uno de ellos mediante su correspondiente valor.

- Variable. Magnitud $\left(\mathrm{x}_{\mathrm{i}}\right)$ que puede tener un valor cualquiera de los comprendidos en un conjunto.

En este estudio, el número de parámetros es de 23; 10 parámetros geométricos, 3 de carga, 3 de coste, 6 de armado y 1 parámetro relacionado con el ambiente de exposición. El problema tendrá 59 variables, que se pueden ordenar en variables geométricas (7), variables relacionadas con la calidad del hormigón (2), con la armadura activa (4) y con la pasiva (46). 


\subsection{Parámetros}

Los parámetros son todos aquellos datos necesarios para definir un problema concreto, siendo invariables en el proceso de cálculo. Mediante la fijación de los parámetros se define un problema determinado dentro del grupo de posibles problemas de optimización. La división en parámetros y variables no es estricta; algunas variables del problema se podrían haber fijado con unos valores determinados y convertirse en parámetros, y en el caso de los parámetros, como algunas de las dimensiones de la sección de las vigas o la separación entre ellas, podrían haberse considerado como variables. La casuística que puede presentar un paso superior es muy amplia, y con el fin de limitar los casos a estudiar, y sin que ello suponga pérdida de generalidad en la investigación, solamente se ha contemplado el caso de tableros de directriz recta y sin esviaje. Los 23 parámetros con los que se ha definido este problema son los siguientes (Tabla 4-1): 
Parámetros

Geométricos (10)

Ancho del tablero

Separación entre vigas

Inclinación alma ( ${ }^{\circ}$ sexagesimales)

Pendiente cartela ala superior (1: ns3)

División base ala superior

Pendiente cartela ala inferior (1: ni3)

División base ala inferior

Luz de la viga

Entrega de la viga

Esbeltez mínima viga
Notación y valores

$$
\begin{aligned}
& \text { b_total }=12.00 \text { metros } \\
& \mathrm{sv}=6.0 \mathrm{~m} . \\
& \mathrm{Ia}=80^{\circ} \\
& \mathrm{ns} 3=3 \\
& \mathrm{~s} 3=3 \\
& \mathrm{ni} 3=3 \\
& \mathrm{i} 4=4 \\
& \mathrm{~L}=35.00 \text { metros } \\
& \text { Ent }=0.47 \text { metros } \\
& \text { Esb }=(1 / 18) \mathrm{L}
\end{aligned}
$$

De carga (3)

Ancho de las barreras

a_bar $=2 \times 0.5 \mathrm{~m}$.

Espesor nominal del pavimento

e_pav $=9 \mathrm{~cm}$.

Carga muerta no procedente del pavimento

$\mathrm{Qm}=2 \times 5.0 \mathrm{kN} / \mathrm{m} 2$

De coste (3)

Distancia transporte (ida)

d_transporte $=20 \mathrm{Km}$.

Despunte armadura activa

$25 \%$

Factor dificultad montaje viga

f_mon $=1.0$

De armado (6 parámetros)

Tipo de acero pasivo (B-500-S)

fyk $=500 \mathrm{~N} / \mathrm{mm} 2$

Tipo de acero activo (Y1860-S7)

$\mathrm{fpk}=1700 \mathrm{~N} / \mathrm{mm} 2$

Diámetro torones acero activo

$\Phi=0.6 "$

Armadura piel viga

$\Phi=8 \mathrm{~mm}$

Fundas torones

Nivel 2 y 3

Esbeltez vertical cercos

200 (longitud / $\Phi$ )

De exposición (1 parámetro)

Ambiente de exposición externo

Aex

Tabla 4-1: Parámetros 


\section{DESCRIPCIÓN:}

\section{Parámetros geométricos (10 parámetros)}

Se fijan como parámetros algunas de las dimensiones de la sección de la viga y de la losa: anchura de la losa, separación entre vigas, inclinación de las almas y de las cartelas, entrega de las vigas, limitación de la esbeltez de la viga. Las cartelas de las vigas arrancan desde la primera división externa de las alas. Los valores para estos parámetros, en el problema tratado, serán los mostrados anteriormente en la Tabla 41. Estos valores se encuentran en un fichero externo que puede modificarse para adaptarse al proceso de fabricación de la viga.

\section{Parámetros de carga (3 parámetros)}

El ancho de las barreras nos determina en los pasos superiores el ancho de la calzada, donde se sitúa la sobrecarga y el tren de cargas de la Norma IAP-98, "Instrucción sobre las acciones a considerar en el proyecto de puentes de carretera" ${ }^{[24]}$. Se considera la carga muerta debida a la capa de aglomerado asfáltico extendida en los 11 metros de ancho de la calzada, y además un peso lineal de pretil más peana de $5.00 \mathrm{kN} / \mathrm{m}$ a cada lado. Estos valores también se encuentran en un fichero externo, y se puede modificar.

\section{Parámetros de coste (3 parámetros)}

Se ha tomado una distancia estándar de transporte de $20 \mathrm{Km}$. El despunte de la armadura activa en las bancadas es importante, tomándose un valor del $25 \%$. Como factor de dificultad del montaje de las vigas sobre los apoyos se ha tomado la unidad. Estos valores también se encuentran en un fichero externo, y se puede modificar en función de las circunstancias que los determinan.

\section{Parámetros de armado (6 parámetros)}

Para la armadura pasiva se toma el acero tipo B-500-S, y para la activa el Y-1860-7S. El diámetro de los torones es de 0.6 pulgadas. Para la armadura de piel de la viga, se utiliza un único diámetro: $\varnothing 8$, siendo su cuantía la mínima que marca la norma $(0.15 \%)$, repartiéndose uniformemente por las caras superiores de las alas y por interiores y exteriores de las almas. Las fundas sólo se pueden disponer en los niveles 2 y 3 . Su diámetro es de $17 \mathrm{~mm}$., y se ha limitado la esbeltez vertical de los cercos de las almas por su dificultad en el montaje.

\section{Parámetros de exposición (1 parámetro)}

Contemplan el ambiente en el que se encuentra construida la estructura, ambiente exterior. El ambiente exterior será IIb según la Norma EHE-08, "Instrucción de Hormigón Estructural", por ser exterior, sin cloruros y precipitación media anual menor de 600 milímetros, siendo el recubrimiento de las armaduras de 30/25 milímetros según hormigón, en todos los casos el tipo de elemento es general y el control de ejecución intenso. 
Los parámetros empleados se muestran en la Figura 4-1:

\section{Semisección}

b total $=12.00 \mathrm{~m}$.

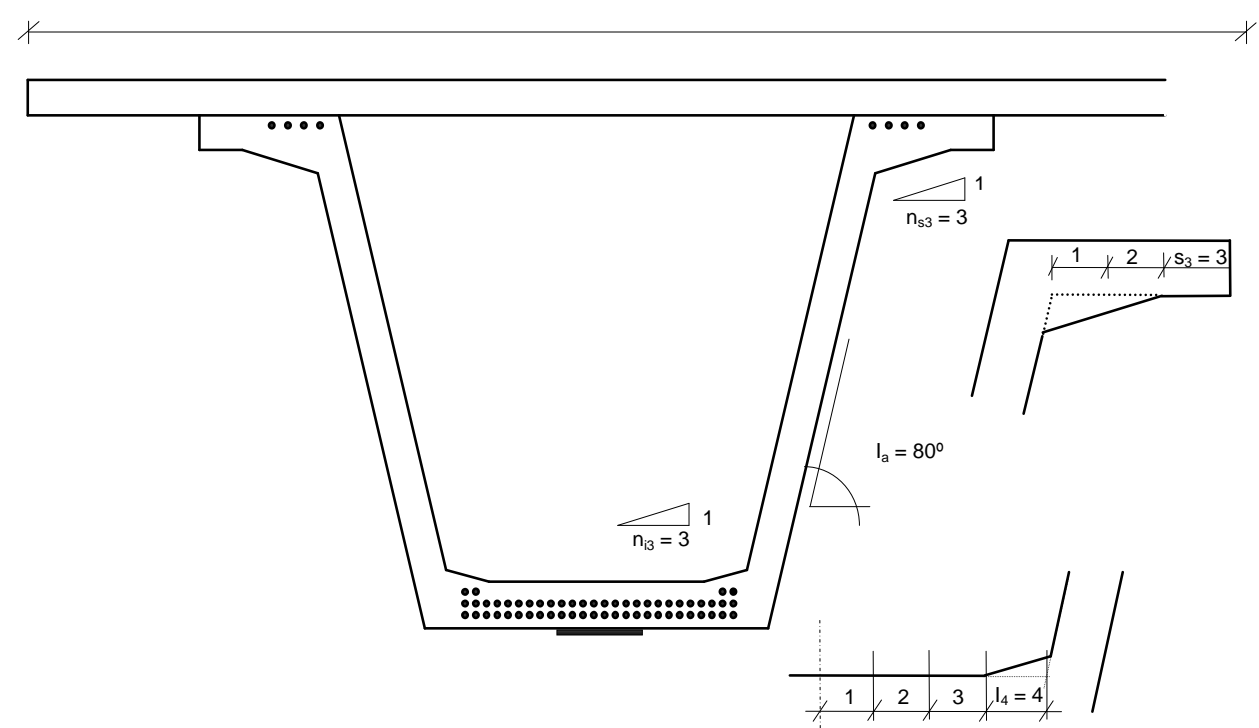

Alzado

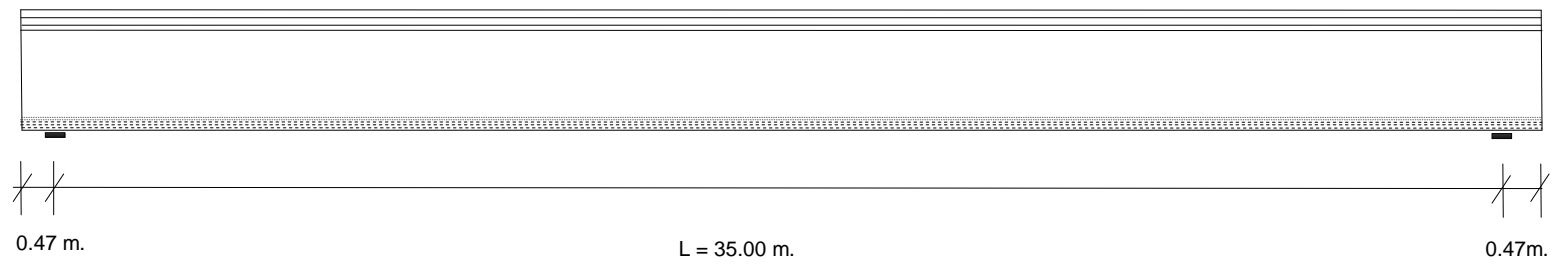

Figura 4-1: Parámetros tablero vigas artesa pretesas 


\subsection{Variables}

Las variables van a permitir completar los valores que definen la estructura, y son modificados por el algoritmo de búsqueda durante el proceso de optimización. Se definen variables discretas para poder construir el tablero con el resultado del proceso de optimización.

Se establecen cuatro grupos de variables: geométricas, calidades del hormigón, armado activo y armado pasivo, siendo su número total de 59.

Variables del tablero (59 variables)

El tablero se encuentra biapoyado. Para su definición y cálculo, se considera su simetría longitudinal y transversal, y se divide el cuadrante en 10 tramos, equivalente a 11 secciones -comenzando por el apoyo y finalizando en el eje de simetría longitudinal-.

Geométricas (7). Definen la geometría de la viga y de la losa. (Ver Figura 4-2).

1. Canto de la viga $h 1$

Valores posibles: desde $50 \mathrm{~cm}$ hasta $194 \mathrm{~cm}$. (de 1 en $1 \mathrm{~cm}$ ). 145 valores distintos. El valor máximo que puede tomar el canto se corresponde con la limitación de 1/18 de la luz

2. Canto de la losa e4

Valores posibles: desde $12 \mathrm{~cm}$ hasta $47 \mathrm{~cm}$. (de 1 en $1 \mathrm{~cm}$ ). 36 valores distintos

3. Ala inferior de la viga

Valores posibles: desde $50 \mathrm{~cm}$ hasta $200 \mathrm{~cm}$ (de 1 en $1 \mathrm{~cm}$ ). 151 valores distintos

4. Alas superiores de la viga (base)

Valores posibles: desde $15 \mathrm{~cm}$ hasta $100 \mathrm{~cm}$ (de 1 en $1 \mathrm{~cm}$ ). 86 valores distintos

5. Canto del ala inferior de la viga e1

Valores posibles: desde $15 \mathrm{~cm}$ hasta $50 \mathrm{~cm}$ (de 1 en $1 \mathrm{~cm}$ ). 36 valores distintos

6. Espesor de las almas de la viga e2

Valores posibles: desde $10 \mathrm{~cm}$ a $50 \mathrm{~cm}$ (de 1 en $1 \mathrm{~cm}$ ). 41 valores distintos

7. Canto de las alas superiores de la viga e3

Valores posibles: desde $10 \mathrm{~cm}$ hasta $40 \mathrm{~cm}$ (de 1 en $1 \mathrm{~cm}$ ). 31 valores distintos. Este valor tiene que ser siempre inferior o igual al ala superior (b3) 
Calidades del Hormigón (2). Definen el tipo de hormigón utilizado en la losa y en la viga.

8. Hormigón en losa $H l$

Valores posibles: HA-25, HA-30, HA-35 y HA-40. 4 valores distintos

9. Hormigón en viga $H v$

Valores posibles: HP-35, HP-40, HP-45 y HP-50. 4 valores distintos

Armado activo(4). Definen la armadura activa de la viga.

10. Pretensado en las alas superiores Nas

Número de torones. Máximo número por ala: 10

11. Pretensado en el ala inferior

Nai

Número de torones. Máximo número: 98 repartido en tres niveles. Se van completando los niveles desde abajo hasta arriba, ajustándose al ancho del ala inferior.

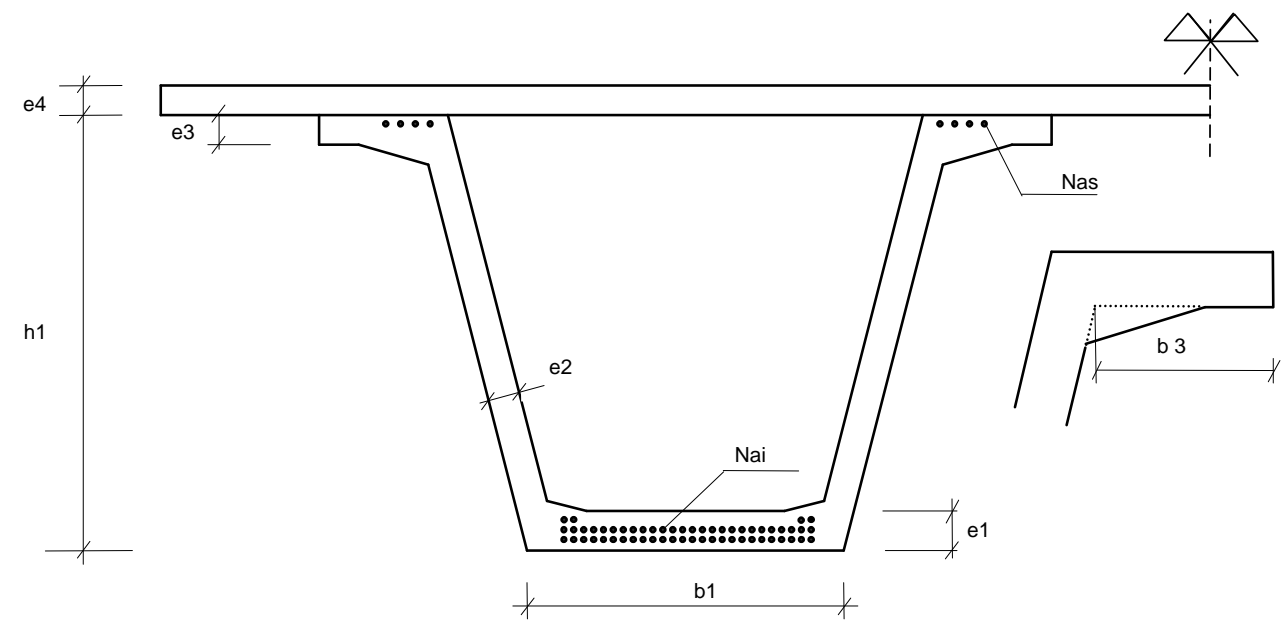

Figura 4-2: Variables geométricas y activa

12. Fundas en nivel 2 $V 2$

Número de tramos con fundas comenzando por el extremo. 8 valores distintos. (Ver Figura 4-3)

13. Fundas en nivel 3 V3

Número de tramos con fundas comenzando por el extremo. 6 valores distintos. Por ser vigas biapoyadas, el valor no supera al del nivel 2. 


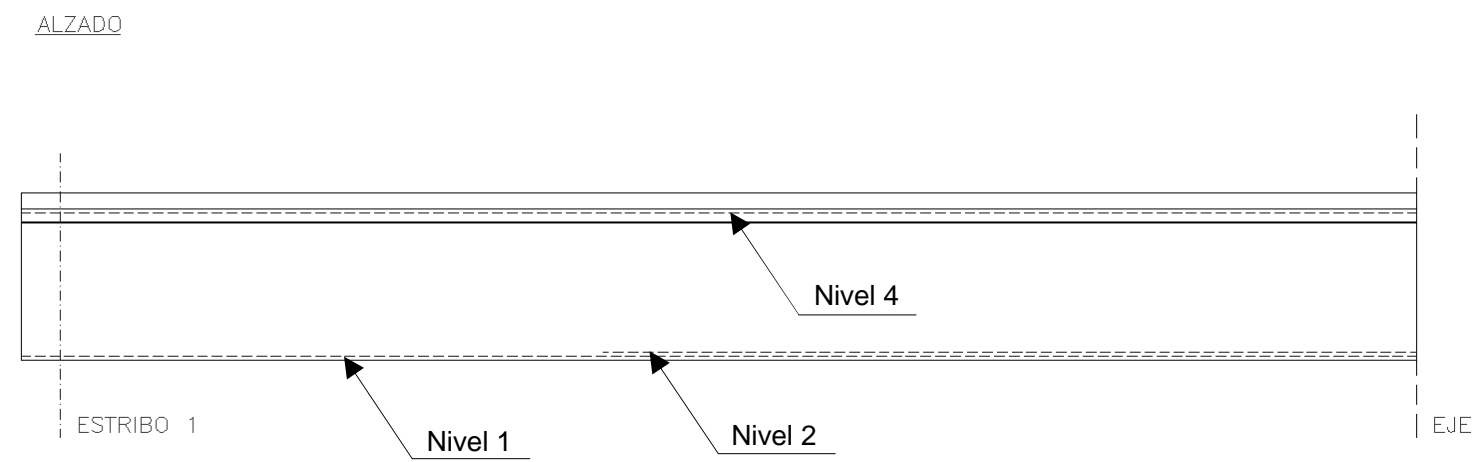

Figura 4-3: Disposición longitudinal de la armadura activa

Armadura pasiva(46). Definen la armadura pasiva del tablero. (Ver Figura 4-4).

14. Longitudinal superior de la losa

As6

Cuantía de acero por metro lineal, uniforme en toda la losa. Ordenado de menor a mayor, desde $\Phi 6 / 25$ hasta $\Phi 32 / 10$. Valores posibles: 56.

15. Longitudinal inferior de la losa

Cuantía de acero por metro lineal, uniforme en toda la losa. Ordenado de menor a mayor, desde $\Phi 6 / 25$ hasta $\Phi 32 / 10$. Valores posibles: 56.

16. Refuerzo longitudinal superior de la losa $A s 61$

Cuantía de acero por metro lineal de refuerzo, que se aplica en los tramos 1 y 2 de la losa. El diámetro de las barras es el mismo que el longitudinal As6, y la separación va desde 25 a $10 \mathrm{~cm} / \mathrm{m}$. Valores posibles: 8 .

17. Separación de los cercos de la viga

Stv

Separación entre cercos de la viga. Ala inferior + Almas + Alas superiores. Puede tomar 4 valores: 200mm / 150mm / 125mm / 100mm. La separación es la misma para los tres tipos de cercos y para todas las secciones.

18. Número de cercos y diámetro ala superior $D t 3,1 \quad$ (sección 1)

Número de cercos (1 ó 2), y diámetro de los cercos de las alas superiores para el tramo 1. Puede tomar 12 valores.

27. Número de cercos y diámetro ala superior Dt3,10 (sección 10)

Número de cercos (1 ó 2), y diámetro de los cercos de las alas superiores para el tramo 10. Puede tomar 12 valores.

28. Número de cercos y diámetro almas $\quad D t 2,1 \quad$ (sección 1)

Número de cercos ( 1 ó 2), y diámetro de los cercos de las almas para el tramo 1 . Puede tomar 12 valores.

37. Número de cercos y diámetro almas

Dt2,10 (sección 10) 
Número de cercos (1 ó 2), y diámetro de los cercos de las almas para el tramo 10. Puede tomar 12 valores.

38. Número de cercos y diámetro ala inferior Dt1,1 (sección 1)

Número de cercos (1 ó 2), y diámetro de los cercos del ala inferior para el tramo 1 . Puede tomar 12 valores.

47. Número de cercos y diámetro ala inferior Dt1,10 (sección 10)

Número de cercos (1 ó 2), y diámetro de los cercos del ala inferior para el tramo 10. Puede tomar 12 valores.

48. Diámetro armadura transversal de la losa Dt4

Diámetro de la armadura transversal superior e inferior de la losa. Puede tomar 8 valores: desde $\Phi 6$ hasta $\Phi 32$.

49. Separación armadura transversal inferior de la losa $\quad S t 5$

Separación entre los redondos de la armadura transversal inferior de la losa. Puede tomar 6 valores: 300mm/250mm/200mm / 150mm / 125mm / 100mm.

50. Separación armadura transversal superior de la losa St4,1 (sección 1)

Separación entre los redondos de la armadura transversal superior de la losa en el tramo 1 . Puede tomar 6 valores: $300 \mathrm{~mm} / 250 \mathrm{~mm} / 200 \mathrm{~mm} / 150 \mathrm{~mm} / 125 \mathrm{~mm} / 100 \mathrm{~mm}$.

59. Separación armadura transversal superior de la losa $\quad S t 4,10 \quad$ (sección 10) Separación entre los redondos de la armadura transversal superior de la losa en el tramo 10 . Puede tomar 6 valores: $300 \mathrm{~mm} / 250 \mathrm{~mm} / 200 \mathrm{~mm} / 150 \mathrm{~mm} / 125 \mathrm{~mm} / 100 \mathrm{~mm}$.

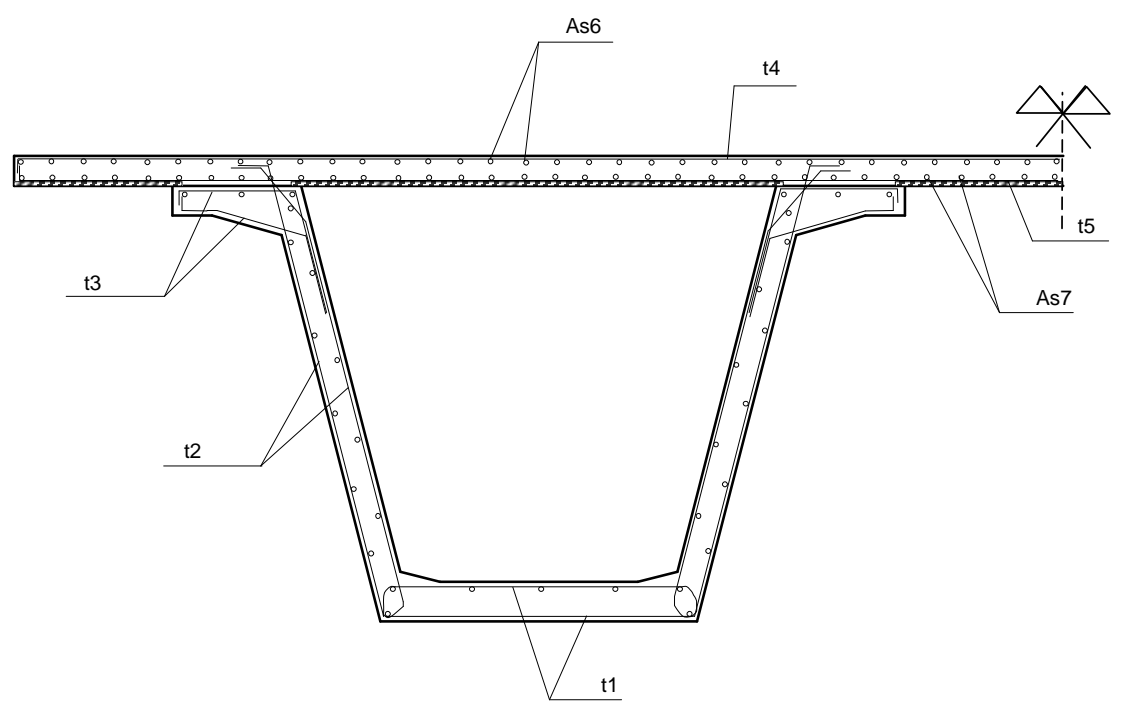

Figura 4-4: Variables armadura pasiva 


\subsection{Dimensión del espacio de soluciones}

Teniendo en cuenta los posibles valores que pueden tomar la 59 variables, el número máximo de soluciones es de:

$145 \times 36 \times 151 \times 86 \times 36 \times 41 \times 31 \times 4 \times 4 \times 10 \times 98 \times 8 \times 6 \times 56 \times 56 \times 8 \times 4 \times 12^{10}$ x $12^{10} \times 12^{10} \times 8 \times 6 \times 6^{10} \approx 1.6 \times 10^{65}$

La comprobación de cada solución requiere un tiempo computacional medio de 0.09 segundos, por lo que el intento de comprobar todo el espacio de soluciones duraría del orden de $4.6 \times 10^{56}$ milenios, haciendo imposible efectuar dicha prueba en la actualidad. 


\subsection{Función objetivo. Coste}

El coste del tablero se evalúa mediante la suma de los precios unitarios de las unidades de obra que lo componen, multiplicada por las mediciones correspondientes a estas unidades. Los precios de las unidades empleadas forman parte de los parámetros del problema, componiéndose mediante los valores definidos en los ficheros precios y transmont. Los precios se entienden como unidades de obra completas. El coste de la estructura que se obtiene a partir de ellos se corresponde con el de ejecución material.

Los precios unitarios considerados se evaluaron a finales del año 2008 después de un muestreo entre constructores de la Comunidad Valenciana, siendo representativos para toda España.

Como consecuencia del proceso constructivo del tablero, se distinguen cuatro partes: Construcción de la viga en la planta de prefabricados, transporte de la viga, montaje de la viga sobre los apoyos y ejecución de la losa in situ.

\subsubsection{Coste de la viga}

Los precios unitarios empleados se muestran en la Tabla siguiente:

\begin{tabular}{ccr}
\hline Unidad en viga & Descripción & Precio unitario \\
\hline Acero (B-500-S) & Material & $2.63[€ / \mathrm{kg}]$ \\
Acero (Y1860-S7) & Material & $3.38[€ / \mathrm{kg}]$ \\
Encofrado & Molde de acero & $75.11[€ / \mathrm{m}]$ \\
Hormigón & HP-35 & $122.25\left[€ / \mathrm{m}^{3}\right]$ \\
& HP-40 & $133.40\left[€ / \mathrm{m}^{3}\right]$ \\
& HP-45 & $142.15\left[€ / \mathrm{m}^{3}\right]$ \\
& HP-50 & $152.89\left[€ / \mathrm{m}^{3}\right]$ \\
\hline
\end{tabular}

Tabla 4-2: Precios unitarios viga

Estos precios incluyen: material + mano de obra + coste indirecto + coste de la estructura productiva + beneficio empresarial. La valoración de las últimas tres componentes dependerá de la empresa de prefabricados, encontrándose entre un $80 \mathrm{y}$ un $90 \%$ de la suma de los dos primeros. En este estudio, se ha tomado como valor $87,80 \%$.

El precio del hormigón incluye su fabricación, transporte, vertido y curado. La tendencia actual respecto a la compactación en prefabricados, es la sustitución del vibrado por la utilización de aditivos en el hormigón, que lo hagan autocompactante. 
El molde utilizado es el mismo para diferentes alturas, por lo que se valora por metro lineal. En cuanto al acero activo, el torón pesa $1.102 \mathrm{Kg} / \mathrm{m}$, y el precio también incluye las fundas de los torones. Para la medición del torón se tiene en cuenta las pérdidas del material por el despunte, que se ha considerado que son del $25 \%$, pudiendo ser modificado por otro valor en el fichero datadeck.

El coste del acero pasivo se valora por $\mathrm{Kg}$, sin embargo el coste debido a su manipulación está en función de la longitud de las barras y por lo tanto lo está de su diámetro. También afecta el suministro de los rollos de la materia prima, no costando lo mismo un $\mathrm{Kg}$ de acero de diámetro 12 que de diámetro 16. Es por ello que deben aplicarse correcciones debidas a estos dos factores. Es las Tablas 4-3 y 4-4 se muestran los coeficientes correctores que se aplican al precio del acero en función del diámetro, tomando como base el diámetro $12 \mathrm{~mm}$. Estos valores aproximados han sido contrastados con fabricantes, pero para su aplicación deberán corregirse en cada momento en el fichero precios, fácilmente modificable y adaptable al fabricante.

\begin{tabular}{ccc}
\hline Unidad en viga & Diámetro & Coeficiente corrector \\
\hline Kg. Acero (B-500-S) & D6 & 1.25 \\
& D8 & 1.17 \\
D10 & 1.075 \\
D12 & 1.00 \\
D16 & 0.98 \\
& D20 & 0.98 \\
\hline
\end{tabular}

Tabla 4-3: Coeficientes correctores de la armadura pasiva en viga. Materia prima

\begin{tabular}{ccc}
\hline Unidad en viga & Diámetro & Coeficiente corrector \\
\hline Kg. Acero (B-500-S) & D6 & 1.40 \\
& D8 & 1.25 \\
& D10 & 1.10 \\
D12 & 1.00 \\
D16 & 0.90 \\
& D20 & 0.90 \\
\hline
\end{tabular}

Tabla 4-4: Coeficientes correctores de la armadura pasiva en viga. Mano de obra 


\subsubsection{Coste del transporte de la viga}

Una vez la viga es fabricada se acopia temporalmente a la espera de ser transportada a la obra. El coste del transporte depende del peso de la viga y de la distancia. Se han considerado distintos tramos en función de los dos factores, mostrándose los valores en la Tabla 4-5, encontrándose en el fichero transmont. En este trabajo se ha tomado una distancia de $20 \mathrm{Km}$, dentro del fichero datadeck.

\begin{tabular}{|c|c|c|}
\hline Peso (hasta Tm) & Distancia (hasta Km) & Precio \\
\hline 55 & 50 & $975 €$ \\
\hline 55 & 100 & $1225 €$ \\
\hline 55 & 150 & $1475 €$ \\
\hline 55 & 200 & $1700 €$ \\
\hline 55 & 400 & $2500 €$ \\
\hline 66 & 50 & $1275 €$ \\
\hline 66 & 100 & $1600 €$ \\
\hline 66 & 150 & $1925 €$ \\
\hline 66 & 200 & $2225 €$ \\
\hline 66 & 400 & $3000 €$ \\
\hline 80 & 50 & $1650 €$ \\
\hline 80 & 100 & $2075 €$ \\
\hline 80 & 150 & $2475 €$ \\
\hline 80 & 200 & $2900 €$ \\
\hline 80 & 400 & $3500 €$ \\
\hline 100 & 50 & $1825 €$ \\
\hline 100 & 100 & $2275 €$ \\
\hline 100 & 150 & $2725 €$ \\
\hline 100 & 200 & $3175 €$ \\
\hline 100 & 400 & $4000 €$ \\
\hline 200 & 50 & $2825 €$ \\
\hline 200 & 100 & $3275 €$ \\
\hline 200 & 150 & $3725 €$ \\
\hline 200 & 200 & $4175 €$ \\
\hline 200 & 400 & $5000 €$ \\
\hline 400 & 50 & $3825 €$ \\
\hline 400 & 100 & $4275 €$ \\
\hline 400 & 150 & $4725 €$ \\
\hline 400 & 200 & $5175 €$ \\
\hline 400 & 400 & $6000 €$ \\
\hline
\end{tabular}

Tabla 4-5: Precios de transporte de la viga 


\subsubsection{Coste de la colocación de la viga}

El coste de la colocación de la viga depende, como en el caso del transporte, de varios factores, como son: la distancia -dietas de los operarios-, longitud de la viga grúa a emplear- y dificultad del montaje. La dificultad del montaje se ha representado como un factor a evaluar en cada caso, y que en nuestro trabajo se ha tomado la unidad. En la siguiente Tabla 4-6 se muestra el precio en función de la distancia y longitud de la viga. Estos datos se encuentran en el fichero transmont.

\begin{tabular}{ccc}
\hline Distancia (hasta Km.) & Longitud (hasta m.) & Precio \\
\hline 50 & 20 & $2900 €$ \\
50 & 25 & $3000 €$ \\
50 & 30 & $5100 €$ \\
50 & 35 & $5200 €$ \\
50 & 40 & $6200 €$ \\
\hline 100 & 20 & $2900 €$ \\
100 & 25 & $3300 €$ \\
100 & 30 & $5100 €$ \\
100 & 35 & $5200 €$ \\
100 & 40 & $6400 €$ \\
\hline 200 & 20 & $3100 €$ \\
200 & 25 & $4900 €$ \\
200 & 30 & $5300 €$ \\
200 & 35 & $5400 €$ \\
200 & 40 & $6400 €$ \\
400 & 20 & $3600 €$ \\
400 & 25 & $5400 €$ \\
400 & 30 & $5800 €$ \\
400 & 35 & $5900 €$ \\
400 & 40 & $6900 €$ \\
\hline & & \\
\hline & 300 & \\
\hline & 300 & \\
\hline
\end{tabular}

Tabla 4-6: Precios de colocación de la viga 


\subsubsection{Coste de la losa}

Los precios unitarios empleados se muestran en la Tabla siguiente:

\begin{tabular}{ccc}
\hline Unidad en losa & Descripción & Precio unitario \\
\hline Acero (B-500-S) & Material & $1.40[€ / \mathrm{kg}]$ \\
Encofrado & Placa autoportante encofrado perdido & $30.00\left[€ / \mathrm{m}^{2}\right]$ \\
Hormigón & HA-25 & $64.99\left[€ / \mathrm{m}^{3}\right]$ \\
& HA-30 & $69.95\left[€ / \mathrm{m}^{3}\right]$ \\
& HA-35 & $74.03\left[€ / \mathrm{m}^{3}\right]$ \\
& HA-40 & $79.12\left[€ / \mathrm{m}^{3}\right]$ \\
\hline
\end{tabular}

Tabla 4-7: Precios unitarios losa

Estos precios incluyen materiales, mano de obra y costes indirectos, y en el caso del hormigón además, el bombeo, nivelación, vibrado y curado. Las placas autoportantes, incluyen los tapes laterales. Como en el caso de la viga, se han tenido en cuenta los coeficientes correctores en función del diámetro de las barras aplicados al suministro y manipulación del material, y sabiendo que son fácilmente modificables en el fichero precios. Estos son los representados en las Tablas 4-8 y 4-9:

\begin{tabular}{ccc}
\hline Unidad en losa & Descripción & Coeficiente corrector \\
\hline Acero (B-500-S) & D6 & 1.25 \\
& D8 & 1.17 \\
& D10 & 1.075 \\
& D12 & 1.00 \\
& D16 & 0.98 \\
& D20 & 0.98 \\
& D25 & 1.00 \\
& D32 & 1.00 \\
\hline
\end{tabular}

Tabla 4-8: Coeficientes correctores de la armadura pasiva en losa. Materia prima

\begin{tabular}{ccc}
\hline Unidad en losa & Descripción & Coeficiente corrector \\
\hline Acero (B-500-S) & D6 & 1.40 \\
& D8 & 1.25 \\
& D10 & 1.10 \\
D12 & 1.00 \\
& D16 & 0.90 \\
& D20 & 0.90 \\
D25 & 0.80 \\
& D32 & 0.80 \\
\hline
\end{tabular}

Tabla 4-9: Coeficientes correctores de la armadura pasiva en losa. Mano de obra 


\subsubsection{Mediciones.}

Se ha tenido en cuenta para todas las mediciones, un incremento de longitud respecto a la luz entre ejes de apoyos, de 0.47 metros a ambos lados, correspondiente a las entregas.

En la medición del acero se han calculado longitudes de anclaje y solapo. Los despuntes de la armadura pasiva van incluidos en el precio, y los de la activa son del $25 \%$ tal como se ha descrito en el punto 4.5.1.

La medición de la cuantía de la armadura total de piel en la viga se ha calculado tomando como valor mínimo el $0.15 \%$ del área de la viga (Art. 51.3. EP-93[1]), y se ha repartido conforme a la Figura 4-5:

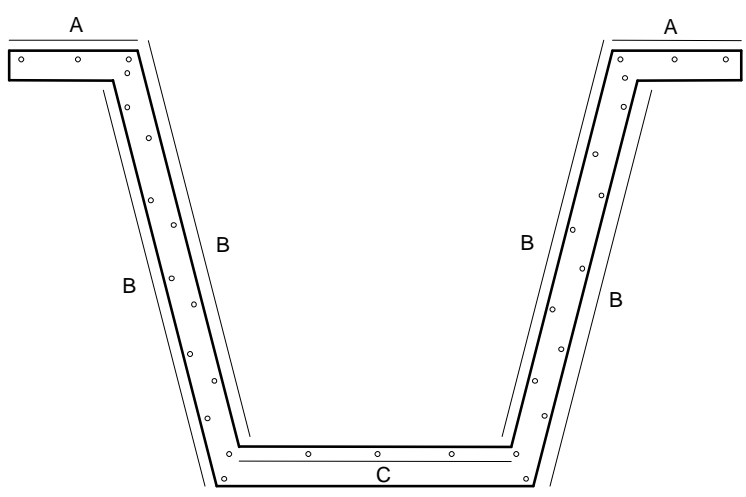

Figura 4-5: Reparto de la armadura de piel

El reparto es proporcional a la longitud de los lados donde se colocan los redondos $\Phi 8$, haciendo coincidir siempre un redondo en los extremos de los lados, y con la separación mínima entre ellos según la EHE-08.

El armado en riostra se considera independiente de las distintas soluciones obtenidas en el proceso de optimización. 


\section{CAPÍTULO 5. COMPROBACIÓN DEL TABLERO}

En este punto se da una descripción de las diferentes acciones que actúan sobre la estructura, la combinación de las mismas de tal manera que produzcan esfuerzos de máximo flector, torsor, cortante y concomitantes, para las que se realizará la comprobación de la viga y del conjunto vigas-losa, además de las características de los materiales empleados con los diferentes diagramas tensión-deformación, el método de integración de secciones y la comprobación de los diferentes estados límite, de acuerdo con la normativa española (EHE-08), la IAP-98[113] y el Eurocódigo EC-2:2[45]. Se han tenido en cuenta algunas prescripciones de las recomendaciones RPX-95 de puentes mixtos[114], y algunas reglas de buena práctica proyectista.

Dado el funcionamiento de las técnicas heurísticas aplicadas, el cálculo del tablero no consiste en su dimensionamiento, sino en la comprobación de una estructura previamente definida. El único cálculo que se realiza como dimensionamiento es el de la armadura de piel de la viga conforme a la anterior EP-93, tal como se ha indicado en el punto 4.5.5.

El proceso es completamente automático dentro del programa de optimización. Se genera aleatoriamente una estructura que se comprueba. Conforme avanza el proceso, la estructura va modificándose conforme a la heurística, y comprobándose mediante un algoritmo su factibilidad. A continuación, se describe el algoritmo de comprobación de la estructura.

\subsection{Acciones y esfuerzos}

Se distinguen las distintas acciones que hay que considerar durante el proceso constructivo, acciones que provienen de la acción del pretensado sobre la propia viga, del peso propio de la viga en la planta de prefabricados, del peso propio de la losa sobre la viga en ejecución de esta última, y el peso propio y acciones variables sobre el conjunto vigas+losa.

Las cargas a considerar con sus valores característicos, sus coeficientes de seguridad y combinación se detallan en la IAP-98.

Las acciones son las siguientes:

- Acciones permanentes:

- Peso propio. Se calcula para las barras longitudinales, como el producto del área bruta por el peso específico del hormigón. 
- Cargas muertas. Debidas al pavimento y a los pretiles, sobre las barras longitudinales.

- Acciones permanentes de valor no constante sobre la viga:

- Pretensado.

- Retracción.

- Fluencia.

- Acciones variables verticales, tren de cargas en puentes de la IAP-98:

- Sobrecarga uniforme. En cada estado individual de cargas se hace actuar una sobrecarga repartida de $4 \mathrm{kN} / \mathrm{m}^{2}$ sobre el área limitada entre dos nodos, el eje de una viga y el extremo del tablero o el eje del mismo (Figura 5-1).

- Una carga puntual de $600 \mathrm{kN}$ del carro. En cada estado de cargas actúa sobre un nodo y a la máxima excentricidad exterior, considerando la separación a los pretiles (Figura 5-2).
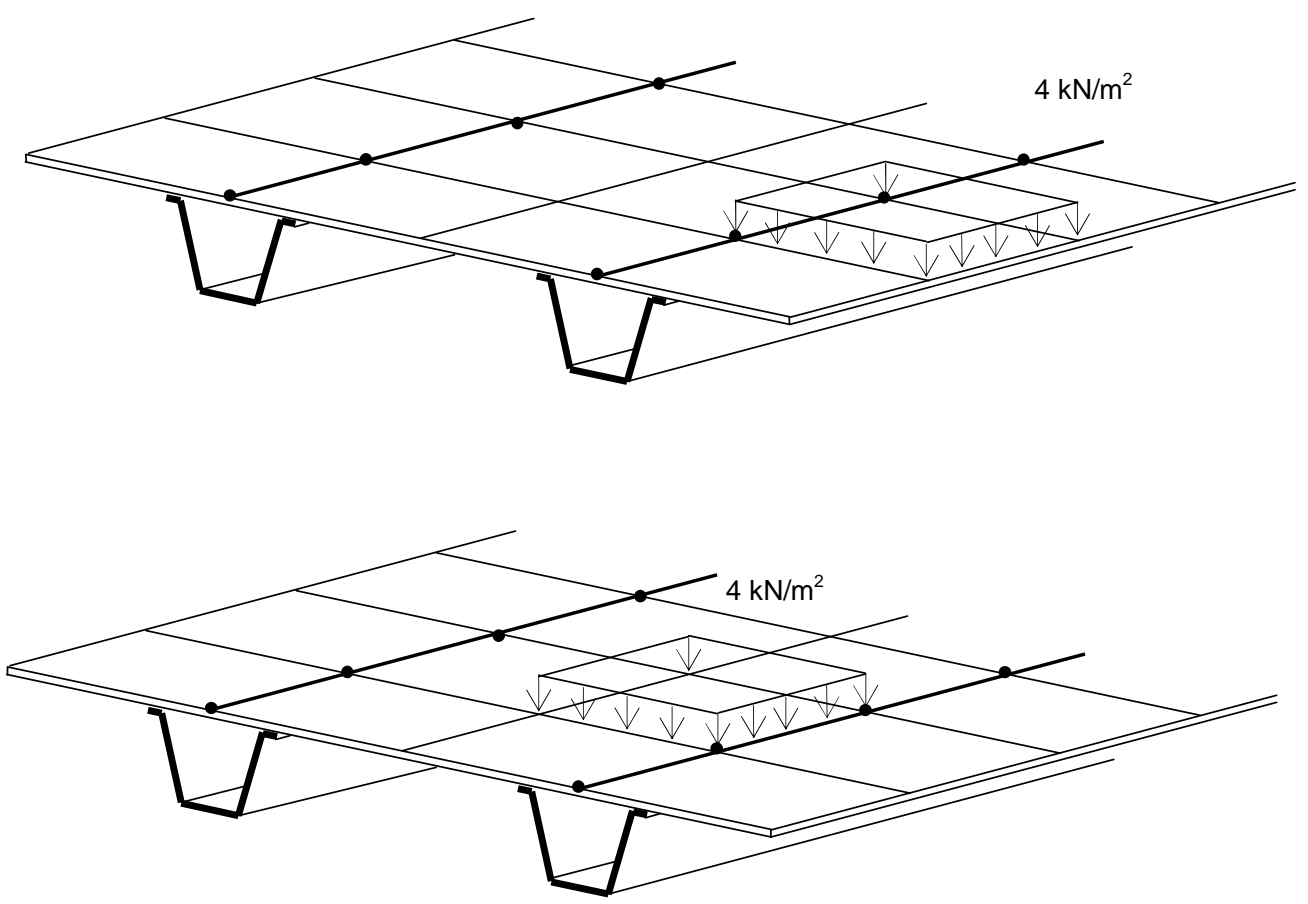

Figura 5-1: Esquema de las posiciones de la sobrecarga uniforme en distintos estados de carga 


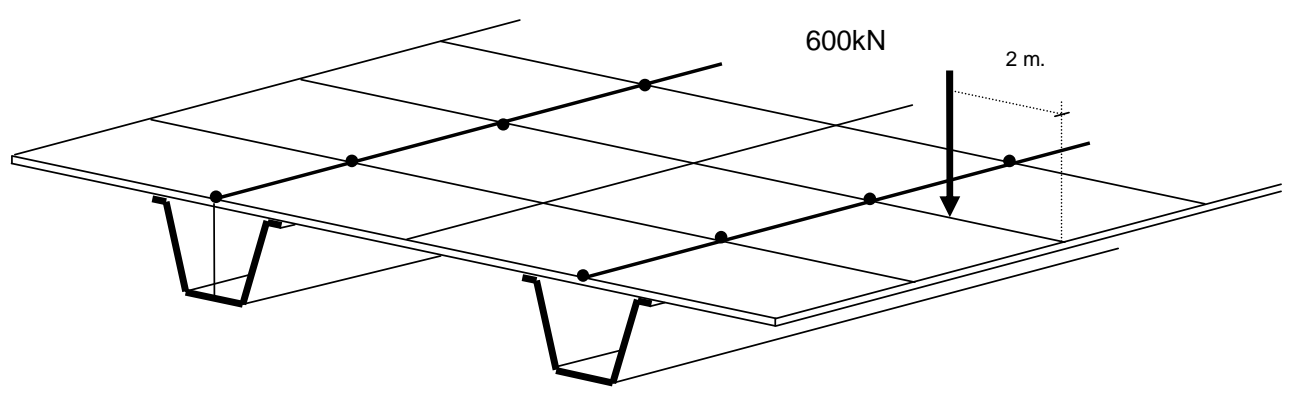

Figura 5-2: Esquema de posiciones del carro en distintos estados de carga

Al encontrarse el tablero biapoyado, no se considerarán gradiente térmico ni descenso de apoyos. Tampoco se ha tomado en cuenta la acción sísmica. La posición excéntrica de las acciones variables permite tener en cuenta los efectos de torsión en la estructura. Dada la simetría de la estructura, se evalúan $10 * 4$ estados individuales de carga repartida que se combinan y 11 posiciones diferentes de carro. Los esfuerzos sobre la losa se evalúan aparte.

\subsection{Modelización de la estructura}

La estructura se ha modelizado como un emparrillado plano, formado por dos vigas paralelas discretizadas en 20 barras cada una, y 21 conexiones entre los nudos de las anteriores barras. Dichas conexiones están formadas por vigas transversales formadas por tres barras cada una de ellas, confiriendo rigidez transversal al conjunto. En total son 103 barras y 84 nudos, tal como se aprecia en la Tabla 5-3.

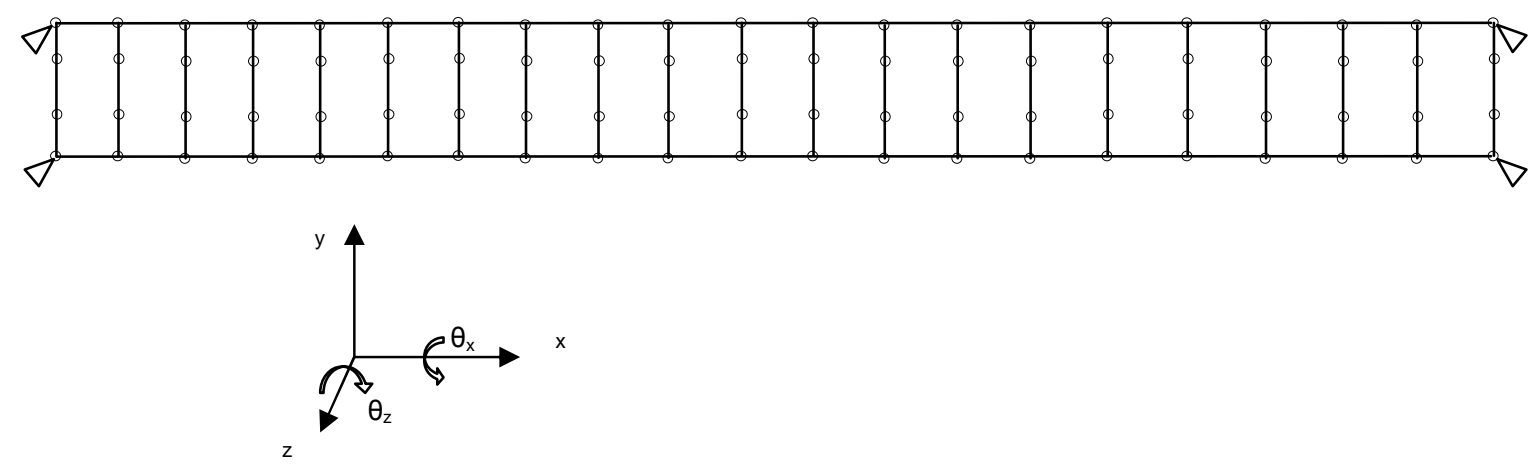

Figura 5-3: Modelización estructura 
Para el cálculo de los desplazamientos de los nodos se aplica la ecuación de matrices: $\{\mathrm{F}\}=[\mathrm{M}] *\{\mathrm{D}\}$; siendo $\{\mathrm{F}\}$ el vector de cargas nodales, $[\mathrm{M}]$ la matriz de rigidez $\mathrm{y}$ $\{\mathrm{D}\}$ el vector de desplazamientos nodales. Para la representación de las características mecánicas de las barras se ha considerado el emparrillado tipo I definido por Manterola[102] "Puentes. Apuntes para su diseño, cálculo y construcción".

La matriz de rigidez de cada barra longitudinal (nudos $\mathrm{i}, \mathrm{j}$ ) es simétrica y de dimensiones $6 * 6$, definida del siguiente modo:

$$
\left[\begin{array}{l}
F_{z i} \\
M_{x i} \\
T_{z i} \\
F_{z j} \\
M_{x j} \\
T_{z j}
\end{array}\right]=\left[\begin{array}{cccccc}
K_{1} & K_{2} & 0 & -K_{1} & K_{2} & 0 \\
-K_{2} & K_{3} & 0 & -K_{2} & K_{3} / 2 & 0 \\
0 & 0 & K & 0 & 0 & -K \\
-K_{1} & -K_{2} & 0 & K_{1} & -K_{2} & 0 \\
K_{2} & K_{3} / 2 & 0 & -K_{2} & K_{3} & 0 \\
0 & 0 & -K & 0 & 0 & K
\end{array}\right] *\left[\begin{array}{c}
z_{i} \\
\theta_{z i} \\
\theta_{x i} \\
z_{j} \\
\theta_{z j} \\
\theta_{x j}
\end{array}\right]
$$

Figura 5-4: Ecuación de rigidez para un elemento longitudinal i,j

con los siguientes valores:

$$
\begin{aligned}
& \mathrm{K}_{1}=12 . * \mathrm{Ec} * \mathrm{R} / \mathrm{T} * * 3 \\
& \mathrm{~K}_{2}=6 . * \mathrm{Ec}^{*} \mathrm{R} / \mathrm{T} * * 2 \\
& \mathrm{~K}_{3}=4 . * \mathrm{Ec}^{*} \mathrm{R} / \mathrm{T} \\
& \mathrm{K}=\mathrm{Gc} * \mathrm{~S} / \mathrm{T}
\end{aligned}
$$

Siendo: $\mathrm{T}=$ Longitud de la barra, $\mathrm{R}=$ Inercia a flexión, $\mathrm{S}=$ Inercia a torsión, $\mathrm{Ec}=$ Módulo de elasticidad del hormigón de la viga y $\mathrm{Gc}=$ Módulo de elasticidad transversal del hormigón de la viga.

La matriz de rigidez en coordenadas globales para cada una de las barras transversales (nudos m,n) también es simétrica y de dimensiones $6 * 6$, definida como:

$$
\left[\begin{array}{l}
F_{z m} \\
M_{x m} \\
T_{z m} \\
F_{z n} \\
M_{x n} \\
T_{z n}
\end{array}\right]=\left[\begin{array}{cccccc}
K_{1} & 0 & -K_{2} & -K_{1} & 0 & -K_{2} \\
0 & K & 0 & 0 & -K & 0 \\
-K_{2} & 0 & K_{3} & K_{2} & 0 & K_{3} / 2 \\
-K_{1} & 0 & K_{2} & K_{1} & 0 & K_{2} \\
0 & -K & 0 & 0 & K & 0 \\
-K_{2} & 0 & K_{3} / 2 & K_{2} & 0 & K_{3}
\end{array}\right] *\left[\begin{array}{l}
z_{m} \\
\theta_{z m} \\
\theta_{x m} \\
z_{n} \\
\theta_{z n} \\
\theta_{x n}
\end{array}\right]
$$

Figura 5-5: Ecuación de rigidez para un elemento transversal m,n 
A continuación, se ensambla la matriz de todas las barras, de dimensión $252 * 252$, y se resuelve el vector $\{\mathrm{D}\}$ aplicando el algoritmo de Cholesky, y a partir del vector desplazamiento, se determinan los esfuerzos en las secciones correspondientes a los nodos.

Para cada estado de carga se han calculado los cortantes, torsores y flectores de cada sección, al igual que los desplazamientos. En el cálculo se han considerado las combinaciones de las acciones anteriores que producen máximo flector, cortante y torsor, para los diferentes estados límite, tanto de servicio como últimos.

\subsection{Envolventes de los esfuerzos}

La instrucción EHE-08 especifica que se determinen los esfuerzos según el denominado valor representativo de la acción, que define mediante la expresión siguiente:

$$
\Psi_{\mathrm{i}} \mathrm{F}_{\mathrm{k}}
$$

debiéndose considerar que, en general para las acciones permanentes, el valor representativo es el característico. Las acciones quedan definidas en el art. 3.4.1. y 3.4.2. de la IAP-98.

Como valor de cálculo de una acción se define el obtenido como producto del valor representativo por un coeficiente parcial de seguridad:

$$
\mathrm{F}_{\mathrm{d}}=\gamma_{\mathrm{f}} \Psi_{\mathrm{i}} \mathrm{F}_{\mathrm{k}}
$$

\subsubsection{Obtención envolventes E.L.U.}

Para las diferentes tipos de acción se aplican los coeficientes parciales de seguridad:

- Pretensado. $\quad$ Favorable: $\quad 1.00$

Desfavorable: $\quad 1.00$

- Peso propio. $\quad$ Favorable: $\quad 1.00$

Desfavorable: $\quad 1.35$

- Carga muerta. Favorable: $\quad 1.00$

Desfavorable: $\quad 1.35$

- Uso (Variable). Favorable: $\quad 0.00$

Desfavorable: $\quad 1.50$

La combinación de acciones se define conforme al criterio de situaciones permanentes o transitorias (art. 13.2. EHE-08):

$$
\sum_{j \geq 1} \gamma_{G, j} G_{k, j}+\gamma_{p} P_{k}+\gamma_{Q, 1} Q_{k, 1}
$$


donde:

$\mathrm{G}_{\mathrm{k}, \mathrm{j}} \quad$ Valor característico de las acciones permanentes

$\mathrm{P}_{\mathrm{k}} \quad$ Valor característico de la acción del pretensado

$\mathrm{Q}_{\mathrm{k}, 1} \quad$ Valor característico de la acción variable determinante

\subsubsection{Obtención envolventes E.L.S.}

Para las diferentes tipos de acción se aplican los coeficientes parciales de seguridad:

- Pretensado. $\quad$ Favorable: 0.95

Desfavorable: $\quad 1.05$

- Peso propio. Favorable: 1.00

Desfavorable: $\quad 1.00$

- Carga muerta. Favorable: $\quad 1.00$

Desfavorable: $\quad 1.00$

- Uso (Variable). Favorable: $\quad 0.00$

Desfavorable: $\quad 1.00$

La combinación de acciones que se consideran son únicamente las situaciones de proyecto permanentes y transitorias. Al estar el tablero biapoyado, no se considera el gradiente térmico, ni los asientos diferenciales, por lo que en los tableros biapoyados sólo se presenta como acción variable la sobrecarga de uso. Por lo tanto se aplican las siguientes combinaciones:

- Combinación frecuente:

$$
\sum_{j \geq 1} \gamma_{G, j} G_{k, j}+\gamma_{p} P_{k}+\gamma_{Q, 1} \Psi_{1,1} Q_{k, 1}
$$

- Combinación cuasipermanente:

$$
\sum_{j \geq 1} \gamma_{G, j} G_{k, j}+\gamma_{p} P_{k}+\gamma_{Q, 1} \Psi_{2,1} Q_{k, 1}
$$

donde:

$\Psi_{1,1} \mathrm{Q}_{\mathrm{k}, 1}$ Valor representativo frecuente de la acción variable determinante

$\Psi_{2,1} \mathrm{Q}_{\mathrm{k}, 1}$ Valores representativos cuasipermanentes de las acciones variables con la acción determinante o con la acción accidental

que en el proceso constructivo toma los siguientes valores:

- Planta de prefabricados:

○ Permanentes: pretensado $+\mathrm{pp}_{\mathrm{viga}}$

- Hormigonado losa:

○ Permanentes: pretensado $+\mathrm{pp}_{\mathrm{viga}}+\mathrm{pp}_{\text {losa }}$ 
- Frecuente: $\quad$ pretensado $+\mathrm{pp}+\mathrm{cm}+$ uso

- Cuasipermanente: pretensado $+\mathrm{pp}+\mathrm{cm}+0.2 *$ uso (Art. 3.3.3. IAP-98)

pp: $\quad$ Peso propio

$\mathrm{cm}$ : Carga muerta

\subsection{Diagramas tensión-deformación}

\subsubsection{Acero. Armadura pasiva}

El diagrama tensión-deformación de cálculo del acero para armaduras pasivas (en tracción o en compresión) será el dispuesto artículo 38.4 de la EHE-08. La deformación del acero en tracción $\varepsilon_{\text {máx }}$ se limita al 10 por 1000 y en compresión $\varepsilon_{\mathrm{cu}}$ al 3.5 por 1000 , ya que debido a la fluencia, la deformación límite del hormigón a compresión será la anterior, y se ha supuesto que existe perfecta adherencia entre el acero y el hormigón. Es un diagrama bilineal con rama horizontal a partir del límite elástico, como módulo de deformación longitudinal del acero se tomará $\mathrm{E}_{\mathrm{s}}=200000$ $\mathrm{N} / \mathrm{mm}^{2}$. El coeficiente de seguridad del acero será de 1.15.

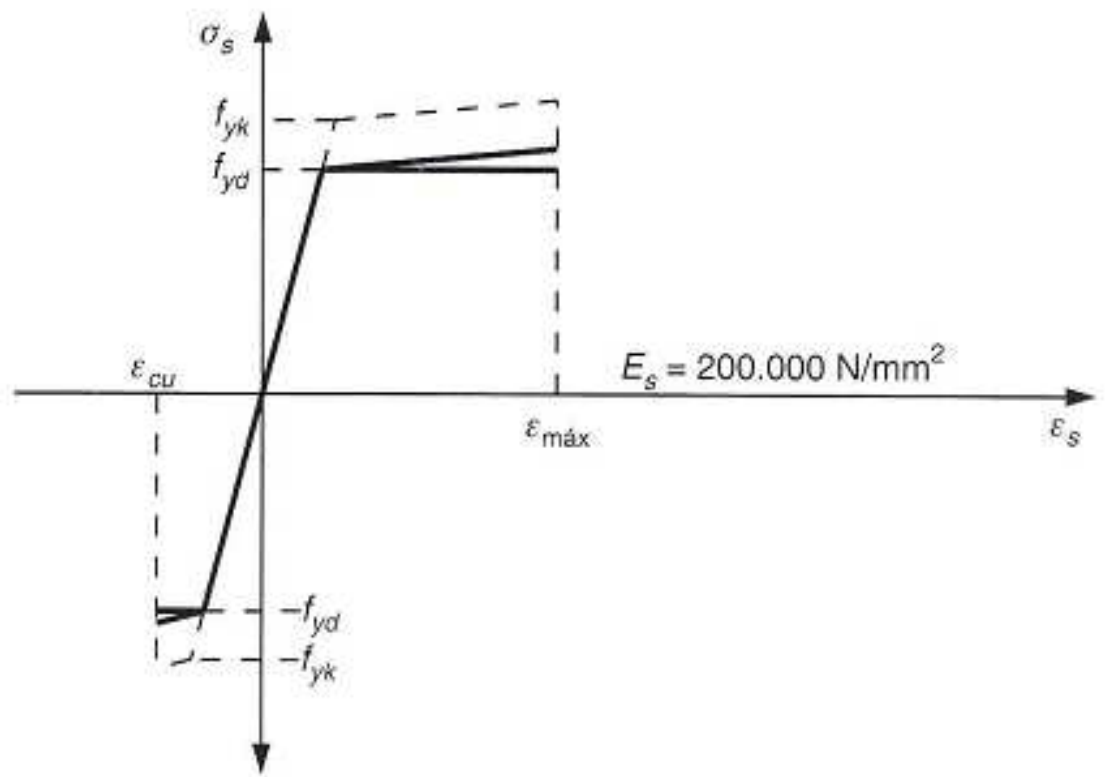

Figura 5-6: Diagrama tensión-deformación de cálculo armaduras pasivas. Fig 38.4 EHE-08

El acero considerado en el estudio es un B-500-S, y su límite elástico, según la tabla 32.2a de la EHE-08, es de $\mathrm{f}_{\mathrm{yk}} \geq 500 \mathrm{~N} / \mathrm{mm}^{2}$. En tirantes, se limita la tensión a $\mathrm{f}_{\mathrm{y} 90 \mathrm{~d}}=$ $400 \mathrm{~N} / \mathrm{mm}^{2}$.

\subsubsection{Acero. Armadura activa}

El diagrama tensión-deformación característico del acero para armaduras activas será el dispuesto artículo 38.5 de la EHE-08. Consta de un primer tramo recto de pendiente $\mathrm{E}_{\mathrm{p}} \mathrm{y}$ un segundo tramo curvo, a partir de $0,7 \mathrm{f}_{\mathrm{pk}}$, definido por la siguiente expresión: 
$\varepsilon_{p}=\frac{\sigma_{p}}{E_{p}}+0,823\left(\frac{\sigma_{p}}{f_{p k}}-0,7\right)^{5} \quad$ para $\sigma_{\mathrm{p}} \geq 0,7 \mathrm{f}_{\mathrm{pk}}$

siendo $\mathrm{E}_{\mathrm{p}}=190000 \mathrm{~N} / \mathrm{mm}^{2}$.

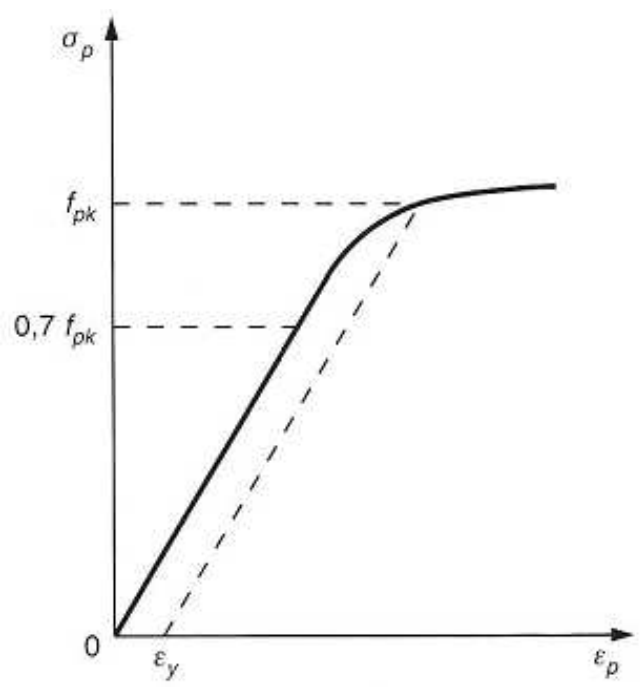

Figura 5-7: Diagrama tensión-deformación característico armaduras activas. Fig 38.5 EHE-08

El diagrama tensión-deformación de cálculo del acero para armaduras activas, se deducirá del correspondiente diagrama característico, mediante una afinidad oblicua, paralela a la recta de Hooke, de razón $1 / \gamma \mathrm{s}$. El coeficiente de seguridad del acero $\gamma_{\mathrm{s}}$ será de 1,15 .

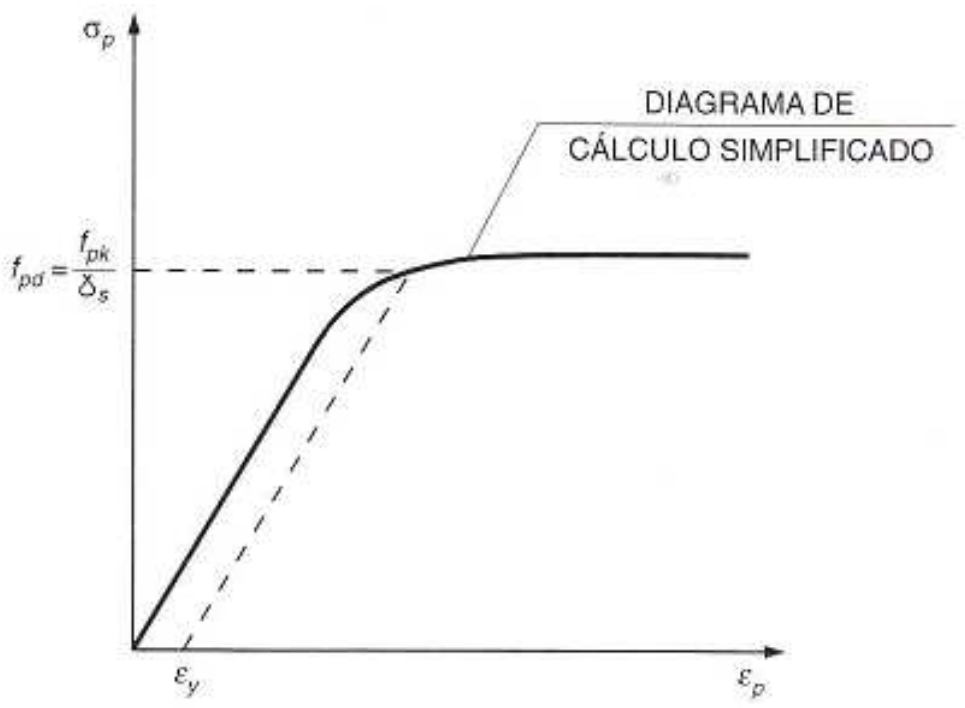

Figura 5-8: Diagrama tensión-deformación de cálculo armaduras activas. Fig 38.7.b EHE-08

La tensión de rotura para el tipo de cordón Y-1860-S7, según la tabla 34.5b de la EHE-08, se toma $\mathrm{f}_{\text {máx }}=1860 \mathrm{~N} / \mathrm{mm}^{2}$. 
El límite elástico característico deducido del catálogo Stronhold es de 1700 N/mm².

El coeficiente de relajación considerado, Art. 38.9 EHE, es del 5\% al millón de horas.

Los torones son de 0.6 " de diámetro, equivalente a $140 \mathrm{~mm}^{2}$ de área.

La fuerza de tesado aplicada por cable es del $72.6 \%$ de la tensión de rotura, equivalente a $189.04 \mathrm{kNw} /$ torón.

\subsubsection{Hormigón}

\subsubsection{Estados Límite Último}

El diagrama utilizado para el cálculo del agotamiento frente solicitaciones normales será el parábola-rectángulo de la instrucción EHE-08, artículo 39.5 .

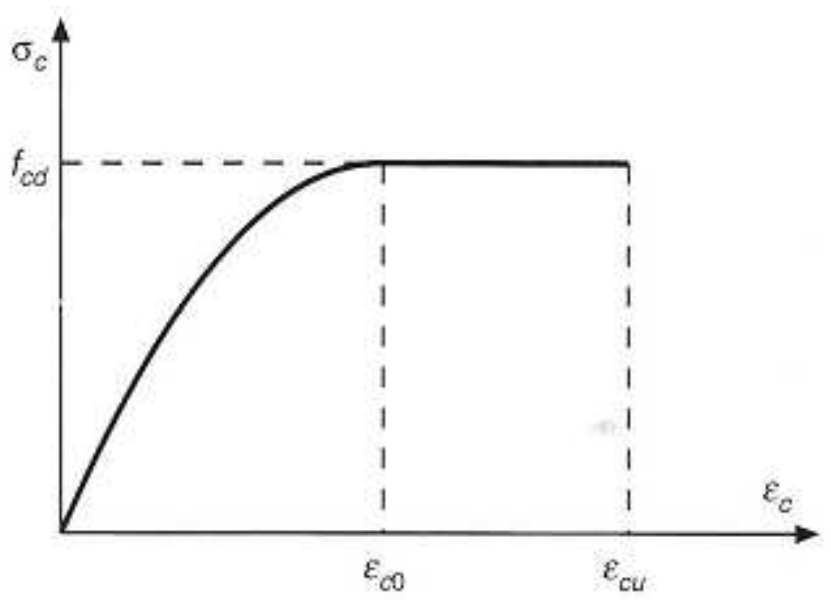

Figura 5-9: Diagrama tensión-deformación del hormigón en ELU. Fig 39.5.a EHE-08

Los parámetros que definen la parábola, los valores de la deformación de rotura a compresión simple $\varepsilon_{0}$, y de la deformación última $\varepsilon_{\mathrm{cu}}$, dependen de la resistencia característica $\mathrm{f}_{\mathrm{ck}}$ del hormigón. Siendo $\mathrm{f}_{\mathrm{cd}}$ la resistencia de cálculo del hormigón a compresión e igual a la resistencia característica $\mathrm{f}_{\mathrm{ck}}$ del hormigón dividida por su coeficiente de seguridad que será de 1.5.

La resistencia característica a tracción también vendrá determinada conforme al artículo 39.1 de la EHE-08, en función de la resistencia característica a compresión.

\subsubsection{Estados Límite de Servicio}

En el cálculo del Estado Límite de Servicio de fisuración se ha supuesto que la respuesta del hormigón es lineal, es decir, tensión y deformación son proporcionales siendo la constante de proporcionalidad el módulo de deformación secante del hormigón Ec, que se tomará: 


$$
E_{c}=8500 * \sqrt[3]{f c m, j}
$$

donde $\mathrm{f}_{\mathrm{cm}, \mathrm{j}}$ será la resistencia media a la edad de j días, Art. 39.6 EHE-08. Para el tesado se admite que el hormigón habrá alcanzado una resistencia estimada de al menos un $80 \%$ de la característica a 28 días, aplicándose un coeficiente corrector $\beta=$ 0.80 .

\subsubsection{Retracción, fluencia y coeficiente de Poisson}

La deformación por retracción a tiempo infinito (art. 39.7 EHE-08) se ha tomado igual a $0.40 \mathrm{~mm} / \mathrm{m}$ por interpolación lineal de las tablas.

El coeficiente de fluencia a tiempo infinito (art. 39.8 EHE-08) se ha tomado igual a 2.5

El coeficiente de Poisson relativo a deformaciones elásticas bajo tensiones normales de utilización (art. 39.9 EHE-08) se ha tomado igual a 0.20

\subsection{Comprobación de las secciones de hormigón}

En cada una de las secciones del hormigón pretensado, se ha de comprobar la resistencia de la misma respecto a cada uno de los esfuerzos máximos reflejados en las envolventes, así como de los correspondientes concomitantes. Para ello, se han seguido las prescripciones impuestas por la instrucción EHE-08, comprobando los estados límites cuyo cumplimiento se requiere.

El uso de dos tipos distintos de hormigón, en viga y en losa, dificultan las comprobaciones, por ello se ha simplificado el proceso mediante la homogenización del hormigón de la losa respecto al de la viga. El coeficiente de homogeneización es igual a la relación entre el módulo de elasticidad de ambos. Este coeficiente, se aplica como un factor sobre el ancho de la losa, manteniéndose el canto. En el caso de la resistencia a torsión, el factor se aplica sobre al canto para el cálculo del espesor de la pared.

La acción que ejerce el pretensado en la viga, se ha desglosado en cuatro niveles, tres en el ala inferior y uno en las alas superiores. Por simplificación de este trabajo, las fundas se colocan sobre todos los torones de un mismo nivel. Cuando en una sección existe uno o varios niveles de fundas, en esos niveles no actúa el pretensado, y no se tiene en cuenta ni en su acción, ni en las características mecánicas de la sección. Las pérdidas de la acción del pretensado sobre toda la viga se calculan por nivel, y se reparten entre las secciones donde la armadura activa es adherente. 


\subsubsection{Análisis estructural del pretensado. Armaduras pretesas}

Las pérdidas instantáneas de fuerza son aquellas que pueden producirse durante la operación de tesado y en el momento del anclaje de las armaduras activas. Su valor en cada sección es:

$$
\Delta \mathrm{P}_{\mathrm{i}}=\Delta \mathrm{P}_{1}+\Delta \mathrm{P}_{2}+\Delta \mathrm{P}_{3}
$$

donde:

$\Delta \mathrm{P}_{1}$ Pérdidas de fuerza, en la sección en estudio, por rozamiento a lo largo del conducto de pretensado. En este caso, el trazado de los torones es completamente recto, por lo que su valor es cero.

$\Delta \mathrm{P}_{2}$ Pérdidas de fuerza, en la sección en estudio, por penetración de cuñas en los anclajes. Una bancada de pretensado, permite fabricar simultáneamente varias vigas a la vez, no produciéndose penetración de cuñas, siendo su valor cero.

$\Delta \mathrm{P}_{3}$ Pérdidas de fuerza, en la sección en estudio, por acortamiento elástico del hormigón. Se calculan mediante la expresión:

$$
\Delta \mathrm{P}_{3}=\sigma_{\mathrm{cp}} * \mathrm{~A}_{\mathrm{p}} * \mathrm{E}_{\mathrm{p}} / \mathrm{E}_{\mathrm{cj}}
$$

donde:

$A_{p}$ Sección total de la armadura activa.

$\sigma_{\mathrm{cp}}$ Tensión de compresión, a nivel del centro de gravedad de las armaduras activas, producida por la fuerza $\mathrm{P}_{0}$ en el momento del tesado.

$\mathrm{E}_{\mathrm{p}}$ Módulo de deformación longitudinal de las armaduras activas.

$E_{\mathrm{cj}}$ Módulo de deformación longitudinal del hormigón para la edad j correspondiente al momento de la puesta en carga de las armaduras activas.

Las pérdidas diferidas se calculan conforme al art. 20.2.2.2. EHE-08, tomando el valor de relajación el definido en el punto 3.3.2, y los de retracción y fluencia del punto 3.3.3.3., partiendo de la acción del pretensado, descontando las pérdidas instantáneas, y considerando el peso propio de la viga y de la losa, más la carga muerta.

\subsubsection{E.L.U. frente a solicitaciones normales}

Respecto a las hipótesis básicas del art. 42.1.2. de la EHE-08, cabe mencionar el efecto producido por las armaduras respecto a la deformación del hormigón que los envuelve.

Las deformaciones de las armaduras pasivas se mantienen iguales a las del hormigón. Las deformaciones totales de las armaduras activas adherentes deben considerar, 
además de la deformación que se produce en la fibra correspondiente en el plano de deformación de agotamiento $\left(\varepsilon_{0}\right)$, la deformación producida por el pretensado y la deformación de descompresión (Figura 5.10) según se define a continuación:

$$
\Delta \varepsilon=\varepsilon_{\mathrm{cp}}+\varepsilon_{\mathrm{p} 0}
$$

donde:

$\varepsilon_{\mathrm{cp}}$ Deformación de descompresión del hormigón al nivel de la fibra de armadura considerada.

$\varepsilon_{\mathrm{p} 0}$ Pre deformación de la armadura activa debida a la acción del pretensado en la fase considerada, teniendo en cuenta las pérdidas que se hayan producido.

El diagrama de cálculo tensión-deformación del hormigón es el definido en el punto 3.3, al igual que los definidos para las armaduras activas y pasivas. No se considerará la resistencia del hormigón a tracción.

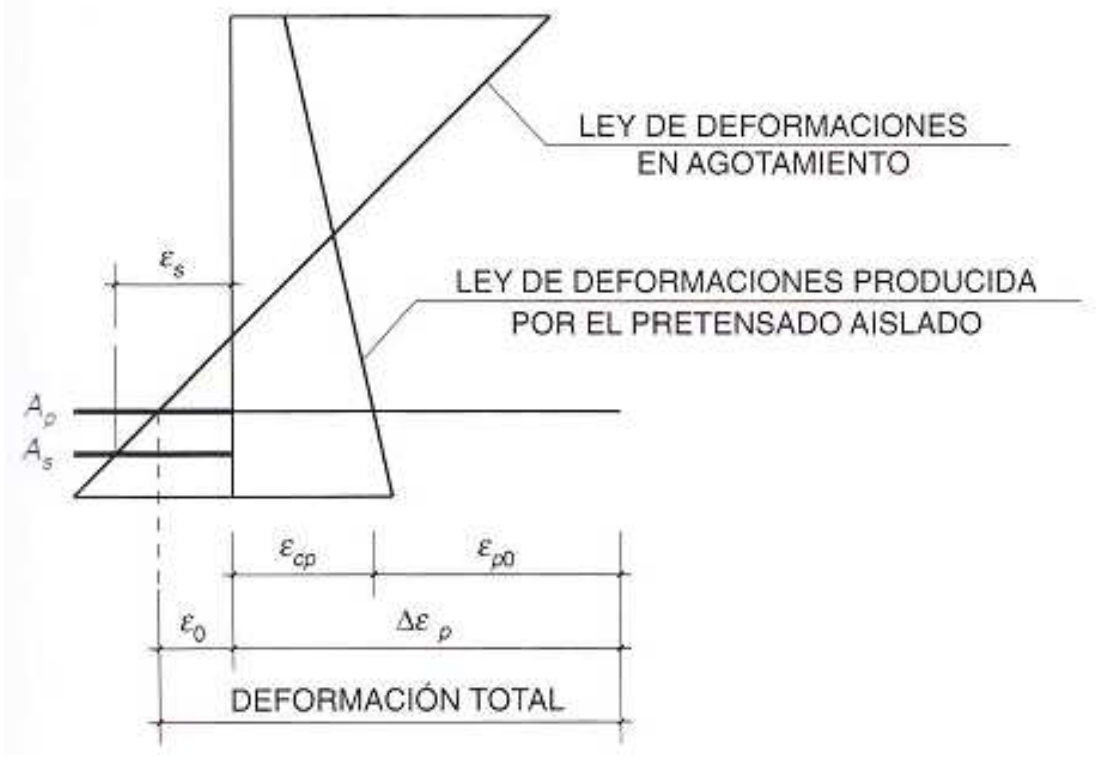

Figura 5-10: Deformación total en una sección. Fig 42.1.2 EHE-08

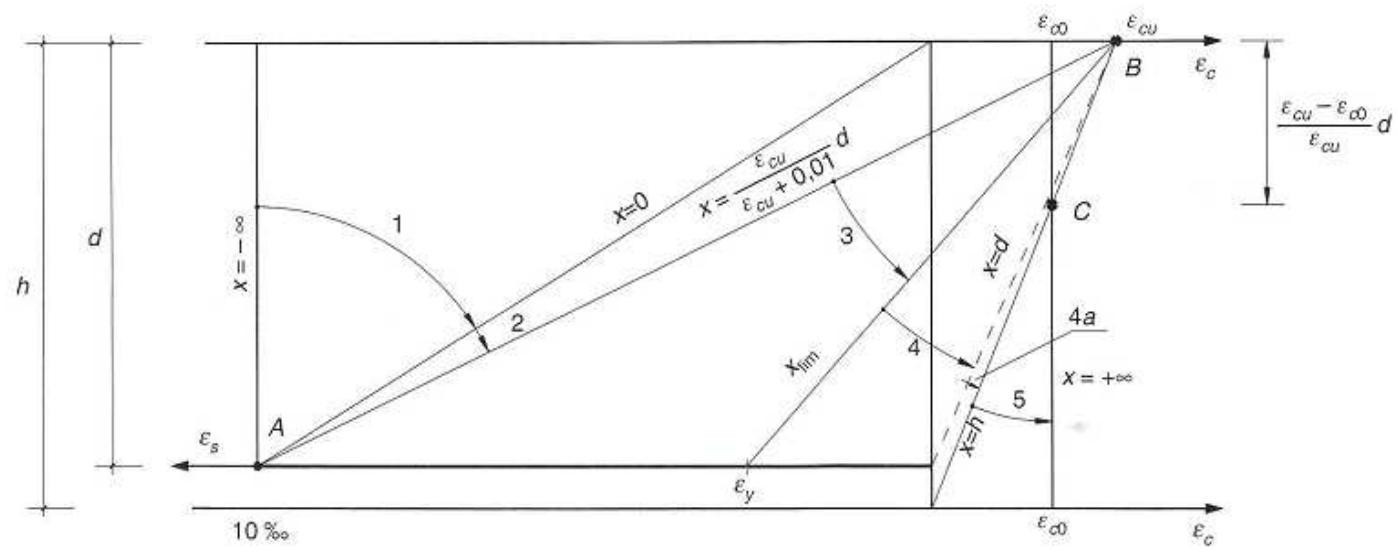

Figura 5-11: Dominios de deformación en una sección. Fig 42.1.3 EHE 
Las fuerzas que solicitan una sección producirán en la misma un plano de deformaciones, este plano generará unas tensiones en el material que equilibrarán las fuerzas aplicadas sobre la misma si éstas son menores a las de rotura de la sección, sino, romperá. El agotamiento de la sección se caracteriza por el valor de la deformación en determinadas fibras, definidas por los dominios de deformación de agotamiento representados en la Figura 5-11. Las tensiones, que este plano generará en los materiales, en un punto concreto de la sección, dependerán de la distancia a la fibra neutra. Cuanto mayor sea esta distancia mayor será su deformación y según los diagramas tensión-deformación anteriormente mostrados la tensión variará. Por lo tanto, cada plano de agotamiento se define por una única variable, que es la profundidad de la fibra neutra.

Para las tensiones de las armaduras, tanto pasivas como activas, se considerarán los diagramas definidos en los puntos 5.4.1 y 5.4.2 considerándose su actuación sobre puntos que tendrán una tensión concreta según la deformación de su centro. Multiplicando las tensiones de las mismas por sus respectivas áreas obtendremos la fuerza a la que están solicitadas y sumando todas las fuerzas obtendremos el axil del acero. Sumando el producto de las fuerzas por las distancias a los ejes de referencia obtendremos los momentos de agotamiento de la sección, que debe ser superior a la solicitación.

División en bandas

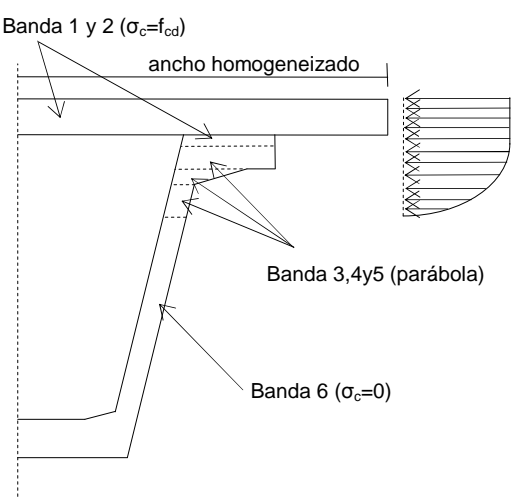

Deformaciones

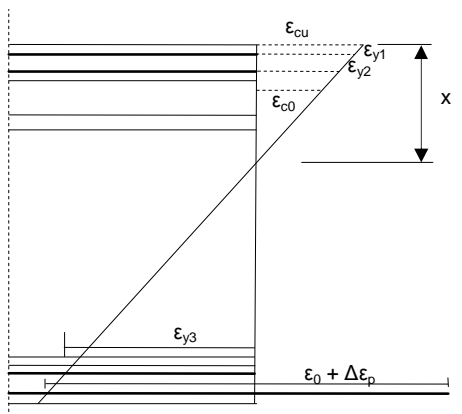

Tensiones

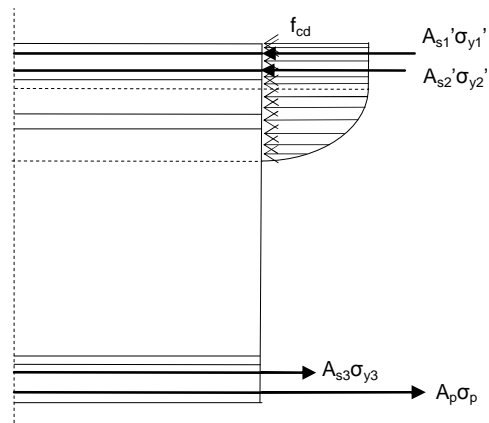

Figura 5-12: Integración de secciones en ELU

El hormigón no se podrá considerar como un elemento puntual, ya que es un continuo y tendrá zonas con distintas deformaciones, y por lo tanto con distintas tensiones. El estado de tensiones del hormigón viene dado por el diagrama parábolarectángulo definido en el punto 5.4.3.1. Para obtener los axiles y momentos que se producen en el hormigón habrá que integrar a lo largo de toda la sección. El problema se reduce a encontrar la profundidad de la fibra neutra tal que anule el axil resultante de todas las tensiones de los aceros y del hormigón (Figura 5-12). 
La integración en la zona de parábola, para las distintas bandas de la sección, se realiza de acuerdo con el método propuesto por Bonet et al.[23], basado en la integración por puntos Gauss. Este método se ha particularizado al caso de secciones en artesa sometidas a flexión cilíndrica, lo que supone que la integración puede realizarse sobre el contorno, en lugar de hacerlo sobre el área. Para ello, la sección se divide en bandas limitadas por las profundidades de los vértices de la sección, la profundidad de la fibra neutra, y la profundidad correspondiente a una deformación del hormigón comprimido $\varepsilon_{\mathrm{c} 0}$. En cada banda, las integrales de tensiones se resuelven por puntos de Gauss. A la suma de todas ellas se les añade la fuerza en los aceros superiores e inferior, y la fuerza en las armaduras activas. El efecto del pretensado se considera a partir de la predeformación de la armadura activa, descontando pérdidas iniciales. Las tensiones se integran considerando la viga maciza, y después se evalúa una sección ficticia formada por el hueco de la viga, restando este resultado al anterior.

Con la integración de tensiones, se encuentra una relación entre la profundidad de la fibra neutra y el axil y momento de agotamiento. Para obtener la profundidad correspondiente al axil nulo se aplica el método iterativo de Müller [120], que consiste en una variante del Régula-Falsi con interpolación parabólica.

\subsubsection{E.L.U. frente a cortante}

La comprobación a cortante se lleva a cabo siguiendo las prescripciones de la EHE08 que está basada en el cálculo por el método de bielas y tirantes que se describe en la misma. El cortante se comprueba en todas las secciones definidas a partir de una nueva sección situada a un canto del eje de apoyo.

Se calcula el esfuerzo cortante de agotamiento por compresión oblicua en el alma $\mathrm{V}_{\mathrm{u}}$ conforme al artículo 44.2.3.1 de la EHE-08. Se calcula el esfuerzo cortante de agotamiento por tracción del alma $\mathrm{V}_{\mathrm{u} 2}$ conforme al artículo 44.2.3.2 de la EHE-08 y para piezas con armadura cortante. Se tiene en cuenta tanto la armadura pasiva, como la activa. Se comprueba que el cortante de cálculo $\mathrm{V}_{\mathrm{rd}}$ no es superior a cada uno de los valores calculados $\mathrm{V}_{\mathrm{u} 1} \mathrm{y} \mathrm{V}_{\mathrm{u} 2}$.

\subsubsection{E.L.U. frente a torsión}

El torsor se comprueba en todas las secciones definidas a partir de la sección situada a un canto del eje de apoyo, conforme al artículo 45 de la EHE-08. Se calculan los máximos momentos torsores que pueden resistir las bielas comprimidas del hormigón $\mathrm{T}_{\mathrm{u} 1}$, las armaduras transversales $\mathrm{T}_{\mathrm{u} 2}$, y las armaduras longitudinales $\mathrm{T}_{\mathrm{u} 3}$. Se comprueba que el torsor de cálculo $\mathrm{T}_{\mathrm{d}}$ no es superior a cada uno de los valores calculados $\mathrm{T}_{\mathrm{u} 1}, \mathrm{~T}_{\mathrm{u} 2}, \mathrm{y} \mathrm{T}_{\mathrm{u} 3}$ 


\subsubsection{E.L.U. disposición armaduras de cortante}

Se comprueba para cada sección, que se cumple la separación mínima entre cercos y la cuantía mínima de acero en los cercos que determina el artículo 44.2.3.4.1 de la EHE-08.

\subsubsection{E.L.U. torsión combinada con cortante}

Se comprueba para cada sección, conforme al artículo 45.3.2.2 de la EHE-08, que los esfuerzos torsores y cortantes de cálculo concomitantes satisfacen la condición para que no se produzcan compresiones excesivas en el hormigón:

$$
\left[\frac{T_{d}}{T_{u 1}}\right]^{\beta}+\left[\frac{V_{r d}}{V_{u 1}}\right]^{\beta} \leq 1
$$

siendo:

$$
\beta=2\left[1-\frac{h_{e}}{b}\right]
$$

donde $h_{e}$ es el espesor eficaz, y

$b$ la suma de los espesores de las almas.

\subsubsection{E.L.U. disposición armaduras de flexión}

Se comprueba para cada sección, que la armadura resistente longitudinal traccionada cumple la limitación del artículo 42.3.2 de la EHE-08.

\subsubsection{E.L.U. rasantes entre ala y alma}

Se comprueba para cada sección la armadura de unión entre ala y alma conforme al artículo 44.2.3.5 de la EHE-08, en los siguientes puntos:

- Voladizo de la losa con el ala superior de la viga

- En el vano interior de la losa con el ala de la viga

- En el ala inferior de la viga con el alma.

El esfuerzo rasante medio de cálculo $S_{d}$, es inferior al esfuerzo rasante de agotamiento por compresión oblicua $S_{\mathrm{u} 1} \mathrm{y}$ también inferior a la contribución de la armadura transversal $\mathrm{S}_{\mathrm{su}}$.

\subsubsection{E.L.U. flexión transversal de la losa}

Se comprueba la resistencia a flexión del voladizo transversal, para cada sección de la losa, en los siguientes puntos: 
$\underline{\text { Sección: Intersección del eje del alma con la cara superior de la losa }}$

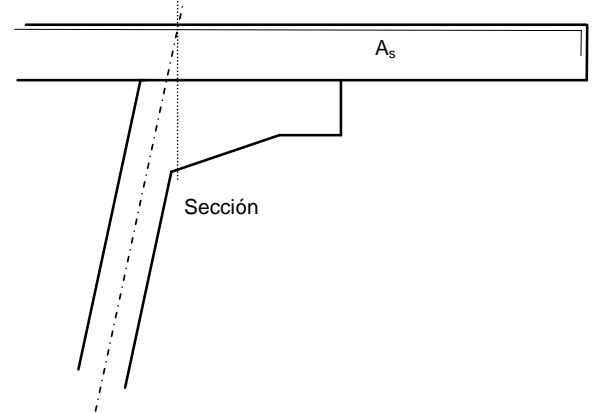

Figura 5-13: Flexión en voladizo transversal I

$\underline{\text { Sección: Voladizo de la losa sobre la viga }}$

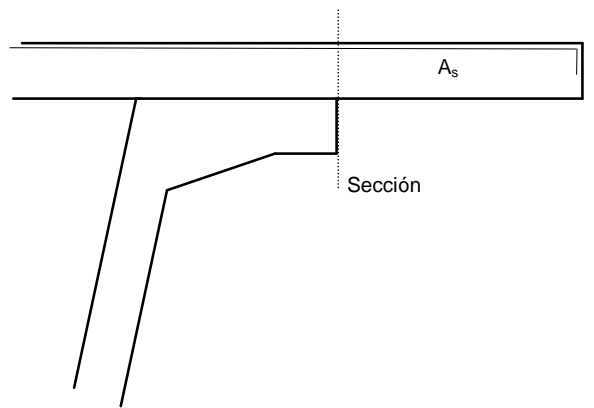

Figura 5-14: Flexión en voladizo transversal II

Sección: Voladizo del ala de la viga en el arranque de la cartela en ejecución losa

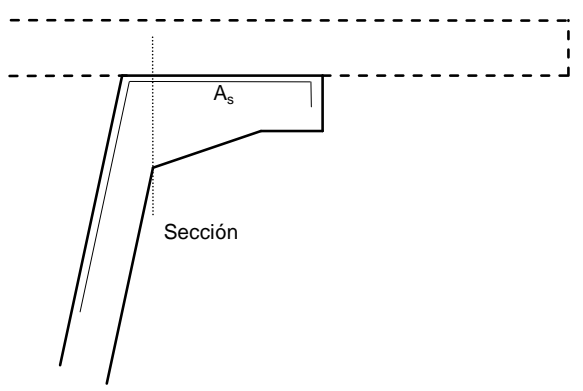

Figura 5-15: Flexión en voladizo transversal III 
Se comprueba en los tres casos anteriores que la armadura $A_{s}$ es superior a la que resulta del equilibrio de la sección de la siguiente Figura, tomando el diagrama tensión-deformación de cálculo del hormigón del art. 39.5.b. de la EHE.

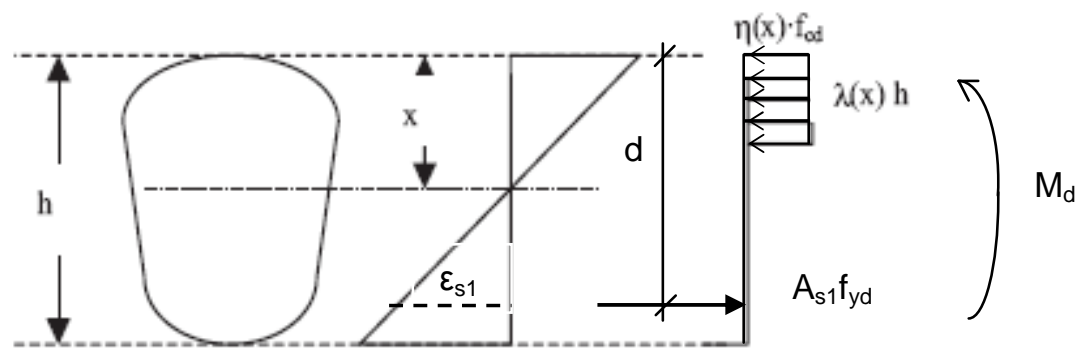

Figura 5-16: Equilibrio de fuerzas y momentos. Figuras 39.5.b EHE-08

Del equilibrio de la sección, se plantea el sistema de ecuaciones:

$\eta(x) f_{c d} * \lambda(x)^{2} / 2 h^{2}-A_{s 1} f_{y d} d+M_{d}=0 ; \quad$ Equilibrio de momentos

$\eta(x) f_{c d} * \lambda(x) h=A_{s 1} f_{y d} ; \quad$ Equilibrio de axiles

Calculando x (profundidad de la fibra neutra) y $A_{s 1}$, debiendo ser $A_{s 1} \leq A_{s}$.

Igualmente, se comprueba la losa en las diferentes secciones del tablero apoyada sobre las vigas. Los puntos de comprobación de la armadura superior e inferior son los siguientes (Figura 5-17): flector negativo (A) en apoyo de losa sobre una viga, flector positivo (B) de losa apoyada sobre una viga, flector negativo (C) de losa apoyada sobre dos vigas y flector positivo (D) de losa apoyada sobre dos vigas.

(C)

(A)

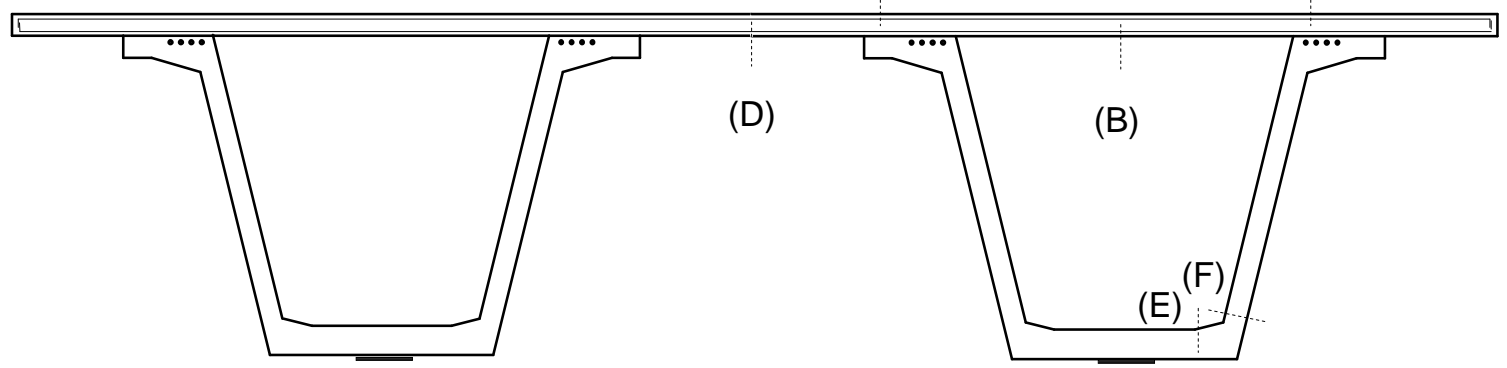

Figura 5-17: Flexión transversal en losa y viga 


\subsubsection{E.L.U. flexión transversal alma y ala inferior de la viga}

Se comprueba al igual que en el punto anterior la armadura transversal de la viga en voladizo solicitada en el momento del hormigonado de la losa. Los puntos de comprobación se corresponde con los flectores negativos del arranque del ala inferior (E) y del alma (F) (Figura 5-17).

\subsubsection{Fatiga}

La comprobación a fatiga es necesario incluirla según se ha efectuado en trabajos anteriores de optimización de estructuras de hormigón. Carrera et al. [34] y Perea et al. [132] observaron que los pórticos y marcos de hormigón armado de carretera optimizados tendían a esbelteces tan altas que incumplían las comprobaciones de fatiga, por lo que ambos autores concluían con la necesidad de incorporar restricciones relacionadas con este estado límite, y para ello se ha empleado el criterio propuesto por el Eurocódigo EC-2:2 [45]. Primeramente, se comprueba la resistencia a fatiga del hormigón sometido a compresión mediante la expresión:

$$
\frac{\sigma_{c, \text { max }}}{f_{c d}} \leq 0.5+0.45 * \frac{\sigma_{c, \min }}{f_{c d}} \leq 0.9
$$

Siendo:

$\sigma_{\mathrm{c} \text {,máx }}$ : Máxima tensión de compresión en una fibra bajo combinación frecuente

$\sigma_{\mathrm{c} \text {,mín }}$ : Mínima tensión de compresión en la misma fibra en que se produce $\sigma_{\mathrm{c} \text {,máx }}$

$f_{c d} \quad$ : Resistencia a compresión simple del hormigón a los 28 días dividida por su coeficiente de seguridad.

Y a continuación, se limitan las tensiones de trabajo del acero a tracción conforme al artículo 38.10 de la EHE-08.

\subsubsection{E.L.S. comprobación de flechas instantáneas en el tablero}

Se aplican los coeficientes del punto 5.3.2. para la envolvente de esfuerzos, resolviendo los desplazamientos de los nodos $\{\mathrm{D}\}$ según la ecuación definida en el punto 5.2. $\{\mathrm{F}\}=[\mathrm{M}] *\{\mathrm{D}\}$; comprobando que la flecha en ninguna de las secciones del tablero supera el valor de 1/250 de la luz del puente, con el objeto de limitar esbelteces excesivas.

\subsubsection{E.L.S. viga en planta}

Se comprueba que en el momento de liberación del tesado de cables en la planta de prefabricados, no se producen tracciones en la fibra superior de la viga, y para evitar 
las fisuras por compresión, que las compresiones $\sigma_{\mathrm{c}}$ en la fibra inferior no superan el $60 \%$ de la resistencia del hormigón $\mathrm{f}_{\mathrm{ck}, \mathrm{j}}$ a los $j$ días (Art. 49.2.1 EHE-08).

\subsubsection{E.L.S. viga con el hormigonado de la losa}

Se comprueba que en el momento del hormigonado de la losa, estando el hormigón fresco, no se producen tracciones en la fibra superior de la viga, y además, que las compresiones $\sigma_{\mathrm{c}}$ en la fibra inferior no superan el $60 \%$ de la resistencia del hormigón $\mathrm{f}_{\mathrm{ck}, \mathrm{j}}$ a los $j$ días (Art. 49.2.1 EHE-08). En este punto, ya se tiene en cuenta el acortamiento elástico del hormigón.

\subsubsection{E.L.S. tablero: compresiones en el hormigón}

Se comprueba que en el tablero en servicio, bajo acciones frecuentes, las compresiones $\sigma_{\mathrm{c}}$ en las fibras inferior y superior no superan el $60 \%$ de la resistencia del hormigón $\mathrm{f}_{\mathrm{ck}}$ a los 28 días (Art. 49.2.1 EHE-08). En este punto, ya se tiene en cuenta las pérdidas diferidas del pretensado.

\subsubsection{E.L.S. tablero: aparición de fisuras}

Se comprueba que en el tablero en servicio, en combinación frecuente, no aparecen fisuras en la fibra inferior de la viga (Art. 49.2.2. EHE-08).

Las comprobaciones correspondientes a que el acero activo se encuentre siempre en la zona comprimida de la sección, y que las armaduras pasivas y activas no plastifiquen, en los tres casos bajo acciones cuasipermanentes, no se realiza dado que antes de llegar a dichas situaciones, se produce la aparición de fisuras.

\subsubsection{E.L.S. comprobación de las flechas diferidas en el tablero}

Se comprueba que la flecha en ninguna de las secciones del tablero, bajo acciones permanentes no supera el valor de 1/1000 de la luz del puente, con el objeto de limitar esbelteces excesivas, siendo un valor similar a la comprobación de la flecha instantánea.

$$
\text { flecha }_{\mathrm{i}} \leq\left(\text { flecha }_{\mathrm{p}, \mathrm{i}} * \mathrm{C}_{\mathrm{p}, \mathrm{i}}+\text { flecha }_{\mathrm{pp}, \mathrm{i}}+\text { flecha }_{\mathrm{cm}, \mathrm{i}}\right) *(1+\text { fluencia }) ;
$$

donde:

flecha $_{\mathrm{i}} \quad$ flecha en la sección i

flecha $_{p, i} \quad$ flecha debida al pretensado en la viga

$\mathrm{C}_{\mathrm{p}, \mathrm{i}} \quad$ coeficiente de pérdidas instantáneas

flecha $_{\mathrm{pp}, \mathrm{i}}$ flecha debida al peso de la losa sobre la viga

flecha $_{\mathrm{cm}, \mathrm{i}}$ flecha en el tablero debida a la carga muerta

fluencia coeficiente de fluencia del hormigón 


\section{CAPÍTULO 6. APLICACIÓN DE LOS MÉTODOS HEURÍSTICOS}

A continuación se describe la aplicación de las técnicas metaheurísticas al problema de optimización expuesto anteriormente. Se basan en la búsqueda de una o varias soluciones iniciales obtenidas de forma aleatoria que se van modificando durante el proceso hasta encontrar un óptimo local con suficiente calidad.

Los algoritmos de búsqueda se parametrizan, y a continuación se presenta un resumen con los resultados experimentales. Finalmente se comparan los resultados respecto a calidad de óptimo encontrado, tiempo de optimización, robustez, etc.

El primer algoritmo que se describe es el random walk strategy (RW), que no es especialmente eficiente, pero nos da información sobre el espacio de soluciones. A continuación se comentarán los resultados obtenidos con el descent local search (DLS) que será el primer método heurístico de búsqueda local empleado, seguidos por el simulated annealing (SA) y finalmente el threshold accepting (TA), y a continuación se tratarán los algoritmos poblacionales y sus correspondientes variaciones: algoritmos genéticos (GA) y meméticos (MA).

La obtención de la primera solución factible siempre es aleatoria. Para ello, inicialmente se ha estudiado que ésta solución fuera válida para una sobrecarga igual a tres veces la de la normativa. Podría pensarse que ello permite una exploración más exhaustiva del espacio de soluciones, dando un margen importante para que las siguientes soluciones, cuya sobrecarga fuese la correcta, pudieran variar considerablemente respecto a la primera, y así no dirigir rápidamente la solución a un mínimo local que impidiesen explorar suficientemente otras soluciones de mejor calidad. Otra de las particularidades del problema consiste en comprobar que en una solución aleatoria sea compatible la geometría de la sección y el pretensado, por lo que se recomienda, para reducir tiempos, comprobar primero la sección y después calcular su coste.

Todas las heurísticas se han aplicado a un tablero cuyos principales parámetros son los definidos en la Tabla 4-1 del Capítulo 4 (12 m. anchura tablero y $35 \mathrm{~m}$. de luz entre apoyos). 


\subsection{Búsqueda aleatoria}

\subsubsection{Descripción del algoritmo}

En este trabajo se ha desarrollado un algoritmo que aplica una técnica de construcción aleatoria -random walk (RW)- a todas las variables del tablero. Para llevarla a cabo se ha procedido de la manera siguiente. En un primer intento, se ha obtenido un valor para cada variable entre una lista de valores posibles para cada una de ellas, y con el objeto de facilitar la obtención de una solución factible se ajusta el número de torones al espacio disponible en el ala inferior y el ala superior, no permitiendo superarlo. Con este único condicionante se han fabricado 200,000 soluciones, comprobándose que ninguna de ellas ha sido factible, empleando un tiempo de proceso de 30,473 segundos. Ante la enorme dificultad en la obtención de una solución factible, se ha optado en una primera fase por ajustar el pretensado a una determinada sección geométrica del hormigón -similar al aplicado por Alcalá[6] -, y en una segunda fase ajustar la armadura transversal -aplicado por Martí[82]-.

Para ello, se ha generado una sección transversal con las variables que la definen de una lista de valores. A continuación se ha definido la armadura activa que se ha hecho encajar en las alas superiores e inferiores de la sección. Se comprueba en flexión longitudinal dicha sección con los torones ajustados. El sistema permite aumentar o disminuir un torón en cada iteración, tanto en ala inferior como en las superiores, en función de los estados límites de servicio o últimos de la viga o conjunto viga+losa. Cuando se comprueba que dicha sección no es factible aumentando o disminuyendo el pretensado, se opta por una nueva sección. Si la sección con su pretensado, cumple los estados límites longitudinales, se fabrican las variables de la armadura transversal del siguiente modo. Se da una solución común por armadura para cada una de las diez secciones distintas, y se comprueba la estructura a cortante y torsor. Para cada comprobación, que se realiza un máximo de 10 veces, en las secciones que no cumplen, se incrementan las armaduras correspondientes. Si finalmente la armadura transversal no es factible, se vuelve a generar una nueva armadura para todas las secciones, hasta un máximo de 250 veces. Si después de haber comprobado la factibilidad longitudinal, no resulta factible la transversal, se vuelve a generar una nueva geometría de la sección. En este segundo intento, el tiempo medio que se necesita para la obtención de una solución es de 317 segundos. El tiempo transcurrido para ajustar el pretensado es muy superior al necesitado para la armadura transversal, y por ello, se propone facilitar dicho ajuste mediante la limitación de la generación del canto de la sección. El valor de la variable correspondiente al canto de la viga se limita entre un 80 y $90 \%$ al valor máximo posible, y el de la losa al 30-40\% del suyo. Con este tercer intento, el tiempo se reduce a un valor medio de 26 segundos. Estos valores límites, se han obtenido a partir de la realización de varias pruebas donde se han tanteado distintos márgenes, dando resultados aceptables y a la vez manteniendo la suficiente aleatoriedad.

Respecto a la validez de este procedimiento, éste puede ser viable para ciertos problemas en los que el número total de posibles soluciones es pequeño. En el random walk la siguiente iteración no depende de la anterior por lo que la exploración es muy extensa y el método de búsqueda no puede quedarse atrapado en un óptimo local. 
Son varias las desventajas al utilizar este método. Por un lado, no se emplea ninguna información obtenida en el proceso de búsqueda. La búsqueda no se intensifica cuando se encuentra una buena solución, el random walk no garantiza encontrar un óptimo local. Y por otro, el espacio de soluciones queda dividido en un subespacio de soluciones factibles y un subespacio de soluciones no factibles. Como se ha comentado anteriormente, debido a las condiciones restrictivas del problema, un gran número de soluciones generadas aleatoriamente son no factibles.

\subsubsection{Aplicación}

El tablero objeto de optimización, tal como se describe en el Capítulo 4, tiene una luz entre apoyos de neopreno de $35 \mathrm{~m}$., y unas entregas de $0.47 \mathrm{~m}$., que convierten la distancia entre ejes en $36 \mathrm{~m}$. Cada viga se apoya sobre un neopreno. El tablero está formado por dos vigas artesa simétricas de HP, separadas $6 \mathrm{~m}$. y una losa de HA de ancho $12 \mathrm{~m}$., con una calzada de $11 \mathrm{~m}$. y barreras de $0.5 \mathrm{~m}$.

La prueba consistió en encontrar 25,000 soluciones factibles, en la Figura 6-1 se muestran los resultados. El tiempo medio para encontrar una solución factible ha sido de $26.30 \mathrm{sg}$, siendo el mínimo de $0.004 \mathrm{sg}$ y el máximo de $729.06 \mathrm{sg}$.

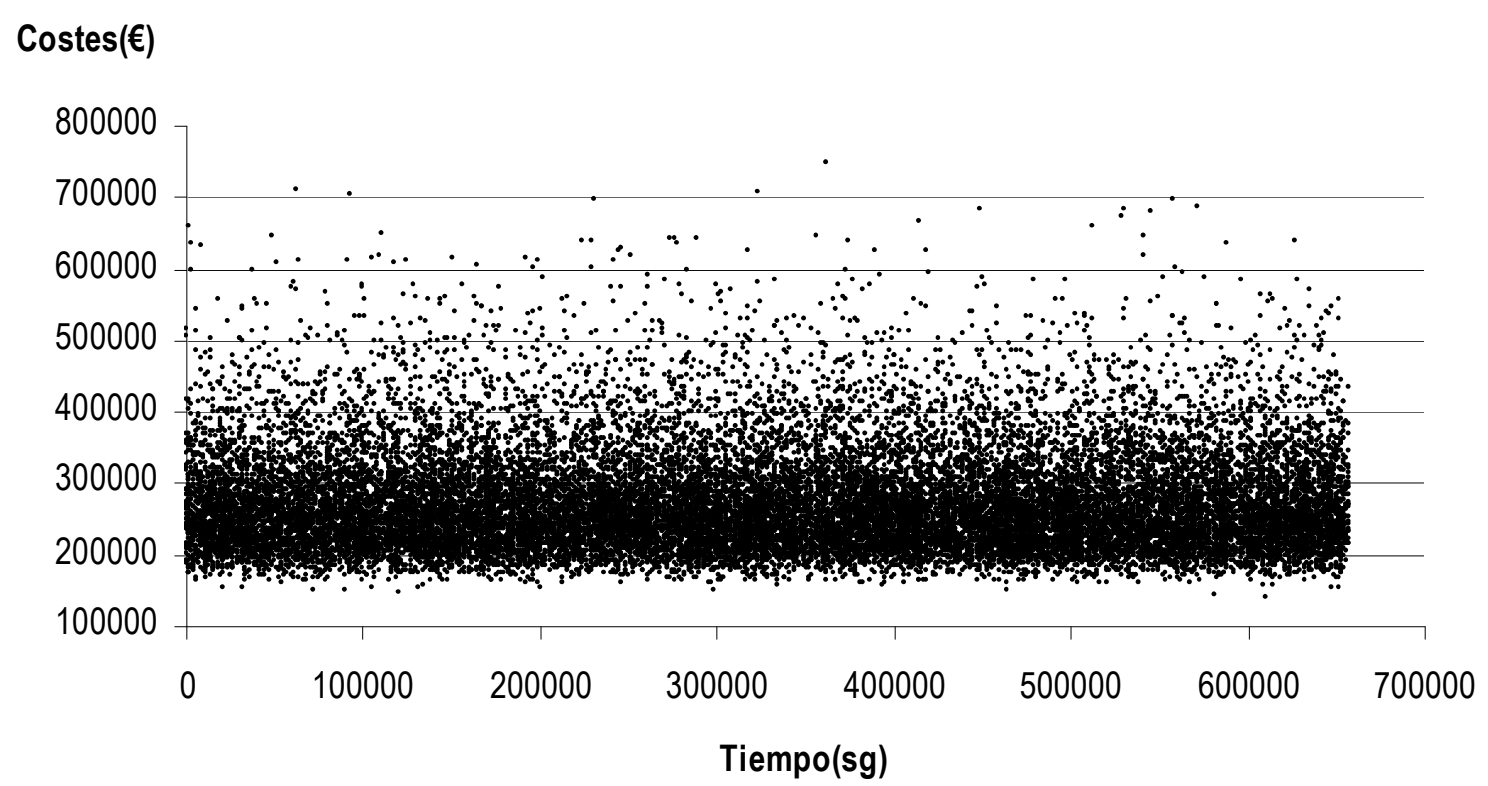

Figura 6-1: Coste de las soluciones factibles encontradas durante un RW de 25,000 soluciones 


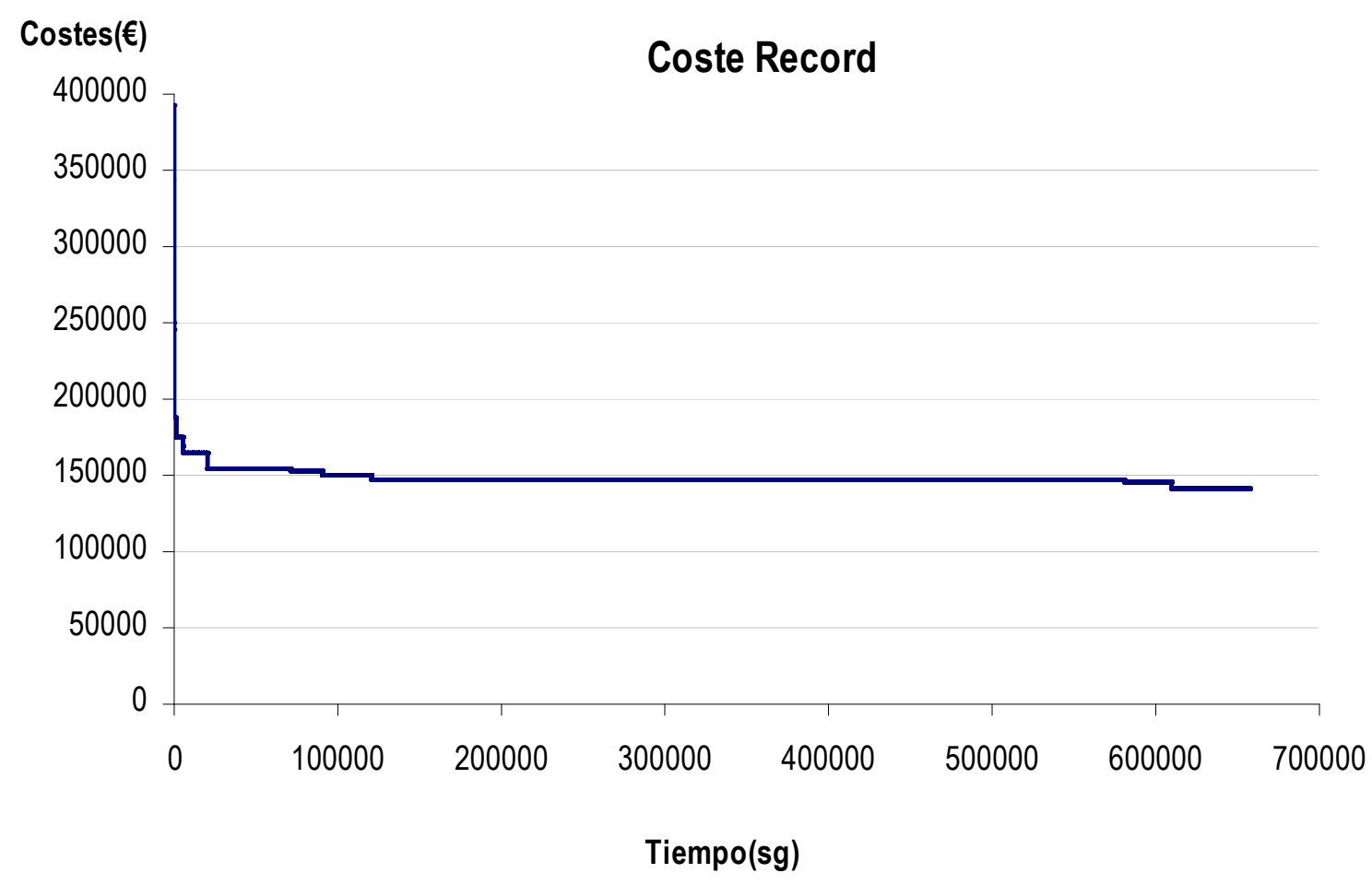

Figura 6-2: Evolución disminución del coste record durante un RW de 25000 soluciones

En la Figura 6-2 se muestra la evolución del proceso de mejora de la solución record, donde ha mejorado un total de 14 veces. Inicialmente la solución mejora rápidamente, produciéndose sucesivas mejoras leves hasta el movimiento 4,540, donde se fabrican 17,597 soluciones que no mejoran el record previo, para finalmente mejorarlo someramente dos veces más hasta el movimiento 25,000; lo cual muestra el escaso rendimiento de este método.

El coste del tablero más barato ha sido de 140,946 €, el más caro de 747,427 €, siendo el coste medio de los tableros de 273,182 €, la desviación de la media respecto al mínimo del $93.8 \%$, lo que nos indica la gran dispersión de resultados obtenidos. 


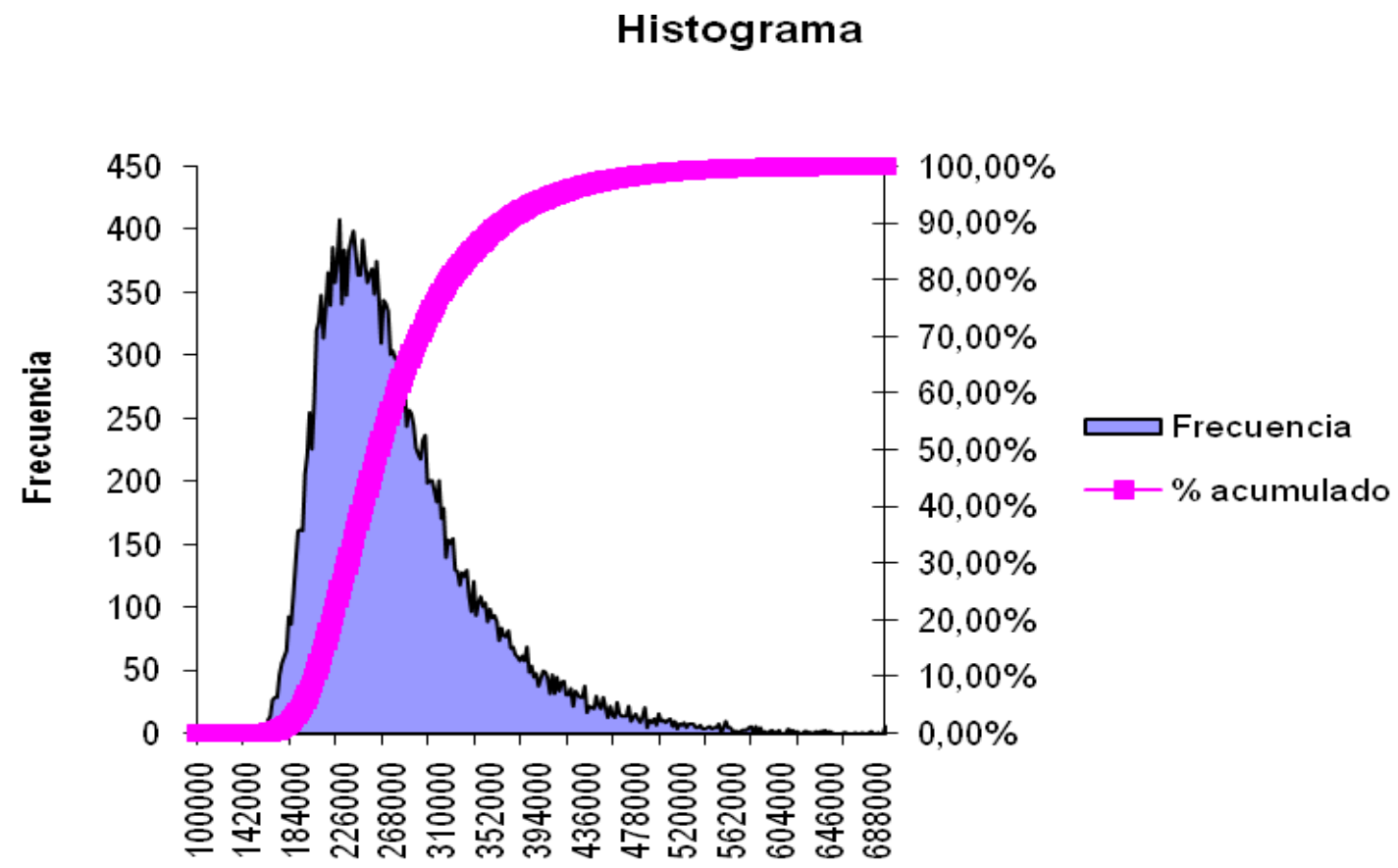

$\operatorname{Costes}(€)$

Figura 6-3: Histograma de frecuencias de las soluciones factibles encontradas

En la Figura 6-3 se muestran, por un lado el número de soluciones factibles entre dos valores determinados, y por el otro, el porcentaje de soluciones con un coste menor. Los costes se han dividido desde el tablero más barato al más caro en intervalos de $2,000 €$, siendo el intervalo de mayor frecuencia el comprendido entre 222,000 € y $224,000 €$ con 408 tableros. Se observa, además, que entre $192,000 €$ y $310,000 €$ se encuentran el $72.02 \%$ de los costes de los tableros obtenidos, teniendo prácticamente todos los rangos frecuencias superiores a 200.

El $99.00 \%$ de los tableros tienen un coste inferior a 512,000€.

Resumiendo, el random walk RW no es un método eficiente para la búsqueda de soluciones, simplemente genera soluciones aleatorias que podrán ser buenas o malas según el azar, generalmente malas, aunque en cambio, nos aporta cierta información sobre el espacio de soluciones, y permite obtener la distribución de la función de costes. En este espacio de soluciones es muy difícil alcanzar una buena solución, ya que éstas se encuentran "muy escondidas", por lo que se necesitan otros métodos de búsqueda más eficaces. La mejor solución se muestra en la Figura 6-4 
SECCION TRANSVERSAL

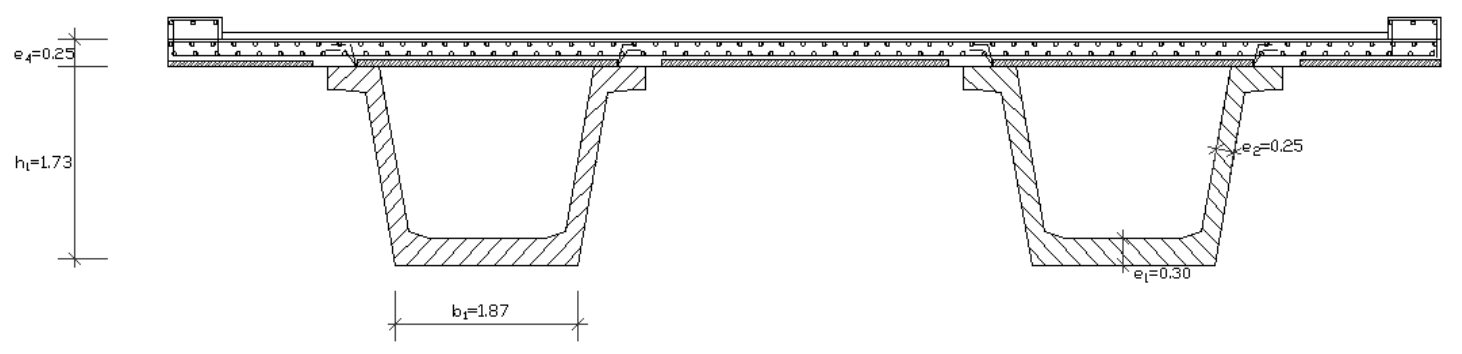

Figura 6-4: Tablero con menor coste en un RW 


\subsection{Búsqueda por gradiente}

\subsubsection{Descripción del algoritmo}

El método de búsqueda por gradiente -descent local search (DLS)-, consiste en seleccionar una solución inicial aleatoria e ir modificándola iterativamente mediante un mecanismo apropiado. Se comprueba la nueva solución, y si es factible se calcula su coste, y si este coste mejora al de la anterior, la nueva solución reemplaza a aquella. Este proceso continúa hasta que no se encuentra ninguna solución factible con precio inferior a la anterior, después de un número determinado de intentos. Uno de los inconvenientes del DLS es que no tiene capacidad de escapar de óptimos locales, por lo que es probable que la convergencia sea prematura, a pesar de ello, no dará tiempos de ejecución cortos y no garantiza que el óptimo encontrado sea el global. Una manera de mejorar el procedimiento es ejecutar el DLS varias veces, partiendo de diferentes soluciones iniciales aleatorias y así obtener mayor número de óptimos locales, con la esperanza de que el mejor de ellos sea el óptimo global. En algunos espacios de soluciones, debido al gran número de óptimos locales, esta estrategia no es efectiva.

Para la aplicación de este método, como se ha mencionado anteriormente en el Capítulo 3, es necesario seleccionar la variación (movimiento) que forme la nueva solución de trabajo. Ese mecanismo, que definirá el entorno de la solución, puede ser: la modificación de varias variables, combinación de variables, etc. Se entenderá como entorno de una solución todas las posibles soluciones que son accesibles desde la anterior.

En espacios de soluciones muy amplios, podrían darse soluciones no relacionadas con la anterior, por lo que se estaría perdiendo la información de esta última dando protagonismo al azar, lo cual se asemeja mucho al Random Walk que se ha comprobado que es poco efectivo. Entornos muy reducidos comprimirán la diversificación de la busqueda local. La selección del mecanismo de modificación, que determina el entorno, es de gran importancia dado que influye en el equilibrio entre diversificación e intensificación de una búsqueda local.

Como se ha indicado en la primera parte del Capítulo 4, en el DLS y en resto de las heurísticas, primero se comprueba la estructura, ajustándose el pretensado a la geometría, y luego se calcula el coste de la solución. En la Figura 6-5 se muestra el esquema seguido en el DLS. En anteriores trabajos[112] se investigó diferenciar las variables en varios grupos: variables de armado y variables de geometría y hormigón, y con ello, modificar las variables con distintas probabilidades. El resultado de esa investigación fue que los mejores resultados se consiguieron variando aleatoriamente un número constante de variables en cada iteración. En este trabajo se han realizado varias pruebas para establecer el número óptimo de variables a modificar en cada movimiento.

Se ha experimentado, modificando el 5\%, 10\%, 15\%, 20\%, 25\% y $30 \%$ de las variables. Lo que corresponde a 3, 6, 9, 12, 15 y 18 variables 


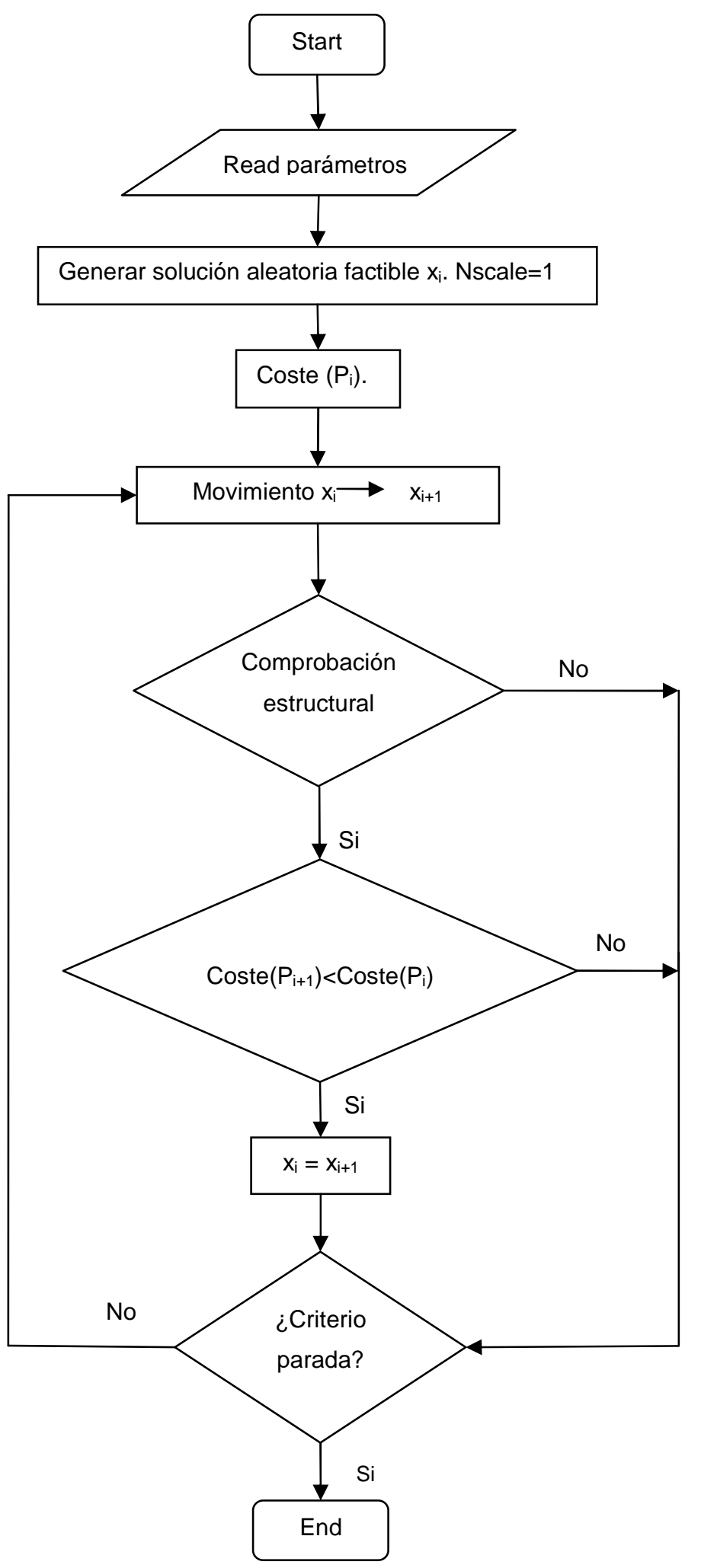

Figura 6-5: Diagrama de flujo de un DLS

Los valores a los que pueden acceder las variables en cada movimiento, son el inmediatamente superior o inferior al que tenían en la solución anterior. Si el valor fuera un extremo sólo podrá acceder al inmediatamente superior si el extremo es 
inferior y al inmediatamente inferior si el extremo es superior. Las variables a modificar se seleccionan aleatoriamente.

\subsubsection{Aplicación.}

Los algoritmos se han realizado 9 veces cada uno partiendo siempre del mismo punto, reiniciándose en cada ocasión para obtener una muestra de resultados. Al obtener nueve resultados puede considerarse al mejor de ellos como representativo del cuantil 10\% de las soluciones obtenidas con este método. En las Figuras 6-6 y 6-7 se representan los resultados medios obtenidos. En las Tablas siguientes 6-1 y 6-2, se muestran todos los resultados de costes medios.

En la Figura 6-6 se representa la evolución del coste según el criterio de parada para los movimientos de las variables, siendo modificadas $3,6,9,12,15$ y 18 de ellas, para 100, 500, 1000, 5000, 10000, 15000, 20000 y 25000 iteraciones sin mejora.

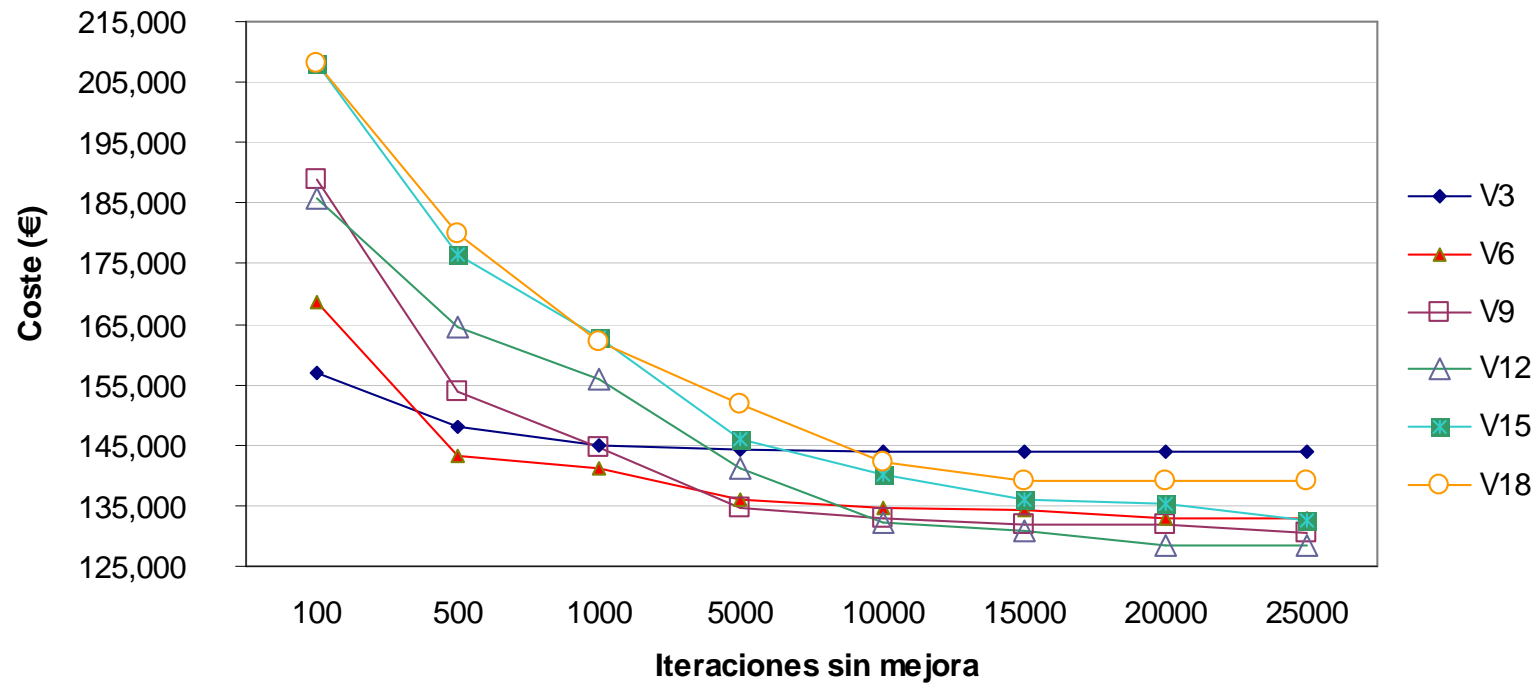

Figura 6-6: Evolución del coste para diferentes movimientos y criterios de parada

A la vista de la Figura 6-6, se puede extraer una primera conclusión. Los movimientos que han generado mejores soluciones son V9 y V12 para un número alto de iteraciones, y V6 y V9 para un número intermedio de iteraciones como pudiera ser 5000. Por otro lado, se empiezan a encontrar soluciones aceptables a partir de 5000 iteraciones, por lo que será difícil encontrar un óptimo local con un número menor. 


\begin{tabular}{|c|c|r|r|r|r|r|}
\hline \multirow{2}{*}{$\begin{array}{c}\text { Itera.sin } \\
\text { mej. }\end{array}$} & \multicolumn{2}{|c|}{ V3 (5\%) } & \multicolumn{2}{c|}{ V6 $(10 \%)$} & \multicolumn{2}{c|}{ V9 (15\%) } \\
\cline { 2 - 7 } & $(€)$ & sg. & $(€)$ & sg. & $(€)$ & \multicolumn{1}{c|}{ sg. } \\
\hline 100 & 156,954 & 85 & 168,651 & 129 & 188,793 & 105 \\
\hline 500 & 147,855 & 150 & 143,355 & 346 & 153,968 & 185 \\
\hline 1000 & 145,004 & 215 & 141,043 & 466 & 144,694 & 368 \\
\hline 5000 & 144,120 & 478 & 136,116 & 1,570 & 134,529 & 790 \\
\hline 10000 & 143,995 & 632 & 134,780 & 2,340 & 133,030 & 1,693 \\
\hline 15000 & 143,995 & 740 & 134,131 & 3,601 & 131,860 & 3,108 \\
\hline 20000 & 143,995 & 847 & 132,951 & 5,217 & 131,852 & 3,518 \\
\hline 25000 & 143,961 & 1,020 & 132,922 & 7,194 & 130,408 & 4,771 \\
\hline
\end{tabular}

Tabla 6-1: Coste medio y tiempos para 5, 10 y $15 \%$ variables.

\begin{tabular}{|c|c|r|r|r|r|r|}
\hline \multirow{2}{*}{$\begin{array}{c}\text { Itera.sin } \\
\text { mej. }\end{array}$} & \multicolumn{2}{|c|}{$\mathrm{V} 12(\mathbf{2 0} \%)$} & \multicolumn{2}{c|}{$\mathrm{V} 15(25 \%)$} & \multicolumn{2}{c|}{$\mathrm{V} 18(30 \%)$} \\
\hline & $(€)$ & \multicolumn{1}{c|}{ sg. } & $(€)$ & \multicolumn{1}{c|}{ sg. } & $(€)$ & \multicolumn{1}{c|}{ sg. } \\
\hline 100 & 185,823 & 106 & 208,149 & 102 & 208,211 & 104 \\
\hline 500 & 164,542 & 148 & 176,646 & 168 & 179,898 & 198 \\
\hline 1000 & 155,961 & 245 & 162,764 & 391 & 161,974 & 376 \\
\hline 5000 & 141,003 & 873 & 146,100 & 1,145 & 151,751 & 1,131 \\
\hline 10000 & 132,344 & 1,360 & 140,053 & 1,723 & 142,306 & 4,332 \\
\hline 15000 & 130,724 & 1,806 & 135,893 & 2,683 & 138,996 & 5,185 \\
\hline 20000 & 128,417 & 7,573 & 135,222 & 2,978 & 138,996 & 5,475 \\
\hline 25000 & 128,309 & 8,122 & 132,432 & 3,786 & 138,993 & 5,815 \\
\hline
\end{tabular}

Tabla 6-2: Coste medio y tiempos para 20, 25 y $30 \%$ variables.

Al analizar las Tablas anteriores, se observa que las soluciones en las que se modifican menos variables llegan generalmente con menores tiempos a un mismo número de iteraciones sin mejora. En el caso de 3 variables (V3), salvo para 500 iteraciones donde V12 se agota antes, siempre llega antes que las demás al final de las iteraciones sin mejora. Las soluciones que tardan más en agotarse se corresponden con V6 y V12, siendo esta última en la que más, y además en ella se obtiene la mejor solución media. Cuando se incrementa el número de iteraciones sin mejora se observa que se mejora la solución, pero a partir de un cierto número de iteraciones se incrementa el tiempo de cálculo considerablemente.

Si se comparan los costes medios con los tiempos de proceso, los contrastes aparecen de un modo mucho más claro. En la Figura 6-7 se muestra la evolución del coste y tiempo para los movimientos de las variables, siendo modificadas 3, 6, 9, 12, 15 y 18 de ellas, para 100, 500, 1000, 5000, 10000, 15000, 20000 y 25000 iteraciones sin mejora. 


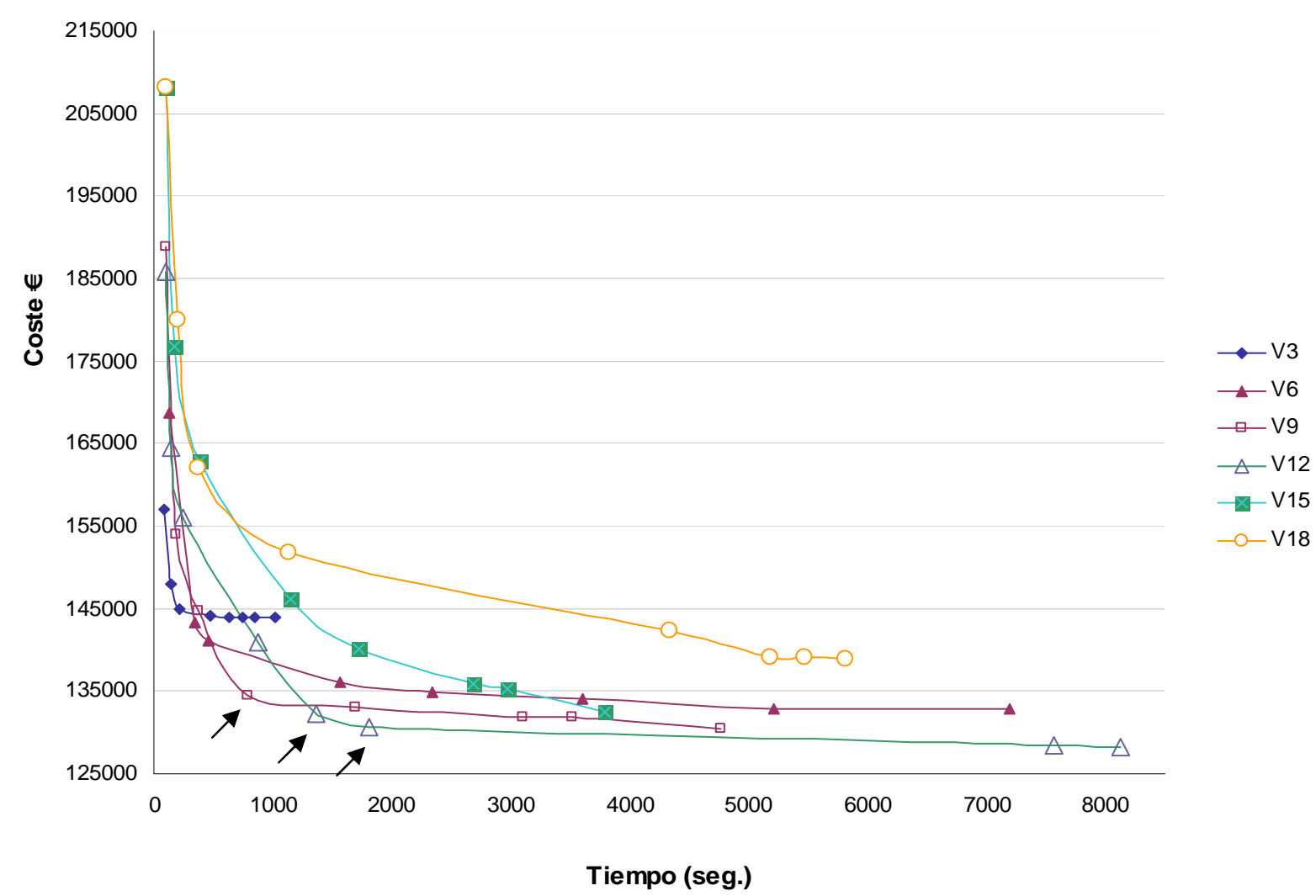

Figura 6-7: Evolución del coste medio para diferentes movimientos y tiempos de ejecución

Los óptimos de Pareto más equilibrados entre coste/tiempo se corresponden con V9 (5000 iteraciones), y V12 (para 10000 y 15000 iteraciones). Se puede apreciar que el movimiento V9 presenta el mejor resultado, pues para este movimiento con 5000 iteraciones sin mejora su coste es de 134,529 € y su tiempo de proceso es de $790 \mathrm{seg}$. A partir de este punto, una mejora del $1.6 \%$ se consigue con un incremento del $72.2 \%$ en tiempos de computación para V12 (10000 it.), y para el siguiente óptimo de Pareto, una mejora del $2.8 \%$ en costes implica un incremento de más del doble en tiempos.

La Tabla 6-3 muestra las desviaciones en las 9 ejecuciones de la media con respecto a la mínima en tanto por cien, en todos los experimentos realizados.

\begin{tabular}{|c|r|r|r|r|r|r|}
\hline $\begin{array}{c}\text { Itera.sin } \\
\text { mej. }\end{array}$ & \multicolumn{1}{|c|}{ V3 } & \multicolumn{1}{c|}{ V6 } & V9 & V12 & \multicolumn{1}{c|}{ V15 } & \multicolumn{1}{c|}{ V18 } \\
\hline 100 & 11.0 & 14.6 & 27.0 & 13.0 & 22.0 & 15.3 \\
\hline 500 & 7.3 & 11.0 & 19.4 & 13.6 & 11.2 & 25.0 \\
\hline 1000 & 9.5 & 9.3 & 12.9 & 17.6 & 9.9 & 12.6 \\
\hline 5000 & 8.8 & 9.5 & 8.3 & 12.4 & 8.0 & 15.1 \\
\hline 10000 & 9.5 & 10.2 & 7.8 & 9.6 & 5.7 & 14.2 \\
\hline 15000 & 9.5 & 10.3 & 6.9 & 12.2 & 9.8 & 14.3 \\
\hline 20000 & 9.5 & 10.0 & 6.9 & 10.8 & 9.7 & 14.3 \\
\hline 25000 & 9.7 & 10.0 & 5.7 & 10.7 & 12.1 & 14.3 \\
\hline
\end{tabular}

Tabla 6-3: Desviaciones (\%) del coste medio respecto al mínimo 
En conjunto, las desviaciones son elevadas, observándose una gran dispersión de resultados, siendo el peor de los casos el V9, entre un 5.7 y un $27.0 \%$, teniendo que llegar hasta 5000 iteraciones para reducir las desviaciones por debajo del 10\%. Esto demuestra que el espacio de soluciones contiene multitud de óptimos locales de baja calidad, y que la búsqueda por medio de este sistema no garantiza la obtención de una buena solución.

La mejor solución encontrada ha sido en una ejecución variando el $15 \%$ de las variables (V9), su coste es de 115,459 € (Figura 6-8). El coste de esta solución es un $18.1 \%$ más económica que la mejor encontrada en el RW, que era de 140,946 €.

Las desviaciones del coste medio con respecto al mínimo eran en el RW del 93,8 \% para el tablero. En el DLS, como podemos apreciar en la Tabla 6-3, independientemente del número de iteraciones sin mejora y de las variables modificadas, está alrededor del 10\%.

Se puede decir como conclusión, que es un método que mejora notablemente al RW, y que proporciona soluciones aceptables en tiempos reducidos, pero con desviaciones elevadas, no siendo por tanto el más adecuado para resolver el problema en el que nos encontramos. Esto se debe a que, por un lado, el método se bloquea al llegar al óptimo local y, por otro lado, a la forma del espacio de soluciones en el que los óptimos locales no son muy parecidos entre sí, por lo que podemos imaginar que tampoco lo son al óptimo global. Es por ello, que se justifica la necesidad de intentar optimizar la estructura mediante heurísticas que admitan la aceptación de soluciones peores, a cambio de salir, en su búsqueda, de óptimos locales de baja calidad.

SECCION TRANSVERSAL

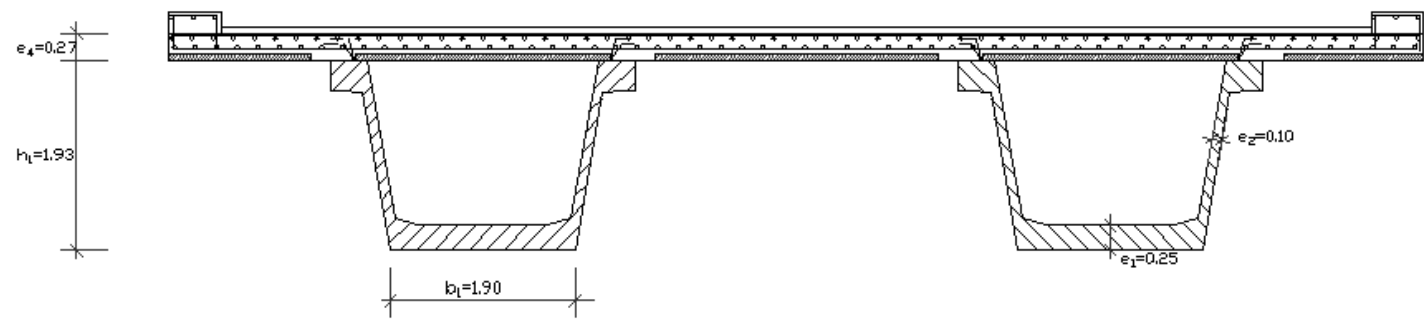

Figura 6-8: Tablero con menor coste en un DLS 


\subsection{Cristalización simulada}

\subsubsection{Descripción del algoritmo}

A diferencia de la búsqueda por gradiente (DLS), el método de la cristalización simulada-simulated annealing (SA)- permite aceptar soluciones con un coste mayor que la anterior siempre que cumpla unas condiciones. Ello permite salir de un óptimo local y buscar un óptimo mejor. Esta metaheurística ha sido descrita en el punto 3.2.2, y en este apartado va a completarse con una descripción del proceso desarrollado.

La aceptación o no de una solución con un coste mayor estará en función de una determinada probabilidad. Esta probabilidad dependerá de la expresión: $e^{-(\Delta C / T)}$, donde $T$ es la temperatura en ese momento y $\Delta C$ representa la variación de coste entre dos soluciones consecutivas. Primero se genera un número aleatorio dentro del intervalo comprendido $[0,1]$ que tenga una distribución uniforme de probabilidad; y segundo, si dicho número es inferior al valor $\mathrm{e}^{-(\Delta \mathrm{C} / \mathrm{T})}$ entonces se acepta la nueva solución. Cada cierto número de iteraciones, se reduce la temperatura. En los estados iniciales las soluciones peores se aceptan con una alta probabilidad, permitiendo adentrarse por el espacio de soluciones, y conforme avanza el proceso de cálculo, reduciéndose la temperatura, se aceptan soluciones peores a la de partida con una menor probabilidad, intensificando la búsqueda por óptimo local. La exploración se detiene cuando se alcanza un criterio de parada.

En la Figura 6-9 se muestra un diagrama de flujo básico del algoritmo del SA. Se selecciona una temperatura inicial que se mantiene constante durante un número de iteraciones. A este número se le denomina cadena de Markov. Al finalizar cada cadena de Markov, si no se cumple el criterio de parada, se reduce la temperatura de forma geométrica, multiplicando la temperatura anterior por un coeficiente denominado coeficiente de enfriamiento. En cada iteración se aplica un movimiento modificado a un número determinado de variables, y si la solución es factible, reemplaza a la anterior si su coste es menor al de la anterior. Si el coste es mayor, se aplica el criterio de aceptación expresado en el párrafo anterior. 


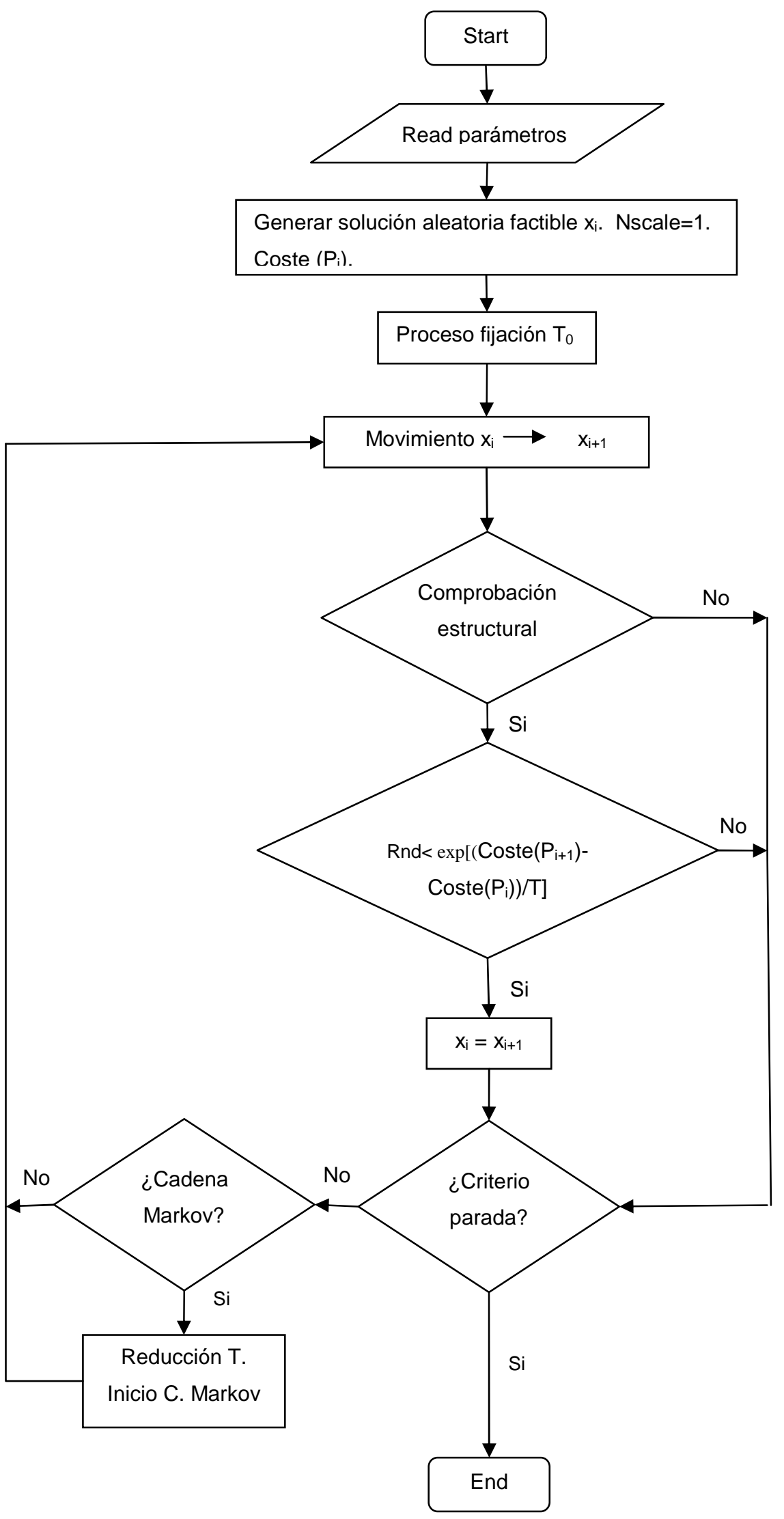

Figura 6-9: Diagrama de flujo de un simulated annealing 
El proceso SA queda definido por los siguientes parámetros:

- Definición del número de variables a modificar

- Selección de la temperatura inicial

- Ritmo de enfriamiento y longitud de cadena de Markov

- Selección de un criterio de parada

\section{Definición del número de variables a modificar}

Se procesará modificando 9 variables del tablero elegidas al azar, esto supone un $15 \%$ del total. Este número de variables modificadas resultó ser la mejor, para nuestro problema, en el estudio realizado para el DLS.

\section{Selección de la temperatura inicial}

El valor de la temperatura inicial depende del orden de magnitud de los resultados de la función objetivo, en concreto, del orden de magnitud de las variaciones de coste que se producen en cada movimiento. Sin embargo, puede establecerse un criterio indicativo de la bondad de una temperatura inicial de acuerdo con el porcentaje de soluciones que son aceptadas (Medina[110],2001).

La selección de la temperatura inicial se ha realizado mediante dicho procedimiento, consistente en:

1. Se selecciona una temperatura arbitraria $\mathrm{T}_{0}$. El valor $\mathrm{T}_{0}$ se ha tomado como valor de partida, el $0.5 \%$ del coste de la primera solución. Con este valor no se ha llegado nunca a necesitar procesar más de 2 cadenas de Markov.

2. Se establece un rango, entre un $20 \%-40 \%$.

3. Se procesa un determinado número de movimientos consecutivos, que se denomina longitud de la cadena, se aplica a cada proceso el criterio de aceptación del SA, y se contabiliza el número de soluciones.

4. Si el número de soluciones aceptadas está por debajo del rango inferior, la nueva temperatura será el doble que el anterior $\mathrm{T}_{0}=2 \mathrm{~T}_{0}$. Si está por encima del rango superior, se reduce a la mitad $\mathrm{T}_{0}=\mathrm{T}_{0} / 2$. Si está dentro del rango, queda establecida la temperatura inicia $\mathrm{T}_{0}$ con dicho valor.

Ritmo de enfriamiento y longitud de cadena de Markov

La temperatura debe disminuir hasta llegar a un valor casi nulo -enfriamiento-, durante el proceso hasta alcanzar un criterio de parada. Para ello, el criterio habitual es el de mantener la temperatura constante durante un determinado número de movimientos, al cabo del cual se aplica algún tipo de reducción. Este número de movimientos se denomina cadena de Markov. La longitud de las cadenas de Markov puede ser variable o constante durante el proceso, siendo éste último caso el más habitual.

El método usado para reducir la temperatura es geométrico, con un coeficiente de enfriamiento constante e inferior a la unidad, que se aplica al final de la cadena de Markov. Este coeficiente debe coordinarse con la longitud de la cadena de Markov, dado que el ritmo de enfriamiento afecta al número de cadenas de Markov que se van 
a ejecutar. Tiempos de computación similares pueden obtenerse con velocidades de enfriamiento lentas y longitud de cadenas cortas, o con enfriamientos rápidos y longitudes más largas.

En la Figura 6-10 se muestra, para un caso de este estudio, como disminuye la temperatura aplicando un descenso geométrico en relación al tiempo de proceso. En la Figura 6-11 se representa la evolución del coste en función al número de soluciones aceptadas.

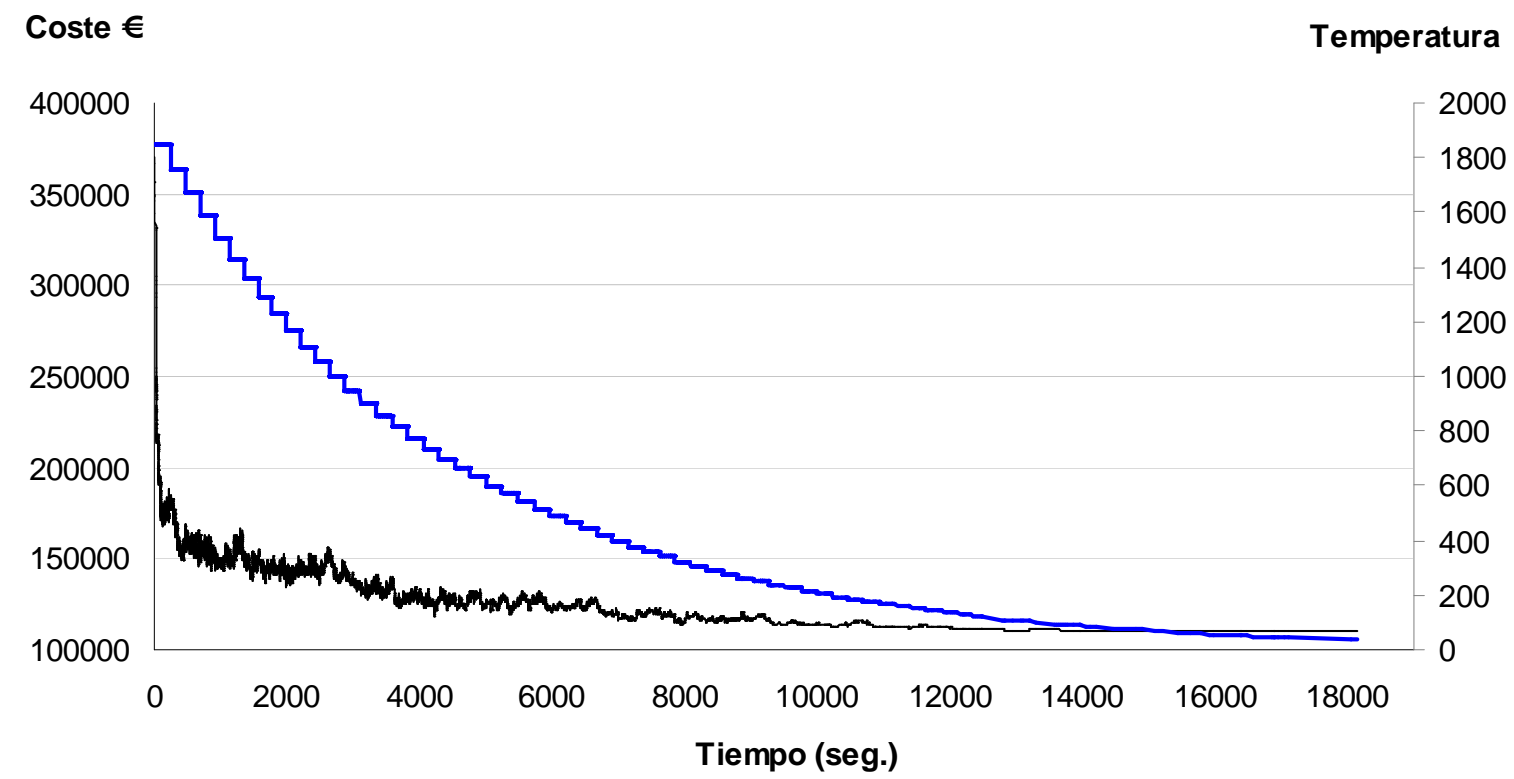

Figura 6-10: Proceso de evolución del coste y disminución de la temperatura relativo al tiempo de proceso

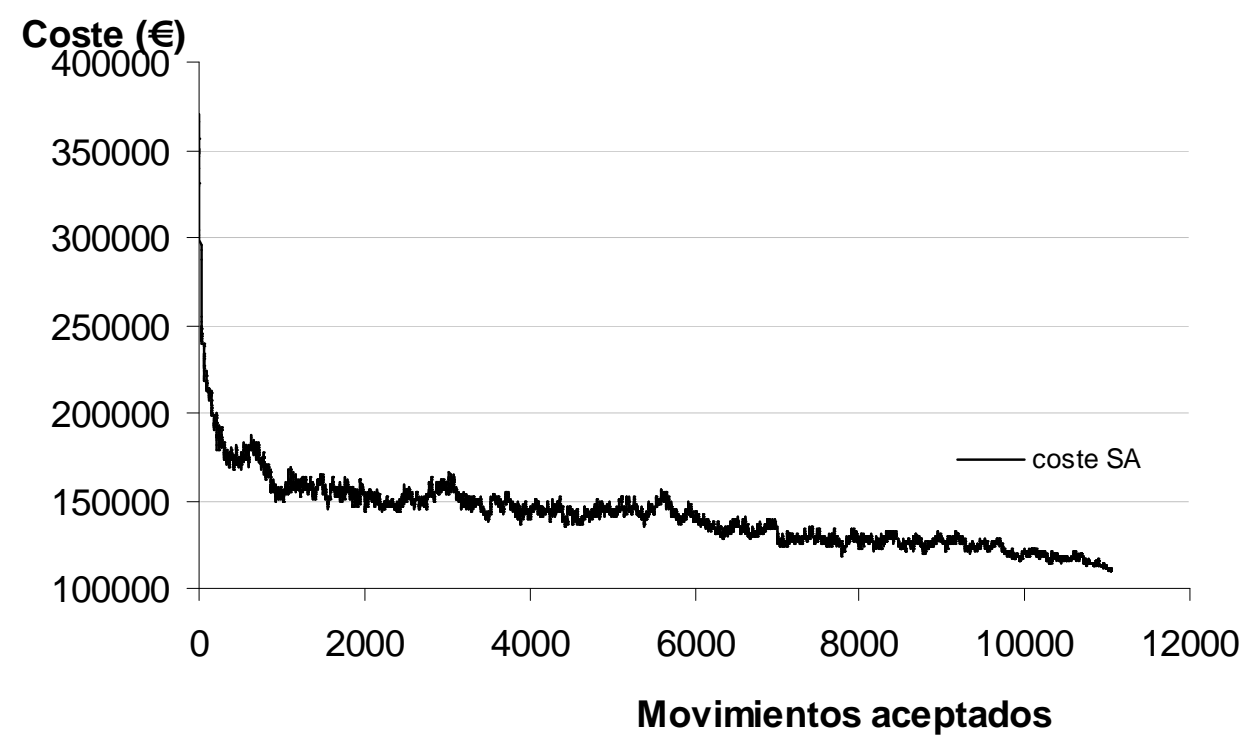

Figura 6-11: Proceso de disminución del coste relativo al número de soluciones aceptadas 
Comparando ambas gráficas, se observa que en la primera al aumentar el tiempo computacional, la variación del coste se minimiza, y sin embargo en la segunda la disminución del coste incluso se incrementa en la fase final de aceptación. Dicha fase puede identificarse como de intensificación.

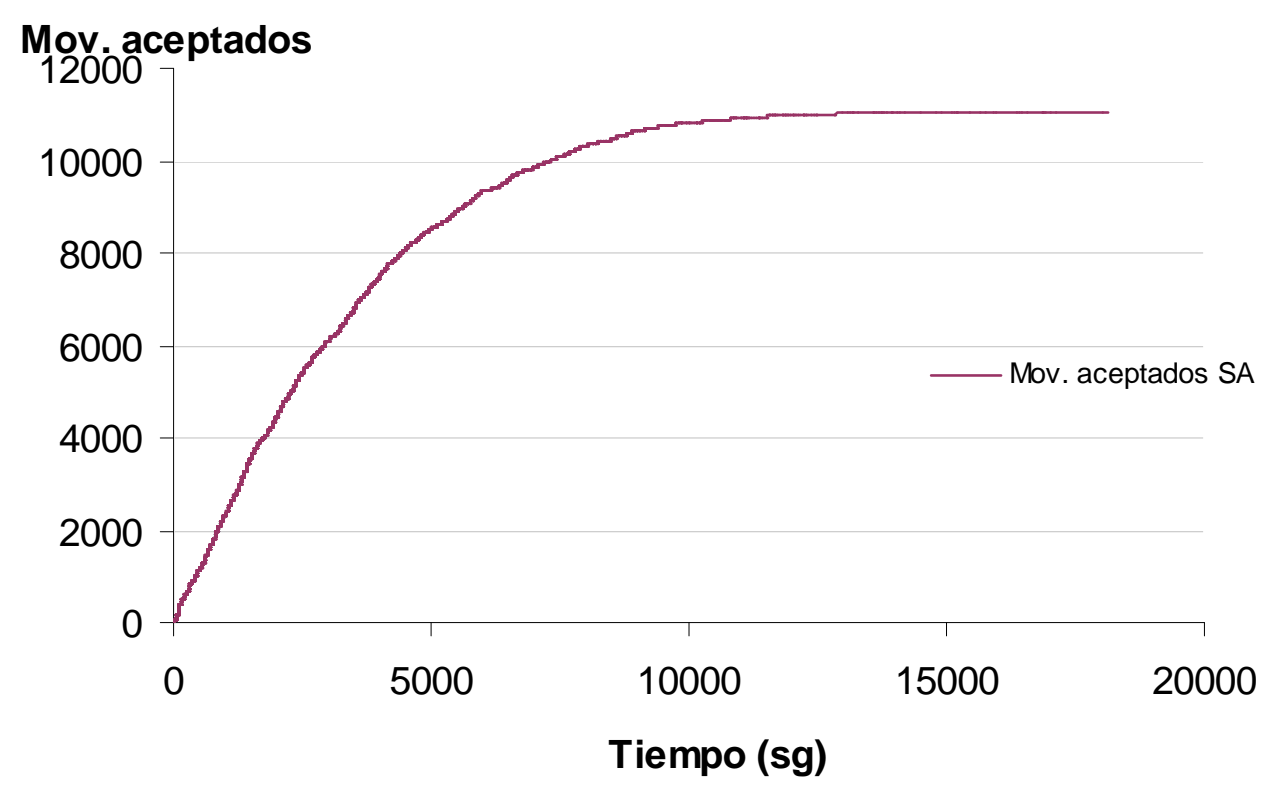

Figura 6-12: Evolución del número de movimientos aceptados respecto al tiempo de proceso

La justificación viene descrita en la Figura 6-12, en la que a partir de cierto momento, existe dificultad en encontrar soluciones aceptadas, observando que la pendiente de la curva se convierte casi en horizontal a partir de aproximadamente 13,000 segundos. Desde ese momento se encuentran 42 soluciones aceptadas en 5,217 seg.; el equivalente a una solución cada $124 \mathrm{seg}$, que contrasta con una solución cada $0.23 \mathrm{seg}$ en las 4,000 primeras soluciones aceptadas.

Los coeficientes de enfriamiento empleados han sido $0.80,0.85,0.90$ y 0.95 . Los valores de las longitudes que definen las cadenas de Markov se han tomado constantes por simplicidad, y porque el adoptar cadenas de longitud variable podrían distorsionar el ritmo geométrico de enfriamiento. Se han empleando longitudes de cadena de 500, 1000, 2500 y 5000, correspondiendo la longitud máxima al valor que para el DLS daba resultados más equilibrados entre coste y tiempo de proceso.

\section{Selección del criterio de parada}

En principio, se podrían establecer distintos criterios de parada. Si el enfriamiento se produjera de forma lineal, el criterio de parada más lógico sería detener el proceso cuando la temperatura se anulara, siempre que no representase tiempos computacionales excesivos. Si el enfriamiento fuera geométrico, la temperatura tendería de forma asintótica, ver Figura anterior 6-10, a un valor nulo, que no se 
alcanzaría nunca; es por ello, que se podría establecer un criterio de parada cuando la temperatura disminuyera por debajo de un umbral.

Otro criterio, podría establecerse deteniendo el proceso cuando durante en una cadena completa de Markov el algoritmo no aceptase ninguna solución. Este criterio es parecido al aplicado en el DSL. Por último, podría detenerse el proceso al fijar un límite del número total de movimientos.

En este estudio, se ha establecido el siguiente criterio de parada, en el que se cumplen las siguientes condiciones, finalizada una cadena:

- La temperatura es menor del $2 \%$ de la temperatura inicial $\mathrm{T}_{0}$. La elección del $2 \%$ viene determinada porque suelen agotarse antes 2 cadenas consecutivas que llegar al $2 \%$ de $\mathrm{T}_{0}$.

- En dos cadenas consecutivas no se encuentra ninguna solución que mejora la de partida.

En el Simulated Annealing, inicialmente se aceptan empeoramientos altos. Conforme avanza el proceso, éstos van reduciéndose, centrando la búsqueda en soluciones con costes parecidos o menores. A continuación (Figura 6-13), se muestra la Figura anterior 6-10, a la que se le extrae la temperatura, y así mostrar la evolución del coste en relación al tiempo de proceso, identificando la fase de intensificación.

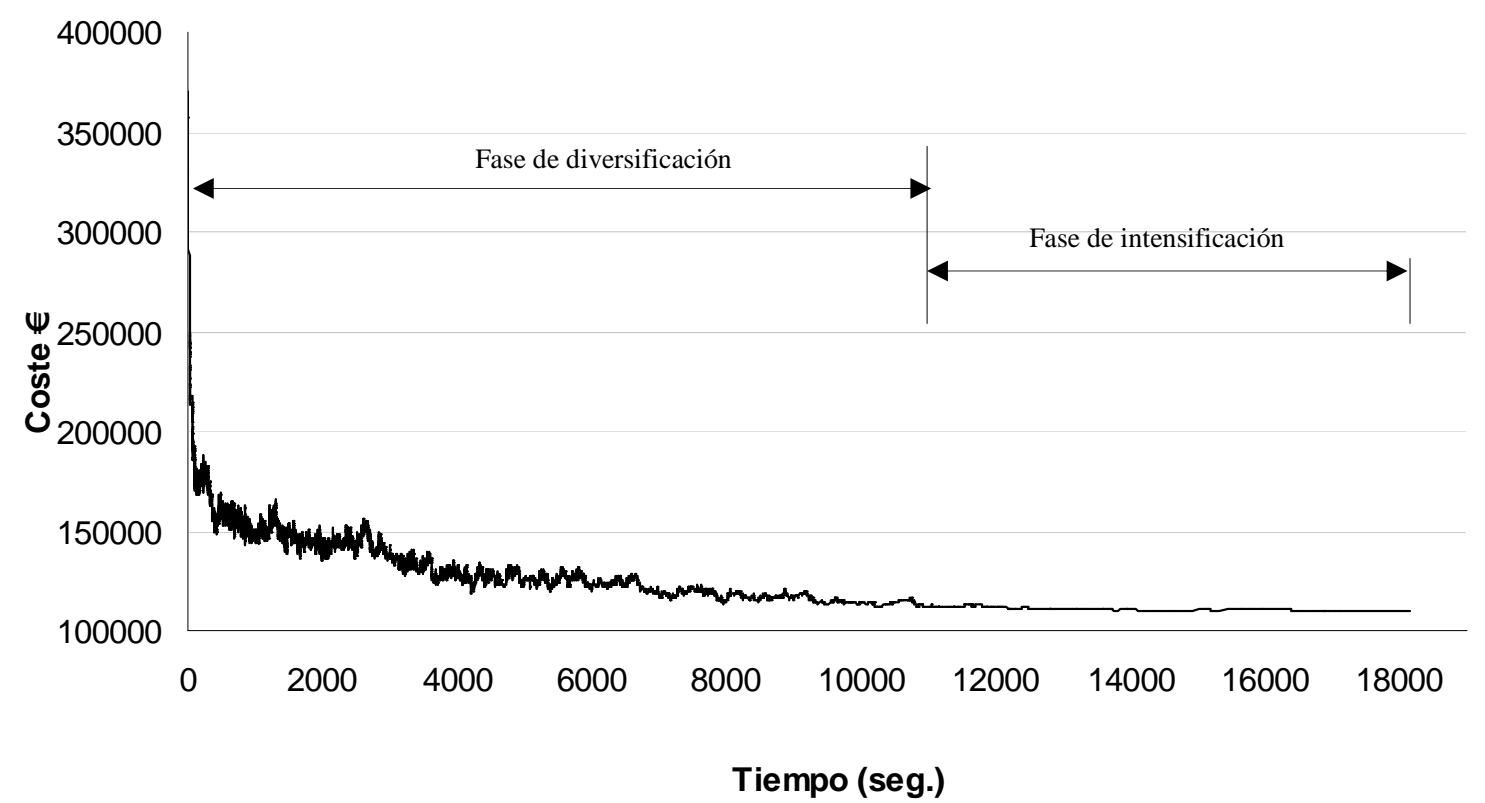

Figura 6-13: Proceso de evolución de un annealing 


\section{Mejoras del algoritmo}

En este punto, se hace una relación de intentos de mejora del algoritmo. Hay dos elementos importantes que afectan a la bondad de las heurísticas para optimizar la función coste en este tipo de estructuras. Por un lado, se tiene el elevado tiempo de proceso necesario para la obtención de una solución factible, tal como se ha descrito anteriormente, y por otro, la dificultad para la aceptación de una solución encontrada.

El primer intento de mejora, ha consistido en intentar obtener una solución con una sección sobredimensionada, tanto en cuanto a la sección de hormigón como en el número de torones. A priori, puede entenderse que bajo estas condiciones se podrá mover con mayor facilidad por el espacio de soluciones mientras el problema está caliente, en la fase de diversificación, y con ello se obtendrán mayor número de soluciones aceptadas que ayuden a conseguir mejores resultados. Para ello, se ha aplicado el método aplicado por Alcalá[6] y otros miembros del GPRC en otros trabajos, que les ha llevado a buenos resultados para la resolución de otros tipos de estructuras de hormigón pretensado, consistente en incrementar la solicitación de las sobrecargas, que obliga a sobredimensionar la estructura para poder cumplir los estados límites. El factor aplicado ha sido 3, con lo cual la carga repartida se convierte en $12 \mathrm{kN} / \mathrm{m}^{2}$, y el carro en $1800 \mathrm{kN}$. Se han contrastado los resultados obtenidos escalando y sin escalar la sobrecarga para la siguiente heurística repetida 9 veces (Tabla 6-4).

\begin{tabular}{|l|c|c|}
\hline & Nscale $=1$ & Nscale $=3$ \\
\hline Coste medio & $110919 €$ & $110864 €$ \\
\hline Coste mínimo & $109117 €$ & $109337 €$ \\
\hline Desviación media & $1.65 \%$ & $1.39 \%$ \\
\hline Tiempo medio primera solución & $25 \mathrm{seg}$ & $1452 \mathrm{seg}$. \\
\hline Movimientos aceptados medios & 19552 & 22203 \\
\hline
\end{tabular}

Tabla 6-4: Resultados escalados. Coef enfriamiento: 0.95, Cadena Markov: 5000

A la vista de estos resultados, no se muestra claramente la conveniencia de utilizar el factor Nscale $=3$. Por un lado el coste medio se mejora sólo un $0.05 \%$, pero por otro, la mejor solución se encuentra en la heurística sin escalar, y el tiempo de proceso para la obtención de la primera solución es muy superior, más de 1,400 segundos. Se analizan los resultados de la evolución de los costes en los dos casos, tomando como ejemplo las mejores soluciones obtenidas en el cuadro anterior. En la primera, la primera solución factible (aleatoria) tiene un coste de 369,996 €, y para la segunda es de 248,899 €, por lo que se desprende que ambas van sobredimensionadas al comienzo. Al finalizar la primera cadena, el coste para la primera es de 167,558 €, y 
para la segunda de 156,658 €. Al comenzar la segunda cadena el factor multiplicativo de la sobrecarga desaparece, siendo la unidad en ambos casos, por lo que ambas parten de soluciones comparables. En cambio, hay un mayor número de movimientos aceptados, como era de prever, para Nscale $=3$, pero este valor no es muy superior, lo que no lo hace determinante en la obtención de mejores soluciones.

El segundo intento de mejora ha consistido en aplicar el método de reparación del pretensado descrito en el RW en cada movimiento aplicado. El objetivo era aumentar el número de soluciones aceptadas. A continuación se contrastan los resultados obtenidos para la siguiente heurística repetida 9 veces (Tabla 6-5).

\begin{tabular}{|l|c|c|}
\hline & Sin Reparación & Con Reparación \\
\hline Coste medio & $112064 €$ & $112550 €$ \\
\hline Coste mínimo & $110049 €$ & $110009 €$ \\
\hline Desviación media & $1.83 \%$ & $2.31 \%$ \\
\hline Tiempo medio & $7442 \mathrm{seg}$ & $79249 \mathrm{seg}$. \\
\hline Movimientos aceptados medios & 2560 & 4573 \\
\hline
\end{tabular}

Tabla 6-5: Resultados con o sin reparación. Coef enfriamiento: 0.95, Cadena Markov: 1000

Se observa que con este método, aumenta considerablemente el número de soluciones aceptadas, pero en contra de lo esperado, el coste medio no mejora, sino que aumenta un $0.43 \%$. El coste mínimo es muy parecido, la variación no llega al 1 por mil. Sin embargo, el factor principal que lleva a descartar este método de reparación del pretensado, es el incremento del tiempo de proceso, que se ve aumentado más de 10 veces.

\subsubsection{Aplicación.}

El algoritmo del SA se ha ejecutado un total de 144 veces, el número de combinaciones de parámetros ha sido 16. Las ejecuciones se han repetido 9 veces para cada combinación estudiada.

En la siguiente Tabla 6-6 se muestran, para las combinaciones de parámetros, el coste y tiempo medios y movimientos aceptados de las 9 ejecuciones, el coste mínimo alcanzado en una ejecución y la desviación del valor medio con respecto al mínimo en tanto por cien. 


\begin{tabular}{|c|c|c|c|c|c|r|r|}
\hline Heurística & $\begin{array}{c}\text { Disminución } \\
\text { temperatura }\end{array}$ & $\begin{array}{c}\text { Cadena } \\
\text { Markov }\end{array}$ & $\begin{array}{c}\text { Coste } \\
\text { medio( }())\end{array}$ & $\begin{array}{c}\text { Tiempo } \\
\text { medio(sg) }\end{array}$ & $\begin{array}{c}\text { Coste } \\
\text { mínimo }(€)\end{array}$ & $\begin{array}{c}\text { Desv. } \\
(\%)\end{array}$ & $\begin{array}{c}\text { Mov. } \\
\text { acep. }\end{array}$ \\
\hline S1 & 0.95 & 500 & 116066 & 3224 & 113284 & 2.46 & 2560 \\
\hline S2 & 0.95 & 1000 & 112064 & 7442 & 110049 & 1.83 & 4336 \\
\hline S3 & 0.95 & 2500 & 110477 & 18322 & 109004 & 1.35 & 11114 \\
\hline S4 & 0.95 & 5000 & 110919 & 36163 & 109117 & 1.65 & 19552 \\
\hline S5 & 0.90 & 500 & 121909 & 1675 & 114905 & 6.10 & 1366 \\
\hline S6 & 0.90 & 1000 & 114839 & 3720 & 111651 & 2.86 & 2161 \\
\hline S7 & 0.90 & 2500 & 111743 & 9147 & 109119 & 2.41 & 5723 \\
\hline S8 & 0.90 & 5000 & 110759 & 18875 & 109810 & 0.86 & 10185 \\
\hline$S 9$ & 0.85 & 500 & 123223 & 1184 & 117632 & 4.75 & 965 \\
\hline$S 10$ & 0.85 & 1000 & 119268 & 2940 & 111143 & 7.31 & 1579 \\
\hline$S 11$ & 0.85 & 2500 & 114308 & 6574 & 109875 & 4.04 & 4021 \\
\hline$S 12$ & 0.85 & 5000 & 111903 & 12986 & 108008 & 3.61 & 7058 \\
\hline$S 13$ & 0.80 & 500 & 125160 & 946 & 114626 & 9.19 & 786 \\
\hline$S 14$ & 0.80 & 1000 & 121381 & 2366 & 109938 & 10.41 & 1162 \\
\hline$S 15$ & 0.80 & 2500 & 115130 & 4967 & 111197 & 3.54 & 3056 \\
\hline$S 16$ & 0.80 & 5000 & 112194 & 10743 & 109134 & 2.80 & 5051 \\
\hline
\end{tabular}

Tabla 6-6: Resultados del SA con las 16 combinaciones con los parámetros seleccionados

Como resumen de los datos de la Tabla anterior, se obtiene el valor medio del coste medio, tiempo medio y movimientos aceptados medios de todas las ejecuciones, el mínimo y la desviación del valor medio con respecto al mínimo:

\begin{tabular}{|l|c|c|c|}
\cline { 2 - 3 } \multicolumn{1}{c|}{} & Coste $(€)$ & Tiempo(sg) & Mov. acep. \\
\hline Media & 115709 & 8830 & 5042 \\
\hline Mínimo & 108008 & 946 & \multicolumn{1}{|c}{} \\
\hline Máximo & 125160 & 36163 & \multicolumn{1}{|c}{} \\
\cline { 1 - 3 } Desviac.(\%) & 7.13 & \multicolumn{2}{c}{} \\
\cline { 1 - 3 }
\end{tabular}

Tabla 6-7: Resultados medios obtenidos en SA

Del análisis de los resultados obtenidos, se tiene que:

- El SA permite saltarse con facilidad óptimos locales en la búsqueda de una mejor solución dentro del espacio de soluciones, sin tener que recurrir como en el DLS, a un número excesivamente largo de iteraciones sin mejora. Como ejemplo, en el SA la peor heurística se corresponde con una longitud de cadena de Markov de 500 (S13), un coste medio de 125,160 €, un tiempo de proceso de 946 segundos y una desviación del 9.2\%, mientras que la mejor del DLS se corresponde con 25,000 iteraciones sin mejora, un coste medio de $128,309 €$, un tiempo de proceso de 8,122 segundos y una desviación del $10.7 \%$. 
- Con coeficientes de disminución de la temperatura más lentos, 0.95, aumenta considerablemente el tiempo de cálculo, sobretodo para longitudes de cadena altas, mejorando ligeramente la solución por estar explorando más minuciosamente el entorno del óptimo local.

- Al aumentar el tamaño de la cadena se posibilita mejorar las soluciones, pero por contrapartida, el tiempo de cálculo aumenta considerablemente.

- El método se muestra realmente robusto para longitudes de cadena altas, igual o superiores a 1000, y con reducciones de la temperatura lentos, del 0.95. En estos casos, las desviaciones varían desde el $1.35 \%$ hasta el $1.83 \%$. La desviación más baja se da para una disminución de la temperatura del 0.90 , y longitud de cadena de 5000. Las desviaciones medias del conjunto de las 16 iteraciones es del $7.13 \%$.

En la Figura 6-14 se representan los valores medios de la Tabla 6-6, marcándose con un círculo tres valores pertenecientes a óptimos de Pareto, correspondientes a costes medios bajos.

La solución con menor coste medio tiene las siguientes características:

- Longitud de cadena:

2500 iteraciones

- Coeficiente disminución temperatura: 0.95

- Coste medio:

$110,477 €$

- Tiempo medio de proceso:

18,322 segundos

- Desviación:

$1.35 \%$

La desviación es buena, se encuentra entre las más bajas de todas las ejecuciones. Sin embargo, el tiempo de cálculo es más elevado debido a que la disminución del umbral es lenta, 0.95, aunque dicho tiempo computacional es perfectamente asumible, tardando aproximadamente 5 horas para obtener una muy buena solución. Los otros dos óptimos de Pareto señalados en la Figura 6-14 se obtienen con tiempos considerablemente menor, pero los costes medios son algo más elevados. Son los casos siguientes:

- Longitud de cadena:

2500 iteraciones

- Coeficiente disminución temperatura: 0.90

- Coste medio:

- Tiempo medio de proceso:

$111,743 €$

- Desviación:

9,147 segundos

(S2)

- Longitud de cadena:

$2.41 \%$

- Coeficiente disminución temperatura: 0.95

- Coste medio:

$112,064 €$

- Tiempo medio de proceso:

- Desviación:

7,442 segundos

$1.83 \%$ 
El coste medio es un $1.1 \%$ superior en (S7) y un $1.4 \%$ en (S2), y el tiempo de proceso se reduce un $50.1 \%$ en (S7) y un $59.4 \%$ en $(\mathrm{S} 2)$.

\section{Coste Medio f(temperatura/cadena)}

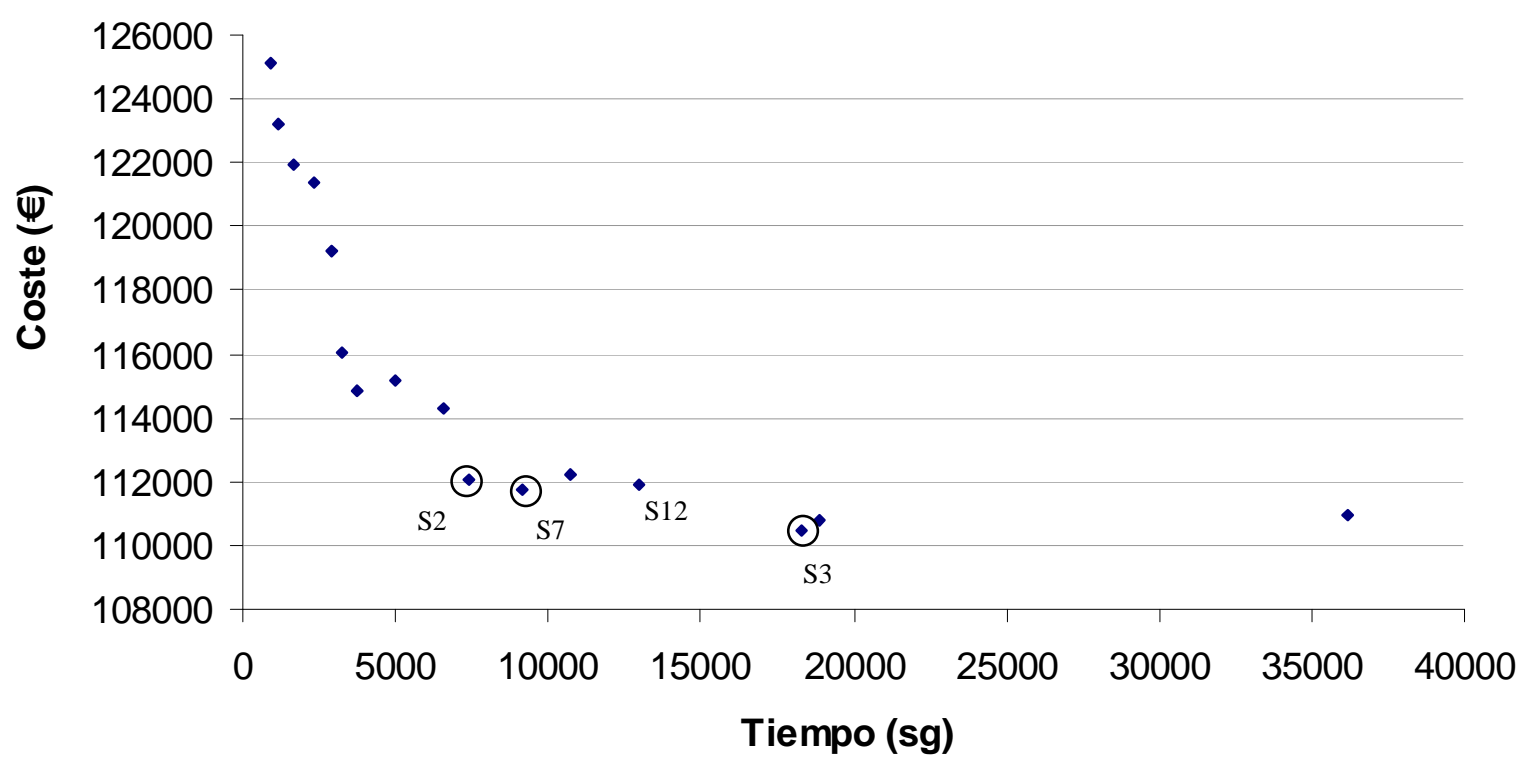

Figura 6-14: Resultados de los valores medios de las 16 combinaciones de los parámetros del SA

La solución de menor coste encontrada (Figura 6-15), heurística S12, no se corresponde con la de menor coste medio, le corresponde un coeficiente de disminución de la temperatura de 0.85 y una longitud de cadena de 5000. Este valor ha sido de $108008 €$, siendo un $6.5 \%$ inferior a la más barata del DLS y un $23.4 \%$ a la del RW.

Además de la mejora en costes, las desviaciones del coste medio respecto al mínimo se han reducido respecto a las obtenidas con el DLS y el RW. En la mayoría de las heurísticas del SA se encuentran las desviaciones alrededor del $2 \%$, mientras que los mejores resultados del DLS se mueven por encima del $10 \%$.

Los tiempos de cálculo son más elevados que en el DLS, del orden de 2 a 3 veces, debido a que se explora más fragmento del espacio de soluciones, siendo éste proporcional a la parte explorada. En cualquier caso, aumentando los tiempos de proceso en el DLS, hasta igualarlos con el SA, no mejorarían apreciablemente los resultados a la vista de la Figura 6-7.

Se puede establecer como conclusión, que este método es mejor que el DLS y proporciona soluciones muy buenas con desviaciones típicas muy bajas, aunque los tiempos de cálculo sean necesariamente más elevados que para los métodos DLS y RW. 
SECCION TRANSVERSAL
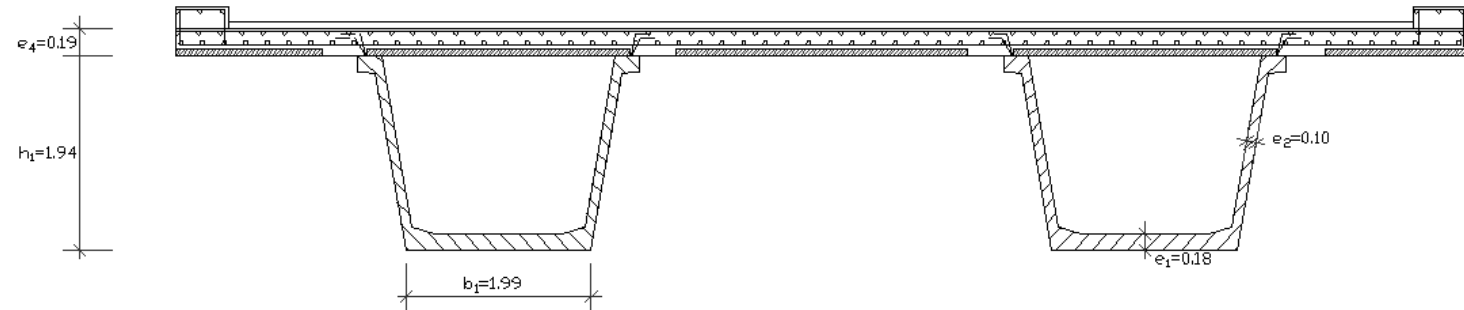

Figura 6-15: Tablero con menor coste en un SA 


\subsection{Aceptación por umbrales}

\subsubsection{Descripción del algoritmo}

Al igual que la cristalización simulada (SA), la aceptación por umbrales -threshold accepting (TA)- se diferencia del descent local search (DLS) en que acepta soluciones con un coste mayor que la anterior siempre que éste sea menor que un determinado umbral. Podría pensarse que este método es una simplificación del SA, donde se sustituye la temperatura por el umbral, la cadena de Markov por longitud de iteraciones, y el criterio probabilístico de la aceptación por el determinista que establece el umbral. Ello permite salir de un óptimo local y buscar un óptimo mejor. El umbral se va reduciendo conforme avanza la búsqueda. Este algoritmo fue propuesto por Gunter Dueck y Tobias Scheuer[55]. En la Figura 6-16 se muestra el diagrama de flujo del algoritmo del TA: 


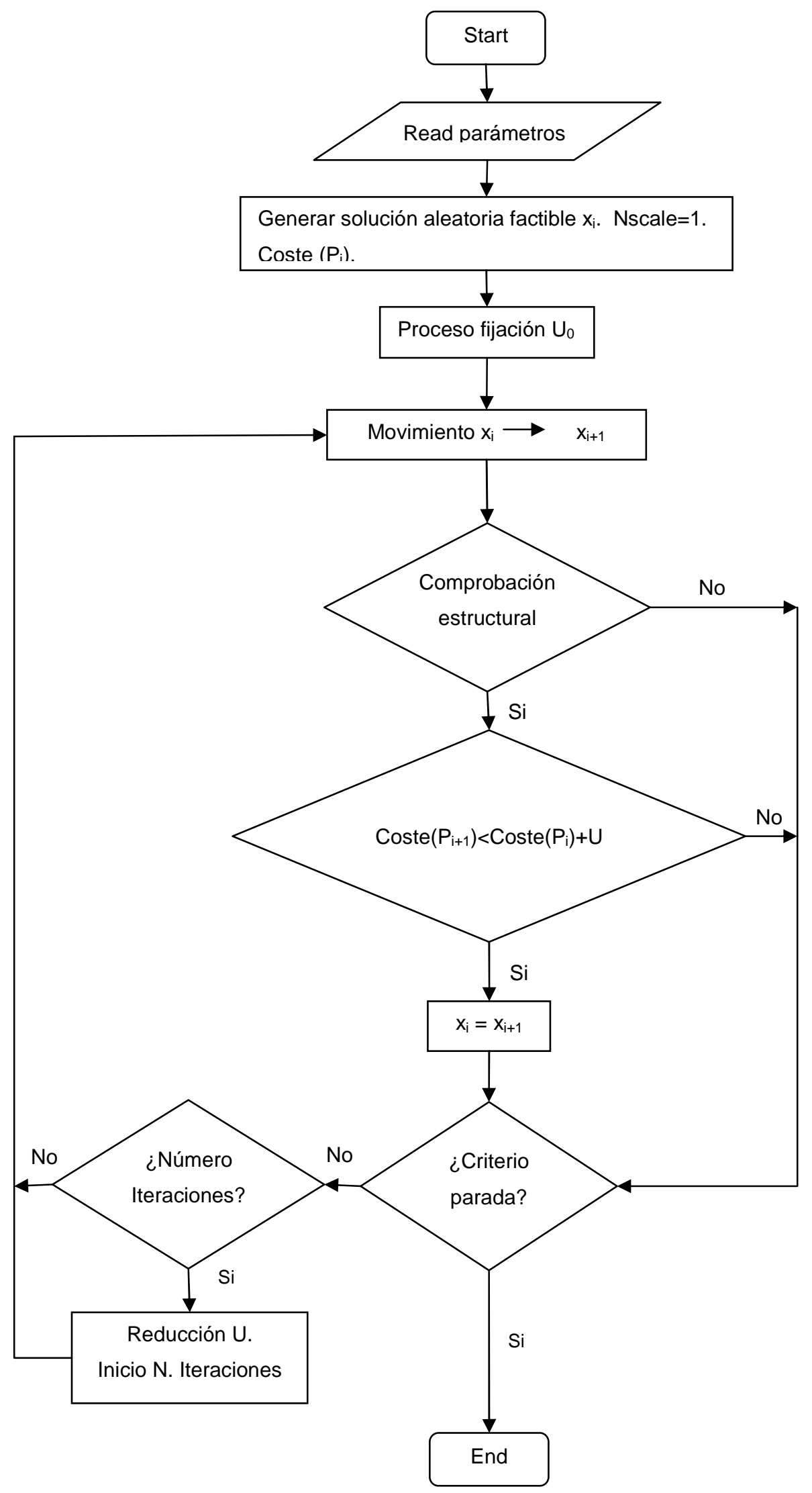

Figura 6-16: Diagrama de flujo de un threshold accepting 
El proceso TA queda definido por los siguientes parámetros:

- Definición del número de variables a modificar

- Selección del umbral inicial

- Proceso de disminución del umbral y cadena de iteraciones

- Selección de un criterio de parada

\section{Definición del número de variables a modificar}

Al igual que para el SA, se procesará modificando 9 variables del tablero, esto supone un $15 \%$ del total.

\section{Selección del umbral inicial}

La selección del umbral inicial se ha realizado mediante un método similar al empleado por Medina[110] para el SA. Este método consiste en:

1. Se selecciona un umbral inicial $\mathrm{T}_{0}$.

2. Se establece un rango: $20-40 \%$.

3. Se fija un determinado número de iteraciones, y se contabiliza el número de soluciones aceptadas.

4. Si el número de soluciones aceptadas está por debajo del rango inferior el nuevo umbral será el doble que el anterior. Si está por encima del rango superior, se reduce a la mitad.

El umbral $\mathrm{T}_{0}$ se ha tomado como valor, al igual que en el SA donde dio buenos resultados, el $0.5 \%$ del coste de la primera solución.

\section{Proceso de disminución del umbral}

A partir de la obtención del umbral inicial, el proceso de su disminución queda definido por dos parámetros: el número de iteraciones y el coeficiente de disminución del umbral. El número de iteraciones se mantiene constante durante todo el proceso, y el proceso de disminución del umbral es geométrico. Para todos los rangos enunciados se han empleado los mismos valores que los aplicados para el SA, es decir, 500, 1000, 2500 y 5000 iteraciones, y coeficientes para disminución del umbral de 0.95, 0.90, 085 y 0.80. Para un caso de este estudio se muestra en la Figura 6-17 como disminuye la temperatura en relación al tiempo de proceso, y en la Figura 6-18 se representa la evolución del coste en función al número de soluciones aceptadas. Esta heurística tiene unos parámetros similares a la análoga del SA, representada en la Figura 6-10, en la que los tiempos de proceso y número de soluciones aceptadas son muy parecidos. 


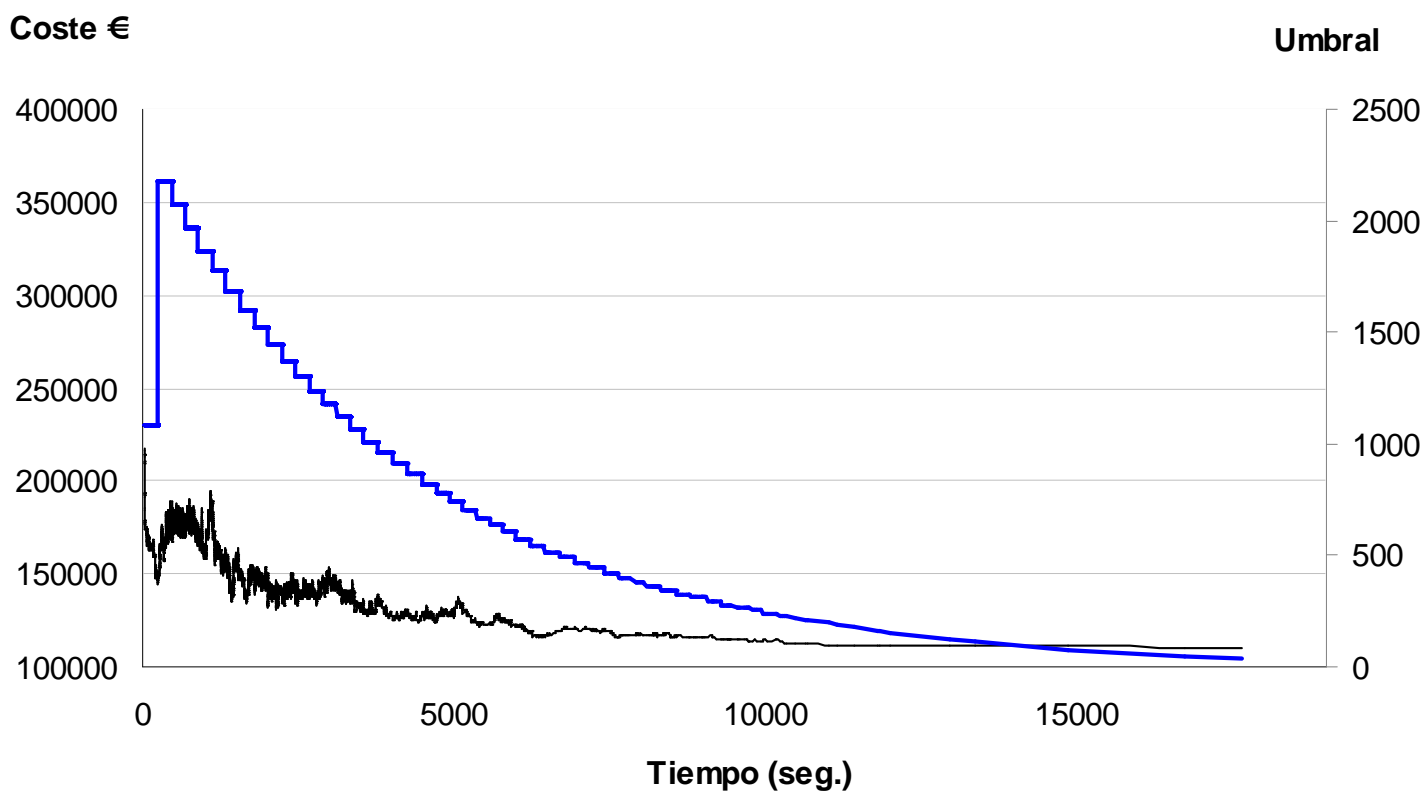

Figura 6-17: Proceso de evolución del coste y disminución del umbral relativo al tiempo de proceso

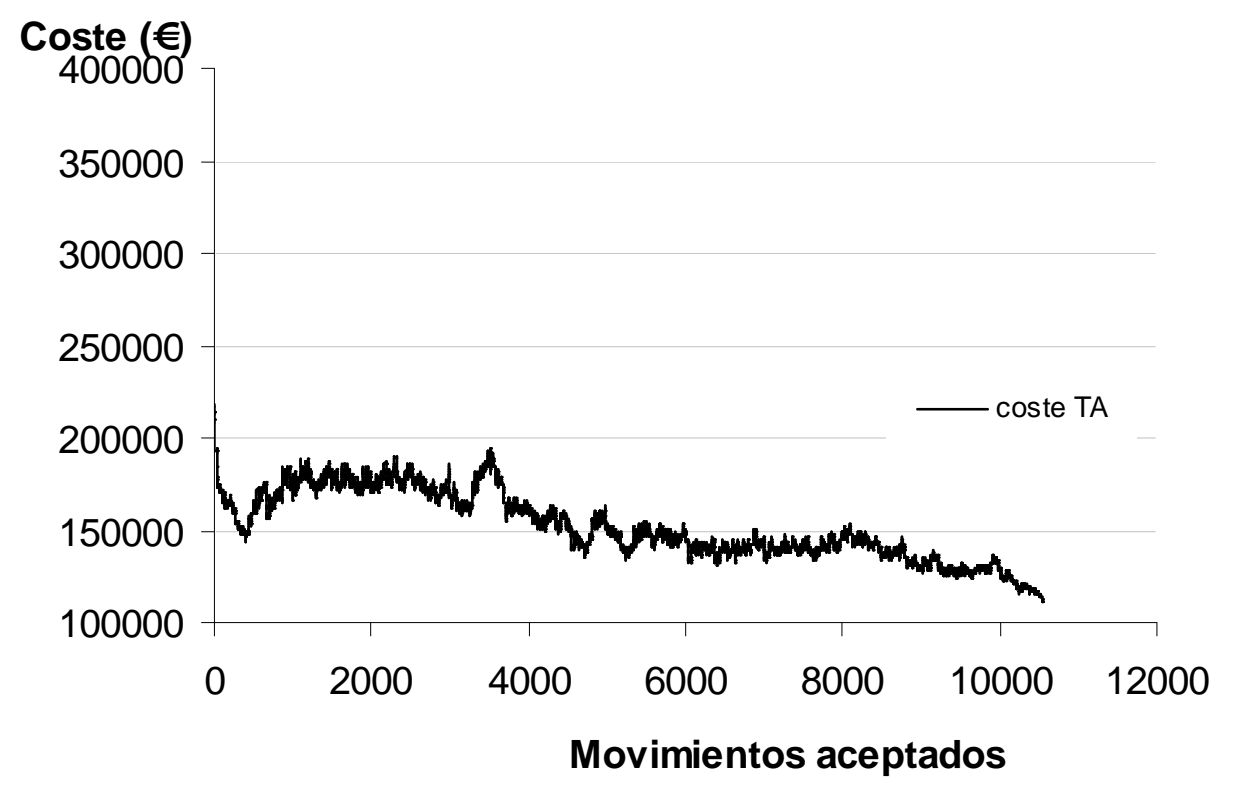

Figura 6-18: Proceso de disminución del coste relativo al número de soluciones aceptadas

Los comentarios realizados a las Figuras 6-10, 6-11 y 6-12 del SA se hacen extensivos para el TA 


\section{Selección del criterio de parada}

Se aplica el mismo criterio que para el SA, es decir, cuando se cumplen simultáneamente las siguientes condiciones, una vez finalizadas las iteraciones:

- El umbral es menor del 2\% inicial.

- En dos cadenas consecutivas no se encuentra ninguna solución que mejora la de partida.

Tanto en el TA como en el SA, inicialmente se aceptan empeoramientos altos. Conforme avanza el proceso éstos van reduciéndose, centrando la búsqueda en soluciones con costes parecidos o menores. La siguiente Figura 6-19 muestra un proceso tipo. En este caso, se aprecia que la fase de diversificación es más corta que en el SA.

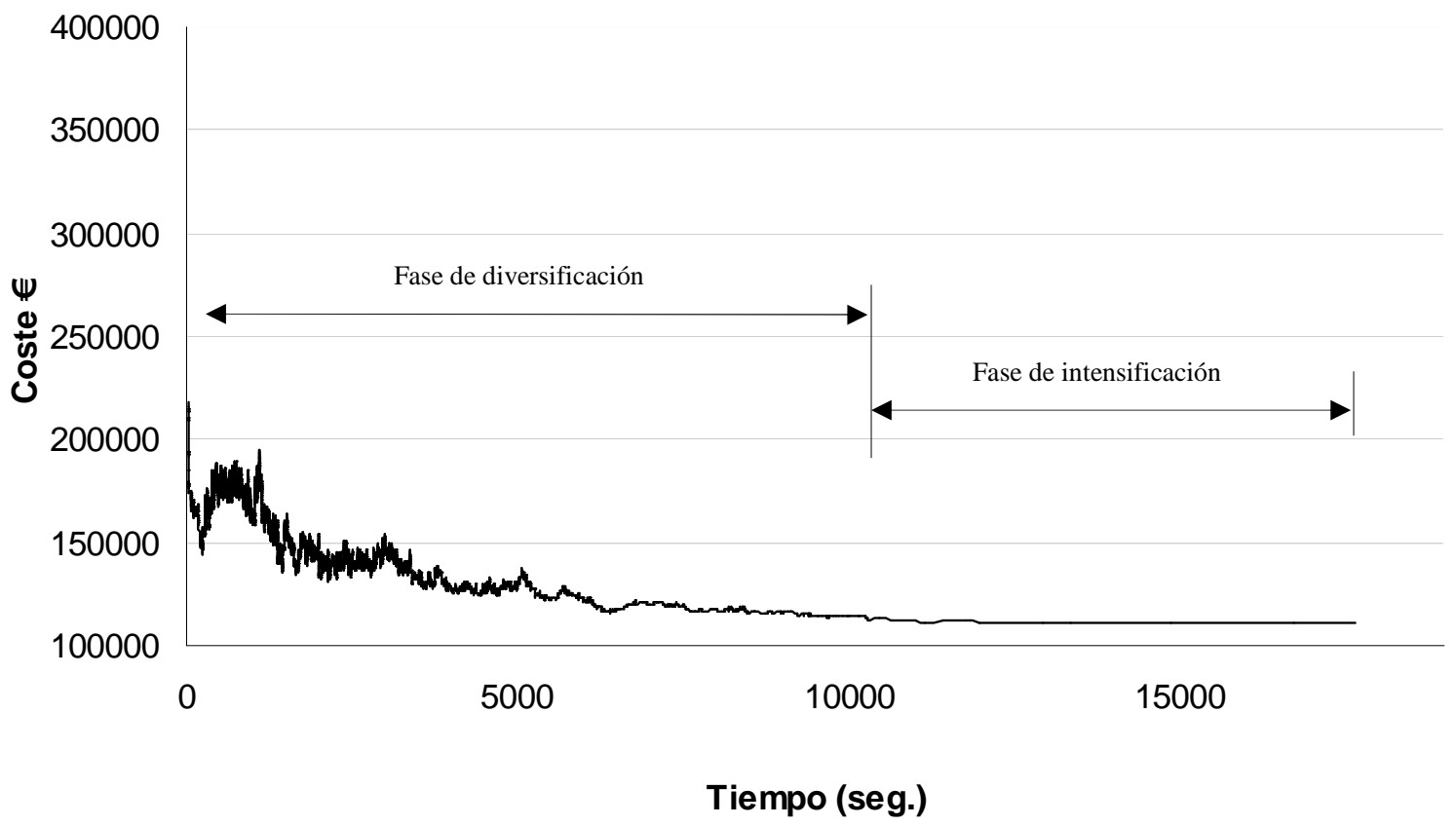

Figura 6-19: Proceso de evolución de un threshold

\subsubsection{Aplicación.}

El algoritmo del TA se ha ejecutado un total de 144 veces, el número de combinaciones de parámetros ha sido 16. Las ejecuciones se han repetido 9 veces para cada combinación estudiada.

En la siguiente Tabla 6-8 se muestran, para las combinaciones de parámetros, el coste y tiempo medio de las 9 ejecuciones, el coste mínimo alcanzado en una ejecución y la desviación del valor medio con respecto al mínimo en tanto por cien. 


\begin{tabular}{|c|c|c|c|c|c|r|r|}
\hline Ejecución & $\begin{array}{c}\text { Disminución } \\
\text { umbral }\end{array}$ & $\begin{array}{c}\text { Longitud } \\
\text { cadena }\end{array}$ & $\begin{array}{c}\text { Coste } \\
\text { medio }(€)\end{array}$ & $\begin{array}{c}\text { Tiempo } \\
\text { medio(sg) }\end{array}$ & $\begin{array}{c}\text { Coste } \\
\text { mínimo }(€)\end{array}$ & $\begin{array}{c}\text { Desv. } \\
(\%)\end{array}$ & $\begin{array}{c}\text { Mov. } \\
\text { acep. }\end{array}$ \\
\hline T1 & 0.95 & 500 & 119641 & 3595 & 111288 & 7.51 & 2531 \\
\hline T2 & 0.95 & 1000 & 114379 & 7317 & 110234 & 3.76 & 5119 \\
\hline T3 & 0.95 & 2500 & 111766 & 18096 & 109894 & 1.70 & 9445 \\
\hline T4 & 0.95 & 5000 & 110865 & 36233 & 108580 & 2.10 & 18347 \\
\hline T5 & 0.90 & 500 & 124150 & 1889 & 116856 & 6.24 & 1396 \\
\hline T6 & 0.90 & 1000 & 118388 & 3631 & 113626 & 4.19 & 2566 \\
\hline T7 & 0.90 & 2500 & 112350 & 9117 & 109689 & 2.43 & 5193 \\
\hline T8 & 0.90 & 5000 & 111758 & 19212 & 110151 & 1.46 & 9898 \\
\hline T9 & 0.85 & 500 & 128841 & 1285 & 121510 & 6.03 & 928 \\
\hline T10 & 0.85 & 1000 & 119157 & 2603 & 114278 & 4.27 & 1896 \\
\hline T11 & 0.85 & 2500 & 113010 & 6274 & 110015 & 2.72 & 3927 \\
\hline T12 & 0.85 & 5000 & 111782 & 12695 & 109998 & 1.62 & 6735 \\
\hline $\mathrm{T} 13$ & 0.80 & 500 & 129649 & 1043 & 118248 & 9.64 & 757 \\
\hline $\mathrm{T} 14$ & 0.80 & 1000 & 121492 & 2030 & 114448 & 6.15 & 1458 \\
\hline $\mathrm{T} 15$ & 0.80 & 2500 & 115292 & 4926 & 111155 & 3.72 & 2868 \\
\hline $\mathrm{T} 16$ & 0.80 & 5000 & 113407 & 9463 & 110571 & 2.56 & 5248 \\
\hline
\end{tabular}

Tabla 6-8: Resultados de las 144 ejecuciones con los parámetros seleccionados en TA

Como resumen de los datos de la Tabla anterior, se obtiene el valor medio del coste medio, tiempo medio y movimientos aceptados medios de todas las ejecuciones, el mínimo y la desviación del valor medio con respecto al mínimo:

\begin{tabular}{|l|c|c|c|}
\cline { 2 - 4 } \multicolumn{1}{c|}{} & Coste(€) & Tiempo(sg) & Mov. acep. \\
\hline Media & 117245 & 8713 & 4895 \\
\hline Mínimo & 108580 & 1043 & \multicolumn{1}{|c}{} \\
\hline Máximo & 129649 & 36233 & \multicolumn{1}{|c}{} \\
\hline Desviac.(\%) & 7.98 & \multicolumn{2}{|c}{} \\
\cline { 1 - 3 }
\end{tabular}

Tabla 6-9: Resultados medios obtenidos en TA

Del análisis de los resultados obtenidos, se tiene que:

- El comportamiento es muy similar al del SA, por lo que podríamos trasladar las mismas conclusiones observadas para las Tablas 6-8 y 6-9.

En la Figura 6-17 se representan los valores medios de la Tabla 6-8, marcándose con un círculo tres valores pertenecientes a óptimos de Pareto, correspondientes a una relación equilibrada entre costes medios bajos y tiempos de proceso. 
La solución con menor coste medio tiene las siguientes características:

(T4)

- Longitud de cadena de iteraciones: 5000 iteraciones

- Coeficiente disminución temperatura: 0.95

- Coste medio:

$110,865 €$

- Tiempo medio de proceso:

36,233 segundos

- Desviación:

$2.11 \%$

La desviación es buena, pero no la más baja de todas las ejecuciones. Sin embargo, el tiempo de cálculo es el más elevado debido a que se conjugan el número máximo de iteraciones y la disminución del umbral más lenta. Esa desproporción de tiempos respecto a otras heurísticas ha hecho que no se incluyera dentro del grupo de óptimos marcados por un círculo -tarda 3 veces más que la T12, y la mejora no llega al 0.9\%-.

De los tres óptimos de Pareto señalados en la Figura 6-20, el que tiene un menor coste medio es el T12, a continuación el T7, y finalmente el T11, encontrándose los tiempos de proceso en orden inverso. Son los casos siguientes:

- Longitud de cadena de iteraciones: 5000 iteraciones

- Coeficiente disminución temperatura: 0.85

- Coste medio:

$111,782 €$

- Tiempo medio de proceso:

- Desviación:

12,695 segundos

$1.62 \%$

(T7)

- Longitud de cadena de iteraciones: 2500 iteraciones

- Coeficiente disminución temperatura: 0.90

- Coste medio:

$112,350 €$

- Tiempo medio de proceso:

9,117 segundos

- Desviación:

$2.43 \%$

(T11)

- Longitud de cadena de iteraciones: 2500 iteraciones

- Coeficiente disminución temperatura: 0.85

- Coste medio:

$113,010 €$

- Tiempo medio de proceso:

6,274 segundos

- Desviación:

$2.72 \%$

El coste medio es un $1.1 \%$ superior en (T11) y un $0.5 \%$ en (T7) respecto a T12, pero en cambio, el tiempo de proceso se reduce un $50.6 \%$ en (T11) y un $28.2 \%$ en (T7).

La heurística con menor desviación, del $1.46 \%$, es la T8. 


\section{Coste Medio f(umbral/iteraciones)}

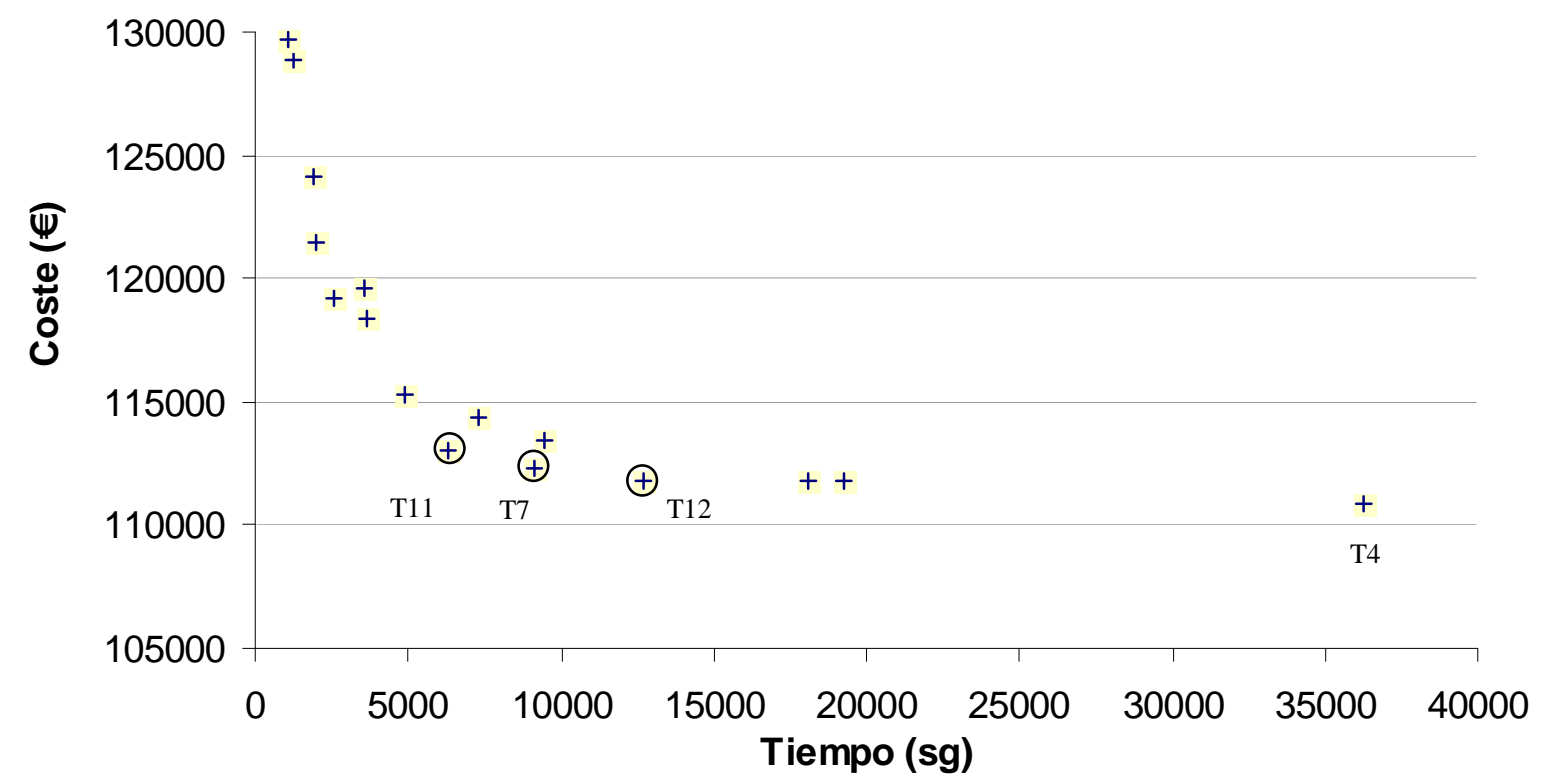

Figura 6-20: Resultados de los valores medios de las 16 combinaciones de los parámetros

La solución de menor coste encontrada (Figura 6-22), corresponde a la heurística T4 indicada. Su coste ha sido 108,580 €, éste valor disminuye en un 6.0\% la más barata del DLS y en un $23.0 \%$ a la del RW. No mejora el menor coste del SA que es de $108,008 €$, quedando la diferencia en un $0.5 \%$.

En este punto, se podrían comparar las dos últimas heurísticas entre sí, dado que ambas son notablemente mejores que el DLS y el RW. De las Tablas 6-6, 6-7, 6-8 y 6-9 se desprende que:

- Los tiempos de proceso son muy similares, tanto para cada heurística como para los valores medios.

- Se puede decir lo mismo para el número de soluciones aceptadas, aunque en valores medios sea superior para el SA.

- También se repite con las desviaciones medias, que son del $7.13 \%$ para el SA y del $7.98 \%$ para el TA.

- El menor coste medio del SA es muy parecido al del TA, variando sólo en un $0.4 \%$.

- Como se ha indicado anteriormente, la diferencia de la mejor solución es del $0.5 \%$.

- Y finalmente, tal como se aprecia en las anteriores figuras que definen la variación de los costes respecto al tiempo de proceso o al número de soluciones aceptadas, se puede concluir que las dos heurísticas tienen un comportamiento similar. En la Figura siguiente, la 6-21, también se observa que las nubes que definen los costes medios de las distintas heurísticas, es muy similar. 


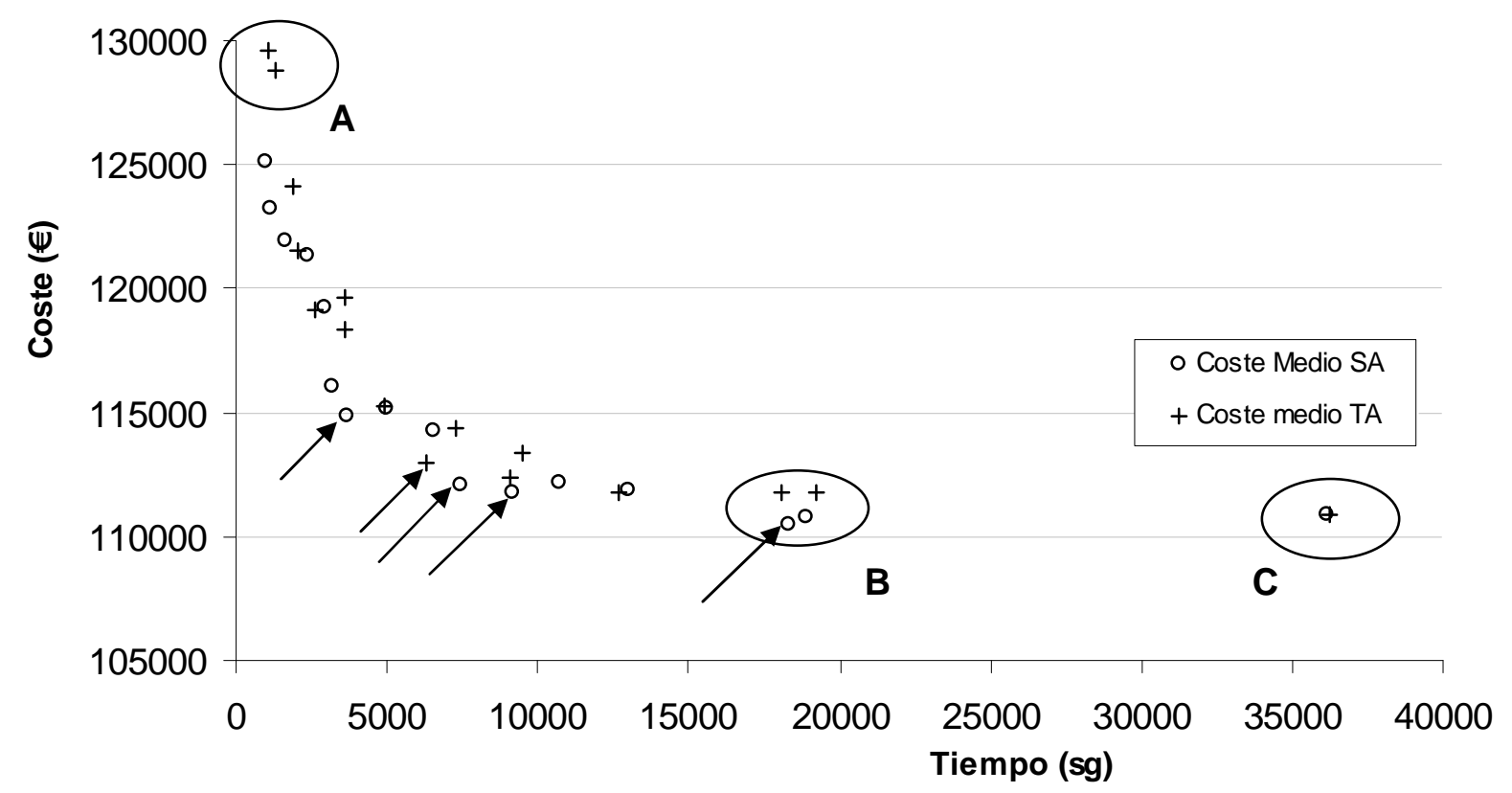

Figura 6-21: Comparativa resultados de los valores medios TA vs SA con las 164 combinaciones de los parámetros

Se señalan cinco óptimos de Pareto con flechas indicativas. Se han agrupado los valores por encima de 126,000 € en "A", y los valores inferiores a 111,000 € en "B" y "C".

De los valores de la Figura se desprende que:

- De los cinco óptimos de Pareto, cuatro de ellos se corresponden al SA. Ello equivale al $80 \%$

- Con el SA, hay 3 procesos de 16, que están por debajo de 111,000 €. Ello equivale al $19 \%$ de los casos.

- Con el TA, hay 1 proceso por debajo de 111,000 €. Equivale al 6\% de los casos.

- Con el TA, hay 2 procesos que están por encima de 126,000 €. Equivale al $13 \%$ de los casos

- Con el SA, hay 0 procesos de 18 están por encima de 126,000 €. El 0\%

Se puede resumir al comparar las dos heurísticas (SA vs TA), lo siguiente:

- El coste más bajo, aunque sea por una magnitud insignificante, le corresponde al SA.

- Los costes medios son algo menores en el SA.

- Las desviaciones de los costes medios son inferiores, aunque escasamente, en el SA.

- Los tiempos medios de proceso son similares en ambos casos. 
Por lo tanto, se puede concluir que ambas heurísticas son similares, decantándose ligeramente por el SA, y que, respecto a las anteriores heurísticas estudiadas, ambas mejoran considerablemente los resultados del DLS y del RW.

SECCION TRANSVERSAL

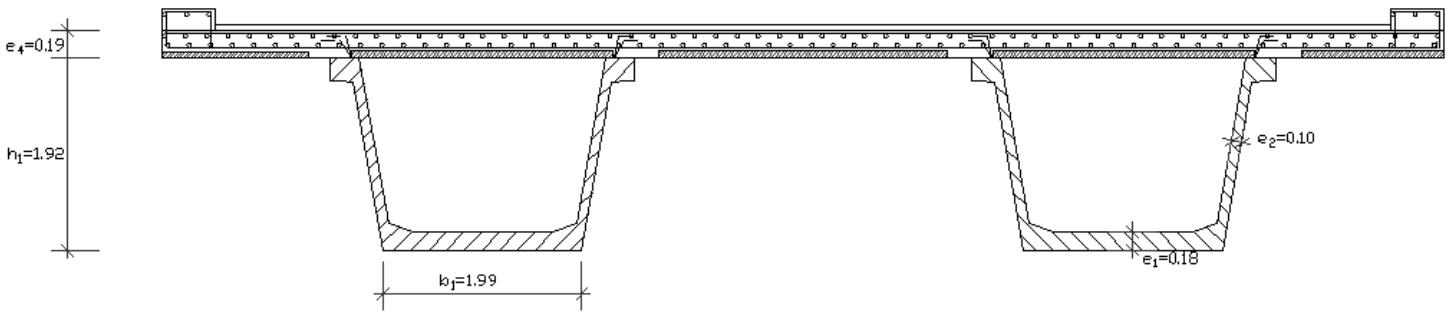

Figura 6-22: Tablero con menor coste en un TA 


\subsection{Algoritmos genéticos}

\subsubsection{Descripción del algoritmo}

A diferencia de los métodos de optimización desarrollados anteriormente, los algoritmos genéticos -genetic algorithms (GA)- son poblacionales y están inspirados en la selección natural basada en la evolución de las especies. Parte con la hipótesis de que la evolución biológica no tiene memoria de las poblaciones anteriores para formar cromosomas, sólo se considera la información de la última generación. En este trabajo, para la codificación de los cromosomas, se ha aplicado la representación natural, que dio mejores resultados a Martínez, F.[105], en la que cada variable es representada por un número. Así, por ejemplo la variable hormigón pretensado posición 2 será el HP-40, ya que, los valores del vector de hormigón en la viga serán el HP-35, HP-40, HP-45, HP-50. Esta codificación permite que no se pierda información en el problema, ya que todas las posiciones de las variables tienen definido un valor, mientras que la codificación binaria, en ciertas ocasiones, no se adapta al número de posibles valores de una variable dando al sistema más información de la necesaria. El cruzamiento se realiza partiendo la cadena de genes por un único punto, utilizando diferentes probabilidades para que se realicen los cruzamientos o no. La mutación se efectúa empezando con probabilidades mayores al inicio del algoritmo, que permiten una mayor exploración del espacio de soluciones, y menores conforme el proceso de cálculo avanza, intensificando la búsqueda centrada en las mejores soluciones. Se realizan ejecuciones sin y con elitismo, de manera que una copia del mejor individuo de una generación se encuentre en la posterior. Se aplica el escalado de las soluciones (fitness scaling) evitando que el algoritmo converja de manera prematura o que no haga distinción entre soluciones buenas y muy buenas al final del proceso. En GA, a diferencia de las heurísticas anteriores, se aceptan soluciones factibles y no factibles empleando una función de penalización para las no factibles. La Figura 5.23 muestra el diagrama de flujo básico de una ejecución del GA. 


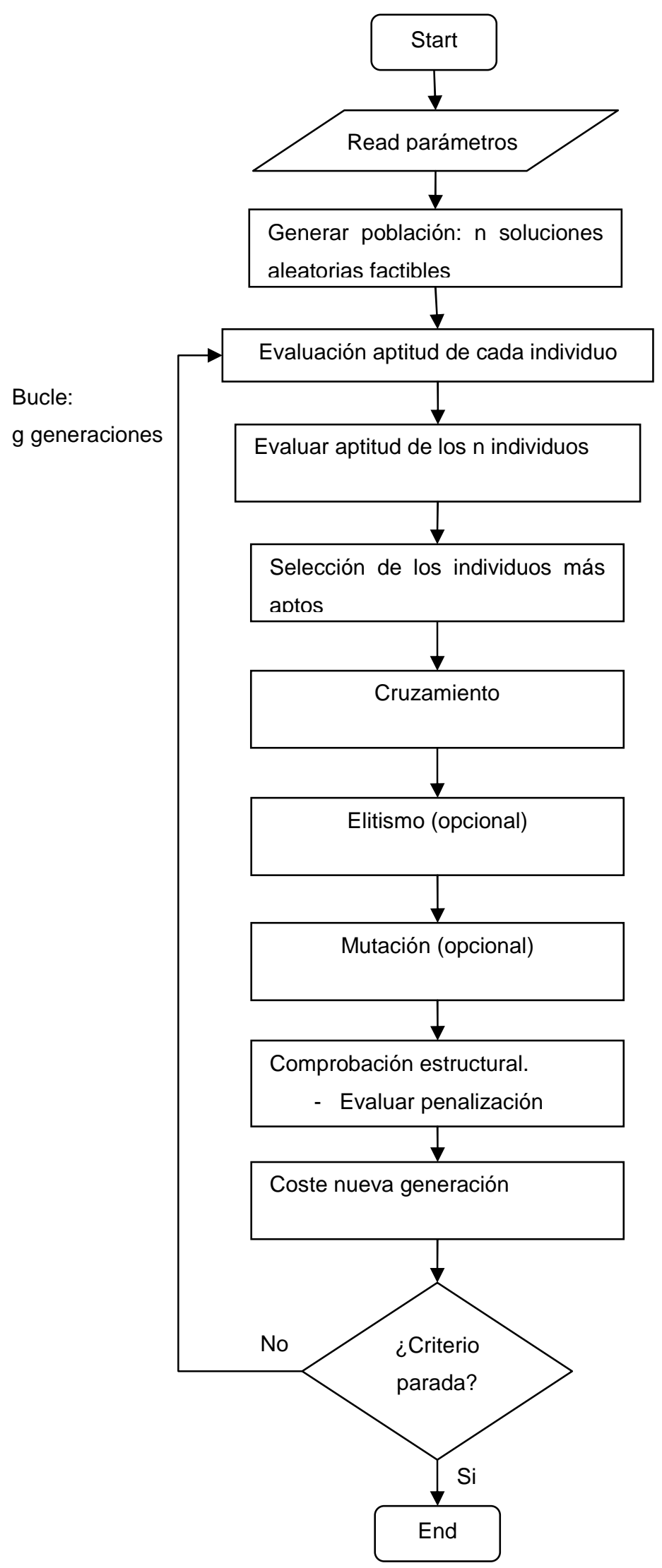

Figura 6-23: Diagrama de flujo de GA 
En la primera etapa, se busca un número de soluciones factibles de manera aleatoria igual al tamaño de la población. A partir de la segunda generación las soluciones se forman mediante cruzamientos y mutaciones de la población, previa selección de éstos mediante una determinada regla. Para ejecutar el algoritmo se deben definir y ajustar los diferentes parámetros del mismo:

1. Terminología genética

2. Función de penalización y aptitud de un individuo

3. Evaluación de la "Aptitud" de un individuo (Fitness)

4. Operador "Selección"

5. Operador "Cruzamiento" (Crossover)

6. Operador "Elitismo"

7. Operador "Mutación"

Terminología genética

El modo original utilizado para codificar una solución fue el sistema binario, según el cual el cromosoma, que es la cadena de datos que contiene la información de una de las variables que definen una solución del problema, tiene la forma $\left(\mathrm{a}_{1}, \ldots, \mathrm{a}_{\mathrm{m}}\right)$ donde cada " $\mathrm{a}_{\mathrm{i}}$ " adopta un valor de "0" ó " 1 ", cada posición de la cadena se le llama gen y, a su vez, un alelo es el valor particular que adopta un gen. El empleo de esta codificación la justificó teóricamente Holland[73] (1975) y Golberg[65] (1989). Además del sistema binario, hay otras formas para codificar la genética, entre las que se encuentra los códigos de Gray, la representación de punto flotante (en la que cada alelo puede tomar un valor entre 0 y 9) o la representación natural (en la que cada gen tiene un único alelo que es un número natural). En Figura 6.24 se puede ver de manera esquemática lo explicado anteriormente.

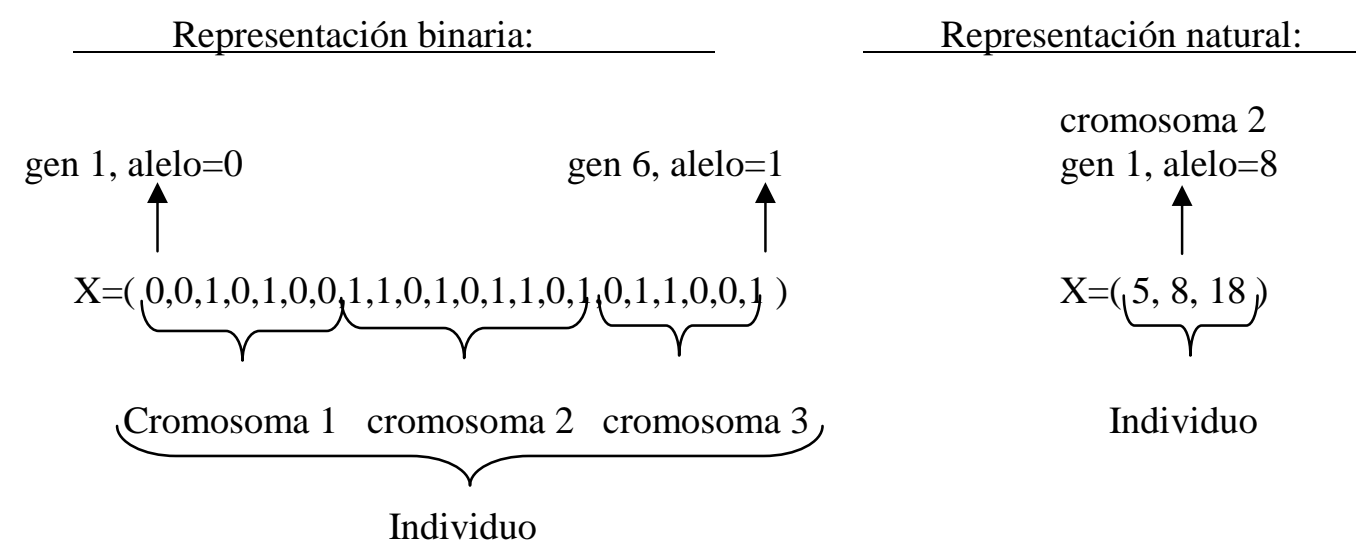

Figura 6-24: Diagrama terminología y ejemplo de representación de soluciones en GA

Para establecer el código binario son necesarios tres pasos: establecer el orden en el que están representadas las variables en la cadena agrupando las variables relacionadas entre ellas y que representan conjuntamente una característica de la 
solución, en el segundo paso se define el número de bits (número de valores necesarios para definir el cromosoma), con una cadena de " $n$ " bits se podrán representar $2^{\mathrm{n}}$ valores de una variable y el tercer paso consiste en asignar a este vector binario los valores de cada una de las variables.

En la representación natural, a cada variable (cromosoma), se le asignan diferentes posiciones, cada una de las cuales significa un valor determinado.

Funciones de penalización y aptitud de un individuo

Una vez obtenida una generación de individuos se debe evaluar su aptitud. Para ello hay que diferenciar entre los que cumplen todos los estados límites de servicio y últimos y los que no satisfacen estas restricciones de la estructura, a los cuales hay que penalizarlos incrementando el valor de su coste según la siguiente expresión:

$$
C p(k)=C(k)\left(1.1+\frac{1}{f}\right)
$$

$\mathrm{Cp}(\mathrm{k})$ será el coste penalizado que se le asignará al individuo " $\mathrm{k}$ " que no satisface todas las restricciones del problema, $\mathrm{C}(\mathrm{k})$ será el coste de dicho individuo sin penalizar, "f" es un factor que depende de la factibilidad de la solución, es decir, será el menor coeficiente de seguridad de los Estados Límite que no se verifiquen, entendiendo que se cumplirían si el coeficiente fuera mayor o igual que 1. El coeficiente "1.1" que se aplica, permite penalizar adicionalmente a las soluciones no factibles un $10 \%$ del coste de la estructura. Además de ésta, se han probado distintas expresiones de penalización, y no todas permitían a la heurística converger a un mínimo. La expresión utilizada ha tenido un buen comportamiento. Como ya se ha señalado, a las soluciones factibles no se les penaliza su coste.

\section{Evaluación de la Aptitud de un individuo (Fitness)}

Después de evaluar los costes de todos los individuos, se escalan (Fitness) para ayudar a que el algoritmo pueda converger teniendo en cuenta que hay que premiar a las mejores soluciones. El nuevo coste escalado que representa a cada una de las soluciones, viene dado por la ecuación:

$$
C e(k)=\frac{C m}{C(k)}
$$

$\mathrm{Ce}(\mathrm{k})$ es el coste escalado para cada individuo de la población, $\mathrm{Cm}$ el menor coste de la población en la generación correspondiente y $\mathrm{C}(\mathrm{k})$ el coste penalizado o sin penalizar que tenía la solución antes de escalarlo.

\section{Operador "Selección"}

El proceso de selección permite dirigir la búsqueda dentro del espacio de soluciones. La selección genera, a partir de una población de partida, otra intermedia reproduciendo con un mayor número de copias a los individuos más aptos y eliminando o asignando un menor número de copias a los menos aptos. Este operador no produce soluciones nuevas, sino que determina cuáles son los individuos 
para el cruzamiento y en definitiva los que sus cualidades van a permanecer en la generación futura.

El método de selección utilizado es el "método de la ruleta" mostrado en la Figura 625 (Coello[38] (1994)). La probabilidad de selección de un individuo será proporcional a su aptitud.

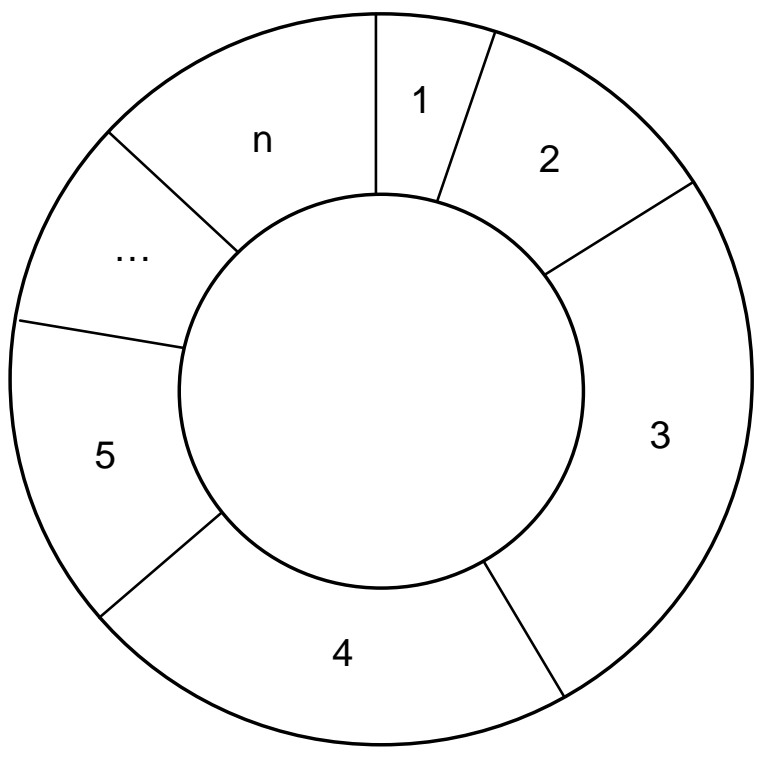

Figura 6-25: Ruleta empleada en la selección de individuos

La circunferencia completa es la suma de los costes escalados de los " $n$ " individuos de una generación. La probabilidad de ser elegidos cada uno de los individuos dependerá de su coste escalado $\mathrm{Ce}(\mathrm{k})$ en relación al de los demás individuos.

\section{Operador “Cruzamiento”(Crossover)}

Este operador permite el intercambio de información entre dos padres produciendo descendientes (hijos) que tendrán las cualidades de sus antecesores. La idea es que, intercambiando información de dos individuos muy aptos de la población, el resultante sea otro individuo de igual calidad o mejor. En el algoritmo se ha utilizado el cruzamiento de un punto, seleccionándose la variable para que se realice el cruzamiento al azar. La Figura 6.26 muestra el esquema con el modo de realizar el cruzamiento.

\section{Padre 1}

Hijo 1

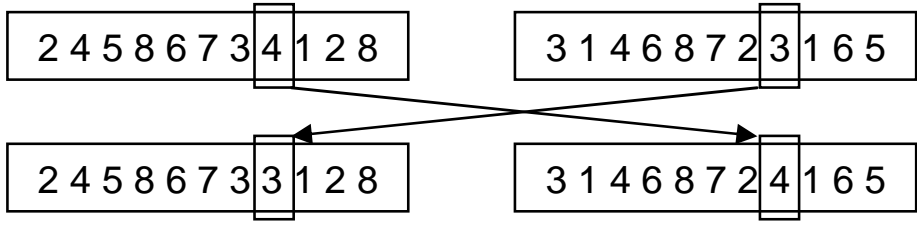

Padre 2

Hijo 2

Figura 6-26: Cruzamiento por un punto 
El operador funciona estableciendo una determinada probabilidad $\mathrm{p}_{\mathrm{c}}$, la cual se establece en $0.25,0.50$ y 0.75 de tal manera que si un número aleatorio $\mathrm{R}$ (cuyos valores posibles van de 0 a 1 ) es menor o igual que la probabilidad de cruzamiento éste se realizará, en caso contrario los hijos serán iguales a los padres.

\section{Operador "Elitismo"}

El método de la ruleta no garantiza en sí la selección de un individuo en particular, incluido el mejor. El operador elitismo se aplica para asegurar que el mejor individuo de la población en una generación $g$ se encuentra en la siguiente $g+1$. Sin embargo, esto no mejora la posibilidad de localizar el óptimo global de una función, aunque su uso se ha revelado necesario para demostrar la convergencia de un GA (Rudolph[148] (1994)).

\section{Operador "Mutación"}

Se entiende como mutación la modificación espontánea de la información genética, que puede ser debida a diversas causas como evolución de las especies, interacción con el medio ambiente, etc. En la representación natural si se produce mutación se modifica el valor de la posición de la variable considerada, aumentando o disminuyendo esta posición en 1 según el valor que tome $\mathrm{R}$, mayor o menor o igual que 0.5. La probabilidad de que se produzca mutación va cambiando a lo largo del proceso, siendo alta al inicio $\left(\mathrm{p}_{\mathrm{m}}=0.1\right.$ en la generación 1$)$ de manera que se permita una exploración del espacio de soluciones, y baja al final del proceso $\left(\mathrm{p}_{\mathrm{m}}=0.01\right) \mathrm{de}$ manera que se intensifique la búsqueda, en las generaciones intermedias será proporcional y lineal a estas probabilidades en función de la generación en que se encuentre el algoritmo. Si el número aleatorio $\mathrm{R}$ (cuyos valores posibles van de 0 a 1 ) es menor o igual que la probabilidad de mutación ésta se producirá. Se puede ocasionar más de una mutación en un movimiento.

\subsubsection{Aplicación.}

Se han realizado 9 ejecuciones con los valores de cruzamiento descritos anteriormente, con tamaños de población de 100, 250 y 500 individuos, y 25, 50, 75, $100,125,150,175$ y 200 generaciones, aplicando o no elitismo. En este primer análisis no se ha aplicado mutación. Los resultados se muestran en las siguientes tablas, donde el coste medio se refiere a la media de los valores mínimos de las 9 ejecuciones.

En las siguientes Figuras 6-27 6-28, se muestra la evolución de dos heurística en el tiempo, una sin elitismo y otra con él, mostrándose el valor medio y mínimo de la generación correspondiente. Se observa que los valores medios rápidamente se acercan al mínimo, son necesarias 100 generaciones en la primera, y de 50 a 75 generaciones en la segunda, y a partir de ese momento, varían muy poco, manteniendo casi constante la diferencia entre ambos. La existencia de elitismo permite reducir en más ocasiones el valor mínimo. 


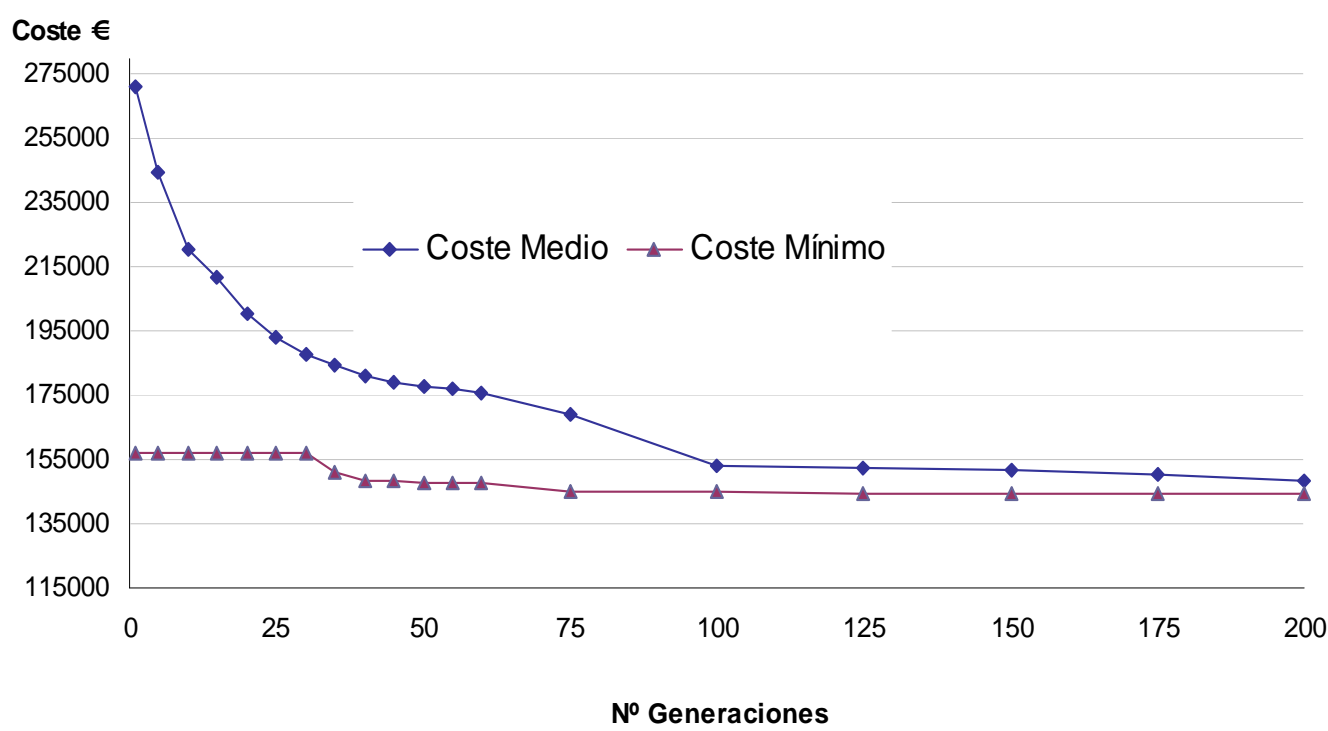

Figura 6-27: Evolución de los costes medios y mínimos en un GA. (Individuos=500 Elitismo=No Cruzamiento $=0.75$ )

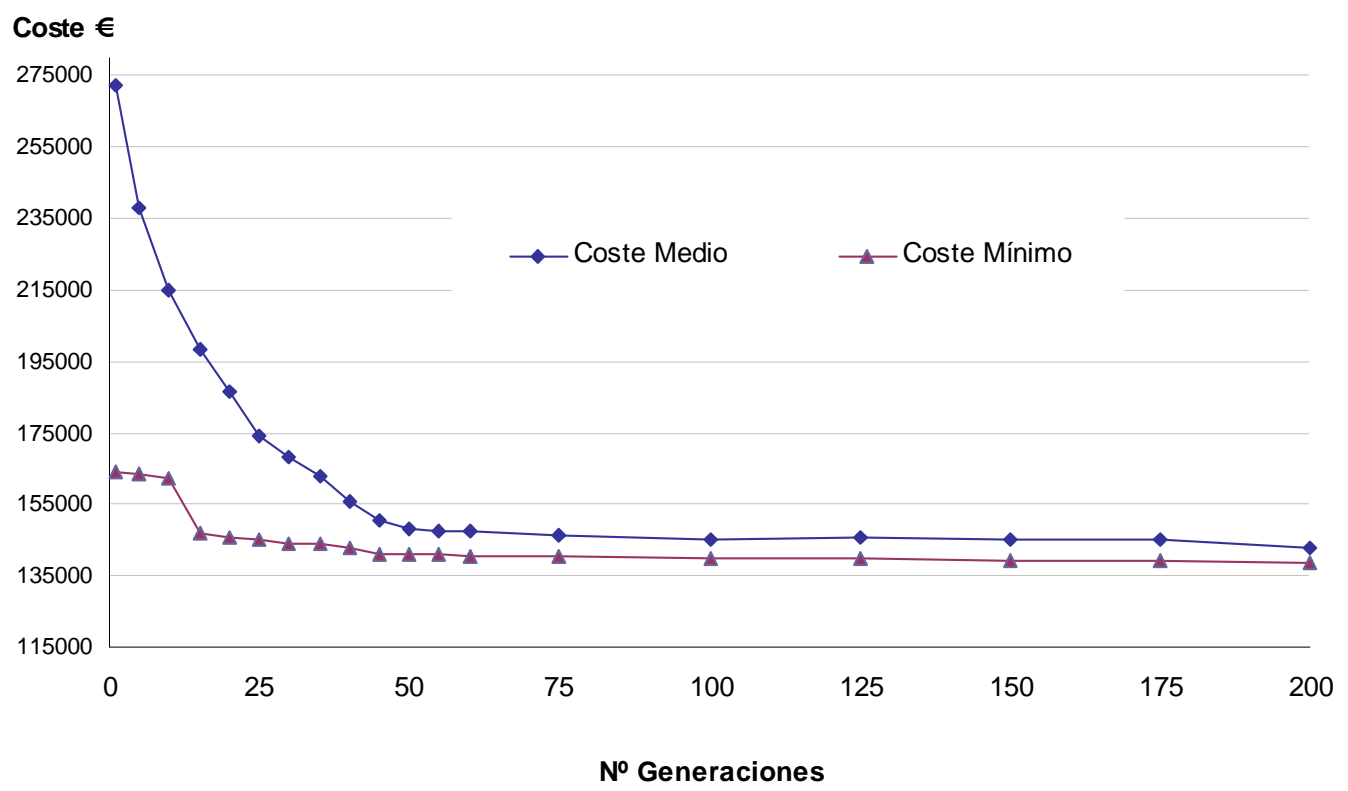

Figura 6-28: Evolución de los costes medios y mínimos en un GA. (Individuos=500 Elitismo=Si Cruzamiento $=0.75$ ) 


\begin{tabular}{|c|c|c|c|c|c|c|}
\hline Individuos & Cruzamiento & Generaciones & Coste medio(€) & sg. & Coste mínimo(€) & $\%$ Desv. \\
\hline \multirow[t]{27}{*}{100} & \multirow[t]{9}{*}{0.25} & 10 & 168,485 & 3,018 & 147,233 & $14.4 \%$ \\
\hline & & 25 & 166,910 & 3,151 & 147,233 & $13.4 \%$ \\
\hline & & 50 & 164,716 & 3,373 & 147,233 & $11.9 \%$ \\
\hline & & 75 & 164,447 & 3,595 & 147,233 & $11.7 \%$ \\
\hline & & 100 & 163,879 & 3,817 & 147,233 & $11.3 \%$ \\
\hline & & 125 & 163,846 & 4,039 & 147,233 & $11.3 \%$ \\
\hline & & 150 & 163,846 & 4,261 & 147,233 & $11.3 \%$ \\
\hline & & 175 & 163,846 & 4,483 & 147,233 & $11.3 \%$ \\
\hline & & 200 & 163,846 & 4,705 & 147,233 & $11.3 \%$ \\
\hline & \multirow[t]{9}{*}{0.50} & 10 & 169,423 & 3,042 & 157,129 & $7.8 \%$ \\
\hline & & 25 & 165,509 & 3,173 & 152,883 & $8.3 \%$ \\
\hline & & 50 & 164,680 & 3,389 & 151,968 & $8.4 \%$ \\
\hline & & 75 & 163,346 & 3,606 & 151,968 & $7.5 \%$ \\
\hline & & 100 & 163,148 & 3,822 & 151,968 & $7.4 \%$ \\
\hline & & 125 & 163,046 & 4,039 & 151,968 & $7.4 \%$ \\
\hline & & 150 & 162,940 & 4,255 & 151,968 & $7.3 \%$ \\
\hline & & 175 & 162,940 & 4,471 & 151,968 & $7.2 \%$ \\
\hline & & 200 & 162,940 & 4,688 & 151,968 & $7.2 \%$ \\
\hline & \multirow[t]{9}{*}{0.75} & 10 & 168,397 & 3,036 & 157,129 & $7.2 \%$ \\
\hline & & 25 & 164,108 & 3,167 & 144,639 & $13.5 \%$ \\
\hline & & 50 & 160,822 & 3,389 & 144,639 & $11.2 \%$ \\
\hline & & 75 & 160,559 & 3,612 & 144,639 & $11.0 \%$ \\
\hline & & 100 & 160,038 & 3,834 & 144,639 & $10.7 \%$ \\
\hline & & 125 & 160,025 & 4,056 & 144,639 & $10.6 \%$ \\
\hline & & 150 & 159,897 & 4,279 & 144,639 & $10.6 \%$ \\
\hline & & 175 & 159,897 & 4,501 & 144,639 & $10.6 \%$ \\
\hline & & 200 & 159,897 & 4,724 & 144,639 & $10.6 \%$ \\
\hline
\end{tabular}

Tabla 6-10: Resultados sin elitismo en GA. 100 individuos 


\begin{tabular}{|c|c|c|c|c|c|c|}
\hline Individuos & Cruzamiento & Generaciones & Coste medio(€) & sg. & Coste mínimo(€) & $\%$ Desv. \\
\hline \multirow[t]{27}{*}{250} & \multirow[t]{9}{*}{0.25} & 10 & 162,614 & 7,116 & 153,613 & $5.9 \%$ \\
\hline & & 25 & 157,336 & 7,449 & 147,518 & $6.7 \%$ \\
\hline & & 50 & 155,312 & 8,007 & 146,656 & $5.9 \%$ \\
\hline & & 75 & 154,468 & 8,473 & 146,656 & $5.3 \%$ \\
\hline & & 100 & 154,204 & 8,938 & 146,656 & $5.2 \%$ \\
\hline & & 125 & 154,035 & 9,404 & 146,656 & $5.0 \%$ \\
\hline & & 150 & 153,976 & 9,869 & 146,656 & $5.0 \%$ \\
\hline & & 175 & 153,837 & 10,334 & 146,656 & $4.9 \%$ \\
\hline & & 200 & 153,837 & 10,800 & 146,656 & $4.9 \%$ \\
\hline & \multirow[t]{9}{*}{0.50} & 10 & 159,843 & 7,134 & 152,407 & $4.9 \%$ \\
\hline & & 25 & 156,982 & 7,468 & 148,042 & $6.0 \%$ \\
\hline & & 50 & 155,434 & 8,029 & 148,042 & $5.0 \%$ \\
\hline & & 75 & 154,970 & 8,591 & 147,330 & $5.2 \%$ \\
\hline & & 100 & 154,713 & 9,152 & 147,330 & $5.0 \%$ \\
\hline & & 125 & 154,641 & 9,714 & 147,330 & $5.0 \%$ \\
\hline & & 150 & 154,626 & 10,275 & 147,193 & $5.1 \%$ \\
\hline & & 175 & 154,527 & 10,836 & 147,193 & $5.0 \%$ \\
\hline & & 200 & 154,403 & 11,398 & 147,193 & $4.9 \%$ \\
\hline & \multirow[t]{9}{*}{0.75} & 10 & 160,208 & 7,095 & 150,152 & $6.7 \%$ \\
\hline & & 25 & 155,575 & 7,428 & 145,719 & $6.8 \%$ \\
\hline & & 50 & 153,243 & 7,985 & 145,719 & $5.2 \%$ \\
\hline & & 75 & 152,502 & 8,543 & 145,719 & $4.7 \%$ \\
\hline & & 100 & 151,857 & 9,100 & 145,719 & $4.2 \%$ \\
\hline & & 125 & 151,218 & 9,658 & 145,719 & $3.8 \%$ \\
\hline & & 150 & 150,757 & 10,215 & 145,719 & $3.5 \%$ \\
\hline & & 175 & 150,497 & 10,772 & 145,482 & $3.5 \%$ \\
\hline & & 200 & 150,373 & 11,330 & 145,005 & $3.7 \%$ \\
\hline
\end{tabular}

Tabla 6-11: Resultados sin elitismo en GA. 250 individuos 


\begin{tabular}{|c|c|c|c|c|c|c|}
\hline Individuos & Cruzamiento & Generaciones & Coste medio(€) & sg. & Coste mínimo(€) & $\%$ Desv. \\
\hline \multirow[t]{27}{*}{500} & \multirow[t]{9}{*}{0.25} & 10 & 157,175 & 13,948 & 147,762 & $6.4 \%$ \\
\hline & & 25 & 154,493 & 14,607 & 144,327 & $7.0 \%$ \\
\hline & & 50 & 150,758 & 15,705 & 144,327 & $4.5 \%$ \\
\hline & & 75 & 149,304 & 16,804 & 144,327 & $3.4 \%$ \\
\hline & & 100 & 147,537 & 17,902 & 141,039 & $4.6 \%$ \\
\hline & & 125 & 146,889 & 19,001 & 136,076 & $7.9 \%$ \\
\hline & & 150 & 146,516 & 20,099 & 136,076 & $7.7 \%$ \\
\hline & & 175 & 146,408 & 21,197 & 136,076 & $7.6 \%$ \\
\hline & & 200 & 146,285 & 22,296 & 135,709 & $7.8 \%$ \\
\hline & \multirow[t]{9}{*}{0.50} & 10 & 156,951 & 13,928 & 149,382 & $5.1 \%$ \\
\hline & & 25 & 151,264 & 14,590 & 145,577 & $3.9 \%$ \\
\hline & & 50 & 148,806 & 15,696 & 139,770 & $6.5 \%$ \\
\hline & & 75 & 147,387 & 16,803 & 139,702 & $5.5 \%$ \\
\hline & & 100 & 146,284 & 17,909 & 139,702 & $4.7 \%$ \\
\hline & & 125 & 145,771 & 19,016 & 138,275 & $5.4 \%$ \\
\hline & & 150 & 145,492 & 20,122 & 138,275 & $5.2 \%$ \\
\hline & & 175 & 145,266 & 21,228 & 138,275 & $5.1 \%$ \\
\hline & & 200 & 145,105 & 22,335 & 137,207 & $7.8 \%$ \\
\hline & \multirow[t]{9}{*}{0.75} & 10 & 156,620 & 13,952 & 149,816 & $4.5 \%$ \\
\hline & & 25 & 151,442 & 14,618 & 133,683 & $13.3 \%$ \\
\hline & & 50 & 147,701 & 15,491 & 127,325 & $16.0 \%$ \\
\hline & & 75 & 145,189 & 16,365 & 127,325 & $14.0 \%$ \\
\hline & & 100 & 144,271 & 17,238 & 126,613 & $14.0 \%$ \\
\hline & & 125 & 144,061 & 18,112 & 126,613 & $13.8 \%$ \\
\hline & & 150 & 143,741 & 18,985 & 126,450 & $13.7 \%$ \\
\hline & & 175 & 143,551 & 19,858 & 126,450 & $13.5 \%$ \\
\hline & & 200 & 143,314 & 20,732 & 126,418 & $13.4 \%$ \\
\hline
\end{tabular}

Tabla 6-12: Resultados sin elitismo en GA. 500 individuos

En las Tablas 6-10, 6-11 y 6-12 se puede observar como para las generaciones sin elitismo, el coste medio mejora conforme aumenta el tamaño de la población, siendo esta diferencia muy significativa entre 100 y 500 individuos. El tiempo crece considerablemente con el tamaño de la población, prácticamente proporcional al mismo. Los resultados muestran una gran dispersión para 100 individuos, entre el 7 y el $11 \%$, una dispersión muy reducida para 250 individuos, entre el 3.5 y el $5 \%$, y un incremento notable en la dispersión para 500 individuos, entre el 8 y el 13\%. Los costes obtenidos están muy lejos de los mínimos logrados en otros algoritmos, y el proceso no va ligado a una convergencia con soluciones de calidad, baste con recordar que el mejor coste obtenido ha sido de 108,008 € con el SA. El mejor coste medio y mínimo se han producido para un tamaño de la población de 500 individuos, 200 generaciones y un cruzamiento máximo del 0.75 , siendo el coste medio de $143,314 €$ y el mínimo de $126,418 €$, un $17 \%$ superior al SA. 


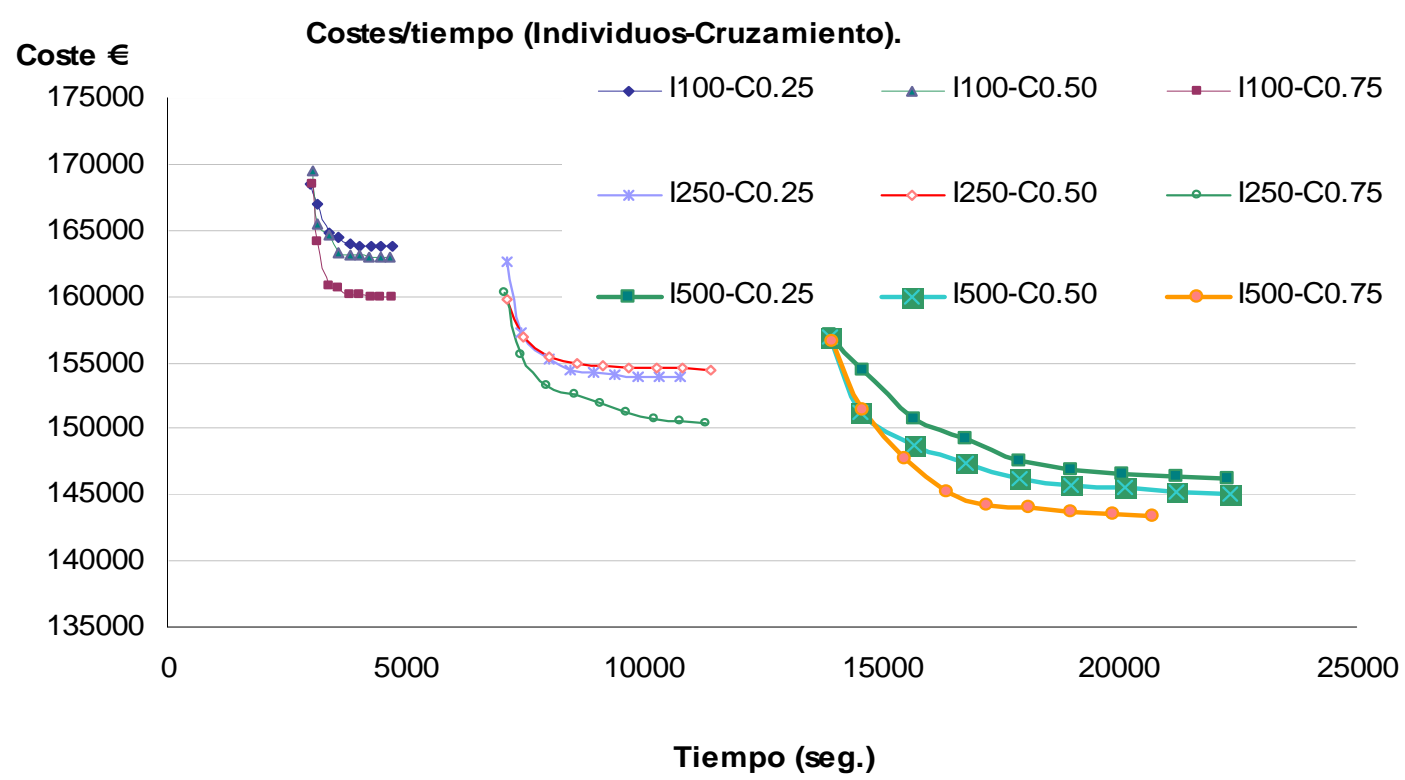

Figura 6-29: Evolución de los costes medios en el tiempo en un GA con elitismo.

\begin{tabular}{|c|c|c|c|c|c|c|}
\hline Individuos & Cruzamiento & Generaciones & $\begin{array}{c}\text { Coste } \\
\text { medio(€) }\end{array}$ & sg. & $\begin{array}{c}\text { Coste } \\
\text { mínimo(€) }\end{array}$ & $\%$ Desv. \\
\hline \multirow[t]{27}{*}{100} & \multirow[t]{9}{*}{0.25} & 10 & 168,505 & 2,848 & 156,912 & $7.4 \%$ \\
\hline & & 25 & 163,368 & 2,983 & 147,293 & $10.9 \%$ \\
\hline & & 50 & 161,265 & 3,308 & 152,015 & $6.1 \%$ \\
\hline & & 75 & 160,881 & 3,537 & 152,015 & $5.8 \%$ \\
\hline & & 100 & 160,849 & 3,766 & 152,015 & $5.8 \%$ \\
\hline & & 125 & 160,849 & 3,995 & 152,015 & $5.8 \%$ \\
\hline & & 150 & 160,849 & 4,224 & 152,015 & $5.8 \%$ \\
\hline & & 175 & 160,849 & 4,454 & 152,015 & $5.8 \%$ \\
\hline & & 200 & 160,849 & 4,683 & 152,015 & $5.8 \%$ \\
\hline & \multirow[t]{9}{*}{0.50} & 10 & 168,217 & 2,856 & 152,827 & $10.1 \%$ \\
\hline & & 25 & 160,518 & 2,992 & 146,020 & $9.9 \%$ \\
\hline & & 50 & 158,154 & 3,317 & 140,694 & $12.4 \%$ \\
\hline & & 75 & 157,154 & 3,547 & 140,515 & $12.0 \%$ \\
\hline & & 100 & 157,395 & 3,777 & 140,515 & $11.9 \%$ \\
\hline & & 125 & 157,168 & 4,007 & 140,515 & $11.8 \%$ \\
\hline & & 150 & 157,116 & 4,237 & 140,515 & $11.8 \%$ \\
\hline & & 175 & 157,116 & 4,467 & 140,515 & $11.8 \%$ \\
\hline & & 200 & 157,116 & 4,697 & 140,515 & $11.8 \%$ \\
\hline & \multirow[t]{9}{*}{0.75} & 10 & 168,509 & 2,836 & 155,601 & $8.3 \%$ \\
\hline & & 25 & 162,535 & 2,971 & 140,589 & $15.6 \%$ \\
\hline & & 50 & 160,614 & 3,294 & 140,287 & $14.5 \%$ \\
\hline & & 75 & 160,156 & 3,523 & 139,915 & $14.5 \%$ \\
\hline & & 100 & 159,991 & 3,751 & 139,915 & $14.3 \%$ \\
\hline & & 125 & 159,911 & 3,979 & 139,915 & $14.3 \%$ \\
\hline & & 150 & 159,969 & 4,207 & 139,915 & $14.3 \%$ \\
\hline & & 175 & 159,941 & 4,436 & 139,915 & $14.3 \%$ \\
\hline & & 200 & 159,941 & 4,664 & 139,915 & $14.3 \%$ \\
\hline
\end{tabular}

Tabla 6-13: Resultados con elitismo en GA. 100 individuos 


\begin{tabular}{|c|c|c|c|c|c|c|}
\hline Individuos & Cruzamiento & Generaciones & $\begin{array}{c}\text { Coste } \\
\text { medio(€) }\end{array}$ & sg. & $\begin{array}{c}\text { Coste } \\
\text { mínimo(€) }\end{array}$ & $\%$ Desv. \\
\hline \multirow[t]{27}{*}{250} & \multirow[t]{9}{*}{0.25} & 10 & 160,393 & 6,872 & 148,167 & $8.3 \%$ \\
\hline & & 25 & 155,063 & 7,184 & 139,176 & $11.4 \%$ \\
\hline & & 50 & 151,627 & 7,744 & 139,176 & $8.9 \%$ \\
\hline & & 75 & 150,925 & 8,303 & 138,997 & $8.6 \%$ \\
\hline & & 100 & 150,635 & 8,862 & 138,997 & $8.4 \%$ \\
\hline & & 125 & 150,497 & 9,422 & 138,997 & $8.3 \%$ \\
\hline & & 150 & 150,303 & 9,981 & 138,997 & $8.1 \%$ \\
\hline & & 175 & 150,303 & 10,541 & 138,997 & $8.1 \%$ \\
\hline & & 200 & 150,303 & 11,100 & 138,997 & $8.1 \%$ \\
\hline & \multirow[t]{9}{*}{0.50} & 10 & 155,983 & 6,920 & 143,169 & $9.0 \%$ \\
\hline & & 25 & 153,469 & 7,235 & 141,616 & $8.4 \%$ \\
\hline & & 50 & 148,779 & 7,787 & 129,669 & $14.7 \%$ \\
\hline & & 75 & 147,167 & 8,340 & 127,969 & $15.0 \%$ \\
\hline & & 100 & 146,872 & 8,892 & 127,969 & $14.8 \%$ \\
\hline & & 125 & 146,618 & 9,444 & 127,969 & $14.6 \%$ \\
\hline & & 150 & 146,520 & 9,997 & 127,969 & $14.5 \%$ \\
\hline & & 175 & 146,461 & 10,549 & 127,969 & $14.5 \%$ \\
\hline & & 200 & 146,370 & 11,102 & 127,969 & $14.4 \%$ \\
\hline & \multirow[t]{9}{*}{0.75} & 10 & 159,466 & 7,084 & 141,921 & $6.7 \%$ \\
\hline & & 25 & 153,431 & 7,419 & 135,176 & $6.8 \%$ \\
\hline & & 50 & 149,998 & 7,978 & 133,636 & $5.2 \%$ \\
\hline & & 75 & 148,408 & 8,538 & 133,636 & $4.7 \%$ \\
\hline & & 100 & 147,620 & 9,097 & 133,636 & $4.2 \%$ \\
\hline & & 125 & 147,110 & 9,657 & 133,636 & $3.8 \%$ \\
\hline & & 150 & 146,854 & 10,216 & 133,636 & $3.5 \%$ \\
\hline & & 175 & 146,743 & 10,775 & 133,636 & $3.5 \%$ \\
\hline & & 200 & 146,647 & 11,335 & 133,636 & $3.7 \%$ \\
\hline
\end{tabular}

Tabla 6-14: Resultados con elitismo en GA. 250 individuos 


\begin{tabular}{|c|c|c|c|c|c|c|}
\hline Individuos & Cruzamiento & Generaciones & $\begin{array}{c}\text { Coste } \\
\text { medio }(€)\end{array}$ & sg. & $\begin{array}{c}\text { Coste } \\
\text { mínimo(€) }\end{array}$ & $\%$ Desv. \\
\hline \multirow[t]{27}{*}{500} & \multirow[t]{9}{*}{0.25} & 10 & 153,560 & 13,758 & 148,212 & $3.6 \%$ \\
\hline & & 25 & 151,048 & 14,412 & 147,340 & $2.5 \%$ \\
\hline & & 50 & 148,592 & 15,519 & 145,186 & $2.4 \%$ \\
\hline & & 75 & 147,512 & 16,627 & 141,425 & $4.3 \%$ \\
\hline & & 100 & 146,825 & 17,734 & 140,179 & $4.7 \%$ \\
\hline & & 125 & 146,567 & 18,842 & 139,137 & $5.3 \%$ \\
\hline & & 150 & 146,433 & 19,949 & 138,290 & $5.9 \%$ \\
\hline & & 175 & 146,337 & 21,056 & 138,290 & $5.8 \%$ \\
\hline & & 200 & 146,160 & 22,164 & 137,442 & $6.3 \%$ \\
\hline & \multirow[t]{9}{*}{0.50} & 10 & 155,907 & 13,745 & 149,470 & $4.3 \%$ \\
\hline & & 25 & 148,711 & 14,397 & 143,798 & $3.4 \%$ \\
\hline & & 50 & 142,763 & 15,477 & 137,458 & $3.9 \%$ \\
\hline & & 75 & 140,890 & 16,558 & 134,412 & $4.8 \%$ \\
\hline & & 100 & 140,117 & 17,638 & 133,377 & $5.1 \%$ \\
\hline & & 125 & 139,830 & 18,719 & 133,332 & $4.9 \%$ \\
\hline & & 150 & 139,285 & 19,799 & 132,618 & $5.0 \%$ \\
\hline & & 175 & 138,885 & 20,879 & 132,618 & $4.7 \%$ \\
\hline & & 200 & 138,743 & 21,960 & 132,618 & $4.6 \%$ \\
\hline & \multirow[t]{9}{*}{0.75} & 10 & 154,134 & 13,763 & 147,789 & $4.3 \%$ \\
\hline & & 25 & 146,274 & 14,414 & 136,229 & $7.4 \%$ \\
\hline & & 50 & 142,436 & 15,498 & 130,041 & $9.5 \%$ \\
\hline & & 75 & 141,470 & 16,583 & 128,945 & $9.7 \%$ \\
\hline & & 100 & 140,903 & 17,667 & 128,618 & $9.6 \%$ \\
\hline & & 125 & 140,574 & 18,752 & 128,293 & $9.6 \%$ \\
\hline & & 150 & 140,354 & 19,836 & 128,261 & $9.4 \%$ \\
\hline & & 175 & 140,217 & 20,920 & 128,261 & $9.3 \%$ \\
\hline & & 200 & 140,084 & 22,005 & 128,261 & $9.2 \%$ \\
\hline
\end{tabular}

Tabla 6-15: Resultados con elitismo en GA. 500 individuos

En las ejecuciones con elitismo de las Tablas 6-13, 6-14 y 6-15 y representadas en la Figura 6-29, donde cada punto representa una generación -desde 10 hasta 200-, se observa un comportamiento muy parecido a sin elitismo. El coste medio mejora conforme aumenta el tamaño de población, el tiempo de proceso aumenta con el tamaño de población y con valores similares a "sin elitismo", y con respecto a la desviación, ésta varía en cada heurística entre valores desde el 3 al 15\%. Los resultados muestran una mejora en la calidad de los mismos, tanto en la reducción de los costes, como en la desviación -considerablemente menor para 500 individuos-. El mejor coste medio se obtiene para un tamaño de la población de 500 individuos y un valor de cruzamiento de 0.50 , siendo dicho valor de $138,863 €$, con una mejora del $3.1 \%$ respecto a sin elitismo. El valor mínimo se obtiene para 250 individuos, cruzamiento del 0.50 y 75 generaciones, siendo de $127,969 €$, superior a "sin elitismo" en $1.2 \%$.

A la mejor heurística -500 individuos, cruzamiento 0.5-, se le ha aplicado el operador "mutación" para comprobar si éste mejora las prestaciones de esta heurística poblacional, aplicando la mutación a una variable, e incrementando su número progresivamente hasta que no mejore la solución. En la siguiente Tabla 6-16 se 
muestran los resultados obtenidos. La columna "\% Mejora" se refiere al coste medio de cada una de las heurísticas respecto al valor con mutación 0 (sin mutación).

\begin{tabular}{|c|c|c|c|r|r|}
\hline $\begin{array}{l}\text { Individuos }= \\
\text { Cruzamiento }=\end{array}$ & $\begin{array}{l}500 \\
\text { Generaciones }=\end{array}$ & $\begin{array}{l}50 \\
200\end{array}$ & \multicolumn{3}{|l|}{} \\
\hline Mutación & Coste medio(€) & sg. & Coste mínimo(€) & $\%$ Desv. & $\%$ Mejora \\
\hline 0 & 138,743 & 21,964 & 132,618 & $4.6 \%$ & - \\
\hline 1 & 134,846 & 22,469 & 130,424 & $3.4 \%$ & $2.8 \%$ \\
\hline 2 & 133,992 & 22,479 & 126,366 & $6.0 \%$ & $3.4 \%$ \\
\hline 3 & 133,091 & 22,487 & 125,187 & $6.3 \%$ & $4.1 \%$ \\
\hline 4 & 132,678 & 22,499 & 123,078 & $7.8 \%$ & $4.4 \%$ \\
\hline 5 & 132,707 & 22,131 & 125,345 & $5.9 \%$ & $4.4 \%$ \\
\hline
\end{tabular}

Tabla 6-16: Aplicación de "mutación” en GA

Como se observa, la mutación mejora los resultados del GA, obteniéndose una reducción del coste medio de hasta el 4.4\%, con mutación 4. Incluso mejora en un $2.6 \%$ el coste mínimo con la misma heurística, siendo su valor de 123,078 €. Los tiempos medios son similares a los resultados anteriores, y la desviación se encuentra entre el 3 y el 7\%. En la siguiente Figura 6-30, se aprecia la evolución de la mejora de la heurística cuando se incrementa la mutación, mostrando un cierto agotamiento a partir de 4 variaciones.

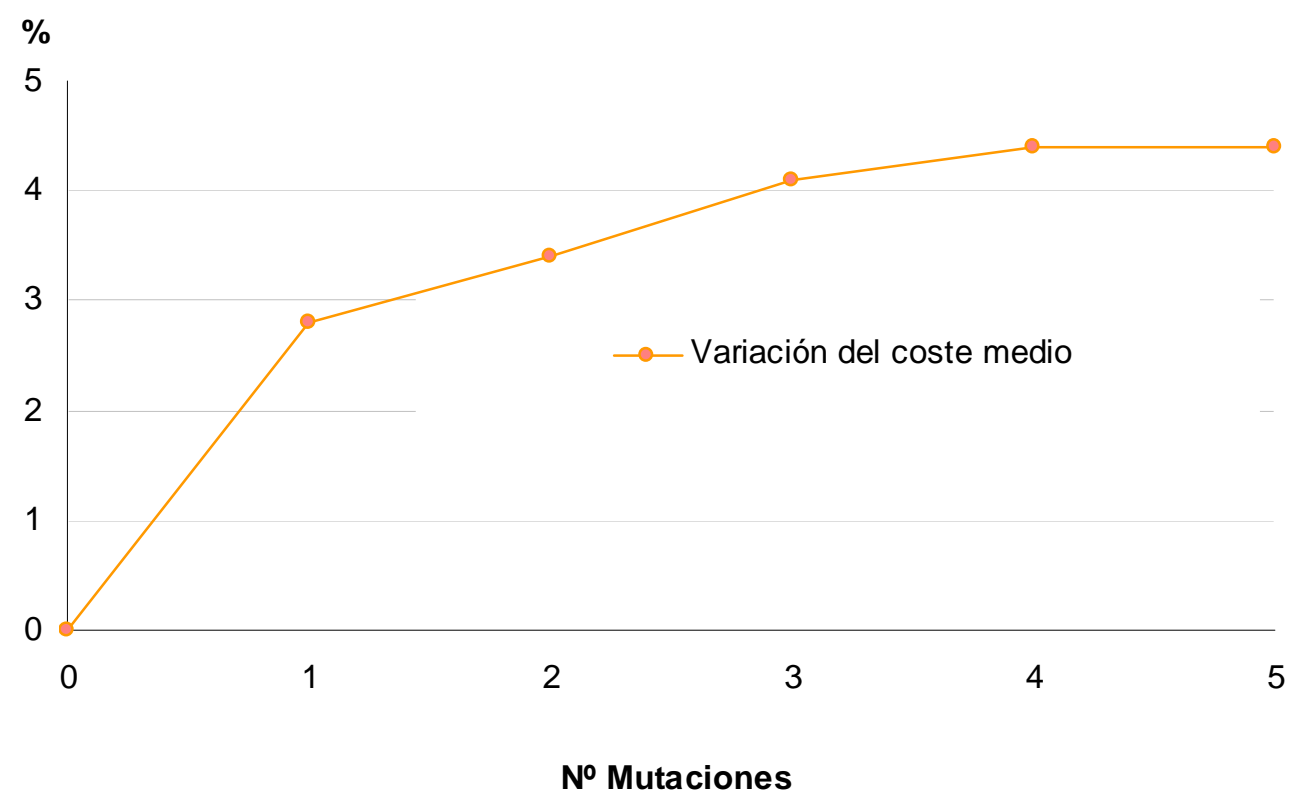

Figura 6-30: \% Reducción coste medio (mutación / no mutación) 


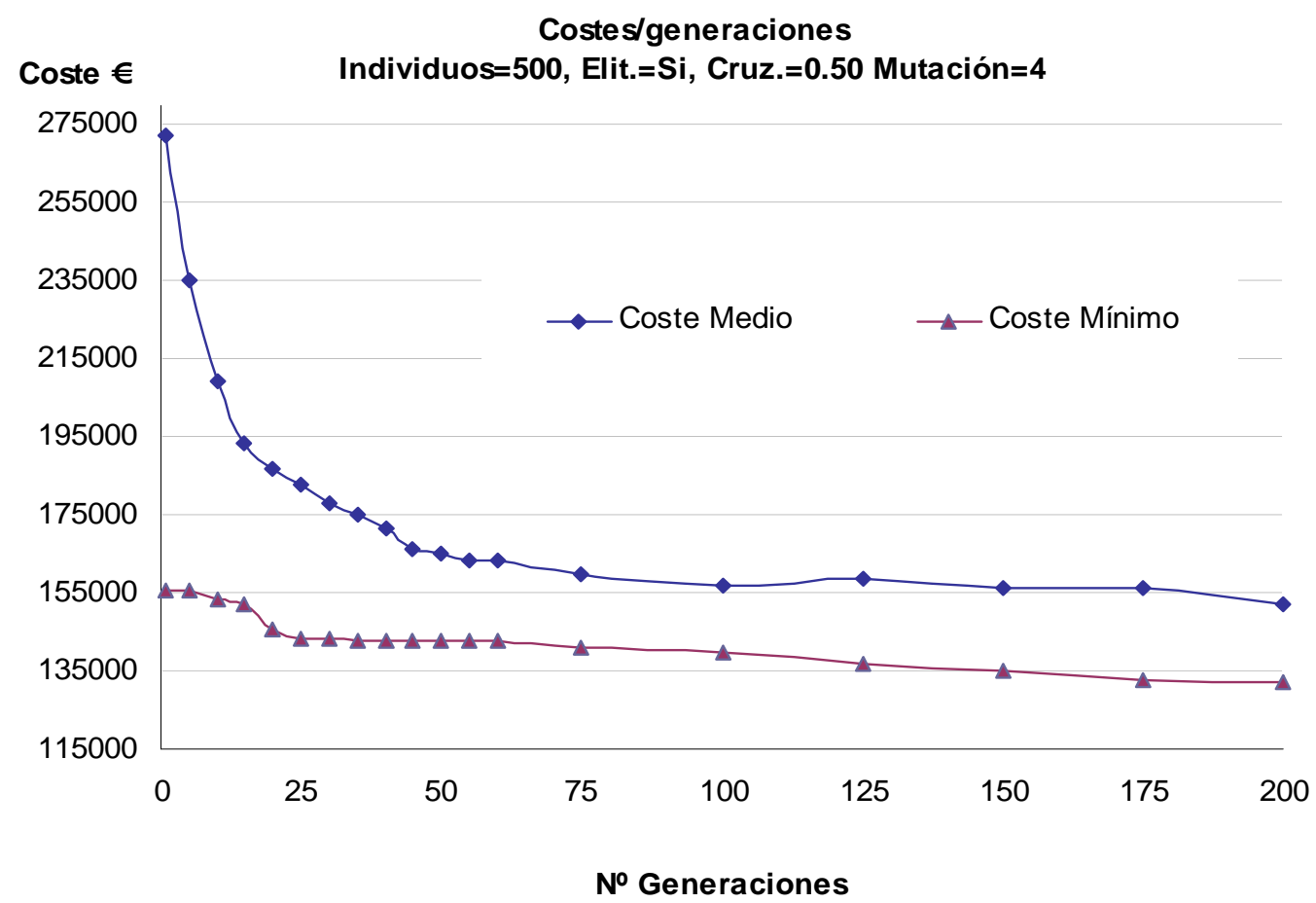

Figura 6-31: Evolución de los costes medios y mínimos en un GA con mutación

En la Figura 6-31 se produce un contraste respecto a las 6-27 y 6-28 vistas anteriormente. En ésta se origina un distanciamiento que permanece más o menos constante, entre la media y el valor mínimo en cada generación. Con esta heurística se ocasiona una mejora en algunos de los individuos, no en la mayoría, mediante una búsqueda en el entorno local.

\section{$\underline{\text { Resumen }}$}

Esta heurística es la primera de carácter poblacional estudiada. Generalizando, se puede afirmar que se obtienen mejores resultados medios cuando aumenta el número de individuos y también el número de generaciones, aunque con éstas, se llega a un momento en el que los individuos se repiten abundantemente. También se observa que el coste se reduce cuando se aplica "elitismo", cuya mejora es del $3.1 \%$ entre las mejores heurísticas. Los tiempos medios giran en ambos entorno a 20,000-22,000 segundos.

El factor mutación permite mejorar la esencia poblacional de esta heurística, añadiendo el efecto de la búsqueda por entornos locales en pequeña escala. El mejor resultado se ha obtenido con mutación 4 . La mejora ha sido del $4.4 \%$ respecto a "elitismo", y del 7.4\% respecto a "sin elitismo". Y en cuanto al tiempo de proceso, su incremento es insignificante. 
Si se compara la mejor heurística de GA con la mejor estudiada hasta el momento, el SA, por un lado los tiempos del SA son similares, del orden de 18,000 segundos, y por otro, se obtiene que el coste medio de la primera es un $20.1 \%$ superior a la segunda, por lo que para este problema, esta heurística GA obtiene resultados de peor calidad que los obtenidos por el SA, el TA, e incluso el DLS.

La solución que presenta un menor coste del tablero se muestra en la Figura 6-32.

SECCION TRANSVERSAL
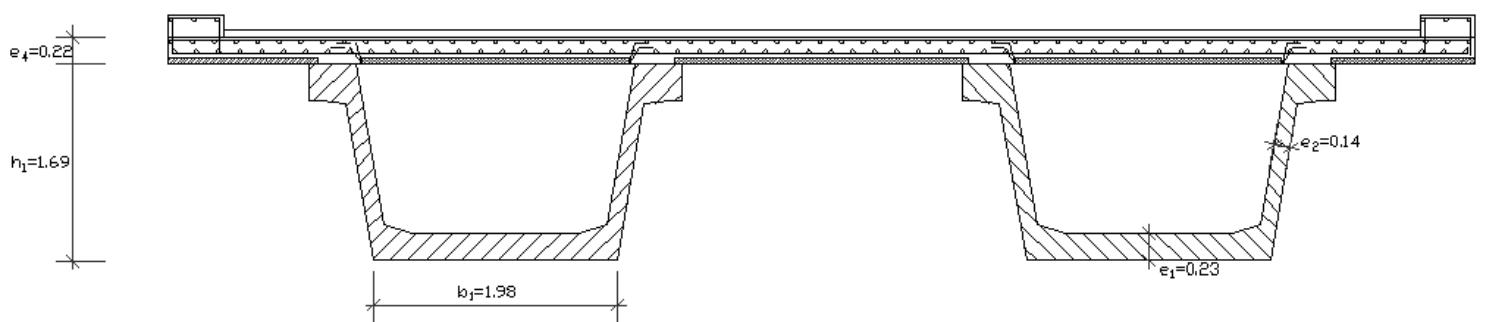

Figura 6-32: Tablero con menor coste en un GA 


\subsection{Algoritmos meméticos}

\subsubsection{Descripción del algoritmo}

Esta heurística poblacional, mantiene la idea de la evolución de las especies desarrollada en GA, pero a diferencia de ésta, que emula una evolución biológica, los algoritmos meméticos -memético algorithms (MA)- intentan representar una evolución cultural. Pablo Moscató[119] (1989) describe la evolución estratégica de los algoritmos meméticos con la simbología de la evolución de las artes marciales, cuyo avance se ha desarrollado en un tiempo muy reducido comparado con la evolución biológica. Este desarrollo no ha sido obra del azar, si no que ha sido un proceso guiado, en el cual solamente los maestros tienen conocimiento suficiente para seguir desarrollando la técnica. El MA combina la búsqueda global basada en una población, y una metaheurística de búsqueda local. Tal como se ha comprobado en GA, cuando se aplicaba mutación -equivalente a una búsqueda local de carácter reducido-, algunas soluciones mejoraban significativamente.

El proceso se inicia con la creación de una población. Los individuos son generados de un modo aleatorio o mediante cualquier otro procedimiento de iniciación. Cada uno de estos individuos es mejorado mediante una búsqueda de carácter local, con el objeto de acercarse a un óptimo local. Con esta nueva población, que debe ser de mejor calidad que la obtenida inicialmente, se procede a la aplicación del GA. La lógica sencilla de este procedimiento se puede expresar del siguiente modo: "con padres biológicamente buenos es más fácil obtener hijos buenos que con padres mediocres". Los miembros obtenidos mediante dicho proceso de selección (búsqueda local), ahora interactúan entre ellos (búsqueda global poblacional). Se aplican los mismos operadores que en el GA, como son, cruzamiento, elitismo y mutación para combinar la información genética de la presente población y así crear una nueva generación. Los algoritmos genéticos tratan de combinar los beneficios propios de GA y los de las búsquedas locales. Los GA se han mostrado particularmente eficientes en encontrar zonas relativamente buenas durante la exploración del espacio de soluciones, pero no así en el acercamiento a un óptimo de carácter local, donde los métodos de búsqueda local son mucho más eficaces. En la Figura 6-33 se muestra un diagrama básico de flujo de MA aplicado en este estudio. 


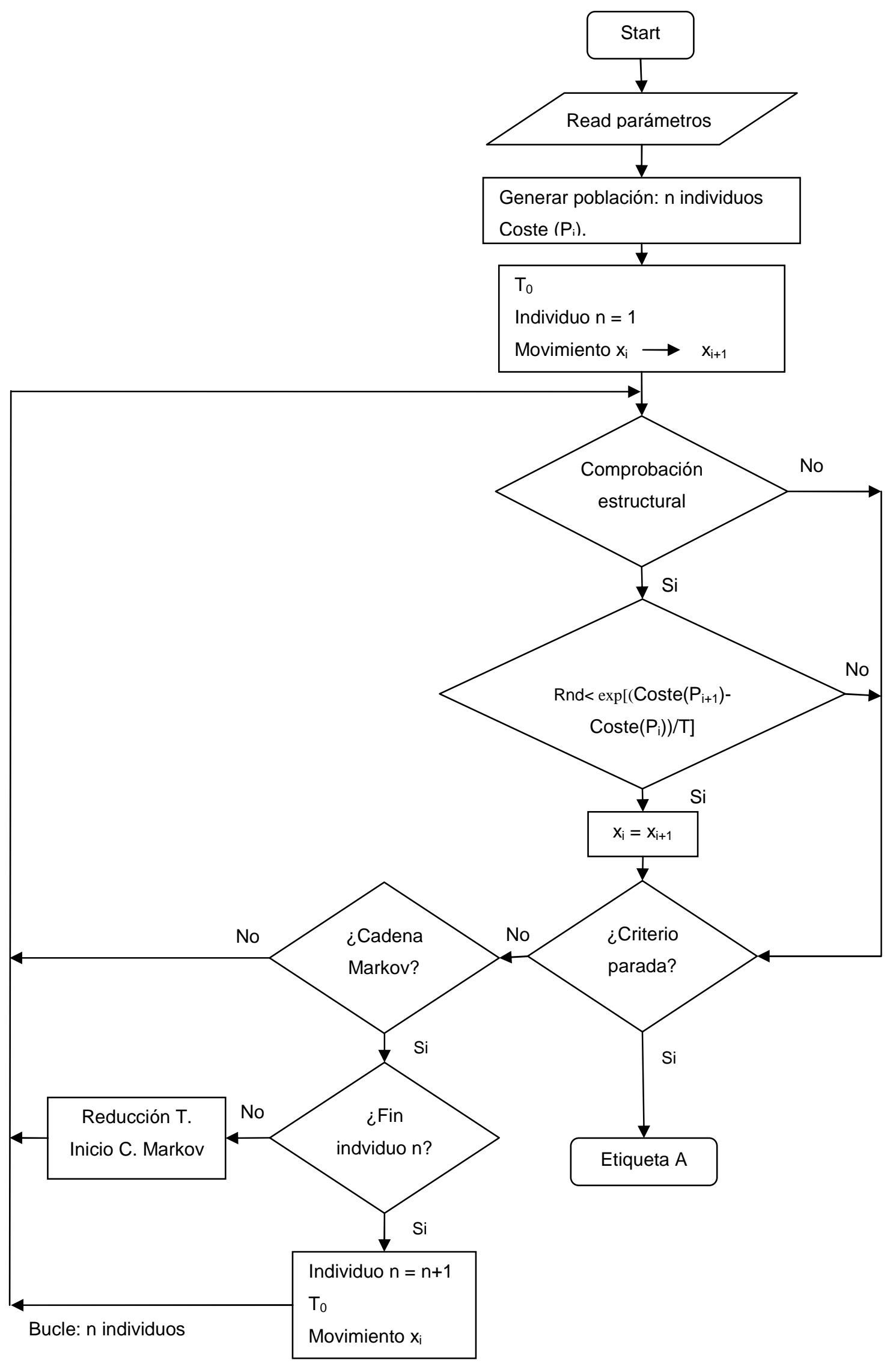




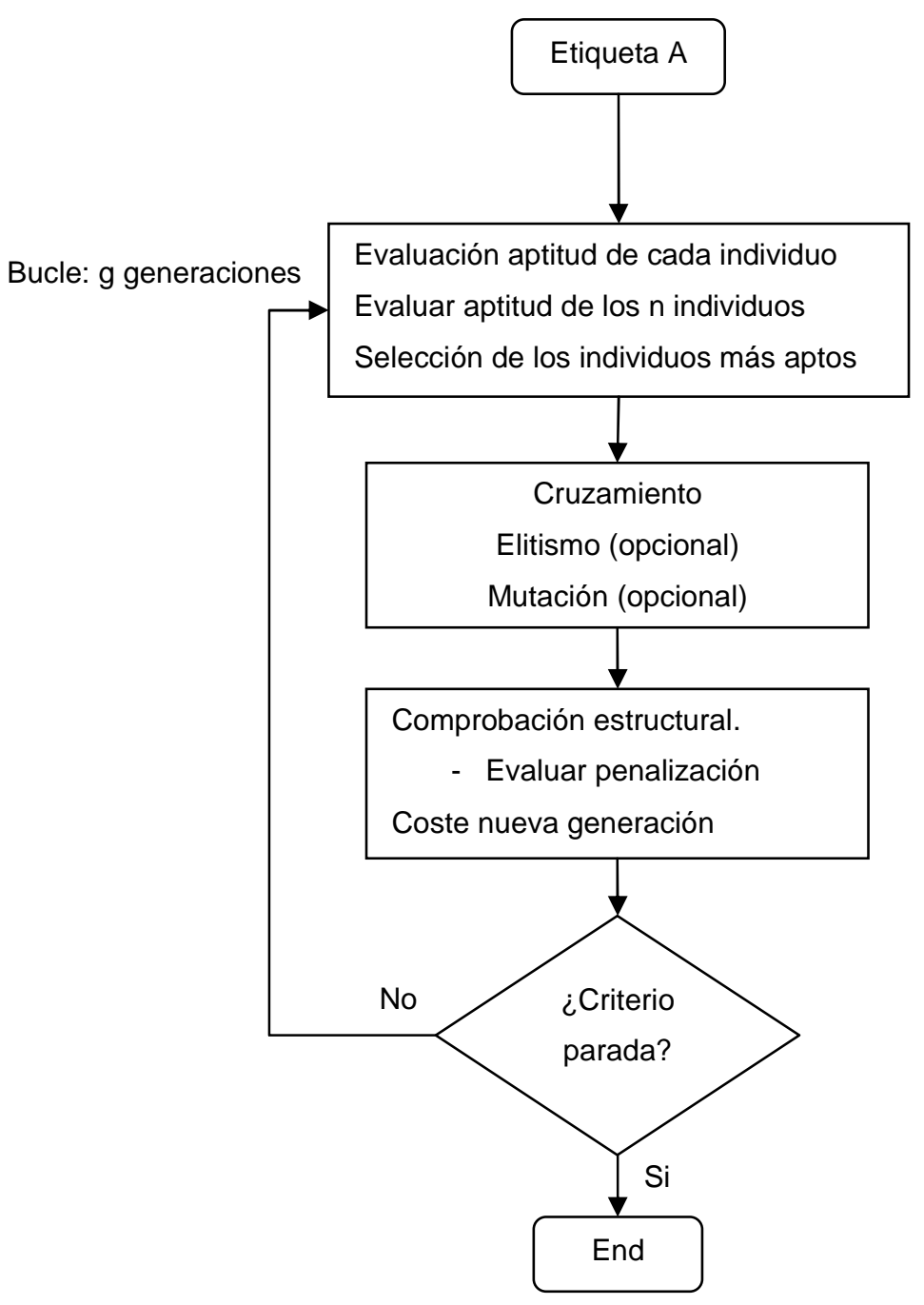

Figura 6-33: Diagrama de flujo de MA

\subsubsection{Aplicación.}

Para la obtención de los primeros padres, se han aplicado tres metaheurísticas de acercamiento local. Se ha utilizado la que mejores resultados ha ofrecido anteriormente, como es el SA, pero con el objeto de no llegar a intensificar dicha búsqueda, se han utilizado los siguientes parámetros: longitud cadena de Markov igual a 50, temperatura inicial $\mathrm{T}_{0}$ igual al 5\% del coste, y criterio de parada 10, $25 \mathrm{y}$ 50 cadenas. Para cada uno de los tres criterios de parada, se han realizado 9 ejecuciones con la mejor heurística obtenida del GA, cuyos valores son: 500 individuos, 200 generaciones, elitismo, cruzamiento igual a 0.50 , mutación $=4$. Los resultados se muestran en la siguiente Tabla 6-17, donde el coste medio se refiere a la media de los valores mínimos de las 9 ejecuciones. 


\begin{tabular}{|c|c|c|c|c|r|r|}
\hline C. Markov & $\begin{array}{c}\text { Num. } \\
\text { Cadenas }\end{array}$ & $\begin{array}{c}\text { Coste } \\
\text { medio(€) }\end{array}$ & sg. & $\begin{array}{c}\text { Coste } \\
\text { mínimo(€) }\end{array}$ & \% Desv. & $\begin{array}{c}\% \\
\text { Mejora/GA }\end{array}$ \\
\hline \multirow{2}{*}{50} & GA & 132,678 & 22,499 & 123,078 & $7.8 \%$ & - \\
\cline { 2 - 7 } & 10 & 129,718 & 43,639 & 124,446 & $4.2 \%$ & $2.2 \%$ \\
\cline { 2 - 7 } & 25 & 124,564 & 76,934 & 119,332 & $4.4 \%$ & $6.1 \%$ \\
\cline { 2 - 7 } & 50 & 119,703 & 129,441 & 116,933 & $2.4 \%$ & $9.8 \%$ \\
\hline
\end{tabular}

Tabla 6-17: Resultados en un MA. (Indiv=500, Elit=Si, Cruz=0.5, Gen=200, Mut=4)

Se aprecia en la Tabla una mejora de los resultados obtenidos previamente con el GA, pero con incrementos de tiempo muy superiores. Se obtienen mejoras en los costes respecto a la mejor heurística del GA del 2 al 10\%, pero incrementando los tiempos desde el $194 \%$ hasta el $575 \%$.

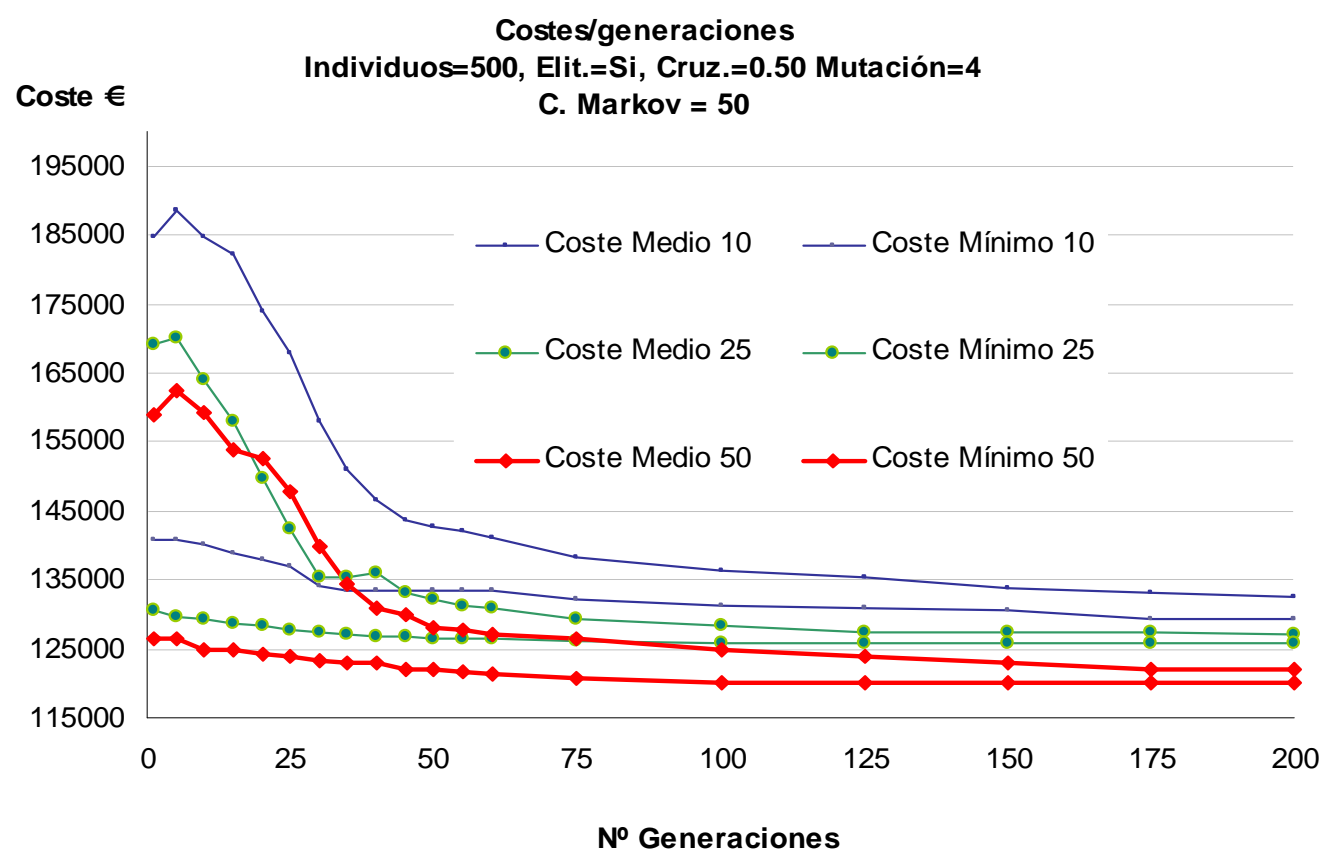

Figura 6-34: Evolución de los costes en un MA según N$^{\circ}$ Cadenas Markov

En la Figura 6-34 se muestra la evolución de los costes medios y mínimos para las heurísticas situadas en la posición media de los 9 procesos. El punto de partida viene determinado por la aproximación a un óptimo local mediante un SA según el criterio de parada utilizado (10, 25 o 25 número de cadenas). Se observa un comportamiento similar para las tres, donde los valores medios van reduciéndose rápidamente al principio, para después ir haciéndolo más lento, y a continuación permanecer prácticamente constante. Hay que resaltar que al principio aumentan los costes respecto a los obtenidos por el SA, pero mínimamente, y a continuación continúan con un comportamiento típico de un GA. Se observa que si el valor de partida es menor, también lo será el resultado final, es decir, si los padres son buenos, las nuevas generaciones también lo serán. 
Si se compara la mejor heurística del MA con la mejor estudiada hasta el momento, el SA, se tiene con respecto al coste, que el menor valor medio, 119,703 €, es un $8.4 \%$ superior al del SA, $110,477 €$, y que el tiempo empleado ha sido más de 7 veces al empleado por el SA. En cuanto al coste mínimo, en el MA es de 116,933 € (Figura 6-35), mientras que en el SA ha sido de 108,008, es decir un $8.3 \%$ superior. Por lo tanto, se deduce que esta heurística MA no es demasiado eficaz, obteniendo resultados de peor calidad que los alcanzados con el SA y el TA, tanto en costes como en tiempos.

SECCION TRANSVERSAL

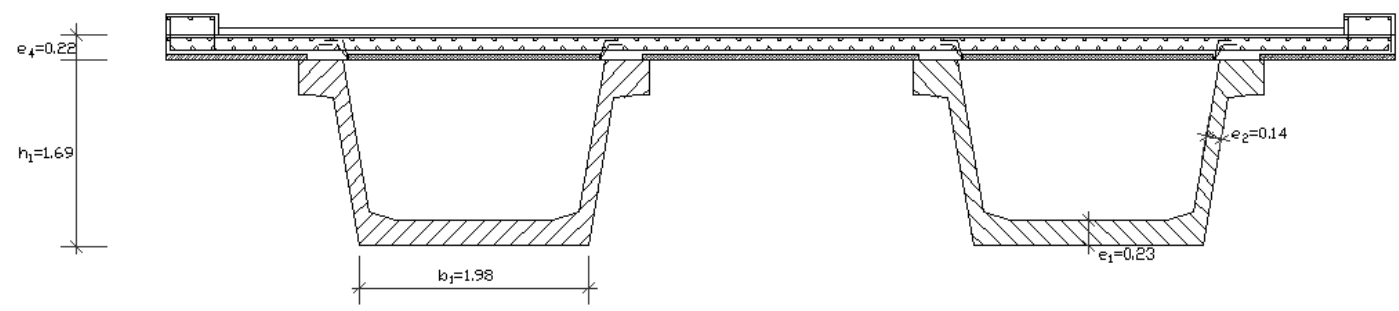

Figura 6-35: Tablero con menor coste en un MA 


\subsection{Resumen de los costes y comparación de las soluciones}

\subsubsection{Resumen de los costes}

En primer lugar se presenta un cuadro resumen con los valores obtenidos en el presente capítulo (Tabla 6-18). En él se incluyen los costes medios y tiempos medios de las mejores heurísticas, el coste mínimo y el \% de los costes medios respecto al menor de ellos -SA-.

\begin{tabular}{|l|c|r|r|r|r|r|r|c|}
\hline & \multicolumn{1}{|c|}{ RW } & \multicolumn{1}{c|}{ DLS } & \multicolumn{1}{c|}{ SA } & \multicolumn{1}{c|}{ TA } & GA $(1)^{*}$ & $\mathrm{GA}(2)^{*}$ & $\mathrm{GA}(3)^{*}$ & MA \\
\hline Coste medio (€) & 273182 & 128309 & 110477 & 110865 & 143414 & 138743 & 132678 & 119703 \\
\hline Coste mínimo (€) & 140946 & 115459 & 108008 & 108580 & 126418 & 127969 & 123078 & 116933 \\
\hline Tiempo medio (seg.) & - & 8122 & 18322 & 36233 & 20732 & 21960 & 22499 & 129441 \\
\hline $\begin{array}{l}\text { \% Coste } \\
\text { medio/record }\end{array}$ & $147.3 \%$ & $16.1 \%$ & $0.0 \%$ & $0.4 \%$ & $29.8 \%$ & $25.6 \%$ & $20.1 \%$ & $8.4 \%$ \\
\hline
\end{tabular}

Tabla 6-18: Tabla resumen de las heurísticas estudiadas

* GA(1) sin elitismo. (2) con elitismo. (3) con elitismo y mutación 4.

Se desprende del cuadro que las heurísticas que han obtenido mejores resultados son el SA y el TA, las cuales destacan sobre las demás. De lo descrito en el punto 5.4, y en especial por la representación de los óptimos de Pareto dentro de la Figura 6-18, nos decantamos por el SA, aunque las diferencias son tan escasas, que podríamos señalar que ambas obtienen resultados similares, y cualquiera de las dos podría ser utilizada en los estudios posteriores de parametrización y variación de precios.

SECCION TRANSVERSAL

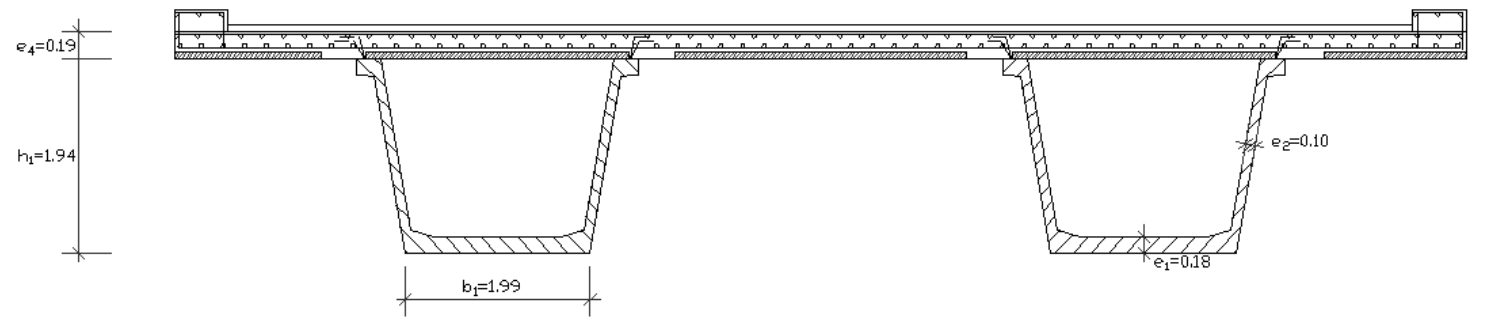

Figura 6-36: Tablero con menor coste (SA) 


\subsubsection{Comparación de las soluciones}

En este apartado se muestran y comparan los resultados de las soluciones que proporcionan los menores costes obtenidos para cada una de las heurísticas estudiadas.

\section{Geometría, hormigones, armadura pasiva y activa}

En los puntos 4.2 y 4.3 se ha realizado una descripción de los parámetros y variables que definen la estructura. A continuación se muestran los valores más significativos de las variables, obtenidos con las heurísticas y que dan, junto con los parámetros, la definición completa del tablero del puente.

\section{Geometría, Hormigones, Activa}

\begin{tabular}{|c|c|r|r|r|r|r|r|r|r|r|r|r|r|r|}
\hline & $\begin{array}{c}\text { Coste } \\
(€)\end{array}$ & $\begin{array}{r}\text { h1 } \\
(\mathrm{m})\end{array}$ & $\begin{array}{r}\mathrm{e} 4 \\
(\mathrm{~m})\end{array}$ & $\begin{array}{r}\mathrm{b} 1 \\
(\mathrm{~m})\end{array}$ & $\begin{array}{r}\mathrm{b} 3 \\
(\mathrm{~m})\end{array}$ & $\begin{array}{r}\mathrm{e} 1 \\
(\mathrm{~m})\end{array}$ & $\begin{array}{r}\mathrm{e} 2 \\
(\mathrm{~m})\end{array}$ & $\begin{array}{r}\mathrm{e} 3 \\
(\mathrm{~m})\end{array}$ & $\begin{array}{r}\mathrm{Hv} \\
(\mathrm{HP})\end{array}$ & $\begin{array}{r}\mathrm{H} \\
(\mathrm{HA})\end{array}$ & $\begin{array}{r}\text { p1 } \\
(\mathrm{n})\end{array}$ & $\begin{array}{r}\text { p2 } \\
(\mathrm{n})\end{array}$ & $\begin{array}{r}\text { p3 } \\
(\mathrm{n})\end{array}$ & $\begin{array}{r}\text { p4 } \\
(\mathrm{n})\end{array}$ \\
\hline RW & 140946 & 1.73 & 0.25 & 1.87 & 1.12 & 0.30 & 0.25 & 0.29 & 45 & 35 & 34 & 34 & 8 & 16 \\
\hline DLS & 115459 & 1.93 & 0.27 & 1.90 & 0.38 & 0.25 & 0.10 & 0.31 & 45 & 40 & 35 & 26 & 0 & 2 \\
\hline SA & 108008 & 1.94 & 0.19 & 1.99 & 0.29 & 0.18 & 0.10 & 0.23 & 40 & 40 & 36 & 20 & 0 & 2 \\
\hline TA & 108580 & 1.92 & 0.19 & 1.99 & 0.29 & 0.18 & 0.10 & 0.23 & 45 & 40 & 36 & 20 & 0 & 2 \\
\hline GA(1) & 126418 & 1.78 & 0.24 & 1.92 & 0.33 & 0.17 & 0.16 & 0.20 & 45 & 30 & 35 & 29 & 0 & 6 \\
\hline GA(2) & 127969 & 1.78 & 0.24 & 1.92 & 0.33 & 0.17 & 0.16 & 0.20 & 45 & 30 & 35 & 29 & 0 & 6 \\
\hline GA(3) & 123078 & 1.69 & 0.22 & 1.98 & 0.41 & 0.23 & 0.14 & 0.32 & 40 & 35 & 36 & 34 & 0 & 6 \\
\hline MA & 116933 & 1.80 & 0.23 & 1.78 & 0.38 & 0.22 & 0.10 & 0.30 & 50 & 35 & 32 & 31 & 0 & 2 \\
\hline
\end{tabular}

Tabla 6-19: Resultados: Geometría, Hormigones y Activas

siendo,

$\begin{array}{ll}\text { h1 } & \text { canto de la viga } \\ \text { e4 } & \text { canto de la losa } \\ \text { b1 } & \text { ancho ala inferior de la viga } \\ \text { b3 } & \text { base ala superior de la viga } \\ \text { e1 } & \text { canto ala inferior de la viga } \\ \text { e2 } & \text { espesor del alma } \\ \text { e3 } & \text { canto ala superior de la viga } \\ \text { Hv } & \text { Hormigón de la viga } \\ \text { H1 } & \text { Hormigón de la losa } \\ \text { p1 } & \text { número de torones nivel 1 ala inferior de } \Phi 0.6 " \\ \text { p2 } & \text { número de torones nivel } 2 \text { ala inferior de } \Phi 0.6 " \\ \text { p3 } & \text { número de torones nivel } 3 \text { ala inferior de } \Phi 0.6 " \\ \text { p4 } & \text { número de torones nivel } 4 \text { ala superior de } \Phi 0.6 "\end{array}$

Los mejores resultados, correspondientes al SA y TA, tienen valores geométricos muy similares, con cantos de viga altos y espesores de losa pequeños; además coinciden con las dimensiones de las alas y el número de torones en todos los niveles. Las dimensiones de las alas inferiores son del orden de magnitud del canto de la viga, ello puede estar asociado a los parámetros del problema (anchura del tablero y número de vigas). La optimización estructural del hormigón va dirigida a dimensionar alas superiores cortas, puesto que no mejoran la resistencia a solicitaciones normales. El menor canto de la viga del TA es compensado con un 
hormigón de mayor resistencia (HP-45). Hay que señalar, que la aplicación en el GA de elitismo o no, no afecta a la geometría de las soluciones de menor coste, puesto que todas las variables de la Tabla coinciden en ambas. Su diferencia en coste viene determinada por la armadura pasiva, tal como se puede ver en la Tabla 6-20.

\section{Pasiva}

\begin{tabular}{|l|c|r|r|r|r|r|r|r|}
\hline Acero & \multicolumn{1}{|c|}{ RW } & \multicolumn{1}{c|}{ DLS } & \multicolumn{1}{|c|}{ SA } & \multicolumn{1}{|c|}{ TA } & GA(1) & GA(2) & GA(3) & \multicolumn{1}{c|}{ MA } \\
\hline en viga $(\mathrm{Kg})$ & 95555 & 6325 & 5562 & 5643 & 8554 & 8496 & 7167 & 6359 \\
\hline en losa $(\mathrm{Kg})$ & 22353 & 17341 & 19561 & 19668 & 22105 & 22181 & 20015 & 19419 \\
\hline Total $(\mathrm{Kg})$ & 117908 & 23666 & 25123 & 25311 & 30659 & 30677 & 27182 & 25778 \\
\hline \% Acero/record & $369.3 \%$ & $-5.8 \%$ & $0.0 \%$ & $0.7 \%$ & $22.0 \%$ & $22.1 \%$ & $8.2 \%$ & $2.6 \%$ \\
\hline
\end{tabular}

Tabla 6-20: Resultados: Armadura pasiva

Las menores cantidades de acero en la viga se corresponden con el DLS y a continuación con el SA y el TA. Los mayores espesores de la losa en el DLS le permiten disponer de menos cantidad de acero en ella que el SA y el TA. En conjunto, son bastante similares en el SA, DLS, TA y MA, todos ellos han dispuesto, al menos en algún momento de su proceso, una búsqueda de aproximación a un óptimo local.

\section{Cuantías de acero}

\begin{tabular}{|l|r|r|r|r|r|r|r|r|}
\hline Cuantías & \multicolumn{1}{|c|}{ RW } & DLS & \multicolumn{1}{c|}{ SA } & \multicolumn{1}{c|}{ TA } & GA(1) & GA(2) & GA(3) & \multicolumn{1}{c|}{ MA } \\
\hline $\mathrm{m}$ 3 Hor / m2 tablero & 0.562 & 0.442 & 0.329 & 0.329 & 0.397 & 0.397 & 0.401 & 0.383 \\
\hline $\mathrm{Kg}$ activas / m3 Hor & 37.50 & 32.63 & 40.34 & 40.42 & 40.40 & 40.40 & 43.43 & 38.84 \\
\hline $\mathrm{Kg}$ activas / m3 Hor viga & 67.48 & 83.58 & 95.15 & 95.61 & 101.85 & 101.99 & 96.07 & 96.88 \\
\hline $\mathrm{Kg}$ activas / m2 tablero & 21.07 & 14.43 & 13.28 & 13.28 & 16.03 & 16.03 & 17.41 & 14.89 \\
\hline $\mathrm{Kg}$ pasiva / m3 Hor & 485.82 & 123.89 & 176.66 & 178.31 & 78.87 & 178.97 & 157.00 & 155.69 \\
\hline $\mathrm{Kg}$ pasiva / m3 Hor viga & 708.44 & 84.82 & 92.25 & 94.04 & 125.98 & 125.12 & 91.59 & 95.80 \\
\hline $\mathrm{Kg}$ pasiva / m2 tablero & 272.94 & 54.78 & 58.16 & 58.59 & 70.97 & 71.01 & 62.93 & 59.67 \\
\hline
\end{tabular}

Tabla 6-21: Resultados: Cuantías de acero

Las menores cuantías de hormigón y de armadura activa se corresponden al SA y TA. Como se ha indicado en el párrafo anterior, la cuantía de armadura pasiva por $\mathrm{m}^{2} \mathrm{de}$ tablero es muy parecida en el DLS, SA, TA y MA.

De los resultados anteriores se desprende que:

- Las tres heurísticas de búsqueda local (DLS, TA y SA), tienden a cantos máximos con espesores mínimos. De hecho, coinciden todos los valores geométricos para los mejores del TA y del SA, sólo cambia el canto de la viga.

- Se tiende a economizar el volumen de hormigón. Hay una búsqueda de una sección que proporcione mayor inercia y sea a la vez lo más ligera posible. 
Ello se refleja en cantos de viga altos y espesores pequeños, y repercute en una necesidad menor de pretensado, por lo que no hay torones en el tercer nivel.

- En el intento de aligerar la estructura se condiciona a utilizar hormigones de mayor resistencia, entre 40 y $50 \mathrm{Mpa}$, en viga. En la losa, cuanto mayor es el canto, menor resistencia del hormigón se necesita, entre 30 y $40 \mathrm{Mpa}$.

- La medición de acero en la armadura pasiva se asemeja en el DLS, SA, TA y MA.

- Los mejores resultados se obtienen con armadura activa mínima en el ala superior, la suficiente para impedir que fisure dicha ala durante el tesado.

- Los espesores menores de losa en el TA y SA, respecto al DLS, se compensa con mayor armadura pasiva.

- La tendencia en la optimización respecto a las alas superiores es dimensionarlas cortas.

- Las mediciones en el TA y el SA son muy similares en cuanto al volumen de hormigón, acero activo y pasivo. Existe la posibilidad de estar cerca del óptimo global. 


\subsection{Número de ejecuciones necesarias}

La cuestión que se plantea es: ¿cuál es en número mínimo necesario de reinicios de un algoritmo para alcanzar, con una probabilidad razonablemente alta, una solución de gran calidad dentro de la muestra?. Este problema es difícilmente cuantificable, puesto que se desconoce el óptimo global. Para responder a esta pregunta se lleva a cabo un estudio estadístico. En él se considera que si tras varios reinicios se obtiene una muestra lo suficientemente representativa de los mejores óptimos locales, la mejor solución de dicha muestra es ejemplo de la mejor solución que se alcanza con dicho algoritmo, por lo que se podrá tomar como resultado final. En ese caso, si continúan los reinicios a partir de esa muestra, lo más probable es que los valores medios de los resultados que se obtengan prácticamente no variarán, por lo que la probabilidad de encontrar soluciones de mejor calidad se verá reducida.

Este estudio estadístico lo planteó Perea et al.[139], basándose en la evolución de la media y de la desviación estándar de una muestra, a medida que se iban reincorporando nuevos resultados. Cuando ambos valores se estabilizaban podría considerarse que dicha muestra era lo suficientemente representativa de la población, y que la mejor solución alcanzada hasta ese momento era lo suficientemente buena como para estimar que no iba a mejorar significativamente en los siguientes reinicios. En dicho estudio, se concluyó que era suficiente entre veinte y treinta y cinco reinicios.

A continuación, en la Tabla 6-22 se representan los resultados obtenidos con cuarenta reinicios, donde se muestra el coste de cada solución, la media acumulada hasta dicha solución, la variación entre las medias acumuladas distanciadas en cinco reinicios, la desviación estándar de las medias acumuladas y la variación de las desviaciones estándar entre cinco reinicios. Se ha aplicado la heurística que mejor resultado del coste medio ha dado, la S3 del SA. 


\begin{tabular}{|r|r|r|r|r|r|}
\hline Ejecución & Coste & $\begin{array}{c}\text { Media } \\
\text { acumulada }\end{array}$ & $\begin{array}{c}\text { variación } \\
\%\end{array}$ & $\begin{array}{c}\text { Desv. } \\
\text { Estándar }\end{array}$ & $\begin{array}{c}\text { variación } \\
\%\end{array}$ \\
\hline 1 & 109912 & 109912 & & 0 & \\
\hline 2 & 111849 & 110881 & & 969 & \\
\hline 3 & 111362 & 111041 & & 823 & \\
\hline 4 & 111219 & 111086 & & 717 & \\
\hline 5 & 109004 & 110669 & & 1051 & \\
\hline 6 & 109034 & 110397 & -0.44 & 1136 & -100.00 \\
\hline 7 & 112868 & 110750 & 0.12 & 1362 & -28.89 \\
\hline 8 & 109770 & 110627 & 0.37 & 1315 & -37.41 \\
\hline 9 & 109277 & 110477 & 0.55 & 1310 & -45.29 \\
\hline 10 & 111388 & 110568 & 0.09 & 1272 & -17.42 \\
\hline 11 & 111872 & 110687 & -0.26 & 1270 & -10.50 \\
\hline 12 & 110614 & 110681 & 0.06 & 1216 & 12.01 \\
\hline 13 & 111783 & 110766 & -0.12 & 1205 & 9.13 \\
\hline 14 & 110637 & 110756 & -0.25 & 1161 & 12.81 \\
\hline 15 & 113132 & 110915 & -0.31 & 1269 & 0.29 \\
\hline 16 & 109777 & 110844 & -0.14 & 1259 & 0.86 \\
\hline 17 & 111173 & 110863 & -0.16 & 1224 & -0.65 \\
\hline 18 & 111602 & 110904 & -0.12 & 1201 & 0.27 \\
\hline 19 & 110770 & 110897 & -0.13 & 1170 & -0.72 \\
\hline 20 & 112317 & 110968 & -0.05 & 1181 & 7.40 \\
\hline 21 & 109833 & 110914 & -0.06 & 1178 & 6.88 \\
\hline 22 & 111567 & 110944 & -0.07 & 1159 & 5.61 \\
\hline 23 & 112447 & 111009 & -0.09 & 1174 & 2.32 \\
\hline 24 & 112439 & 111069 & -0.15 & 1184 & -1.24 \\
\hline 25 & 109942 & 111024 & -0.05 & 1181 & 0.00 \\
\hline 26 & 109390 & 110961 & -0.04 & 1200 & -1.85 \\
\hline 27 & 108830 & 110882 & 0.06 & 1245 & -6.89 \\
\hline 28 & 110686 & 110875 & 0.12 & 1223 & -3.97 \\
\hline 29 & 111339 & 110891 & 0.16 & 1204 & -1.66 \\
\hline 30 & 109930 & 110859 & 0.15 & 1197 & -1.29 \\
\hline 31 & 110922 & 110861 & 0.09 & 1177 & 1.95 \\
\hline 32 & 109098 & 110806 & 0.07 & 1199 & 3.83 \\
\hline 33 & 109083 & 110754 & 0.11 & 1217 & 0.49 \\
\hline 34 & 111308 & 110770 & 0.11 & 1202 & 0.17 \\
\hline 35 & 110582 & 110764 & 0.09 & 1185 & 0.95 \\
\hline 36 & 110031 & 110744 & 0.11 & 1175 & 0.19 \\
\hline 37 & 110409 & 110735 & 0.06 & 1160 & 3.30 \\
\hline 39 & 110393 & 110726 & 0.02 & 1146 & 6.14 \\
\hline & 113153 & 110788 & -0.02 & 1195 & 0.63 \\
\hline & & 110800 & -0.03 & 1182 & 0.29 \\
\hline
\end{tabular}

Tabla 6-22: Resultados obtenidos con 40 reinicios

En la Figura 6-37 se muestran representados los valores de la Tabla, tanto en lo que respecta a las medias como a las desviaciones estándar de los costes. Se aprecia una significativa variación inicial de la media hasta llegar a siete reinicios, donde se estabiliza. La desviación estándar tarda un poco más en estabilizarse, a partir de nueve. 


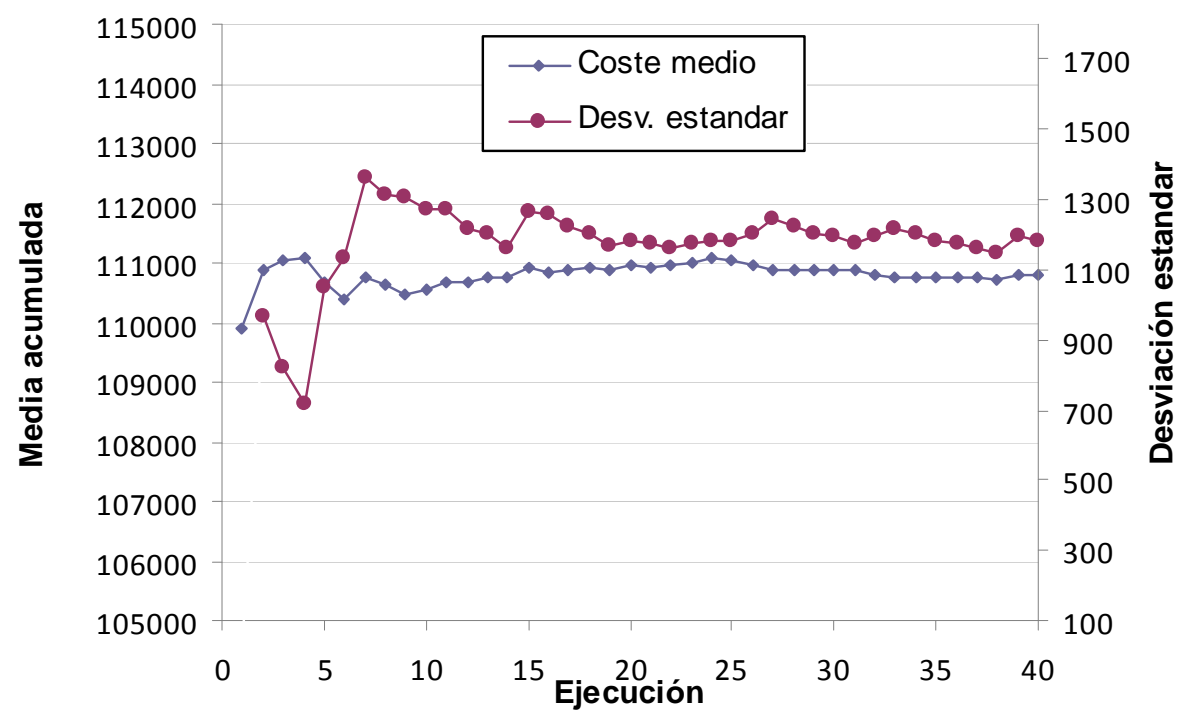

Figura 6-37: Evolución de las medias y desviaciones estándar con el número de ejecuciones

Para determinar el número mínimo de reinicios considerando la variación que se produce en la media acumulada entre cinco reinicios, cuando no difieran sus valores en más del $0.10 \%$, éste se produce a partir del reinicio nueve. Si tenemos en cuenta la misma operación con la desviación estándar cuando la variación de ésta es menor a un $10 \%$, esto se produce en el reinicio trece $(9.13 \%)$, y si seguimos con el análisis, toma un valor muy reducido $(0.29 \%)$ para el reinicio quince, que aumenta en el reinicio veinte $(7.40 \%)$. Por lo tanto, con estos resultados, se podría establecer de forma general como metodología para obtener una muestra significativa de óptimos locales de alta calidad, aplicar entre nueve y veinte reinicios. Con los tiempos de proceso del ordenador empleado en éste estudio, son necesarias 45 horas (menos de 2 días) para nueve reinicios, 75 horas (algo más de 3 días) para quince reinicios y 100 horas (algo más de 4 días) para veinte reinicios.

Si se observan los valores de la Tabla 6-22, el óptimo global del problema se obtiene en el reinicio 27, con un tiempo computacional de 135 horas (5 días y medio), siendo la variación de los valores medios del $0.06 \%$ y de las desviaciones estándar del $6.89 \%$. Esta posición no es significativa, viene determinada por la aleatoriedad de la muestra, encontrándose hasta ese momento el valor mínimo en el reinicio cinco. Este segundo valor se mantiene en ese orden hasta el final de la muestra, compuesta por los cuarenta reinicios. La diferencia entre estos dos costes es muy pequeña ( $0.16 \%)$. Para tomar de decisión del número de reinicios a aplicar en el estudio paramétrico de las luces del tablero, se han contrastado los siguientes valores 9, 15 y 27. El menor de los costes medios se produce para el reinicio 9, tomando un valor escasamente menor a los otros dos $(0.40 \%$ y $0.37 \%)$, siendo los tiempos de proceso para estos últimos un $66.7 \%$ y un $200 \%$ superiores al primero, lo que es determinante para aplicar 9 reinicios en los estudios paramétricos. 


\subsection{Variación de precios unitarios}

En este apartado se analiza la sensibilidad del problema a la variación de los precios unitarios. Nos planteamos cómo se adapta la solución ante un incremento en el precio del mercado del acero, o del hormigón. En cuánto aumenta el coste de la solución óptima, en cuánto se reduce la cantidad del material que incrementa el precio y en cuánto aumenta la que lo mantiene.

Para este estudio se han considerado incrementos del precio del acero, que incluye ambas armaduras activa y pasiva, en un 5, 10, 15 y 20\% manteniendo el precio del hormigón, y la reciprocidad en el incremento del precio del hormigón, tanto armado como pretensado, también en un 5, 10, 15 y 20\%. Se ha aplicado la heurística con menor coste medio, la S3 del SA. En el Apéndice B se muestran los resultados completos obtenidos de la geometría, los valores medios y las distintas gráficas que los representan.

\subsubsection{Costes}

En la siguiente Tabla, 6-23, se muestran los resultados para nueve ejecuciones, de los costes obtenidos con variaciones de precios del acero y del hormigón, desde el 5 hasta el $20 \%$ del incremento en precio. Se obtienen costes medios, coste mínimo y variación del incremento del coste medio.

\begin{tabular}{|c|c|c|c|c|c|r|r|r|r|}
\hline Ejec. & $\begin{array}{c}20 \% \\
\text { acero }\end{array}$ & $\begin{array}{c}15 \% \\
\text { acero }\end{array}$ & $\begin{array}{c}10 \% \\
\text { acero }\end{array}$ & $\begin{array}{c}5 \% \\
\text { acero }\end{array}$ & $\begin{array}{c}5 \% \\
\text { hormigón }\end{array}$ & $\begin{array}{c}10 \% \\
\text { hormigón }\end{array}$ & $\begin{array}{c}15 \% \\
\text { hormigón }\end{array}$ & $\begin{array}{c}20 \% \\
\text { hormigón }\end{array}$ \\
\hline 1 & 124872 & 123487 & 115403 & 112515 & 109912 & 111093 & 113670 & 110966 & 117123 \\
\hline 2 & 123773 & 121820 & 117298 & 113150 & 111849 & 114902 & 110225 & 112915 & 116470 \\
\hline 3 & 123369 & 122117 & 118189 & 115703 & 111362 & 111500 & 115634 & 113209 & 115292 \\
\hline 4 & 124206 & 119172 & 115901 & 112415 & 111219 & 112015 & 113046 & 111226 & 115197 \\
\hline 5 & 121137 & 121570 & 118234 & 114316 & 109004 & 113112 & 110208 & 112491 & 113898 \\
\hline 6 & 122817 & 120044 & 117844 & 114999 & 109034 & 111527 & 110762 & 111885 & 114784 \\
\hline 7 & 122327 & 120796 & 123370 & 113583 & 112868 & 111833 & 112002 & 113557 & 113741 \\
\hline 8 & 123752 & 119843 & 117712 & 114432 & 109770 & 111931 & 113661 & 116441 & 114606 \\
\hline 9 & 125578 & 122239 & 115761 & 114151 & 109277 & 112085 & 114162 & 112867 & 114931 \\
\hline Media & 123537 & 121232 & 117746 & 113918 & 110477 & 112222 & 112597 & 112840 & 115116 \\
\hline Mínimo & 121137 & 119172 & 115403 & 112415 & 109912 & 111093 & 110208 & 110966 & 113741 \\
\hline $\begin{array}{c}\text { Var. } \\
\text { s/ 0\% }\end{array}$ & $11.82 \%$ & $9.73 \%$ & $6.58 \%$ & $3.11 \%$ & $0.00 \%$ & $1.58 \%$ & $1.92 \%$ & $2.14 \%$ & $4.20 \%$ \\
\hline
\end{tabular}

Tabla 6-23: Resultados obtenidos con la variación de precios (€)

En la Figura 6-38 se representan los resultados obtenidos con los costes medios. 


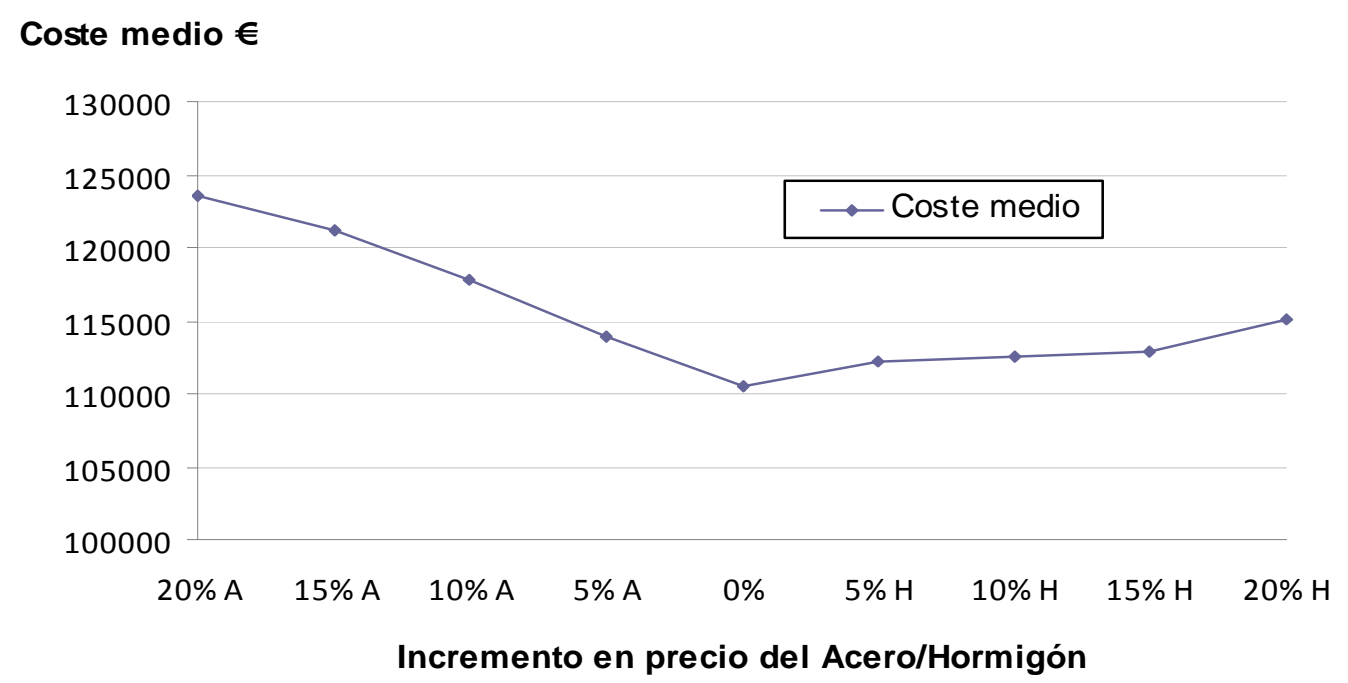

Figura 6-38: Variación costes medios respecto al incremento de precios acero/ hormigón

Se observa que es mucho más acusada la repercusión sobre el coste cuando el incremento en precio se produce en el acero. En el caso extremo, cuando se incrementa el $20 \%$ el precio del acero, el coste se ve aumentado en un $11.82 \%$; mientras que si dicho incremento se ocasiona en el hormigón, el coste del tablero aumenta un $4.20 \%$, equivalente a 2.8 veces menos.

A continuación se analiza la adaptabilidad de la heurística y la del propio problema a los incrementos del precio, haciendo especial observación a la variación que se produce en las cuantías del acero y del hormigón para optimizar los costes.

\subsubsection{Geometría}

Analizando los valores y gráficas recogidos en el Apéndice B se comprueba el comportamiento de la geometría del tablero ante la variación de los precios.

\section{Canto de la viga}

El canto de la viga se mantiene prácticamente constante. Su valor medio es de 1.91 metros, con una esbeltez próxima al 1/18 de la luz.

\section{Espesor de la losa}

El espesor de la losa tiene tendencia escasa a reducirse cuando aumenta el precio del hormigón, y a aumentar cuando lo hace el acero.

\section{Armadura activa}

El número de torones permanece prácticamente constante, con un valor medio de 58 torones. 


\section{Hormigones}

Tanto los hormigones de la viga como de la losa toman valores muy uniformes, siendo las resistencias características medias de 48 Mpa. y de 38 Mpa., respectivamente.

\section{Alas de la viga}

Los valores correspondientes al espesor y a la anchura del ala inferior y de las alas superiores no siguen una correlación clara con la variación de los precios.

Como se ve, prácticamente todos los valores geométricos, incluido el pretensado, permanecen inalterables, salvo una ligera variación en el espesor de la losa que sí se adapta a la variación de los precios. Esta rigidez geométrica vendría determinada por la dificultad en encajar una geometría de la sección con su pretensado, ante las solicitaciones longitudinales en los estados límite y de servicio.

\subsubsection{Cuantías de acero.}

Con respecto a la sensibilidad de la cuantía del acero en función de la variación de precios, se ha tomado como primera aproximación, la suma de la cuantía del acero activo y del pasivo. Se pretende conocer si la cuantía se altera ante los nuevos parámetros del problema. En la Tabla, 6-24, se muestran los resultados obtenidos para nueve ejecuciones con el valor medio.

\begin{tabular}{|c|c|c|c|c|c|r|r|r|r|}
\hline Ejec. & $\begin{array}{c}20 \% \\
\text { acero }\end{array}$ & $\begin{array}{c}15 \% \\
\text { acero }\end{array}$ & $\begin{array}{c}10 \% \\
\text { acero }\end{array}$ & $\begin{array}{c}5 \% \\
\text { acero }\end{array}$ & \multicolumn{1}{c|}{$0 \%$} & $\begin{array}{c}5 \% \\
\text { hormigón }\end{array}$ & $\begin{array}{c}10 \% \\
\text { hormigón }\end{array}$ & $\begin{array}{c}15 \% \\
\text { hormigón }\end{array}$ & $\begin{array}{c}20 \% \\
\text { hormigón }\end{array}$ \\
\hline 1 & 79.65 & 75.56 & 72.04 & 74.27 & 76.01 & 74.02 & 77.02 & 75.24 & 74.70 \\
\hline 2 & 74.69 & 67.42 & 67.03 & 75.42 & 63.49 & 74.76 & 78.94 & 67.13 & 77.33 \\
\hline 3 & 63.48 & 73.89 & 77.69 & 73.52 & 70.28 & 77.52 & 78.27 & 64.25 & 72.90 \\
\hline 4 & 67.98 & 73.59 & 75.19 & 77.78 & 72.54 & 74.22 & 72.86 & 75.90 & 77.64 \\
\hline 5 & 62.77 & 67.56 & 65.00 & 67.57 & 73.08 & 75.44 & 71.81 & 78.24 & 74.04 \\
\hline 6 & 76.75 & 63.48 & 63.41 & 62.65 & 76.86 & 66.48 & 69.98 & 72.95 & 68.95 \\
\hline 7 & 62.70 & 78.33 & 77.55 & 76.15 & 79.02 & 73.98 & 70.46 & 75.47 & 67.35 \\
\hline 8 & 74.30 & 66.88 & 73.59 & 63.98 & 66.56 & 69.90 & 71.92 & 73.74 & 70.27 \\
\hline 9 & 77.16 & 67.46 & 74.87 & 75.04 & 75.27 & 63.51 & 69.56 & 72.13 & 72.83 \\
\hline Media & 71.05 & 70.46 & 71.82 & 71.82 & 72.57 & 72.20 & 73.42 & 72.78 & 72.89 \\
\hline
\end{tabular}

Tabla 6-24: Cuantías del acero en $\mathrm{Kg} / \mathrm{m}^{2}$ tablero, obtenidas con la variación de precios

En la Figura 6-39 se representa el resumen de dicha Tabla. Se han combinado dos gráficas para apreciar mejor la variación del comportamiento según se incremente el precio del acero o del hormigón. A cada una de ellas se les ha añadido la línea de tendencia, en la que se observa como la heurística en su objetivo de optimizar el coste adapta, con cierta lógica, la cuantía del acero a la variación del precio del acero y del hormigón, reduciéndola cuando aumenta el precio del acero, y aumentándola cuando se incrementa el precio del hormigón. Si se presta atención, y se comparan las pendientes de las ecuaciones de las rectas, el efecto es más acusado con la variación del precio del acero. 


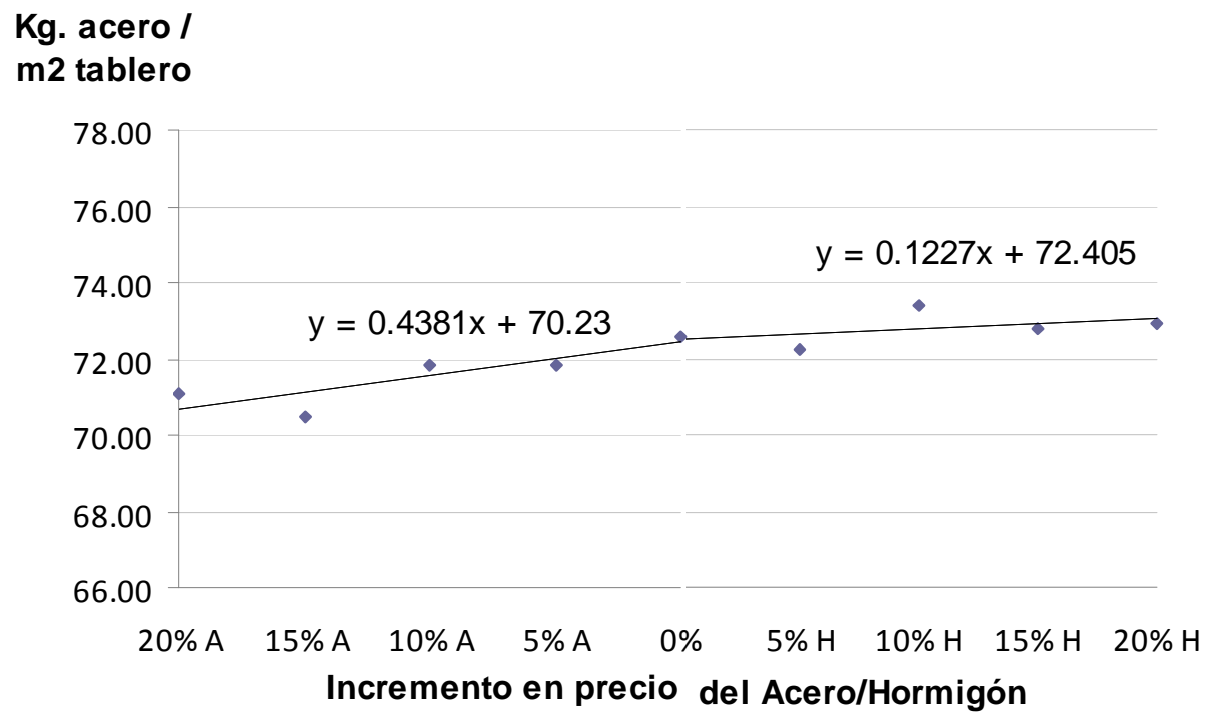

Figura 6-39: Cuantías acero en $\mathrm{Kg} / \mathrm{m}^{2}$ tablero sobre incremento precios acero/ hormigón

\subsubsection{Cuantías de hormigón.}

La respuesta de la heurística respecto a la sensibilidad mostrada por la cuantía del hormigón en función de la variación de precios del acero y del hormigón sigue el mismo comportamiento que el expresado en el punto anterior. En la Tabla, 6-25, se muestran los resultados obtenidos para nueve ejecuciones con el valor medio.

\begin{tabular}{|c|c|c|c|c|c|r|r|r|r|}
\hline Ejec. & $\begin{array}{c}20 \% \\
\text { acero }\end{array}$ & $\begin{array}{c}15 \% \\
\text { acero }\end{array}$ & $\begin{array}{c}10 \% \\
\text { acero }\end{array}$ & $\begin{array}{c}5 \% \\
\text { acero }\end{array}$ & $\begin{array}{c}0 \% \\
\text { hormigón }\end{array}$ & $\begin{array}{c}10 \% \\
\text { hormigón }\end{array}$ & $\begin{array}{c}15 \% \\
\text { hormigón }\end{array}$ & $\begin{array}{c}20 \% \\
\text { hormigón }\end{array}$ \\
\hline 1 & 0.339 & 0.336 & 0.333 & 0.341 & 0.329 & 0.336 & 0.330 & 0.320 & 0.346 \\
\hline 2 & 0.340 & 0.374 & 0.381 & 0.328 & 0.404 & 0.331 & 0.341 & 0.413 & 0.328 \\
\hline 3 & 0.416 & 0.336 & 0.363 & 0.341 & 0.366 & 0.339 & 0.318 & 0.410 & 0.346 \\
\hline 4 & 0.365 & 0.343 & 0.330 & 0.326 & 0.329 & 0.340 & 0.329 & 0.332 & 0.328 \\
\hline 5 & 0.413 & 0.396 & 0.416 & 0.380 & 0.329 & 0.335 & 0.329 & 0.341 & 0.330 \\
\hline 6 & 0.341 & 0.416 & 0.413 & 0.413 & 0.335 & 0.382 & 0.366 & 0.323 & 0.379 \\
\hline 7 & 0.430 & 0.327 & 0.354 & 0.341 & 0.329 & 0.341 & 0.380 & 0.331 & 0.401 \\
\hline 8 & 0.331 & 0.400 & 0.334 & 0.414 & 0.408 & 0.392 & 0.329 & 0.330 & 0.366 \\
\hline 9 & 0.321 & 0.407 & 0.322 & 0.333 & 0.340 & 0.412 & 0.374 & 0.322 & 0.371 \\
\hline Media & 0.366 & 0.371 & 0.361 & 0.357 & 0.352 & 0.356 & 0.344 & 0.347 & 0.355 \\
\hline
\end{tabular}

Tabla 6-25: Cuantías del hormigón en $\mathrm{m}^{3} / \mathrm{m}^{2}$ tablero, obtenidas con la variación de precios

En la Figura 6-40 se representa el resumen de dicha Tabla. Se ha actuado igual que en el caso de la cuantía del acero al combinar dos gráficas y añadir las líneas de tendencia. En este caso, se observa también como la heurística adapta la cuantía del hormigón a la variación del precio del acero y del hormigón, aumentándola cuando aumenta el precio del acero, y reduciéndola cuando se incrementa el precio del hormigón. Igualmente, el efecto es más acusado con la variación del precio del acero. 


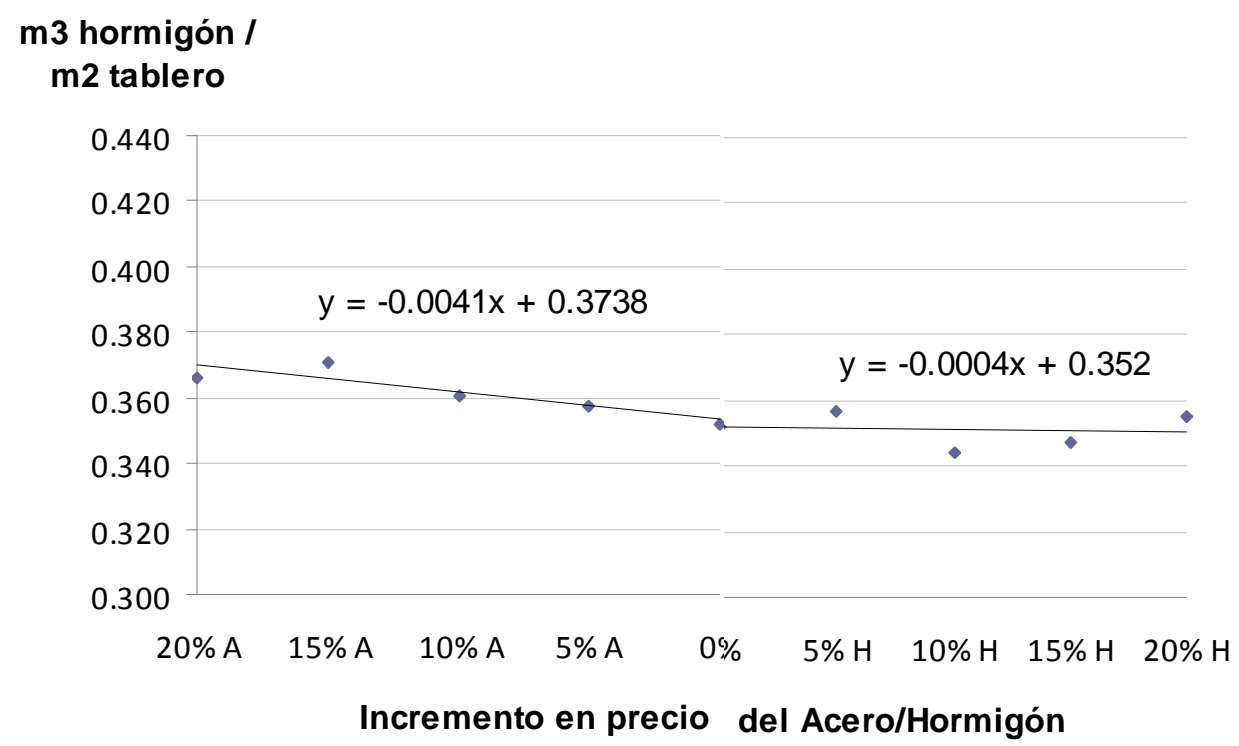

Figura 6-40: Cuantías hormigón en $\mathrm{m}^{3} / \mathrm{m}^{2}$ tablero sobre incremento precios acero/ hormigón 


\subsection{Conclusiones}

A continuación, se resumen las principales conclusiones de este capítulo en los siguientes puntos:

- Se han aplicado seis heurísticas -RW, DLS, SA, TA, GA y MA-, para el diseño automatizado y optimizado de tableros para puentes con vigas artesa pretensadas, siendo las más eficaces el SA y el TA. Los tiempos de proceso para estas dos últimas son suficientemente aceptables como para poder utilizarse de un modo práctico en casos reales.

- La heurística que mejores resultados ha obtenido se corresponde con el algoritmo SA, con cadenas de Markov de 2500 iteraciones y coeficiente de enfriamiento de 0.95 -S3-. Su tiempo de proceso es de unas cinco horas con el procesador utilizado para este trabajo. Como resultado del estudio, se recomienda ejecutar dicho algoritmo 9 veces para obtener una muestra de estructuras optimizadas de alta calidad.

- El modelo se adapta a la variación de precios para optimizar el coste de la estructura. Al incrementarse el precio del acero, disminuye su cuantía y aumenta la del hormigón, y al aumentar el precio del hormigón, ocurre lo contrario. Es coste es más sensible a la variación del precio del acero que del hormigón, del orden de tres veces. Sin embargo, prácticamente no se ve afectada la geometría de la sección, incluido el pretensado, salvo una ligera adaptación del canto de la losa. Ello muestra la rigidez de la sección y del pretensado ante las variaciones de los precios. Consecuentemente, la esbeltez de la viga tampoco varía, manteniéndose en el límite del 1/18 de la luz. 


\section{CAPÍTULO 7. ESTUDIO PARAMÉTRICO DE TABLEROS}

\subsection{Introducción}

El presente capítulo repasa el comportamiento del algoritmo diseñado para este trabajo, empleándolo sobre una variedad de luces de tableros y analizando los resultados obtenidos, para poder aplicar las conclusiones al diseño de tableros proyectados con vigas pretesas. El único de los parámetros que varía es la luz, manteniendo constante el ancho del tablero -12 metros- y la separación entre ejes de vigas -6 metros-. Para ello, se utiliza una parametrización de la estructura con las siguientes luces: $20,25,30,35$ y 40 metros. Se analiza su influencia sobre el coste total, la geometría, los tipos de hormigón y las cuantías de los aceros y del hormigón.

Se mantiene el límite de la relación canto/luz = 1/18. Además de la problemática que genera el transporte de vigas de gran canto, el límite viene condicionado por razones estéticas. En otros estudios (Martí et al. [104]) comprobó que la optimización se acercaba al límite superior impuesto en el canto, que llegaba a relaciones del orden de $1 / 13.5$.

\subsection{Coste de los tableros}

El cálculo de las estructuras, tal como se ha indicado en el apartado anterior, se ha realizado con la heurística que mejores resultados ha obtenido en este trabajo, la S3. Cada heurística se procesa 9 veces, tal como se determina en el apartado 6.8. En la siguiente Tabla se presentan los resultados de los costes medios, el mínimo y la desviación.

\begin{tabular}{|c|r|r|r|}
\hline \multirow{2}{*}{ LUZ } & \multicolumn{3}{|c|}{ HP } \\
\cline { 2 - 4 } & Xm $(€)$ & \multicolumn{1}{|c|}{ Xmin $(€)$} & \multicolumn{1}{|c|}{ Desv \% } \\
\hline 20 & 62,262 & 61,676 & 1.0 \\
\hline 25 & 75,045 & 74,326 & 1.0 \\
\hline 30 & 92,601 & 91,412 & 1.3 \\
\hline 35 & 110,477 & 109,004 & 1.4 \\
\hline 40 & 128,755 & 126,035 & 2.2 \\
\hline
\end{tabular}

Tabla 7-1: Costes obtenidos para distintas luces.

Se aprecia en primer lugar, que las desviaciones de los valores medios respecto al mínimo son muy pequeñas, en la mayoría de las luces alrededor del 1\%. En la Figura 7-1 se representan los valores medio y los mínimos, apreciándose la cercanía 
existente de ambos valores para cada luz de vano. En el Apéndice $\mathrm{C}$ se muestran los planos de los mejores resultados obtenidos para cada caso. En la Figura 7-2 aparecen los valores medio, y se aprecia una correlación claramente cuadrática, aunque sensiblemente lineal -el coeficiente de determinación $\mathrm{R}^{2}$ es ligeramente superior en el primer caso-. Fácilmente se percibe en la ecuación que el valor de A (807.86) que afecta a " $\mathrm{x}$ ", es muy inferior a B (11995.00) que afecta a " $x$ ", y además, examinando la Figura, se ve que para un incremento de luz de 20 a 40 metros (200\%), el coste medio aumenta prácticamente lo mismo, un $206 \%$.

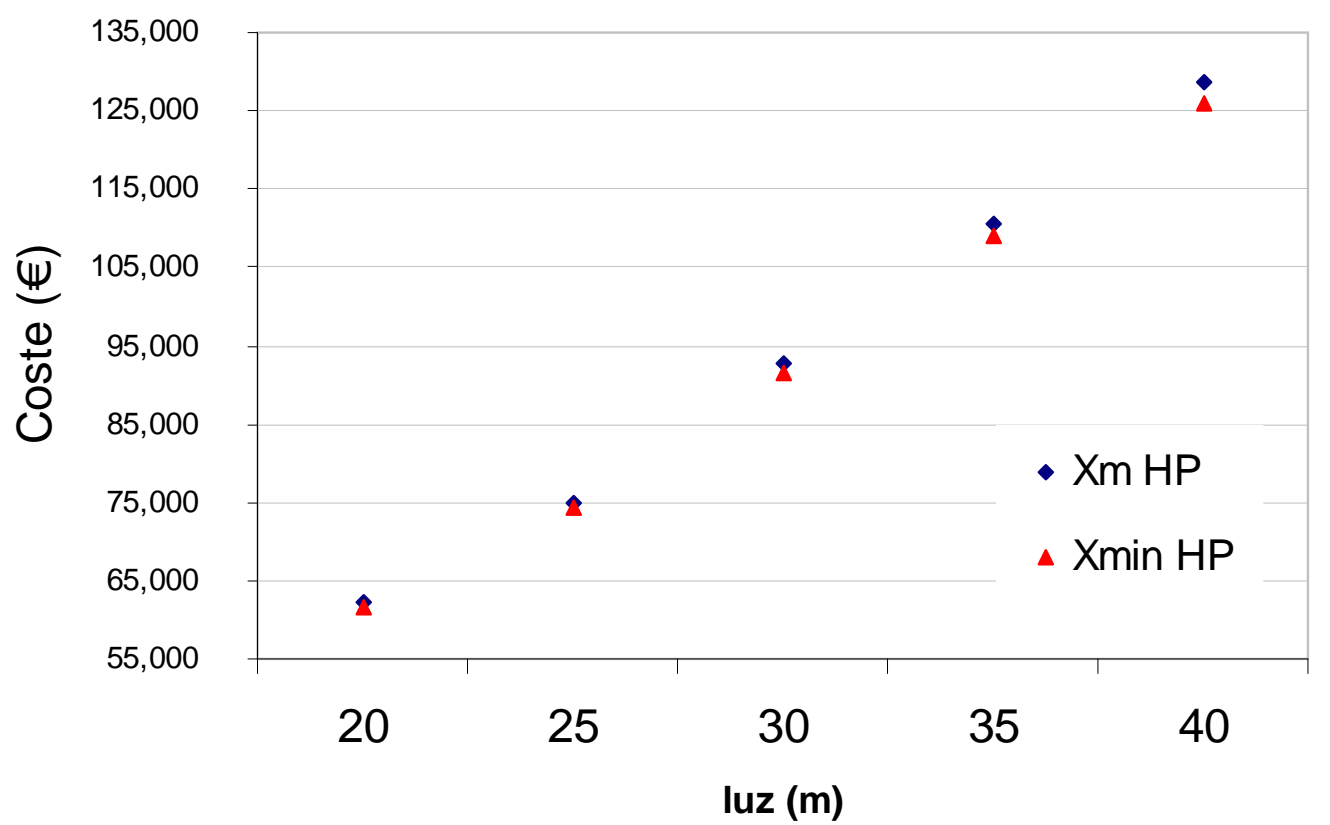

Figura 7-1: Coste medio y mínimo de los tableros en función de la luz.

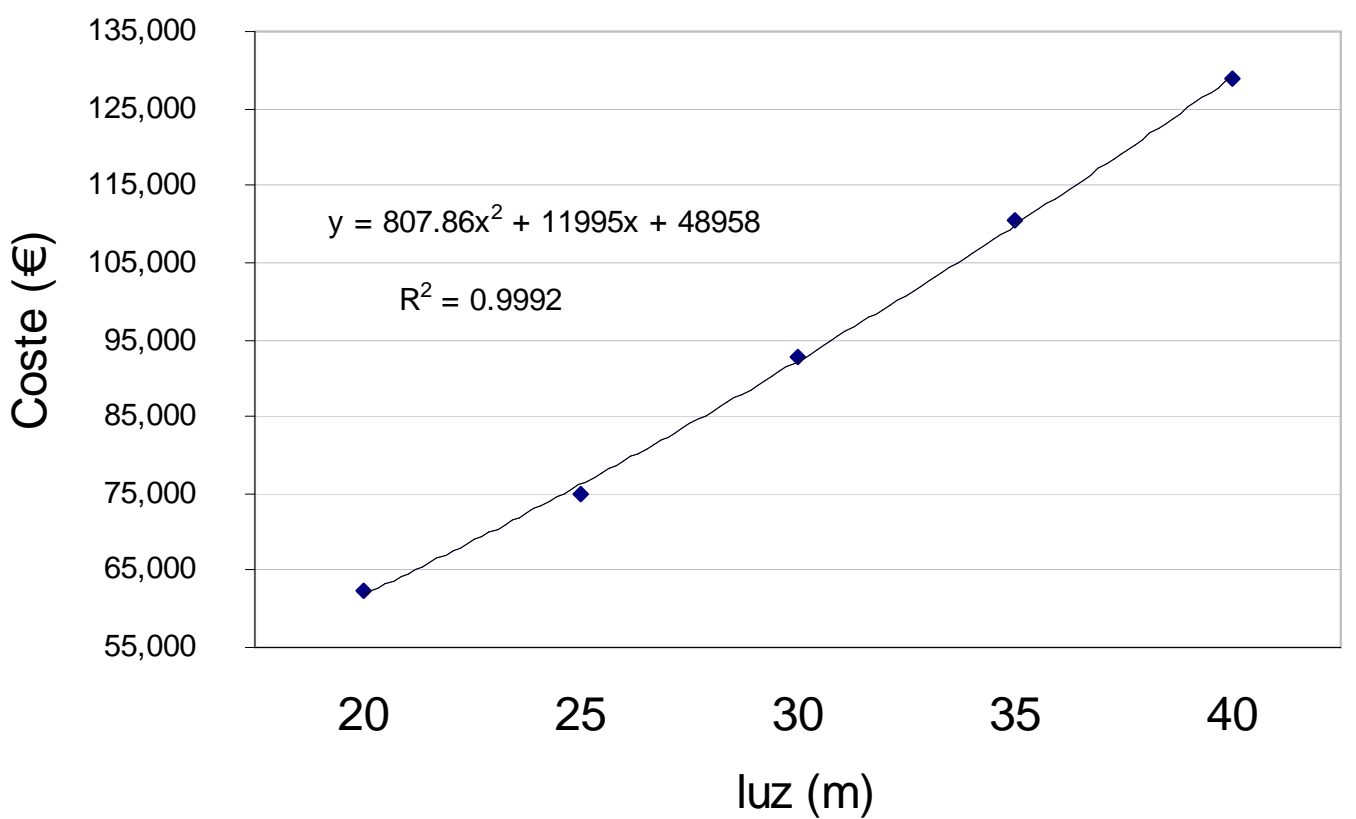

Figura 7-2: Función del coste medio de los tableros en función de la luz. 


\subsection{Resultados geométricos}

En las siguientes Tablas se presentan los resultados geométricos obtenidos.

\begin{tabular}{|c|c|c|c|c|c|c|c|c|c|}
\hline & Presupuesto & h1 & $\mathrm{e} 4$ & b1 & b3 & $\mathrm{e} 1$ & $\mathrm{e} 2$ & e3 Hv H1 p1 p2 p3 p4 T & \\
\hline 9 & 129089.156 & 2.20 & 0.19 & 1.91 & 0.29 & 0.18 & 0.10 & $0.2345403528 \quad 0 \quad 2 / 0.6$ & $\mathrm{Luz}=40 \mathrm{~m}$ \\
\hline 8 & 126434.117 & 2.22 & 0.19 & 1.99 & 0.28 & 0.18 & 0.10 & $0.2245403627 \quad 0 \quad 2 / 0.6$ & \\
\hline 7 & 128347.703 & 2.20 & 0.19 & 2.00 & 0.27 & 0.18 & 0.10 & $0.2145403726 \quad 0 \quad 2 / 0.6$ & \\
\hline 6 & 138398.984 & 2.20 & 0.19 & 2.00 & 0.28 & 0.18 & 0.10 & $0.2245403728 \quad 0 \quad 2 / 0.6$ & \\
\hline 5 & 126035.688 & 2.22 & 0.19 & 1.99 & 0.28 & 0.21 & 0.10 & $0.2240403628 \quad 0 \quad 2 / 0.6$ & \\
\hline 4 & 127418.625 & 2.20 & 0.19 & 2.00 & 0.28 & 0.18 & 0.10 & $0.2245403726 \quad 0 \quad 2 / 0.6$ & \\
\hline 3 & 127202.141 & 2.19 & 0.19 & 1.98 & 0.28 & 0.17 & 0.10 & $0.2245403627 \quad 0 \quad 2 / 0.6$ & \\
\hline 2 & 128571.086 & 2.22 & 0.19 & 1.99 & 0.25 & 0.20 & 0.10 & $0.1845403627 \quad 0 \quad 2 / 0.6$ & \\
\hline 1 & 127307.719 & 2.20 & 0.19 & 2.00 & 0.27 & 0.18 & 0.10 & $0.2145403726 \quad 0 \quad 2 / 0.6$ & \\
\hline
\end{tabular}

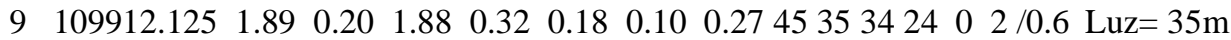
$\begin{array}{lllllllllllllll}8 & 111849.727 & 1.94 & 0.28 & 1.97 & 0.27 & 0.16 & 0.10 & 0.20 & 50 & 35 & 36 & 22 & 0 & 2 / 0.6\end{array}$

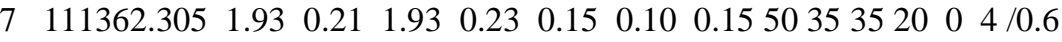

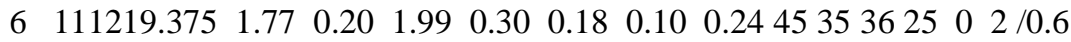
$\begin{array}{lllllllllllll}5 & 109004.859 & 1.94 & 0.19 & 1.99 & 0.29 & 0.18 & 0.10 & 0.2340 & 4036 & 20 & 0 & 2 / 0.6\end{array}$

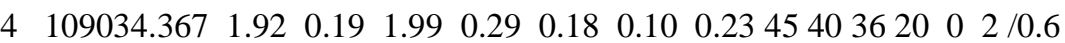

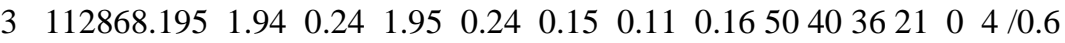

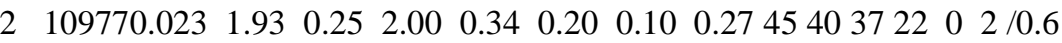
$\begin{array}{llllllllllllll}1 & 109277.883 & 1.94 & 0.21 & 1.95 & 0.23 & 0.15 & 0.10 & 0.15 & 5035 & 36 & 20 & 0 & 4\end{array}$

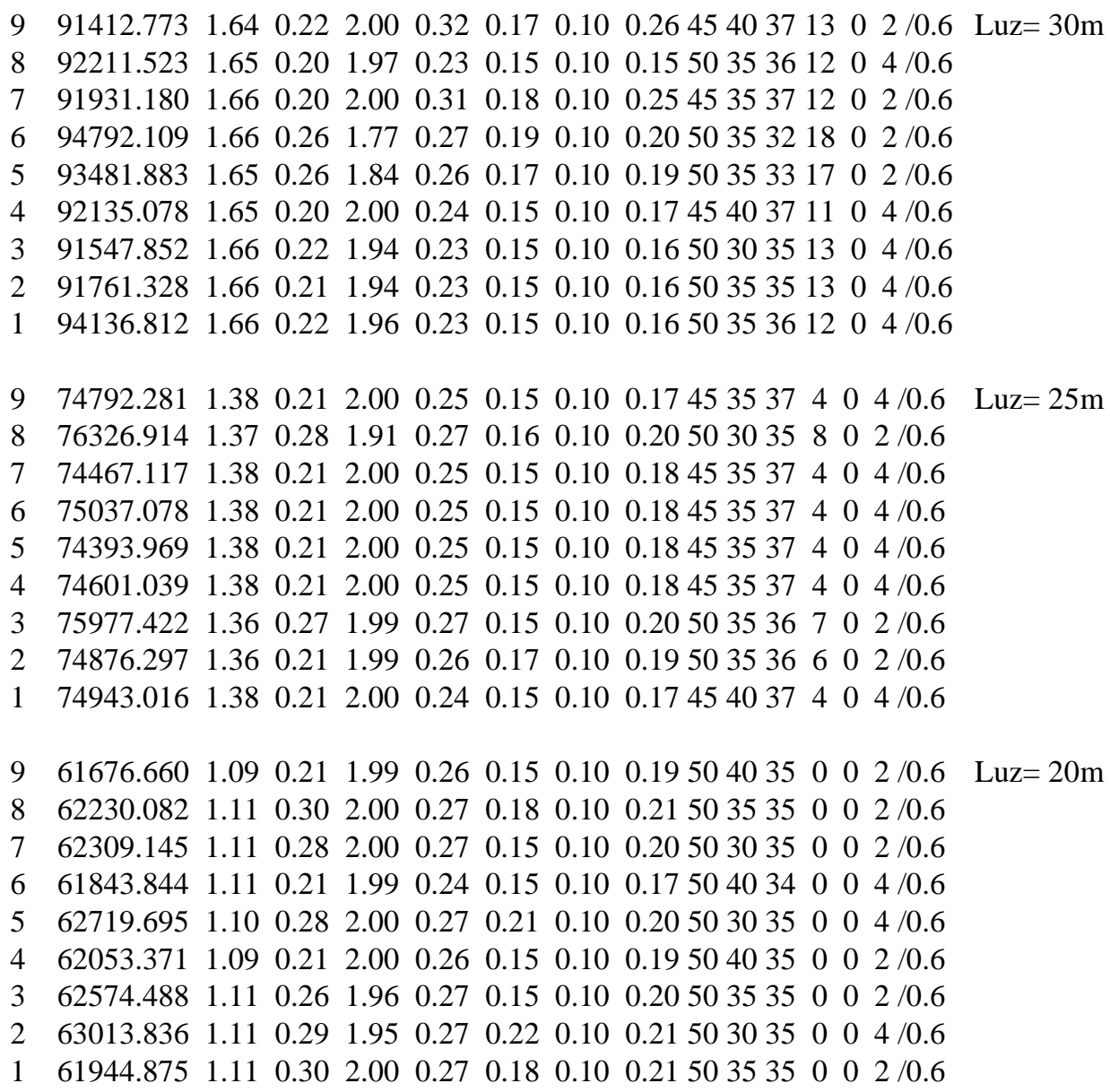

Tabla 7-2: Resultados geométricos hormigón sin fibras HP 
Siendo las siguientes unidades:
Coste h1
e4
b1 b3
$\begin{array}{lll}\text { e1 } & \text { e2 } & \text { e3 }\end{array}$
$\mathrm{Hv} \quad \mathrm{Hl}$
p1 p2 p3p4
(€)
(m.) (m.)
(m.)
(m.) (m.) (m.) (m.)
(HP) (HA)
(n) (n) (n) (n)

Y siendo su significado:

h1 canto de la viga

e4 canto de la losa

b1 ancho ala inferior de la viga

b3 base ala superior de la viga

e1 canto ala inferior de la viga

e2 espesor del alma

e3 canto ala superior de la viga

Hv Hormigón de la viga: HP/HPF

Hl Hormigón de la losa: HA

p1 número de torones nivel 1 ala inferior de $\Phi 0.6$ "

p2 número de torones nivel 2 ala inferior de $\Phi 0.6$ "

p3 número de torones nivel 3 ala inferior de Ф0.6"

p4 número de torones nivel 4 ala superior de $\Phi 0.6$ "

fR3k resistencia residual a tracción

A continuación se valoran distintas variables que conforman la geometría de la sección del tablero:

\section{Canto de la viga, espesor de la losa y pretensado}

Como se ha dicho anteriormente, para la resolución del problema se ha limitado la esbeltez de la viga a 1/18 de su luz, por lo que los valores máximos posibles son los siguientes:

\begin{tabular}{|l|c|c|c|c|c|}
\cline { 2 - 6 } \multicolumn{1}{c|}{} & \multicolumn{5}{c|}{ Luz $(\mathrm{m})}$. \\
\cline { 2 - 6 } \multicolumn{1}{c|}{} & 20 & 25 & 30 & 35 & 40 \\
\hline Canto máx. $(\mathrm{m})$ & 1.111 & 1.389 & 1.667 & 1.944 & 2.222 \\
\hline
\end{tabular}

Tabla 7-3: Limitación cantos máximos de la viga en función de la luz

A continuación, en el siguiente tabla se presentan los resultados medios obtenidos con la heurística:

\begin{tabular}{|c|r|r|r|}
\hline \multirow{2}{*}{ LUZ } & \multicolumn{3}{|c|}{ HP } \\
\cline { 2 - 4 } & $\begin{array}{c}\text { Canto Viga } \\
\mathrm{h}(\mathrm{m})\end{array}$ & $\begin{array}{c}\text { Espesor losa } \\
\text { e4(m) }\end{array}$ & No torones \\
\hline 20 & 1.104 & 0.260 & 34.89 \\
\hline 25 & 1.374 & 0.224 & 41.56 \\
\hline 30 & 1.654 & 0.221 & 48.78 \\
\hline 35 & 1.911 & 0.219 & 57.33 \\
\hline 40 & 2.206 & 0.190 & 63.33 \\
\hline
\end{tabular}

Tabla 7-4: Resultados geométricos: Canto viga, losa $\mathbf{y} \mathbf{n}^{\circ}$ torones 
En las siguientes Figuras 7-3, 7-4 y 7-5 se representan los resultados en función de la luz:

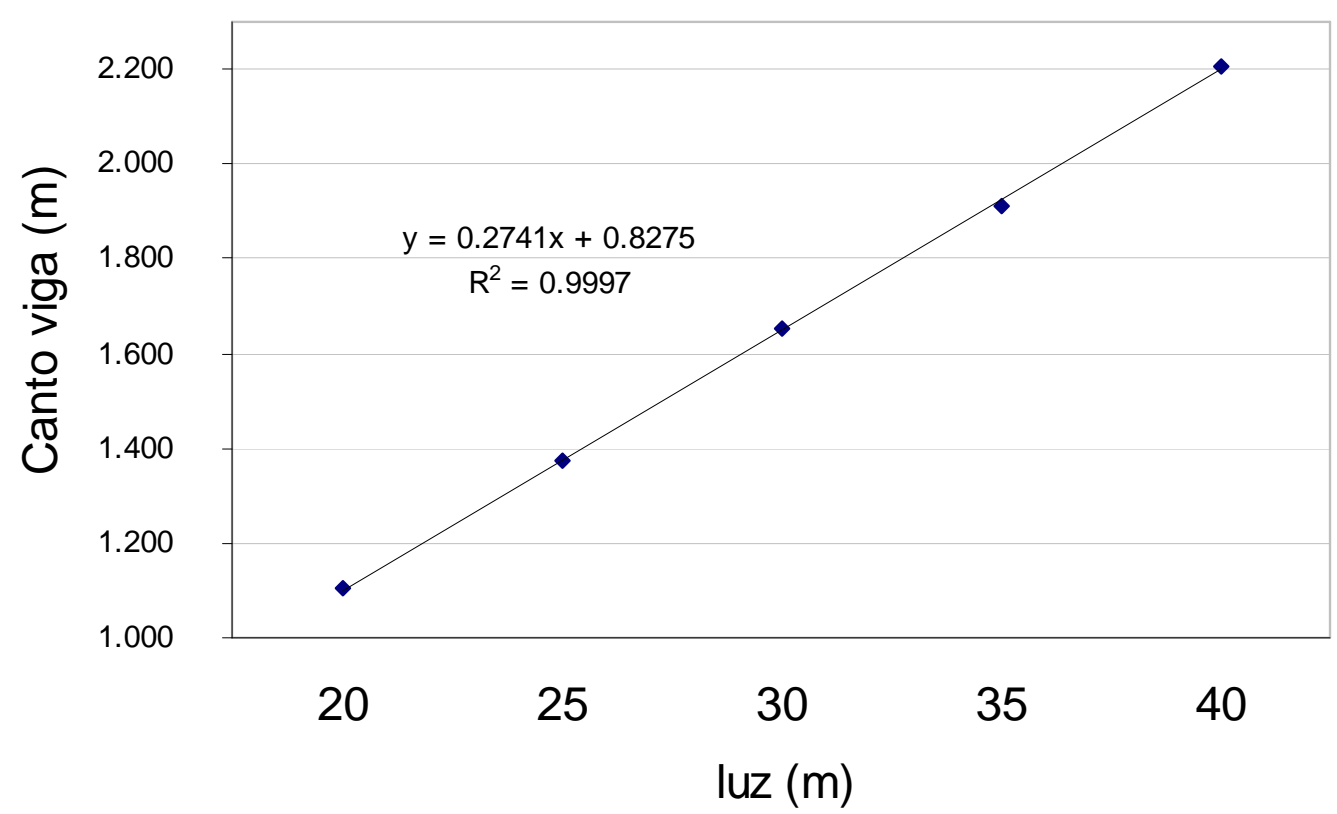

Figura 7-3: Canto de la viga en función de la luz.

Se observa que la variación del canto de la viga con respecto a la luz sigue una correlación claramente lineal, tomando $\mathrm{R}^{2}$ un valor muy próximo a la unidad.

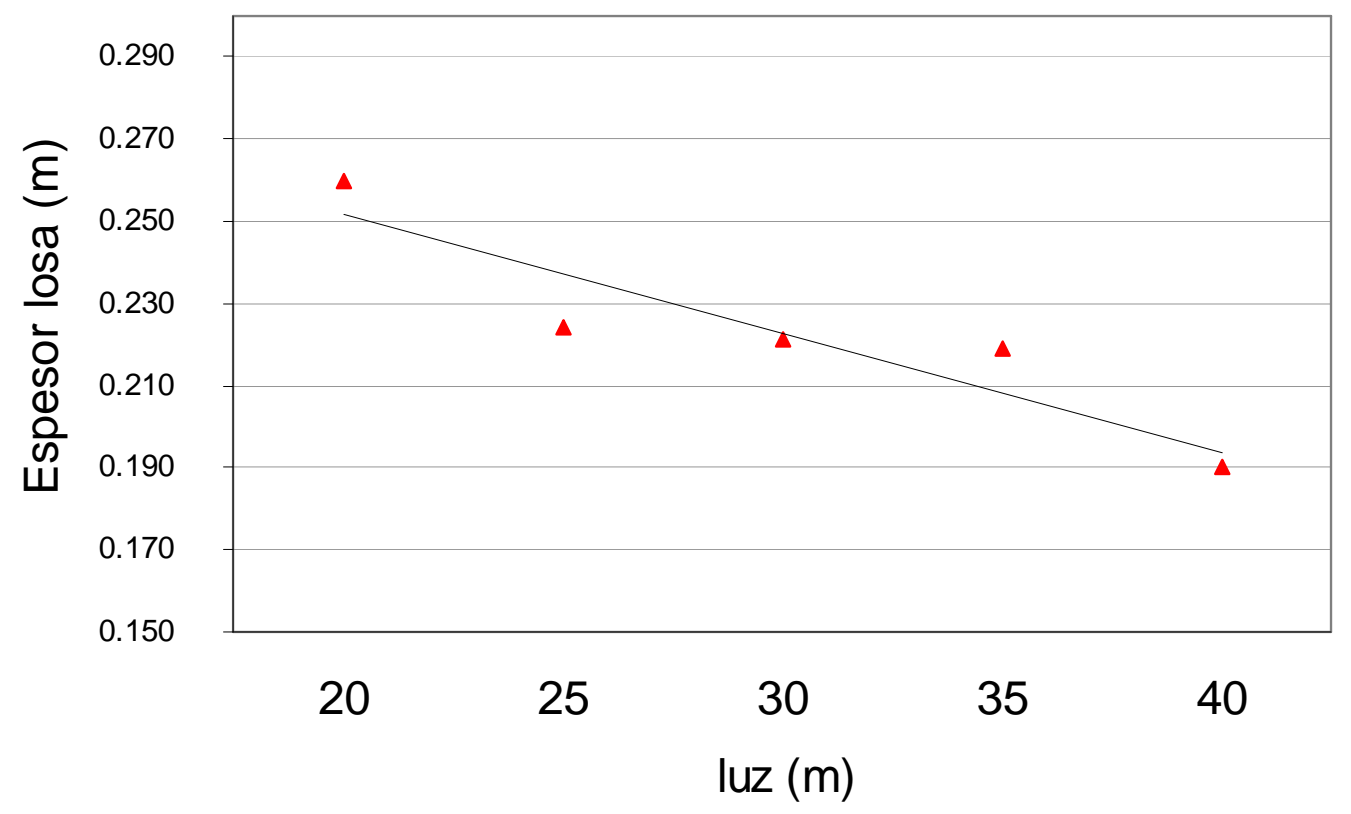

Figura 7-4: Espesor de la losa en función de la luz.

Por otro lado, se observa cierta tendencia a reducir el espesor de la losa al aumentar la luz. 


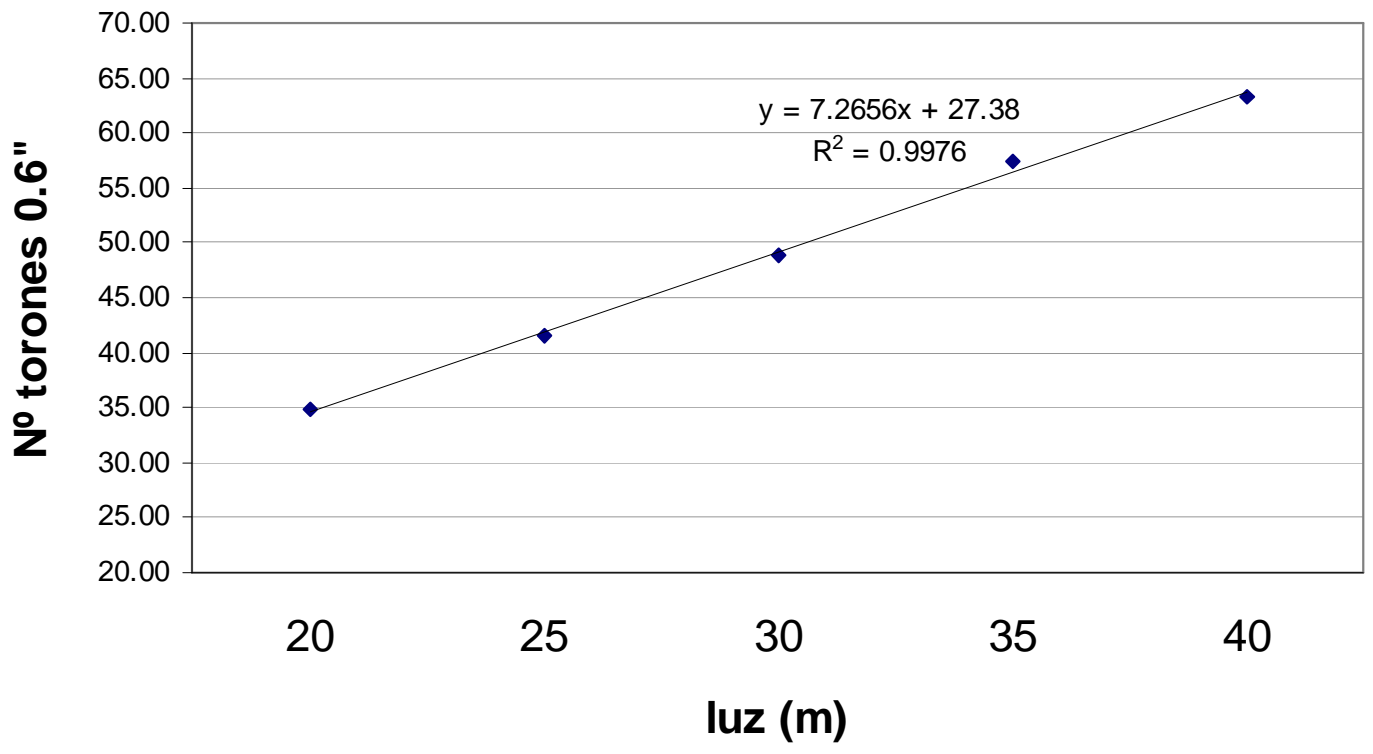

Figura 7-5: Número de torones medio en función de la luz.

Aquí se aprecia un incremento lineal de la fuerza de pretensado conforme aumenta la luz, tomando $\mathrm{R}^{2}$ un valor alto.

El producto del canto (brazo mecánico) por el número de torones (fuerza de pretensado), que se convierten en una función cuadrática -ver Figura 7-6-, permite compensar las solicitaciones longitudinales del tablero, también de carácter cuadrático en función de la luz. Consecuentemente, el incremento lineal del canto y del pretensado podría explicar el también incremento cuasilineal del coste respecto la luz -Figura 7-2-.

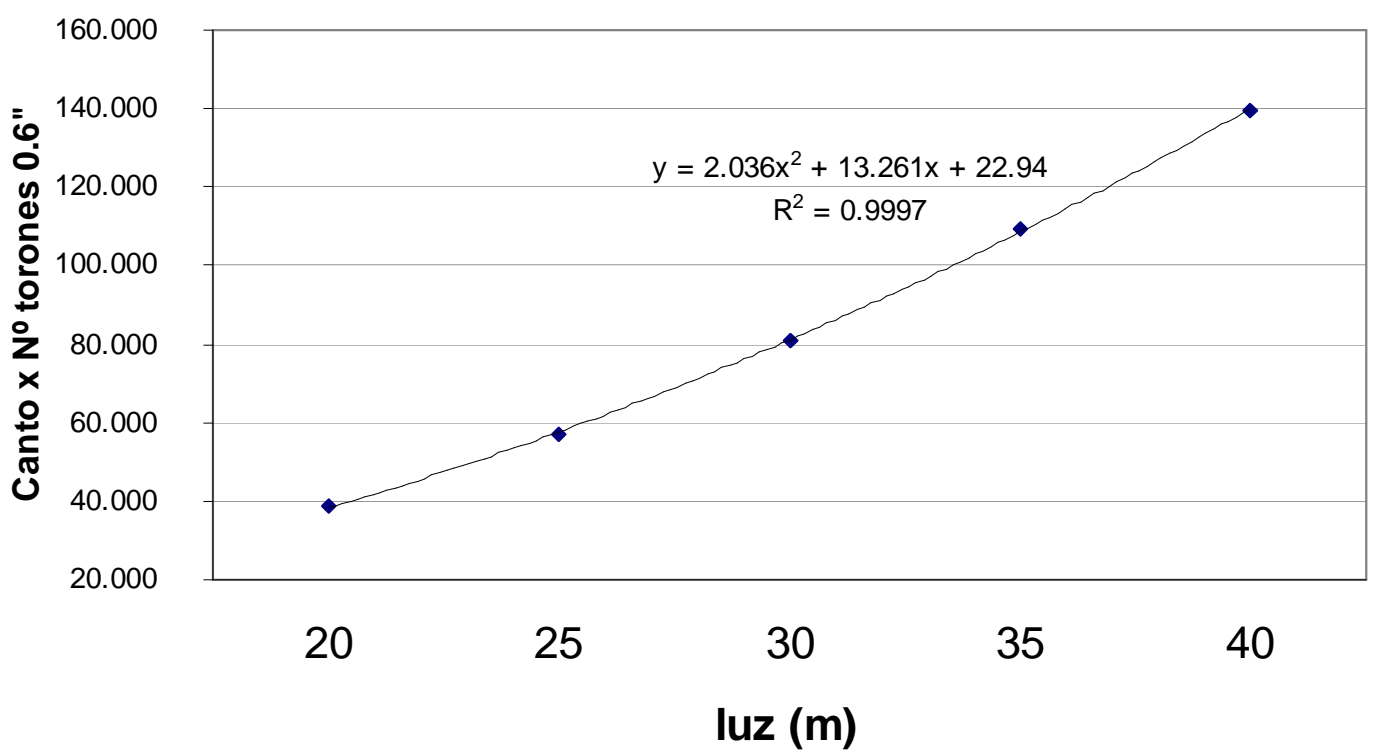

Figura 7-6: Producto del canto de la viga $\mathrm{x}$ el número de torones en función de la luz. 


\section{Tipos de hormigón}

En el siguiente Tabla se presentan los valores medios del resultado de los hormigones:

\begin{tabular}{|c|c|c|c|}
\hline \multirow{2}{*}{ LUZ } & \multicolumn{3}{|c|}{ HP } \\
\cline { 2 - 4 } & Hviga & Hlosa & - \\
\hline 20 & 50.00 & 35.00 & \\
\hline 25 & 46.67 & 35.00 & \\
\hline 30 & 48.33 & 35.56 & \\
\hline 35 & 46.67 & 37.22 & \\
\hline 40 & 44.44 & 40.00 & \\
\hline
\end{tabular}

Tabla 7-5: Resultados hormigones: Viga y losa

En las siguientes Figuras 7-6 y 7-7 se representan dichos resultados en función de la luz:

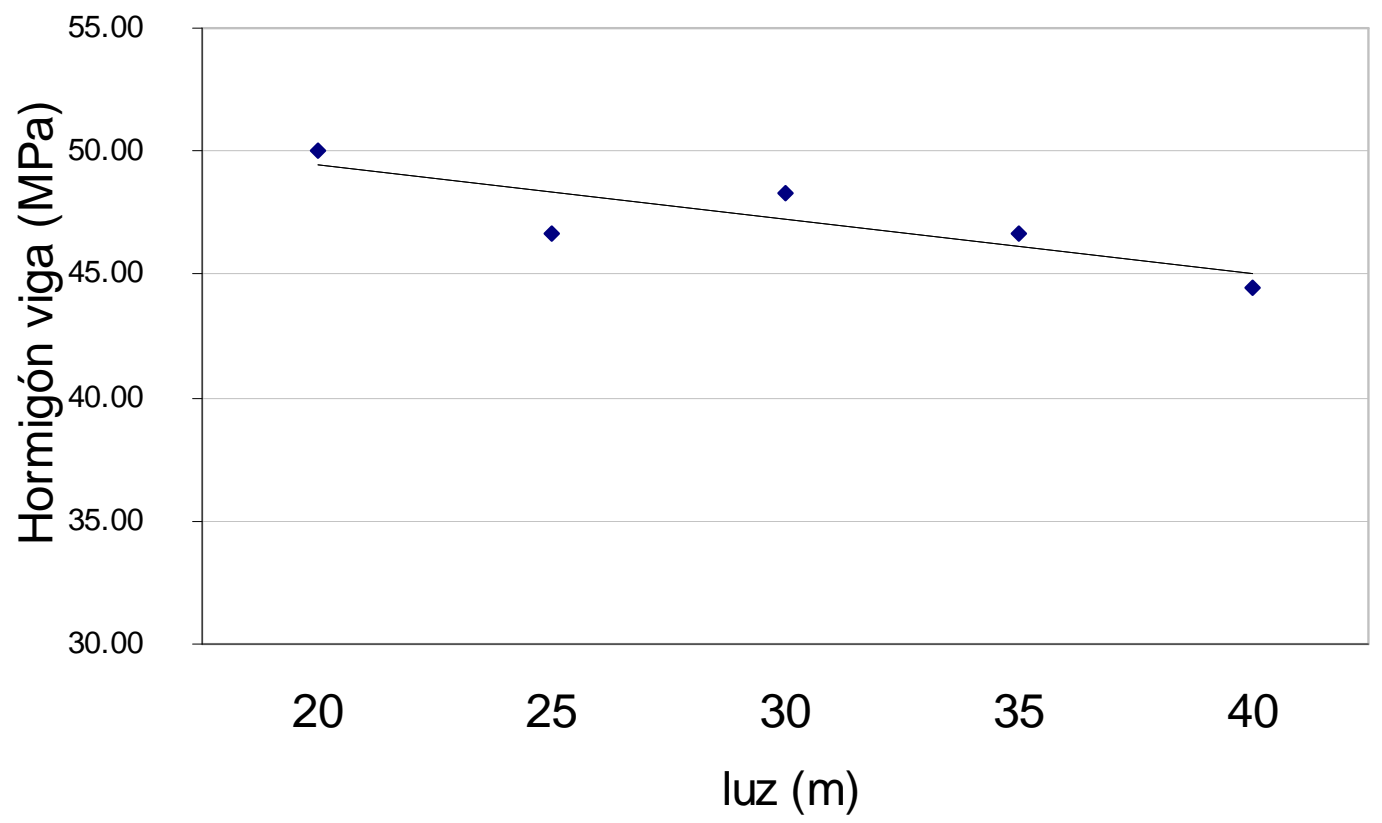

Figura 7-7: Hormigones medios en viga en función de la luz.

Los tipos de hormigón que se aplican son de resistencias elevadas (la más alta considerada como variable del problema es de 50MPa), aunque hay una leve tendencia hacia una reducción de resistencia conforme aumenta la luz. 


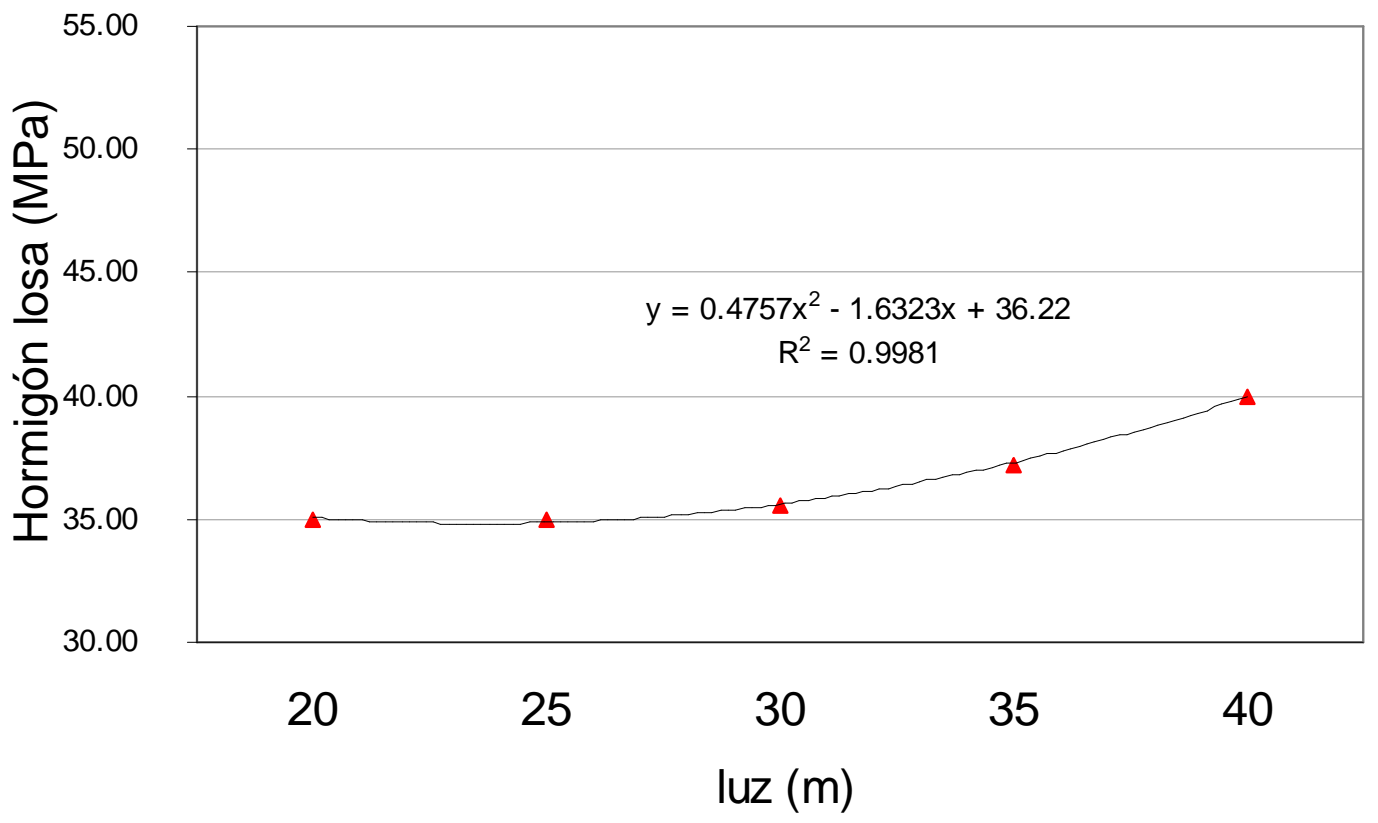

Figura 7-8: Hormigones medios en losa en función de la luz.

Como en el caso anterior, la tendencia es a utilizar hormigones de resistencias elevadas (la más elevada en losa es de 40MPa.), pero ahora, la representación se puede realizar mediante una función cuadrática con una correlación suficientemente alta.

\section{Espesor del alma de la viga}

En todos los casos toma el valor mínimo de dicha variable $-10 \mathrm{~cm}$.-

\section{Ancho y espesor del ala superior de la viga}

Los valores medios del ancho se encuentran entre 25 y $28 \mathrm{~cm}$, siendo la media considerando todas las luces, de $27 \mathrm{~cm}$. No existe una clara correlación de este valor con la luz del tablero.

Lo mismo ocurre con el espesor, donde los valores van de 18 a $21 \mathrm{~cm}$, siendo la media de $20 \mathrm{~cm}$., no existiendo correlación alguna.

\section{Geometría del ala inferior}

En el siguiente Tabla se presentan los valores medios de la geometría del ala inferior:

\begin{tabular}{|c|r|r|r|}
\hline \multirow{2}{*}{ LUZ } & \multicolumn{3}{|c|}{ HP } \\
\cline { 2 - 4 } & Ancho b1 & \multicolumn{1}{|c|}{ Canto e1 } & - \\
\hline 20 & 1.988 & 0.171 & \\
\hline 25 & 1.988 & 0.150 & \\
\hline 30 & 1.936 & 0.162 & \\
\hline 35 & 1.961 & 0.170 & \\
\hline 40 & 1.873 & 0.182 & \\
\hline
\end{tabular}

Tabla 7-6: Resultados geométricos ala inferior: Ancho y canto 
En las siguientes Figuras 7-9, 7-10 se representan los resultados en función de la luz:

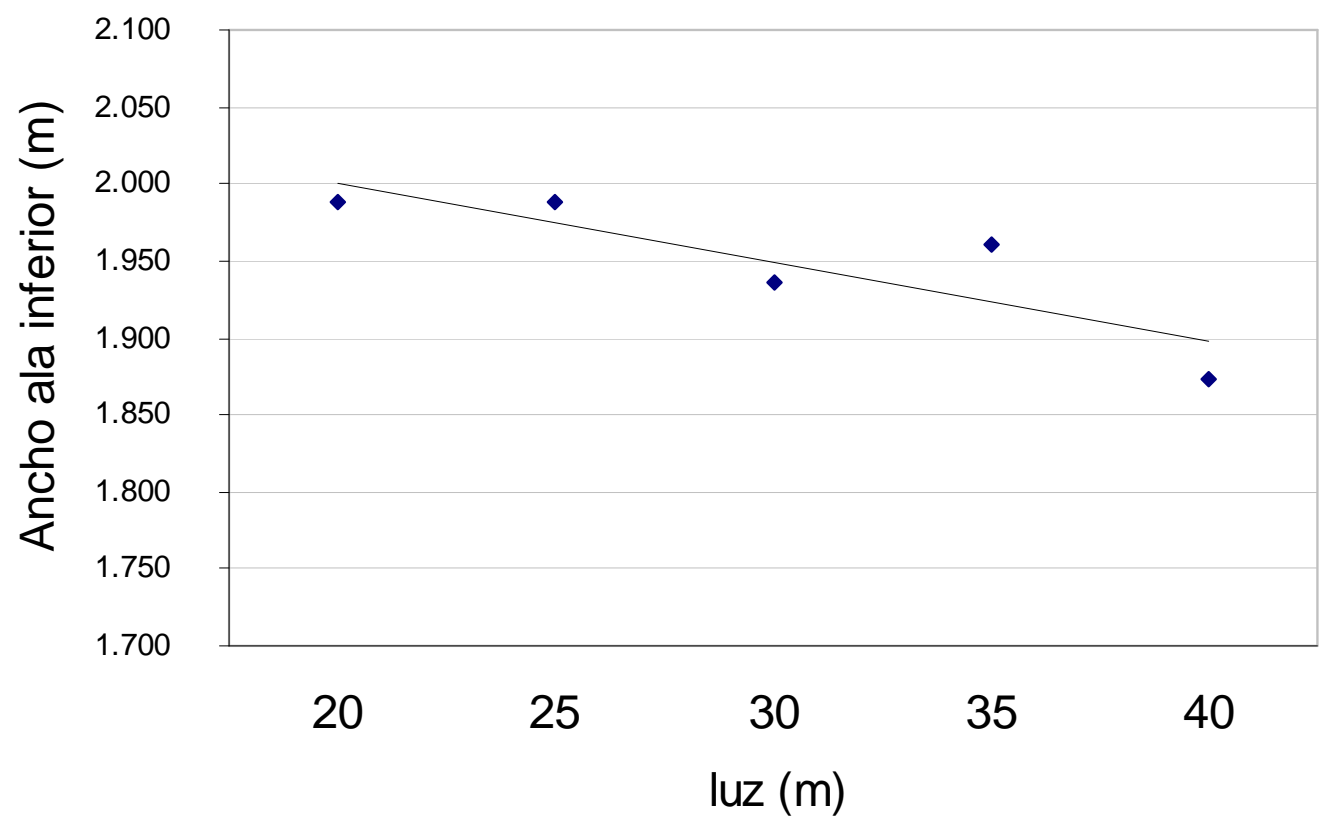

Figura 7-9: Ancho medio del ala inferior en función de la luz.

Se aprecia una leve tendencia a reducir el ancho del ala inferior al aumentar la luz del tablero.

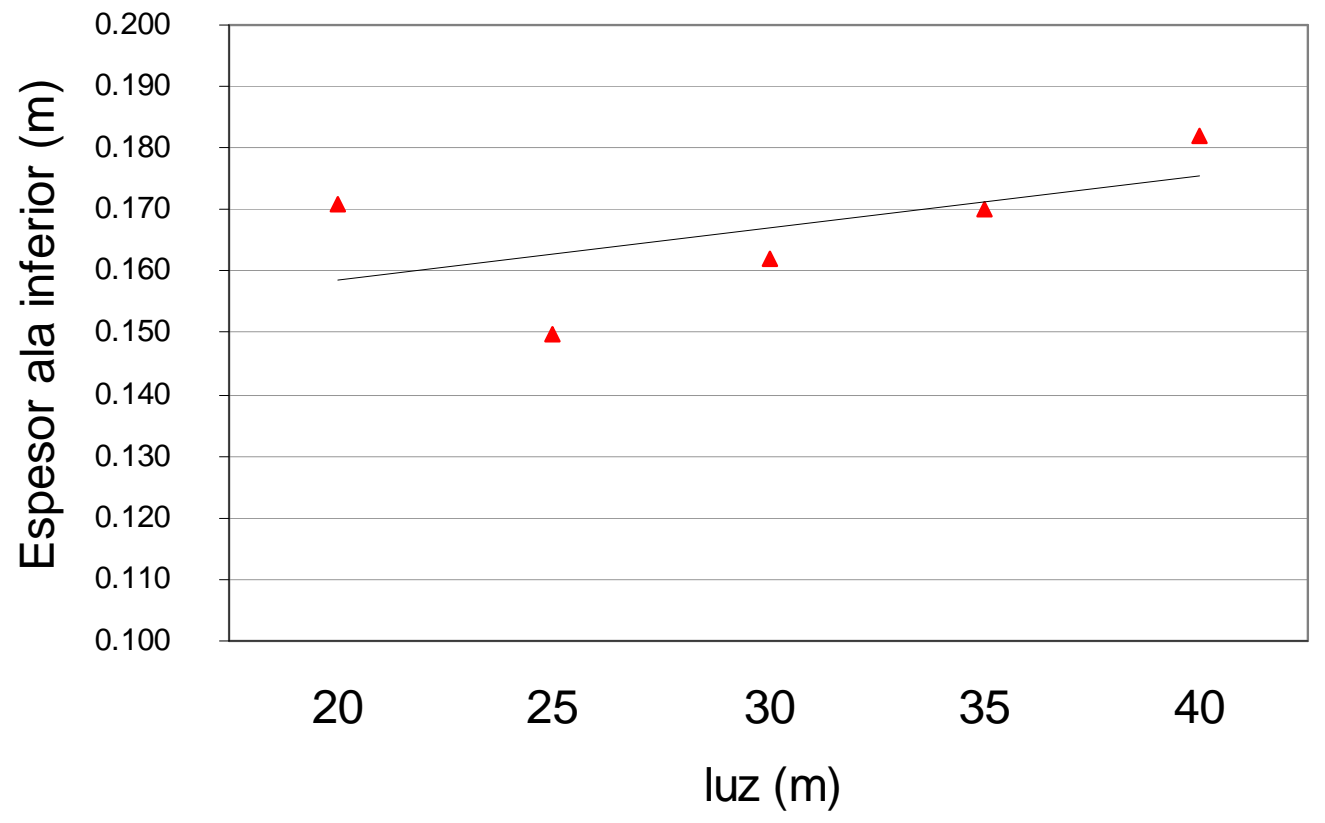

Figura 7-10: Espesor medio del ala inferior en función de la luz.

Observando la gráfica, no se justifica que exista una correlación entre el espesor del ala inferior y la luz del tablero. Sin embargo, se puede ver como desde una luz de 25 
metros hasta 40 metros pueda parecer que si existe un ajuste lineal en el que se incrementa el canto conforme aumenta la luz.

\subsection{Cuantías de hormigón y acero}

En la siguiente Tabla se presentan los resultados de las cuantías de hormigón y acero pasivo por metro cuadrado de tablero.

\begin{tabular}{|c|c|c|c|}
\hline \multirow{2}{*}{ LUZ } & \multicolumn{3}{|c|}{ HP } \\
\cline { 2 - 4 } & $\begin{array}{c}\mathrm{m} 3 \mathrm{H} / \mathrm{m} 2 \\
\text { tablero }\end{array}$ & $\begin{array}{c}\text { Kg pasivas/m2 } \\
\text { tablero }\end{array}$ & - \\
\hline 20 & 0.364 & 55.725 & \\
\hline 25 & 0.330 & 57.353 & \\
\hline 30 & 0.339 & 59.636 & \\
\hline 35 & 0.352 & 58.825 & \\
\hline 40 & 0.337 & 61.228 & \\
\hline
\end{tabular}

Tabla 7-7: Cuantías de hormigón y acero pasivo.

A continuación se representan las Figuras correspondientes a la Tabla:

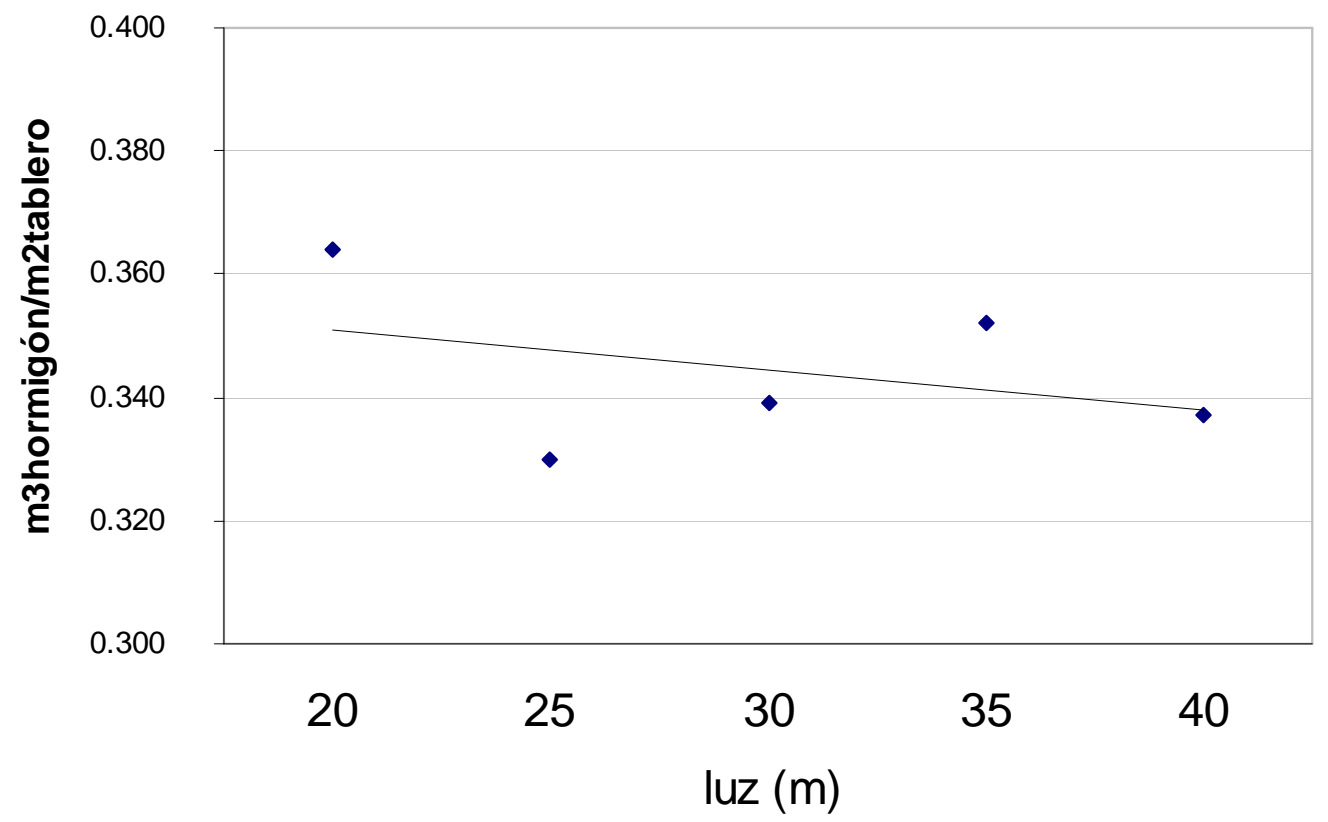

Figura 7-11: Cuantía de hormigón por $\mathbf{m}^{2}$ de tablero.

Se observa cierta tendencia a reducir la cuantía de hormigón al aumentar la luz. Se recibe con cierta curiosidad, puesto que parece lógico que fuera alrevés, es decir, al aumenta la luz debería también aumentar la cuantía puesto que la viga tiene más canto. Sin embargo tal como se observa en la Figura 7-4, al aumentar la luz, se reducía el espesor de la losa, y también, en la Figura 7-8, al aumentar la luz se reducía el ancho del ala inferior de la viga. Ambas geometrías podrían justificar dicha reducción de cuantía. 


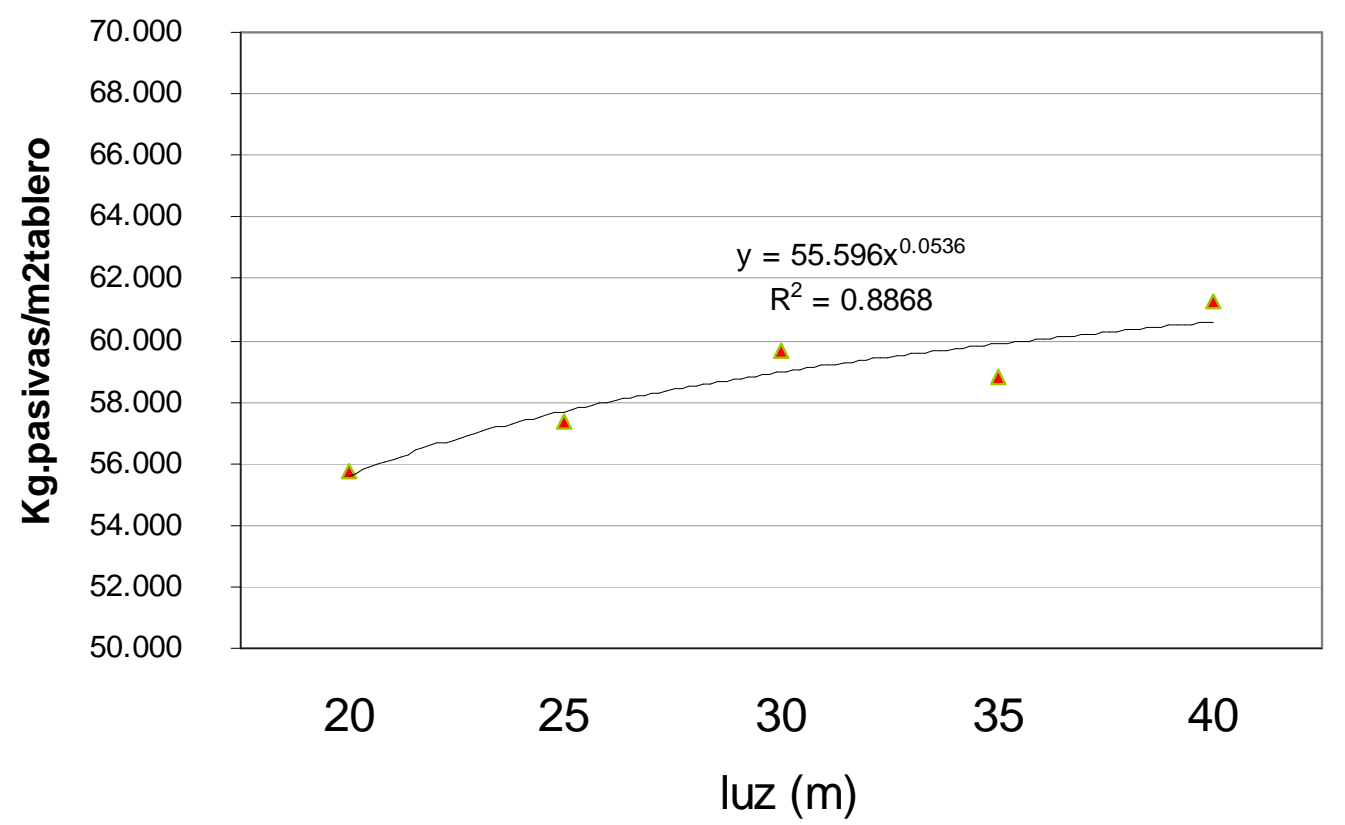

Figura 7-12: Cuantía de acero pasivo por $\mathbf{m}^{2}$ de tablero.

En la Figura 7-11 se observa, como era de esperar, que aumenta la cuantía de acero pasivo al incrementarse la luz. La función representativa es potencial aunque con una correlación no demasiado elevada.

\subsection{Conclusiones}

A continuación, se resumen las principales conclusiones de este estudio paramétrico:

- La dependencia del coste del tablero respecto a la luz del vano muestra una correlación cuadrática, aunque sensiblemente lineal. Las variables canto de la viga y número de torones afectan al carácter lineal del coste.

- El canto de la viga y el número de torones, muestran una fuerte correlación lineal con coeficientes de determinación muy próximos a la unidad.

- El canto de la viga está muy cerca del límite superior del parámetro establecido en el trabajo (1/18 de la luz). El valor medio obtenido es de $1 / 18.176$.

- La elección de la resistencia del hormigón en losa respecto a la luz, es una función fuertemente cuadrática, con un valor de $\mathrm{R}^{2}$ elevado. Sin embargo, sólo se puede afirmar que existe cierta tendencia a reducir la resistencia del hormigón de la viga conforme aumenta la luz.

- El resto de variables geométricas, sólo muestran tendencias, con coeficientes de determinación bajos, así se puede decir que al aumentar la luz: el ancho del ala inferior tiende a reducirse, mientras su espesor tiende a aumentar, el espesor del alma toma el mínimo dispuesto en este problema, y que el ancho 
y espesor del ala superior toman valores que varían muy poco entre sí y que no muestran tendencia alguna.

- La cuantía del hormigón respecto a la superficie del tablero muestra una ligera tendencia a la reducción de ésta al incrementarse la luz, pero no existe una clara correlación al respecto. Sin embargo, si existe cierta correlación de carácter potencial en cuanto al incremento de la cuantía de la armadura pasiva con respecto a la luz. 


\section{CAPÍTULO 8. APLICACIÓN A VIGAS REFORZADAS CON FIBRAS DE ACERO}

En este capítulo se introduce la posibilidad de aplicar las fibras al hormigón utilizado en la fabricación de las vigas pretesas. Se aprovecha la inclusión de este tipo de hormigón en la normativa de la EHE-08, donde en su anejo 14 se establecen recomendaciones específicas y complementarias en su aplicación. A continuación, se relacionan los elementos adicionales necesarios para poder realizar el nuevo estudio:

- Nuevas variables de la heurística.

- Precio unitario de las fibras.

- Características mecánicas del hormigón con fibras (HPF)

- Aspectos tenidos en cuenta en la comprobación de las secciones del tablero.

Con los nuevos condicionantes para este tipo de hormigón HPF, se aplica la mejor heurística estudiada -S3-. Los resultados obtenidos se comparan con los del hormigón HP en viga. Para ello, se utiliza una parametrización de la estructura con distintas luces, desde 20 hasta 40 metros, de 5 en 5 metros. Finalmente se obtienen algunas conclusiones. 


\subsection{Condicionantes sobre el modelo}

\subsubsection{Variables}

La variable que va a permitir completar los valores que definen la estructura pertenece al grupo de calidades del hormigón:

- Fibras de acero

$f R 3 d$

Valores posibles: $3,3.5,4,4.5,5,5.5,6,6.5,7$

\subsubsection{Coste de la viga}

El coste de la viga viene condicionado por el precio de las fibras de acero. La Tabla queda actualizada con el nuevo precio, mostrándose a continuación:

\begin{tabular}{|l|l|c|}
\hline Unidad en viga & Descripción & Precio unitario \\
\hline Acero (B-500-S) & Material y Mano de obra & $2.63[€ / \mathrm{kg}]$ \\
\hline Acero (Y1860-S7) & Material y Mano de obra & $3.38[€ / \mathrm{kg}]$ \\
\hline Encofrado & Molde de acero & $75.11[€ / \mathrm{m}]$ \\
\hline Hormigón & HP-35 & $122.25\left[€ / \mathrm{m}^{3}\right]$ \\
\hline & HP-40 & $133.40\left[€ / \mathrm{m}^{3}\right]$ \\
\hline & HP-45 & $142.15\left[€ / \mathrm{m}^{3}\right]$ \\
\hline & HP-50 & $152.89\left[€ / \mathrm{m}^{3}\right]$ \\
\hline & Fibras de acero & $1.00[€ / \mathrm{kg}]$ \\
\hline
\end{tabular}

Tabla 8-1: Precios unitarios viga

\subsubsection{Características mecánicas del hormigón con fibras HPF.}

Para el hormigón con fibras traccionado, se aplica el diagrama rectangular con valor fctR,d del Anejo 14 de la EHE-08. Los valores aplicados de obtienen a partir de los trabajos de investigación del Profesor Pedro Serna[155] del ICITECH de la UPV, dicha información ha sido facilitada por PREVALESA. Los trabajos están realizados sobre "placas", obteniéndose el siguiente resumen:

Hormigones con dosificaciones de fibras entre 40 y $60 \mathrm{~kg} / \mathrm{m}^{3}$, pueden conducir a valores medios de resistencia residual a tracción de entre 3,0 y 7,0 MPa. 
A partir de aquí:

\begin{tabular}{|c|c|c|}
\hline$f_{c t, R, m}(\mathrm{Mpa})$ & $f_{c t, R, k}=0,7^{*} f_{c t, R, m}(\mathrm{Mpa})$ & $f_{c t, R, d}=f_{c t, R, k} / \gamma_{c}(\mathrm{Mpa})$ \\
\hline 3.0 & 2.1 & 1.40 \\
\hline 4.0 & 2.8 & 1.87 \\
\hline 5.0 & 3.5 & 2.33 \\
\hline 6.0 & 4.2 & 2.80 \\
\hline 7.0 & 4.9 & 3.27 \\
\hline
\end{tabular}

$f_{c t, R, d}=1.40 \mathrm{MPa} \rightarrow \tau_{f d}=0.70 \mathrm{MPa}$

\begin{tabular}{|c|c|c|c|}
\hline Canto placa & $\xi$ & $d(\mathrm{~cm})$ & $V_{\text {fu }}(\mathrm{kN})$ \\
\hline 15 & 2 & 12 & 55.57 \\
\hline 20 & 2 & 17 & 78.72 \\
\hline 26 & 1.93 & 23 & 102.77 \\
\hline
\end{tabular}

$f_{c t, R, d}=1.87 \mathrm{MPa} \rightarrow \tau_{f d}=0.93 \mathrm{MPa}$

\begin{tabular}{|c|c|c|c|}
\hline Canto placa & $\xi$ & $d(\mathrm{~cm})$ & $V_{\text {fu }}(\mathrm{kN})$ \\
\hline 15 & 2 & 12 & 74.22 \\
\hline 20 & 2 & 17 & 105.14 \\
\hline 26 & 1.93 & 23 & 137.27 \\
\hline
\end{tabular}

$f_{c t, R, d}=2.33 \mathrm{MPa} \rightarrow \tau_{f d}=1.16 \mathrm{MPa}$

\begin{tabular}{|c|c|c|c|}
\hline Canto placa & $\xi$ & $d(\mathrm{~cm})$ & $V_{f u}(\mathrm{kN})$ \\
\hline 15 & 2 & 12 & 92.48 \\
\hline 20 & 2 & 17 & 131.01 \\
\hline 26 & 1.93 & 23 & 171.03 \\
\hline
\end{tabular}

$f_{c t, R, d}=2.80 \mathrm{MPa} \rightarrow \tau_{f d}=1.40 \mathrm{MPa}$

\begin{tabular}{|c|c|c|c|}
\hline Canto placa & $\xi$ & $d(\mathrm{~cm})$ & $V_{f u}(\mathrm{kN})$ \\
\hline 15 & 2 & 12 & 111.12 \\
\hline 20 & 2 & 17 & 157.44 \\
\hline 26 & 1.93 & 23 & 205.54 \\
\hline
\end{tabular}

$f_{c t, R, d}=3.27 \mathrm{MPa} \rightarrow \tau_{f d}=1.63 \mathrm{MPa}$

\begin{tabular}{|c|c|c|c|}
\hline Canto placa & $\xi$ & $d(\mathrm{~cm})$ & $V_{f u}(\mathrm{kN})$ \\
\hline 15 & 2 & 12 & 129.79 \\
\hline 20 & 2 & 17 & 183.86 \\
\hline 26 & 1.93 & 23 & 240.04 \\
\hline
\end{tabular}

UNE-EN 14651:2007 Método de ensayo para hormigón con fibras metálicas. Anejo 2 - 361 -

Determinación de la resistencia a la tracción por flexión (límite de proporcionalidad (LOP), resistencia residual) 


\subsubsection{Comprobación de las secciones del tablero.}

La utilización del hormigón con fibras como material de las vigas exige la aplicación del anejo 14 de la EHE-08, teniéndose en cuenta a flexión y cortante, no aplicándose por el momento al torsor. Resumiendo, a continuación se relatan los puntos que presentan un aspecto diferencial del resto de los artículos de la EHE-08.

Se aplica la serie de las resistencias residuales a flexotracción características en $\mathrm{N} / \mathrm{mm}^{2}: 3,0-3,5-4,0-4,5-5,0-5,5-6,0-6,5-7,0$. (Art. 39.2)

Se considera el diagrama rectangular tensión-deformación de la Figura A.14.2. Donde $\mathrm{f}_{\mathrm{ctR}, \mathrm{d}}=0,33 \mathrm{f}_{\mathrm{R}, 3, \mathrm{~d}}$, y se toma la deformación máxima $\varepsilon_{\mathrm{lim}}=20 \%$, correspondiente a secciones sometidas a flexión.

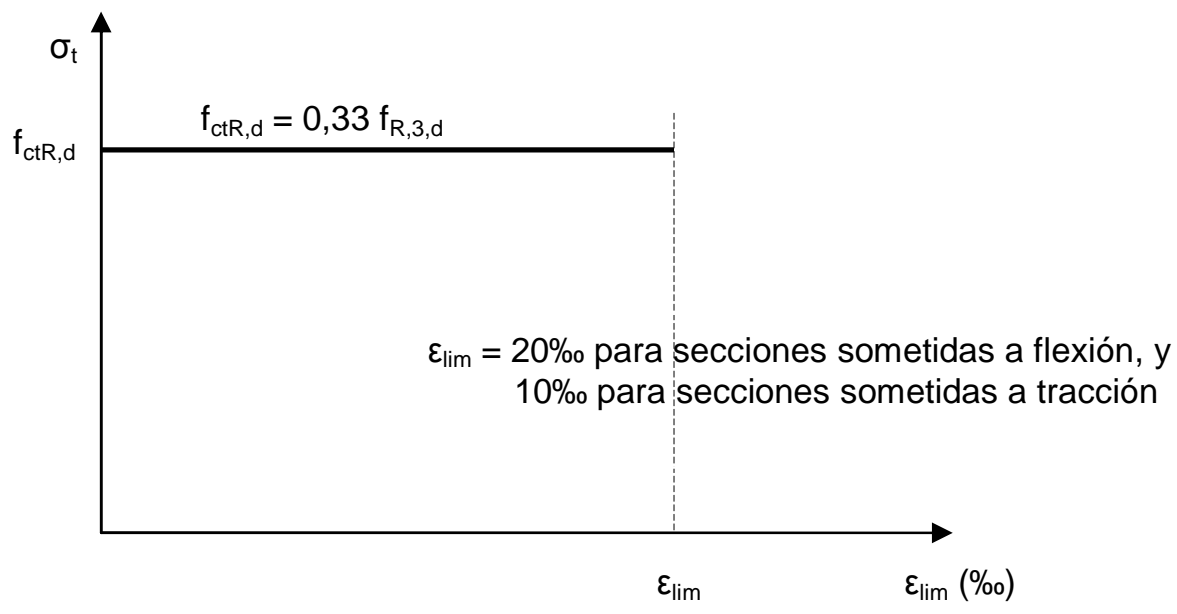

Figura 8-1: Diagrama tensión-deformación en tracción de cálculo del hormigón con fibras. Se corresponde con la Figura A.14.2 de la EHE-08

Se produce un efecto de confinamiento del hormigón que permite prescindir de la armadura de piel longitudinal.

Se tiene en cuenta la contribución de las fibras en la resistencia de la sección a flexión simple (art. 42.3.2), cumpliéndose la siguiente limitación:

$A_{p} f_{p d}\left(d_{p} / d_{s}\right)+A_{s} f_{y d}+\left(z_{f} / z\right) A_{c t} f_{c t R, d}>\left(W_{1} / z\right) f_{c t m}+(P / z)\left(\left(W_{1} / A\right)+e\right)$

siendo:

$\mathrm{Z}_{\mathrm{f}} \mathrm{A}_{\mathrm{ct}} \mathrm{f}_{\mathrm{ctR}, \mathrm{d}}$ contribución de las fibras

$\mathrm{Z}_{\mathrm{f}} \quad$ brazo mecánico de la tracción del hormigón

$\mathrm{A}_{\mathrm{ct}} \quad$ área traccionada del hormigón

$\mathrm{f}_{\mathrm{ctR}, \mathrm{d}} \quad$ resistencia residual a tracción de cálculo en el diagrama rectangular

Se puede reducir (art. 42.3.5) las cuantías geométricas mínimas en una cuantía mecánica equivalente a :

$$
\mathrm{A}_{\mathrm{ct}} \mathrm{f}_{\mathrm{ctR}, \mathrm{d}}
$$


En el estado límite de agotamiento a cortante, el esfuerzo cortante de agotamiento por tracción en el alma vale (art. 44.2.3.2.3):

$$
\mathrm{V}_{\mathrm{u} 2}=\mathrm{V}_{\mathrm{cu}}+\mathrm{V}_{\mathrm{su}}+\mathrm{V}_{\mathrm{fu}}
$$

Siendo $\mathrm{V}_{\mathrm{fu}}$ la contribución de las fibras de acero a la resistencia a esfuerzo cortante,

$$
\mathrm{V}_{\mathrm{fu}}=0,7 \xi \tau_{\mathrm{fd}} \mathrm{b}_{0} \mathrm{~d},
$$

$\operatorname{con} \tau_{\mathrm{fd}}=0,5 \mathrm{f}_{\mathrm{ctR}, \mathrm{d}}$

La cuantía mínima de esfuerzo cortante (art. 44.2.3.4.1) se verifica siempre que se cumpla la relación:

$$
\mathrm{V}_{\mathrm{su}}+\mathrm{V}_{\mathrm{fu}}>\left(\mathrm{f}_{\mathrm{ct}, \mathrm{m}} / 7,5\right) \mathrm{b}_{0} \mathrm{~d}
$$

En cuanto a armaduras longitudinales (Art, 44.2.3.4.2) en lugar de $\mathrm{V}_{\text {su }}$ debe considerarse la suma $\mathrm{V}_{\mathrm{su}}+\mathrm{V}_{\mathrm{fu}}$

\subsection{Comparación tableros con vigas de HP vs HPF.}

En este apartado se pretende comparar los tableros constituidos por vigas HP con los de vigas HPF. Se han aplicado los valores y la heurística de la parametrización del capítulo 7, procesándose cada heurística 9 veces. Se analiza el coste económico, la geometría de las soluciones, y las cuantías del hormigón y del acero.

\subsubsection{Coste de los tableros}

En la siguiente Tabla se presentan los resultados de los costes medios, el mínimo y la desviación.

\begin{tabular}{|c|c|r|r|r|r|r|}
\hline & \multicolumn{3}{|c|}{ HP } & \multicolumn{3}{c|}{ HPF } \\
\hline$\underline{\text { LUZ }}$ & Xm $(€)$ & Xmin $(€)$ & \multicolumn{1}{|c|}{ Desv \% } & \multicolumn{1}{|c|}{ Xm $(€)$} & \multicolumn{1}{|c|}{ Xmin $(€)$} & \multicolumn{1}{c|}{ Desv \% } \\
\hline 20 & 62,262 & 61,676 & 1.0 & 61,539 & 60,923 & 1.0 \\
\hline 25 & 75,045 & 74,326 & 1.0 & 75,171 & 74,254 & 1.2 \\
\hline 30 & 92,601 & 91,412 & 1.3 & 91,462 & 90,745 & 0.8 \\
\hline 35 & 110,477 & 109,004 & 1.4 & 110,092 & 109,127 & 0.9 \\
\hline 40 & 128,755 & 126,035 & 2.2 & 128,014 & 126,866 & 0.9 \\
\hline
\end{tabular}

Tabla 8-2: Resultados obtenidos para hormigón pretensado sin o con fibras.

Se puede apreciar en primer lugar, que para el HPF las desviaciones de los valores medios respecto al mínimo son muy pequeñas, alrededor del 1\%. Tanto los valores medios como los mínimos son muy similares para los dos tipos de hormigones. No se aprecia mucha diferencia, aunque normalmente son algo más económicos los tableros con HPF, del orden del 1\% o algo menos. En las siguientes Figuras 8-2 y 8-3 se aprecia con claridad. 


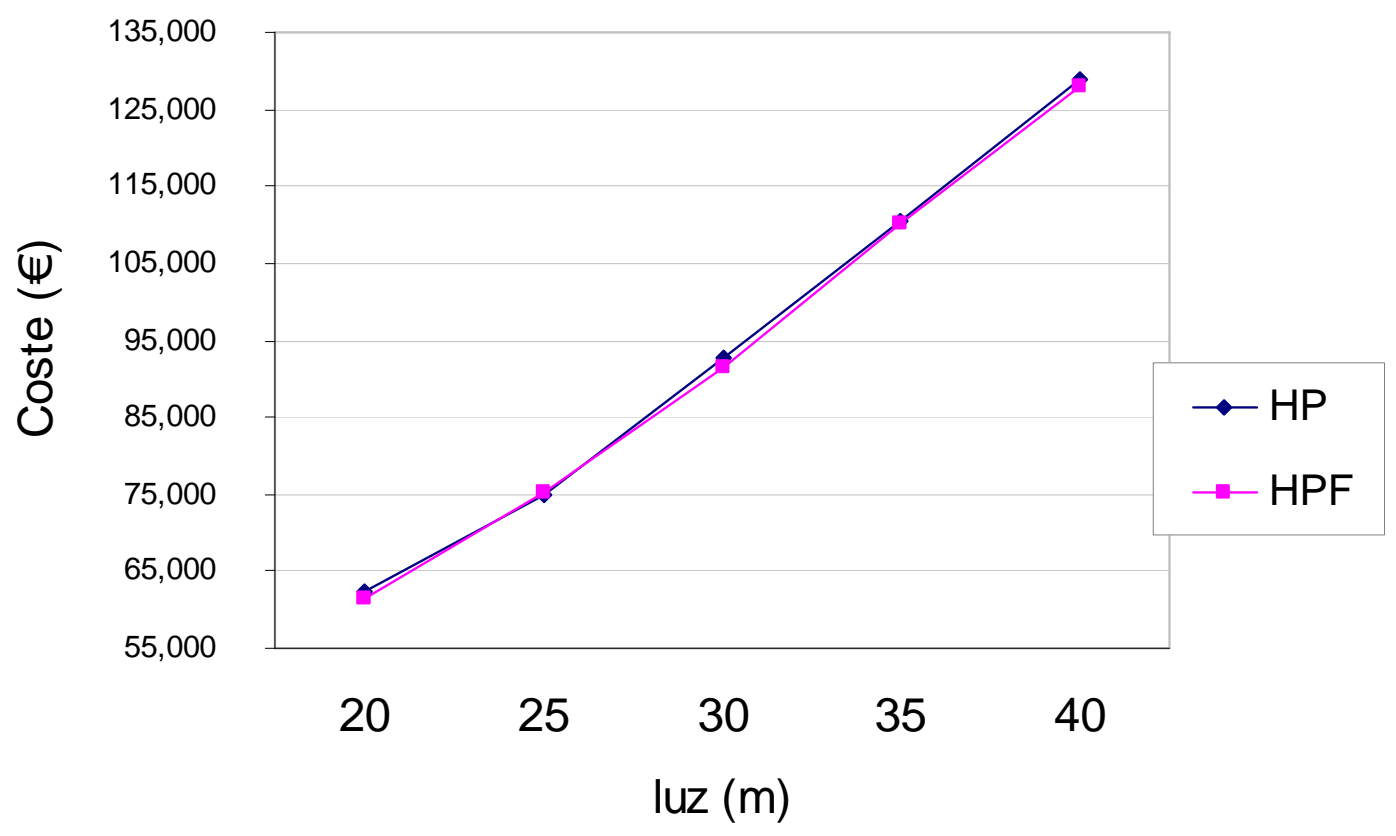

Figura 8-2: Coste medio de los tableros en función de la luz.

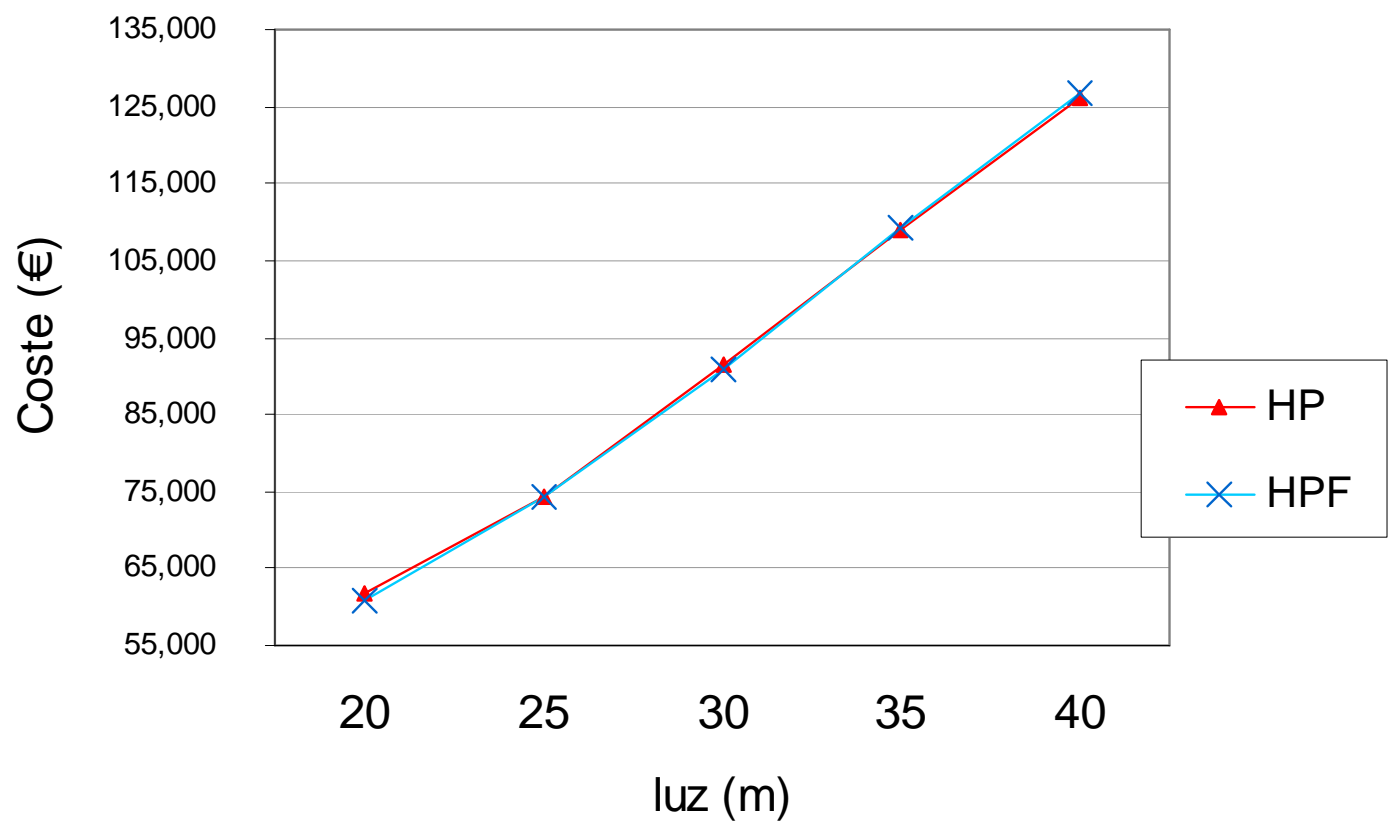

Figura 8-3: Coste mínimo de los tableros en función de la luz.

En el Apéndice C se muestran los planos de los mejores resultados obtenidos para cada luz, tanto para el HP como para el HPF.

A continuación se realiza un análisis comparativo entre los dos tipos de hormigones, para los valores geométricos obtenidos, cuantías y armaduras pasivas, en función de la luz del tablero. 


\subsubsection{Resultados geométricos}

En la siguiente Tabla se presentan los resultados geométricos obtenidos para el HPF. Los valores para el HP se han visto en el punto 7.3.

\begin{tabular}{l}
$\mathrm{N}$ \\
\hline
\end{tabular}

$\begin{array}{lllllllllllllllll}9 & 127820.547 & 2.13 & 0.19 & 1.94 & 0.28 & 0.15 & 0.10 & 0.22 & 45 & 40 & 35 & 23 & 0 & 2 / 0.6 & 6.00\end{array}$

$\begin{array}{llllllllllllllll}8 & 127518.211 & 2.21 & 0.19 & 1.98 & 0.28 & 0.18 & 0.10 & 0.22 & 40 & 4036 & 19 & 0 & 2 / 0.6 & 7.00\end{array}$

$\begin{array}{llllllllllllllll}7 & 126866.602 & 2.09 & 0.19 & 1.96 & 0.28 & 0.15 & 0.10 & 0.22 & 45 & 40 & 36 & 22 & 0 & 2 / 0.6 & 7.00\end{array}$

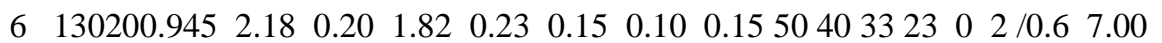

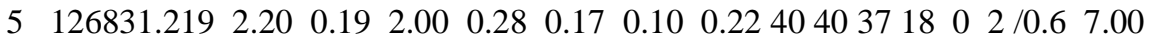

$\begin{array}{llllllllllllllll}4 & 129245.680 & 2.13 & 0.20 & 1.86 & 0.23 & 0.15 & 0.10 & 0.15 & 504034 & 23 & 0 & 2 / 0.6 & 7.00\end{array}$

$\begin{array}{llllllllllllllll}3 & 128580.492 & 2.15 & 0.19 & 1.94 & 0.28 & 0.15 & 0.10 & 0.2245 & 4035 & 22 & 0 & 2 / 0.6 & 6.50\end{array}$

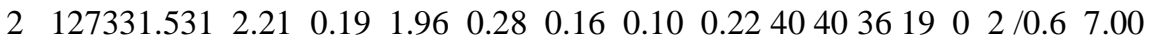

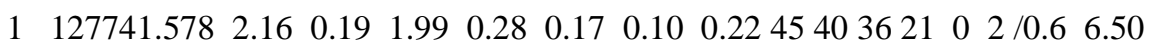

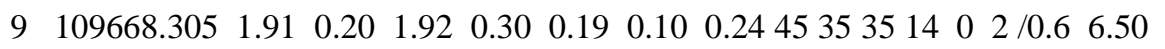

$\begin{array}{lllllllllllllll}8 & 109701.297 & 1.94 & 0.20 & 1.92 & 0.23 & 0.15 & 0.10 & 0.1645 & 40 & 35 & 13 & 0 & 2 / 0.6 & 7.00\end{array}$

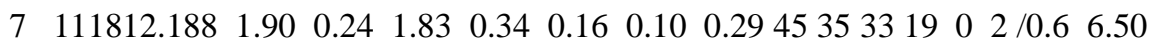

$\begin{array}{llllllllllllll}6 & 110001.031 & 1.90 & 0.20 & 1.99 & 0.30 & 0.18 & 0.10 & 0.24 & 453536 & 13 & 0 & 2 / 0.6 & 6.00\end{array}$

$\begin{array}{llllllllllllll}5 & 109667.852 & 1.90 & 0.19 & 1.98 & 0.30 & 0.18 & 0.10 & 0.24 & 454036 & 14 & 0 & 2 / 0.6 & 6.00\end{array}$

$\begin{array}{lllllllllllllll}4 & 109127.586 & 1.91 & 0.21 & 1.83 & 0.24 & 0.15 & 0.10 & 0.1745 & 35 & 33 & 16 & 0 & 2 / 0.6 & 7.00\end{array}$

$\begin{array}{lllllllllllllll}3 & 109257.898 & 1.86 & 0.20 & 1.97 & 0.30 & 0.15 & 0.10 & 0.24 & 4535 & 36 & 15 & 0 & 2 / 0.6 & 7.00\end{array}$

$\begin{array}{lllllllllllllllll}2 & 111562.852 & 1.88 & 0.24 & 1.88 & 0.25 & 0.15 & 0.10 & 0.18 & 50 & 40 & 34 & 18 & 0 & 2 / 0.6 & 6.50\end{array}$

$\begin{array}{lllllllllllllll}1 & 110037.445 & 1.94 & 0.20 & 1.93 & 0.24 & 0.15 & 0.10 & 0.1745 & 4035 & 14 & 0 & 2 / 0.6 & 6.00\end{array}$

$\begin{array}{lllllllllllllll}9 & 90992.102 & 1.63 & 0.21 & 1.89 & 0.23 & 0.15 & 0.10 & 0.16 & 45 & 4034 & 8 & 0 & 2 / 0.6 & 6.50\end{array}$

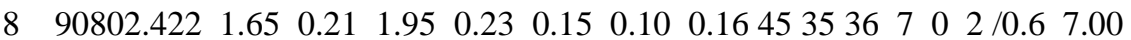

$\begin{array}{llllllllllllllll}7 & 90938.133 & 1.66 & 0.22 & 1.92 & 0.24 & 0.15 & 0.10 & 0.17 & 45 & 30 & 35 & 7 & 0 & 2 / 0.6 & 6.50\end{array}$

$\begin{array}{llllllllllllllll}6 & 91326.422 & 1.66 & 0.24 & 1.95 & 0.26 & 0.15 & 0.10 & 0.1945 & 40 & 36 & 6 & 0 & 2 / 0.6 & 7.00\end{array}$

$\begin{array}{lllllllllllllll}5 & 91835.188 & 1.66 & 0.20 & 1.99 & 0.23 & 0.16 & 0.10 & 0.1645 & 4036 & 5 & 0 & 2 / 0.6 & 7.00\end{array}$

$\begin{array}{lllllllllllllll}4 & 91351.445 & 1.65 & 0.21 & 1.89 & 0.23 & 0.15 & 0.10 & 0.16 & 45 & 4034 & 8 & 0 & 2 / 0.6 & 6.50\end{array}$

$\begin{array}{llllllllllllllll}3 & 92322.828 & 1.61 & 0.24 & 1.99 & 0.24 & 0.15 & 0.10 & 0.17 & 504036 & 7 & 0 & 2 & / 0.6 & 7.00\end{array}$

$\begin{array}{lllllllllllllll}2 & 90745.281 & 1.66 & 0.21 & 1.97 & 0.23 & 0.15 & 0.10 & 0.1645 & 35 & 36 & 5 & 0 & 2 / 0.6 & 7.00\end{array}$

$\begin{array}{llllllllllllllll}1 & 92850.141 & 1.65 & 0.21 & 1.94 & 0.24 & 0.19 & 0.10 & 0.17 & 45 & 35 & 35 & 8 & 0 & 2 / 0.6 & 6.50\end{array}$

$9 \begin{array}{llllllllllllll}74276.453 & 1.37 & 0.21 & 2.00 & 0.23 & 0.16 & 0.10 & 0.1645 & 40 & 35 & 0 & 0 & 2 / 0.6 & 6.50\end{array}$

$\begin{array}{lllllllllllllll}8 & 74254.727 & 1.37 & 0.21 & 2.00 & 0.23 & 0.16 & 0.10 & 0.164540 & 35 & 0 & 0 & 2 / 0.6 & 6.50\end{array}$

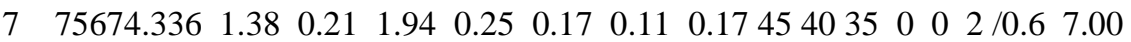

$\begin{array}{llllllllllllllll}6 & 74446.250 & 1.38 & 0.22 & 1.97 & 0.25 & 0.15 & 0.11 & 0.17 & 40 & 35 & 35 & 0 & 0 & 2 / 0.6 & 7.00\end{array}$

$\begin{array}{llllllllllllllll}5 & 74537.711 & 1.36 & 0.21 & 2.00 & 0.23 & 0.16 & 0.10 & 0.1645 & 40 & 35 & 0 & 0 & 2 / 0.6 & 7.00\end{array}$

$\begin{array}{lllllllllllllll}4 & 77437.188 & 1.37 & 0.26 & 1.93 & 0.28 & 0.22 & 0.12 & 0.1945 & 35 & 35 & 0 & 0 & 2 / 0.6 & 7.00\end{array}$

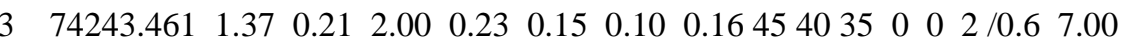

$\begin{array}{lllllllllllllll}2 & 76397.398 & 1.36 & 0.28 & 1.91 & 0.27 & 0.16 & 0.10 & 0.205030 & 55 & 1 & 0 & 2 / 0.6 & 6.50\end{array}$

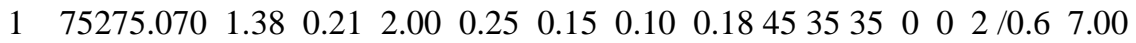

$\begin{array}{llllllllllllllll}9 & 61274.922 & 1.11 & 0.29 & 2.00 & 0.30 & 0.22 & 0.13 & 0.21 & 40 & 40 & 28 & 0 & 0 & 2 / 0.6 & 6.00\end{array}$

$\begin{array}{llllllllllllllll}8 & 61664.734 & 1.11 & 0.21 & 2.00 & 0.24 & 0.16 & 0.10 & 0.17 & 50 & 40 & 29 & 0 & 0 & 2 / 0.6 & 7.00\end{array}$

$\begin{array}{llllllllllllllll}7 & 61430.176 & 1.11 & 0.21 & 1.98 & 0.24 & 0.18 & 0.10 & 0.17 & 50 & 40 & 28 & 0 & 0 & 2 / 0.6 & 7.00\end{array}$

$\begin{array}{lllllllllllllll}6 & 61439.531 & 1.11 & 0.28 & 2.00 & 0.27 & 0.17 & 0.10 & 0.20 & 503030 & 0 & 0 & 2 / 0.6 & 5.50\end{array}$

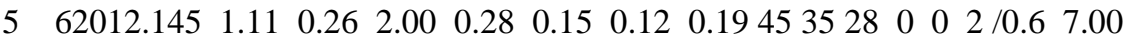

$\begin{array}{llllllllllllllll}4 & 61342.047 & 1.11 & 0.21 & 1.97 & 0.24 & 0.15 & 0.10 & 0.17 & 50 & 40 & 28 & 0 & 0 & 2 / 0.6 & 7.00\end{array}$

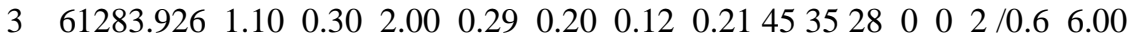

$\begin{array}{llllllllllllllll}2 & 60923.270 & 1.11 & 0.21 & 1.97 & 0.24 & 0.15 & 0.10 & 0.17 & 50 & 40 & 28 & 0 & 0 & 2 / 0.6 & 7.00\end{array}$

$\begin{array}{llllllllllllll}1 & 62492.898 & 1.11 & 0.26 & 2.00 & 0.26 & 0.21 & 0.10 & 0.195035 & 31 & 0 & 0 & 2 / 0.6 & 5.50\end{array}$

$\mathrm{Luz}=40 \mathrm{~m}$

$\mathrm{Luz}=35 \mathrm{~m}$

$\mathrm{Luz}=30 \mathrm{~m}$

$\mathrm{Luz}=25 \mathrm{~m}$

$\mathrm{Luz}=20 \mathrm{~m}$

Tabla 8-3: Resultados geométricos hormigón con fibras HPF 
Siendo las siguientes unidades:
Coste h1
e4
b1 b3
e1 e2
e3 $\mathrm{Hv} \quad \mathrm{Hl}$ p1 p2 p3p4
fR3k
$(€)$
(m.) (m.)
(m.)
(m.) (m.)
(m.) (m.) (HP/HPF)
(n) (n) (n) (n)
(MPa)

Y siendo su significado:

h1 canto de la viga

e4 canto de la losa

b1 ancho ala inferior de la viga

b3 base ala superior de la viga

e1 canto ala inferior de la viga

e2 espesor del alma

e3 canto ala superior de la viga

Hv Hormigón de la viga: HP/HPF

Hl Hormigón de la losa: HA

p1 número de torones nivel 1 ala inferior de $\Phi 0.6 "$

p2 número de torones nivel 2 ala inferior de $\Phi 0.6$ "

p3 número de torones nivel 3 ala inferior de $Ф 0.6$ "

p4 número de torones nivel 4 ala superior de $\Phi 0.6$ "

fR3k resistencia residual a tracción

A continuación se valoran distintas variables que conforman la geometría de la sección del tablero:

\section{Canto de la viga, espesor de la losa y pretensado}

Al igual que para el HP, se ha limitado la esbeltez de la viga a 1/18 de su luz, por lo que los valores máximos posibles son los mismos de la Tabla 7-3.

A continuación, en el siguiente Tabla se presentan los resultados medios obtenidos con la heurística:

\begin{tabular}{|c|c|c|r|r|r|r|}
\hline \multirow{2}{*}{ LUZ } & \multicolumn{3}{|c|}{ HP } & \multicolumn{3}{c|}{ HPF } \\
\cline { 2 - 7 } & $\begin{array}{c}\text { Canto Viga } \\
\mathrm{h}(\mathrm{m})\end{array}$ & $\begin{array}{c}\text { Espesor losa } \\
\mathrm{e} 4(\mathrm{~m})\end{array}$ & № torones & $\begin{array}{c}\text { Canto Viga } \\
\mathrm{h}(\mathrm{m})\end{array}$ & $\begin{array}{c}\text { Espesor losa } \\
\text { e4 }(\mathrm{m})\end{array}$ & № torones \\
\hline 20 & 1.104 & 0.260 & 34.89 & 1.109 & 0.248 & 28.67 \\
\hline 25 & 1.374 & 0.224 & 41.56 & 1.371 & 0.224 & 35.11 \\
\hline 30 & 1.654 & 0.221 & 48.78 & 1.648 & 0.217 & 42.11 \\
\hline 35 & 1.911 & 0.219 & 57.33 & 1.904 & 0.209 & 49.89 \\
\hline 40 & 2.206 & 0.190 & 63.33 & 2.162 & 0.192 & 56.44 \\
\hline
\end{tabular}

Tabla 8-4: Resultados geométricos: Canto viga, losa $y \mathrm{n}^{0}$ torones 
En las siguientes Figuras 8-4, 8-5 y 8-6 se representan dichos resultados en función de la luz:

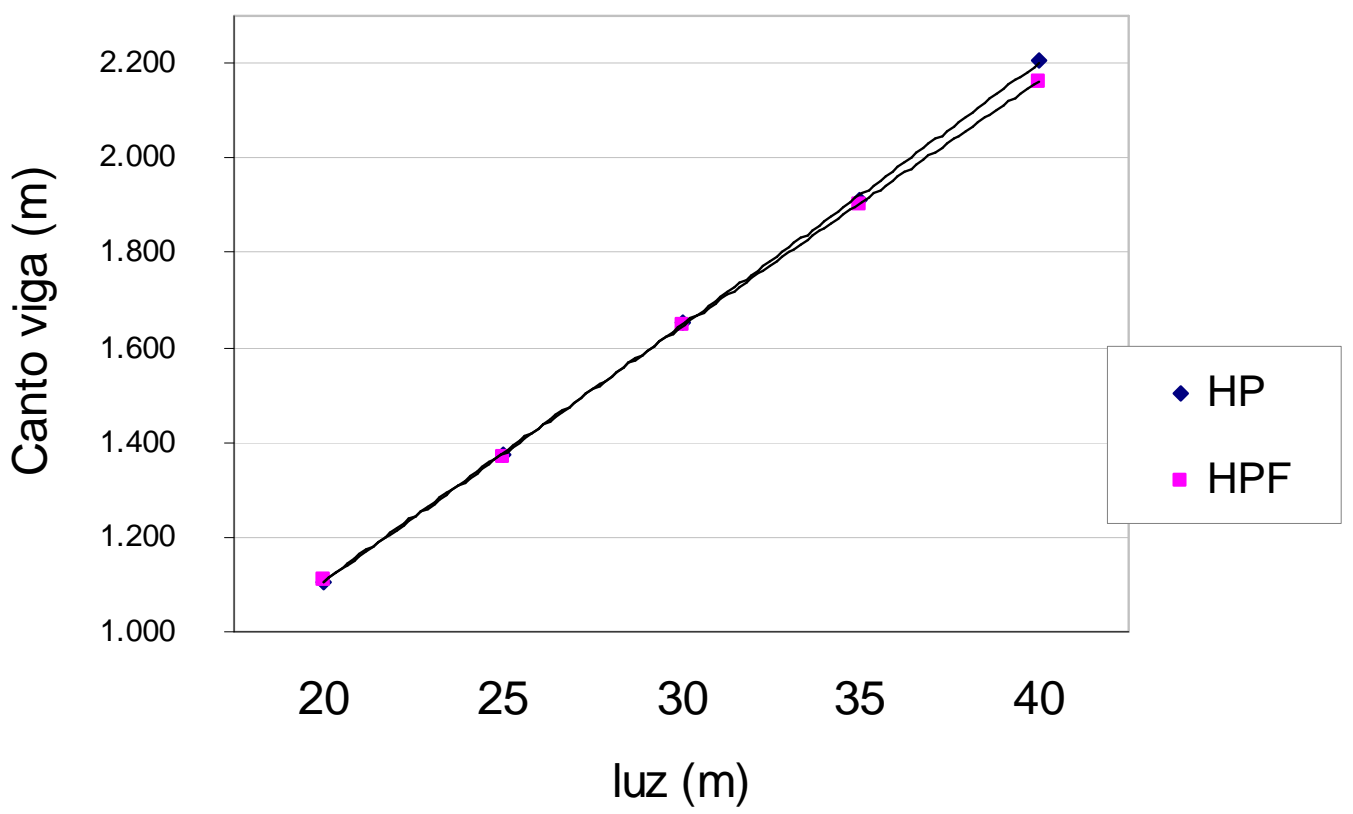

Figura 8-4: Canto de la viga en función de la luz.

Se observa que el canto de la viga es prácticamente independiente del tipo de hormigón. Solamente se observa una pequeña variación, del orden del $2 \%$ para una luz de $40 \mathrm{~m}$.

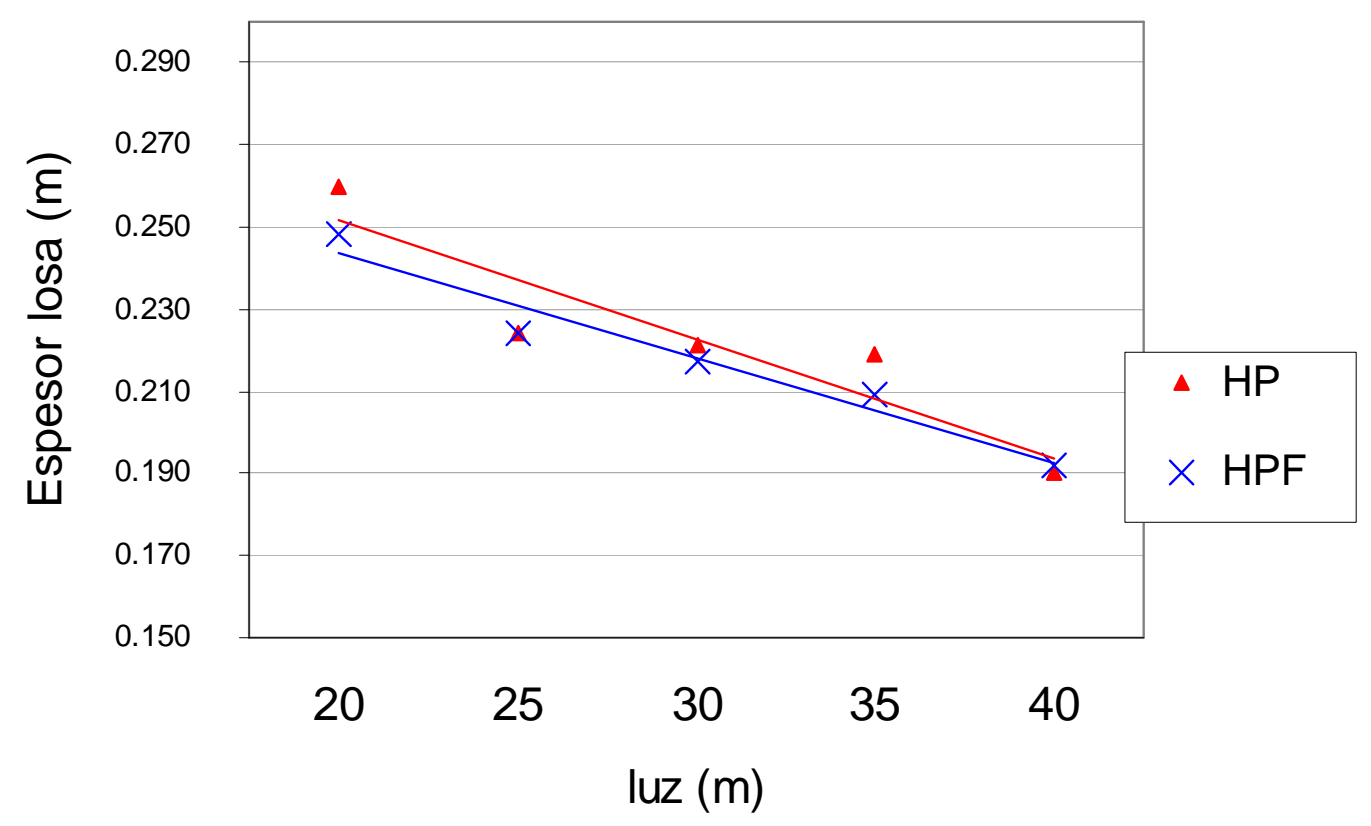

Figura 8-5: Espesor de la losa en función de la luz. 
Se observa una tendencia, en ambos tipos de hormigones, a reducir el espesor de la losa al aumentar la luz.

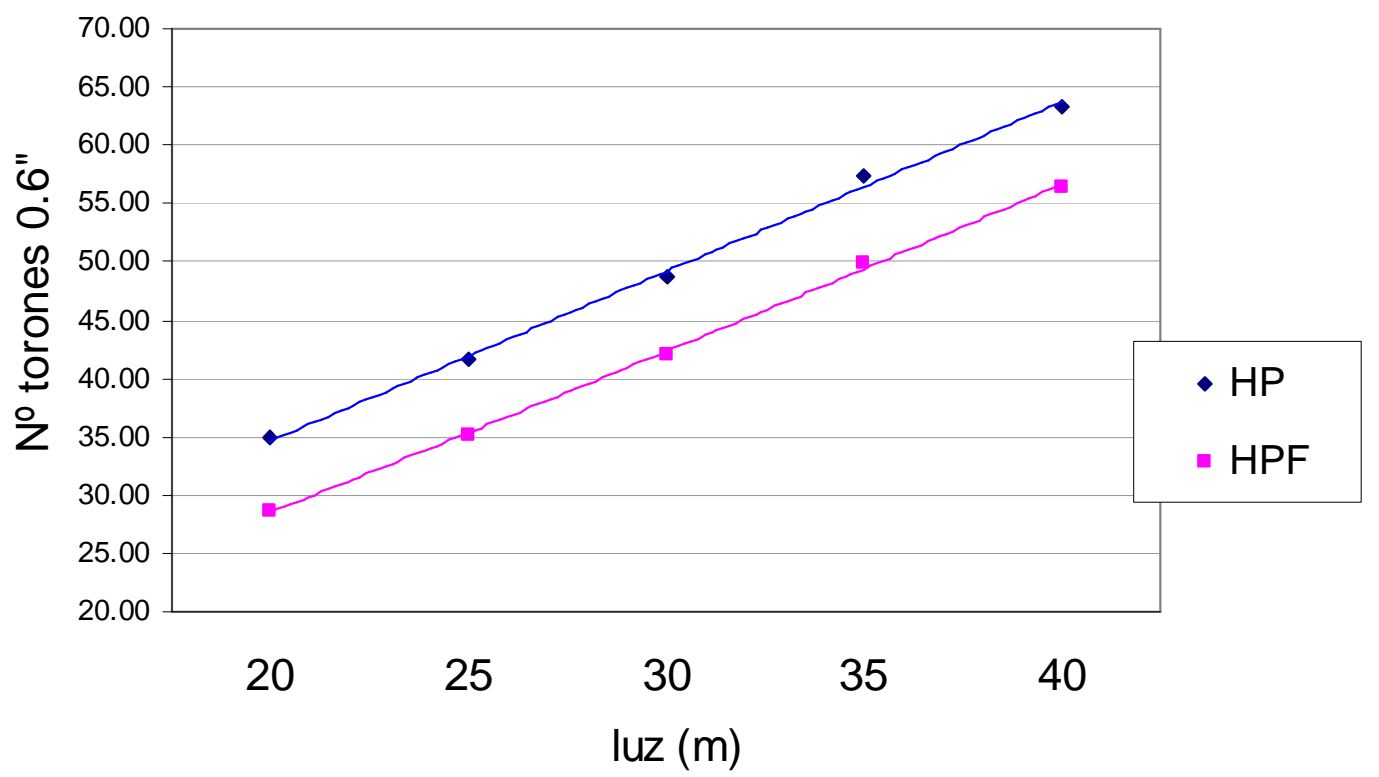

Figura 8-6: Número de torones medio en función de la luz.

Aquí se vislumbra una diferencia significativa entre los dos tipos de hormigón. La consideración de la resistencia del HPF a tracción, permite liberar parte de la acción del pretensado. Esa diferencia permanece prácticamente constante para las distintas luces, con un valor medio de "6.748 torones", equivalente a $1275,64 \mathrm{KNw}$ (cada torón se tesa a $189,04 \mathrm{KNw}$ ).

\section{Tipos de hormigón}

En el siguiente Tabla se presentan los valores medios del resultado de los hormigones:

\begin{tabular}{|c|c|c|c|r|r|r|}
\hline \multirow{2}{*}{ LUZ } & \multicolumn{3}{|c|}{ HP } & \multicolumn{3}{c|}{ HPF } \\
\cline { 2 - 7 } & Hviga & Hlosa & - & Hviga & Hlosa & fR3k \\
\hline 20 & 50.00 & 35.00 & & 47.78 & 37.22 & 6.44 \\
\hline 25 & 46.67 & 35.00 & & 45.00 & 37.22 & 6.83 \\
\hline 30 & 48.33 & 35.56 & & 45.56 & 37.22 & 6.78 \\
\hline 35 & 46.67 & 37.22 & & 45.56 & 37.22 & 6.50 \\
\hline 40 & 44.44 & 40.00 & & 44.44 & 40.00 & 6.78 \\
\hline
\end{tabular}

Tabla 8-5: Resultados hormigones: Viga, losa, fibras 
En las siguientes Figuras 8-7, 8-8 y 8-9 se representan los resultados en función de la luz:

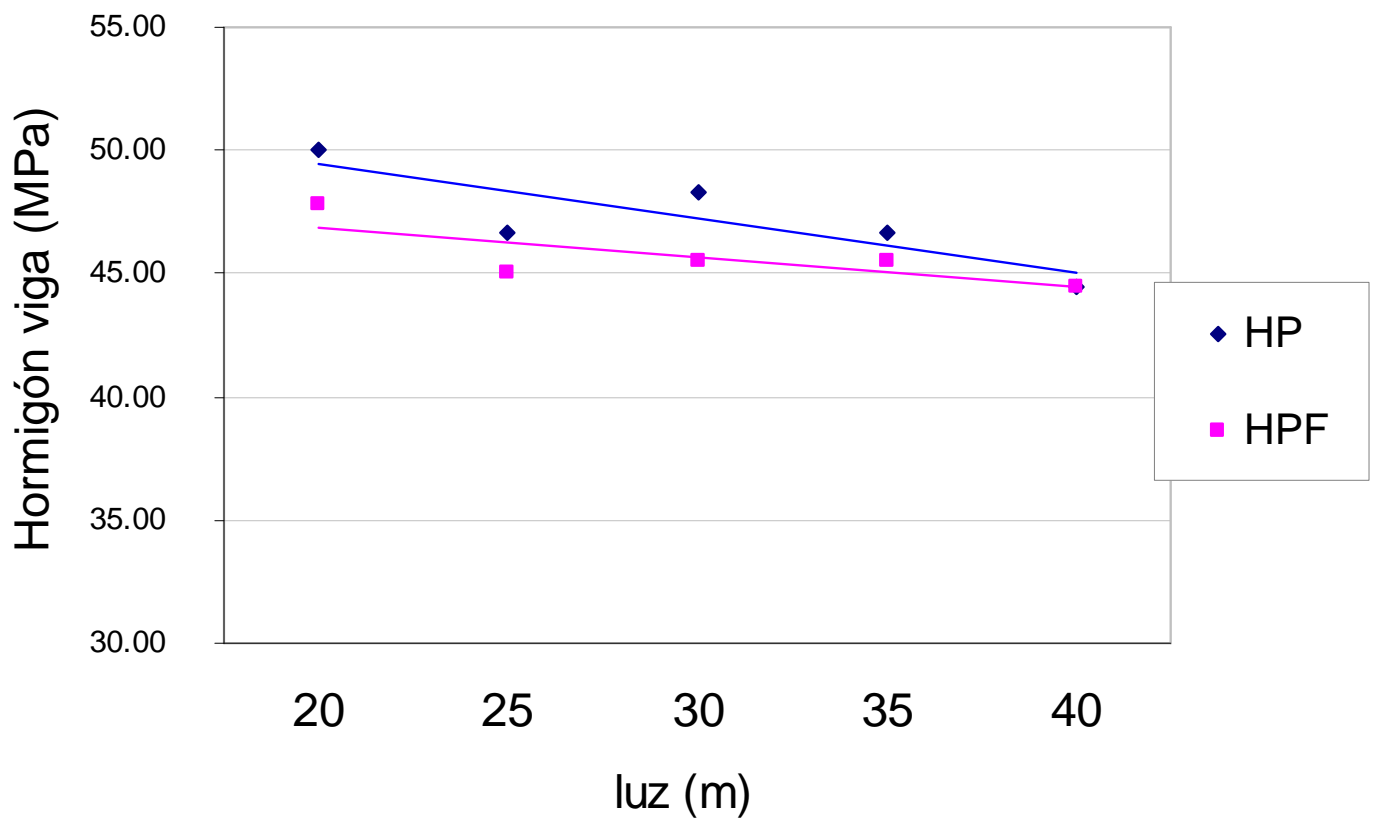

Figura 8-7: Hormigones medios en viga en función de la luz.

En ambos casos, los tipos de hormigón que se aplican son de resistencias elevadas (el límite está establecido en 50MPa), aunque hay una leve tendencia hacia una reducción de resistencia conforme aumenta la luz.

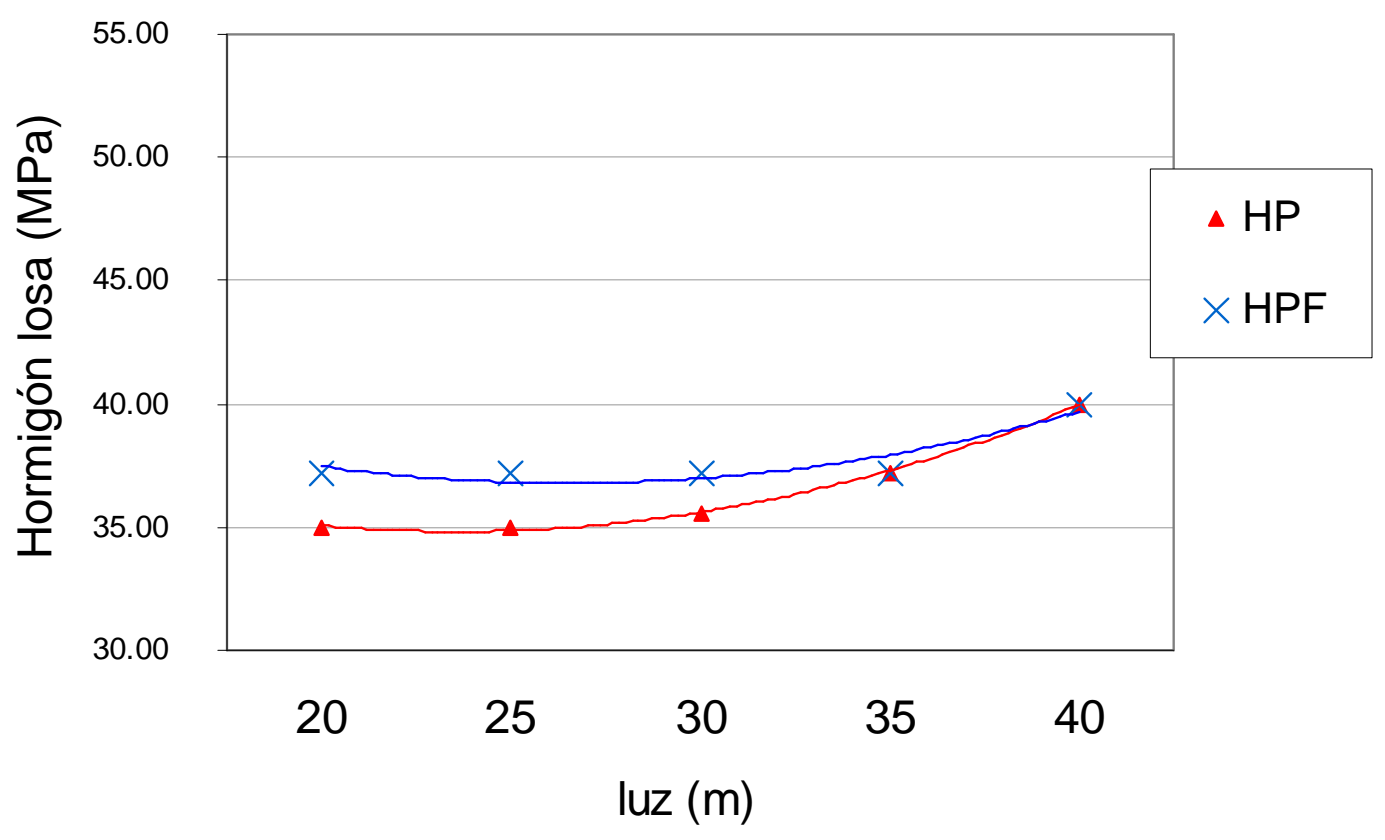

Figura 8-8: Hormigones medios en losa en función de la luz. 
Como en el caso anterior, en ambos tipos de hormigón su tendencia es a utilizar resistencias elevadas (la más elevada en losa es de 40MPa.).

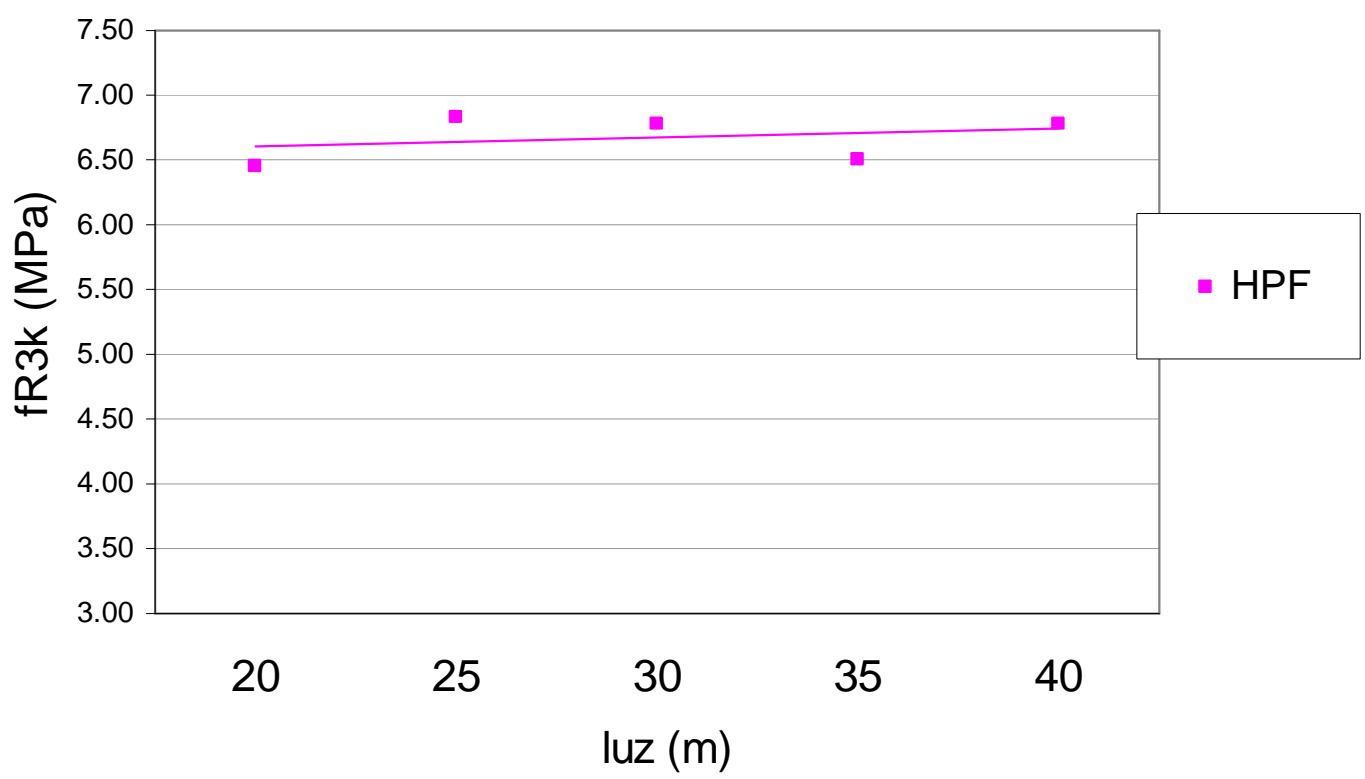

Figura 8-9: Resistencias residuales medias a tracción en función de la luz.

En este caso, la estructura busca aprovechar al máximo la aportación de las fibras de acero para compensar la reducción del pretensado. El valor máximo posible es de 7 $\mathrm{MPa}$, y los valores obtenidos se encuentran entre 6.5 y $7 \mathrm{MPa}$. Este comportamiento debe estar muy condicionado por el precio de las fibras de acero. Si su precio fuera mayor, es probable que el comportamiento cambiase. De aquí se desprende la necesidad de realizar un estudio de la sensibilidad de la optimización en función de la variación del precio de las fibras.

\section{Geometría del ala inferior}

En la siguiente Tabla se presentan los valores medios de la geometría del ala inferior:

\begin{tabular}{|c|r|r|r|r|r|r|}
\hline \multirow{2}{*}{ LUZ } & \multicolumn{3}{|c|}{ HP } & \multicolumn{3}{c|}{ HPF } \\
\cline { 2 - 7 } & Ancho b1 & Canto e1 & - & Ancho b1 & Canto e1 & - \\
\hline 20 & 1.988 & 0.171 & & 1.991 & 0.177 & \\
\hline 25 & 1.988 & 0.150 & & 1.972 & 0.164 & \\
\hline 30 & 1.936 & 0.162 & & 1.943 & 0.156 & \\
\hline 35 & 1.961 & 0.170 & & 1.917 & 0.162 & \\
\hline 40 & 1.873 & 0.182 & & 1.939 & 0.150 & \\
\hline
\end{tabular}

Tabla 8-6: Resultados geométricos ala inferior: Ancho y canto 
En las siguientes Figuras 8-10, 8-11 se representan los resultados en función de la luz:

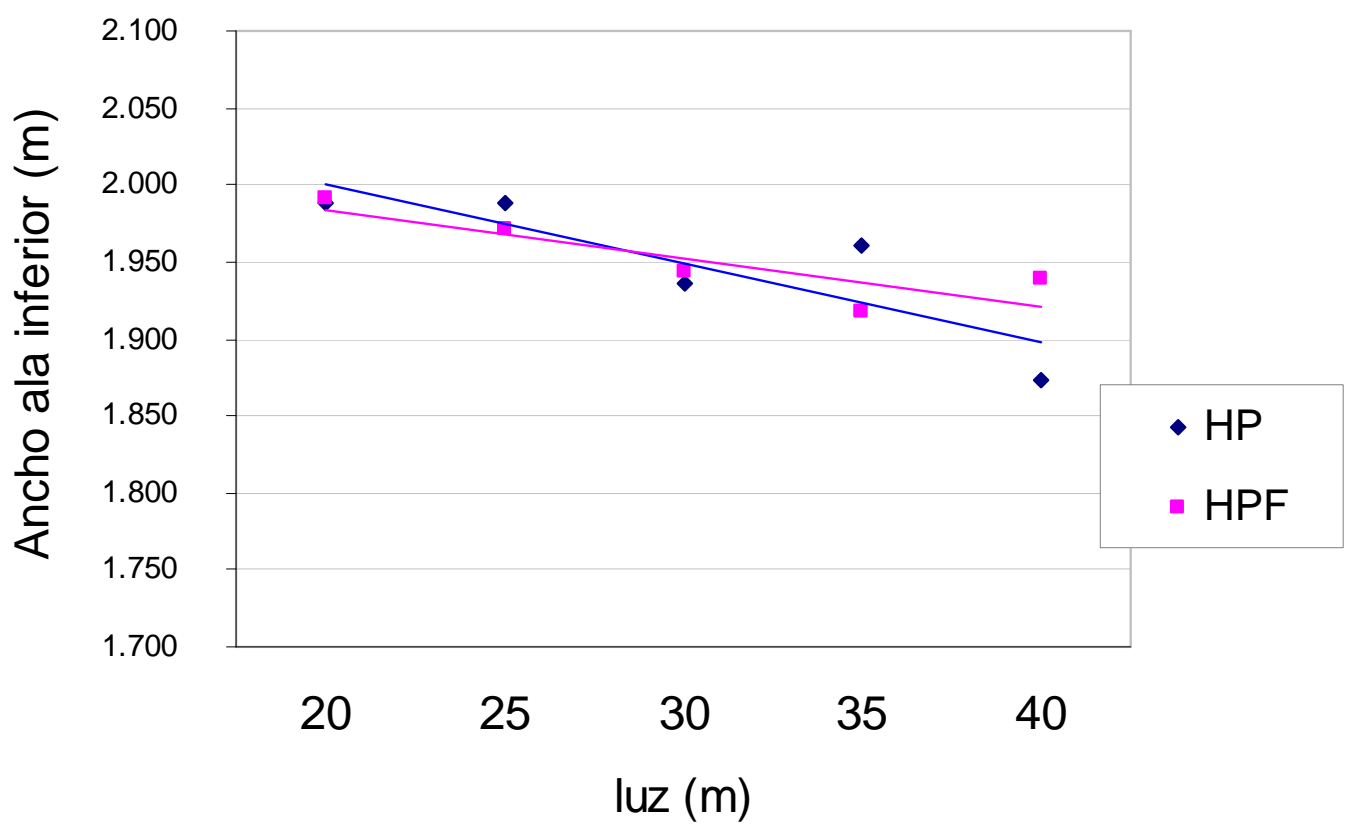

Figura 8-10: Ancho medio del ala inferior en función de la luz.

Se aprecia una leve tendencia en ambos casos a reducir el ancho del ala inferior al aumentar la luz del tablero.

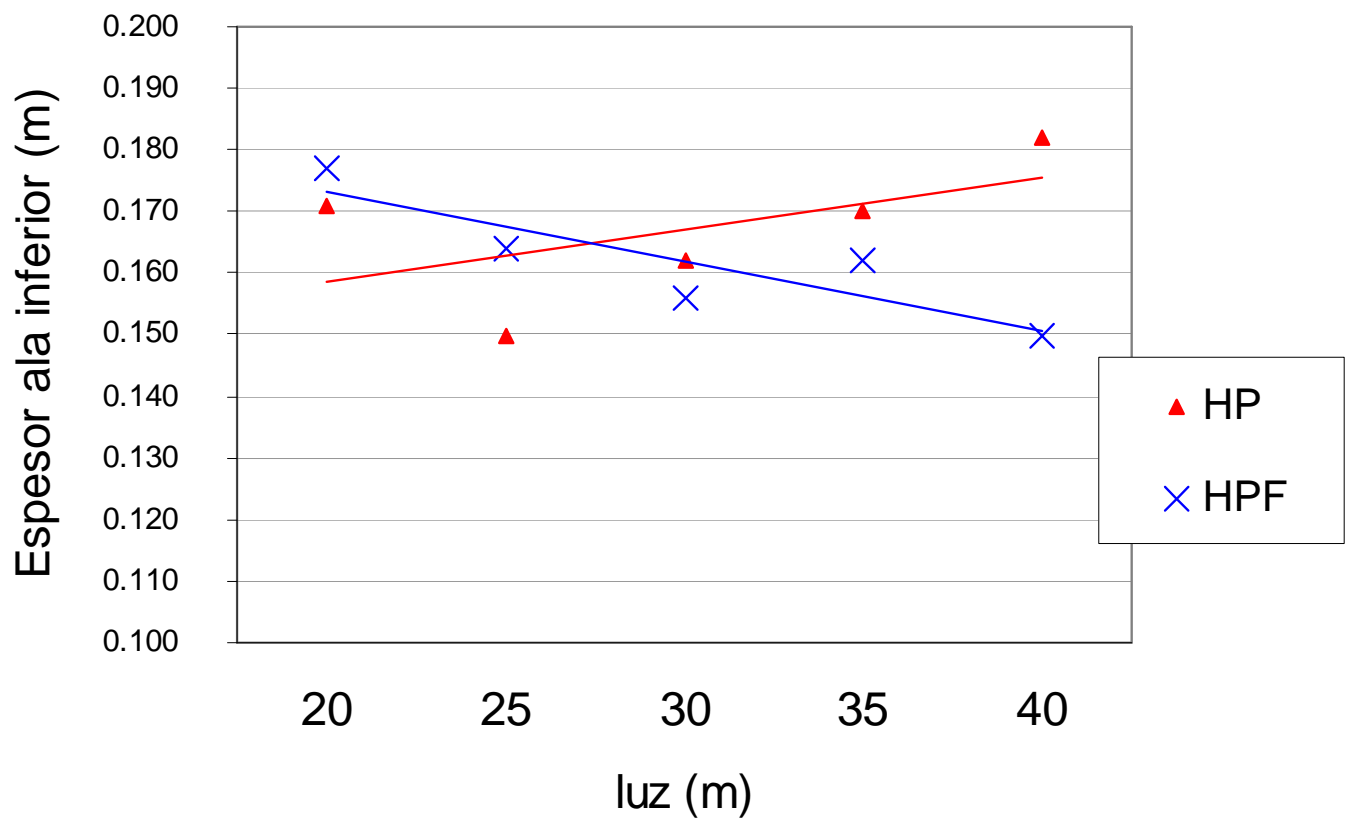

Figura 8-11: Espesor medio del ala inferior en función de la luz.

Si en el punto 7.3 se indicaba que existía una clara correlación lineal creciente en el HP a partir de una luz de 25 metros, observando la gráfica anterior, se desprende un 
comportamiento contrario, ya que existe cierta tendencia a reducir el espesor del ala inferior en el HPH al incrementarse la luz del tablero.

\subsubsection{Cuantías de hormigón y acero}

En la siguiente Tabla se presentan los resultados de las cuantías de hormigón y acero pasivo por metro cuadrado de tablero.

\begin{tabular}{|c|c|c|c|c|c|c|}
\hline \multirow{2}{*}{ LUZ } & \multicolumn{3}{|c|}{ HP } & \multicolumn{3}{c|}{ HPF } \\
\cline { 2 - 7 } & $\begin{array}{c}\mathrm{m} 3 \mathrm{H} / \mathrm{m} 2 \\
\text { tablero }\end{array}$ & $\begin{array}{c}\text { Kg pasivas/m2 } \\
\text { tablero }\end{array}$ & - & $\begin{array}{c}\mathrm{m} 3 \mathrm{H} / \mathrm{m} 2 \\
\text { tablero }\end{array}$ & $\begin{array}{c}\text { Kg pasivas/m2 } \\
\text { tablero }\end{array}$ & - \\
\hline 20 & 0.364 & 55.725 & & 0.355 & 57.929 & \\
\hline 25 & 0.330 & 57.353 & & 0.333 & 60.574 & \\
\hline 30 & 0.339 & 59.636 & & 0.329 & 62.678 & \\
\hline 35 & 0.352 & 58.825 & & 0.338 & 64.497 & \\
\hline 40 & 0.337 & 61.228 & & 0.328 & 66.346 & \\
\hline
\end{tabular}

Tabla 8-7: Cuantías de hormigón y acero pasivo.

A continuación se representan las Figuras correspondientes a la Tabla:

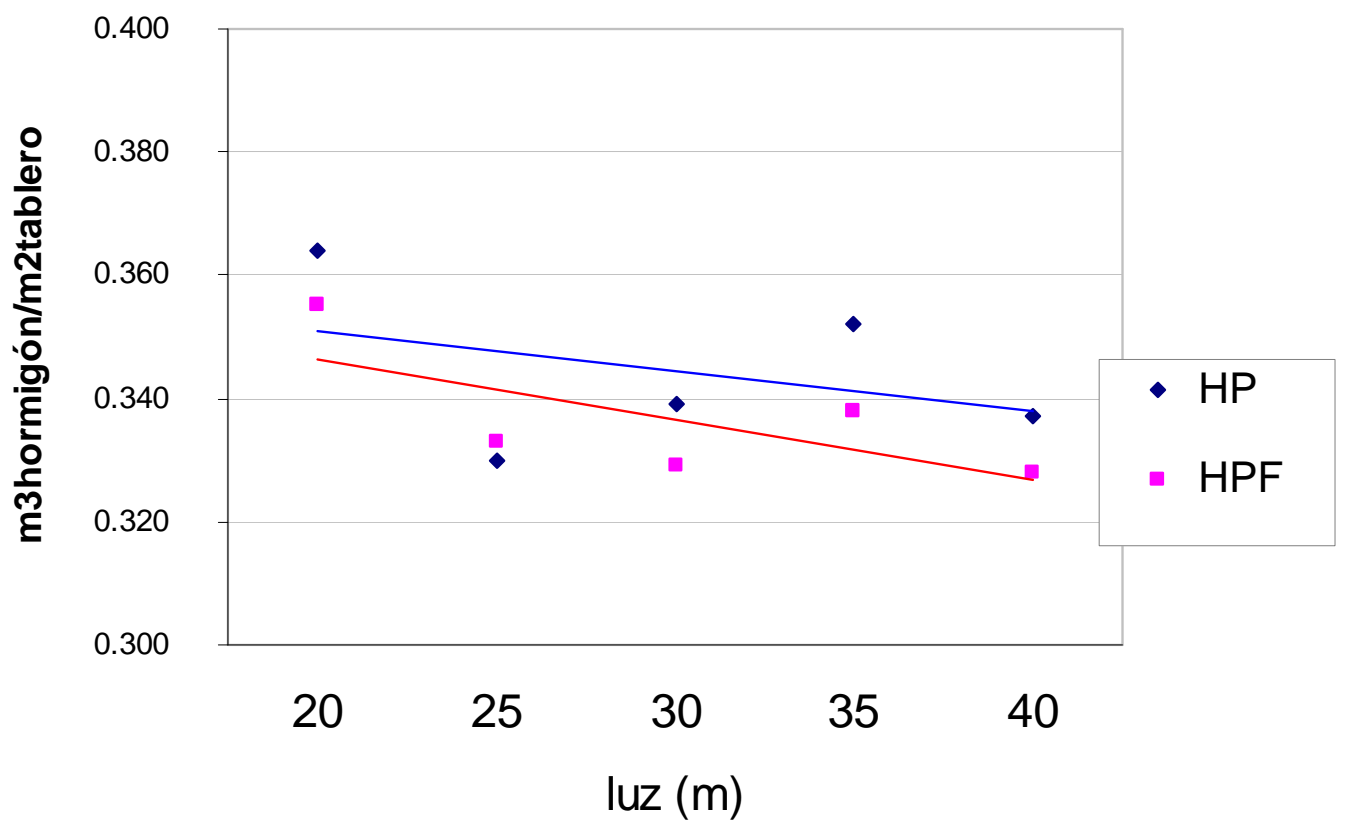

Figura 8-12: Cuantía de hormigón por $\mathrm{m}^{2}$ de tablero.

Se aprecia cierta tendencia a reducir la cuantía de hormigón al aumentar la luz. Se recibe con cierta curiosidad, puesto que parece lógico que fuera alrevés, es decir, al aumenta la luz debería también aumentar la cuantía puesto que la viga tiene más canto. Sin embargo, ya se observaba en la Figura 8-5, que al aumentar la luz, se reducía el espesor de la losa, y también, en la Figura 8-10, al aumentar la luz se reducía el ancho del ala inferior de la viga. Ambas disminuciones justifican finalmente la reducción de cuantía del hormigón. 


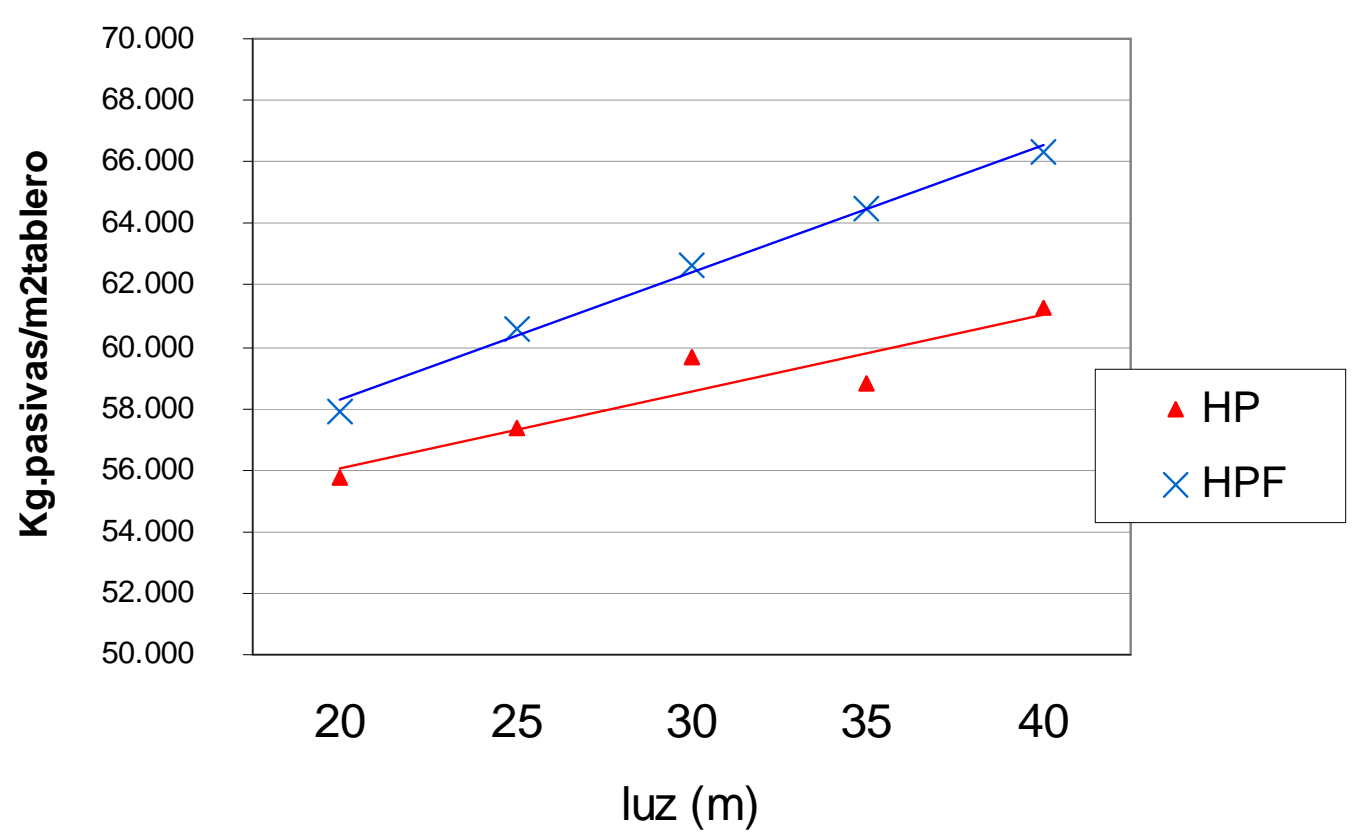

Figura 8-13: Cuantía de acero pasivo por $\mathrm{m}^{2}$ de tablero.

En la Figura 8-13 se observa, como era de esperar, que aumenta la cuantía de acero pasivo al incrementarse la luz. Lo que en un principio no parece lógico es que la cuantía del acero pasivo sea superior con el HPF que con el HP, dado que en el HPF, las fibras de acero contribuyen a la resistencia a flexión longitudinal y a cortante, por lo que aparentemente, debería ser menor. Vamos a intentar responder a esta paradoja. Tomemos el caso del precio del hormigón en viga, por ejemplo para un HPF-45 es de $142.15 € / \mathrm{m} 3$. Si se utilizan fibras de fR3k igual a 7 , se necesitan $60 \mathrm{Kg}$ de acero por $\mathrm{m}^{3}$ de hormigón, y a $1 € / \mathrm{Kg}$ que es su precio, repercute en que el hormigón cueste $202,15 € / \mathrm{m} 3$, un $42.2 \%$ más caro. Por lo tanto, la optimización económica buscará reducir al máximo el volumen de hormigón en la viga. Las fibras contribuyen a la resistencia a cortante de la sección transversal, pero al reducirse el área de la sección, su aportación no es suficiente, y obliga a que el acero pasivo deba contribuir más, sale más económico. Por otro lado, no hay que reducir en demasía la sección del hormigón, puesto que la contribución de las fibras permite reducir el número de torones, tal como se ha visto en la Figura 8-6, y el coste de la armadura activa es elevado, por lo que deberá buscarse la máxima inercia, y eso se consigue reduciendo espesores del ala inferior. Resumiendo, para llegar a un equilibrio reduciendo costes, se obliga a:

Reducir el volumen de hormigón

Reducir el número de torones

Aumentar la armadura pasiva transversal 


\subsection{Conclusiones}

A continuación, se resumen en dos grupos, heurísticas y hormigones, las principales conclusiones de este estudio comparativo entre el HPF y el HP.

\subsubsection{Heurísticas}

- La metaheurística empleada en el trabajo -SA-, adaptándola a las nuevas características del hormigón con fibras HPF, también se ha demostrado eficaz para el diseño automatizado y optimizado de tableros para pasos superiores con vigas artesa pretensadas. Las desviaciones producidas son muy pequeñas, del orden del 1\%, y los tiempos de proceso son similares a los del HP, pudiendo utilizarse también de un modo práctico en casos reales.

\subsubsection{Hormigones con fibras}

- Los resultados muestran que, para los precios unitarios empleados en este estudio, el tablero se optimiza mediante secciones de canto alto y espesores mínimos, dotando de gran esbeltez a la sección transversal. Los cantos de las vigas tienden a agotar la relación canto/luz =1/18.

- En cuanto al comparativo de costes mediante el uso de HPF, para los precios considerados, y en especial el correspondiente al coste de las fibras, sale algo más económico el uso de éstas que con HP, entorno al 1\%. Éste margen es muy estrecho, lo que hace que sea muy sensible el resultado a una leve variación de precios. Pero lo que sí se puede afirmar es que el uso de fibras es económicamente competitivo y que, mediante un buen control de este material, puede aplicarse indistintamente donde se aplica el hormigón tradicional HP.

- Con los precios aplicados a los hormigones, la tendencia es a utilizarlos de elevada resistencia.

- Con los precios de las fibras, y su dosificación, la tendencia es a utilizar hormigones con altas resistencias a tracción.

- La inclusión de las fibras metálicas en el hormigón, permite considerar cierta resistencia a tracción de la viga en la zona situada por debajo de la fibra neutra, reduciéndose la necesidad del uso de armadura activa.

- El uso de fibras, incrementa considerablemente el precio del hormigón (del orden del $40 \%$ ), por lo que la heurística, en su proceso de optimización económica, reduce el volumen de hormigón. Ante las solicitaciones normales se considera la aportación de las fibras a esfuerzo cortante, pero se necesita incrementar la armadura pasiva transversal para compensar la reducción de la aportación de resistencia de la sección de hormigón.

- Al igual que para el HP, se puede adaptar el modelo al proceso de fabricación de las distintas industrias de prefabricados, condicionando variables y modificando parámetros, de un modo sencillo. 


\section{CAPÍTULO 9. CONCLUSIONES Y FUTURAS LÍNEAS DE INVESTIGACIÓN}

El objeto de esta tesis ha sido la aplicación de métodos heurísticos en el diseño óptimo económico automatizado de tableros de pasos superiores constituidos con vigas artesa de hormigón pretensado. Con ello, se pretende ofrecer a los ingenieros proyectistas de puentes una herramienta eficaz en su trabajo, y además, poner a disposición de la industria del prefabricado del hormigón un instrumento que permita adaptar sus diseños a la fluctuación de los precios de las materias primas con el fin de consumir menos recursos materiales y empresariales.

Para la obtención del diseño, el procedimiento establece dos partes fundamentales: la comprobación estructural y el método de optimización utilizado. Se obtienen los resultados para distintas las heurísticas aplicadas, y con la que mejor se adapta al problema se realiza un estudio paramétrico y posteriormente un comparativo con la aplicación del hormigón reforzado con fibras de acero en la viga. Además, por su novedad se introduce este trabajo dentro de la bibliografía del momento (estado del arte). A continuación se resumen las principales conclusiones a las que se llega a lo largo de los capítulos anteriores desde los puntos de vista descritos: estado del arte, medios de optimización empleados, estructura objeto del estudio, estudio paramétrico y análisis comparativo con el hormigón con fibras HPF. Finalmente se presentan las posibles futuras líneas de investigación.

\subsection{Conclusiones}

\subsubsection{Estado del arte}

De la revisión bibliográfica realizada se concluye que:

- La mayoría de los trabajos de optimización realizados por los investigadores han sido de estructuras metálicas, dedicándose para las de hormigón una pequeña parte de los mismos. De éstos últimos, una porción reducida hacen referencia a tableros de puentes. Se han publicado, algo más de una treintena de trabajos relacionados con la optimización de estructuras de hormigón pretensado, y entre ellas unas pocas hacen referencia a tableros de puentes. En cuanto a optimizaciones heurísticas de estructuras de hormigón pretensado sólo se han encontrado cinco referencias, no correspondiendo ninguna de ellas a la estructura de esta tesis, ni tampoco a HPF. 
- Generalmente, en los trabajos relacionados con el hormigón pretensado, se busca una técnica matemática de optimización exacta, y para ello se simplifica el comportamiento de la estructura, no considerando efectos importantes como el cortante, torsor, fatiga, rasante, flecha, etc. Esto comporta la realización de trabajos muy teóricos, que adolecen de una aplicación práctica inmediata.

- De otro lado, cuando se realiza la optimización de tableros de vigas se acaba reduciendo el problema a diseñar un pretensado que controle las tensiones en servicio y la fisuración, y una armadura pasiva por secciones que soporte los estados últimos. Además, buscan obtener soluciones óptimas respecto a la cuantía de los materiales empleados, esbelteces, pretensado, etc., sin tener en cuenta los costes económicos.

\subsubsection{Optimización heurística}

En el Capítulo 6 se implementaron diversos algoritmos basados en técnicas heurísticas y metaheurísticas para la optimización de la estructura del presente trabajo. Hay que señalar que algunas de estas técnicas han sido recientemente aplicadas para tableros de hormigón pretensado, y al igual que en ellos, se ha encontrado un buen funcionamiento de los algoritmos en la optimización. De la comparación entre los distintos métodos aplicados se desprenden las siguientes conclusiones:

- La factibilidad de las soluciones en las estructuras con vigas artesa pretensadas es muy baja. Las correcciones del pretensado y de la armadura transversal para una sección dada, ayudan a encontrar una solución inicial de partida. En este caso, partir de una solución intencionadamente sobredimensionada, no mejora el rendimiento de la heurística.

- El número de variables que deben modificarse, para conseguir el movimiento más eficaz en las metaheurísticas basadas en la búsqueda secuencial por entornos, es del $15 \%$.

- Se han aplicado las siguientes heurísticas, incluyendo variantes de éstas: RW, DLS, SA, TA, GA y MA. Las dos últimas, de carácter poblacional, no han resultado ser competitivas respecto a las de búsqueda por entornos, siendo las más eficaces y distanciadas de las demás el SA y el TA. La calidad de dichos algoritmos es elevada a tenor de las escasas desviaciones que se producen con los mejores ajustes, incluso para un número alto de reinicios -desviación del $1.78 \%$ para 40 reinicios-. Si a esto se le añade que los tiempos de proceso son suficientemente aceptables, ambas heurísticas se pueden utilizar de un modo práctico en casos reales.

- La heurística que mejores resultados ha presentado se corresponde con el algoritmo SA, con cadenas de Markov de 2500 iteraciones y coeficiente de enfriamiento de 0.95 -S3-. Su tiempo de proceso es de unas cinco horas con el procesador utilizado para este trabajo. Además, se propone, dentro de la 
metodología de la aplicación de esta heurística, una reiniciación de 9 veces consecutivas.

- El modelo diseñado se adapta correctamente a la variación de los precios del hormigón y del acero para optimizar el coste de la estructura. Al incrementarse el precio del acero, disminuye su cuantía y aumenta la del hormigón, y al aumentar el precio del hormigón, ocurre lo contrario. El coste del tablero es más sensible a la variación del precio del acero que del hormigón, del orden de tres veces. Sin embargo, no afecta a los cambios en la geometría de la sección, incluido el pretensado, salvo una ligera adaptación del canto de la losa. Ello muestra que el canto de la sección y el pretensado están más condicionados por su capacidad resistente ante las solicitaciones longitudinales que ante las variaciones de los precios. Consecuentemente, la esbeltez de la viga no varía, manteniéndose alrededor del límite impuesto del $1 / 18$ de la luz.

- La heurística elegida para la realización del estudio paramétrico y de la comparación de resultados respecto al uso de vigas de HPF, de los Capítulos 7 y 8 , ha sido el S3, debido tanto a los buenos resultados obtenidos por el mismo y por la baja dispersión entre ellos, como por los tiempos de ejecución.

\subsubsection{Tableros con vigas artesa}

Del análisis comparativo de los resultados obtenidos en el Capítulo 6, para luces de 35 metros, se pueden obtener las siguientes conclusiones referidas a la optimización de los tableros isostáticos con vigas artesa en puentes de carreteras:

- Por economía del volumen de hormigón hay una búsqueda de una sección que proporcione mayor inercia y sea a la vez lo más ligera posible. Ello conlleva cantos de viga altos y espesores pequeños en alas y almas, por lo que repercute en una necesidad menor de pretensado, no siendo necesario en este problema los torones del tercer nivel. En la losa, la reducción de su espesor se compensa estructuralmente con una mayor cuantía de acero pasivo. El canto de la viga lo determina el límite de esbeltez impuesto -en este caso de (1/18)Luz-.

- El intento de aligerar la estructura conduce a utilizar hormigones de mayor resistencia característica, entre 40 y $50 \mathrm{Mpa}$, en viga. En la losa, cuanto mayor es el canto, menor resistencia del hormigón se necesita, entre 30 y 40 Mpa.

- Respecto a las alas superiores de la viga, se tiende a dimensiones cortas y con armadura activa mínima, la suficiente para impedir que fisure dicha ala durante el tesado. 


\subsubsection{Estudio paramétrico}

Del estudio comparativo de los tableros con vigas pretesas de HP de distintas luces en apoyos isostáticos se pueden extraer las siguientes conclusiones:

- El canto de la viga y el número de torones, muestran una fuerte correlación lineal, con coeficientes de determinación muy próximos a la unidad.

- Los cantos de las vigas tienden a agotar la relación canto/luz $=1 / 18$, siendo muy uniforme, independientemente de la luz. Dicha esbeltez, se mantiene sin variaciones apreciables aunque cambien los precios del hormigón y del acero.

- Existe una tendencia a reducir la resistencia característica del hormigón de la viga al aumentar la luz, comportando una correlación fuertemente cuadrática.

- El resto de variables geométricas, sólo muestran tendencias, con coeficientes de determinación bajos, así se puede decir que al aumentar la luz: el ancho del ala inferior tiende a reducirse, mientras su espesor tiende a aumentar, el espesor del alma toma el mínimo dispuesto en este problema, y que el ancho y espesor del ala superior toman valores que varían muy poco entre sí y que no muestran tendencia alguna.

- La cuantía del hormigón respecto a la superficie del tablero muestra una ligera tendencia a la reducción de ésta al incrementarse la luz, pero no existe una clara correlación al respecto. Sin embargo, si existe cierta correlación de carácter potencial en cuanto al incremento de la cuantía de la armadura pasiva con respecto a la luz.

\subsubsection{Estudio comparativo HP vs HPF}

De la utilización de hormigón con fibras de acero HPF en la fabricación de las vigas y del estudio parametrizado comparativo con las vigas de HP se pueden extraer las siguientes conclusiones:

- La metaheurística empleada en el trabajo -SA-, también se ha demostrado eficaz para el diseño automatizado y optimizado de tableros para pasos superiores con vigas artesa pretensadas de HPF, pudiendo utilizarse también de un modo práctico en casos reales.

- Los resultados muestran que el tablero se optimiza, al igual que con HP, mediante secciones de canto alto y espesores mínimos, dotando de gran esbeltez a la sección transversal. La esbeltez longitudinal de la viga es también muy uniforme, independientemente de la luz, con valores que agotan el límite impuesto de 1/18.

- Respecto al comparativo de costes, sale algo más económico el uso de HPF, entorno al 1\%. Éste margen es muy estrecho, lo que hace que sea muy sensible el resultado a una leve variación de precios. Se puede afirmar que el 
uso de fibras es económicamente competitivo y que, mediante un buen control de este material, puede aplicarse indistintamente donde se aplica el hormigón tradicional HP.

- La tendencia es a utilizar hormigones de elevada resistencia, tanto en la viga como en la losa.

- Con la inclusión de fibras metálicas en el hormigón, la normativa permite considerar cierta resistencia a tracción de la viga en la zona situada por debajo de la fibra neutra, reduciéndose la necesidad del uso de armadura activa.

- El uso de fibras, incrementa considerablemente el precio global del hormigón (del orden de un 40\%), por lo que la heurística, en su proceso de optimización económica, tiende a reducir el volumen de hormigón. Ante solicitaciones normales se puede considerar la aportación de las fibras a esfuerzo cortante, pero se necesita incrementar la armadura pasiva transversal para compensar la reducción de la aportación de resistencia de la sección de hormigón.

- Al igual que para el HP, se puede adaptar el modelo al proceso de fabricación de las distintas industrias de prefabricados, condicionando variables y modificando parámetros, de un modo sencillo.

\subsubsection{Resumen}

- Se aplica un método que obtiene diseños óptimo económicos automatizados de tableros de pasos superiores constituidos con vigas artesa de hormigón pretensado.

- Se pone a disposición de la industria del prefabricado del hormigón, una herramienta que permite adaptar sus diseños de tableros de puentes a la fluctuación de precios de un mercado globalizado, obteniendo estructuras que consumen menos recursos materiales y empresariales.

\subsection{Futuras líneas de investigación}

Por último, como líneas de investigación que pueden plantearse a partir de este trabajo pueden citarse las siguientes:

- Realizar estudios multiobjetivo que tengan en cuenta además del coste de los materiales y del proceso constructivo otros aspectos como la sostenibilidad medioambiental -emisiones de $\mathrm{CO}_{2-}$, vida útil -coste de mantenimiento y reposición-, etc.

- Completar el estudio de la sensibilidad a la variación de precios afectando la fluctuación a un mayor número de precios: hormigón viga, hormigón losa, 
acero activo, acero pasivo en viga y acero pasivo en losa. Realizar el mismo trabajo para el HPF con una variación del precio de las fibras.

- Realizar el mismo estudio paramétrico tomando como variable la separación entre las vigas. Cambiar la disposición y geometría de las armaduras pasivas adaptadas a otros fabricantes de vigas.

- Adaptar el modelo para más de dos vigas.

- Adaptar el modelo para puentes de ferrocarril.

- Adaptar el modelo como viga continua.

- Empleo de las mismas técnicas heurística u otras, con movimientos más sofisticados que permitan acelerar la convergencia. 


\section{REFERENCIAS}

[1] "Instrucción para el proyecto y la ejecución de obras de hormigón pretensado EP-93”. Ministerio de Obras Públicas y Transporte. Madrid 1997.

[2] Aarts, E.H..L.; Van Laarhoven, P.J.M. "Statistical cooling: a general approach to combinatorial optimisation problems". Philips Journal of Research, 40:193226.

[3] Adeli, H. (Ed.). “Advances in Design Optimization”. Chapman \& Hall, Londres, 1994.

[4] Adeli, H.; Cheng, N.T. "Augmented Lagrangian Genetic Algorithm for structural optimization”. ASCE Journal of Aerospace Engineering 7(1): 104118. 1994.

[5] Adeli, H.; Kumar, S. "Distributed Genetic Algorithm for structural optimization”. ASCE Journal of Aerospace Engineering 8(3): 156-163. 1995.

[6] Alcalá González, Julián "Optimización heurística económica de tableros de puentes losa pretensados". Tesis Doctoral. Universidad Politécnica de Valencia, Dpto. Ingeniería de la Construcción, 292 pp. 2010.

[7] Alcalá, Carrera, Glez. Vidosa y Yepes (2005), “Cristalización simulada aplicada a la optimización económica de muros ménsula de contención de hormigón armado", Hormigón y Acero, No. 236, pp 97-108, abril-junio 2005.

[8] Alcalá, Carrera, Perea, Yepes y González Vidosa, "Cristalización simulada aplicada a la optimización económica de muros de contención", Actas III Congreso de ACHE de Puentes y Estructuras, Zaragoza, noviembre 2005, pp. 693-705.

[9] Alcalá, J.; Carrera, M.A.; González-Vidosa, F.; Yepes, V. “Cristalización simulada aplicada a la optimización económica de muros ménsula de contención de hormigón armado". Hormigón y Acero, 236: 97-108. 2005.

[10] Alfonso Carbonell Lombardero (2005), "Optimización heurística de bóvedas de paso de carretera", Informe interno CST/GPRC-05, Universidad Politécnica de Valencia, Dep. Ingeniería de la Construcción, Diciembre 2005, 306 pp. 
[11] Al-Gahtani, A.S.; Al-Saadoun, S.S.; Abul-Feilat, E.A. "Design optimization of continuous partially prestressed concrete beams". Computers \& Structures, 55(2): 365-370. 1995.

[12] Al-Harthy, A.A.; Frangopol, D.M. "Reliability-based design of prestressed concrete beams". Journal of Structural Engineering, 120(11): 3156-3177. 1994.

[13] Ali, N.; Behdinan K.; Fawaz, Z. "Applicability and viability of a GA based finite element analysis architecture for structural design optimization". Computer and Structures, 81: 2251-2271. 2003.

[14] Althöfer, I.; Koschnick, K-U. "On the Convergence of "Threshold Accepting"'. Applied Mathematics and Optimization 24:183-195. 1991.

[15] Aparicio, A C.; Casas, J.R.; Ramos, G. "Computer aided design of prestressed concrete highway Bridges”. Computers \& Structures, 60(6): 957-969. 1996.

[16] Arenas, Juan J.; Villegas, Luis "Análisis en teoría de segundo orden de las pilas del viaducto de Cruzul” Hormigón y Acero 171: 35-55 (1989).

[17] Atabay, Ş. "Cost optimization of three-dimensional beamless reinforced concrete shear-wall systems via genetic algorithm”. Expert systems with Applications 36:3555-3561. 2009.

[18] Balling, R.; "Optimal Steel frame design by Simulated Annealing”. ASCE Journal of Structural Engineering 117(6): 1780-1795.1991.

[19] Balling, R.; Yao, X. "Optimization of reinforced concrete frames". ASCE Journal of Structural Engineering 123 (2): 193-202. 1997.

[20] Barakat, S.; Altoubat, S. "Application of evolutionary global optimization techniques in the design of RC water tanks". Engineering Structures, 31(2): 332-334. 2009.

[21] Barakat, S.; Bani-Hani, K.; Taha, M.Q. "Multi-objective reliability-based optimization of prestressed concrete beams". Structural Safety, 26(3): 311-342. 2004.

[22] Barakat, S.; Kallas, N; Taha, M.Q. "Single objective reliability-based optimization of prestressed concrete beams". Computers \& Structures, 81(2627): 2601-2512. 2003.

[23] Bonet, J.L.; Romero, M.L.; Miguel, P.F.; Fernandez, M.A. “A fast stress integration algorithm for reinforced concrete sections with axial loads and biaxial bending". Computers \& Structures, 82(2-3): 213-225. 2004. 
[24] Brotchie, J. F. "A criterion for optimal design of plates". Journal ACI, 66(11): 898-906. 1969.

[25] Camp, C.; Bichon, B. "Design of space trusses using Ant Colony optimization" ASCE Journal of Structural Engineering 131(3): 369-379. 2004.

[26] Camp, C.; Pezeshk, S.; Hansson, H. "Flexural design of reinforced concrete frames using a genetic algorithm". ASCE Journal of Structural Engineering 130 (5): 741-751. 2003.

[27] Carbonell Lombardero, Alfonso "Optimización heurística de bóvedas de paso de hormigón armado”. Tesis Doctoral. Universidad Politécnica de Valencia, Dpto. Ingeniería de la Construcción, 283 pp. 2009.

[28] Carbonell, A.; Alcalá, J.; González-Vidosa, F.; Yepes, V. "Optimización heurística de bóvedas de hormigón armado para pasos de carretera”. Ingeniería Civil (145): 79-88. 2007.

[29] Carbonell, A.; Martínez, F.J.; Yepes, V.; Hospitaler, A.; González-Vidosa, F. "Threshold accepting optimization of road vaults and rectangular hollow bridge piers", Proceedings of the Tenth International Conference on Computer Aided Optimum Design in Engineering (OPTI-2007), Ed. WIT Press, Myrtle Beach (South Carolina, USA), 7-9 May, 2007, pp. 141-150.

[30] Carbonell, A.; Yepes, V.; González-Vidosa, F. "Heuristic optimization of reinforced concrete road vault underpasses". Proceedings Sixth International Conference on Engineering Computational Technology. Athens (Greece), 2-5 September, 2008.

[31] Carbonell, Alcalá, González-Vidosa y Yepes, “Optimización heurística de bóvedas de hormigón armado para pasos de carretera”, revista Ingeniería Civil, No. 145, pp. 79-88, 2007.

[32] Carrera, Alcalá, Yepes y Glez. Vidosa (2005), "Optimización heurística de pórticos de paso de carretera de hormigón armado", Hormigón y Acero, No. 236, pp 85-95, abril-junio 2005.

[33] Carrera, Alcalá, Yepes y González Vidosa, "Optimización por cristalización simulada de pórticos de paso de carretera de hormigón armado", Actas III Congreso de ACHE de Puentes y Estructuras, Zaragoza, noviembre 2005, pp. 707-717.

[34] Carrera, M.A.; Alcalá, J.; Yepes, V.; González-Vidosa, F. "Optimización heurística de pórticos de paso de carretera de hormigón armado", Hormigón y Acero, 236: 85-95. 2005. 
[35] Ceranic, B.; Fryer, C. and Bines, R. W. "An application of simulated annealing to the optimum design of reinforced concrete retaining structures". Computers and Structures, 79:1569-1581. 2001.

[36] Černy, V. "A thermodynamical approach to the travelling salesman problem: an efficient simulation algorithm". Journal of Optimization Theory and Applications, 45:41-51, 1985.

[37] Clark, C.; Yi Wu, Z. "Integrated hydraulic model and genetic algorithm optimization for informed analysis of a real water system". ASCE 8th Annual International Symposium on Water Distribution System Analysis, Cincinnati, Ohio, August 27-30. 2006.

[38] Coello, C. A. "Uso de Algoritmos Genéticos para el Diseño Optimo de Armaduras" Congreso Nacional de Informática "Herramientas Estratégicas para los Mercados Globales", pp. 290-305, Fundación Arturo Rosenblueth, México, D.F., 14 al 17 de junio de 1994.

[39] Coello, C.A.; Christiansen, A. D.; Hernández, F.S. “A simple Genetic Algorithm for the Design of Reinforced Concrete Beams", Engineering with Computers, Vol. 13, 185-196. 1997.

[40] Cohn, M.Z., Dinovitzer, A.S. (1994). "Application of structural optimization" Struct. Engrg. J., ASCE, 120(2), 617-650.

[41] Cohn, M.Z.; Lounis, Z. "Optimal design of structural concrete bridge systems". Journal of Structural Engineering, 120(9): 2653-2674. 1994.

[42] Cohn, M.Z.; Lounis, Z. "Optimum limit design of continuous prestressed concrete beams”. Journal of Structural Engineering, 119(12): 3551-3570. 1993.

[43] Cohn, M.Z.; MacRae, A.J. "Optimization of structural concrete beams". Journal of Structural Engineering, 110(7): 1573-1588. 1984.

[44] Comisión Permanente del Hormigón "Instrucción de Hormigón Estructural (EHE-08)“. Ministerio de Fomento. Madrid. 2008.

[45] Comité Europeo de Normalización. Eurocódigo EC-2: Proyecto de Estructuras de Hormigón. Parte 2: Reglas de diseño en puentes de hormigón. UNE-ENV 1992-2. AENOR. 1992.

[46] Cristian Perea de Dios (2004), "Heuristic optimization of reinforced concrete road box frames", Informe interno CST/GPRC-03, Universidad Politécnica de Valencia, Dep. Ingeniería de la Construcción, 308 pp., julio 2004. Premio tesina fin de carrera Fundación Friedrich-Wilhelm, Universidad RWTH Aachen (Alemania). 
[47] Chakrabarty, B.K. "A model for optimal design of reinforced concrete beam", Struct. Engrg. J., ASCE, 118(1), 3238-3242. 1992.

[48] Chau, K. W.; Albermani, F.; "Knowledge-based system on optimum design of liquid retaining structures with Genetic Algorithms". ASCE Journal of Structural Engineering 129 (10): 1312-1321. 2003.

[49] Dawkins, R. "El gen egoísta". Salvat Editores SA. 408 pp. 1976.

[50] De Leao, M.T.P. "Marginal cost computation using simulated annealing" Electric Power Engineering, 1999. PowerTech Budapest 99. International Conference.

[51] Degertekin, S.O.; Saka, M.P.; Hayalioglu, M.S. "Optimal load and resistance factor design of geometrically nonlinear stell space frames via tabu search and genetic algorithm". Article in press. Accepted In Engineering Structures 22 March 2007.

[52] Degertekina, S.O.; Sakab, M.P. y Hayaioglu, M.S. "Optimal load and resistence factor design of geometrically nonlinear steel space frames via tabu search and genetic algorithm”. Engineering Structures, 30(1):197-205, 2008

[53] Dorigo, M.; Maniezzo, V.; Colorni, A. "The ant system: optimization by a colony of cooperating agents", IEEE Transactions on Systems, Man, and Cybernetics, Part B, vol. 26, no. 1, pp. 29-41, 1996.

[54] Dueck, G. "The great deluge algorithm and the record to record travel", Journal of Computation Physics, No 104, pp. 86, 1993.

[55] Dueck, G.; Scheuer, T. "Threshold Accepting: A general Purpose Optimization Algorithm appearing Superior to Simulated Annealing". Journal of Computational Physics 90:161-175. 1990.

[56] Erbatur, F.; Al Zaid, R., Dahman, N.A. "Optimization and sensitivity of prestressed concrete beams", Computers \& Structures, 45(5-6): 881-886. 1992.

[57] Galilei Linceo, Galileo. "Discorsi e dimostrazioni matematiche, intorno à due nuove scienze attenenti alla mecanica i movimenti locali” Leida. 1638.

[58] Gasch, M.S. "Optimización de estructuras de forjados reticulares”. Tesis doctoral. Universidad Politécnica de Valencia. 1991.

[59] Glover, F. "Future Paths for Integer Programming and Links to Artificial Intelligence", Computers and Operations Research 13:5, 533-549, 1986 
[60] Glover, F. "Tabu Search, Part I", ORSA Journal on Computing, 1, pp. 190206. 1983

[61] Glover, F., Laguna and M., Martí, R. "New Ideas and Aplications of Scatter Search and Path Relinking: Advances and Aplications", to appear in Theory and Applications of Evolutionary Computation: Recent Trends, A. Ghosh and S. Tsutsui (Eds.), Springer-Verlang. 2002.

[62] Glover, F., Laguna and M., Martí, R. "Scatter Search", to appear in Theory and Applications of Evolutionary Computation: Recent Trends, A. Ghosh and S. Tsutsui (Eds.), Springer-Verlang. 2002.

[63] Glover, F."A template for Scatter Search and Path Relinking”, Artificial Evolution, Lecture Notes in Computer Science 1363, J.-K. Hao, E. Lutton, E. Ronald, M. Schoenauer and D. Snyers (Eds.), Springer, pp. 13-54. 1998.

[64] Goble, G.G.; LaPay, W.S. "Optimum design of prestressed beams". Journals Proceedings ACI, 68(9): 712-718. 1971.

[65] Goldberg, D.E. "Genetic Algorithms in search, optimization and machine learning”. Addison-Wesley. Boston. 1989.

[66] Goldberg, D.E.; Samtani, M.P. "Engineering optimization via genetic algorithms". ASCE Proceedings of the Ninth Conference on Electronic Computation, pp. 471-482. 1986.

[67] Gong, Y.; Li, Q.; Xiao, Z. "The Application of Ant Algorithm in Structural Optimization" 6th World Congresses of Structural and Multidisciplinary Optimization" Rio de Janeiro, Brazil. 2005.

[68] Govindaraj, V.; Ramasamy, J.V. "Optimum detailed design of reinforced concrete continuous beams using Genetic Algorithms", Computers and Structures, Vol. 84, 34-48. 2005.

[69] Hajela, P. "Genetic Search - An Approach to the Nonconvex Optimization Problem". AIAA Journal, 26(7): 1205-1210. 1990.

[70] Hajela, P. "Genetic algorithms in automated structural synthesis". NATO Advanced Study Institute on Optimization and Decision Support Systems, Edinburgh (UK). June 26-July 7, 1989.

[71] Hasancebi, O.; Erbatur, F. "Layout optimization of trusses using Simulated Annealing". Advances in Engineering Software, 33: 681-696.2002. 
[72] Hernandez, S.; Fontan, A.N.; Diaz, J.; Marcos, D. "VTOP. An improved software for design optimization of pretressed concrete beams". Advances in Engineering Software, 41: 415-421. 2010.

[73] Holland, J. “Adaptation In Natural and Artificial Systems.", The University of Michigan Press, Ann Arbour, 1975.

[74] Hooke R.; Jeeves TA. Direct search solution of numerical and statistical problems. J Assoc Comput. March 1961; 8:212-29.

[75] Ignacio Payá Zaforteza (2005), "Optimización heurística de pórticos de edificación de hormigón armado”, Informe interno CST/GPRC-04, Universidad Politécnica de Valencia, Dep. Ingeniería de la Construcción, 290 pp., abril 2005.

[76] Ignacio Payá Zaforteza (2007), "Optimización heurística de pórticos de edificación de hormigón armado", tesis doctoral mención europea, publicación CST/GPRC-16, Universidad Politécnica de Valencia, Dep. Ingeniería de la Construcción, 265 pp. (lectura 24 septiembre 2007).

[77] J.P. Hart and A.W. Shogan (1987) Semi-greedy heuristics: An empirical study. Operations Research Letters, 6:107-114, 1987.

[78] Jenkins W.M. "Towards structural optimisation via the genetic algorithm". Computers \& Structures, 40(5): 1321-1327. 1991.

[79] Jenkins, W.M. "Structural optimisation with the genetic algorithm". The Structural Engineer, 69(24): 418-422. 1991.

[80] Johnson Jr., F.R. "An interactive design algorithm for prestressed concrete girders”. Computers \& Structures, 2(5-6): 1075-1088. 1972.

[81] Jones, H.L. "Minimum cost prestressed concrete beam design”. Journal of Structural Engineering, 111(11): 2464-2478. 1985.

[82] José Vicente Martí Albiñana, "Optimización heurística de pasarelas pretensadas de viga artesa prefabricada", Informe interno CST/GPRC-14, Universidad Politécnica de Valencia, Dep. Ingeniería de la Construcción, Febrero 2008, 248 pp.

[83] Karush, W. "Minima of Functions of Several Variables with Inequalities as Side Constraints". M.Sc. Dissertation 1939. Dept. of Mathematics, Univ. of Chicago, Chicago, Illinois.

[84] Kaveh, A.; Talatahari, S. "An improved ant colony optimization for design of planar steel frames". Engineering Structures 32, 864-873. 2010. 
[85] Kirkpatrick, S.; Gelatt, C.D.; Vecchi, M.P. "Optimization by simulated annealing”. Science 220: 671-680. 1983.

[86] Kirsch, U. "Optimized prestressing by linear programming". International Journal for Numerical Methods in Engineering, 7(2): 125-136. 1973.

[87] Kirsch, U. "Optimum design of prestressed beams". Computers \& Structures, 2(4): 573-583. 1972.

[88] Klein, B. "Direct use of extremal principles in solving certain optimization problems involving inequalities". Journal of the Operations Research Society of America, 3: 168-175. 1955.

[89] Kocer, FY y Arora, J S. "Design of prestressed Concrete Transmission Poles: Optimization Approach”. Journal of Structural Engineering, 122(7):804-814, 1996.

[90] Kong, J. "Optimal drift design of tall reinforced concrete buildings using genetic algorithms". Proc. Computational Mechanic. WCCM VI in conjunction with APCOM’04. Beijing, China. 2004.

[91] Kuhn, H. W.; Tucker, A. W. "Nonlinear programming”. Proceedings of 2nd Berkeley Symposium. University of California Press, Berkeley. 1951.

[92] Lagaros, N.; Charmpis, D.; Tsompanakis, Y.; Papadrakakis M. "Structural design in the framework of casacade evolutionary optimization". Proc. $6^{\text {th }}$ Congress of Structural and Multidisciplinary Optimization. Rio de Janeiro. 2005 .

[93] Lee, C. ; Ahn, J. "Flexural design of reinforced concrete frames by Genetic Algorithm”. ASCE Journal of Structural Engineering 129 (6): 762-773. 2003.

[94] Leite, J.P.B.; Topping, B. H. V. "Improves genetic operators for structural engineering optimization”. Advances in Engineering Software, 29(7-9): 529562. 1998.

[95] Leps, M.; Sejnoha, M. "New approach to optimization of reinforced concrete beams". Computers and Structures, Volume 81, Number 18: 1957-1966. 2003.

[96] Levy, M.M. "La statique graphique et ses applications aux constructions". Gauthier-Villars, Paris.1874.

[97] Lin, T.Y. "Load-balancing method for design and analysis of prestressed concrete structures". Journal Proceedings ACI, 60(6): 719-742. 1963. 
[98] Lounis, Z.; Cohn, M.Z. "Multiobjective optimization of prestressed concrete structures”. Journal of Structural Engineering, 119(3): 794-808. 1993.

[99] Lounis, Z.; Mirza, M. S.; Cohn, M.Z. "Segmental and conventional precast prestressed concrete I-bridge girders". Journal of bridge engineering, 2(3): 7382. 1997.

[100] Malekly, H., et al. "A fuzzy integrated methodology for evaluating conceptual bridge design”. Expert Systems with Applications. Doi:10.1016. 2010.

[101] Manoharan, S.; Shanmuganathan S. "A comparison of search mechanisms for structural optimization”. Computers and Structures, Volume 73: 363-372. 2003.

[102] Manterola, J "Puentes. Apuntes para su diseño, cálculo y construcción”. Vol II, pp. 814-816. Colegio de Ingenieros de Caminos, Canales y Puertos. 2006.

[103] Martí, JV (ponente); González-Vidosa, F y Alcalá, J. “Optimización heurística de pasarelas pretensadas de viga artesa prefabricada", IX Congreso Iberoamericano de Ingeniería Mecánica, 17-20 Noviembre 2009, Las Palmas de Gran Canaria, España. Las palmas

[104] Martí, JV; González-Vidosa, F (ponente) and Alcalá, J. "Heuristic optimization of prestressed concrete precast pedestrian bridges", Proceedings of the Eleventh International Conference on Optimum Design of Structures and Materials in Engineering (OPTI-2009), Ed. WIT Press, Algarve (Portugal), 810 June 2009.

[105] Martínez Martín, Francisco "Optimización heurística de pilas rectangulares huecas de hormigón armado". Tesis Doctoral. Universidad Politécnica de Valencia, Dpto. Ingeniería de la Construcción, 327 pp. 2008.

[106] Martínez, F.J.; González-Vidosa F.; Hospitaler, A. "Design of high speed railways bridge piers by ant colony optimization". Proceedings of the First International Conference on Soft Computing Technology in Civil, Structural and Environmental Engineering. Funchal, Madeira (Portugal), September 2009.

[107] Martínez, F.J.; Yepes, V.; Hospitaler A.; González-Vidosa, F. “Ant colony optimization of reinforced concrete bridge piers of rectangular hollow section". Proceedings of the Ninth International Conference on the Application of Artificial Intelligence to Civil, Structural and Environmental Engineering (AICC-2007). St. Julians (Malta), 18-21 September, 2007. 
[108] Martínez, FJ; González-Vidosa, F; Hospitaler, A y Yepes, V.:'Heuristic optimization of RC bridge piers with rectangular hollow sections". Computers and Structures 88 (2010) 375-386.

[109] Maxwell, C. Scientific Papers, 2, Dover Publications, New York, 1952): 175177. 1869.

[110] Medina, J.R. "Estimation of incident and reflected waves using simulated annealing". ASCE Journal of Waterway, Port, Coastal and Ocean Engineering 127(4):213-221. 2001.

[111] Metropolis, N.; Rosenbluth, A.W.; Rosenbluth, M.N.; Teller, A.H.; Teler, E. "Equation of state calculation by Fast Computing Machines". Journal of Chemical Physics, 21: 1087-1092. 1953.

[112] Miguel Carrera Hueso (2004), "Optimización heurística de pórticos de paso de carretera", Informe interno CST/GPRC-02, Universidad Politécnica de Valencia, Dep. Ingeniería de la Construcción, 205 pp., abril 2004.

[113] Ministerio de Fomento. "Instrucción sobre las acciones a considerar en el proyecto de puentes de carretera (IAP)". Madrid, 1998.

[114] Ministerio de Fomento. "Recomendaciones para el proyecto de puentes mixtos para puentes de carretera RPX-95". Madrid, 1995.

[115] Mitchell, A.G.M. "The Limits of Economy of Material in Frame Structures". Philosophical Magazine, Series 6, 8(47): 589-597. 1904.

[116] Miyamoto, A.; Nakamura, H.; Kruszka, L. "Application of the Improved Immune Algorithm to Structural Design Support System" ASCE. Journal of Structural Engineering. 130 (1): 108-119. (2004).

[117] Mladenovich, N. and Hansen, P., "Variable Neighborhood Search", Journal of Computation Physics, No 24, pp. 1097-1100, 1997.

[118] Moragues, J. "Diseño óptimo de estructuras aporticadas de hormigón armado". Tesis doctoral. Universidad Politécnica de Valencia. 1980.

[119] Moscato, P., "On evolution, search, optimization, genetic algorithms and Martial Arts: Towards Memetic Algorithms“, Caltech Concurrent Computation Program, C3P Report 826, (1989).

[120] Müller, D. E. "A Method for Solving Algebraic Equations Using an Automatic Computer". Committee on Mathematical Tables and Other Aids to Computation, 10: 208-215. 1956. 
[121] Naaman, A.E. "Minimum cost versus minimum weight of prestressed slabs". Journal of the Structural Division ASCE, 102(7): 1493-1505. 1976.

[122] Navarrina Martínez, Fermín Luis “Una metodología general para optimización estructural en diseño asistido por ordenador". Tesis Doctoral, 1987.

Universidad Politécnica de Cataluña.

[123] O.C. 11/2002. "Criterios a tener en cuenta en el proyecto y construcción de puentes con elementos prefabricados de hormigón estructural". D.G.C. O.C. 11/2002 (BOE 27/11/02)

[124] O.C. 302/89T. "Obras de paso de nueva construcción. Conceptos generales". D.G.C. 2000 (mayo).

[125] Payá, I.; Yepes, V.; González-Vidosa F; Hospitaler, A. "Cost versus sustainability of reinforced concrete building frames by multiobjective optimization". Proceedings First International Symposium on Life Cycle Civil Engineering (IALCCE-08). Varenna (Italy), 11-14 June, 2008.

[126] Payá, I.; Yepes, V.; González-Vidosa, F. "Diseño optimizado de estructuras de edificación considerando su coste económico y su facilidad constructiva", Proceedings XXXIII Jornadas Sudamericanas de Ingeniería Estructural. Santiago (Chile), 26-30 Mayo 2008.

[127] Payá, I.; Yepes, V.; González-Vidosa, F.; Hospitaler, A. "Multiobjective optimization of reinforced concrete building frames by simulated annealing". Computer-Aided Civil and Infrastructure Engineering, 23(8): 575-589. 2008.

[128] Payá, I.; Yepes, V.; Hospitaler, A.; González-Vidosa, F. " $\mathrm{CO}_{2}$-optimization of reinforced concrete frames by simulated annealing". Engineering Structures (article in press). 2009.

[129] Payá, I.; Yepes, V.; Hospitaler, A.; González-Vidosa, F. “On the statistical estimation of the minimum cost of RC frames designed by heuristic optimization". Proceedings Second Conference on Multidisciplinary Design Optimization and Applications (ASMDO-2008). Gijón (Spain), 2-5 September, 2008.

[130] Payá, Yepes, Clemente y Glez.Vidosa, "Optimización heurística de pórticos de edificación de hormigón armado", aceptado Revista Internacional de Métodos Numéricos para Cálculo y Diseño en Ingeniería, Vol. 22, No. 3, pp. 241-259, Septiembre 2006.

[131] Perea de Dios, Cristian (2006), "Optimización heurística de marcos de carretera de hormigón armado”, Informe interno CST/GPRC-07, Universidad 
Politécnica de Valencia, Dep. Ingeniería de la Construcción, 107 pp., mayo 2006.

[132] Perea de Dios, Cristian "Heuristic optimization of reinforced concrete frame bridges". Tesis Doctoral. Universidad Politécnica de Valencia, Dpto. Ingeniería de la Construcción, 202 pp. 2007.

[133] Perea, Alcalá, Martínez, Yepes y González-Vidosa (2006), "Optimum design of the box frame tunnel of the Underground Line Palma - UIB", Proceedings X International Conference on Project Engineering, Valencia, 12-15 septiembre 2006.

[134] Perea, Alcalá, Yepes y González Vidosa, "Optimización por cristalización simulada de marcos de carretera de hormigón armado", Actas III Congreso de ACHE de Puentes y Estructuras, Zaragoza, noviembre 2005, pp. 719-728.

[135] Perea, Alcalá, Yepes, González-Vidosa y Hospitaler, "Design of reinforced concrete bridge frames by heuristic optimization", aceptado Advances in Engineering Software 6 julio 2007, doi:10.1016/j. advengsoft.2007.07.007.

[136] Perea, C., Alcalá, J., Yepes, V., González-Vidosa, F.; Hospitaler, A. "Design of reinforced concrete bridge frames by heuristic optimization". Advances in Engineering Software, 39, No.8, 676-688. 2008.

[137] Perea, C.; Alcalá, J.; Yepes, V.; González-Vidosa, F.; Hospitaler, A. "Design of reinforced concrete bridge frames by heuristic optimization", Advances in Engineering Software, 39, No.8, pp.676-688. 2008.

[138] Perea, C.; Baitsch, M.; González-Vidosa, F.; Hartmann, D. "Optimization of reinforced concrete frame bridges by parallel genetic and memetic algorithms". Proceedings of the Third International Conference on Structural Engineering, Mechanics and Computation (SEMC-2007). Cape Town (South Africa), 10-12 September 2007.

[139] Perea, C.; Yepes, V.; Alcalá, J.; Hospitaler, A.; González-Vidosa, F. “On the Optimum Design of Road Frame Bridges by Local Search Algorithms". Advances in Engineering Software (in press).

[140] Perea, Payá, Yepes y Glez.Vidosa, "Heuristic optimization of reinforced concrete road bridges and frames", Proceedings Segundo Congreso FIB, Nápoles, 5-8 junio 2006, 9 pp.

[141] Perea, Yepes, Alcalá, Hospitaler y González-Vidosa, "Heuristic optimization of reinforced concrete road bridge frames", Proceedings Eighth International Conference on Computational Structures Technology, Las Palmas, 12-15 septiembre 2006. 
[142] Rafiq, M.Y.; Packham, I.S.J.; Easterbrook, D.J.; Denham, S.L. "Visualizing Search and Solution Spaces in the Optimum Design of Biaxial Columns" ASCE. Journal of computing in civil engineering 88-98 (2006).

[143] Rafiq, M.Y.; Sothcombe, C. "Genetic algorithms in optimal design and detailing of reinforced concrete columns supported by a declarative approach for capacity checking”. Computers and Structures 69: 443-457. 1998.

[144] Rajeev, S. ; Krishnamoorthy, C.S. "Genetic Algorithm-Based Methodology for Design Optimization of Reinforced Concrete Frames". Computer-Aided Civil and Infrastructure Engineering, Vol. 13, 63-74. 1998.

[145] Rajeev, S.; Krishnamoorthy, C. S. "Discrete optimization of structures using genetic algorithms". Journal of Structural Engineering, 118(5): 1233-1250. 1992.

[146] Ramamurthy, S. "Optimum design of prestressed concrete slabs using primal geometric programming". International Journal for Numerical Methods in Engineering. Vol. 13(2): 229-246. 1978.

[147] Rodrigues Junior, S.; Eloy Vaz, L.; Barbosa Guimaraes, G. "Optimum design of tall buildings in reinforced concrete subjected to wind forces". Proc. of the 6th Congress of Structural and Multidisciplinary Optimization. Rio de Janeiro. 2005.

[148] Rudolph G. "Convergence analysis of canonical genetic algorithms". IEEE Transactions on Neuronal Networks, 5: 96-101. 1994.

[149] Sahab M.G.; Ashour A.F.; Toropov, V.V. "A hybrid genetic algorithm for reinforced concrete flat buildings". Computers and Structures, 83(8): 551-559. 2004.

[150] Sahab M.G.; Ashour A.F.; Toropov, V.V. "Cost optimization of reinforced concrete flat buildings". Engineering Structures, 27(3) :313-322. 2005.

[151] Samartín A.; Utrilla, M.A. "Optimization of prestressed concrete bridge decks (technical note)". Computers \& Structures, 41(3): 553-559. 1991.

[152] Saouma, V.E.; Murad, R.S. "Partially prestressed concrete beam optimization". Journal of Structural Engineering, 110(3): 589-604. 1984.

[153] Schmidt, H.; Thierauf, G. "A combined optimization technique". Advances in Engineering Software, 36: 11-19. 2005.

[154] Schmidt, L. A. "Structural design by systematic synthesis". Proceedings, 2nd Conference on Electronic Computation, ASCE. New York, pp. 105-132. 1960. 
[155] Serna, P. "Informe proyecto de investigación UPV-Prevalesa Desarrollo de una Tecnología del H.A.C. con fibras". Financiada por el IMPIVA. 2009-2010.

[156] Sirca Jr., G. F.; Adeli, H. "Cost optimization of prestressed concrete bridges". Journal of Structural Engineering, 131(3): 380-388. 2005.

[157] Srinivas, V.; Ramanjaneyulu, K. "An integrated approach for optimum design of bridge decks using genetic algorithms and artificial neural networks". Advances in Engineering Software, 38: 475-487. 2007.

[158] Torrano, M. "Diseño óptimo de secciones y elementos estructurales de hormigón armado". Tesis Doctoral. Universidad Politécnica de Cartagena. 2003.

[159] Torres, G.G.B.; Brotchie, J.F.; Cornell, C.A. "A program for the optimum design of prestressed concrete highway bridges". PCI Journal, 11(3): 63-71. 1966.

[160] Yepes, Alcalá, Perea y Glez. Vidosa, "A parametric study of optimum earth retaining walls by simulated annealing", aceptado Engineering Structures mayo 2007.

[161] Yepes, V. "Optimización heurística económica aplicada a las redes de transporte del tipo VRPTW". Tesis Doctoral. Universidad Politécnica de Valencia, Dpto. Ingeniería del Transporte. 352 pp. 2002.

[162] Yepes, V.; Alcalá, J.; Perea, C.; González-Vidosa, F. "Estudio paramétrico de muros óptimos de contención de hormigón armado". Revista Ingeniería Civil 142:129-139. 2006.

[163] Yepes, V.; Alcalá, J.; Perea, C.; Vidosa, F. G. "A parametric study of optimum earth-retaining walls by simulated annealing". Engineering Structures, 30(3): 821-830. 2008. 


\section{APENDICE A. DATOS DE ENTRADA}

\section{1.- Fichero "Parámetros del Tablero"}

\begin{tabular}{|l|l|}
\hline 1 & :numero de vanos \\
18 & :esbeltez máxima: $1 /$ esbeltez $(1 / 18)$ \\
35.00 & :luz del vano (metros) \\
12.00 & :anchura del tablero (metros) \\
11.00 & :anchura de la calzada (metros) \\
9.00 & :espesor nominal de pavimento(centímetros) \\
10.00 & :carga muerta -otros- (kN/m lineal) \\
25.00 & :despunte armadura activa en \% \\
20.00 & :distancia de ida en Km \\
200.00 & :límite esbeltez armadura vertical cercos: $1 /$ esbeltez $(1 / 200)$ \\
80 & :inclinación alma en grados sexagesimales (valor $\left.80^{\circ}, 80 / 90\right)$ \\
3 & :pendiente cartela ala sup. "1:ns3" valor igual a 3 \\
3 & :división base ala sup. Arranca desde la primera división extrema. s3 valor igual a 3 \\
3 & :pendiente cartela ala inf. "1:ni3" valor igual a 3 \\
4 & :división superficie ala inf. Arranca desde la primera división extrema. i4 valor igual a 4 \\
0.47 & :entrega viga (metros)
\end{tabular}




\section{2.- Fichero "Precios de viga y losa"}

\begin{tabular}{|c|c|}
\hline 30.00 & : Placas autoportantes encofrado perdido (m2) \\
\hline 75.11 & : Molde Viga (m.) \\
\hline 122.25 & : Hormigón 35 en viga $\left(\mathrm{m}^{3}\right)$ \\
\hline 133.40 & : Hormigón 40 en viga $\left(\mathrm{m}^{3}\right)$ \\
\hline 142.15 & : Hormigón 45 en viga $\left(\mathrm{m}^{3}\right)$ \\
\hline 152.89 & : Hormigón 50 en viga $\left(\mathrm{m}^{3}\right)$ \\
\hline 64.99 & : Hormigón 25 en losa $\left(\mathrm{m}^{3}\right)$ \\
\hline 69.95 & : Hormigón 30 en losa $\left(\mathrm{m}^{3}\right)$ \\
\hline 74.03 & : Hormigón 35 en losa $\left(\mathrm{m}^{3}\right)$ \\
\hline 79.12 & : Hormigón 40 en losa $\left(\mathrm{m}^{3}\right)$ \\
\hline 3.38 & : Acero activo $(\mathrm{Kg})$ \\
\hline 2.63 & : Acero pasivo en viga $(\mathrm{Kg})$ \\
\hline 1.40 & : Acero pasivo en losa $(\mathrm{Kg})$ \\
\hline 1.25 & : Coeficientes de corrección del precio del acero en VIGA por diámetro (D6) \\
\hline 1.17 & : Coeficientes de corrección del precio del acero en VIGA por diámetro (D8) \\
\hline 1.075 & : Coeficientes de corrección del precio del acero en VIGA por diámetro (D10) \\
\hline 1.0 & : Coeficientes de corrección del precio del acero en VIGA por diámetro (D12) \\
\hline 0.98 & : Coeficientes de corrección del precio del acero en VIGA por diámetro (D16) \\
\hline 0.98 & : Coeficientes de corrección del precio del acero en VIGA por diámetro (D20) \\
\hline 1.25 & : Coeficientes de corrección del precio del acero en LOSA por diámetro (D6) \\
\hline 1.17 & : Coeficientes de corrección del precio del acero en LOSA por diámetro (D8) \\
\hline 1.075 & : Coeficientes de corrección del precio del acero en LOSA por diámetro (D10) \\
\hline 1.00 & : Coeficientes de corrección del precio del acero en LOSA por diámetro (D12) \\
\hline 0.98 & : Coeficientes de corrección del precio del acero en LOSA por diámetro (D16) \\
\hline 0.98 & : Coeficientes de corrección del precio del acero en LOSA por diámetro (D20) \\
\hline 1.0 & : Coeficientes de corrección del precio del acero en LOSA por diámetro (D25) \\
\hline 1.0 & : Coeficientes de corrección del precio del acero en LOSA por diámetro (D32) \\
\hline 1.40 & : Coeficientes de corrección del precio del acero en VIGA por mano de obra (D6) \\
\hline 1.25 & : Coeficientes de corrección del precio del acero en VIGA por mano de obra (D8) \\
\hline 1.1 & : Coeficientes de corrección del precio del acero en VIGA por mano de obra (D10) \\
\hline 1.0 & : Coeficientes de corrección del precio del acero en VIGA por mano de obra (D12) \\
\hline 0.90 & : Coeficientes de corrección del precio del acero en VIGA por mano de obra (D16) \\
\hline 0.90 & : Coeficientes de corrección del precio del acero en VIGA por mano de obra (D20) \\
\hline 1.40 & : Coeficientes de corrección del precio del acero en LOSA por mano de obra (D6) \\
\hline 1.25 & : Coeficientes de corrección del precio del acero en LOSA por mano de obra (D8) \\
\hline 1.10 & : Coeficientes de corrección del precio del acero en LOSA por mano de obra (D10) \\
\hline 1.0 & : Coeficientes de corrección del precio del acero en LOSA por mano de obra (D12) \\
\hline 0.90 & : Coeficientes de corrección del precio del acero en LOSA por mano de obra (D16) \\
\hline 0.90 & : Coeficientes de corrección del precio del acero en LOSA por mano de obra (D20) \\
\hline 0.80 & : Coeficientes de corrección del precio del acero en LOSA por mano de obra (D25) \\
\hline 0.80 & : Coeficientes de corrección del precio del acero en LOSA por mano de obra (D32) \\
\hline
\end{tabular}




\section{3.- Fichero "Precios de transporte y montaje"}

\begin{tabular}{|c|c|}
\hline 6 & :Número de tramos de pesos para transporte 6 \\
\hline 5 & :Número de tramos de distancias transporte 5 \\
\hline 55.00 & :Hasta $55 \mathrm{Tm}$ \\
\hline 66.00 & :Hasta 66 Tm \\
\hline 80.00 & :Hasta $80 \mathrm{Tm}$ \\
\hline 100.00 & :Hasta $100 \mathrm{Tm}$ \\
\hline 200.00 & :Hasta $200 \mathrm{Tm}$ \\
\hline 400.00 & :Hasta $400 \mathrm{Tm}$ \\
\hline 50.00 & :Hasta $50 \mathrm{Km}$ \\
\hline 100.00 & :Hasta $100 \mathrm{Km}$ \\
\hline 150.00 & :Hasta $150 \mathrm{Km}$ \\
\hline 200.00 & :Hasta $200 \mathrm{Km}$ \\
\hline 400.00 & :Hasta $400 \mathrm{Km}$ \\
\hline 975.00 & :(€) $55 \mathrm{Tm}-50 \mathrm{Km}$ \\
\hline 1225.00 & :(€) $55 \mathrm{Tm}-100 \mathrm{Km}$ \\
\hline 1475.00 & :(€) $55 \mathrm{Tm}-150 \mathrm{Km}$ \\
\hline 1700.00 & :(€) $55 \mathrm{Tm}-200 \mathrm{Km}$ \\
\hline 2500.00 & :(€)55Tm - 400Km \\
\hline 1275.00 & :(€) $66 \mathrm{Tm}-50 \mathrm{Km}$ \\
\hline 1600.00 & :(€) $66 \mathrm{Tm}-100 \mathrm{Km}$ \\
\hline 1925.00 & :(€) $66 \mathrm{Tm}-150 \mathrm{Km}$ \\
\hline 2225.00 & :(€) $66 \mathrm{Tm}-200 \mathrm{Km}$ \\
\hline 3000.00 & :(€) $66 \mathrm{Tm}-400 \mathrm{Km}$ \\
\hline 1650.00 & :(€) $80 \mathrm{Tm}-50 \mathrm{Km}$ \\
\hline 2075.00 & :(€) $80 \mathrm{Tm}-100 \mathrm{Km}$ \\
\hline 2475.00 & :(€) $80 \mathrm{Tm}-150 \mathrm{Km}$ \\
\hline 2900.00 & :(€) $80 \mathrm{Tm}-200 \mathrm{Km}$ \\
\hline 3500.00 & :(€) $80 \mathrm{Tm}-400 \mathrm{Km}$ \\
\hline 1825.00 & :(€) $100 \mathrm{Tm}-50 \mathrm{Km}$ \\
\hline 2275.00 & $:(€) 100 \mathrm{Tm}-100 \mathrm{Km}$ \\
\hline 2725.00 & $:(€) 100 \mathrm{Tm}-150 \mathrm{Km}$ \\
\hline 3175.00 & :(€) $100 \mathrm{Tm}-200 \mathrm{Km}$ \\
\hline 4000.00 & :(€) $100 \mathrm{Tm}-400 \mathrm{Km}$ \\
\hline 2825.00 & $:(€)$ 200Tm - 50Km \\
\hline 3275.00 & :(€) $200 \mathrm{Tm}-100 \mathrm{Km}$ \\
\hline 3725.00 & $:(€) 200 \mathrm{Tm}-150 \mathrm{Km}$ \\
\hline 4175.00 & :(€) $200 \mathrm{Tm}-200 \mathrm{Km}$ \\
\hline 5000.00 & :(€) $200 \mathrm{Tm}-400 \mathrm{Km}$ \\
\hline 3825.00 & $:(€)$ 400Tm - 50Km \\
\hline 4275.00 & $:(€) 400 \mathrm{Tm}-100 \mathrm{Km}$ \\
\hline 4725.00 & $:(€) 400 \mathrm{Tm}-150 \mathrm{Km}$ \\
\hline 5175.00 & :(€) $400 \mathrm{Tm}-200 \mathrm{Km}$ \\
\hline 6000.00 & :(€) 400Tm - 400Km \\
\hline 1.00 & :Factor dificultad en el montaje \\
\hline 4 & :Número de tramos Km \\
\hline 5 & :Número de tramos Longitud viga (luz) \\
\hline 50. & :hasta $50 \mathrm{Km}$ \\
\hline 100. & :de 50 hasta $100 \mathrm{Km}$ \\
\hline 200. & :de 100 hasta $200 \mathrm{Km}$ \\
\hline 400. & :de 200 hasta $400 \mathrm{Km}$ \\
\hline 20. & :hasta $20 \mathrm{~m}$ \\
\hline 25. & :de 20 a $25 \mathrm{~m}$ \\
\hline 30. & :de 25 a $30 \mathrm{~m}$ \\
\hline 35. & :de 30 a $35 \mathrm{~m}$ \\
\hline 40. & :de 35 a $40 \mathrm{~m}$ \\
\hline 2900. & $:(€)$ coste $50 \mathrm{Km} 20 \mathrm{~m}$ \\
\hline
\end{tabular}




\begin{tabular}{|l|l|}
\hline Cont. & Cont..... \\
3000. & $:()$ coste $50 \mathrm{Km} 25 \mathrm{~m}$ \\
5100. & $:(€)$ coste $50 \mathrm{Km} 30 \mathrm{~m}$ \\
5200. & $:(€)$ coste $50 \mathrm{Km} 35 \mathrm{~m}$ \\
6200. & $:()$ coste $50 \mathrm{Km} 40 \mathrm{~m}$ \\
2900. & $:(€)$ coste $100 \mathrm{Km} 20 \mathrm{~m}$ \\
3300. & $:(€)$ coste $100 \mathrm{Km} 25 \mathrm{~m}$ \\
5100. & $:(€)$ coste $100 \mathrm{Km} 30 \mathrm{~m}$ \\
5200. & $:(€)$ coste $100 \mathrm{Km} 35 \mathrm{~m}$ \\
6400. & $:(€)$ coste $100 \mathrm{Km} 40 \mathrm{~m}$ \\
3100. & $:(€)$ coste $200 \mathrm{Km} 20 \mathrm{~m}$ \\
4900. & $:(€)$ coste $200 \mathrm{Km} 25 \mathrm{~m}$ \\
5300. & $:(€)$ coste $200 \mathrm{Km} 30 \mathrm{~m}$ \\
5400. & $:(€)$ coste $200 \mathrm{Km} 35 \mathrm{~m}$ \\
6400. & $:(€)$ coste $200 \mathrm{Km} 40 \mathrm{~m}$ \\
3600. & $:(€)$ coste $400 \mathrm{Km} 20 \mathrm{~m}$ \\
5400. & $:(€)$ coste $400 \mathrm{Km} 25 \mathrm{~m}$ \\
5800. & $:()$ coste $400 \mathrm{Km} 30 \mathrm{~m}$ \\
5900. & $:(€)$ coste $400 \mathrm{Km} 35 \mathrm{~m}$ \\
6900. & $:(€)$ coste $400 \mathrm{Km} 40 \mathrm{~m}$ \\
\hline
\end{tabular}




\section{APENDICE B. RESULTADO VARIACIÓN DE PRECIOS}

\section{B.I Valores numéricos}

Los resultados geométricos obtenidos en la variación de los precios, para una luz de 35 metros, son los siguientes:

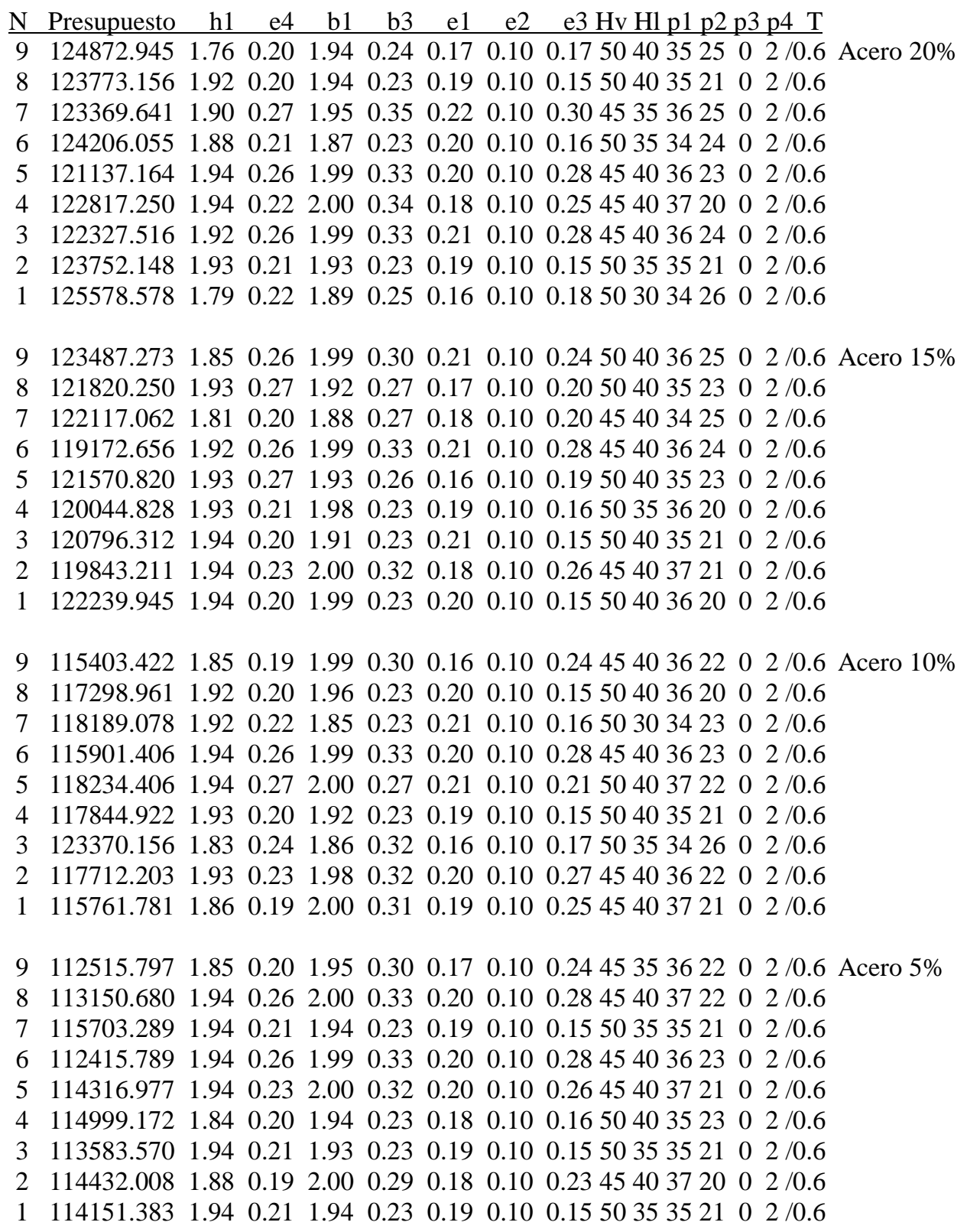


$\begin{array}{lllllllll}\mathrm{N} & \text { Presupuesto } & \text { h1 } & \text { e4 } & \text { b1 } & \text { b3 } & \text { e1 } & \text { e2 } & \text { e3 Hv Hl p1 p2 p3 p4 T }\end{array}$

$\begin{array}{llllllllllllllll}9 & 109912.125 & 1.89 & 0.20 & 1.88 & 0.32 & 0.18 & 0.10 & 0.27 & 45 & 35 & 34 & 24 & 0 & 2 / 0.6 & 0 \%\end{array}$

$\begin{array}{llllllllllllll}8 & 111849.727 & 1.94 & 0.28 & 1.97 & 0.27 & 0.16 & 0.10 & 0.20 & 5035 & 36 & 22 & 0 & 2\end{array} / 0.6$

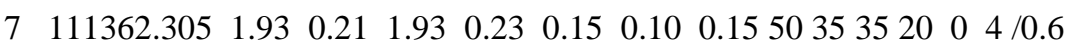

$\begin{array}{llllllllllllll}6 & 111219.375 & 1.77 & 0.20 & 1.99 & 0.30 & 0.18 & 0.10 & 0.24 & 4535 & 36 & 25 & 0 & 2 / 0.6\end{array}$

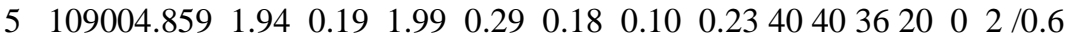

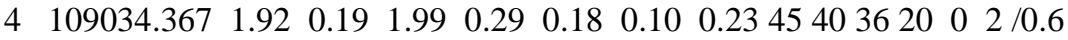

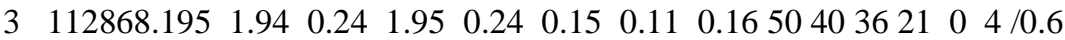

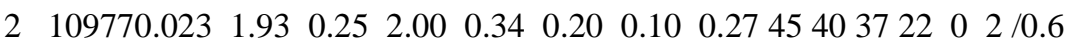

$\begin{array}{llllllllllllll}1 & 109277.883 & 1.94 & 0.21 & 1.95 & 0.23 & 0.15 & 0.10 & 0.15 & 5035 & 36 & 20 & 0 & 4\end{array}$

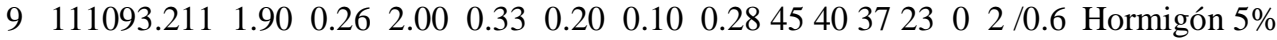

$\begin{array}{llllllllllllll}8 & 114902.539 & 1.94 & 0.25 & 1.95 & 0.26 & 0.21 & 0.10 & 0.1950 & 4036 & 22 & 0 & 2 / 0.6\end{array}$

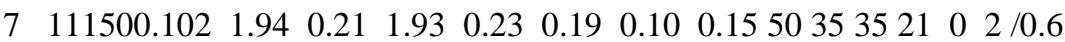

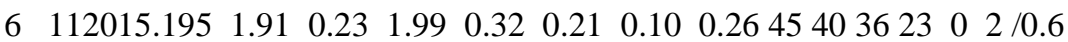

$\begin{array}{lllllllllllllll}5 & 113112.453 & 1.90 & 0.20 & 1.98 & 0.26 & 0.19 & 0.10 & 0.19 & 45 & 40 & 36 & 21 & 0 & 2 / 0.6\end{array}$

$\begin{array}{llllllllllllll}4 & 111527.133 & 1.93 & 0.21 & 1.93 & 0.23 & 0.19 & 0.10 & 0.15 & 5035 & 35 & 21 & 0 & 2 / 0.6\end{array}$

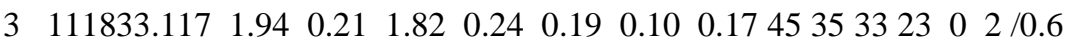

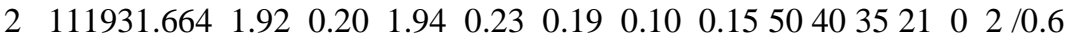

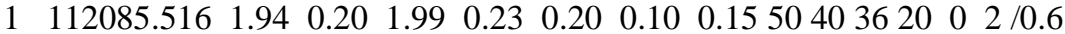

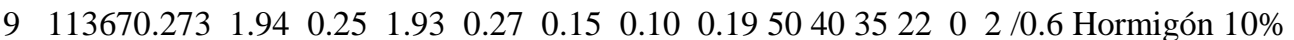

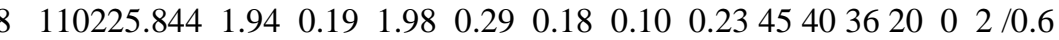

$\begin{array}{llllllllllllll}7 & 115634.266 & 1.82 & 0.24 & 1.99 & 0.28 & 0.20 & 0.10 & 0.22 & 5035 & 36 & 25 & 0 & 2 / 0.6\end{array}$

$\begin{array}{lllllllllllll}6 & 113046.438 & 1.89 & 0.24 & 1.97 & 0.26 & 0.16 & 0.10 & 0.195035 & 36 & 22 & 0 & 2 / 0.6\end{array}$

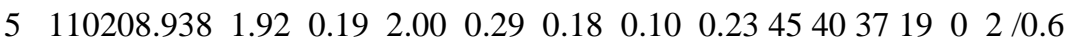

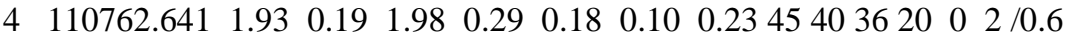

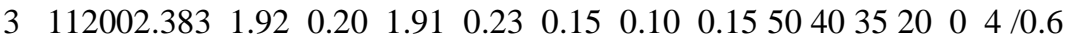

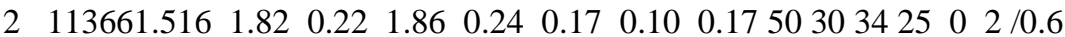

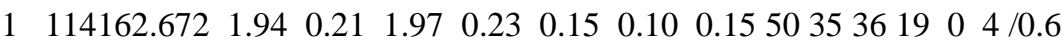

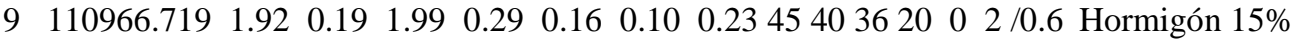

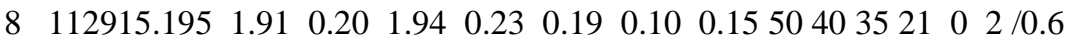

$\begin{array}{lllllllllllll}7 & 113209.734 & 1.93 & 0.21 & 1.97 & 0.23 & 0.15 & 0.10 & 0.165035 & 36 & 19 & 0 & 4 / 0.6\end{array}$

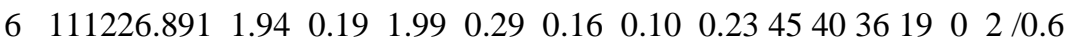

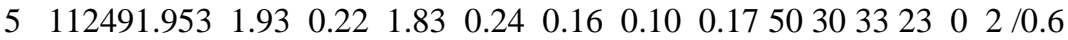

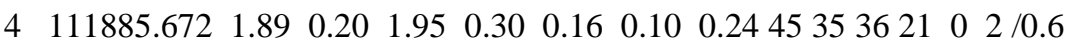

$\begin{array}{llllllllllllll}3 & 113557.516 & 1.94 & 0.25 & 1.99 & 0.35 & 0.21 & 0.10 & 0.3045 & 40 & 36 & 23 & 0 & 2\end{array} / 0.6$

$\begin{array}{llllllllllllll}2 & 116441.109 & 1.71 & 0.27 & 2.00 & 0.35 & 0.18 & 0.10 & 0.3050 & 4037 & 29 & 0 & 2 / 0.6\end{array}$

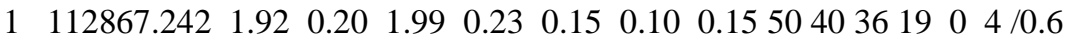

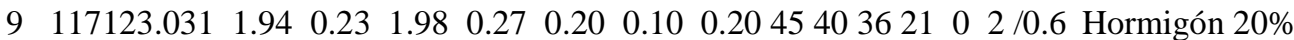

$\begin{array}{llllllllllllll}8 & 116470.711 & 1.88 & 0.23 & 1.99 & 0.28 & 0.18 & 0.10 & 0.22454036 & 23 & 0 & 2 / 0.6\end{array}$

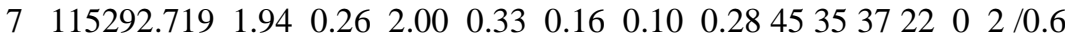

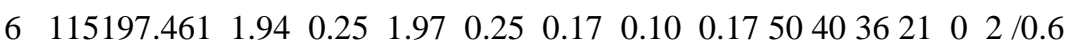

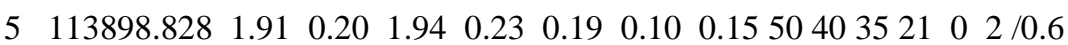

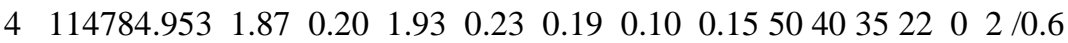

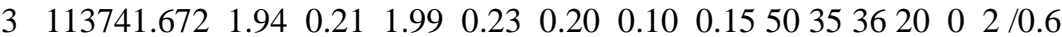

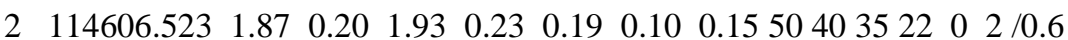

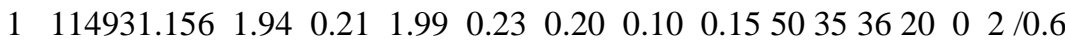


Los valores medios obtenidos para los distintos incrementos son:

$\begin{array}{lllllllll}\text { Presupuesto } & \text { h1 } & \text { e4 } & \text { b1 } & \text { b3 } & \text { e1 } & \text { e2 } & \text { e3 Hv H1 p1 p2 p3 p4 T }\end{array}$

$\begin{array}{lllllllllllll}123537 & 1.89 & 0.23 & 1.94 & 0.28 & 0.19 & 0.10 & 0.2148 & 37 & 35 & 23 & 0 & 2\end{array} / 0.6$ Acero $20 \%$

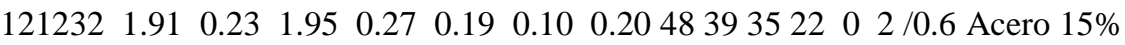

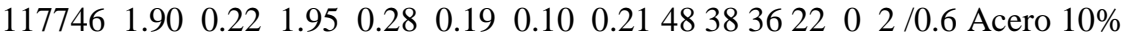

$\begin{array}{llllllllllll}113918 & 1.91 & 0.22 & 1.97 & 0.28 & 0.19 & 0.10 & 0.214738 & 36 & 22 & 0 & 2\end{array} / 0.6$ Acero $5 \%$

$\begin{array}{lllllllllllll}110477 & 1.92 & 0.22 & 1.96 & 0.25 & 0.17 & 0.10 & 0.2147373622 & 0 & 2 / 0.6 & 0 \%\end{array}$

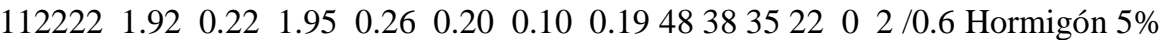

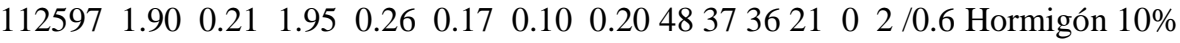

$\begin{array}{lllllllllllll}112840 & 1.90 & 0.21 & 1.96 & 0.28 & 0.17 & 0.10 & 0.2148 & 38 & 36 & 22 & 0 & 2\end{array} / 0.6$ Hormigón $15 \%$

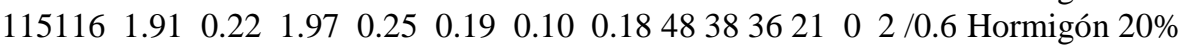




\section{B.II Gráficos}

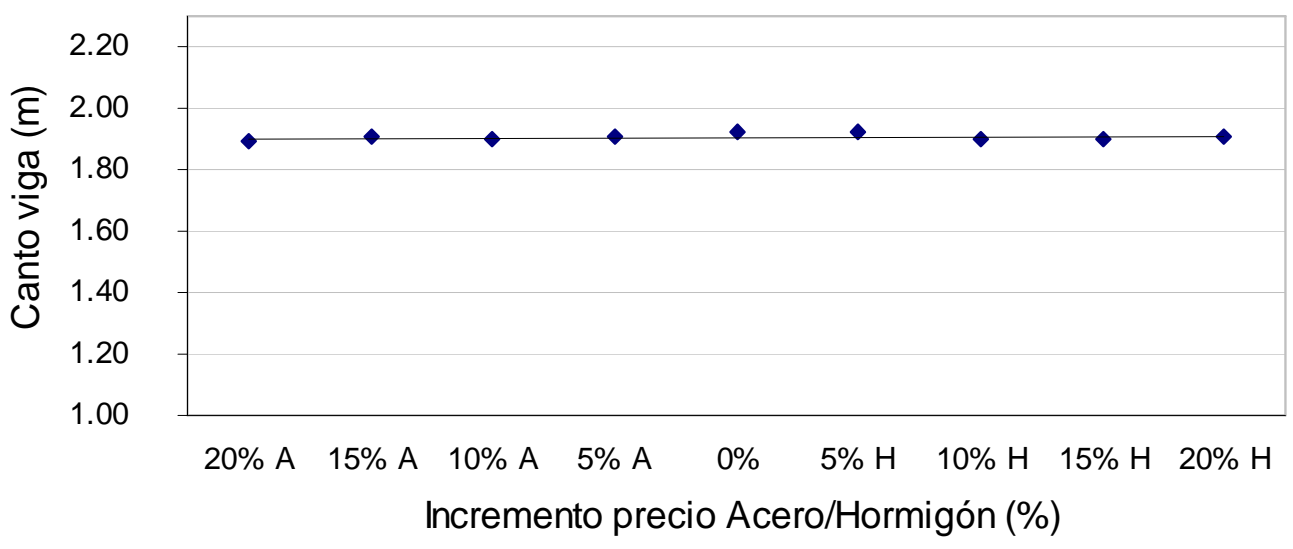

Figura 0-1: Canto medio de la viga respecto al incremento de precios acero/ hormigón

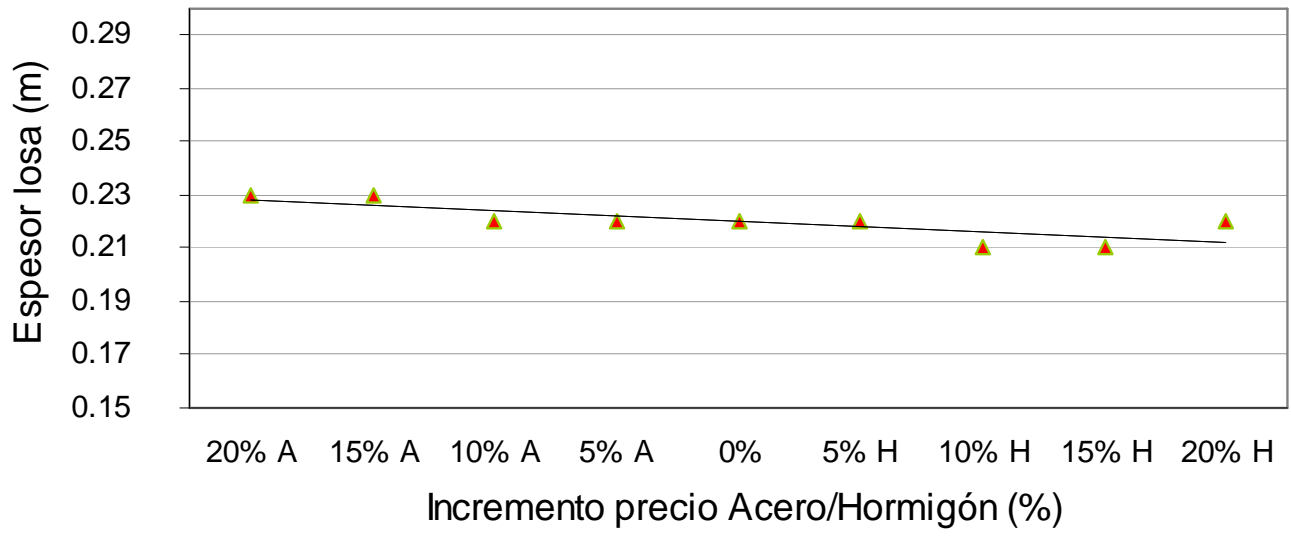

Figura 0-2: Espesor medio de la losa respecto al incremento de precios acero/ hormigón

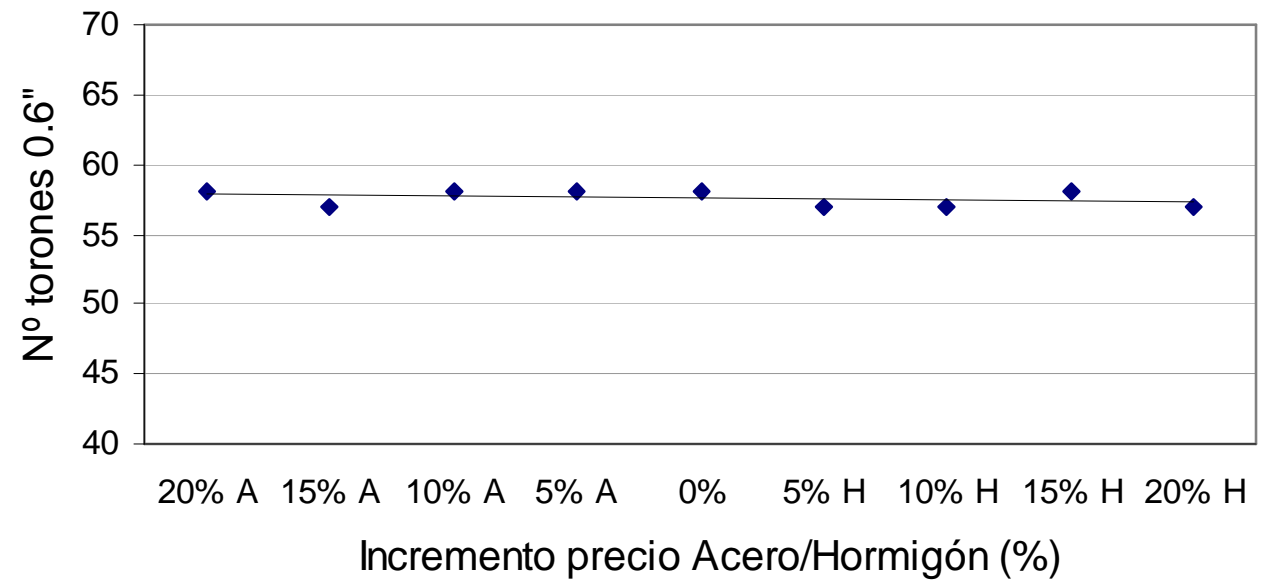

Figura 0-3: $\mathrm{N}^{\text {o }}$ torones medio respecto al incremento de precios acero/ hormigón 


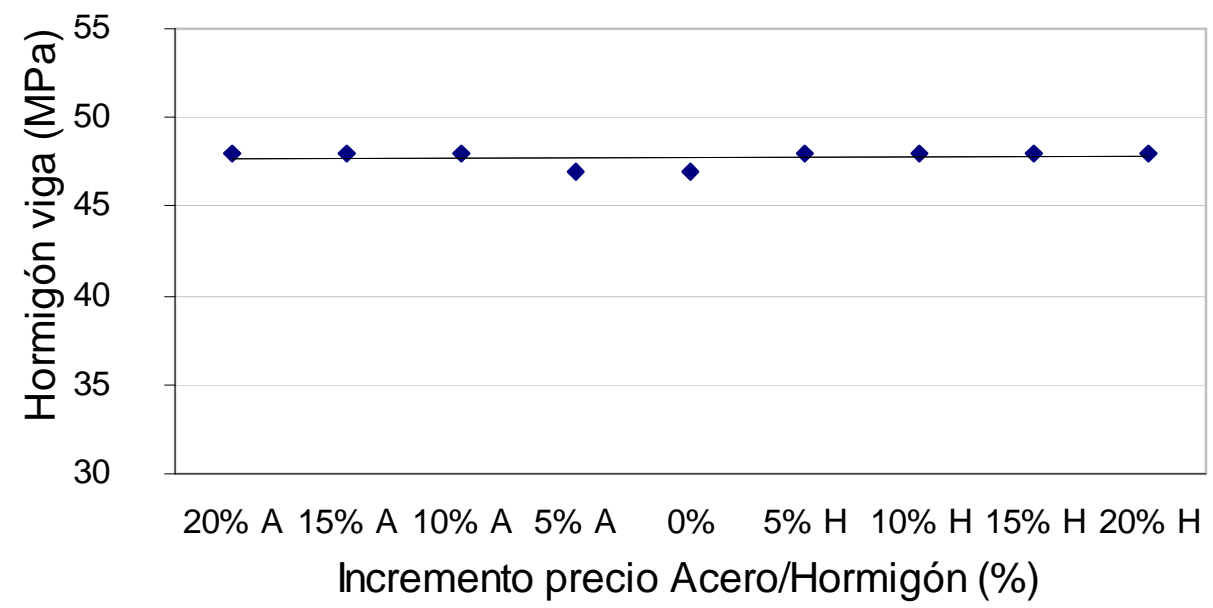

Figura 0-4: Resistencia característica media del hormigón de la viga respecto al incremento de precios acero/ hormigón

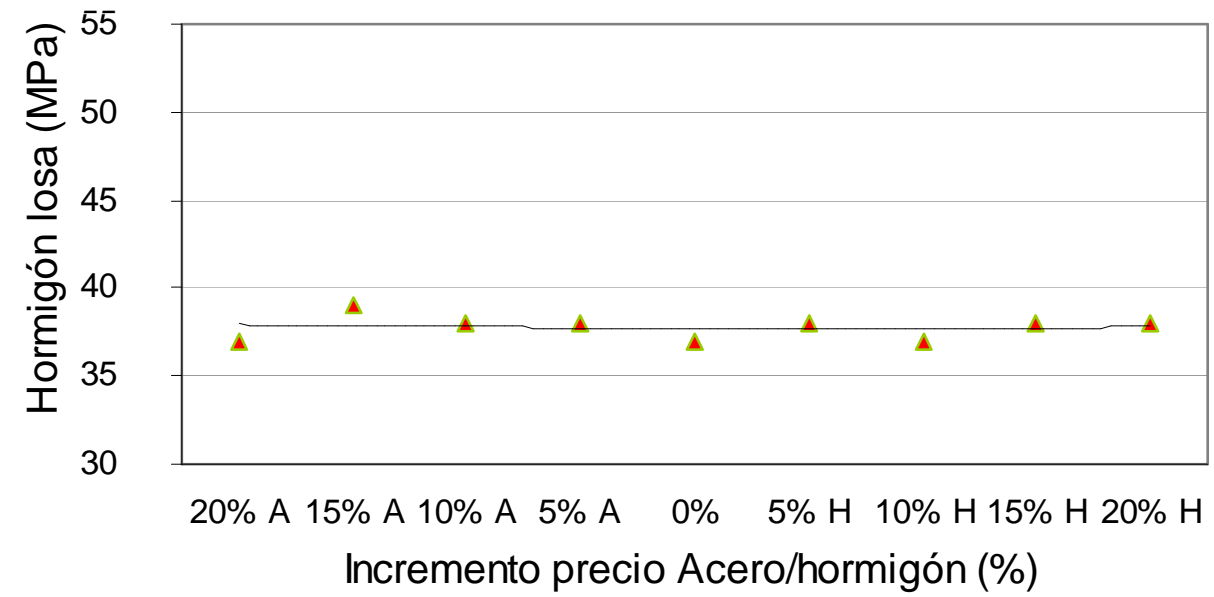

Figura 0-5: Resistencia característica media del hormigón de la losa respecto al incremento de precios acero/ hormigón

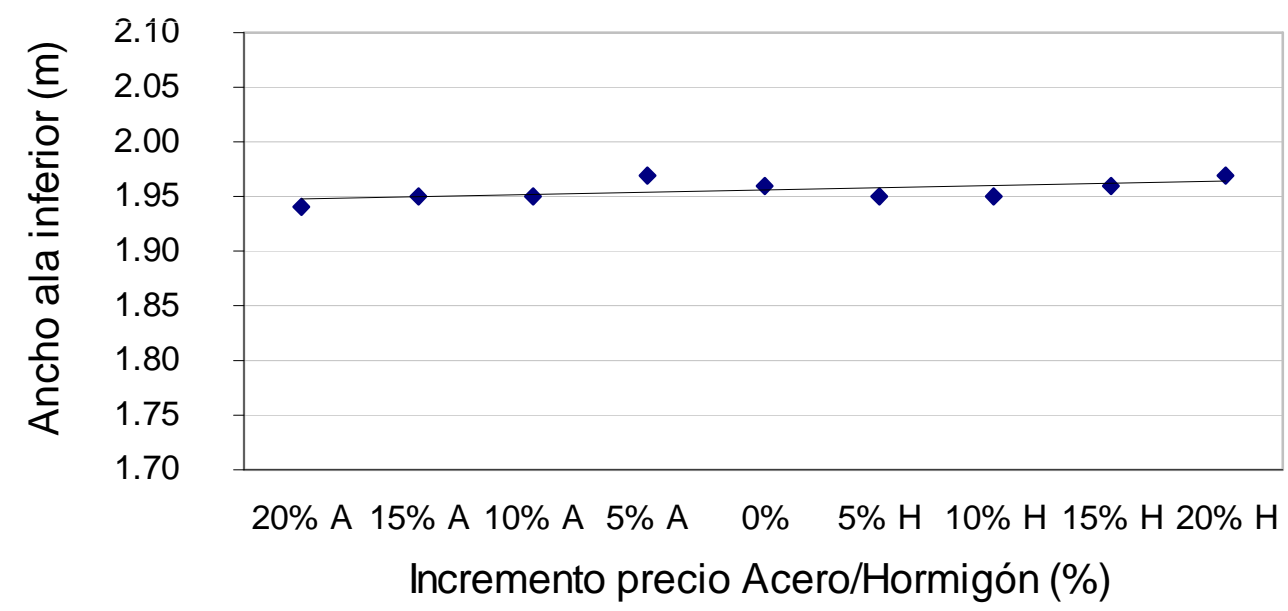

Figura 0-6: Ancho medio del ala inferior respecto al incremento de precios acero/ hormigón 


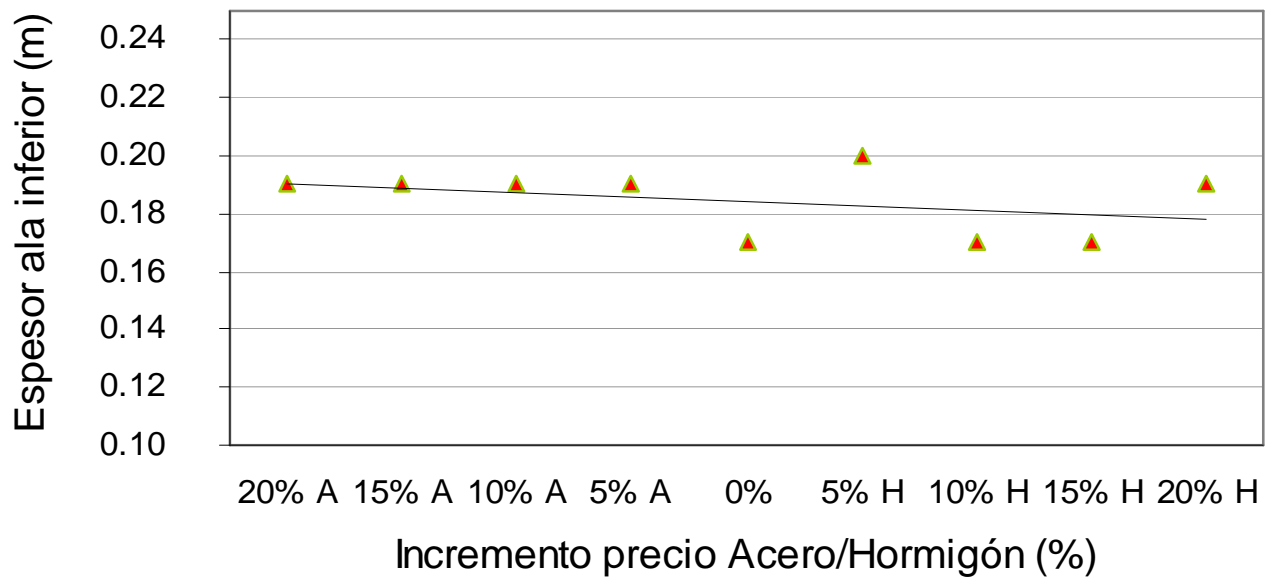

Figura 0-7: Espesor medio del ala inferior respecto al incremento de precios acero/ hormigón

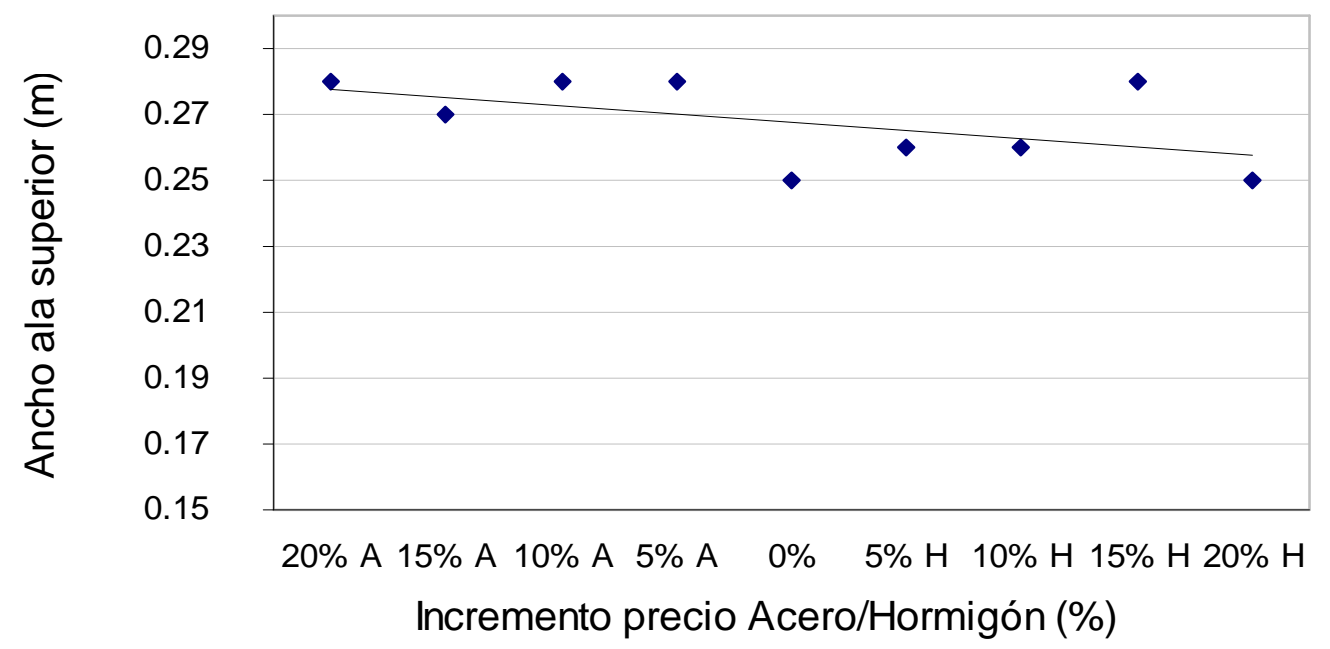

Figura 0-8: Ancho medio del ala superior respecto al incremento de precios acero/ hormigón

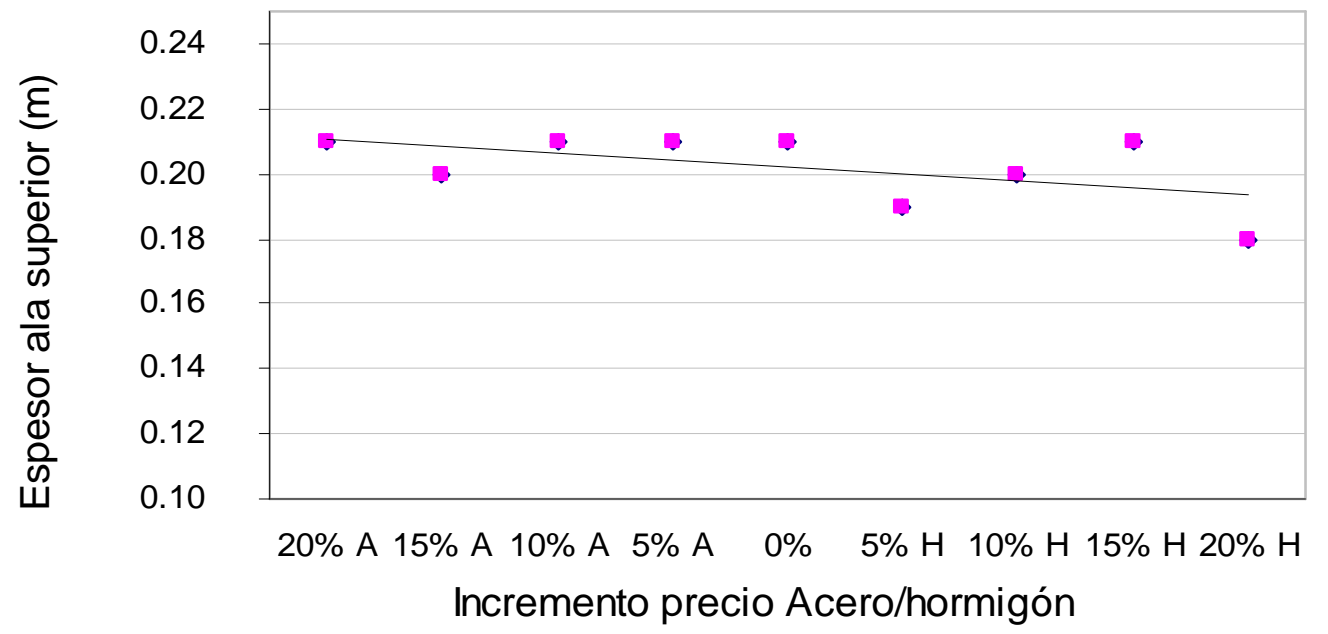

Figura 0-9: Espesor medio del ala superior respecto al incremento de precios acero/ hormigón 


\title{
APENDICE C. RESULTADOS DE LOS EJEMPLOS NUMÉRICOS
}

\author{
C.I Métodos heurísticos
}


RW (Búsqueda aleatoria)

Luz $=35 \mathrm{~m}$

ARMADURA PASIVA

Armadura transversal

№ $\quad L 11 \quad L 2 \quad L 3 \quad L \quad L \quad L$ total $\quad D$ peso

$\mathrm{sec}$ barras $\langle\ldots \ldots[\mathrm{m}] \cdots[\mathrm{mm}][\mathrm{kg} / \mathrm{m}] \quad[\mathrm{kg}]$

$\begin{array}{rrrrrrr}+++ & \text { Cerco } & \text { ala inf.semi-viga } & & & \\ 1 & 8 & 3.10 & 0.32 & -- & 3.42 & 30.729 \\ 2 & 8 & 3.10 & 0.32 & -- & 3.42 & 29.925 \\ 3 & 8 & 3.10 & 0.32 & -- & 3.42 & 29.925 \\ 4 & 8 & 3.10 & 0.32 & -- & 3.42 & 29.925 \\ 5 & 8 & 3.10 & 0.32 & -- & 3.42 & 29.925 \\ 6 & 8 & 3.10 & 0.32 & -- & 3.42 & 29.925 \\ 7 & 8 & 3.10 & 0.32 & -- & 3.42 & 29.925 \\ 8 & 8 & 3.10 & 0.32 & -- & 3.42 & 29.925 \\ 9 & 8 & 3.10 & 0.32 & -- & 3.42 & 29.925 \\ 10 & 8 & 3.10 & 0.32 & -- & 3.42 & 29.925\end{array}$

+++ Horquillas alma semi-viga

$\begin{array}{rrrrrr}1 & 17 & 1.55 & 1.70 & 0.28 & 11.02 \\ 2 & 17 & 1.54 & 1.70 & 0.35 & 11.28 \\ 3 & 17 & 1.55 & 1.70 & 0.28 & 11.02 \\ 4 & 17 & 1.55 & 1.70 & 0.28 & 11.02 \\ 5 & 17 & 1.55 & 1.70 & 0.28 & 11.02 \\ 6 & 17 & 1.55 & 1.70 & 0.28 & 11.02 \\ 7 & 17 & 1.55 & 1.70 & 0.28 & 11.02 \\ 8 & 17 & 1.55 & 1.70 & 0.28 & 11.02 \\ 9 & 17 & 1.55 & 1.70 & 0.28 & 11.02 \\ 10 & 17 & 1.55 & 1.70 & 0.28 & 11.02\end{array}$

+++ Cerco alas semi-viga

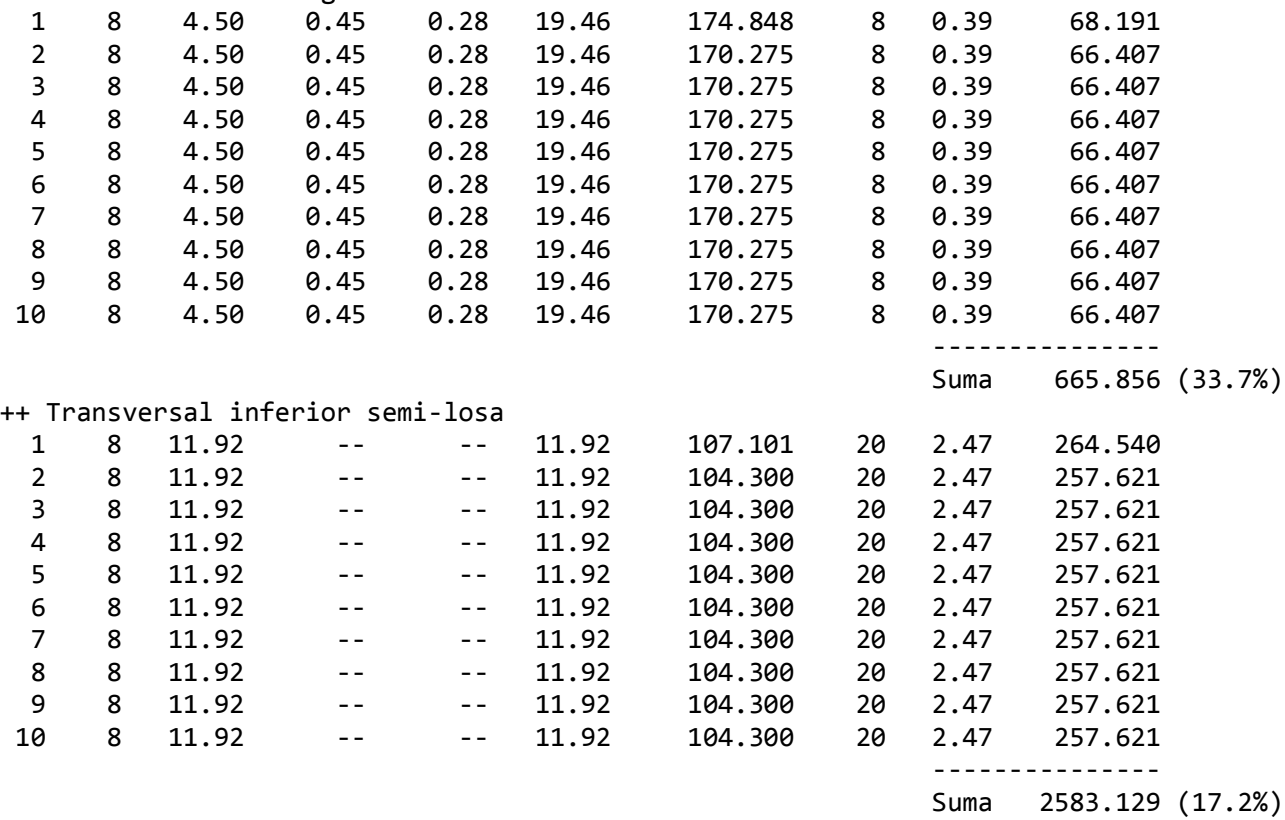




$\begin{array}{rrrrrrrrrr}+++ \\ 1 & 8 & 11.92 & -- & -- & 11.92 & 107.101 & 20 & 2.47 & 264.540 \\ 2 & 8 & 11.92 & -- & -- & 11.92 & 104.300 & 20 & 2.47 & 257.621 \\ 3 & 8 & 11.92 & -- & -- & 11.92 & 104.300 & 20 & 2.47 & 257.621 \\ 4 & 8 & 11.92 & -- & -- & 11.92 & 104.300 & 20 & 2.47 & 257.621 \\ 5 & 8 & 11.92 & -- & -- & 11.92 & 104.300 & 20 & 2.47 & 257.621 \\ 6 & 8 & 11.92 & -- & -- & 11.92 & 104.300 & 20 & 2.47 & 257.621 \\ 7 & 8 & 11.92 & -- & -- & 11.92 & 104.300 & 20 & 2.47 & 257.621 \\ 8 & 8 & 11.92 & -- & -- & 11.92 & 104.300 & 20 & 2.47 & 257.621 \\ 9 & 8 & 11.92 & -- & -- & 11.92 & 104.300 & 20 & 2.47 & 257.621 \\ 10 & 8 & 11.92 & -- & -- & 11.92 & 104.300 & 20 & 2.47 & 257.621 \\ & & & & & & & & & ------------- \\ \end{array}$

Armadura longitudinal total tablero

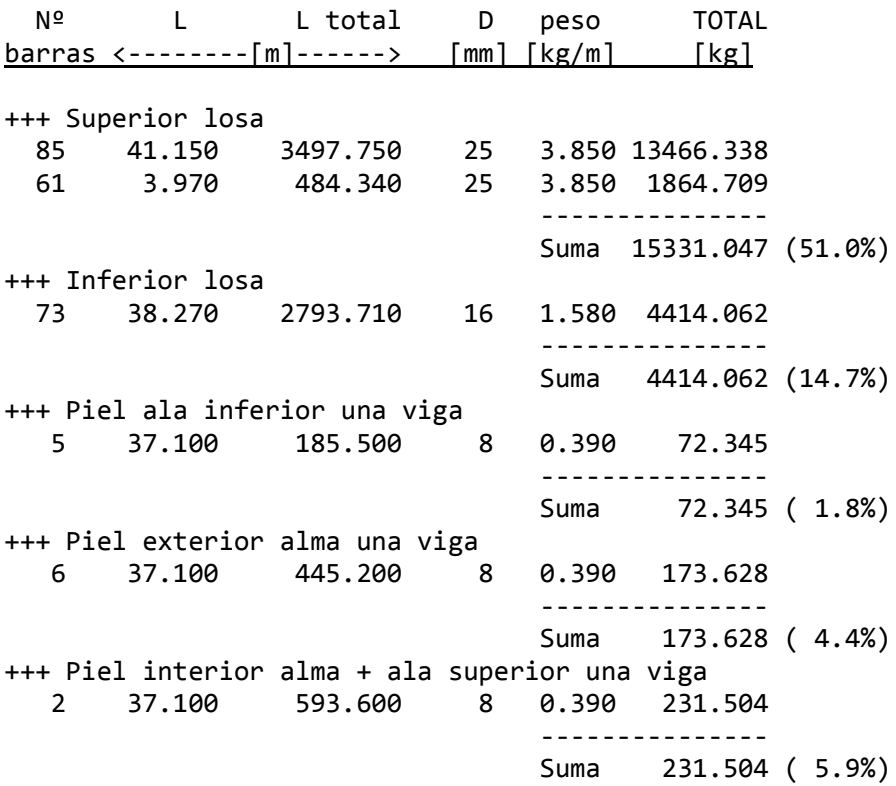

\section{ESTADO DE MEDICIONES}

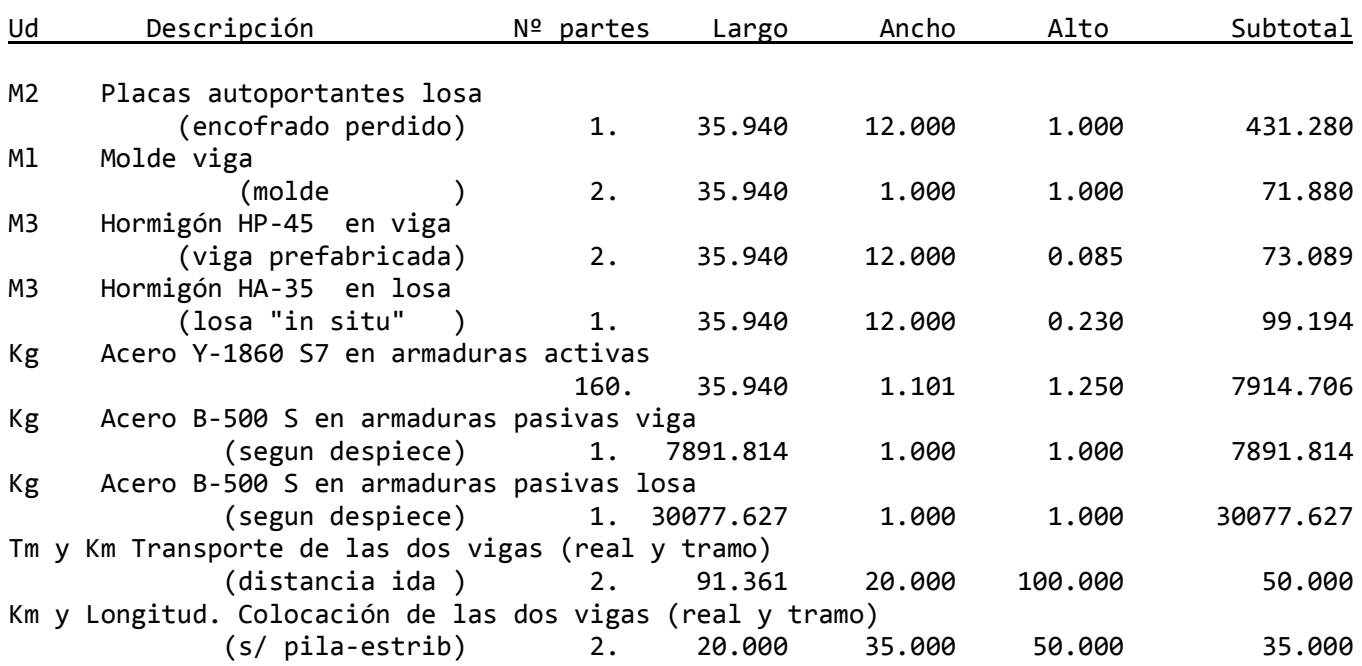




\section{CUANTIAS RESULTANTES}

0.399 M3 hormigon / M2 tablero

$45.940 \mathrm{~kg}$ activas / M3 hormigón

$108.289 \mathrm{~kg}$ activas / M3 hormigón VIGA

$18.321 \mathrm{~kg}$ activas / M2 tablero

$220.390 \mathrm{~kg}$ pasivas / M3 hormigón

$107.976 \mathrm{~kg}$ pasivas / M3 hormigón VIGA

$87.892 \mathrm{~kg}$ pasivas / M2 tablero

\section{CALCULO DEL COSTE}

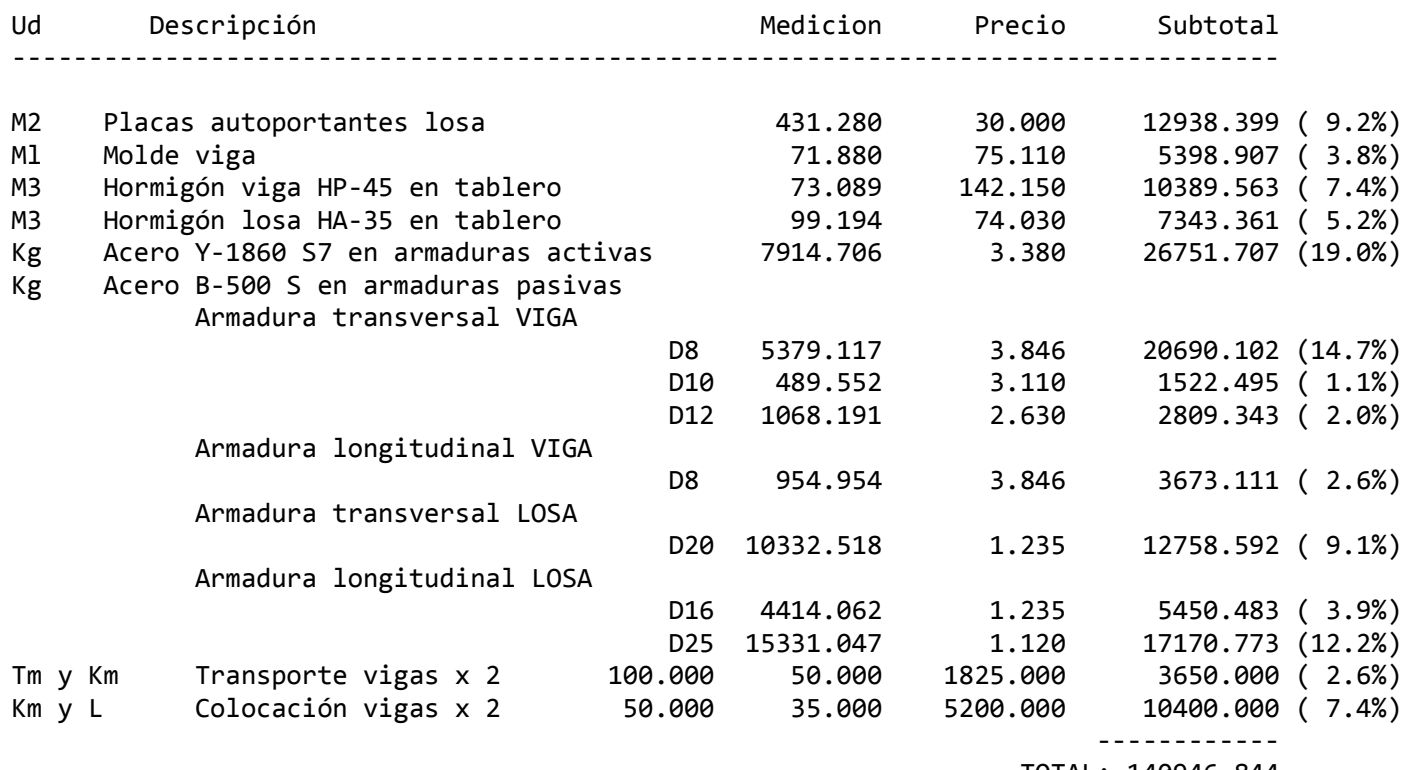

326. 27 EUROS / M2 tablero 


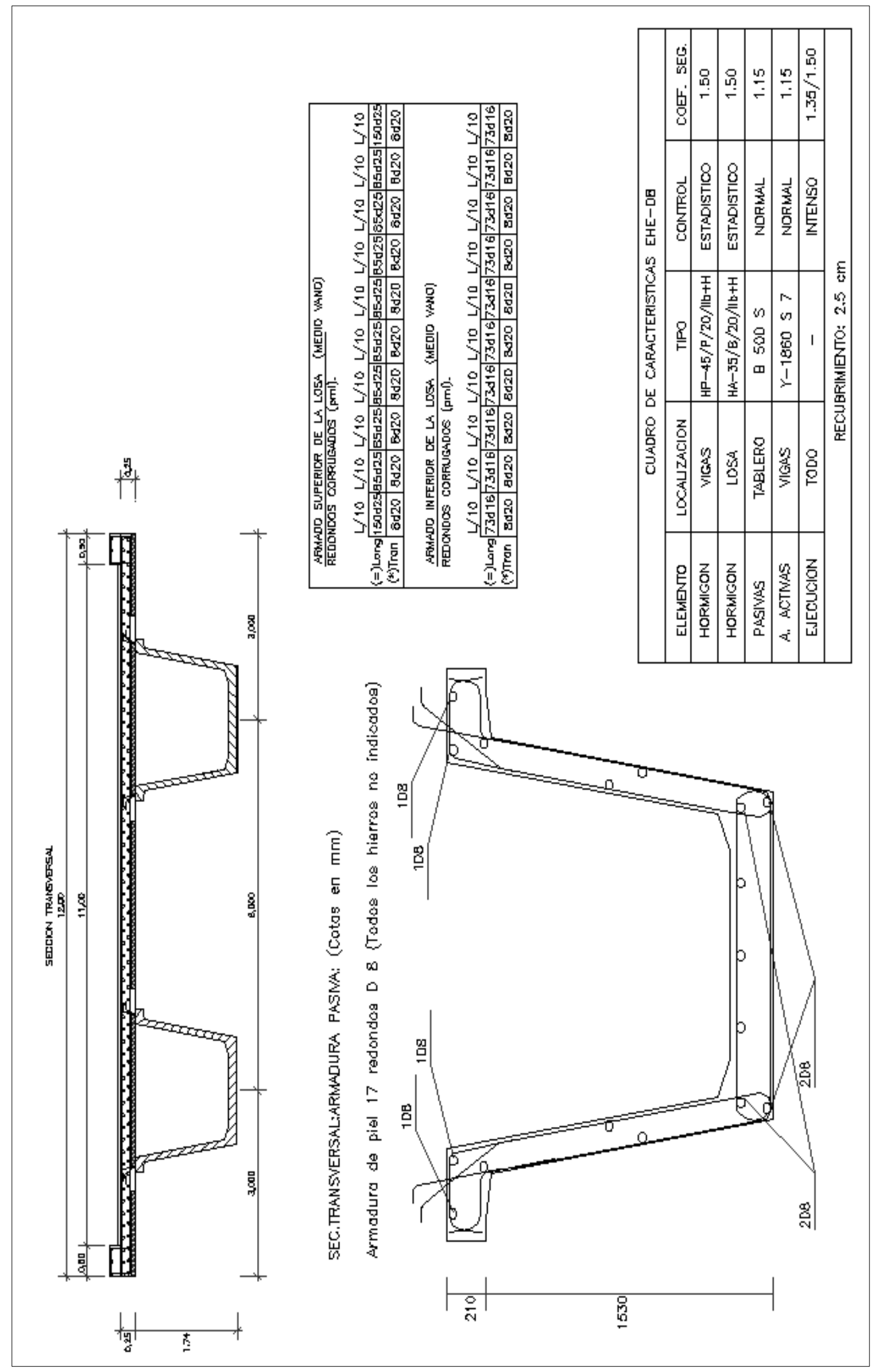




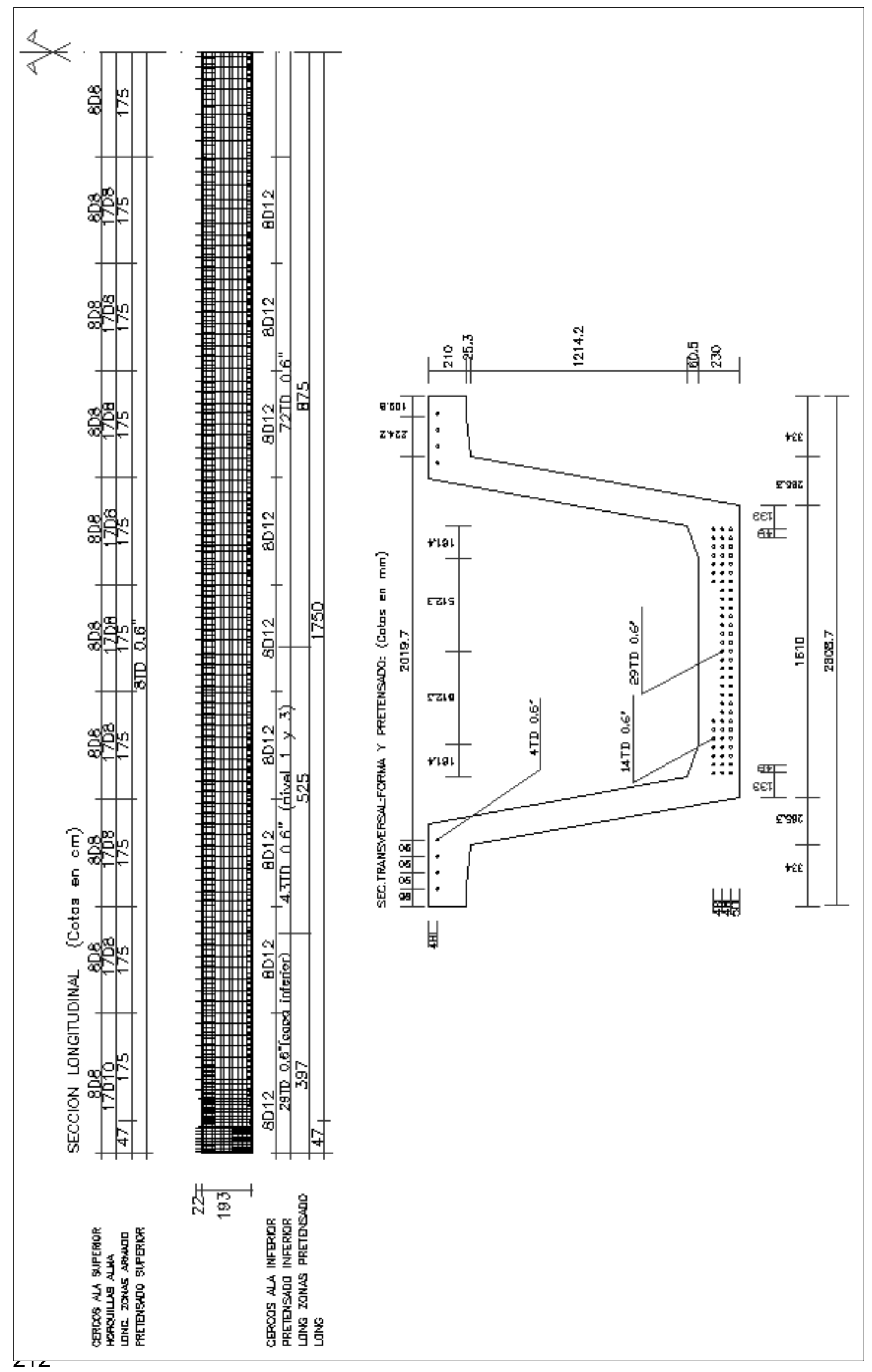


DLS (Búsqueda por gradiente)

Luz $=35 \mathrm{~m}$.

ARMADURA PASIVA

Armadura transversal

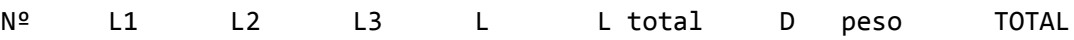

$\mathrm{sec}$ barras $\langle\ldots[\mathrm{mm}][\mathrm{kg} / \mathrm{m}] \quad[\mathrm{kg}]$

+++ Cerco ala inf.semi-viga

$\begin{array}{rrrrrrrrrr}1 & 8 & 3.68 & 0.36 & -- & 4.04 & 36.299 & 12 & 0.89 & 32.306 \\ 2 & 8 & 3.68 & 0.36 & -- & 4.04 & 35.350 & 12 & 0.89 & 31.461\end{array}$

$\begin{array}{llllllllll}4 & 17 & 3.70 & 0.38 & -- & 4.08 & 71.400 & 6 & 0.22 & 15.708\end{array}$

$\begin{array}{llllllllll}5 & 17 & 3.70 & 0.38 & -- & 4.08 & 71.400 & 6 & 0.22 & 15.708\end{array}$

$\begin{array}{llllllllll}6 & 8 & 3.68 & 0.38 & -- & 4.06 & 35.525 & 10 & 0.62 & 22.025\end{array}$

$\begin{array}{llllllllll}7 & 8 & 3.70 & 0.38 & -- & 4.08 & 35.700 & 8 & 0.39 & 13.923\end{array}$

$\begin{array}{llllllllll}8 & 17 & 3.70 & 0.38 & -- & 4.08 & 71.400 & 8 & 0.39 & 27.846\end{array}$

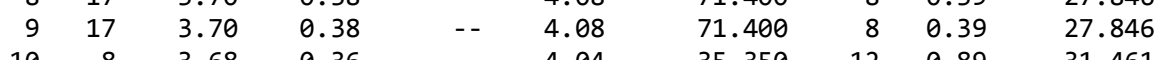

$\begin{array}{llllllllll}10 & 8 & 3.68 & 0.36 & - & 4.04 & 35.350 & 12 & 0.89 & 31.461\end{array}$

+++ Horquillas alma semi-viga

$\begin{array}{rrrrrr}1 & 8 & 0.94 & 1.89 & 0.42 & 11.12 \\ 2 & 8 & 0.94 & 1.89 & 0.42 & 11.12 \\ 3 & 8 & 0.94 & 1.89 & 0.42 & 11.12 \\ 4 & 8 & 0.94 & 1.89 & 0.35 & 10.84 \\ 5 & 8 & 0.94 & 1.89 & 0.35 & 10.84 \\ 6 & 8 & 0.94 & 1.89 & 0.35 & 10.84 \\ 7 & 8 & 0.94 & 1.89 & 0.35 & 10.84 \\ 8 & 8 & 0.94 & 1.89 & 0.35 & 10.84 \\ 9 & 8 & 0.94 & 1.89 & 0.35 & 10.84 \\ 10 & 8 & 0.94 & 1.89 & 0.35 & 10.84\end{array}$

+++ Cerco alas semi-viga

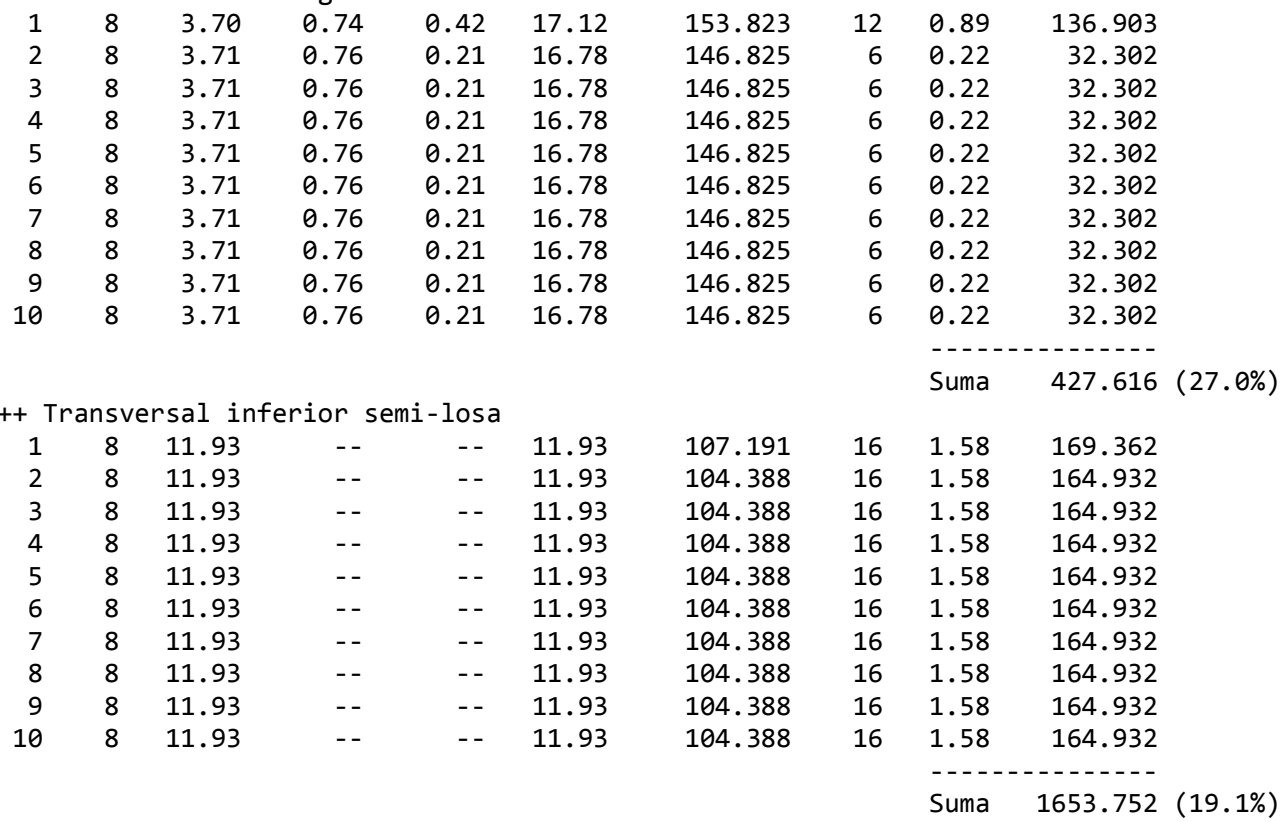




\begin{tabular}{|c|c|c|c|c|c|c|c|c|c|}
\hline 1 & 8 & 11.93 & -- & -- & 11.93 & 107.191 & 16 & 1.58 & 169.362 \\
\hline 2 & 8 & 11.93 & -- & -- & 11.93 & 104.388 & 16 & 1.58 & 164.932 \\
\hline 3 & 8 & 11.93 & -- & -- & 11.93 & 104.388 & 16 & 1.58 & 164.932 \\
\hline 4 & 8 & 11.93 & -- & -- & 11.93 & 104.388 & 16 & 1.58 & 164.932 \\
\hline 5 & 8 & 11.93 & -- & -- & 11.93 & 104.388 & 16 & 1.58 & 164.932 \\
\hline 6 & 8 & 11.93 & -- & -- & 11.93 & 104.388 & 16 & 1.58 & 164.932 \\
\hline 7 & 8 & 11.93 & -- & -- & 11.93 & 104.388 & 16 & 1.58 & 164.932 \\
\hline 8 & 8 & 11.93 & -- & -- & 11.93 & 104.388 & 16 & 1.58 & 164.932 \\
\hline 9 & 8 & 11.93 & - - & -- & 11.93 & 104.388 & 16 & 1.58 & 164.932 \\
\hline \multirow[t]{2}{*}{10} & 8 & 11.93 & -- & -- & 11.93 & 104.388 & 16 & 1.58 & 164.932 \\
\hline & & & & & & & & Suma & 1653.752 \\
\hline
\end{tabular}

Armadura longitudinal total tablero

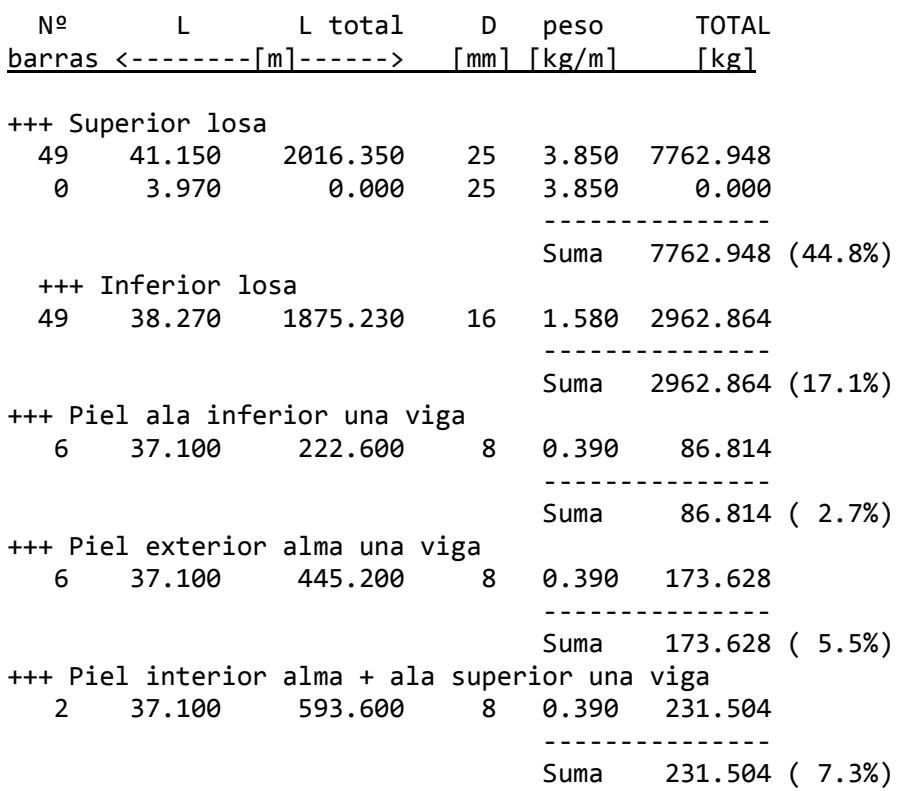

\section{ESTADO DE MEDICIONES}

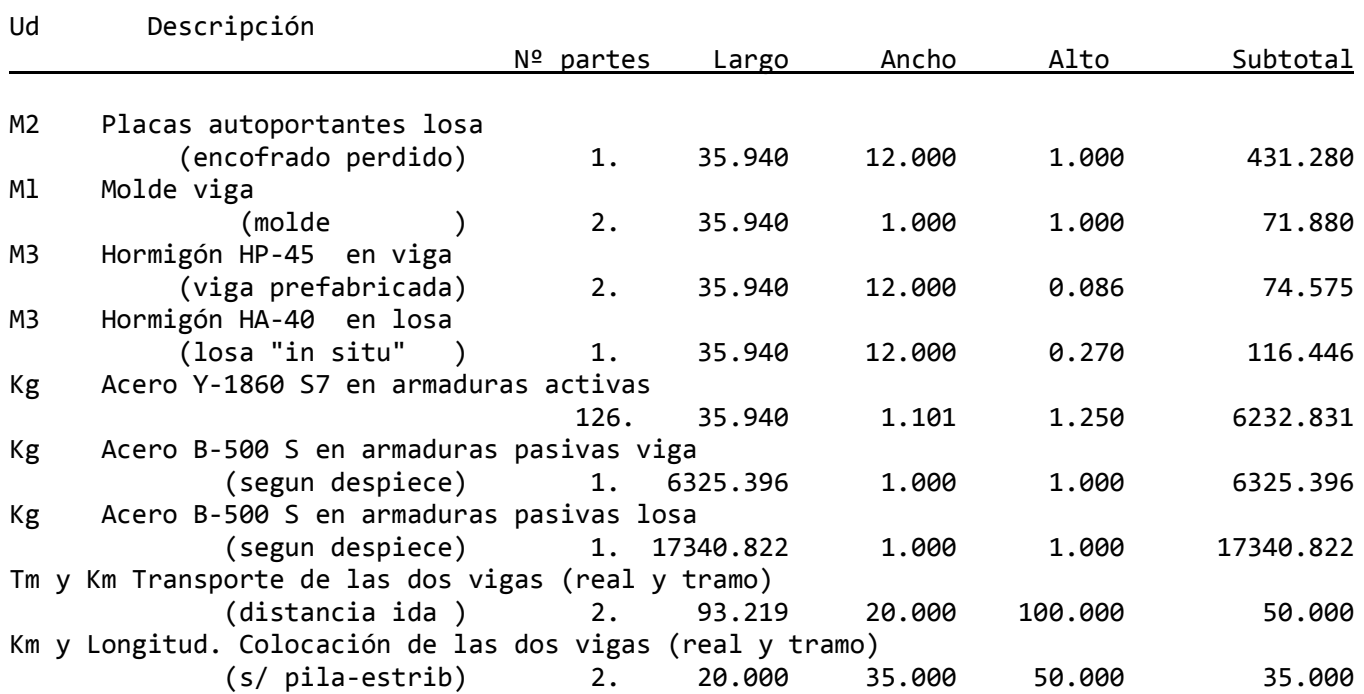




\section{CUANTIAS RESULTANTES}

0.442 M3 hormigon / M2 tablero

$32.629 \mathrm{~kg}$ activas / M3 hormigón

$83.578 \mathrm{~kg}$ activas / M3 hormigón VIGA

$14.428 \mathrm{~kg}$ activas / M2 tablero

$123.893 \mathrm{~kg}$ pasivas / M3 hormigón

$84.819 \mathrm{~kg}$ pasivas / M3 hormigón VIGA

$54.783 \mathrm{~kg}$ pasivas / M2 tablero

\section{CALCULO DEL COSTE}

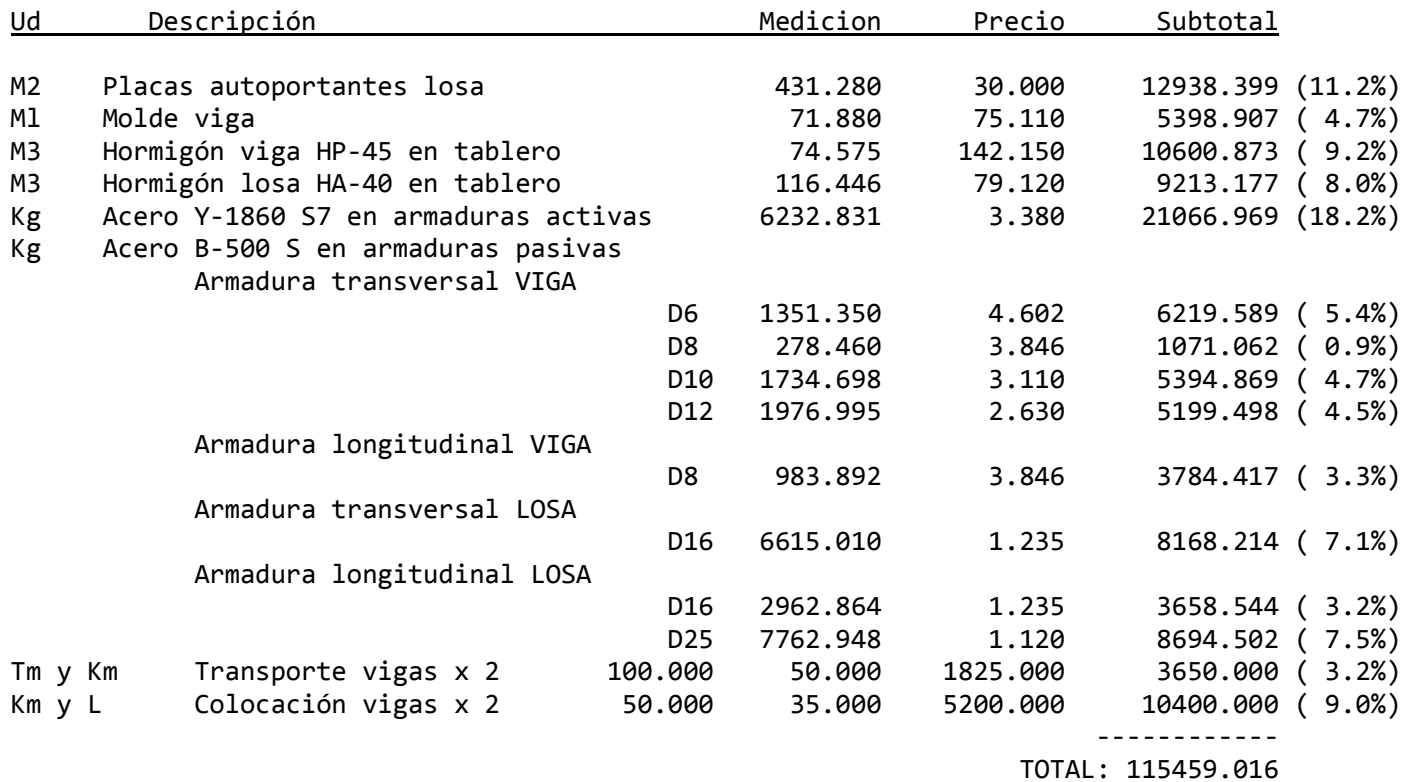

267.27 EUROS / M2 tablero 


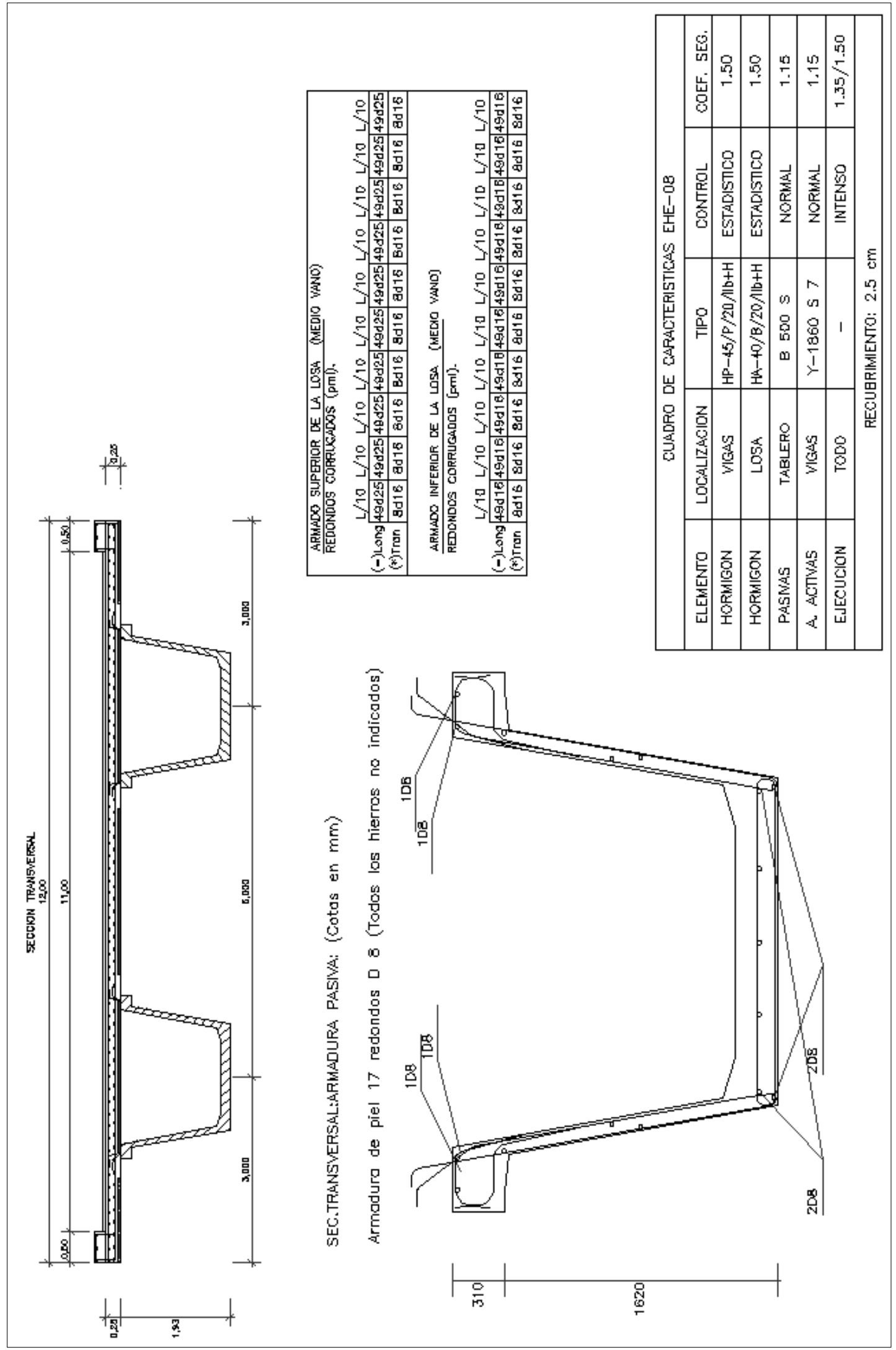




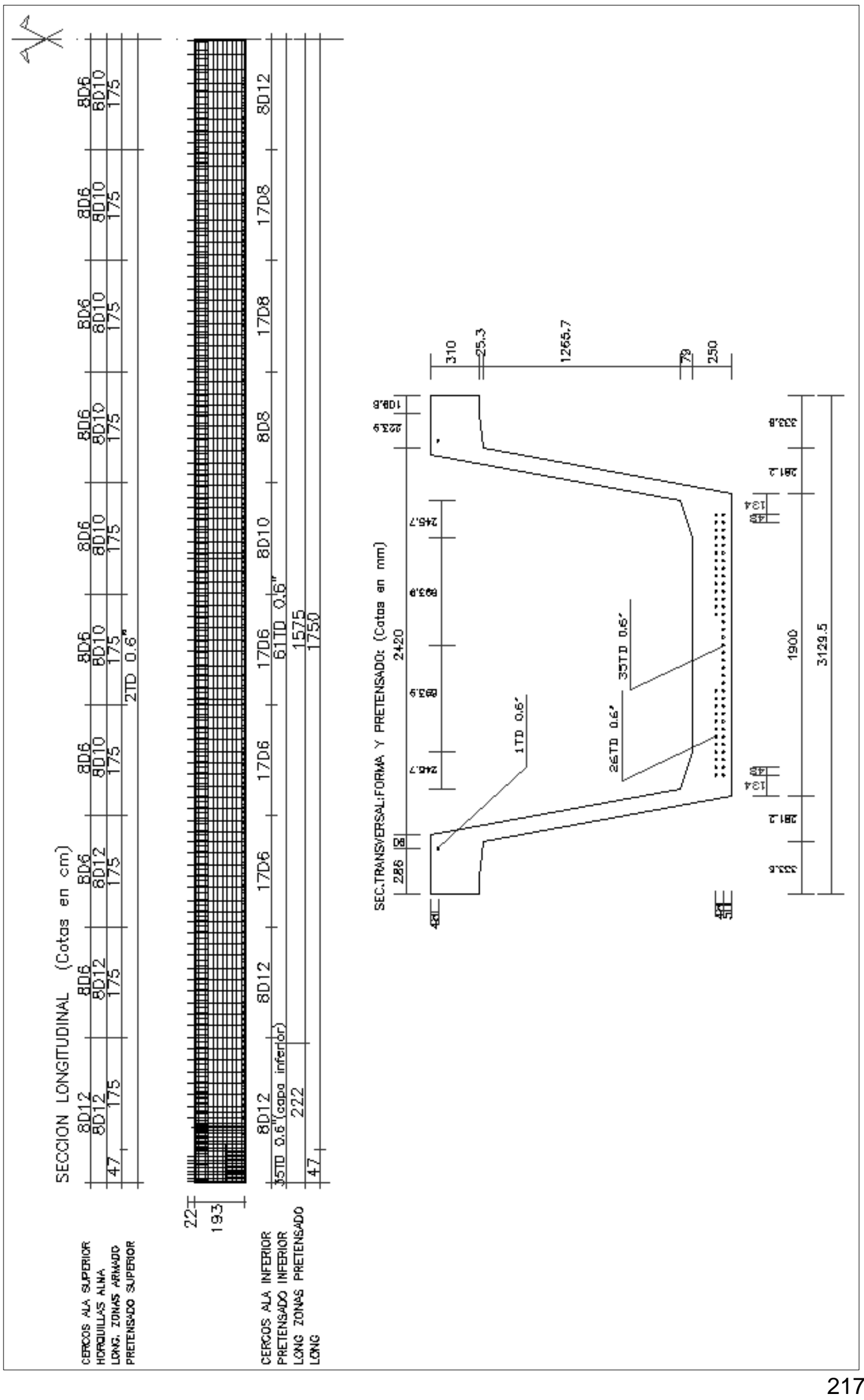


TA (Aceptación por umbrales)

Luz $=35 \mathrm{~m}$

ARMADURA PASIVA

Armadura transversal

№ $\quad \mathrm{L} 1 \quad \mathrm{~L} 2 \quad \mathrm{~L} 3 \quad \mathrm{~L} \quad \mathrm{~L}$ total $\quad \mathrm{D}$ peso

sec barras $<\cdots[\mathrm{mm}][\mathrm{kg} / \mathrm{m}] \quad[\mathrm{kg}]$

+++ Cerco ala inf.semi-viga

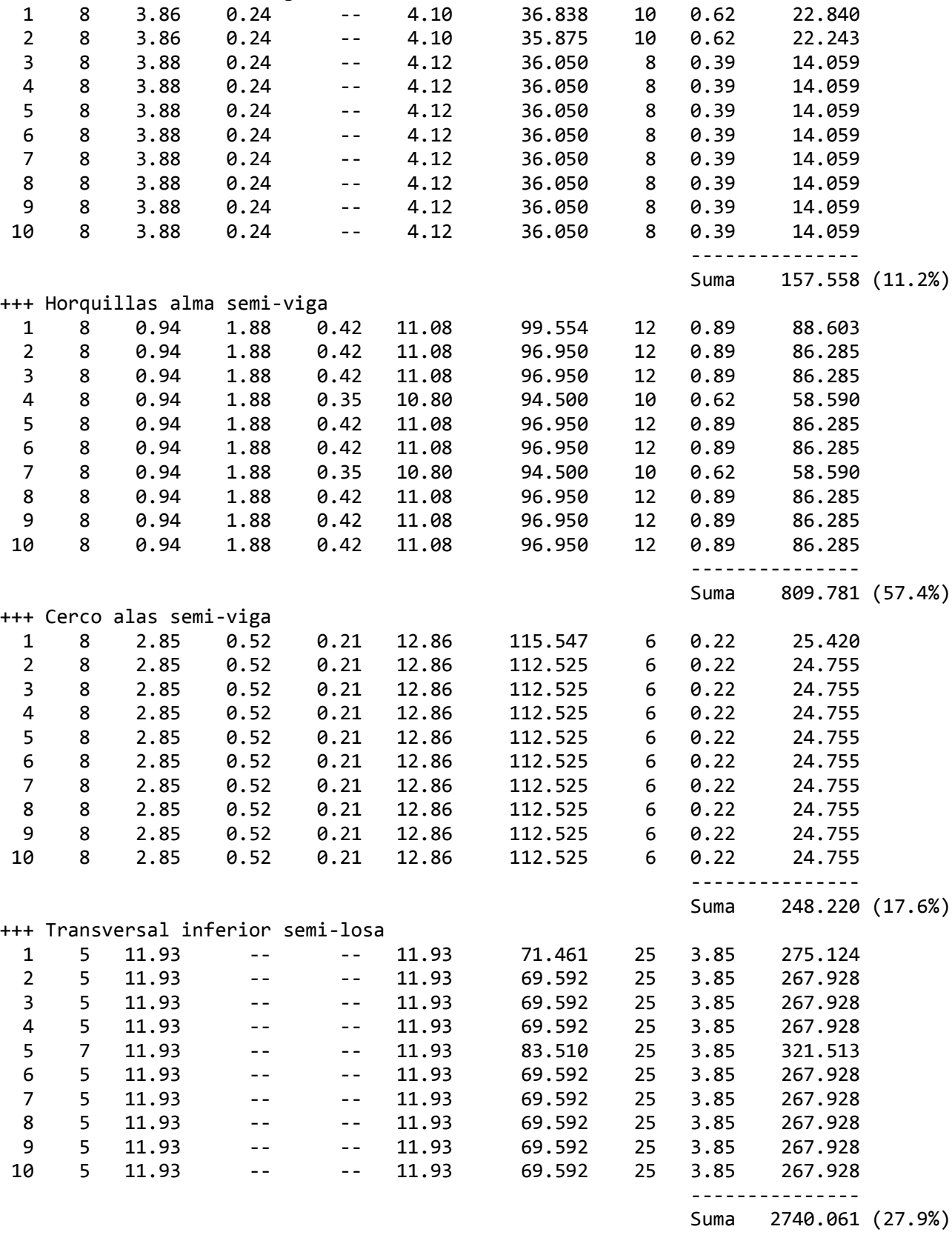




$\begin{array}{rrrrrrrrrr}+++ \\ 1 & 5 & 11.93 & -- & -- & 11.93 & 71.461 & 25 & 3.85 & 275.124 \\ 2 & 5 & 11.93 & -- & -- & 11.93 & 69.592 & 25 & 3.85 & 267.928 \\ 3 & 5 & 11.93 & -- & -- & 11.93 & 69.592 & 25 & 3.85 & 267.928 \\ 4 & 5 & 11.93 & -- & -- & 11.93 & 69.592 & 25 & 3.85 & 267.928 \\ 5 & 5 & 11.93 & -- & -- & 11.93 & 69.592 & 25 & 3.85 & 267.928 \\ 6 & 5 & 11.93 & -- & -- & 11.93 & 69.592 & 25 & 3.85 & 267.928 \\ 7 & 5 & 11.93 & -- & -- & 11.93 & 69.592 & 25 & 3.85 & 267.928 \\ 8 & 5 & 11.93 & -- & -- & 11.93 & 69.592 & 25 & 3.85 & 267.928 \\ 9 & 5 & 11.93 & -- & -- & 11.93 & 69.592 & 25 & 3.85 & 267.928 \\ 10 & 5 & 11.93 & -- & -- & 11.93 & 69.592 & 25 & 3.85 & 267.928 \\ & & & & & & & & & \\ \end{array}$

Armadura longitudinal total tablero

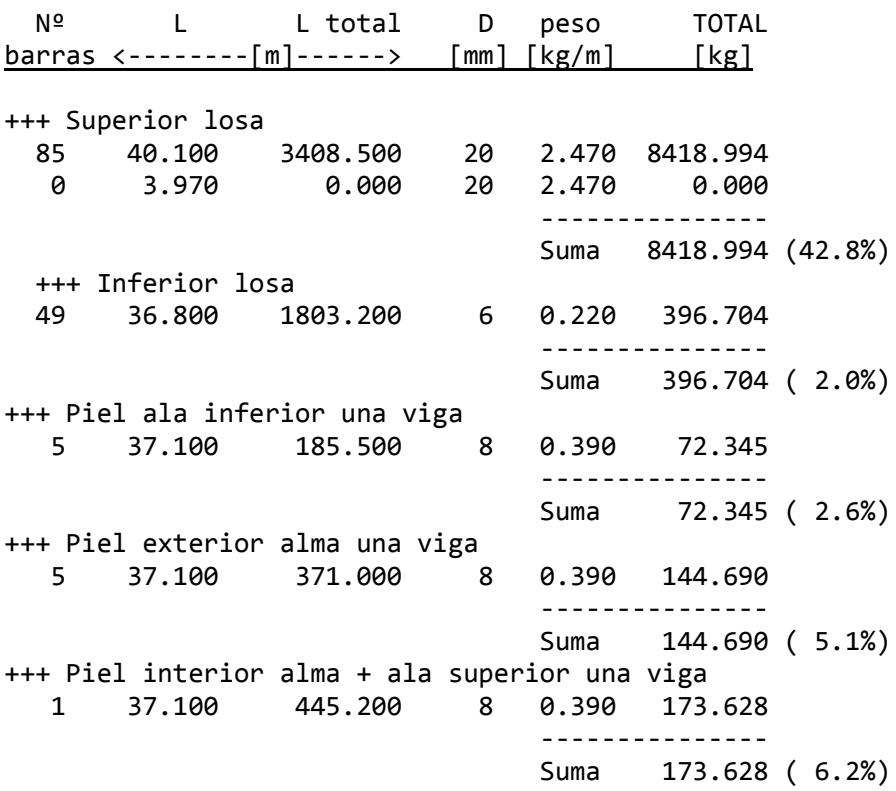

\section{ESTADO DE MEDICIONES}

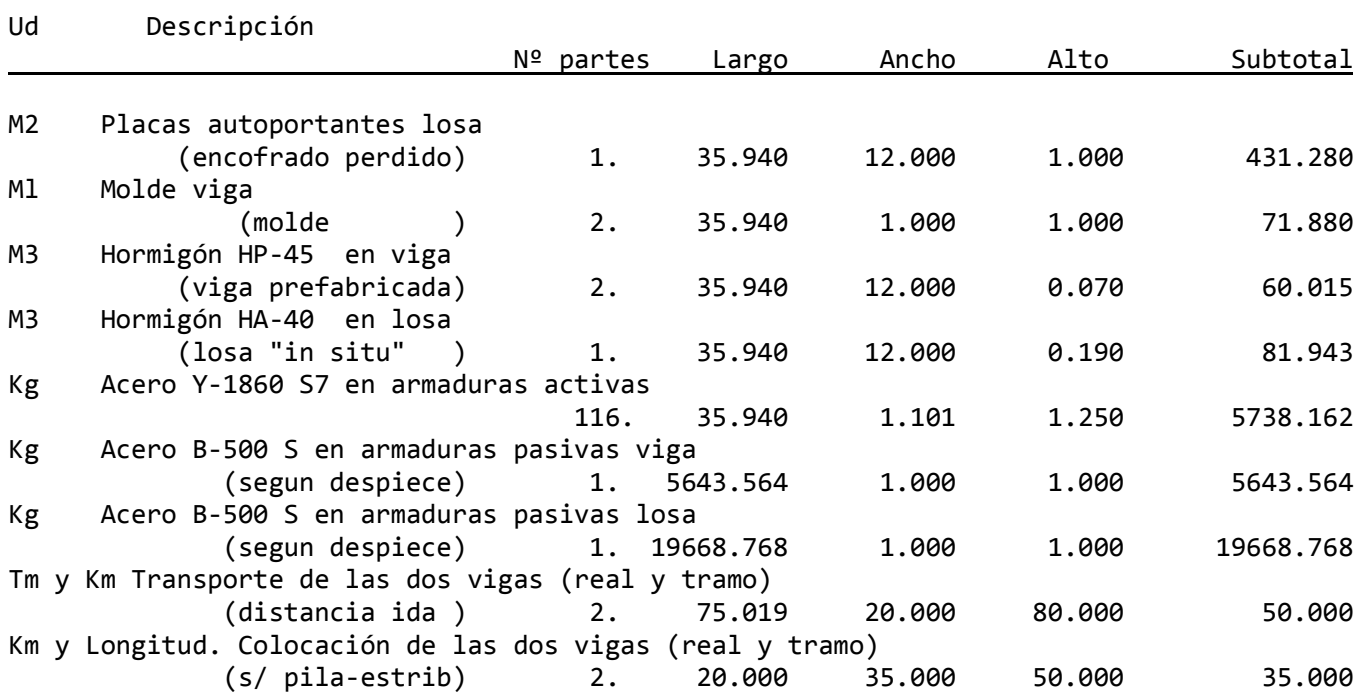




\section{CUANTIAS RESULTANTES}

0.329 M3 hormigon / M2 tablero

$40.421 \mathrm{~kg}$ activas / M3 hormigón

$95.612 \mathrm{~kg}$ activas / M3 hormigón VIGA

$13.283 \mathrm{~kg}$ activas / M2 tablero

178. $308 \mathrm{~kg}$ pasivas / M3 hormigón

$94.036 \mathrm{~kg}$ pasivas / M3 hormigón VIGA

$58.593 \mathrm{~kg}$ pasivas / M2 tablero

\section{CALCULO DEL COSTE}

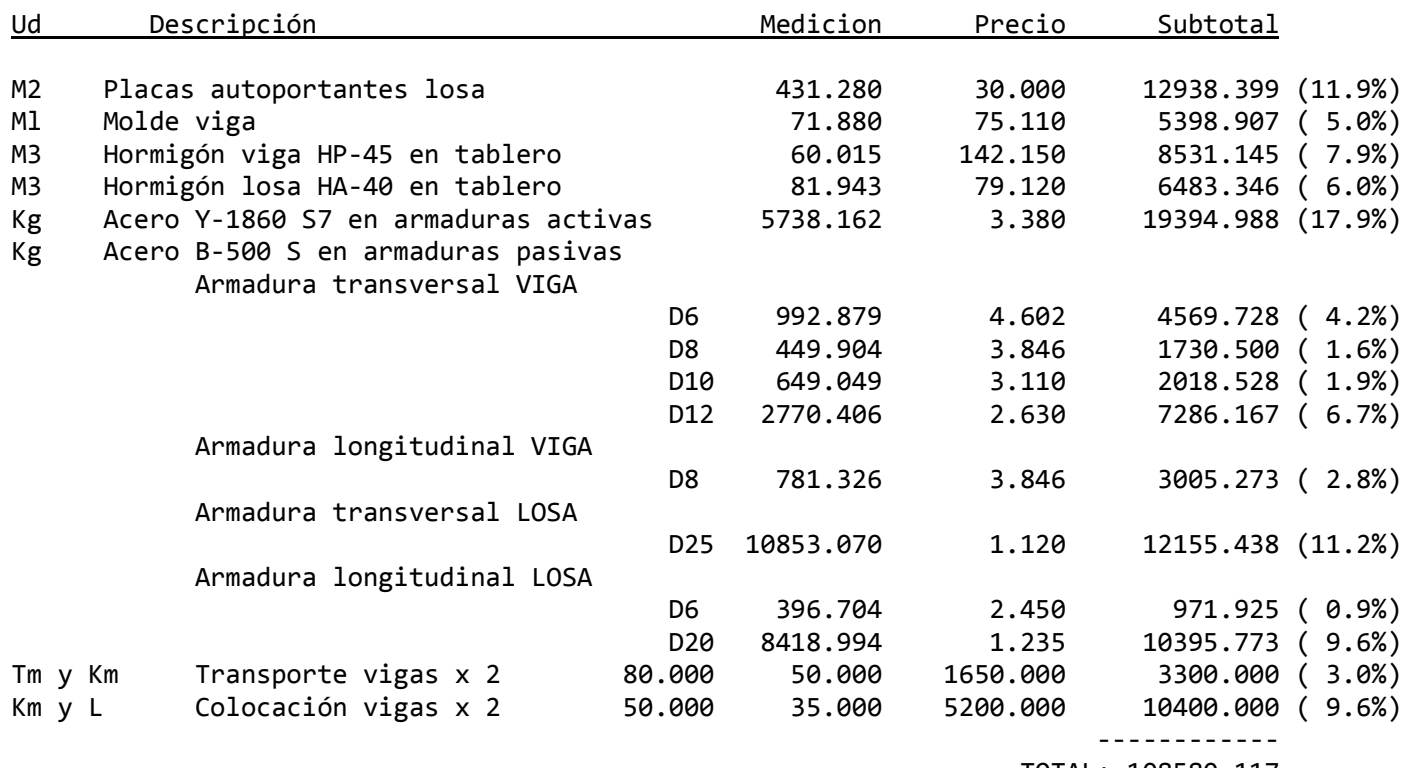

TOTAL: 108580.117

251.34 EUROS / M2 tablero 


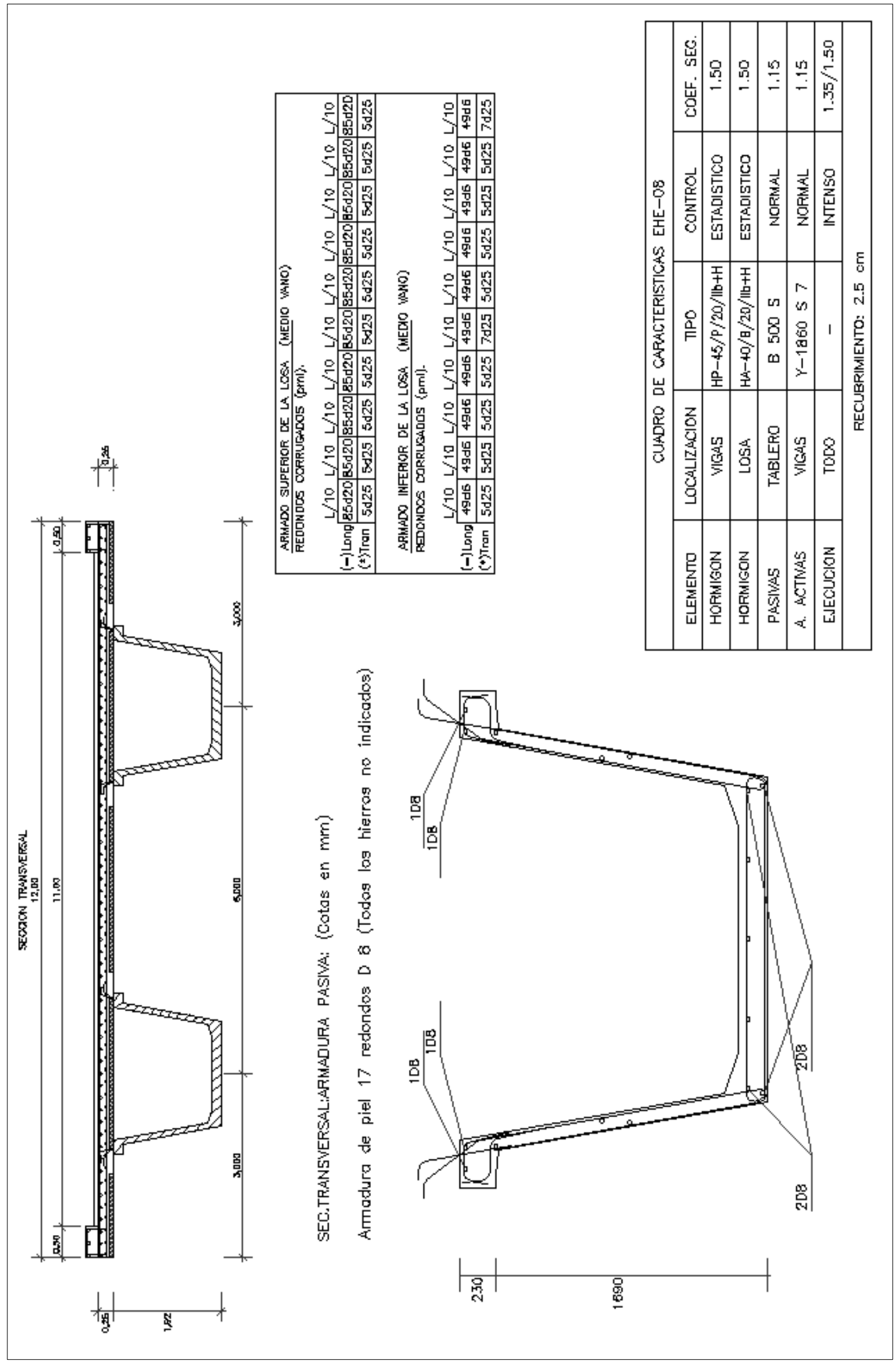




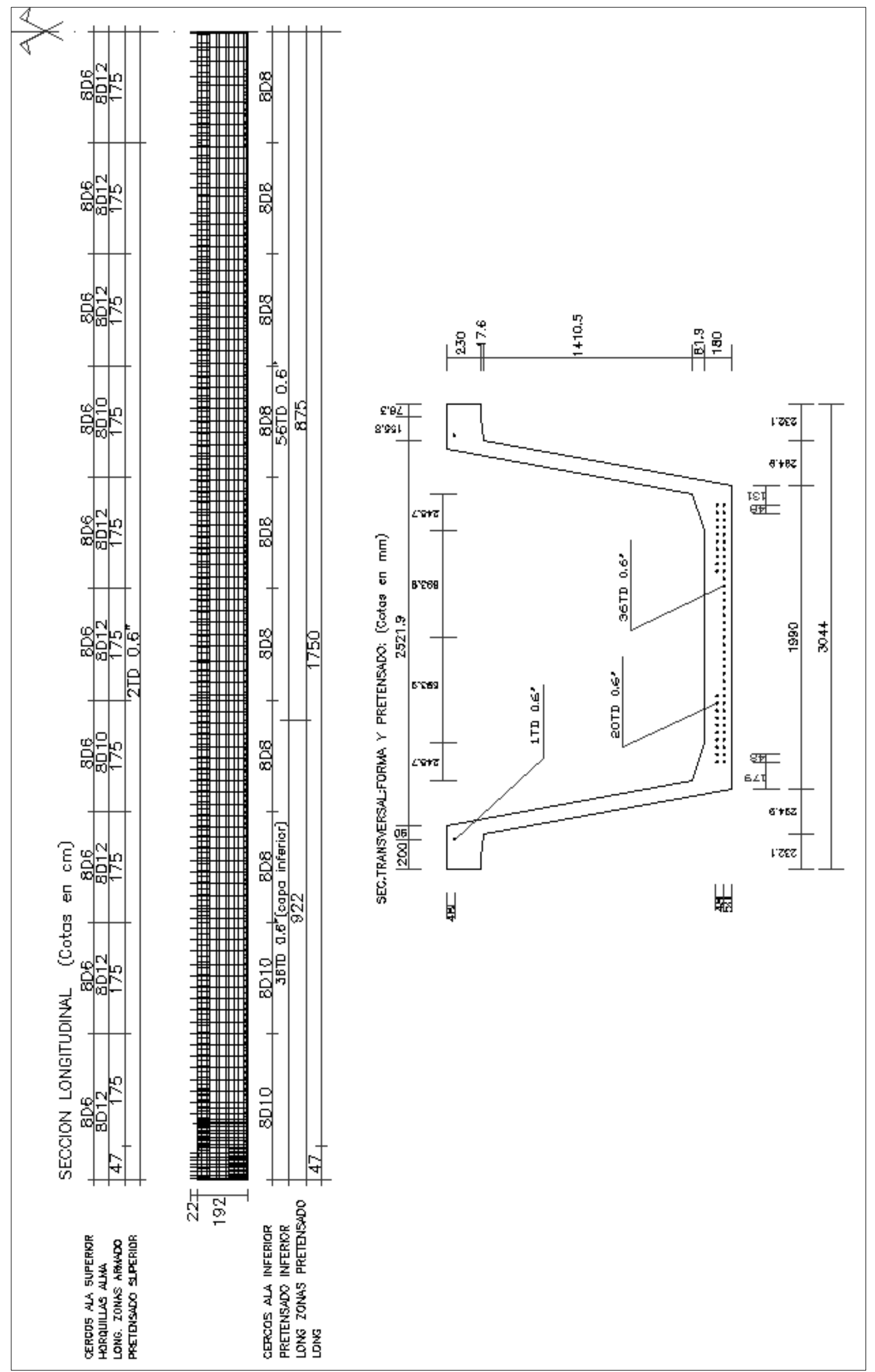


GA (Algoritmos genéticos)

Luz $=35 \mathrm{~m}$.

\section{ARMADURA PASIVA}

Armadura transversal

\begin{tabular}{|c|c|c|c|c|c|c|c|c|c|c|}
\hline $\mathrm{sec}$ & $\begin{array}{c}\text { № } \\
\text { barras }\end{array}$ & $\begin{array}{c}\mathrm{L} 1 \\
\mathrm{~S}<----- \\
\end{array}$ & $\begin{array}{c}\mathrm{L} 2 \\
-\end{array}$ & $\begin{array}{c}\text { L3 } \\
---[n\end{array}$ & $\begin{array}{c}\mathrm{L} \\
-\end{array}$ & L total & $\begin{array}{c}\mathrm{D} \\
{[\mathrm{mm}]} \\
\end{array}$ & $\begin{array}{c}\text { peso } \\
{[\mathrm{kg} / \mathrm{m}]}\end{array}$ & $\begin{array}{l}\text { TOTAL } \\
{[\mathrm{kg}]}\end{array}$ & \\
\hline \multicolumn{11}{|c|}{ o ala inf.semi-viga } \\
\hline 1 & 8 & 3.84 & 0.32 & - - & 4.16 & 37.378 & 12 & 0.89 & 33.266 & \\
\hline 2 & 8 & 3.84 & 0.32 & - & 4.16 & 36.400 & 12 & 0.89 & 32.396 & \\
\hline 3 & 8 & 3.84 & 0.32 & -- & 4.16 & 36.400 & 12 & 0.89 & 32.396 & \\
\hline 4 & 8 & 3.84 & 0.32 & - & 4.16 & 36.400 & 12 & 0.89 & 32.396 & \\
\hline 5 & 17 & 3.84 & 0.34 & - & 4.18 & 73.150 & 10 & 0.62 & 45.353 & \\
\hline 6 & 8 & 3.84 & 0.34 & -- & 4.18 & 36.575 & 10 & 0.62 & 22.676 & \\
\hline 7 & 8 & 3.84 & 0.32 & -- & 4.16 & 36.400 & 12 & 0.89 & 32.396 & \\
\hline 8 & 8 & 3.84 & 0.32 & - & 4.16 & 36.400 & 12 & 0.89 & 32.396 & \\
\hline 9 & 8 & 3.84 & 0.32 & - & 4.16 & 36.400 & 12 & 0.89 & 32.396 & \\
\hline \multirow[t]{3}{*}{10} & 8 & 3.84 & 0.32 & -- & 4.16 & 36.400 & 12 & 0.89 & 32.396 & \\
\hline & & & & & & & \multicolumn{4}{|c|}{ - - - - - - - - - - - } \\
\hline & & & & & & & & Suma & 328.068 & $(18.3 \%)$ \\
\hline \multicolumn{11}{|c|}{ +++ Horquillas alma semi-viga } \\
\hline 1 & 17 & 1.34 & 1.65 & 0.35 & 10.68 & 191.920 & 10 & 0.62 & 118.990 & \\
\hline 2 & 17 & 1.34 & 1.65 & 0.35 & 10.68 & 186.900 & 10 & 0.62 & 115.878 & \\
\hline 3 & 8 & 1.34 & 1.65 & 0.42 & 10.96 & 95.900 & 12 & 0.89 & 85.351 & \\
\hline 4 & 8 & 1.34 & 1.65 & 0.42 & 10.96 & 95.900 & 12 & 0.89 & 85.351 & \\
\hline 5 & 8 & 1.34 & 1.65 & 0.35 & 10.68 & 93.450 & 10 & 0.62 & 57.939 & \\
\hline 6 & 8 & 1.34 & 1.65 & 0.42 & 10.96 & 95.900 & 12 & 0.89 & 85.351 & \\
\hline 7 & 8 & 1.34 & 1.65 & 0.35 & 10.68 & 93.450 & 10 & 0.62 & 57.939 & \\
\hline 8 & 8 & 1.34 & 1.65 & 0.42 & 10.96 & 95.900 & 12 & 0.89 & 85.351 & \\
\hline 9 & 8 & 1.34 & 1.65 & 0.42 & 10.96 & 95.900 & 12 & 0.89 & 85.351 & \\
\hline \multirow[t]{3}{*}{10} & 8 & 1.34 & 1.65 & 0.42 & 10.96 & 95.900 & 12 & 0.89 & 85.351 & \\
\hline & & & & & & & & \multicolumn{3}{|c|}{ - - - - - - - - - - - - } \\
\hline & & & & & & & & Suma & 862.852 & $(48.2 \%)$ \\
\hline \multicolumn{11}{|c|}{ +++ Cerco alas semi-viga } \\
\hline 1 & 8 & 4.00 & 0.79 & 0.21 & 18.00 & 161.730 & 6 & 0.22 & 35.581 & \\
\hline 2 & 8 & 4.00 & 0.79 & 0.21 & 18.00 & 157.500 & 6 & 0.22 & 34.650 & \\
\hline 3 & 8 & 4.00 & 0.79 & 0.21 & 18.00 & 157.500 & 6 & 0.22 & 34.650 & \\
\hline 4 & 8 & 4.00 & 0.79 & 0.21 & 18.00 & 157.500 & 6 & 0.22 & 34.650 & \\
\hline 5 & 8 & 4.00 & 0.79 & 0.21 & 18.00 & 157.500 & 6 & 0.22 & 34.650 & \\
\hline 6 & 8 & 4.00 & 0.79 & 0.21 & 18.00 & 157.500 & 6 & 0.22 & 34.650 & \\
\hline 7 & 8 & 4.00 & 0.79 & 0.21 & 18.00 & 157.500 & 6 & 0.22 & 34.650 & \\
\hline 8 & 8 & 4.00 & 0.79 & 0.21 & 18.00 & 157.500 & 6 & 0.22 & 34.650 & \\
\hline 9 & 8 & 4.00 & 0.79 & 0.21 & 18.00 & 157.500 & 6 & 0.22 & 34.650 & \\
\hline \multirow[t]{3}{*}{10} & 8 & 4.00 & 0.79 & 0.21 & 18.00 & 157.500 & 6 & 0.22 & 34.650 & \\
\hline & & & & & & & & \multicolumn{3}{|c|}{$--1-1-1-1$} \\
\hline & & & & & & & & Suma & 347.431 & $(19.4 \%)$ \\
\hline \multicolumn{11}{|c|}{ +++ Transversal inferior semi-losa } \\
\hline 1 & 8 & 11.92 & -- & -- & 11.92 & 107.101 & 20 & 2.47 & 264.540 & \\
\hline 2 & 8 & 11.92 & -- & -- & 11.92 & 104.300 & 20 & 2.47 & 257.621 & \\
\hline 3 & 8 & 11.92 & -- & -- & 11.92 & 104.300 & 20 & 2.47 & 257.621 & \\
\hline 4 & 8 & 11.92 & -- & -- & 11.92 & 104.300 & 20 & 2.47 & 257.621 & \\
\hline 5 & 8 & 11.92 & - & - & 11.92 & 104.300 & 20 & 2.47 & 257.621 & \\
\hline 6 & 11 & 11.92 & - & - & 11.92 & 139.067 & 20 & 2.47 & 343.495 & \\
\hline 7 & 8 & 11.92 & - & - & 11.92 & 104.300 & 20 & 2.47 & 257.621 & \\
\hline 8 & 8 & 11.92 & -- & -- & 11.92 & 104.300 & 20 & 2.47 & 257.621 & \\
\hline 9 & 11 & 11.92 & -- & - & 11.92 & 139.067 & 20 & 2.47 & 343.495 & \\
\hline \multirow[t]{2}{*}{10} & 8 & 11.92 & -- & -- & 11.92 & 104.300 & 20 & 2.47 & 257.621 & \\
\hline & & & & & & & & \multicolumn{3}{|c|}{------------} \\
\hline
\end{tabular}




$\begin{array}{rrrrrrrrrr}+++ \\ 1 & 7 & 11.92 & -- & -- & 11.92 & 85.681 & 20 & 2.47 & 211.632 \\ 2 & 7 & 11.92 & -- & -- & 11.92 & 83.440 & 20 & 2.47 & 206.097 \\ 3 & 7 & 11.92 & -- & -- & 11.92 & 83.440 & 20 & 2.47 & 206.097 \\ 4 & 7 & 11.92 & -- & -- & 11.92 & 83.440 & 20 & 2.47 & 206.097 \\ 5 & 7 & 11.92 & -- & -- & 11.92 & 83.440 & 20 & 2.47 & 206.097 \\ 6 & 7 & 11.92 & -- & -- & 11.92 & 83.440 & 20 & 2.47 & 206.097 \\ 7 & 7 & 11.92 & -- & -- & 11.92 & 83.440 & 20 & 2.47 & 206.097 \\ 8 & 7 & 11.92 & -- & -- & 11.92 & 83.440 & 20 & 2.47 & 206.097 \\ 9 & 7 & 11.92 & -- & -- & 11.92 & 83.440 & 20 & 2.47 & 206.097 \\ 10 & 7 & 11.92 & -- & -- & 11.92 & 83.440 & 20 & 2.47 & 206.097 \\ & & & & & & & & & ------------- \\ \end{array}$

Armadura longitudinal total tablero

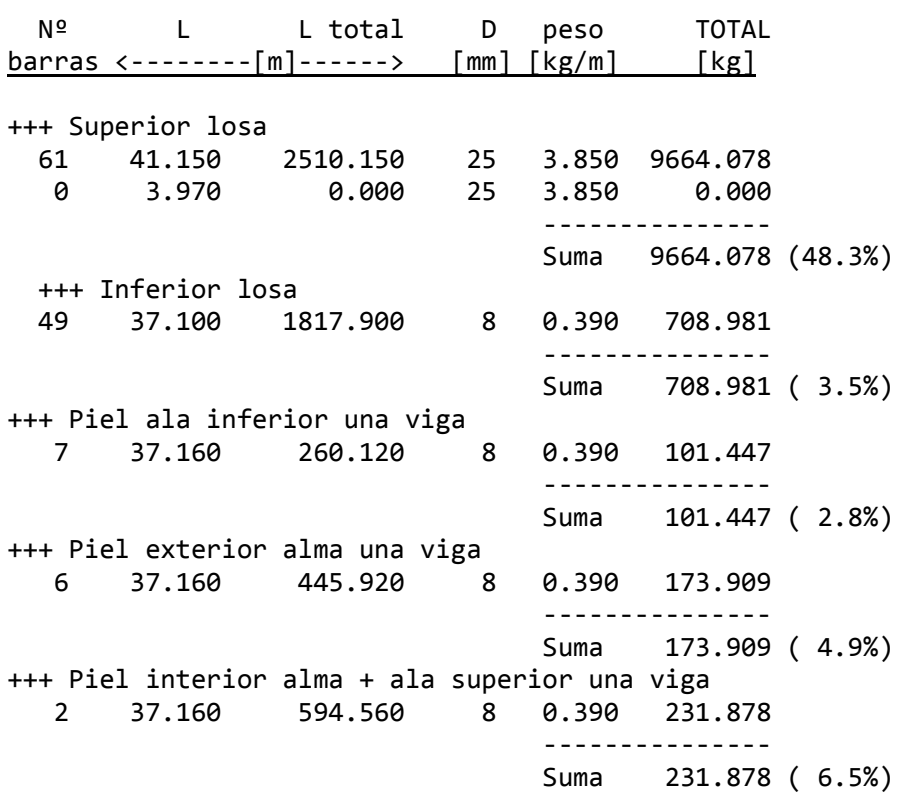

\section{ESTADO DE MEDICIONES}

\begin{tabular}{|c|c|c|c|c|c|c|}
\hline Ud & Descripción & o partes & Largo & Ancho & Alto & Subtotal \\
\hline \multirow[t]{2}{*}{ M2 } & Placas autoportantes losa & & & & & \\
\hline & $\begin{array}{l}\text { (encofrado perdido) } \\
\text { Molde viga }\end{array}$ & 1. & 35.940 & 12.000 & 1.000 & 431.280 \\
\hline M1 & (molde $\quad$ ) & 2. & 35.940 & 1.000 & 1.000 & 71.880 \\
\hline M3 & $\begin{array}{l}\text { Hormigón HP-40 en viga } \\
\text { (viga prefabricada) }\end{array}$ & 2. & 35.940 & 12.000 & 0.091 & 78.263 \\
\hline M3 & $\begin{array}{l}\text { Hormigón HA-35 en losa } \\
\text { (losa "in situ" ) }\end{array}$ & 1 & 35.940 & 12.000 & 0.220 & 94.882 \\
\hline Kg & Acero Y-1860 S7 en armaduras & $\begin{array}{l}\text { activas } \\
152\end{array}$ & 35.940 & 1.101 & 1.250 & 7518.971 \\
\hline Kg & $\begin{array}{c}\text { Acero } \mathrm{B}-500 \mathrm{~S} \text { en armaduras } \mathrm{p} \\
\text { (segun despiece) }\end{array}$ & $\begin{array}{c}\text { pasivas v } \\
1 .\end{array}$ & 167.869 & 1.000 & 1.000 & 7167.869 \\
\hline Kg & $\begin{array}{l}\text { Acero B-500 S en armaduras } \mathrm{p} \\
\text { (segun despiece) } \\
\text { Km Transporte de las dos viga }\end{array}$ & $\begin{array}{c}\text { oasivas } 10 \\
1 . \\
\text { as (real })\end{array}$ & $\begin{array}{l}215.816 \\
\text { tramo) }\end{array}$ & 1.000 & 1.000 & 20015.816 \\
\hline Tm & $\begin{array}{l}\text { (distancia ida ) } \\
\text { Longitud. Colocación de las d }\end{array}$ & $\begin{array}{l}2 . \\
\text { tos vigas }\end{array}$ & $\begin{array}{l}97.829 \\
\text { real y } t\end{array}$ & 20.000 & 100.000 & 50.000 \\
\hline & (s/ pila-estrib) & 2. & 20.000 & 35.000 & 50.000 & 35.000 \\
\hline
\end{tabular}




\section{CUANTIAS RESULTANTES}

0.401 M3 hormigon / M2 tablero

43.426 kg activas / M3 hormigón

$96.073 \mathrm{~kg}$ activas / M3 hormigón VIGA

$17.405 \mathrm{~kg}$ activas / M2 tablero

$157.000 \mathrm{~kg}$ pasivas / M3 hormigón

$91.587 \mathrm{~kg}$ pasivas / M3 hormigón VIGA

$62.925 \mathrm{~kg}$ pasivas / M2 tablero

\section{CALCULO DEL COSTE}

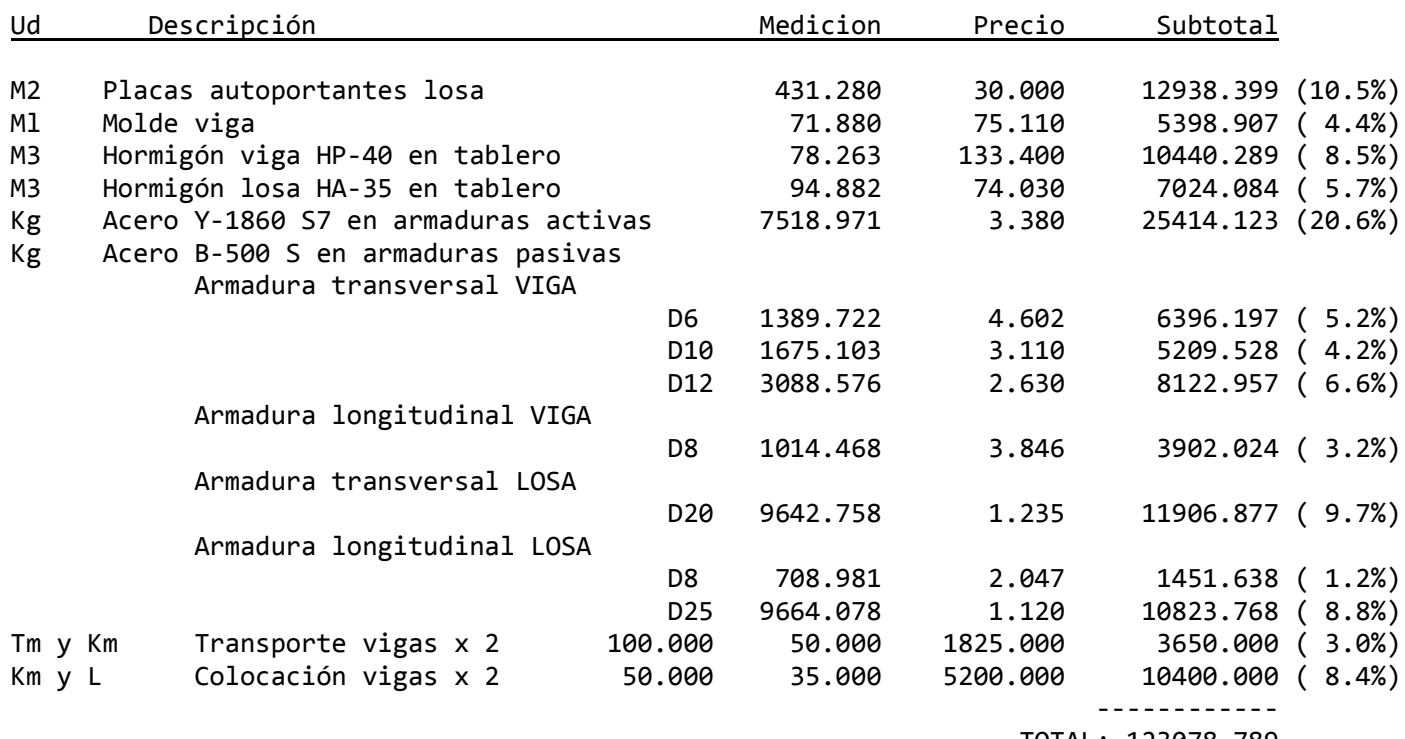

TOTAL: 123078.789 


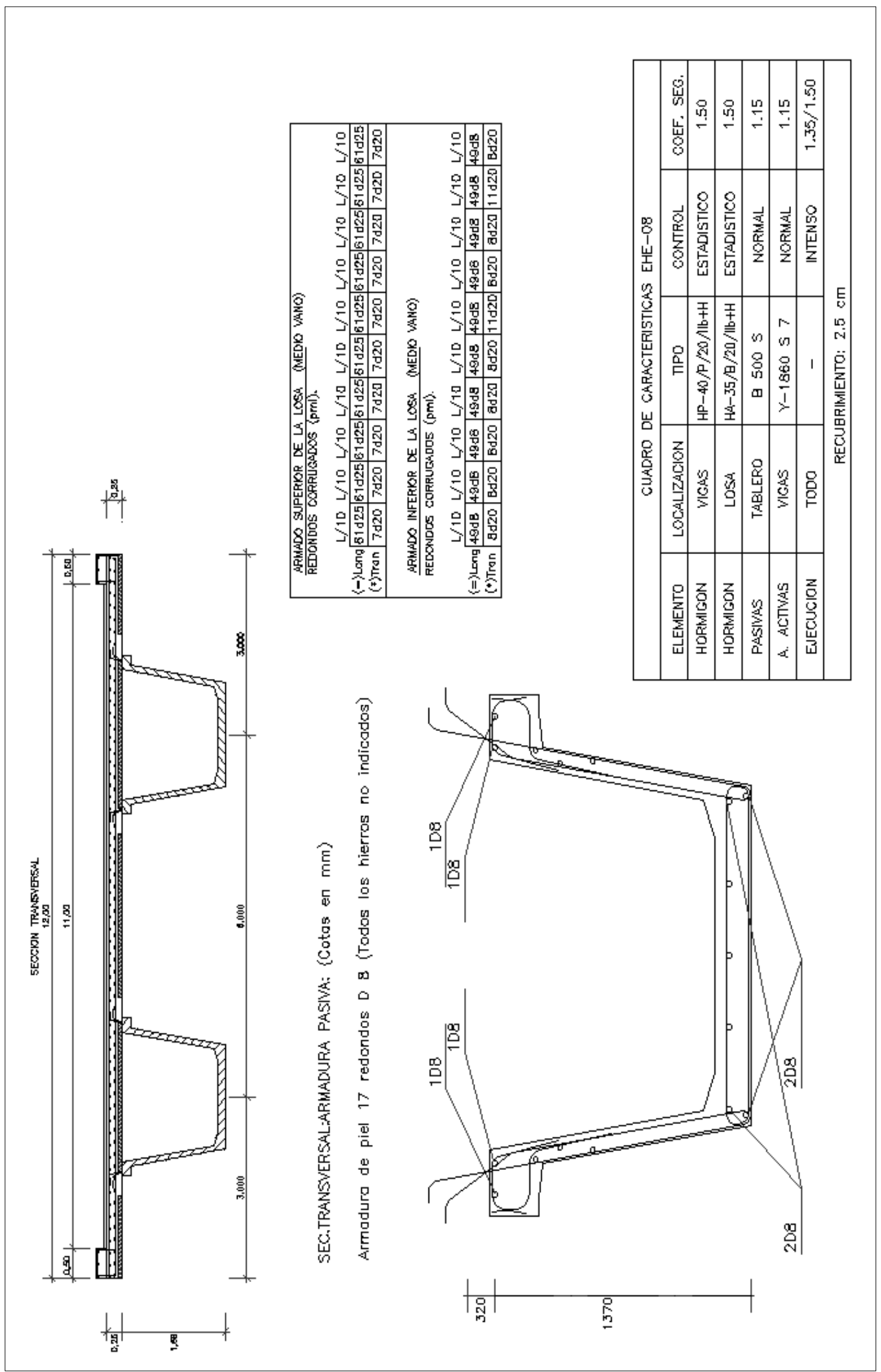




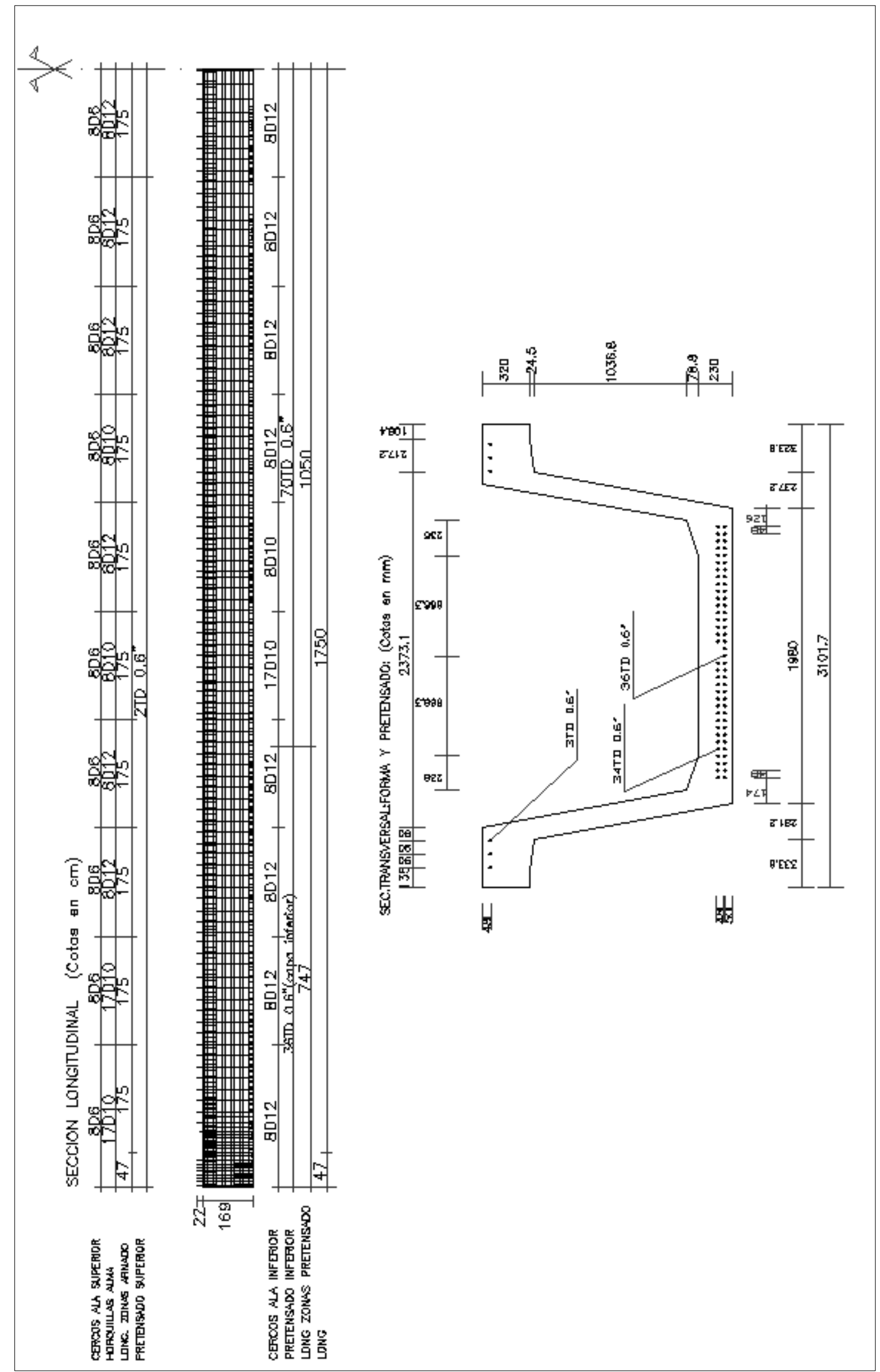


MA (Algoritmos meméticos)

Luz $=35 \mathrm{~m}$.

ARMADURA PASIVA

Armadura transversal

№ $\quad L 1$ L $\quad L 2 \quad L 3 \quad L \quad L \quad L$ total $\quad D$ peso $\quad$ TOTAL

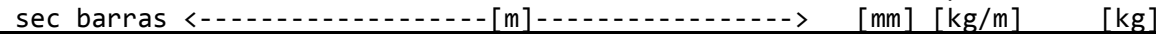

+++ Cerco ala inf.semi-viga

$\begin{array}{llllllllll}1 & 8 & 3.72 & 0.34 & -- & 4.06 & 36.479 & 10 & 0.62 & 22.617 \\ 2 & 8 & 3.72 & 0.34 & -- & 4.06 & 35.525 & 10 & 0.62 & 22.025\end{array}$

$\begin{array}{llllllllll} & 8 & 3.72 & 0.34 & - & 4.06 & 35.525 & 10 & 0.62 & 22.025 \\ 4 & 8 & 3.72 & 0.32 & - & 4.04 & 35.350 & 12 & 0.89 & 31.461\end{array}$

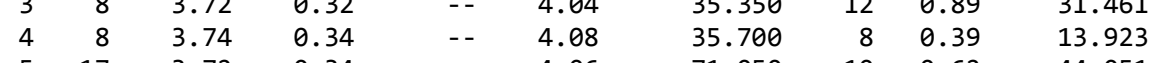

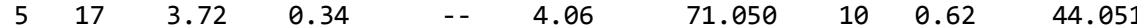

$\begin{array}{lrllllllll}6 & 8 & 3.72 & 0.34 & -- & 4.06 & 35.525 & 10 & 0.62 & 22.025\end{array}$

$\begin{array}{llllllllll}7 & 17 & 3.74 & 0.34 & -- & 4.08 & 71.400 & 8 & 0.39 & 27.846\end{array}$

$\begin{array}{llllllllll}8 & 17 & 3.74 & 0.34 & -- & 4.08 & 71.400 & 8 & 0.39 & 27.846\end{array}$

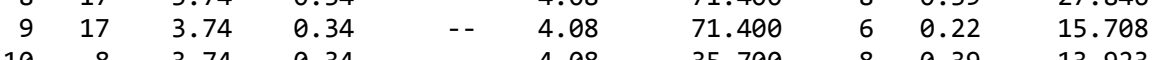

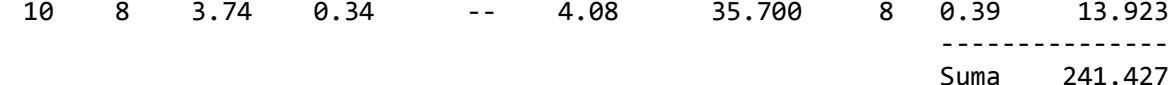

+++ Horquillas alma semi-viga

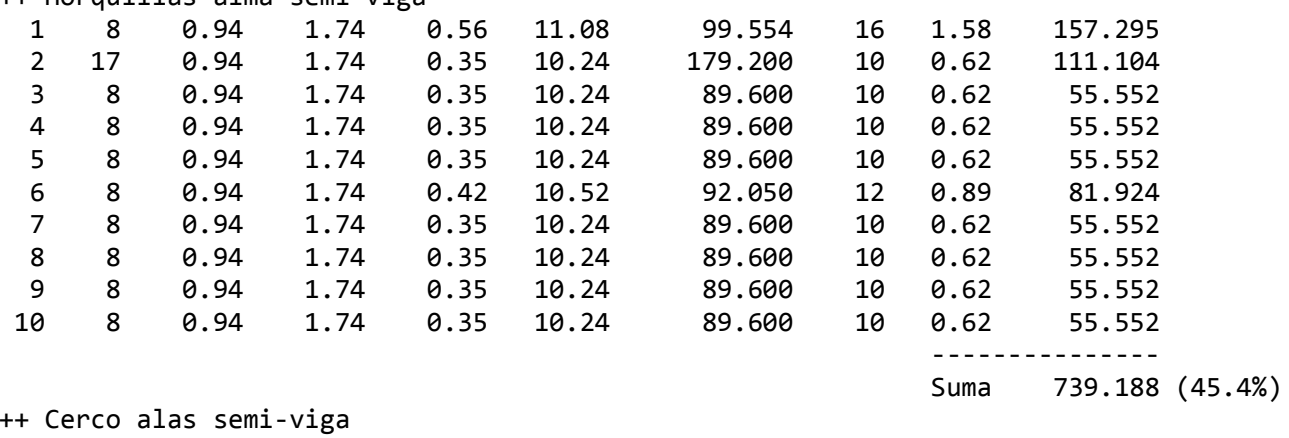

\begin{tabular}{|c|c|c|c|c|c|c|c|c|c|}
\hline 1 & 8 & 3.63 & 0.72 & 0.28 & 16.52 & 148.432 & 8 & 0.39 & 57889 \\
\hline 2 & 8 & 3.63 & 0.73 & 0.21 & 16.40 & 143.500 & 6 & 0.22 & 31.570 \\
\hline 3 & 8 & 3.62 & 0.72 & 0.35 & 16.62 & 145.425 & 10 & 0.62 & 90.163 \\
\hline 4 & 8 & 3.63 & 0.73 & 0.21 & 16.40 & 143.500 & 6 & 0.22 & 31.570 \\
\hline 5 & 8 & 3.63 & 0.73 & 0.21 & 16.40 & 143.500 & 6 & 0.22 & 31.570 \\
\hline 6 & 8 & 3.63 & 0.73 & 0.21 & 16.40 & 143.500 & 6 & 0.22 & 31.570 \\
\hline 7 & 8 & 3.63 & 0.73 & 0.21 & 16.40 & 143.500 & 6 & 0.22 & 31.570 \\
\hline 8 & 8 & 3.63 & 0.73 & 0.21 & 16.40 & 143.500 & 6 & 0.22 & 31.570 \\
\hline 9 & 8 & 3.63 & 0.73 & 0.21 & 16.40 & 143.500 & 6 & 0.22 & 31.570 \\
\hline 10 & 8 & 3.63 & 0.73 & 0.21 & 16.40 & 143.500 & 6 & 0.22 & 31.570 \\
\hline & & & & & & & & Suma & $400.612(24.6 \%)$ \\
\hline +++ T & insv & rsal i & ior $s$ & - los & & & & & \\
\hline 1 & 7 & 11.93 & -- & -- & 11.93 & 85.753 & 20 & 2.47 & 211.810 \\
\hline 2 & 8 & 11.93 & - & -- & 11.93 & 104.388 & 20 & 2.47 & 257.837 \\
\hline 3 & 5 & 11.93 & - & -- & 11.93 & 69.592 & 20 & 2.47 & 171.891 \\
\hline 4 & 7 & 11.93 & -- & -- & 11.93 & 83.510 & 20 & 2.47 & 206.270 \\
\hline 5 & 5 & 11.93 & -- & -- & 11.93 & 69.592 & 20 & 2.47 & 171.891 \\
\hline 6 & 7 & 11.93 & -- & -- & 11.93 & 83.510 & 20 & 2.47 & 206.270 \\
\hline 7 & 8 & 11.93 & -- & -- & 11.93 & 104.388 & 20 & 2.47 & 257.837 \\
\hline 8 & 5 & 11.93 & -- & -- & 11.93 & 69.592 & 20 & 2.47 & 171.891 \\
\hline 9 & 7 & 11.93 & -- & -- & 11.93 & 83.510 & 20 & 2.47 & 206.270 \\
\hline 10 & 7 & 11.93 & -- & -- & 11.93 & 83.510 & 20 & 2.47 & 206.270 \\
\hline & & & & & & & & Suma & 2068.237 (24.1\%) \\
\hline
\end{tabular}




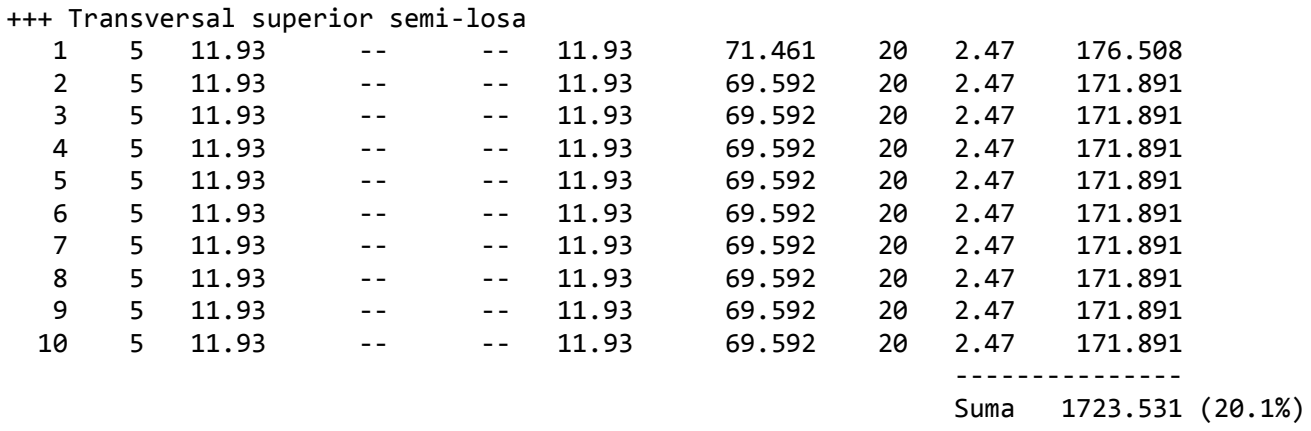

Armadura longitudinal total tablero

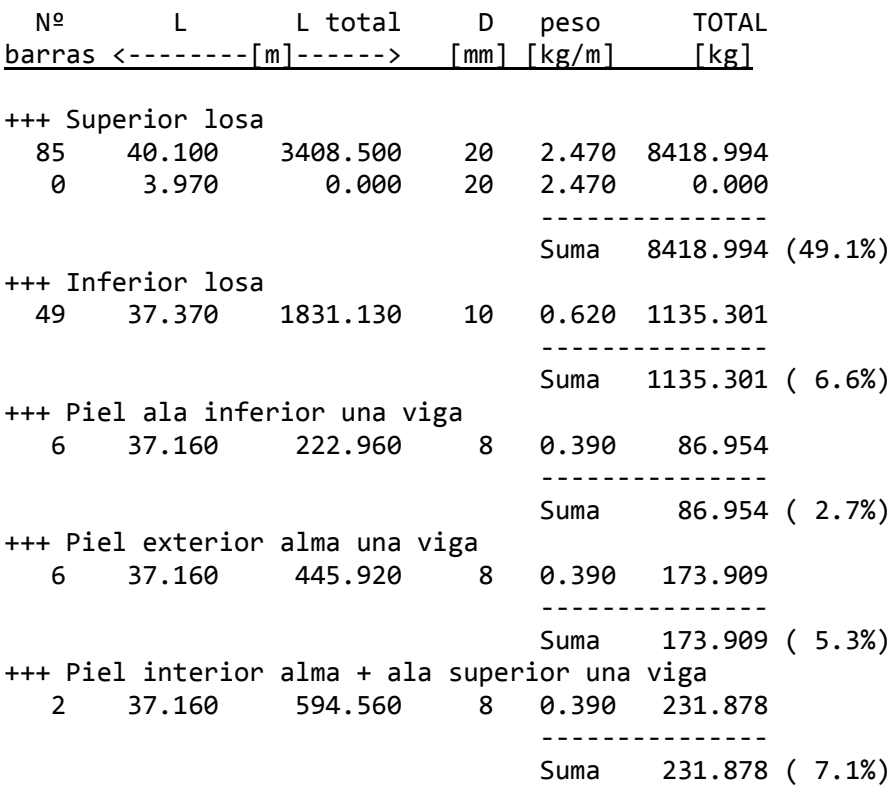

\section{ESTADO DE MEDICIONES}

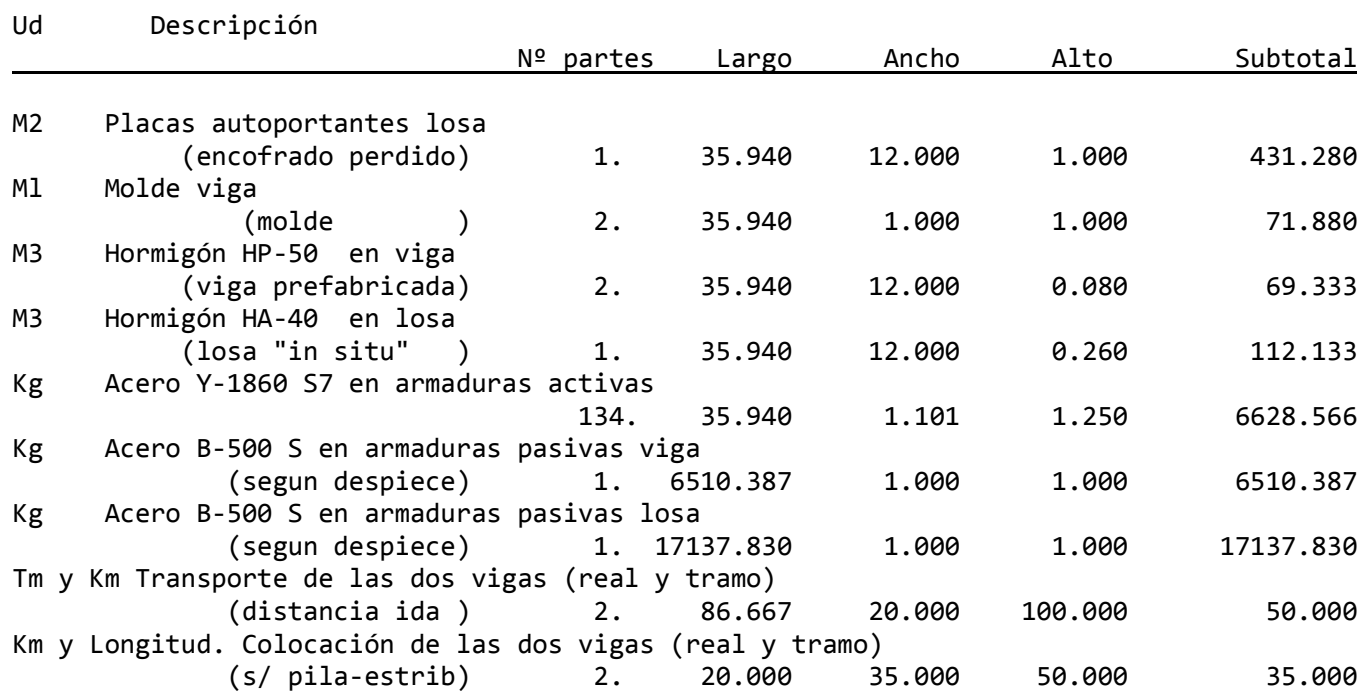


CUANTIAS RESULTANTES

0.420 M3 hormigon / M2 tablero

$36.528 \mathrm{~kg}$ activas / M3 hormigón

$95.604 \mathrm{~kg}$ activas / M3 hormigón VIGA

$15.344 \mathrm{~kg}$ activas / M2 tablero

$130.318 \mathrm{~kg}$ pasivas / M3 hormigón

$93.900 \mathrm{~kg}$ pasivas / M3 hormigón VIGA

$54.741 \mathrm{~kg}$ pasivas / M2 tablero

\section{CALCULO DEL COSTE}

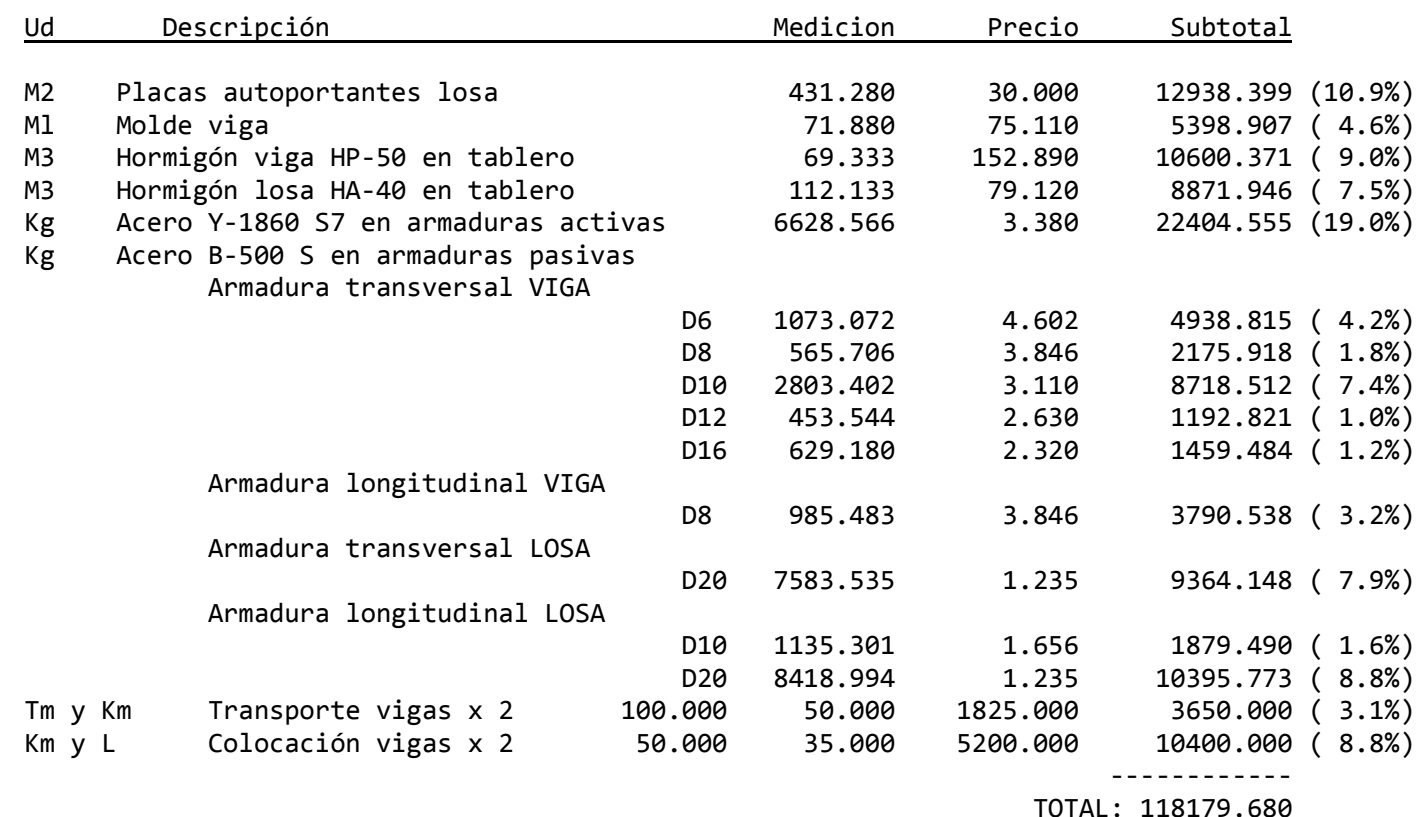

273.56 EUROS / M2 tablero 


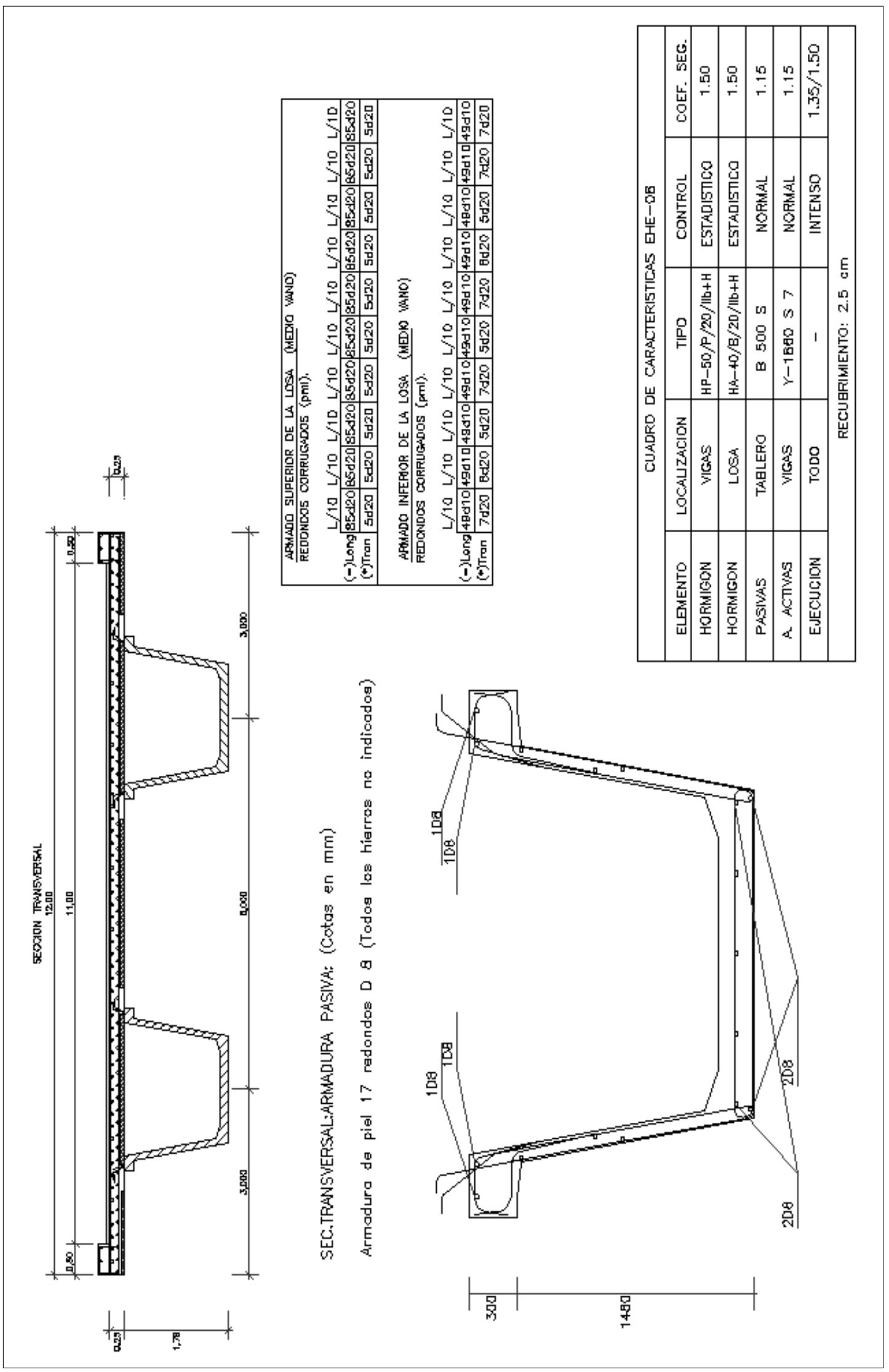




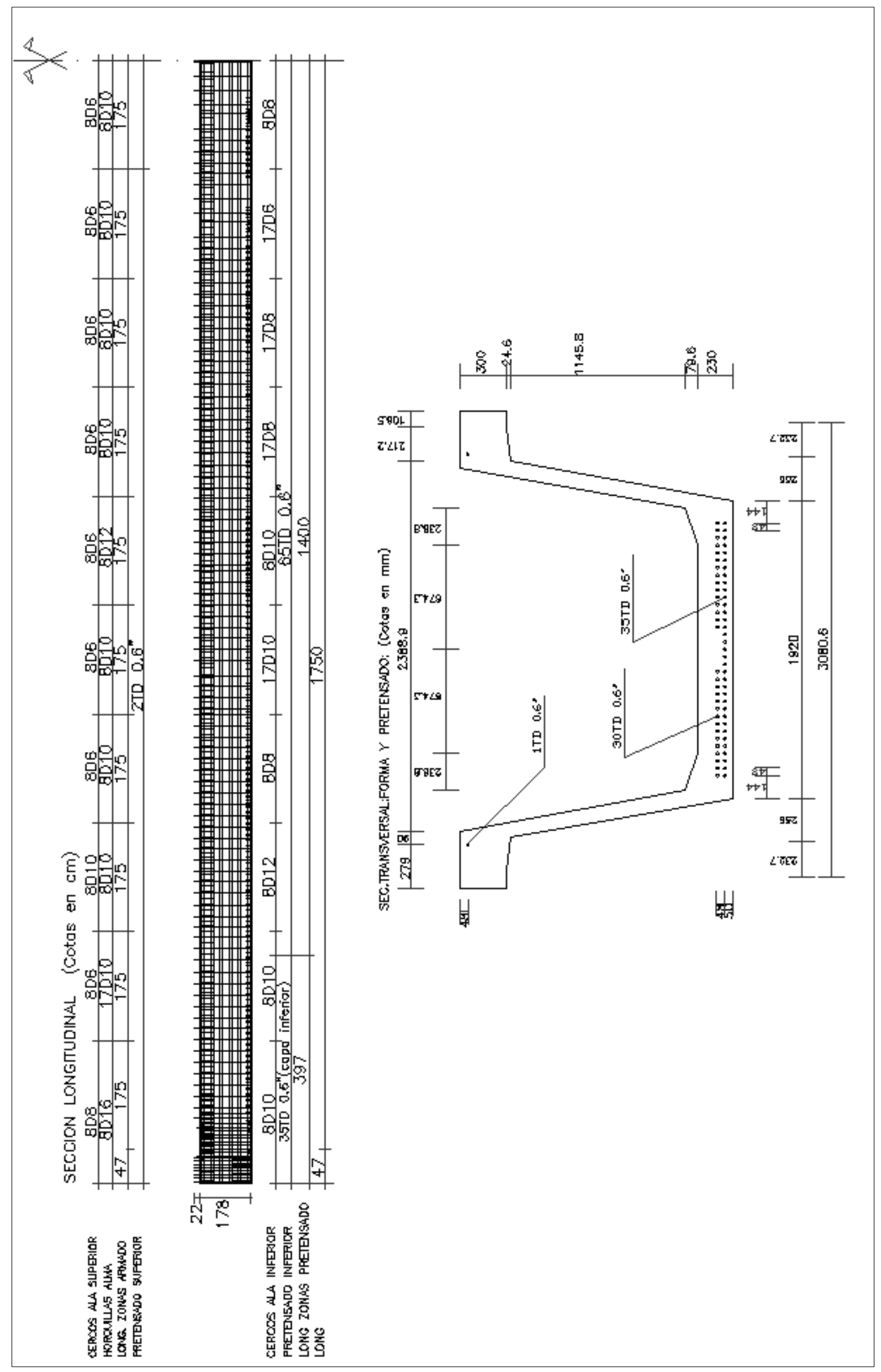


C.II Estudio paramétrico (SA) 
$\mathrm{L}=20 \mathrm{~m} . \quad \mathrm{HP}$

\section{ARMADURA PASIVA}

Armadura transversal

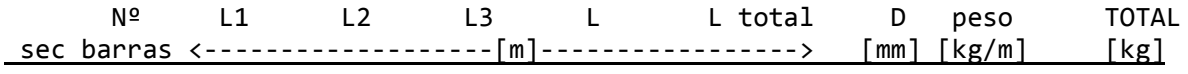

\begin{tabular}{|c|c|c|c|c|c|c|c|c|c|c|}
\hline 1 & 10 & 3.88 & 0.18 & - - & 406 & 42508 & 8 & a 30 & 16578 & \\
\hline 2 & 10 & 3.88 & 0.18 & _- & 496 & 49690 & 8 & a 39 & 15834 & \\
\hline 3 & 5 & 3.86 & 0.18 & - - & 4.04 & 20.200 & 10 & 0.62 & 12.524 & \\
\hline 4 & 5 & 3.86 & 0.18 & -- & 4.04 & 20.200 & 10 & 0.62 & 12.524 & \\
\hline 5 & 5 & 3.86 & 0.18 & - & 4.04 & 20.200 & 10 & 0.62 & 12.524 & \\
\hline 6 & 5 & 3.86 & 0.18 & - & 4.04 & 20.200 & 10 & 0.62 & 12.524 & \\
\hline 7 & 5 & 3.86 & 0.18 & - & 4.04 & 20.200 & 10 & 0.62 & 12.524 & \\
\hline 8 & 5 & 3.86 & 0.16 & - & 4.02 & 20.100 & 12 & 0.89 & 17.889 & \\
\hline 9 & 5 & 3.86 & 0.18 & -- & 4.04 & 20.200 & 10 & 0.62 & 12.524 & \\
\hline \multirow[t]{3}{*}{10} & 5 & 3.86 & 0.18 & - & 4.04 & 20.200 & 10 & 0.62 & 12.524 & \\
\hline & & & & & & & & \multicolumn{3}{|c|}{ 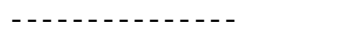 } \\
\hline & & & & & & & & Suma & 137.969 & $(22.5 \%)$ \\
\hline \multicolumn{11}{|c|}{ +++ Horquillas alma semi-viga } \\
\hline 1 & 10 & 0.94 & 1.04 & 0.35 & 7.44 & 77.897 & 10 & 0.62 & 48.296 & \\
\hline 2 & 10 & 0.94 & 1.04 & 0.35 & 7.44 & 74.400 & 10 & 0.62 & 46.128 & \\
\hline 3 & 5 & 0.95 & 1.04 & 0.28 & 7.18 & 35.900 & 8 & 0.39 & 14.001 & \\
\hline 4 & 5 & 0.95 & 1.04 & 0.28 & 7.18 & 35.900 & 8 & 0.39 & 14.001 & \\
\hline 5 & 5 & 0.95 & 1.04 & 0.21 & 6.90 & 34.500 & 6 & 0.22 & 7.590 & \\
\hline 6 & 5 & 0.95 & 1.04 & 0.21 & 6.90 & 34.500 & 6 & 0.22 & 7.590 & \\
\hline 7 & 5 & 0.95 & 1.04 & 0.21 & 6.90 & 34.500 & 6 & 0.22 & 7.590 & \\
\hline 8 & 5 & 0.95 & 1.04 & 0.21 & 6.90 & 34.500 & 6 & 0.22 & 7.590 & \\
\hline 9 & 5 & 0.95 & 1.04 & 0.21 & 6.90 & 34.500 & 6 & 0.22 & 7.590 & \\
\hline \multirow[t]{3}{*}{10} & 5 & 0.95 & 1.04 & 0.21 & 6.90 & 34.500 & 6 & 0.22 & 7.590 & \\
\hline & & & & & & & & \multicolumn{3}{|c|}{$-\ldots-\ldots-1--\ldots$} \\
\hline & & & & & & & & Suma & 167.966 & $(27.4 \%)$ \\
\hline \multicolumn{11}{|c|}{ +++ Cerco alas semi-viga } \\
\hline 1 & 5 & 2.53 & 0.39 & 0.28 & 11.46 & 59.993 & 8 & 0.39 & 23.397 & \\
\hline 2 & 5 & 2.53 & 0.39 & 0.28 & 11.46 & 57.300 & 8 & 0.39 & 22.347 & \\
\hline 3 & 5 & 2.53 & 0.39 & 0.28 & 11.46 & 57.300 & 8 & 0.39 & 22.347 & \\
\hline 4 & 5 & 2.53 & 0.39 & 0.28 & 11.46 & 57.300 & 8 & 0.39 & 22.347 & \\
\hline 5 & 5 & 2.53 & 0.39 & 0.28 & 11.46 & 57.300 & 8 & 0.39 & 22.347 & \\
\hline 6 & 5 & 2.53 & 0.39 & 0.28 & 11.46 & 57.300 & 8 & 0.39 & 22.347 & \\
\hline 7 & 5 & 2.53 & 0.39 & 0.28 & 11.46 & 57.300 & 8 & 0.39 & 22.347 & \\
\hline 8 & 5 & 2.53 & 0.39 & 0.28 & 11.46 & 57.300 & 8 & 0.39 & 22.347 & \\
\hline 9 & 5 & 2.53 & 0.39 & 0.28 & 11.46 & 57.300 & 8 & 0.39 & 22.347 & \\
\hline \multirow[t]{3}{*}{10} & 5 & 2.53 & 0.39 & 0.28 & 11.46 & 57.300 & 8 & 0.39 & 22.347 & \\
\hline & & & & & & & & \multicolumn{3}{|c|}{$------1---$} \\
\hline & & & & & & & & Suma & 224.520 & $(36.6 \%)$ \\
\hline \multicolumn{11}{|c|}{ +++ Transversal inferior semi-losa } \\
\hline 1 & 3 & 11.93 & -- & -- & 11.93 & 41.636 & 25 & 3.85 & 160.297 & \\
\hline 2 & 3 & 11.93 & -- & -- & 11.93 & 39.767 & 25 & 3.85 & 153.102 & \\
\hline 3 & 3 & 11.93 & -- & -- & 11.93 & 39.767 & 25 & 3.85 & 153.102 & \\
\hline 4 & 3 & 11.93 & - & -- & 11.93 & 39.767 & 25 & 3.85 & 153.102 & \\
\hline 5 & 3 & 11.93 & - & -- & 11.93 & 39.767 & 25 & 3.85 & 153.102 & \\
\hline 6 & 3 & 11.93 & -- & - - & 11.93 & 39.767 & 25 & 3.85 & 153.102 & \\
\hline 7 & 3 & 11.93 & - - & - - & 11.93 & 39.767 & 25 & 3.85 & 153.102 & \\
\hline 8 & 3 & 11.93 & -- & -- & 11.93 & 39.767 & 25 & 3.85 & 153.102 & \\
\hline 9 & 3 & 11.93 & - & - & 11.93 & 39.767 & 25 & 3.85 & 153.102 & \\
\hline \multirow[t]{3}{*}{10} & 3 & 11.93 & -- & -- & 11.93 & 39.767 & 25 & 3.85 & 153.102 & \\
\hline & & & & & & & & \multirow{2}{*}{\multicolumn{3}{|c|}{ 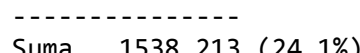 }} \\
\hline & & & & & & & & & & \\
\hline
\end{tabular}




$\begin{array}{rlllllllll}+++ \\ 1 & 3 & 11.93 & -- & -- & 11.93 & 41.636 & 25 & 3.85 & 160.297 \\ 2 & 3 & 11.93 & -- & -- & 11.93 & 39.767 & 25 & 3.85 & 153.102 \\ 3 & 3 & 11.93 & -- & -- & 11.93 & 39.767 & 25 & 3.85 & 153.102 \\ 4 & 3 & 11.93 & -- & -- & 11.93 & 39.767 & 25 & 3.85 & 153.102 \\ 5 & 3 & 11.93 & -- & -- & 11.93 & 39.767 & 25 & 3.85 & 153.102 \\ 6 & 3 & 11.93 & -- & -- & 11.93 & 39.767 & 25 & 3.85 & 153.102 \\ 7 & 3 & 11.93 & -- & -- & 11.93 & 39.767 & 25 & 3.85 & 153.102 \\ 8 & 3 & 11.93 & -- & -- & 11.93 & 39.767 & 25 & 3.85 & 153.102 \\ 9 & 3 & 11.93 & -- & -- & 11.93 & 39.767 & 25 & 3.85 & 153.102 \\ 10 & 3 & 11.93 & -- & -- & 11.93 & 39.767 & 25 & 3.85 & 153.102 \\ & & & & & & & & & ----------\end{array}$

Armadura longitudinal total tablero

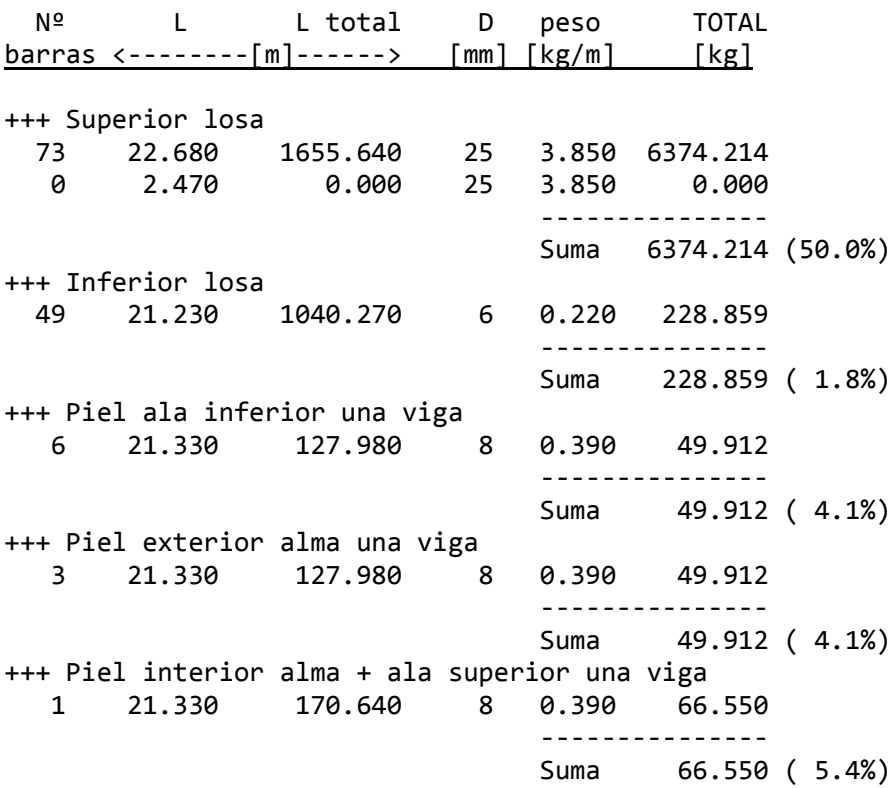

\section{ESTADO DE MEDICIONES}

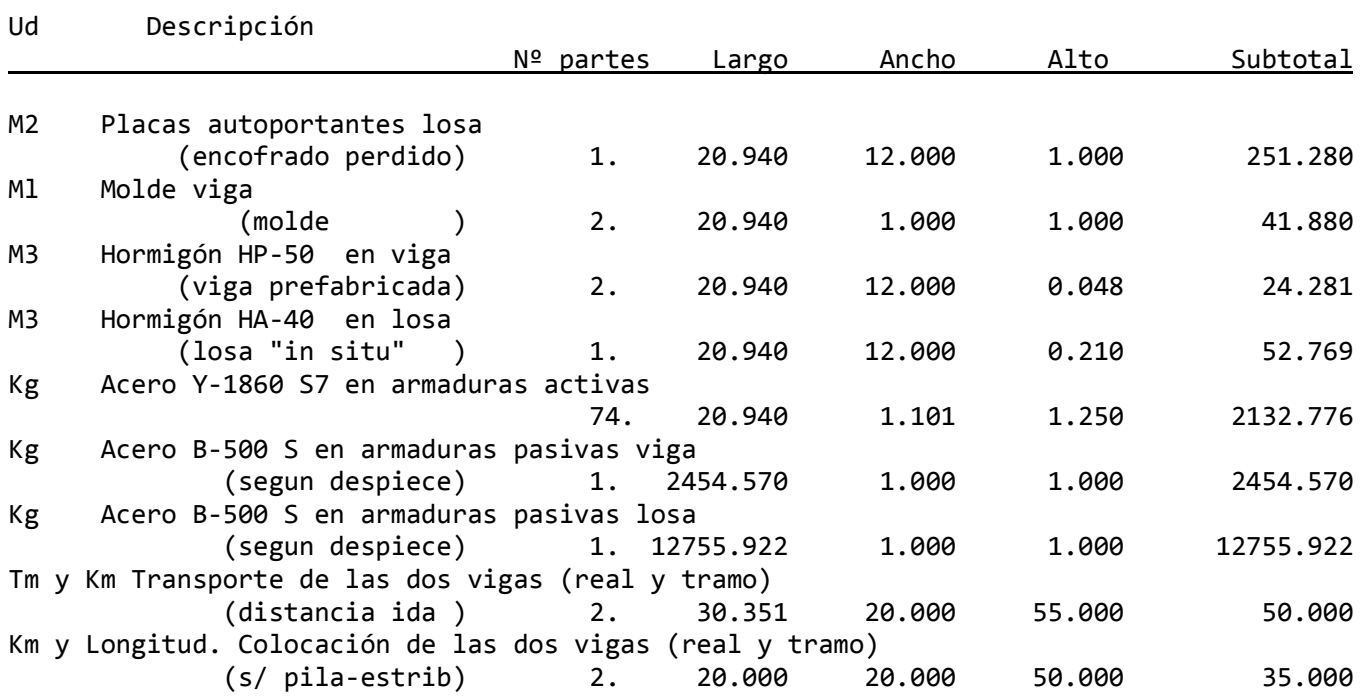




\section{CUANTIAS RESULTANTES}

0.306 M3 hormigon / M2 tablero

$27.681 \mathrm{~kg}$ activas / M3 hormigón

$87.839 \mathrm{~kg}$ activas / M3 hormigón VIGA

$8.463 \mathrm{~kg}$ activas / M2 tablero

$197.412 \mathrm{~kg}$ pasivas / M3 hormigón

$101.092 \mathrm{~kg}$ pasivas / M3 hormigón VIGA

$60.359 \mathrm{~kg}$ pasivas / M2 tablero

\section{CALCULO DEL COSTE}

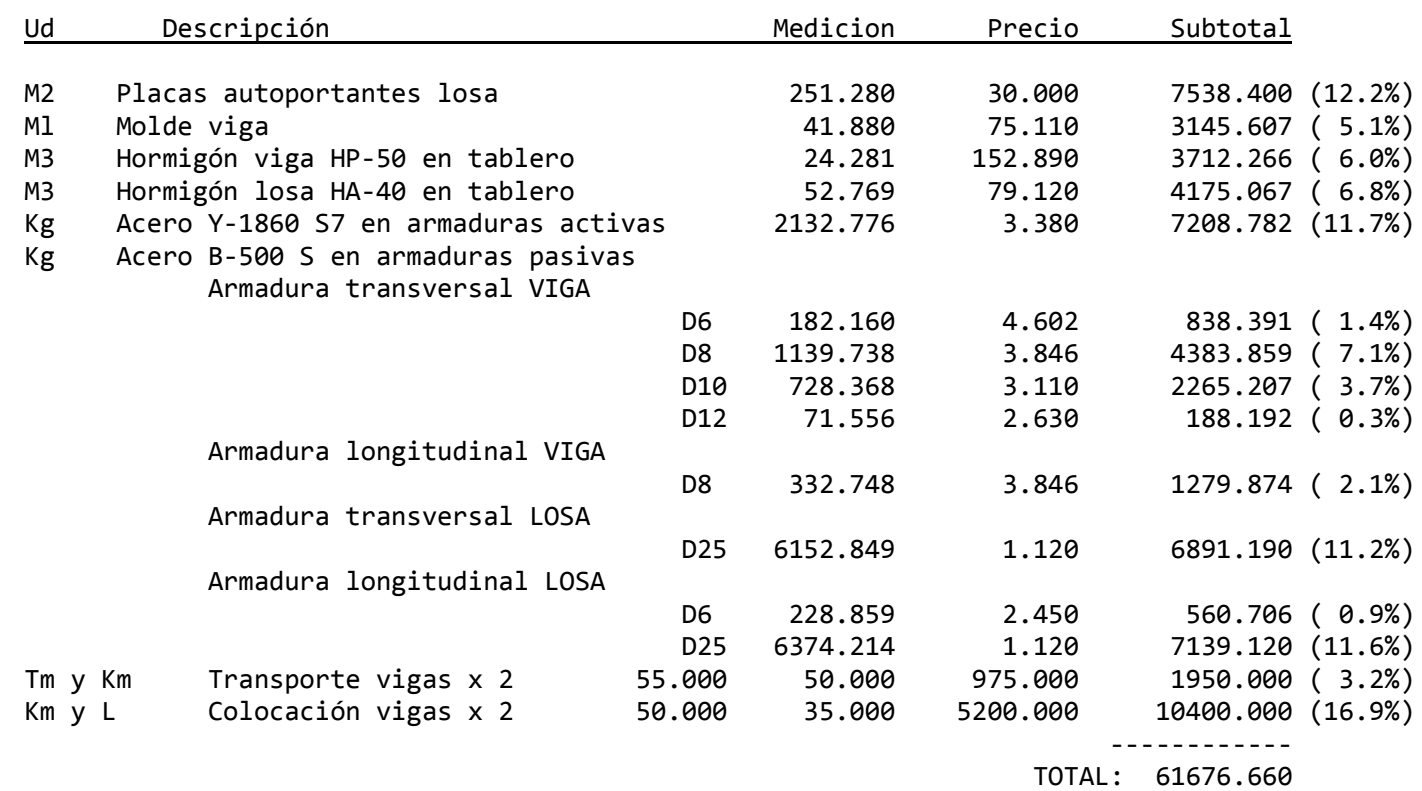

244.75 EUROS / M2 tablero 


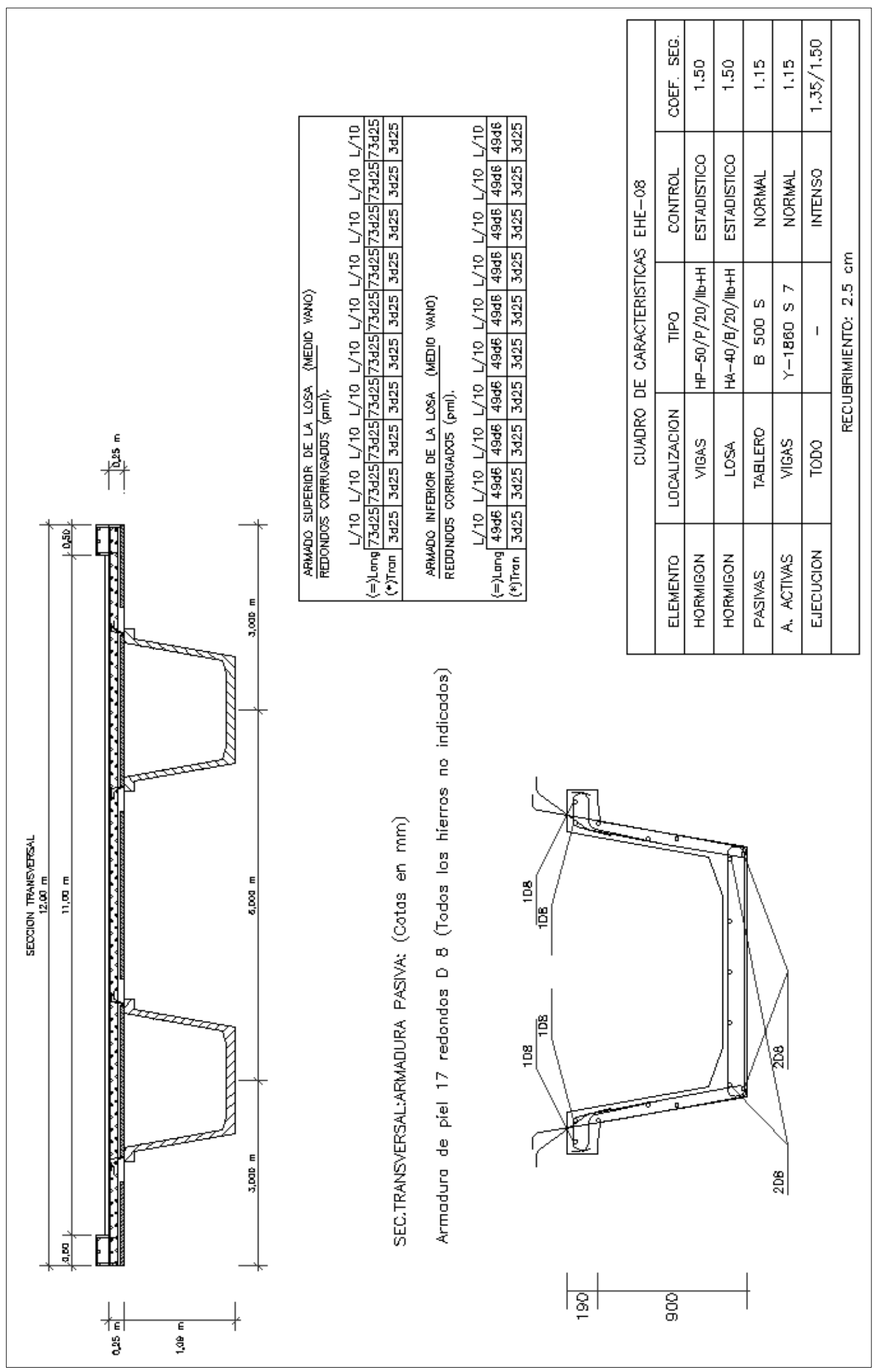




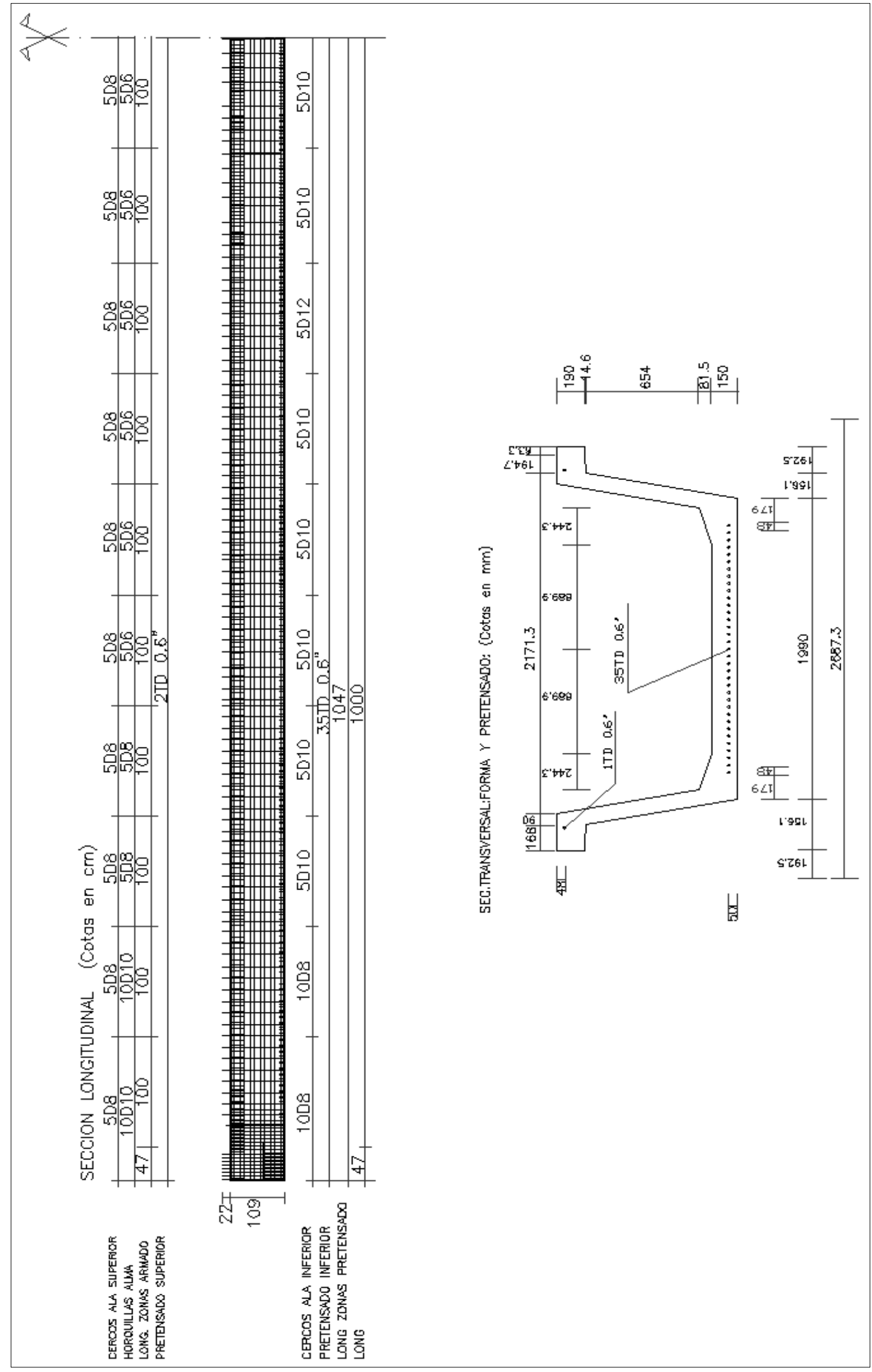


$\mathrm{L}=20 \mathrm{~m} . \mathrm{HPF}$

ARMADURA PASIVA

Armadura transversal

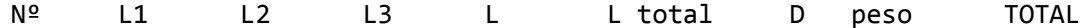

$\mathrm{sec}$ barras $<\ldots[\mathrm{mm}][\mathrm{kg} / \mathrm{m}] \quad[\mathrm{kg}]$

\begin{tabular}{|c|c|c|c|c|c|c|c|c|c|c|}
\hline 1 & 5 & 3.82 & 0.16 & -- & 3.98 & 20.835 & 12 & 0.89 & 18.543 & \\
\hline 2 & 5 & 3.82 & 0.16 & -- & 3.98 & 19.900 & 12 & 0.89 & 17.711 & \\
\hline 3 & 5 & 3.82 & 0.18 & -- & 4.00 & 20.000 & 10 & 0.62 & 12.400 & \\
\hline 4 & 10 & 3.84 & 0.18 & -- & 4.02 & 40.200 & 8 & 0.39 & 15.678 & \\
\hline 5 & 5 & 3.82 & 0.18 & -- & 4.00 & 20.000 & 10 & 0.62 & 12.400 & \\
\hline 6 & 5 & 3.82 & 0.18 & -- & 4.00 & 20.000 & 10 & 0.62 & 12.400 & \\
\hline 7 & 5 & 3.82 & 0.18 & -- & 4.00 & 20.000 & 10 & 0.62 & 12.400 & \\
\hline 8 & 5 & 3.82 & 0.16 & -- & 3.98 & 19.900 & 12 & 0.89 & 17.711 & \\
\hline 9 & 5 & 3.82 & 0.18 & - - & 4.00 & 20.000 & 10 & 0.62 & 12.400 & \\
\hline \multirow[t]{2}{*}{10} & 5 & 3.82 & 0.18 & -- & 4.00 & 20.000 & 10 & 0.62 & 12.400 & \\
\hline & & & & & & & & Suma & 144.043 & $(28.0 \%)$ \\
\hline \multicolumn{11}{|c|}{ +++ Horquillas alma semi-viga } \\
\hline 1 & 5 & 0.94 & 1.06 & 0.42 & 7.80 & 40.833 & 12 & 0.89 & 36.341 & \\
\hline 2 & 5 & 0.94 & 1.06 & 0.42 & 7.80 & 39.000 & 12 & 0.89 & 34.710 & \\
\hline 3 & 5 & 0.95 & 1.06 & 0.28 & 7.26 & 36.300 & 8 & 0.39 & 14.157 & \\
\hline 4 & 5 & 0.95 & 1.06 & 0.28 & 7.26 & 36.300 & 8 & 0.39 & 14.157 & \\
\hline 5 & 5 & 0.95 & 1.07 & 0.21 & 7.02 & 35.100 & 6 & 0.22 & 7.722 & \\
\hline 6 & 5 & 0.95 & 1.07 & 0.21 & 7.02 & 35.100 & 6 & 0.22 & 7.722 & \\
\hline 7 & 5 & 0.95 & 1.07 & 0.21 & 7.02 & 35.100 & 6 & 0.22 & 7.722 & \\
\hline 8 & 5 & 0.95 & 1.07 & 0.21 & 7.02 & 35.100 & 6 & 0.22 & 7.722 & \\
\hline 9 & 5 & 0.95 & 1.07 & 0.21 & 7.02 & 35.100 & 6 & 0.22 & 7.722 & \\
\hline \multirow[t]{3}{*}{10} & 5 & 0.94 & 1.06 & 0.35 & 7.52 & 37.600 & 10 & 0.62 & 23.312 & \\
\hline & & & & & & & & --- & - - - - - - & \\
\hline & & & & & & & & Suma & 161.287 & $(31.4 \%)$ \\
\hline \multicolumn{11}{|c|}{ +++ Cerco alas semi-viga } \\
\hline 1 & 5 & 2.36 & 0.33 & 0.28 & 10.66 & 55.805 & 8 & 0.39 & 21.764 & \\
\hline 2 & 5 & 2.36 & 0.33 & 0.28 & 10.66 & 53.300 & 8 & 0.39 & 20.787 & \\
\hline 3 & 5 & 2.36 & 0.33 & 0.28 & 10.66 & 53.300 & 8 & 0.39 & 20.787 & \\
\hline 4 & 5 & 2.36 & 0.33 & 0.28 & 10.66 & 53.300 & 8 & 0.39 & 20.787 & \\
\hline 5 & 5 & 2.36 & 0.33 & 0.28 & 10.66 & 53.300 & 8 & 0.39 & 20.787 & \\
\hline 6 & 5 & 2.36 & 0.33 & 0.28 & 10.66 & 53.300 & 8 & 0.39 & 20.787 & \\
\hline 7 & 5 & 2.36 & 0.33 & 0.28 & 10.66 & 53.300 & 8 & 0.39 & 20.787 & \\
\hline 8 & 5 & 2.36 & 0.33 & 0.28 & 10.66 & 53.300 & 8 & 0.39 & 20.787 & \\
\hline 9 & 5 & 2.36 & 0.33 & 0.28 & 10.66 & 53.300 & 8 & 0.39 & 20.787 & \\
\hline \multirow[t]{3}{*}{10} & 5 & 2.36 & 0.33 & 0.28 & 10.66 & 53.300 & 8 & 0.39 & 20.787 & \\
\hline & & & & & & & & ---- & - - - - - & \\
\hline & & & & & & & & Suma & 208.847 & $(40.6 \%)$ \\
\hline \multicolumn{11}{|c|}{ +++ Transversal inferior semi-losa } \\
\hline 1 & 3 & 11.93 & -- & -- & 11.93 & 41.636 & 25 & 3.85 & 160.297 & \\
\hline 2 & 4 & 11.93 & -- & -- & 11.93 & 47.720 & 25 & 3.85 & 183.722 & \\
\hline 3 & 3 & 11.93 & -- & -- & 11.93 & 39.767 & 25 & 3.85 & 153.102 & \\
\hline 4 & 3 & 11.93 & -- & -- & 11.93 & 39.767 & 25 & 3.85 & 153.102 & \\
\hline 5 & 4 & 11.93 & -- & -- & 11.93 & 47.720 & 25 & 3.85 & 183.722 & \\
\hline 6 & 4 & 11.93 & -- & -- & 11.93 & 47.720 & 25 & 3.85 & 183.722 & \\
\hline 7 & 3 & 11.93 & -- & -- & 11.93 & 39.767 & 25 & 3.85 & 153.102 & \\
\hline 8 & 3 & 11.93 & -- & -- & 11.93 & 39.767 & 25 & 3.85 & 153.102 & \\
\hline 9 & 3 & 11.93 & - - & - - & 11.93 & 39.767 & 25 & 3.85 & 153.102 & \\
\hline \multirow[t]{2}{*}{10} & 3 & 11.93 & -- & -- & 11.93 & 39.767 & 25 & 3.85 & 153.102 & \\
\hline & & & & & & & & Suma & 1630.073 & $(23.7 \%)$ \\
\hline
\end{tabular}




$\begin{array}{rrrrrrrrrr}+++ \\ 1 & 3 & 11.93 & -- & -- & 11.93 & 41.636 & 25 & 3.85 & 160.297 \\ 2 & 3 & 11.93 & -- & -- & 11.93 & 39.767 & 25 & 3.85 & 153.102 \\ 3 & 3 & 11.93 & -- & -- & 11.93 & 39.767 & 25 & 3.85 & 153.102 \\ 4 & 3 & 11.93 & -- & -- & 11.93 & 39.767 & 25 & 3.85 & 153.102 \\ 5 & 3 & 11.93 & -- & -- & 11.93 & 39.767 & 25 & 3.85 & 153.102 \\ 6 & 3 & 11.93 & -- & -- & 11.93 & 39.767 & 25 & 3.85 & 153.102 \\ 7 & 3 & 11.93 & -- & -- & 11.93 & 39.767 & 25 & 3.85 & 153.102 \\ 8 & 3 & 11.93 & -- & -- & 11.93 & 39.767 & 25 & 3.85 & 153.102 \\ 9 & 3 & 11.93 & -- & -- & 11.93 & 39.767 & 25 & 3.85 & 153.102 \\ 10 & 3 & 11.93 & -- & -- & 11.93 & 39.767 & 25 & 3.85 & 153.102 \\ & & & & & & & & & \\ \end{array}$

Armadura longitudinal total tablero

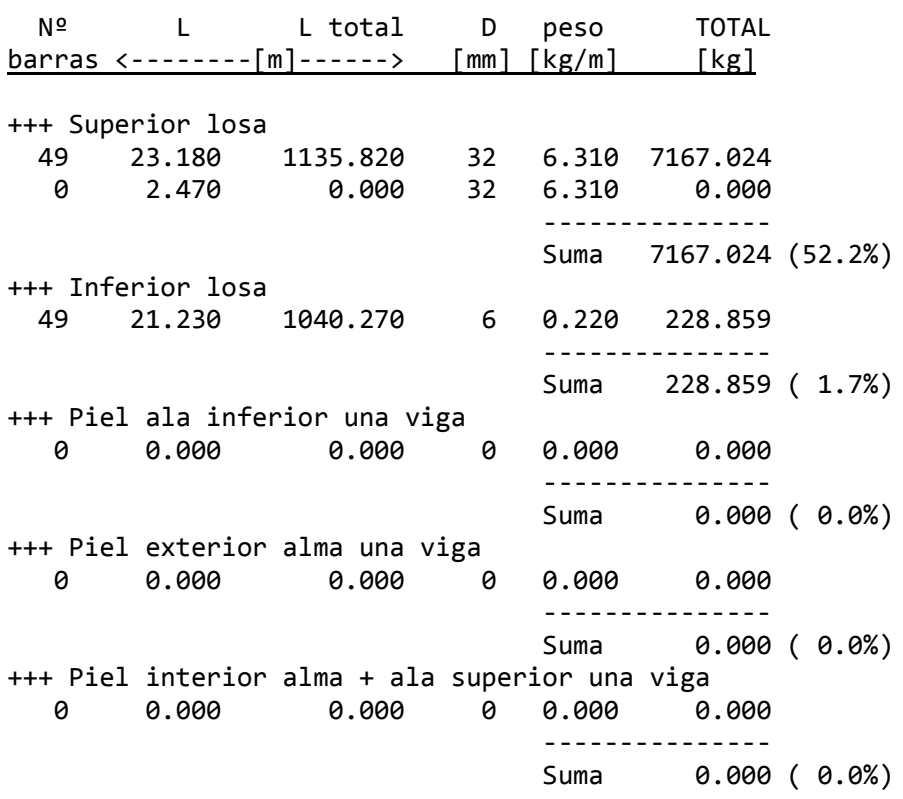

ESTADO DE MEDICIONES

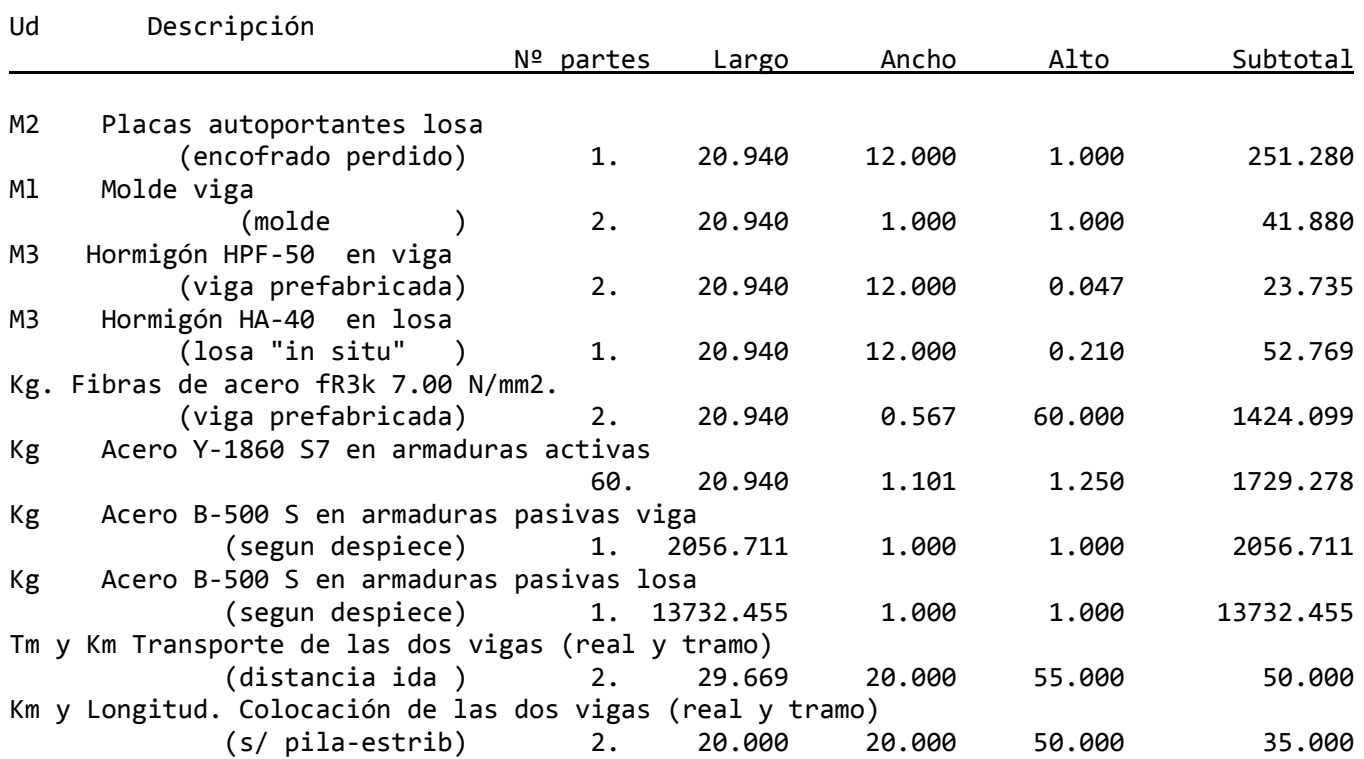




\section{CUANTIAS RESULTANTES}
$60.000 \mathrm{Kg} . / \mathrm{M} 3$ hormigon
0.304 M3 hormigon / M2 tablero
$22.604 \mathrm{~kg}$ activas / M3 hormigón
$72.858 \mathrm{~kg}$ activas / M3 hormigón VIGA
$6.862 \mathrm{~kg}$ activas / M2 tablero
206.384 kg pasivas / M3 hormigón
$86.653 \mathrm{~kg}$ pasivas / M3 hormigón VIGA
$62.655 \mathrm{~kg}$ pasivas / M2 tablero

\section{CALCULO DEL COSTE}

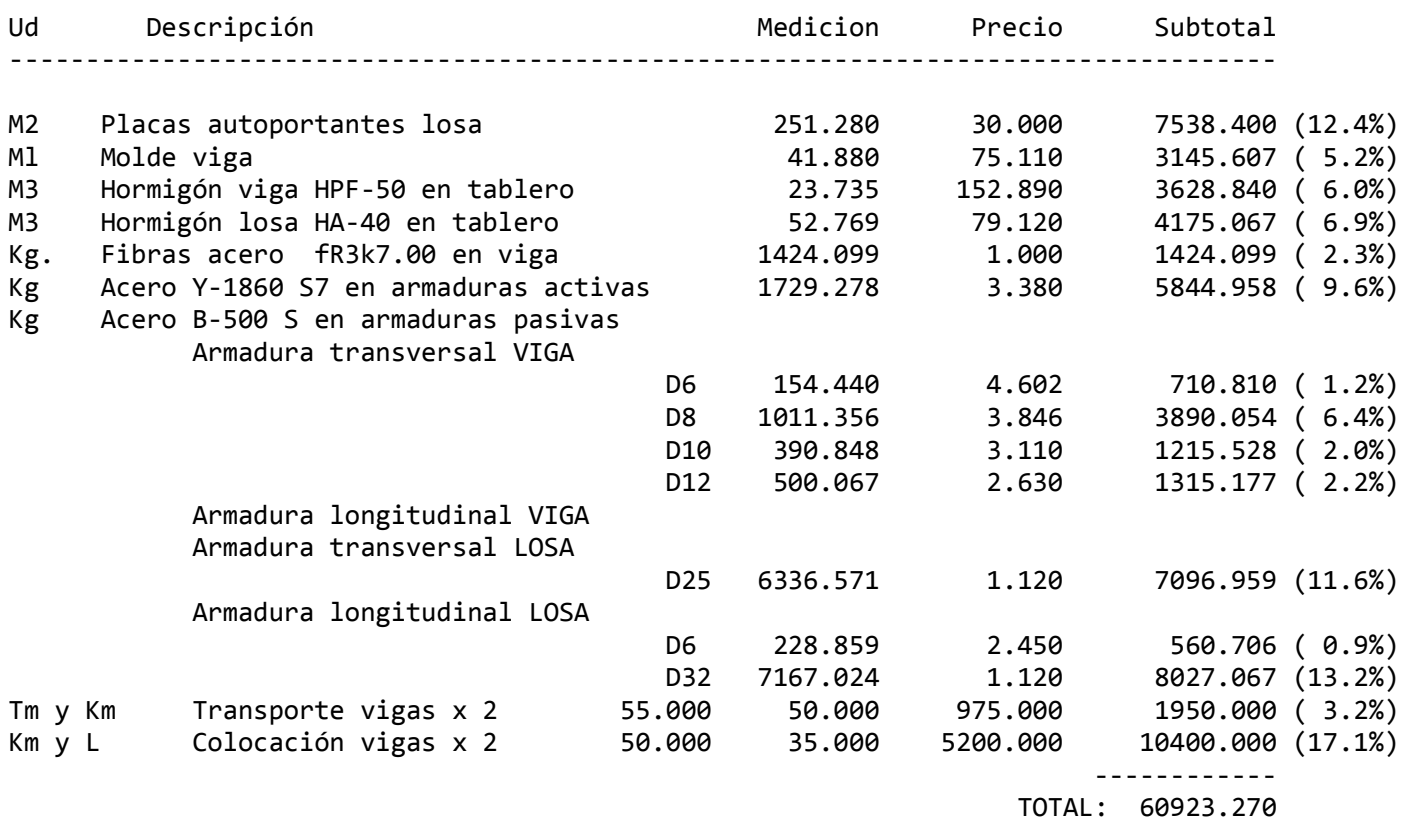

241.76 EUROS / M2 tablero 


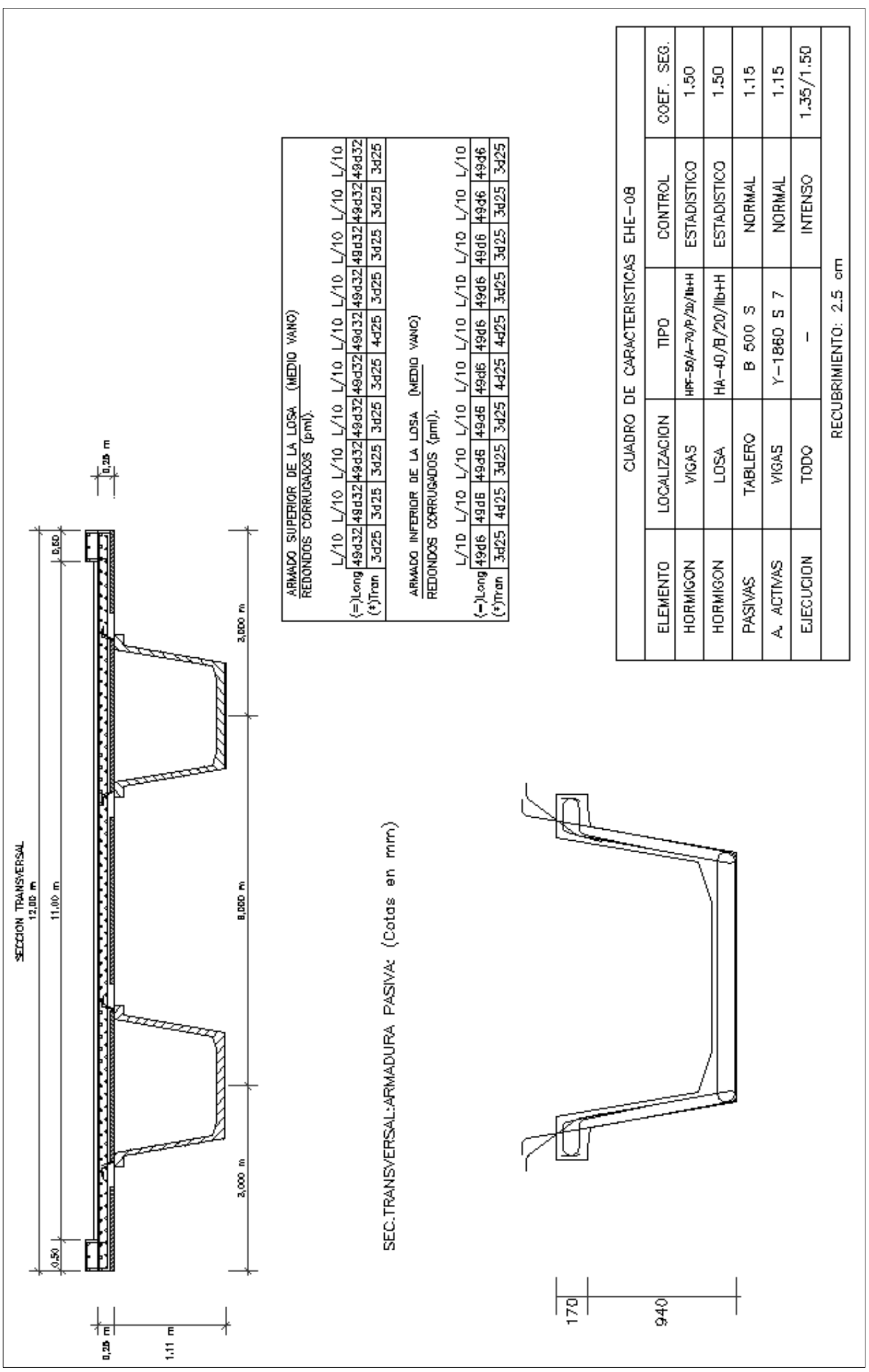




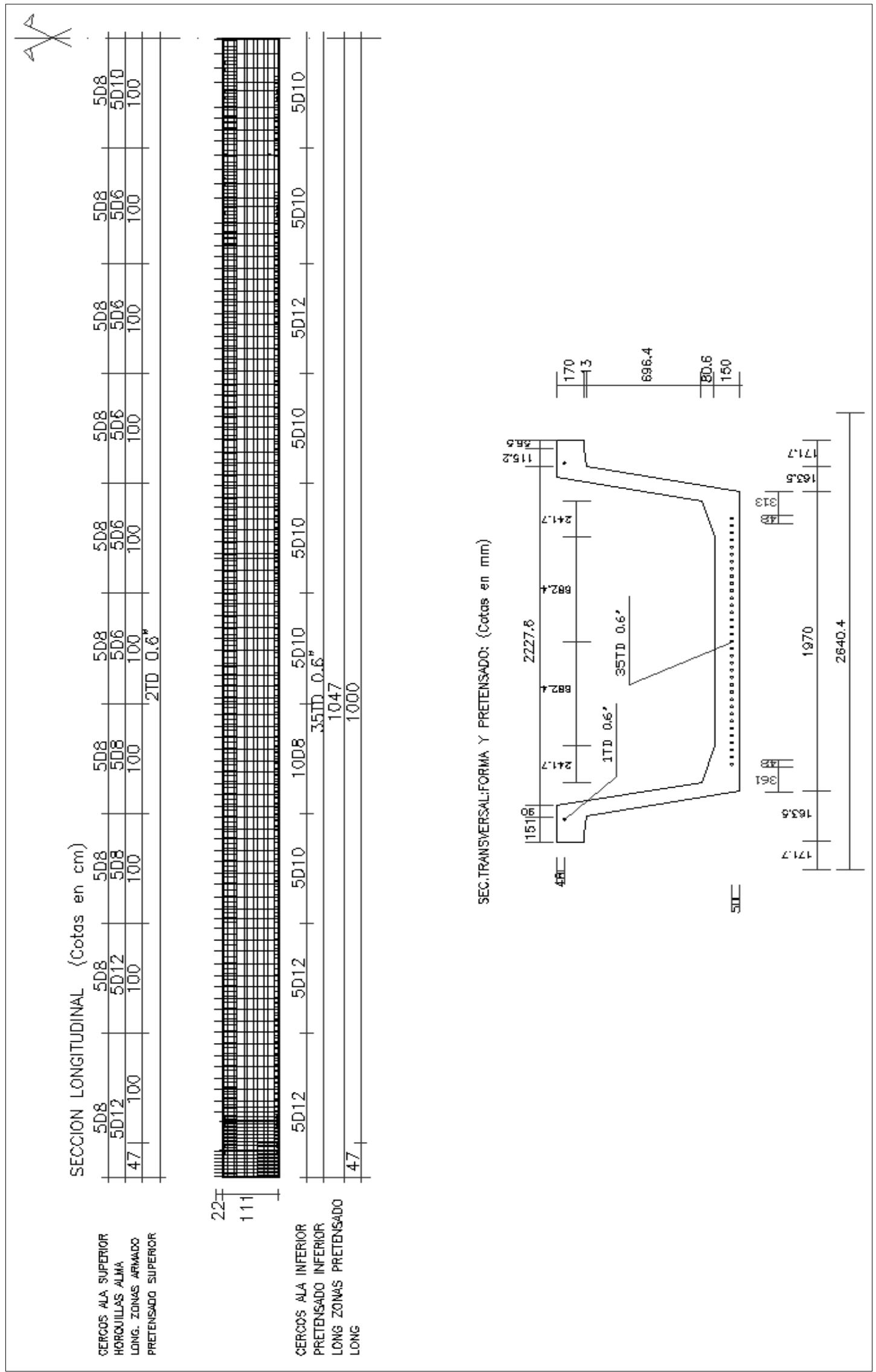


$\mathrm{L}=25 \mathrm{~m} . \quad \mathrm{HP}$

ARMADURA PASIVA

Armadura transversal

$\begin{array}{lllllllll}\text { No } & \mathrm{L} 1 & \mathrm{~L} 2 & \mathrm{~L} 3 & \mathrm{~L} & \mathrm{~L} \text { total } & \mathrm{D} & \text { peso } & \text { TOTAL }\end{array}$

$\mathrm{sec}$ barras $<\ldots-\ldots[\mathrm{m}]-\ldots[\mathrm{mm}][\mathrm{kg} / \mathrm{m}][\mathrm{kg}]$

\begin{tabular}{|c|c|c|c|c|c|c|c|c|c|c|}
\hline 1 & 6 & 3.88 & 0.16 & -- & 4.04 & 26.199 & 12 & 0.89 & 23.317 & \\
\hline 2 & 6 & 3.88 & 0.16 & - - & 4.04 & 25.250 & 12 & 0.89 & 22.472 & \\
\hline 3 & 6 & 3.88 & 0.16 & -- & 4.04 & 25.250 & 16 & 1.58 & 39.895 & \\
\hline 4 & 6 & 3.88 & 0.18 & -- & 4.06 & 25.375 & 10 & 0.62 & 15.733 & \\
\hline 5 & 6 & 3.88 & 0.18 & -- & 4.06 & 25.375 & 10 & 0.62 & 15.733 & \\
\hline 6 & 6 & 3.88 & 0.16 & -- & 4.04 & 25.250 & 12 & 0.89 & 22.472 & \\
\hline 7 & 6 & 3.88 & 0.18 & -- & 4.06 & 25.375 & 10 & 0.62 & 15.733 & \\
\hline 8 & 6 & 3.88 & 0.16 & -- & 4.04 & 25.250 & 12 & 0.89 & 22.472 & \\
\hline 9 & 6 & 3.88 & 0.18 & -- & 4.06 & 25.375 & 10 & 0.62 & 15.733 & \\
\hline \multirow[t]{2}{*}{10} & 6 & 3.88 & 0.18 & -- & 4.06 & 25.375 & 10 & 0.62 & 15.733 & \\
\hline & & & & & & & & \multicolumn{2}{|c|}{ 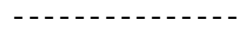 } & $(25.9 \%)$ \\
\hline \multicolumn{11}{|c|}{ +++ Horquillas alma semi-viga } \\
\hline 1 & 6 & 0.94 & 1.33 & 0.42 & 8.88 & 57.587 & 12 & 0.89 & 51.252 & \\
\hline 2 & 6 & 0.94 & 1.33 & 0.42 & 8.88 & 55.500 & 12 & 0.89 & 49.395 & \\
\hline 3 & 12 & 0.95 & 1.34 & 0.21 & 8.10 & 101.250 & 6 & 0.22 & 22.275 & \\
\hline 4 & 6 & 0.95 & 1.34 & 0.28 & 8.38 & 52.375 & 8 & 0.39 & 20.426 & \\
\hline 5 & 6 & 0.95 & 1.34 & 0.21 & 8.10 & 50.625 & 6 & 0.22 & 11.138 & \\
\hline 6 & 6 & 0.95 & 1.34 & 0.21 & 8.10 & 50.625 & 6 & 0.22 & 11.138 & \\
\hline 7 & 6 & 0.95 & 1.34 & 0.21 & 8.10 & 50.625 & 6 & 0.22 & 11.138 & \\
\hline 8 & 6 & 0.95 & 1.34 & 0.21 & 8.10 & 50.625 & 6 & 0.22 & 11.138 & \\
\hline 9 & 6 & 0.95 & 1.34 & 0.21 & 8.10 & 50.625 & 6 & 0.22 & 11.138 & \\
\hline \multirow[t]{3}{*}{10} & 6 & 0.95 & 1.34 & 0.21 & 8.10 & 50.625 & 6 & 0.22 & 11.138 & \\
\hline & & & & & & & & \multicolumn{3}{|c|}{-------------} \\
\hline & & & & & & & & Suma & 210.173 & $(26.0 \%)$ \\
\hline \multicolumn{11}{|c|}{ +++ Cerco alas semi-viga } \\
\hline 1 & 6 & 2.44 & 0.36 & 0.28 & 11.04 & 71.594 & 8 & 0.39 & 27.922 & \\
\hline 2 & 6 & 2.44 & 0.36 & 0.28 & 11.04 & 69.000 & 8 & 0.39 & 26.910 & \\
\hline 3 & 6 & 2.44 & 0.36 & 0.28 & 11.04 & 69.000 & 8 & 0.39 & 26.910 & \\
\hline 4 & 6 & 2.44 & 0.36 & 0.28 & 11.04 & 69.000 & 8 & 0.39 & 26.910 & \\
\hline 5 & 6 & 2.44 & 0.36 & 0.28 & 11.04 & 69.000 & 8 & 0.39 & 26.910 & \\
\hline 6 & 6 & 2.44 & 0.36 & 0.28 & 11.04 & 69.000 & 8 & 0.39 & 26.910 & \\
\hline 7 & 6 & 2.44 & 0.36 & 0.28 & 11.04 & 69.000 & 8 & 0.39 & 26.910 & \\
\hline 8 & 6 & 2.44 & 0.36 & 0.28 & 11.04 & 69.000 & 8 & 0.39 & 26.910 & \\
\hline 9 & 6 & 2.44 & 0.36 & 0.28 & 11.04 & 69.000 & 8 & 0.39 & 26.910 & \\
\hline \multirow[t]{3}{*}{10} & 6 & 2.44 & 0.36 & 0.28 & 11.04 & 69.000 & 8 & 0.39 & 26.910 & \\
\hline & & & & & & & & \multicolumn{3}{|c|}{-------------} \\
\hline & \multicolumn{7}{|c|}{ +++ Transversal inferior semi-losa } & Suma & 210.112 & $(33.4 \%)$ \\
\hline 1 & 4 & 11.92 & -- & -- & 11.92 & 51.534 & 25 & 3.85 & 198.406 & \\
\hline 2 & 4 & 11.92 & -- & -- & 11.92 & 49.667 & 25 & 3.85 & 191.217 & \\
\hline 3 & 4 & 11.92 & -- & -- & 11.92 & 49.667 & 25 & 3.85 & 191.217 & \\
\hline 4 & 4 & 11.92 & -- & -- & 11.92 & 49.667 & 25 & 3.85 & 191.217 & \\
\hline 5 & 4 & 11.92 & -- & -- & 11.92 & 49.667 & 25 & 3.85 & 191.217 & \\
\hline 6 & 4 & 11.92 & -- & - - & 11.92 & 49.667 & 25 & 3.85 & 191.217 & \\
\hline 7 & 5 & 11.92 & -- & -- & 11.92 & 59.600 & 25 & 3.85 & 229.460 & \\
\hline 8 & 4 & 11.92 & -- & -- & 11.92 & 49.667 & 25 & 3.85 & 191.217 & \\
\hline 9 & 4 & 11.92 & -- & -- & 11.92 & 49.667 & 25 & 3.85 & 191.217 & \\
\hline \multirow[t]{3}{*}{10} & 4 & 11.92 & -- & -- & 11.92 & 49.667 & 25 & 3.85 & 191.217 & \\
\hline & & & & & & & & \multirow{2}{*}{\multicolumn{3}{|c|}{ Suma $1957.600(26.2 \%)$}} \\
\hline & & & & & & & & & & \\
\hline
\end{tabular}




$\begin{array}{rrrrrrrrrr}+++ \\ 1 & 4 & 11.92 & -- & -- & 11.92 & 51.534 & 25 & 3.85 & 198.406 \\ 2 & 4 & 11.92 & -- & -- & 11.92 & 49.667 & 25 & 3.85 & 191.217 \\ 3 & 4 & 11.92 & -- & -- & 11.92 & 49.667 & 25 & 3.85 & 191.217 \\ 4 & 4 & 11.92 & -- & -- & 11.92 & 49.667 & 25 & 3.85 & 191.217 \\ 5 & 4 & 11.92 & -- & -- & 11.92 & 49.667 & 25 & 3.85 & 191.217 \\ 6 & 4 & 11.92 & -- & -- & 11.92 & 49.667 & 25 & 3.85 & 191.217 \\ 7 & 4 & 11.92 & -- & -- & 11.92 & 49.667 & 25 & 3.85 & 191.217 \\ 8 & 4 & 11.92 & -- & -- & 11.92 & 49.667 & 25 & 3.85 & 191.217 \\ 9 & 4 & 11.92 & -- & -- & 11.92 & 49.667 & 25 & 3.85 & 191.217 \\ 10 & 4 & 11.92 & -- & -- & 11.92 & 49.667 & 25 & 3.85 & 191.217 \\ & & & & & & & & & ------------ \\ \end{array}$

Armadura longitudinal total tablero

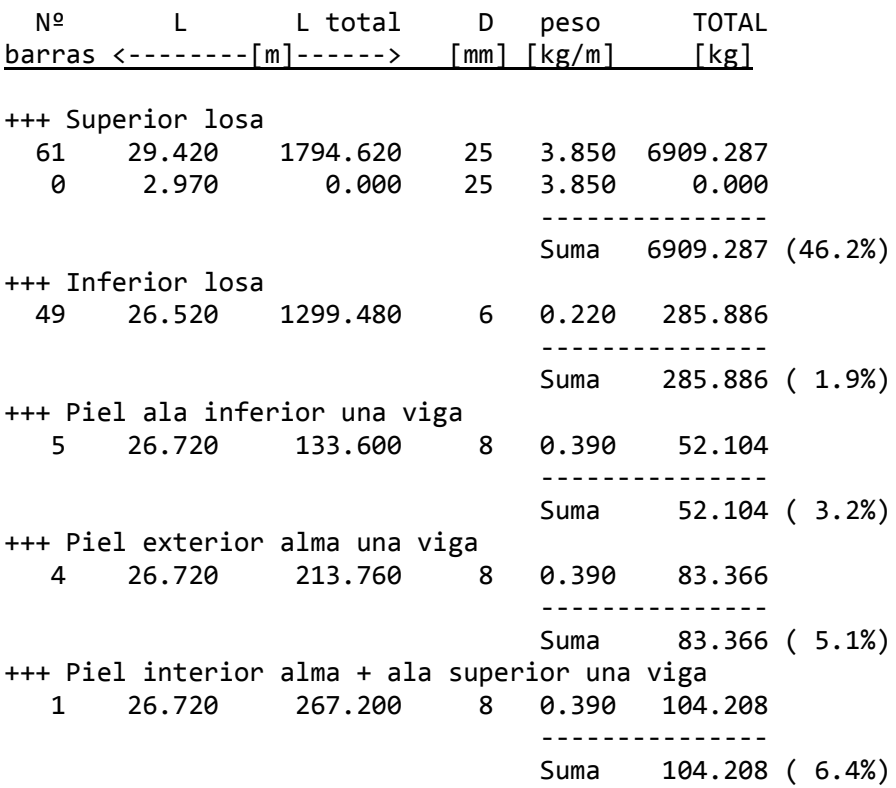

\section{ESTADO DE MEDICIONES}

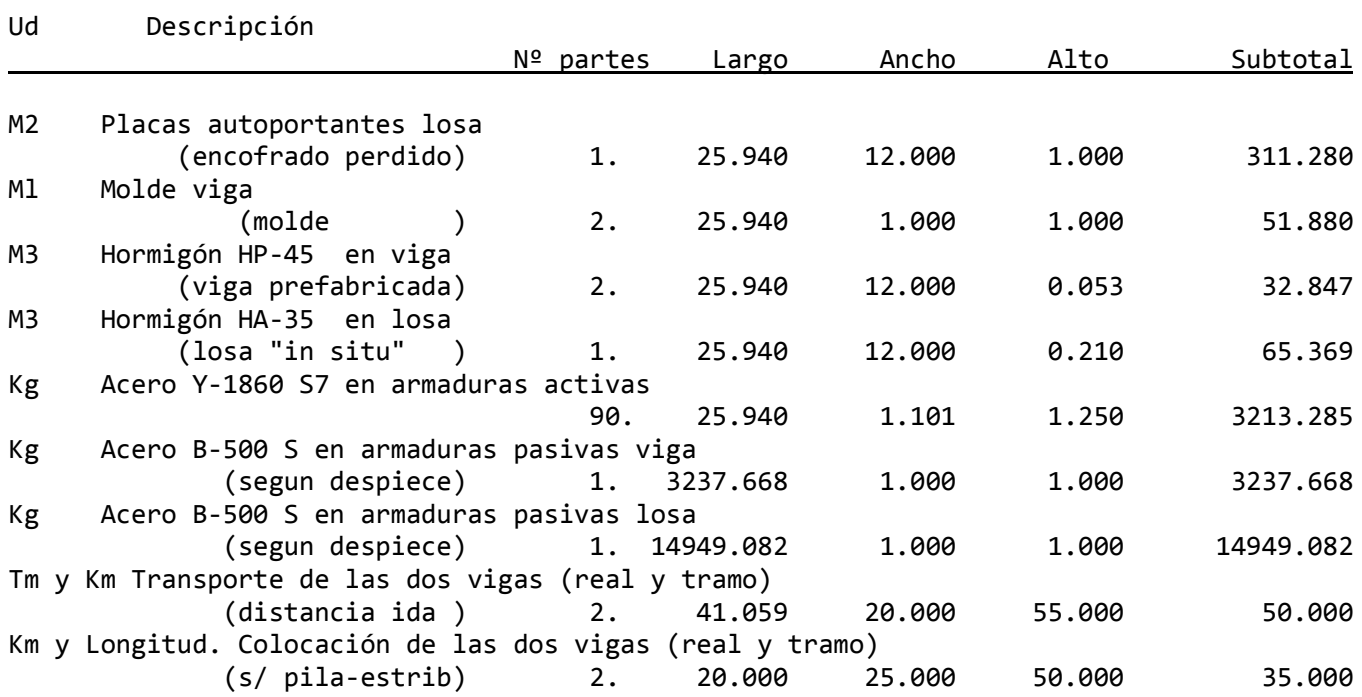




\section{CUANTIAS RESULTANTES}

0.315 M3 hormigon / M2 tablero

$32.716 \mathrm{~kg}$ activas / M3 hormigón

$97.825 \mathrm{~kg}$ activas / M3 hormigón VIGA

$10.299 \mathrm{~kg}$ activas / M2 tablero

$185.171 \mathrm{~kg}$ pasivas / M3 hormigón

$98.567 \mathrm{~kg}$ pasivas / M3 hormigón VIGA

$58.291 \mathrm{~kg}$ pasivas / M2 tablero

\section{CALCULO DEL COSTE}

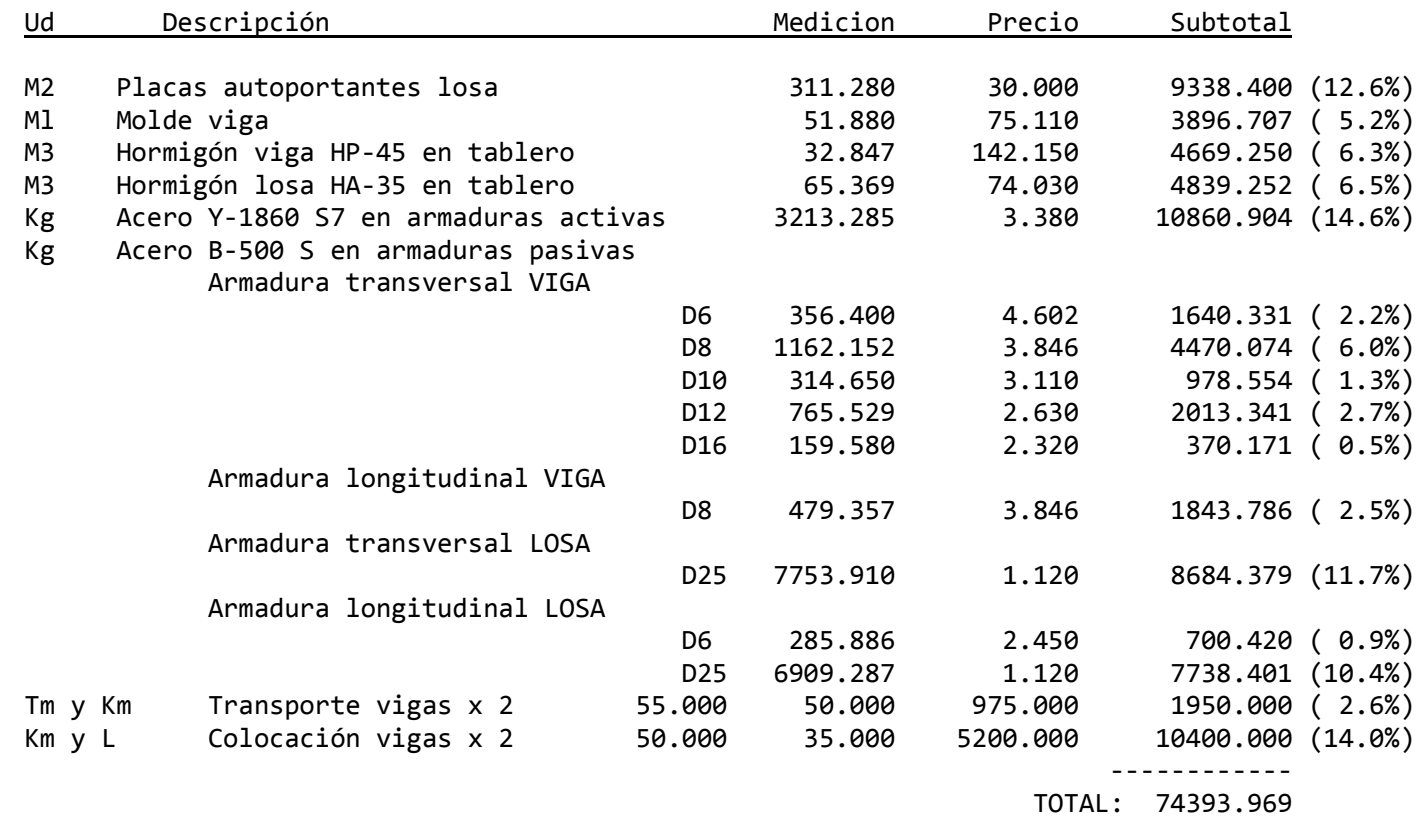

238.44 EUROS / M2 tablero 


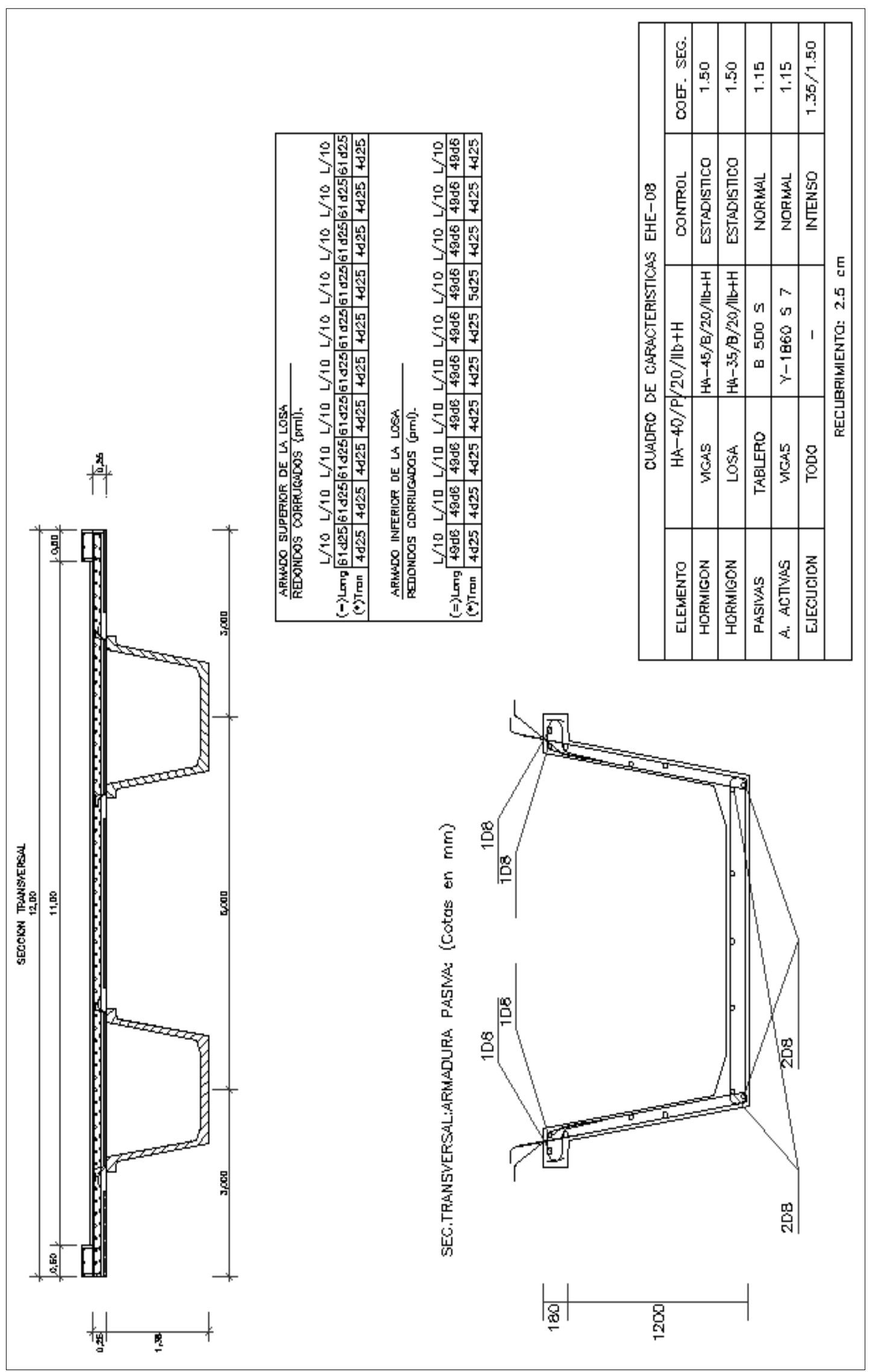




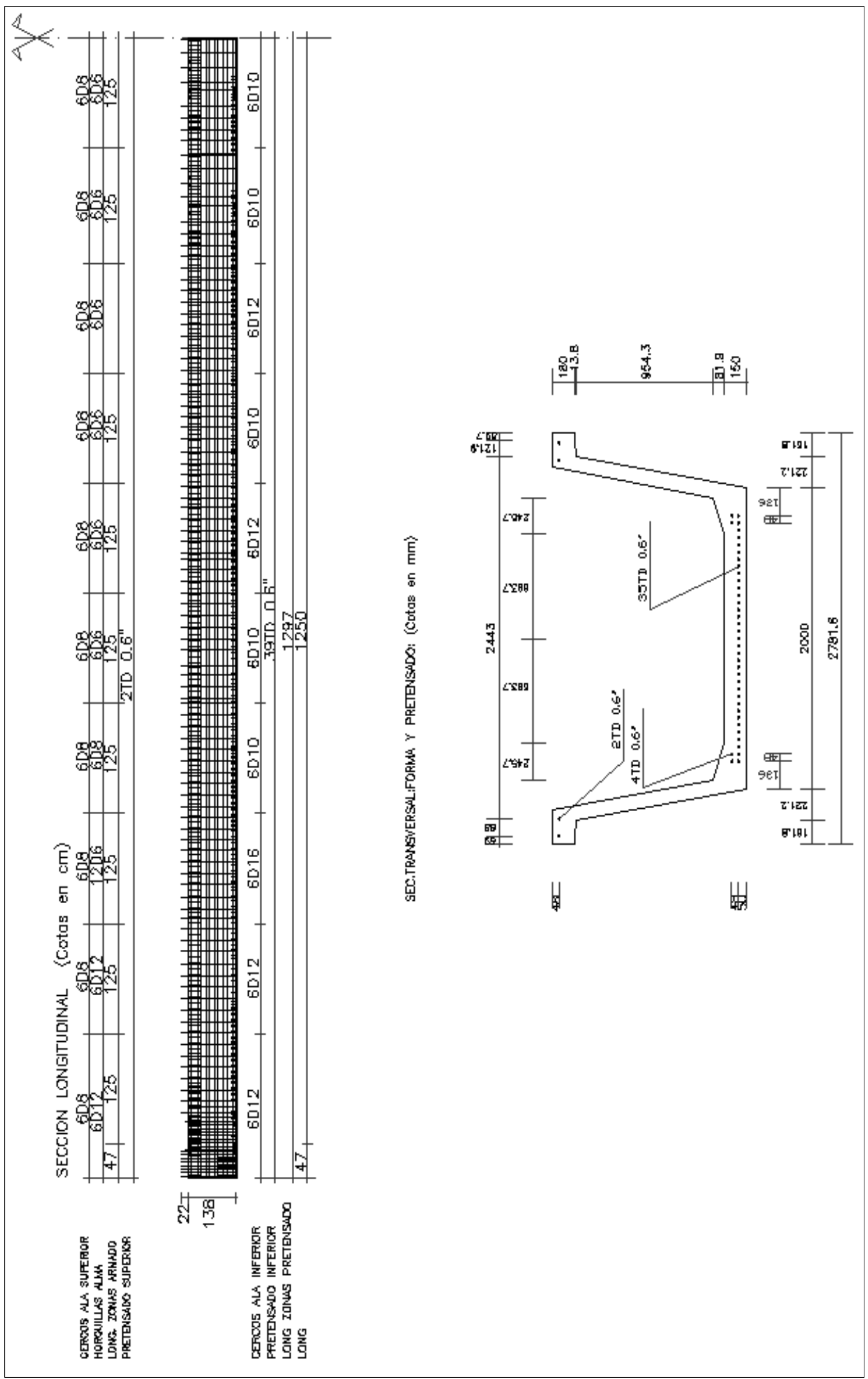


$\mathrm{L}=25 \mathrm{~m} . \mathrm{HPF}$

ARMADURA PASIVA

Armadura transversal

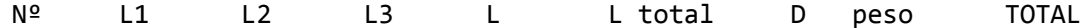

$\mathrm{sec}$ barras $<\ldots[\mathrm{mm}][\mathrm{kg} / \mathrm{m}] \quad[\mathrm{kg}]$

+++ Cerco ala inf.semi-viga

$\begin{array}{llllllllll}1 & 6 & 3.88 & 0.16 & -- & 4.04 & 26.199 & 12 & 0.89 & 23.317\end{array}$

$\begin{array}{llllllllll}2 & 6 & 3.88 & 0.16 & -- & 4.04 & 25.250 & 12 & 0.89 & 22.472\end{array}$

$\begin{array}{llllllllll}4 & 6 & 3.88 & 0.18 & -- & 4.06 & 25.375 & 10 & 0.62 & 15.733\end{array}$

$\begin{array}{llllllllll}5 & 6 & 3.88 & 0.18 & -- & 4.06 & 25.375 & 10 & 0.62 & 15.733\end{array}$

$\begin{array}{llllllllll}6 & 6 & 3.88 & 0.18 & -- & 4.06 & 25.375 & 10 & 0.62 & 15.733\end{array}$

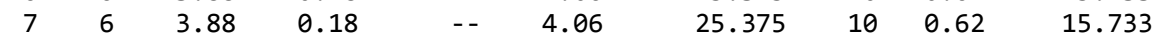

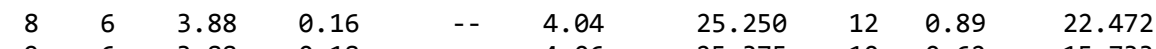

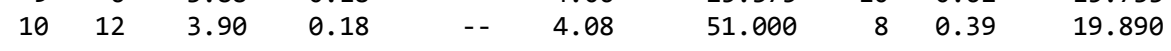

+++ Horquillas alma semi-viga

$\begin{array}{llllllllll}1 & 6 & 0.94 & 1.32 & 0.42 & 8.84 & 57.327 & 12 & 0.89 & 51.021\end{array}$

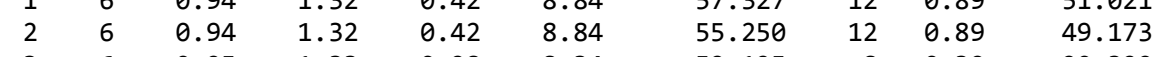

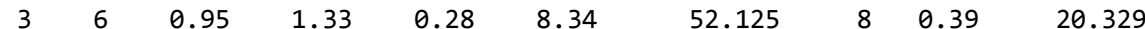

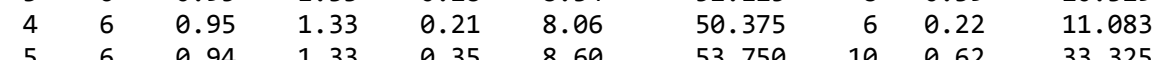

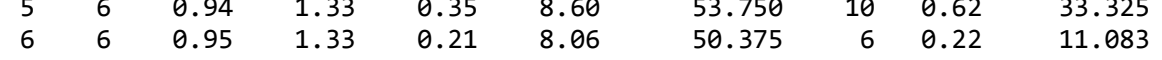

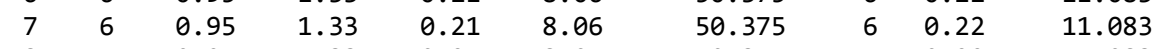

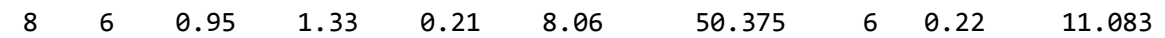

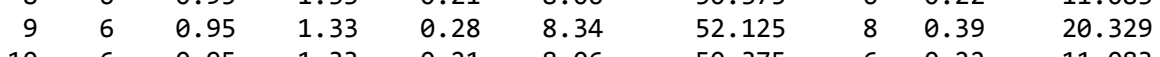

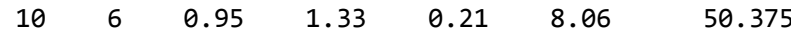

+++ Cerco alas semi-viga

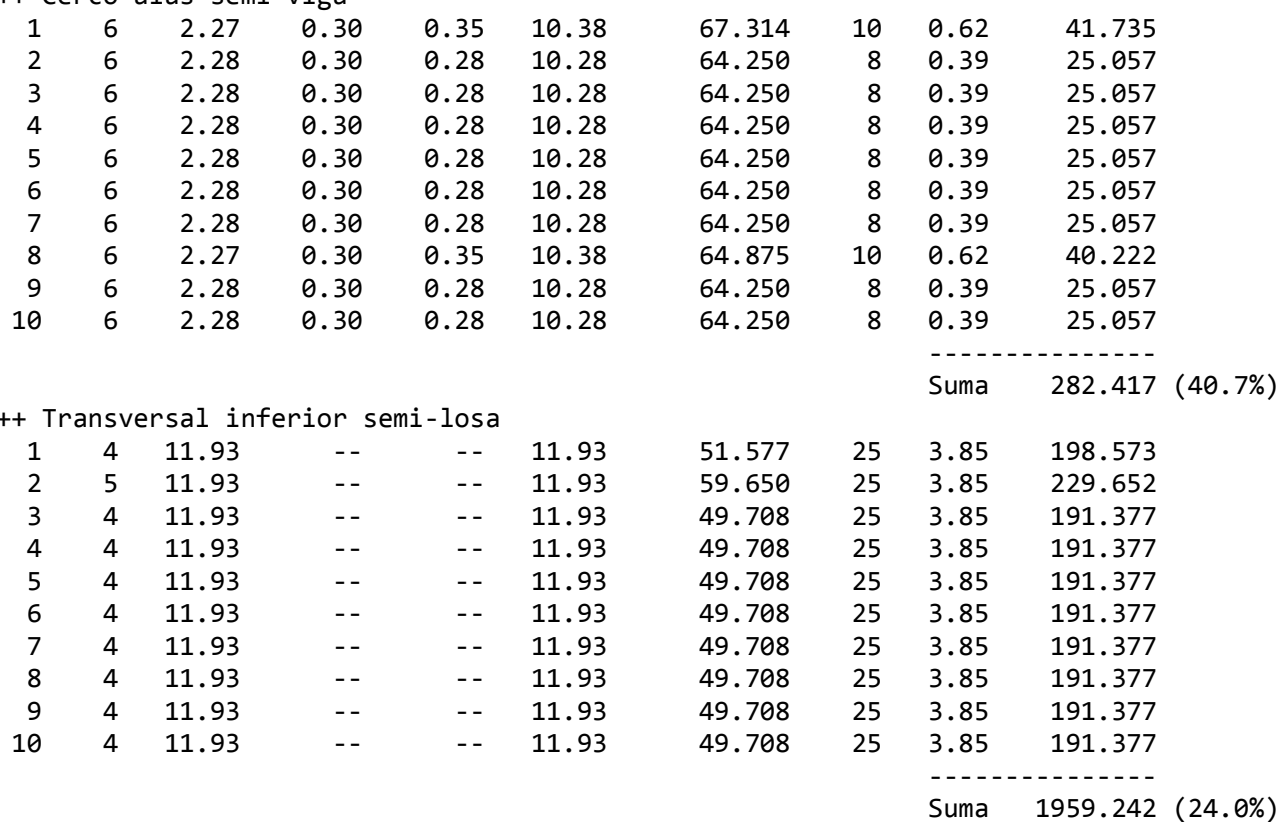




$\begin{array}{rrrrrrrrrr}+++ \\ 1 & 4 & 11.93 & -- & -- & 11.93 & 51.577 & 25 & 3.85 & 198.573 \\ 2 & 4 & 11.93 & -- & -- & 11.93 & 49.708 & 25 & 3.85 & 191.377 \\ 3 & 4 & 11.93 & -- & -- & 11.93 & 49.708 & 25 & 3.85 & 191.377 \\ 4 & 4 & 11.93 & -- & -- & 11.93 & 49.708 & 25 & 3.85 & 191.377 \\ 5 & 4 & 11.93 & -- & -- & 11.93 & 49.708 & 25 & 3.85 & 191.377 \\ 6 & 4 & 11.93 & -- & -- & 11.93 & 49.708 & 25 & 3.85 & 191.377 \\ 7 & 4 & 11.93 & -- & -- & 11.93 & 49.708 & 25 & 3.85 & 191.377 \\ 8 & 4 & 11.93 & -- & -- & 11.93 & 49.708 & 25 & 3.85 & 191.377 \\ 9 & 4 & 11.93 & -- & -- & 11.93 & 49.708 & 25 & 3.85 & 191.377 \\ 10 & 4 & 11.93 & -- & -- & 11.93 & 49.708 & 25 & 3.85 & 191.377 \\ & & & & & & & & & ------------- \\ \end{array}$

Armadura longitudinal total tablero

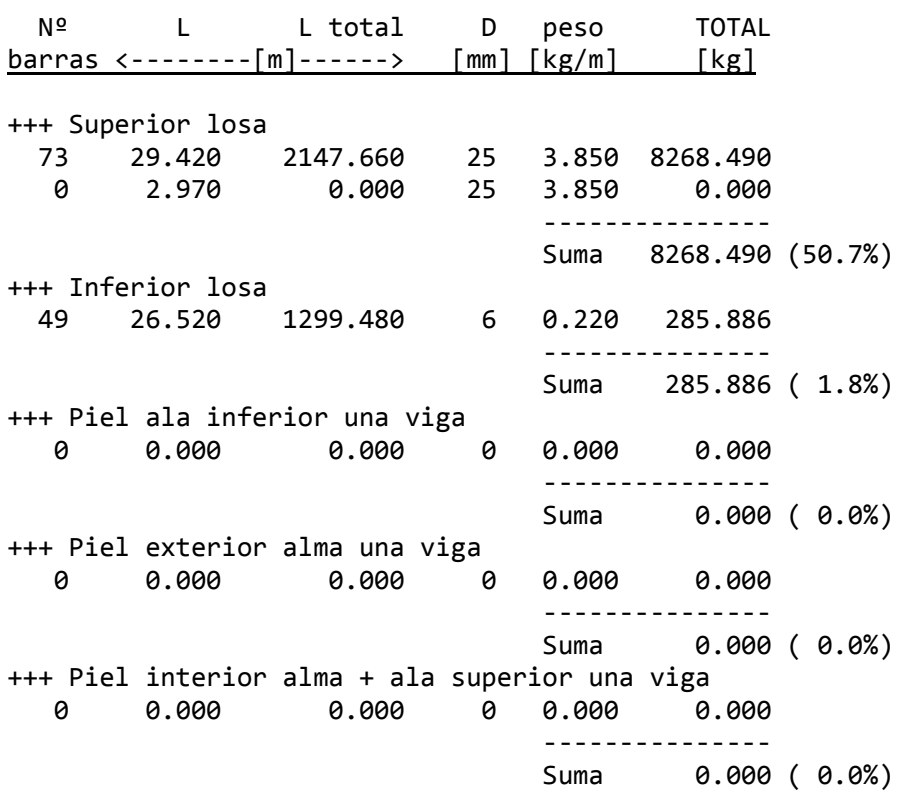

ESTADO DE MEDICIONES

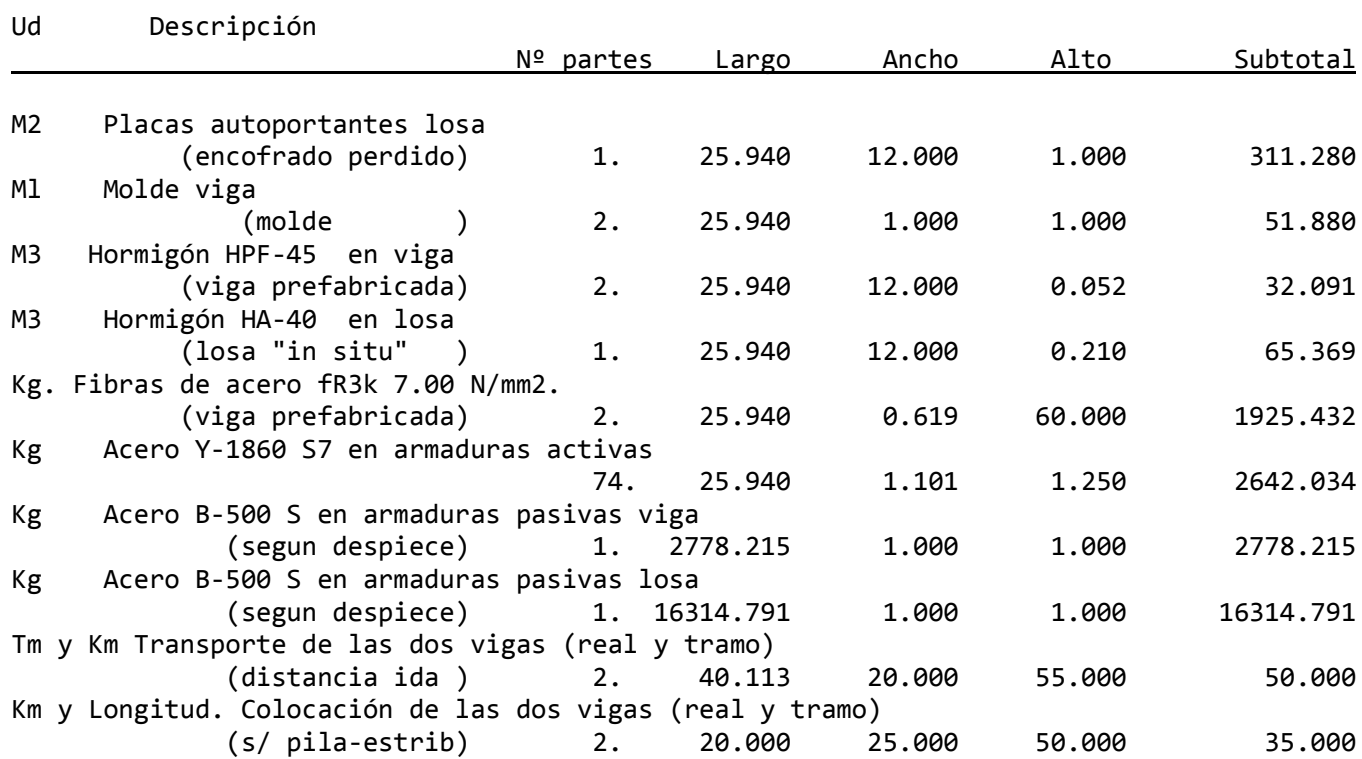




\section{CUANTIAS RESULTANTES}
$60.000 \mathrm{Kg} . / \mathrm{M3}$ hormigon
0.312 M3 hormigon / M2 tablero
$27.109 \mathrm{~kg}$ activas / M3 hormigón
$82.331 \mathrm{~kg}$ activas / M3 hormigón VIGA
$8.468 \mathrm{~kg}$ activas / M2 tablero
$195.907 \mathrm{~kg}$ pasivas / M3 hormigón
$86.574 \mathrm{~kg}$ pasivas / M3 hormigón VIGA
$61.196 \mathrm{~kg}$ pasivas / M2 tablero

\section{CALCULO DEL COSTE}

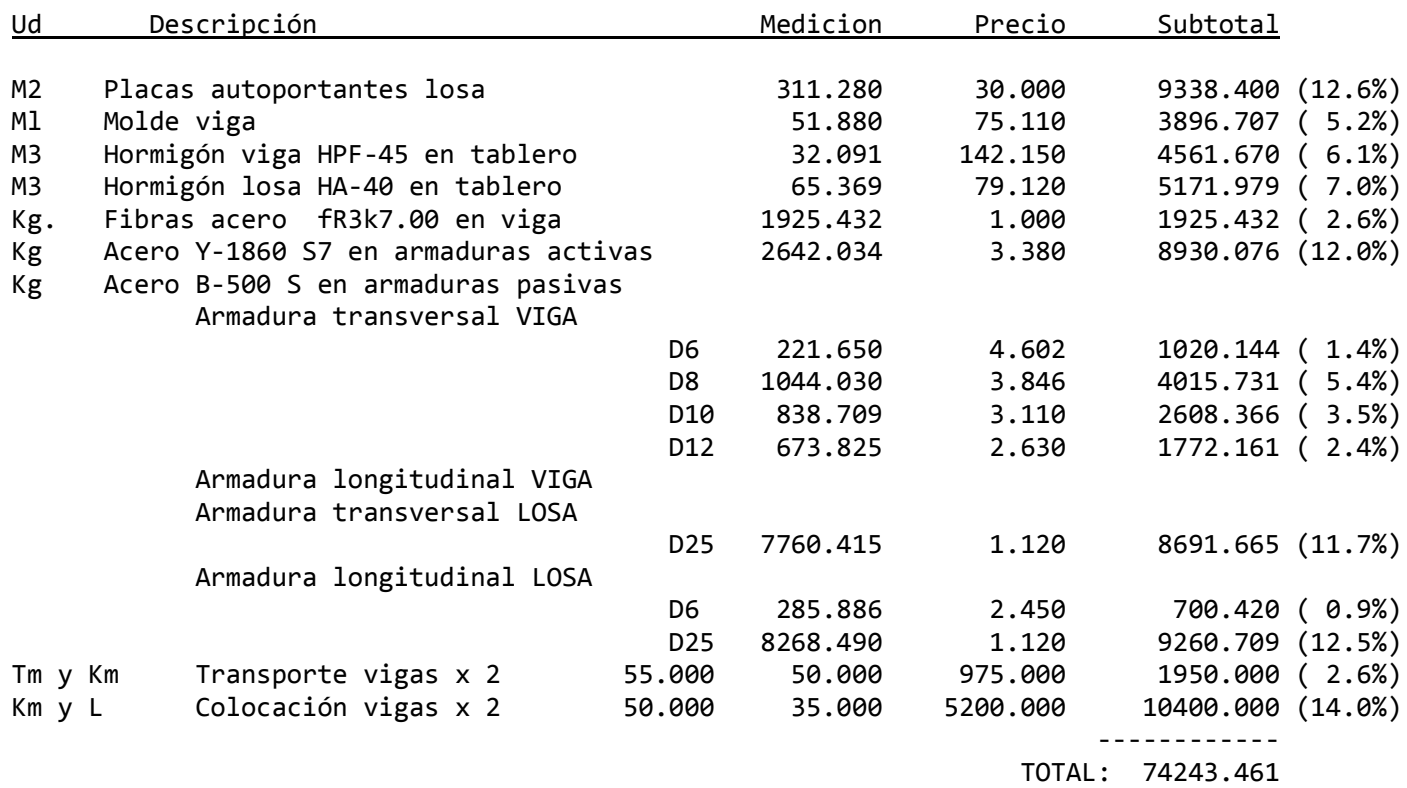

237.96 EUROS / M2 tablero 


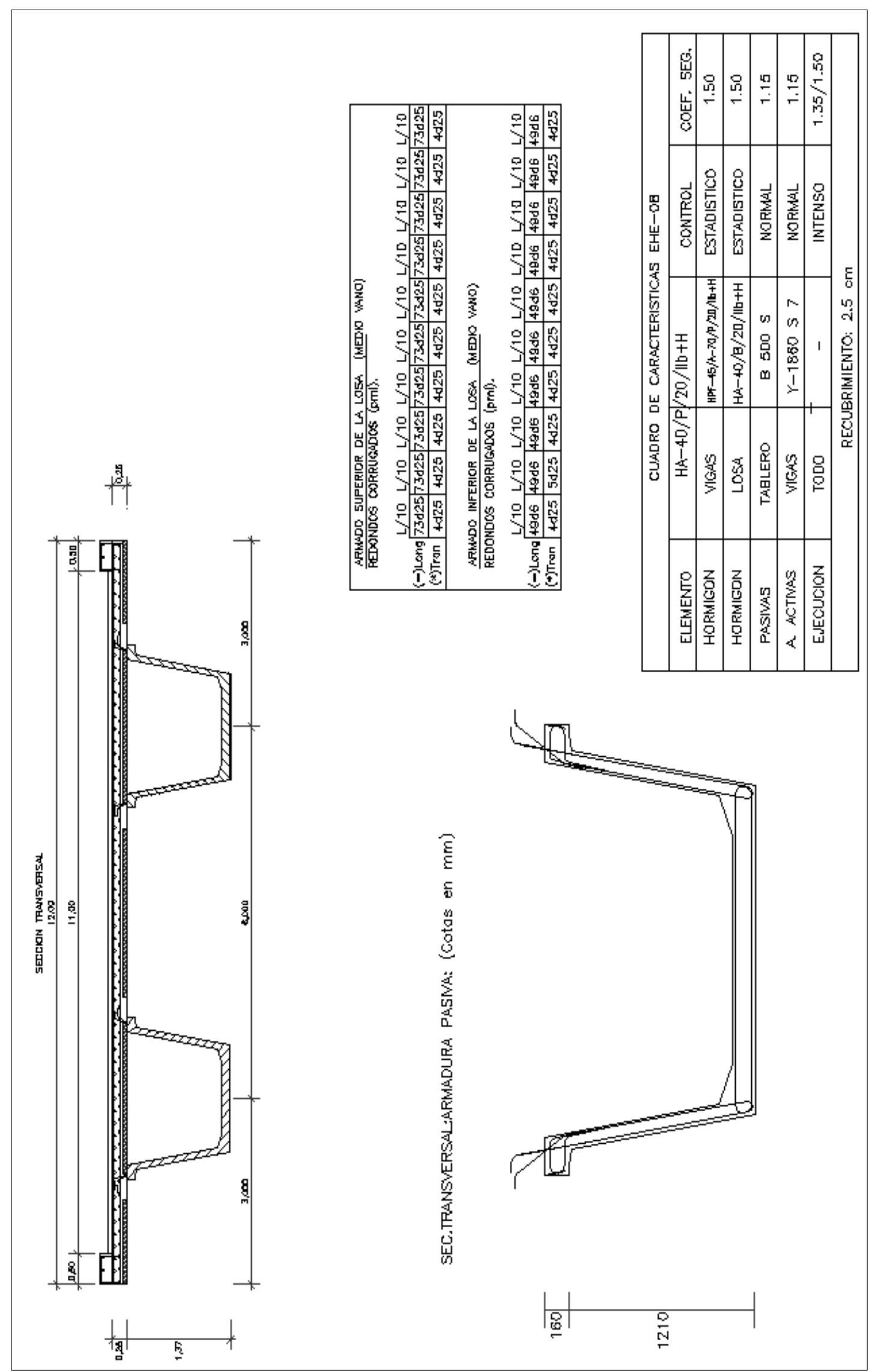




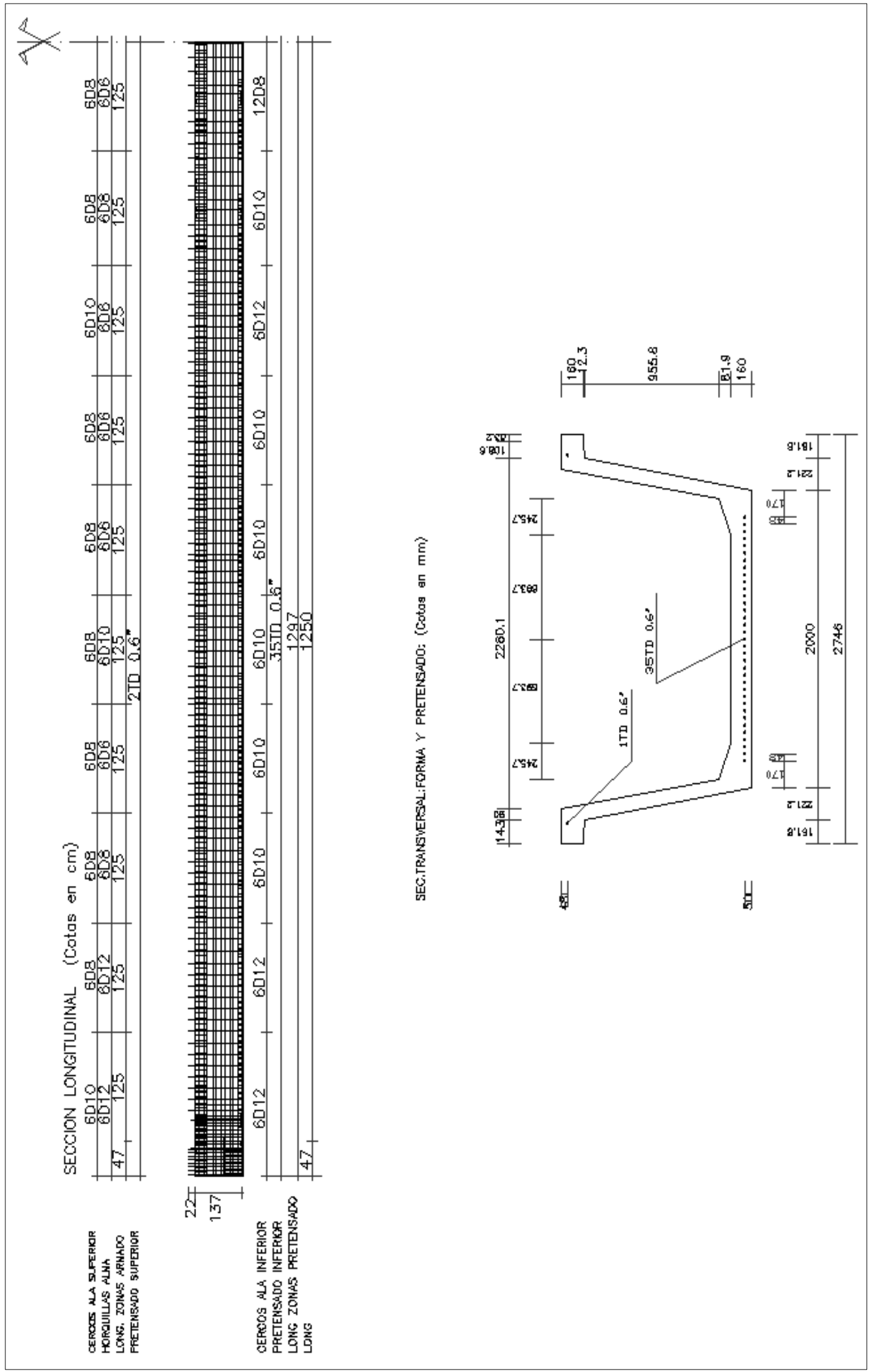


$\mathrm{L}=30 \mathrm{~m} . \quad \mathrm{HP}$

ARMADURA PASIVA

Armadura transversal

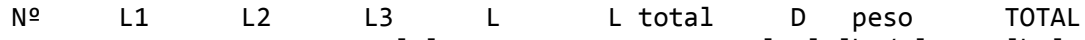

$\mathrm{sec}$ barras $<\ldots-\ldots[\mathrm{m}]-\ldots[\mathrm{mm}][\mathrm{kg} / \mathrm{m}][\mathrm{kg}]$

\begin{tabular}{|c|c|c|c|c|c|c|c|c|c|c|}
\hline 1 & 7 & 3.88 & 0.20 & -- & 4.08 & 31.559 & 12 & 0.89 & 28.087 & \\
\hline 2 & 7 & 3.88 & 0.22 & -- & 4.10 & 30.750 & 10 & 0.62 & 19.065 & \\
\hline 3 & 7 & 3.88 & 0.22 & -- & 4.10 & 30.750 & 10 & 0.62 & 19.065 & \\
\hline 4 & 7 & 3.88 & 0.22 & -- & 4.10 & 30.750 & 10 & 0.62 & 19.065 & \\
\hline 5 & 7 & 3.88 & 0.22 & -- & 4.10 & 30.750 & 10 & 0.62 & 19.065 & \\
\hline 6 & 7 & 3.88 & 0.22 & - - & 4.10 & 30.750 & 10 & 0.62 & 19.065 & \\
\hline 7 & 7 & 3.88 & 0.22 & -- & 4.10 & 30.750 & 10 & 0.62 & 19.065 & \\
\hline 8 & 15 & 3.88 & 0.22 & -- & 4.10 & 61.500 & 10 & 0.62 & 38.130 & \\
\hline 9 & 7 & 3.88 & 0.22 & - - & 4.10 & 30.750 & 10 & 0.62 & 19.065 & \\
\hline \multirow[t]{2}{*}{10} & 7 & 3.88 & 0.20 & -- & 4.08 & 30.600 & 12 & 0.89 & 27.234 & \\
\hline & & & & & & & & Suma & 226.906 & $(21.5 \%)$ \\
\hline \multicolumn{11}{|c|}{ +++ Horquillas alma semi-viga } \\
\hline 1 & 7 & 0.94 & 1.60 & 0.42 & 9.96 & 77.041 & 12 & 0.89 & 68.566 & \\
\hline 2 & 7 & 0.94 & 1.60 & 0.42 & 9.96 & 74.700 & 12 & 0.89 & 66.483 & \\
\hline 3 & 7 & 0.95 & 1.60 & 0.28 & 9.42 & 70.650 & 8 & 0.39 & 27.553 & \\
\hline 4 & 7 & 0.94 & 1.60 & 0.35 & 9.68 & 72.600 & 10 & 0.62 & 45.012 & \\
\hline 5 & 7 & 0.94 & 1.60 & 0.35 & 9.68 & 72.600 & 10 & 0.62 & 45.012 & \\
\hline 6 & 7 & 0.95 & 1.60 & 0.28 & 9.42 & 70.650 & 8 & 0.39 & 27.553 & \\
\hline 7 & 7 & 0.95 & 1.60 & 0.28 & 9.42 & 70.650 & 8 & 0.39 & 27.553 & \\
\hline 8 & 7 & 0.95 & 1.60 & 0.28 & 9.42 & 70.650 & 8 & 0.39 & 27.553 & \\
\hline 9 & 7 & 0.94 & 1.60 & 0.35 & 9.68 & 72.600 & 10 & 0.62 & 45.012 & \\
\hline \multirow[t]{3}{*}{10} & 7 & 0.94 & 1.60 & 0.35 & 9.68 & 72.600 & 10 & 0.62 & 45.012 & \\
\hline & & & & & & & & ---- & - - - - - - & \\
\hline & & & & & & & & Suma & 425.311 & $(40.2 \%)$ \\
\hline \multicolumn{11}{|c|}{ +++ Cerco alas semi-viga } \\
\hline 1 & 7 & 3.10 & 0.61 & 0.21 & 14.04 & 108.599 & 6 & 0.22 & 23.892 & \\
\hline 2 & 7 & 3.10 & 0.61 & 0.21 & 14.04 & 105.300 & 6 & 0.22 & 23.166 & \\
\hline 3 & 7 & 3.10 & 0.61 & 0.21 & 14.04 & 105.300 & 6 & 0.22 & 23.166 & \\
\hline 4 & 7 & 3.10 & 0.61 & 0.21 & 14.04 & 105.300 & 6 & 0.22 & 23.166 & \\
\hline 5 & 7 & 3.10 & 0.61 & 0.21 & 14.04 & 105.300 & 6 & 0.22 & 23.166 & \\
\hline 6 & 7 & 3.10 & 0.61 & 0.21 & 14.04 & 105.300 & 6 & 0.22 & 23.166 & \\
\hline 7 & 7 & 3.10 & 0.61 & 0.21 & 14.04 & 105.300 & 6 & 0.22 & 23.166 & \\
\hline 8 & 7 & 3.10 & 0.61 & 0.21 & 14.04 & 105.300 & 6 & 0.22 & 23.166 & \\
\hline 9 & 7 & 3.10 & 0.61 & 0.21 & 14.04 & 105.300 & 6 & 0.22 & 23.166 & \\
\hline \multirow[t]{3}{*}{10} & 7 & 3.10 & 0.61 & 0.21 & 14.04 & 105.300 & 6 & 0.22 & 23.166 & \\
\hline & & & & & & & & --- & - - - - - - & \\
\hline & & & & & & & & Suma & 232.386 & $(22.0 \%)$ \\
\hline \multicolumn{11}{|c|}{ +++ Transversal inferior semi-losa } \\
\hline 1 & 12 & 11.93 & -- & -- & 11.93 & 147.646 & 16 & 1.58 & 233.280 & \\
\hline 2 & 10 & 11.93 & -- & -- & 11.93 & 119.300 & 16 & 1.58 & 188.494 & \\
\hline 3 & 12 & 11.93 & -- & -- & 11.93 & 143.160 & 16 & 1.58 & 226.193 & \\
\hline 4 & 10 & 11.93 & -- & -- & 11.93 & 119.300 & 16 & 1.58 & 188.494 & \\
\hline 5 & 10 & 11.93 & -- & -- & 11.93 & 119.300 & 16 & 1.58 & 188.494 & \\
\hline 6 & 12 & 11.93 & -- & -- & 11.93 & 143.160 & 16 & 1.58 & 226.193 & \\
\hline 7 & 10 & 11.93 & -- & -- & 11.93 & 119.300 & 16 & 1.58 & 188.494 & \\
\hline 8 & 12 & 11.93 & -- & -- & 11.93 & 143.160 & 16 & 1.58 & 226.193 & \\
\hline 9 & 10 & 11.93 & -- & - - & 11.93 & 119.300 & 16 & 1.58 & 188.494 & \\
\hline \multirow[t]{2}{*}{10} & 10 & 11.93 & -- & -- & 11.93 & 119.300 & 16 & 1.58 & 188.494 & \\
\hline & & & & & & & & Suma & 2042.823 & $(26.7 \%)$ \\
\hline
\end{tabular}




$\begin{array}{rrrrrrrrrr}+++ \\ 1 & 10 & 11.93 & -- & -- & 11.93 & 123.038 & 16 & 1.58 & 194.400 \\ 2 & 10 & 11.93 & -- & -- & 11.93 & 119.300 & 16 & 1.58 & 188.494 \\ 3 & 10 & 11.93 & -- & -- & 11.93 & 119.300 & 16 & 1.58 & 188.494 \\ 4 & 10 & 11.93 & -- & -- & 11.93 & 119.300 & 16 & 1.58 & 188.494 \\ 5 & 10 & 11.93 & -- & -- & 11.93 & 119.300 & 16 & 1.58 & 188.494 \\ 6 & 10 & 11.93 & -- & -- & 11.93 & 119.300 & 16 & 1.58 & 188.494 \\ 7 & 10 & 11.93 & -- & -- & 11.93 & 119.300 & 16 & 1.58 & 188.494 \\ 8 & 10 & 11.93 & -- & -- & 11.93 & 119.300 & 16 & 1.58 & 188.494 \\ 9 & 10 & 11.93 & -- & -- & 11.93 & 119.300 & 16 & 1.58 & 188.494 \\ 10 & 10 & 11.93 & -- & -- & 11.93 & 119.300 & 16 & 1.58 & 188.494 \\ & & & & & & & & & ------------ \\ \end{array}$

Armadura longitudinal total tablero

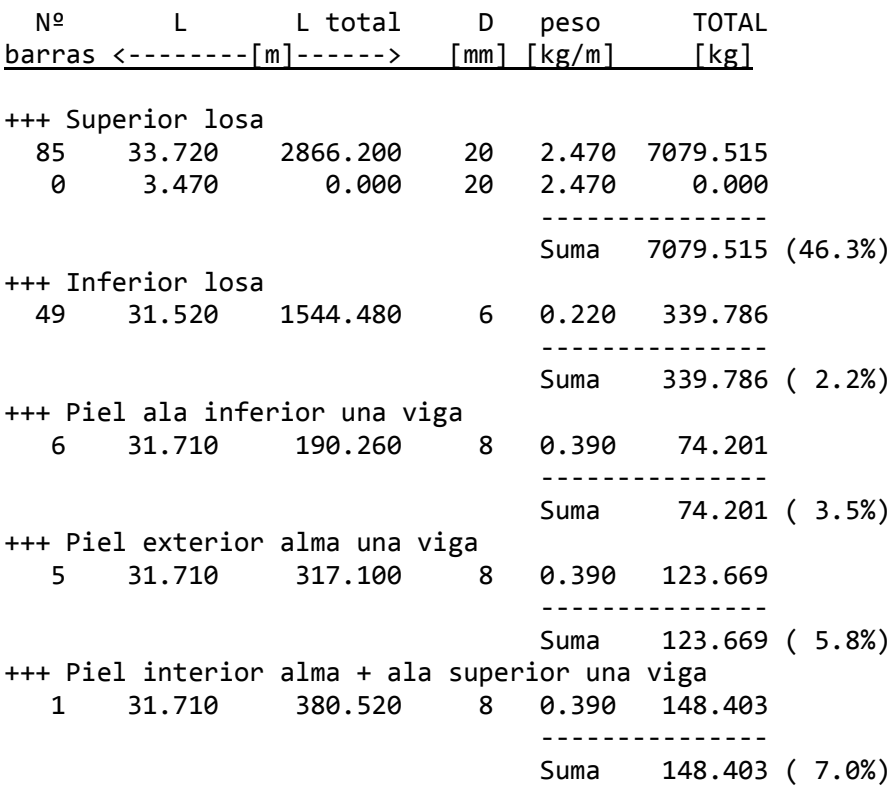

\section{ESTADO DE MEDICIONES}

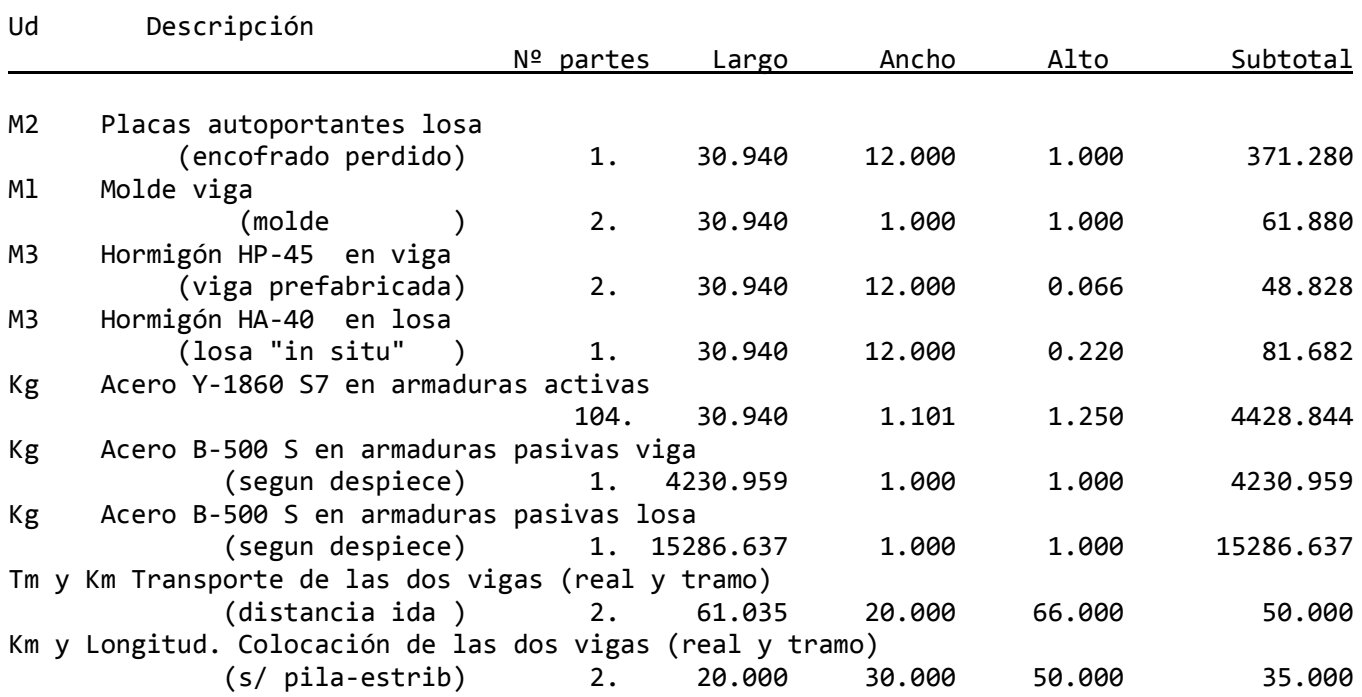




\section{CUANTIAS RESULTANTES}

0.351 M3 hormigon / M2 tablero

$33.935 \mathrm{~kg}$ activas / M3 hormigón

$90.703 \mathrm{~kg}$ activas / M3 hormigón VIGA

$11.905 \mathrm{~kg}$ activas / M2 tablero

$149.549 \mathrm{~kg}$ pasivas / M3 hormigón

$86.650 \mathrm{~kg}$ pasivas / M3 hormigón VIGA

$52.467 \mathrm{~kg}$ pasivas / M2 tablero

\section{CALCULO DEL COSTE}

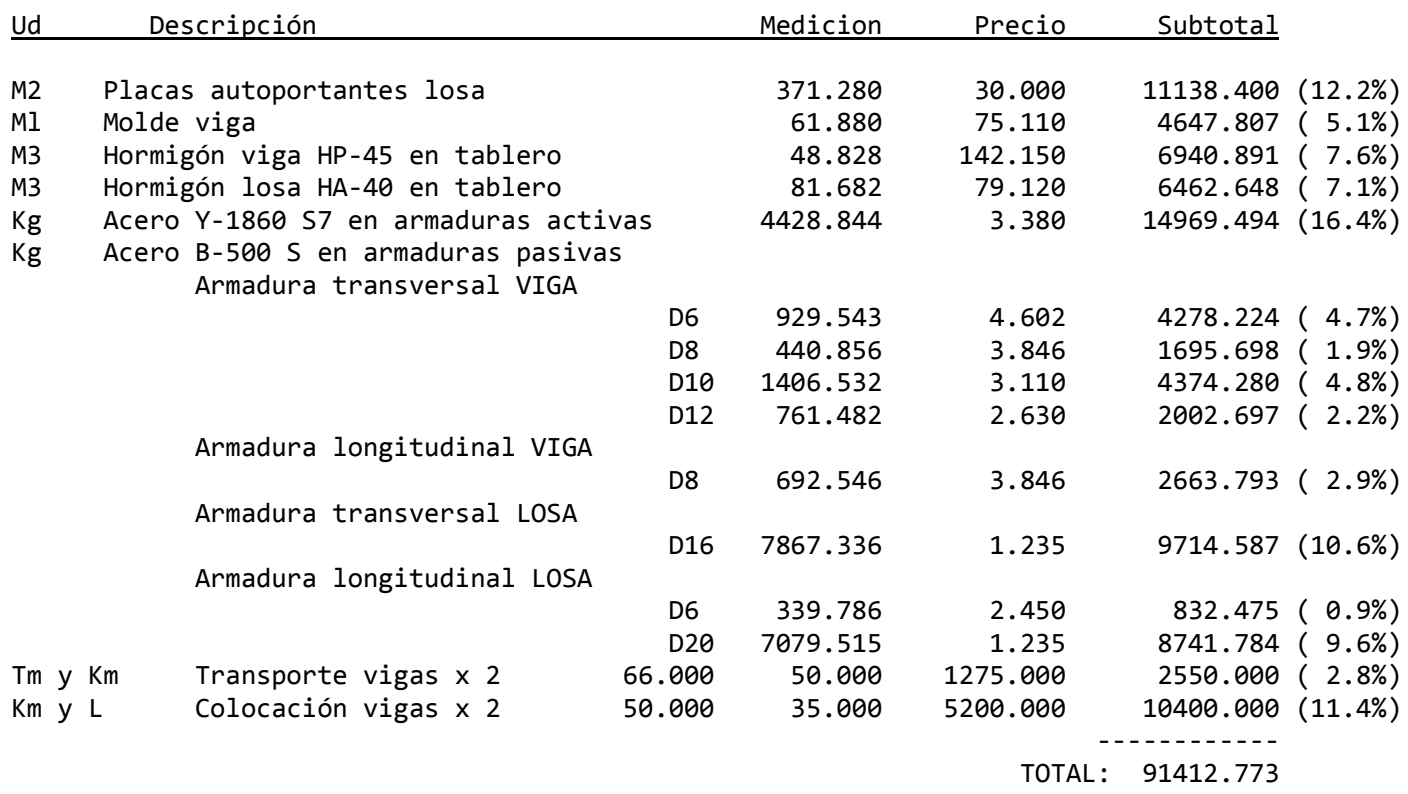

245.73 EUROS / M2 tablero 


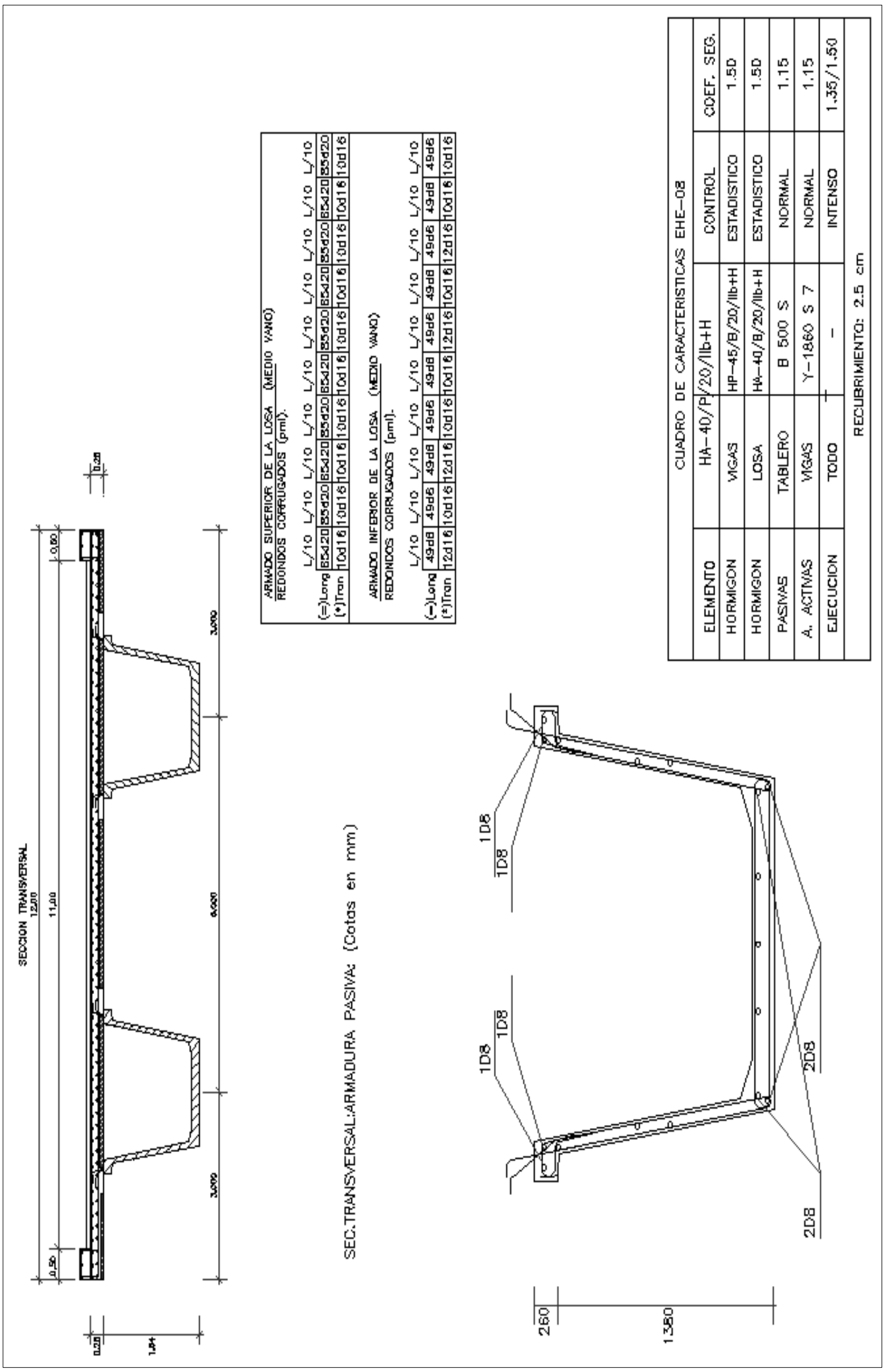




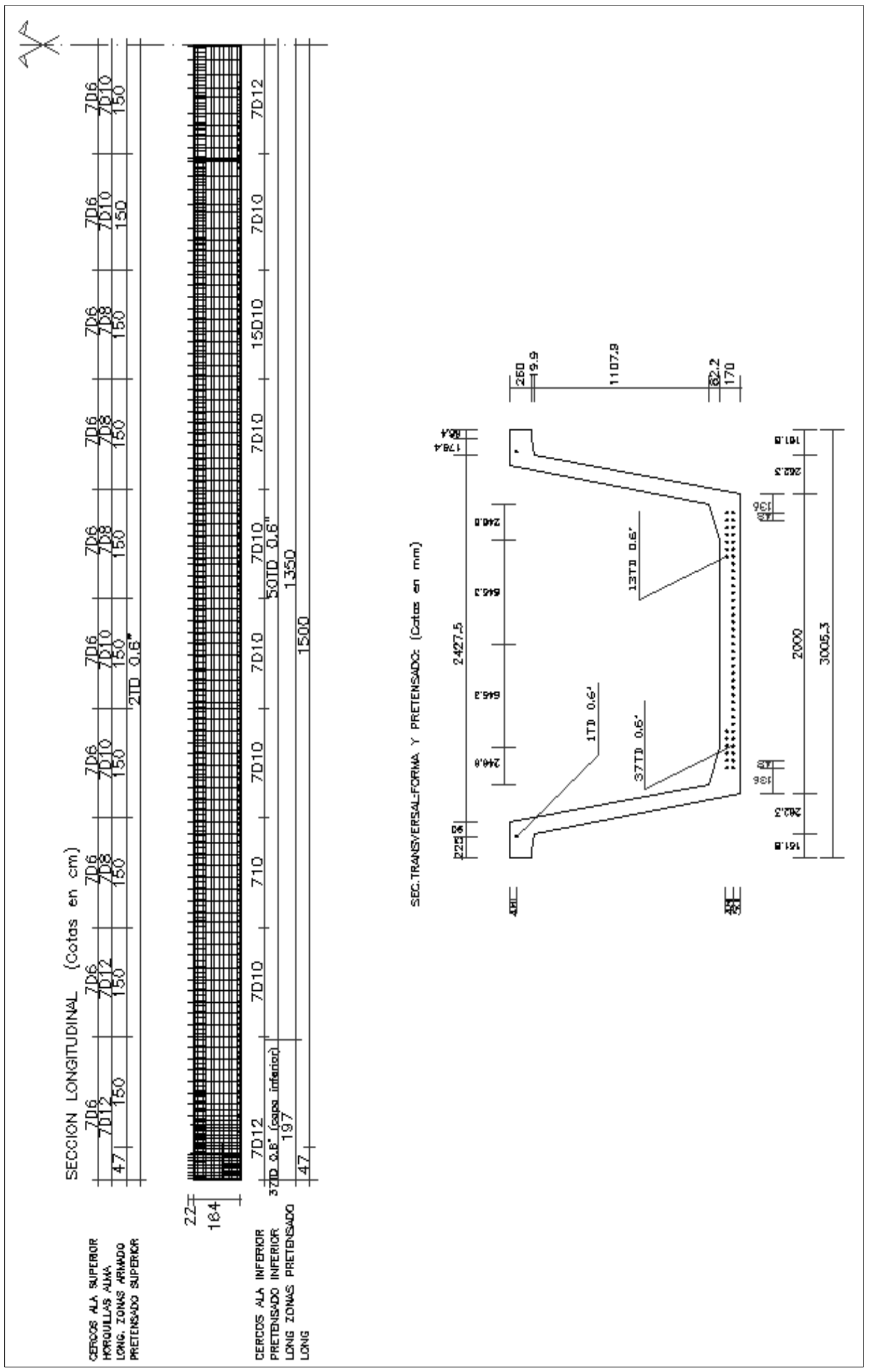


$\mathrm{L}=30 \mathrm{~m} . \quad \mathrm{HPF}$

ARMADURA PASIVA

Armadura transversal

№ $\quad \mathrm{L} 1 \quad \mathrm{~L} 2 \quad \mathrm{L3} \quad \mathrm{L} \quad \mathrm{L}$ total $\mathrm{D}$ peso

sec barras

\begin{tabular}{|c|c|c|c|c|c|c|c|c|c|c|}
\hline 1 & 7 & 3.82 & 0.16 & _- & 398 & 30785 & 12 & a 89 & 27399 & \\
\hline 2 & 7 & 3.82 & 0.18 & _- & 4.00 & 30.000 & 10 & 0.62 & 18,600 & \\
\hline 3 & 7 & 3.82 & 0.18 & - - & 4.00 & 30.000 & 10 & 0.62 & 18.600 & \\
\hline 4 & 7 & 3.82 & 0.18 & -- & 4.00 & 30.000 & 10 & 0.62 & 18.600 & \\
\hline 5 & 7 & 3.82 & 0.18 & - & 4.00 & 30.000 & 10 & 0.62 & 18.600 & \\
\hline 6 & 7 & 3.82 & 0.16 & -- & 3.98 & 29.850 & 12 & 0.89 & 26.566 & \\
\hline 7 & 7 & 3.82 & 0.18 & -- & 4.00 & 30.000 & 10 & 0.62 & 18.600 & \\
\hline 8 & 7 & 3.82 & 0.16 & -- & 3.98 & 29.850 & 12 & 0.89 & 26.566 & \\
\hline 9 & 15 & 3.84 & 0.18 & - - & 4.02 & 60.300 & 8 & 0.39 & 23.517 & \\
\hline \multirow[t]{2}{*}{10} & 7 & 3.82 & 0.16 & -- & 3.98 & 29.850 & 12 & 0.89 & 26.566 & \\
\hline & & & & & & & & Suma & 223.615 & $(20.4 \%)$ \\
\hline \multicolumn{11}{|c|}{ +++ Horquillas alma semi-viga } \\
\hline 1 & 7 & 0.94 & 1.62 & 0.42 & 10.04 & 77.659 & 12 & 0.89 & 69.117 & \\
\hline 2 & 7 & 0.94 & 1.62 & 0.42 & 10.04 & 75.300 & 12 & 0.89 & 67.017 & \\
\hline 3 & 7 & 0.94 & 1.62 & 0.42 & 10.04 & 75.300 & 12 & 0.89 & 67.017 & \\
\hline 4 & 7 & 0.94 & 1.62 & 0.35 & 9.76 & 73.200 & 10 & 0.62 & 45.384 & \\
\hline 5 & 7 & 0.95 & 1.62 & 0.28 & 9.50 & 71.250 & 8 & 0.39 & 27.787 & \\
\hline 6 & 7 & 0.94 & 1.62 & 0.35 & 9.76 & 73.200 & 10 & 0.62 & 45.384 & \\
\hline 7 & 7 & 0.94 & 1.62 & 0.35 & 9.76 & 73.200 & 10 & 0.62 & 45.384 & \\
\hline 8 & 7 & 0.94 & 1.62 & 0.35 & 9.76 & 73.200 & 10 & 0.62 & 45.384 & \\
\hline 9 & 7 & 0.94 & 1.62 & 0.42 & 10.04 & 75.300 & 12 & 0.89 & 67.017 & \\
\hline \multirow[t]{3}{*}{10} & 7 & 0.94 & 1.62 & 0.42 & 10.04 & 75.300 & 12 & 0.89 & 67.017 & \\
\hline & & & & & & & & --- & - - - - - - & \\
\hline & & & & & & & & Suma & 546.508 & $(49.8 \%)$ \\
\hline \multicolumn{11}{|c|}{ +++ Cerco alas semi-viga } \\
\hline 1 & 7 & 2.28 & 0.30 & 0.28 & 10.28 & 79.516 & 8 & 0.39 & 31.011 & \\
\hline 2 & 7 & 2.28 & 0.30 & 0.28 & 10.28 & 77.100 & 8 & 0.39 & 30.069 & \\
\hline 3 & 7 & 2.28 & 0.30 & 0.28 & 10.28 & 77.100 & 8 & 0.39 & 30.069 & \\
\hline 4 & 7 & 2.28 & 0.30 & 0.28 & 10.28 & 77.100 & 8 & 0.39 & 30.069 & \\
\hline 5 & 7 & 2.28 & 0.30 & 0.28 & 10.28 & 77.100 & 8 & 0.39 & 30.069 & \\
\hline 6 & 15 & 2.28 & 0.31 & 0.21 & 10.16 & 152.400 & 6 & 0.22 & 33.528 & \\
\hline 7 & 7 & 2.28 & 0.30 & 0.28 & 10.28 & 77.100 & 8 & 0.39 & 30.069 & \\
\hline 8 & 7 & 2.27 & 0.30 & 0.35 & 10.38 & 77.850 & 10 & 0.62 & 48.267 & \\
\hline 9 & 15 & 2.28 & 0.31 & 0.21 & 10.16 & 152.400 & 6 & 0.22 & 33.528 & \\
\hline \multirow[t]{3}{*}{10} & 7 & 2.28 & 0.30 & 0.28 & 10.28 & 77.100 & 8 & 0.39 & 30.069 & \\
\hline & & & & & & & & --- & ------ & \\
\hline & & & & & & & & Suma & 326.748 & $(29.8 \%)$ \\
\hline \multicolumn{11}{|c|}{ +++ Transversal inferior semi-losa } \\
\hline 1 & 5 & 11.92 & - - & -- & 11.92 & 61.467 & 25 & 3.85 & 236.650 & \\
\hline 2 & 5 & 11.92 & - & -- & 11.92 & 59.600 & 25 & 3.85 & 229.460 & \\
\hline 3 & 5 & 11.92 & -- & -- & 11.92 & 59.600 & 25 & 3.85 & 229.460 & \\
\hline 4 & 5 & 11.92 & -- & -- & 11.92 & 59.600 & 25 & 3.85 & 229.460 & \\
\hline 5 & 5 & 11.92 & -- & -- & 11.92 & 59.600 & 25 & 3.85 & 229.460 & \\
\hline 6 & 5 & 11.92 & -- & -- & 11.92 & 59.600 & 25 & 3.85 & 229.460 & \\
\hline 7 & 6 & 11.92 & - & -- & 11.92 & 71.520 & 25 & 3.85 & 275.352 & \\
\hline 8 & 5 & 11.92 & -- & -- & 11.92 & 59.600 & 25 & 3.85 & 229.460 & \\
\hline 9 & 5 & 11.92 & - & -- & 11.92 & 59.600 & 25 & 3.85 & 229.460 & \\
\hline \multirow[t]{3}{*}{10} & 5 & 11.92 & -- & -- & 11.92 & 59.600 & 25 & 3.85 & 229.460 & \\
\hline & & & & & & & & --- & - - - - - - & \\
\hline & & & & & & & & Suma & 2347.682 & $(24.3 \%)$ \\
\hline
\end{tabular}




\begin{tabular}{|c|c|c|c|c|c|c|c|c|c|}
\hline 1 & 5 & 11.92 & -- & - & 11.92 & 61.467 & 25 & 3.85 & 236.650 \\
\hline 2 & 5 & 11.92 & - - & - - & 11.92 & 59.600 & 25 & 3.85 & 229.460 \\
\hline 3 & 5 & 11.92 & - & - & 11.92 & 59.600 & 25 & 3.85 & 229.460 \\
\hline 4 & 5 & 11.92 & - & - & 11.92 & 59.600 & 25 & 3.85 & 229.460 \\
\hline 5 & 5 & 11.92 & - & - & 11.92 & 59.600 & 25 & 3.85 & 229.460 \\
\hline 6 & 5 & 11.92 & -- & -- & 11.92 & 59.600 & 25 & 3.85 & 229.460 \\
\hline 7 & 5 & 11.92 & -- & -- & 11.92 & 59.600 & 25 & 3.85 & 229.460 \\
\hline 8 & 5 & 11.92 & - & - & 11.92 & 59.600 & 25 & 3.85 & 229.460 \\
\hline 9 & 5 & 11.92 & - & - & 11.92 & 59.600 & 25 & 3.85 & 229.460 \\
\hline \multirow[t]{2}{*}{10} & 5 & 11.92 & - & -- & 11.92 & 59.600 & 25 & 3.85 & 229.460 \\
\hline & & & & & & & & \multicolumn{2}{|c|}{ Suma 2301.790} \\
\hline
\end{tabular}

Armadura longitudinal total tablero

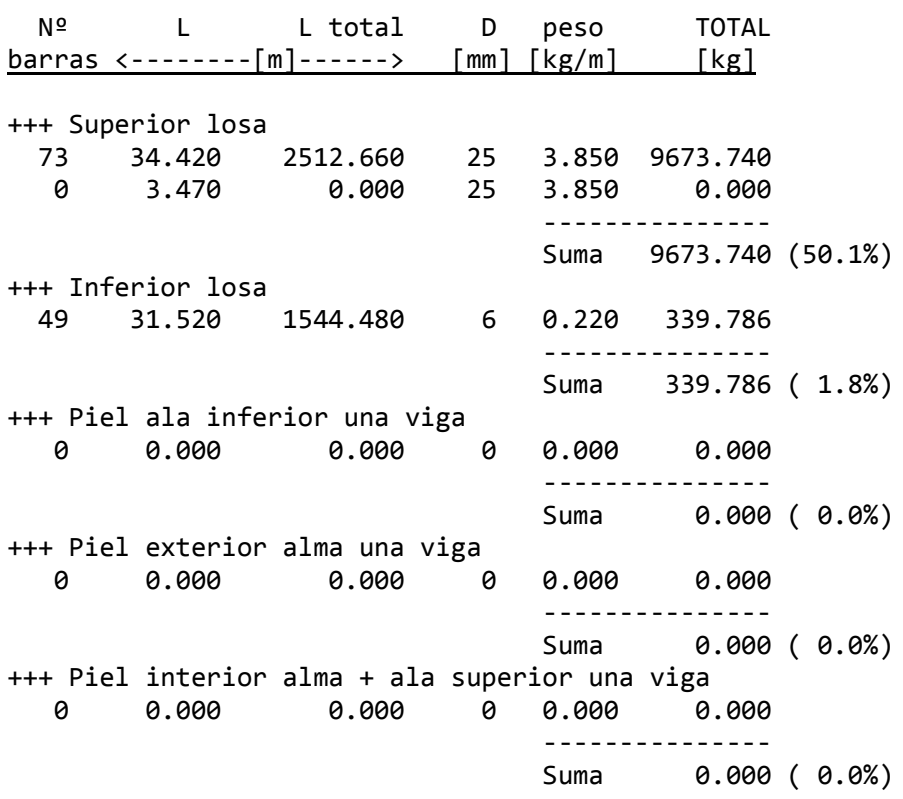

ESTADO DE MEDICIONES

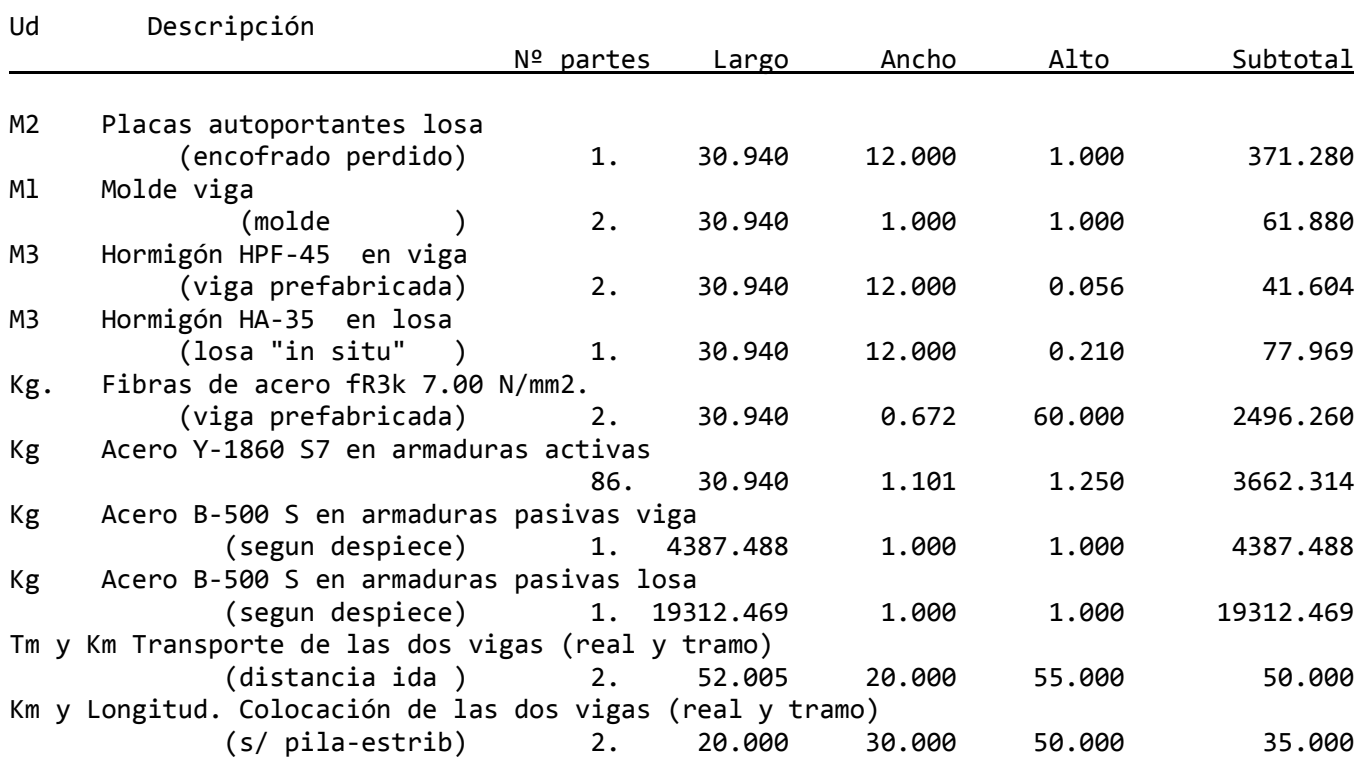




\section{CUANTIAS RESULTANTES}
$60.000 \mathrm{Kg} . / \mathrm{M3}$ hormigon
0.321 M3 hormigon / M2 tablero
$30.628 \mathrm{~kg}$ activas / M3 hormigón
$88.027 \mathrm{~kg}$ activas / M3 hormigón VIGA
$9.845 \mathrm{~kg}$ activas / M2 tablero
198.205 kg pasivas / M3 hormigón
$105.457 \mathrm{~kg}$ pasivas / M3 hormigón VIGA
$63.710 \mathrm{~kg}$ pasivas / M2 tablero

\section{CALCULO DEL COSTE}

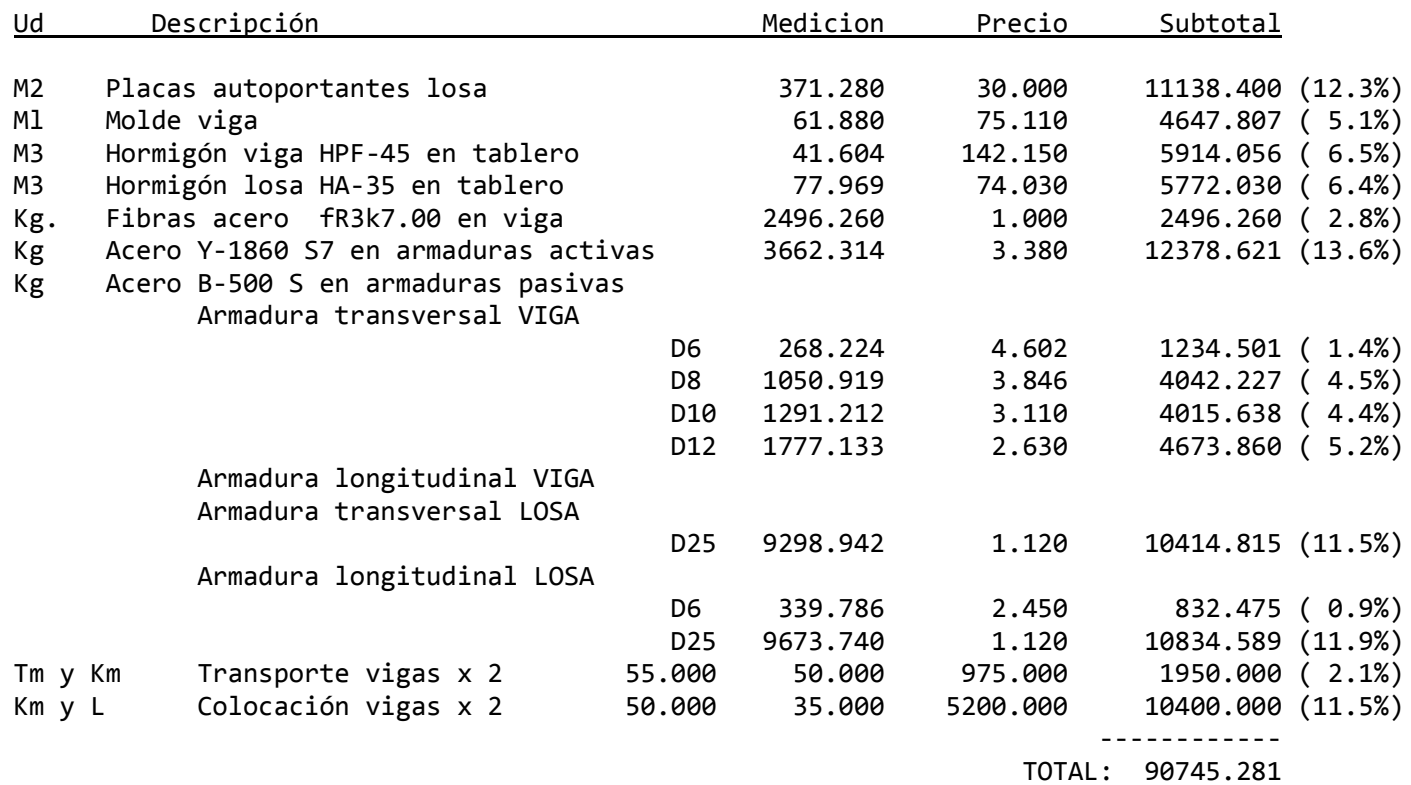

243.94 EUROS / M2 tablero 


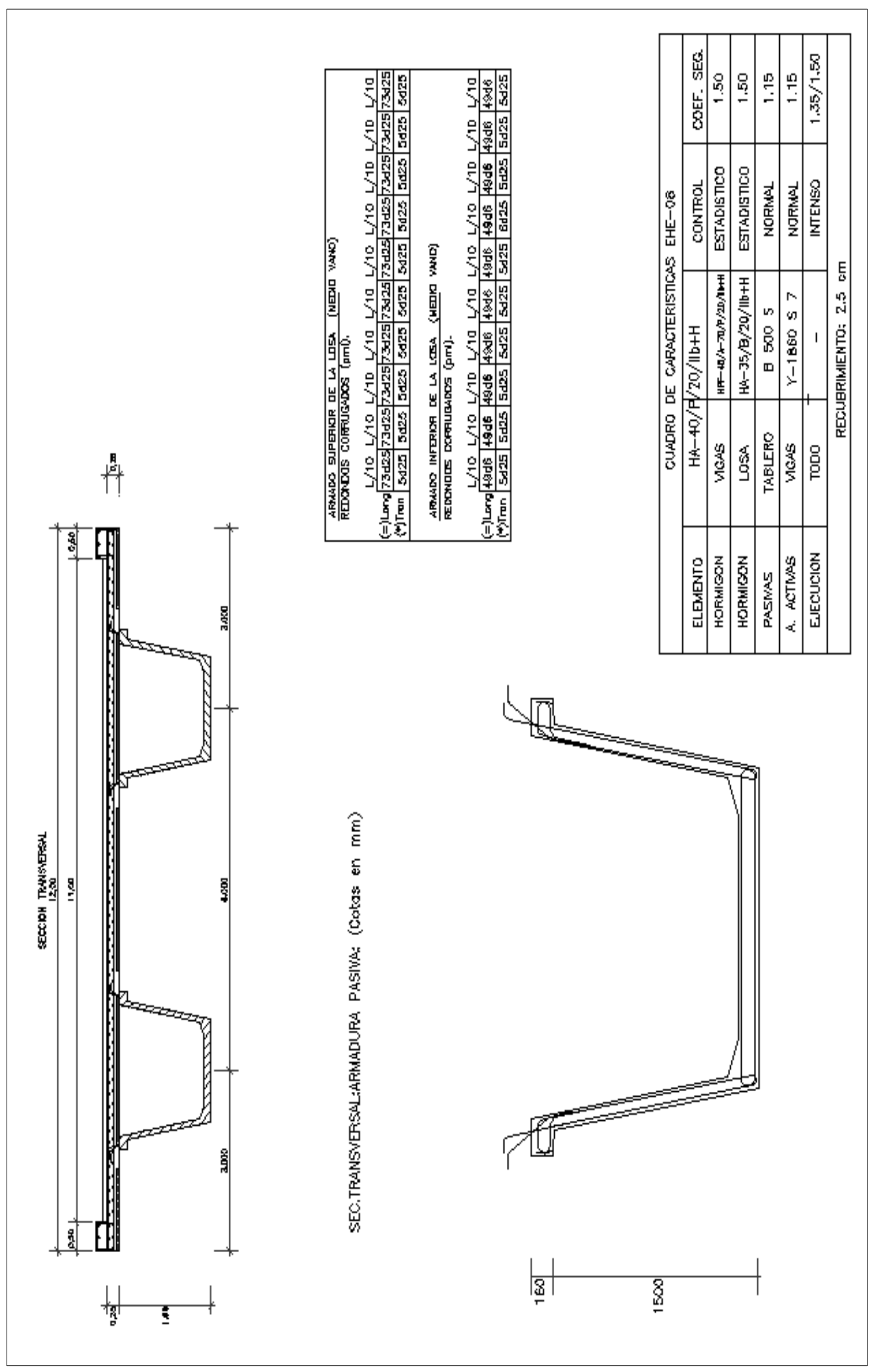




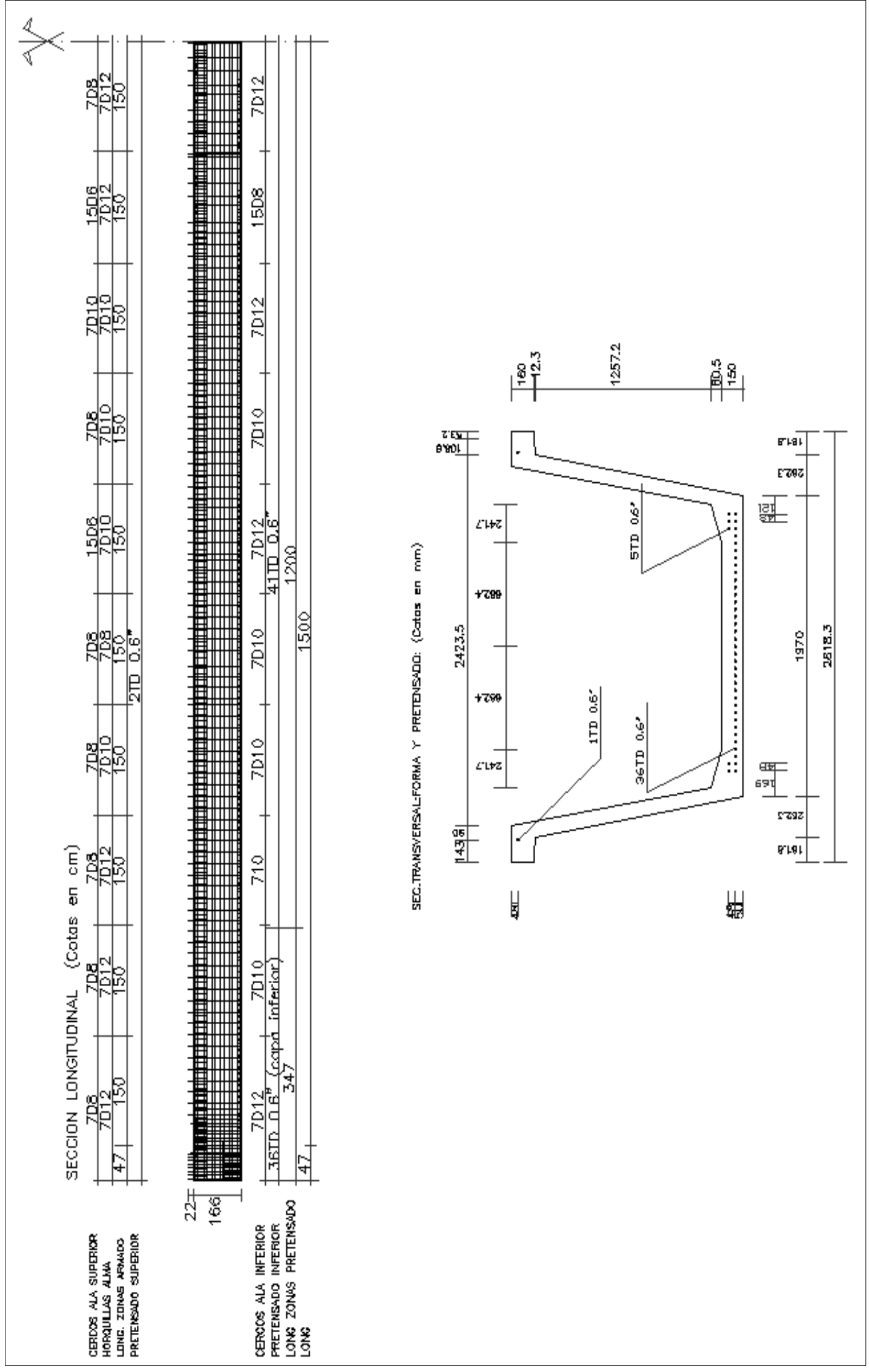


$\mathrm{L}=35 \mathrm{~m} . \quad \mathrm{HP}$

ARMADURA PASIVA

Armadura transversal

\begin{tabular}{|c|c|c|c|c|c|c|c|c|c|c|}
\hline sec & $\begin{array}{c}\text { № } \\
\text { barras }\end{array}$ & $\begin{array}{c}\text { L1 } \\
<<--\end{array}$ & $\begin{array}{c}\text { L2 } \\
- \\
\end{array}$ & $\begin{array}{c}\text { L3 } \\
---[\mathrm{m}\end{array}$ & $\begin{array}{c}\mathrm{L} \\
-\end{array}$ & L total & $\begin{array}{c}\mathrm{D} \\
{[\mathrm{mm}]} \\
\end{array}$ & $\begin{array}{c}\text { peso } \\
{[\mathrm{kg} / \mathrm{m}]}\end{array}$ & $\begin{array}{l}\text { TOTAL } \\
{[\mathrm{kg}]}\end{array}$ & \\
\hline \multicolumn{11}{|c|}{+++ Cerco ala inf.semi-viga } \\
\hline 1 & 8 & 3.86 & 0.22 & -- & 4.08 & 36.659 & 16 & 1.58 & 57.921 & \\
\hline 2 & 8 & 3.86 & 0.22 & -- & 4.08 & 35.700 & 12 & 0.89 & 31.773 & \\
\hline 3 & 8 & 3.86 & 0.22 & -- & 4.08 & 35.700 & 12 & 0.89 & 31.773 & \\
\hline 4 & 8 & 3.86 & 0.24 & -- & 4.10 & 35.875 & 10 & 0.62 & 22.243 & \\
\hline 5 & 8 & 3.88 & 0.24 & -- & 4.12 & 36.050 & 8 & 0.39 & 14.059 & \\
\hline 6 & 8 & 3.88 & 0.24 & - & 4.12 & 36.050 & 8 & 0.39 & 14.059 & \\
\hline 7 & 8 & 3.86 & 0.24 & -- & 4.10 & 35.875 & 10 & 0.62 & 22.243 & \\
\hline 8 & 8 & 3.88 & 0.24 & -- & 4.12 & 36.050 & 8 & 0.39 & 14.059 & \\
\hline 9 & 8 & 3.88 & 0.24 & -- & 4.12 & 36.050 & 8 & 0.39 & 14.059 & \\
\hline \multirow[t]{3}{*}{10} & 8 & 3.86 & 0.22 & - - & 4.08 & 35.700 & 12 & 0.89 & 31.773 & \\
\hline & & & & & & & & ---- & - - - - - - - & \\
\hline & & & & & & & & Suma & 253.963 & $(16.5 \%)$ \\
\hline \multicolumn{11}{|c|}{ +++ Horquillas alma semi-viga } \\
\hline 1 & 8 & 0.94 & 1.90 & 0.42 & 11.16 & 100.273 & 12 & 0.89 & 89.243 & \\
\hline 2 & 8 & 0.94 & 1.90 & 0.42 & 11.16 & 97.650 & 12 & 0.89 & 86.909 & \\
\hline 3 & 8 & 0.94 & 1.90 & 0.42 & 11.16 & 97.650 & 12 & 0.89 & 86.909 & \\
\hline 4 & 8 & 0.94 & 1.90 & 0.42 & 11.16 & 97.650 & 12 & 0.89 & 86.909 & \\
\hline 5 & 8 & 0.94 & 1.90 & 0.35 & 10.88 & 95.200 & 10 & 0.62 & 59.024 & \\
\hline 6 & 8 & 0.94 & 1.90 & 0.42 & 11.16 & 97.650 & 12 & 0.89 & 86.909 & \\
\hline 7 & 8 & 0.94 & 1.90 & 0.42 & 11.16 & 97.650 & 12 & 0.89 & 86.909 & \\
\hline 8 & 8 & 0.94 & 1.90 & 0.42 & 11.16 & 97.650 & 12 & 0.89 & 86.909 & \\
\hline 9 & 8 & 0.94 & 1.90 & 0.42 & 11.16 & 97.650 & 12 & 0.89 & 86.909 & \\
\hline \multirow[t]{3}{*}{10} & 8 & 0.94 & 1.90 & 0.42 & 11.16 & 97.650 & 12 & 0.89 & 86.909 & \\
\hline & & & & & & & & ----- & ------ & \\
\hline & & & & & & & & Suma & 843.535 & $(54.7 \%)$ \\
\hline \multicolumn{11}{|c|}{ +++ Cerco alas semi-viga } \\
\hline 1 & 8 & 2.85 & 0.52 & 0.21 & 12.86 & 115.547 & 6 & 0.22 & 25.420 & \\
\hline 2 & 8 & 2.85 & 0.52 & 0.21 & 12.86 & 112.525 & 6 & 0.22 & 24.755 & \\
\hline 3 & 8 & 2.85 & 0.52 & 0.21 & 12.86 & 112.525 & 6 & 0.22 & 24.755 & \\
\hline 4 & 8 & 2.85 & 0.52 & 0.21 & 12.86 & 112.525 & 6 & 0.22 & 24.755 & \\
\hline 5 & 8 & 2.85 & 0.52 & 0.21 & 12.86 & 112.525 & 6 & 0.22 & 24.755 & \\
\hline 6 & 8 & 2.85 & 0.52 & 0.21 & 12.86 & 112.525 & 6 & 0.22 & 24.755 & \\
\hline 7 & 8 & 2.85 & 0.52 & 0.21 & 12.86 & 112.525 & 6 & 0.22 & 24.755 & \\
\hline 8 & 8 & 2.85 & 0.52 & 0.21 & 12.86 & 112.525 & 6 & 0.22 & 24.755 & \\
\hline 9 & 8 & 2.85 & 0.52 & 0.21 & 12.86 & 112.525 & 6 & 0.22 & 24.755 & \\
\hline \multirow[t]{3}{*}{10} & 8 & 2.85 & 0.52 & 0.21 & 12.86 & 112.525 & 6 & 0.22 & 24.755 & \\
\hline & & & & & & & & $-\ldots$ &.------ & \\
\hline & & & & & & & & Suma & 248.220 & $(16.1 \%)$ \\
\hline \multicolumn{11}{|c|}{ +++ Transversal inferior semi-losa } \\
\hline 1 & 5 & 11.93 & -- & -- & 11.93 & 71.461 & 25 & 3.85 & 275.124 & \\
\hline 2 & 5 & 11.93 & -- & -- & 11.93 & 69.592 & 25 & 3.85 & 267.928 & \\
\hline 3 & 5 & 11.93 & -- & -- & 11.93 & 69.592 & 25 & 3.85 & 267.928 & \\
\hline 4 & 5 & 11.93 & -- & -- & 11.93 & 69.592 & 25 & 3.85 & 267.928 & \\
\hline 5 & 5 & 11.93 & -- & -- & 11.93 & 69.592 & 25 & 3.85 & 267.928 & \\
\hline 6 & 5 & 11.93 & -- & -- & 11.93 & 69.592 & 25 & 3.85 & 267.928 & \\
\hline 7 & 5 & 11.93 & -- & -- & 11.93 & 69.592 & 25 & 3.85 & 267.928 & \\
\hline 8 & 5 & 11.93 & -- & -- & 11.93 & 69.592 & 25 & 3.85 & 267.928 & \\
\hline 9 & 5 & 11.93 & -- & -- & 11.93 & 69.592 & 25 & 3.85 & 267.928 & \\
\hline \multirow[t]{2}{*}{10} & 7 & 11.93 & -- & -- & 11.93 & 83.510 & 25 & 3.85 & 321.513 & \\
\hline & & & & & & & & $\begin{array}{l}\text { Suma } \\
\text { Sum }\end{array}$ & 2740.061 & (27.9\%) \\
\hline
\end{tabular}




$\begin{array}{rrrrrrrrrr}+++ \\ 1 & 5 & 11.93 & -- & -- & 11.93 & 71.461 & 25 & 3.85 & 275.124 \\ 2 & 5 & 11.93 & -- & -- & 11.93 & 69.592 & 25 & 3.85 & 267.928 \\ 3 & 5 & 11.93 & -- & -- & 11.93 & 69.592 & 25 & 3.85 & 267.928 \\ 4 & 5 & 11.93 & -- & -- & 11.93 & 69.592 & 25 & 3.85 & 267.928 \\ 5 & 5 & 11.93 & -- & -- & 11.93 & 69.592 & 25 & 3.85 & 267.928 \\ 6 & 5 & 11.93 & -- & -- & 11.93 & 69.592 & 25 & 3.85 & 267.928 \\ 7 & 5 & 11.93 & -- & -- & 11.93 & 69.592 & 25 & 3.85 & 267.928 \\ 8 & 5 & 11.93 & -- & -- & 11.93 & 69.592 & 25 & 3.85 & 267.928 \\ 9 & 5 & 11.93 & -- & -- & 11.93 & 69.592 & 25 & 3.85 & 267.928 \\ 10 & 5 & 11.93 & -- & -- & 11.93 & 69.592 & 25 & 3.85 & 267.928 \\ & & & & & & & & & \\ \end{array}$

Armadura longitudinal total tablero

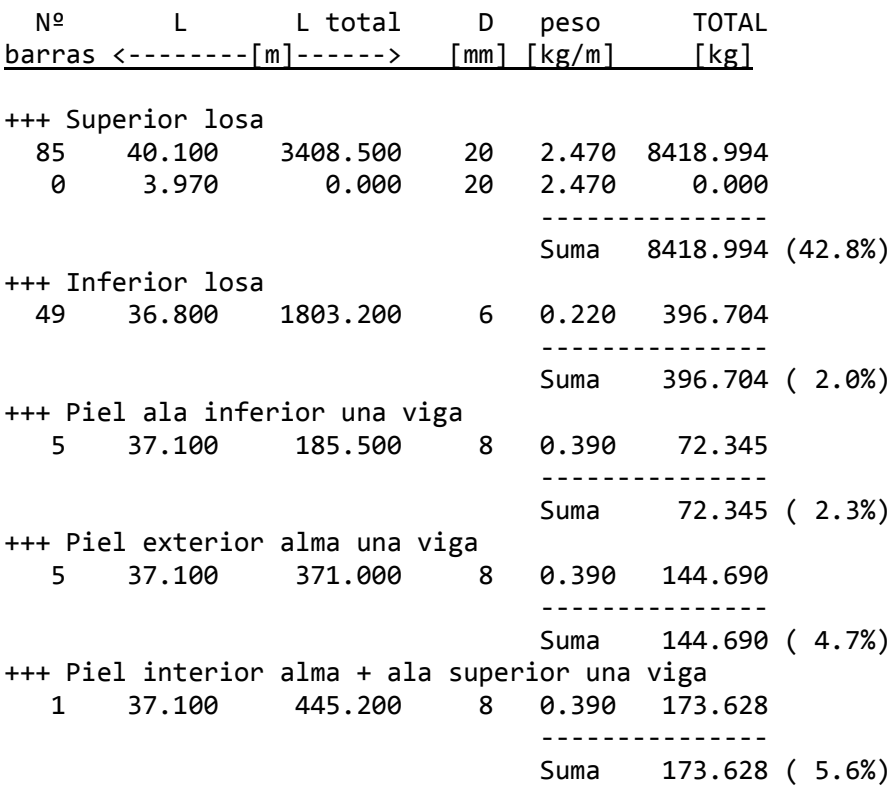

\section{ESTADO DE MEDICIONES}

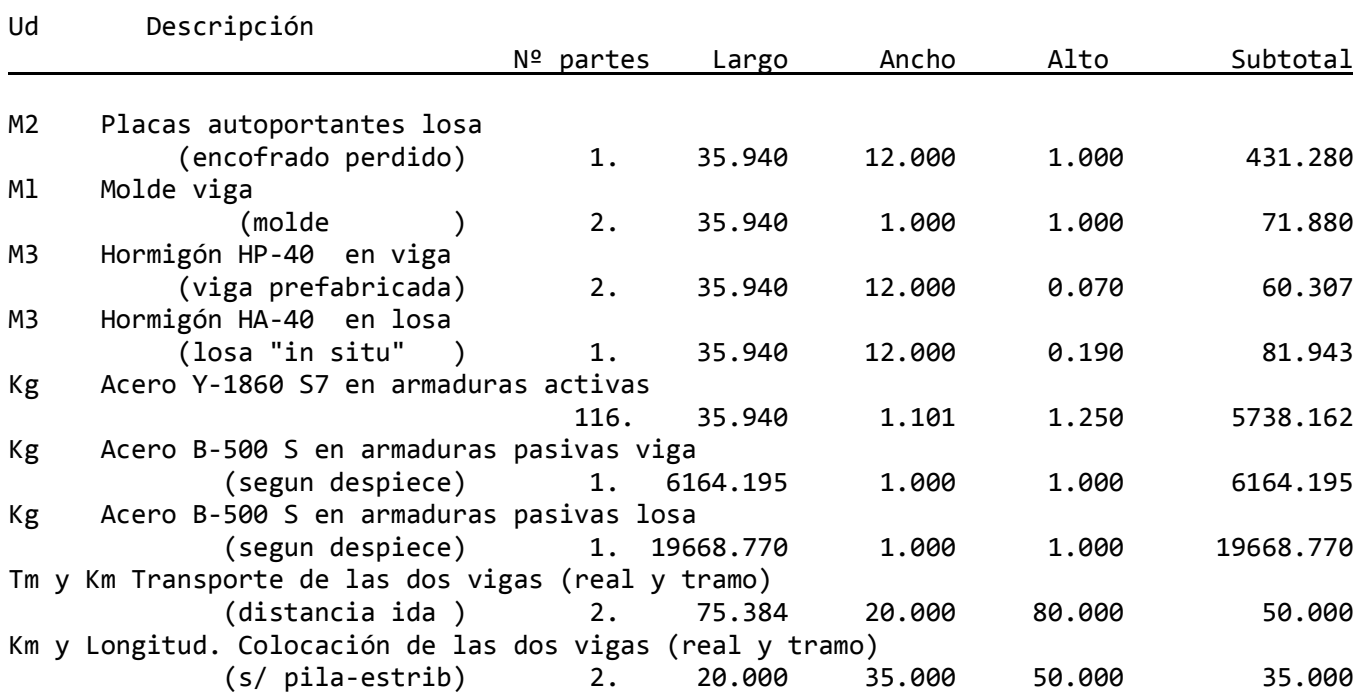




\section{CUANTIAS RESULTANTES}

0.329 M3 hormigon / M2 tablero

$40.339 \mathrm{~kg}$ activas / M3 hormigón

$95.149 \mathrm{~kg}$ activas / M3 hormigón VIGA

$13.283 \mathrm{~kg}$ activas / M2 tablero

$181.602 \mathrm{~kg}$ pasivas / M3 hormigón

$102.214 \mathrm{~kg}$ pasivas / M3 hormigón VIGA

$59.799 \mathrm{~kg}$ pasivas / M2 tablero

\section{CALCULO DEL COSTE}

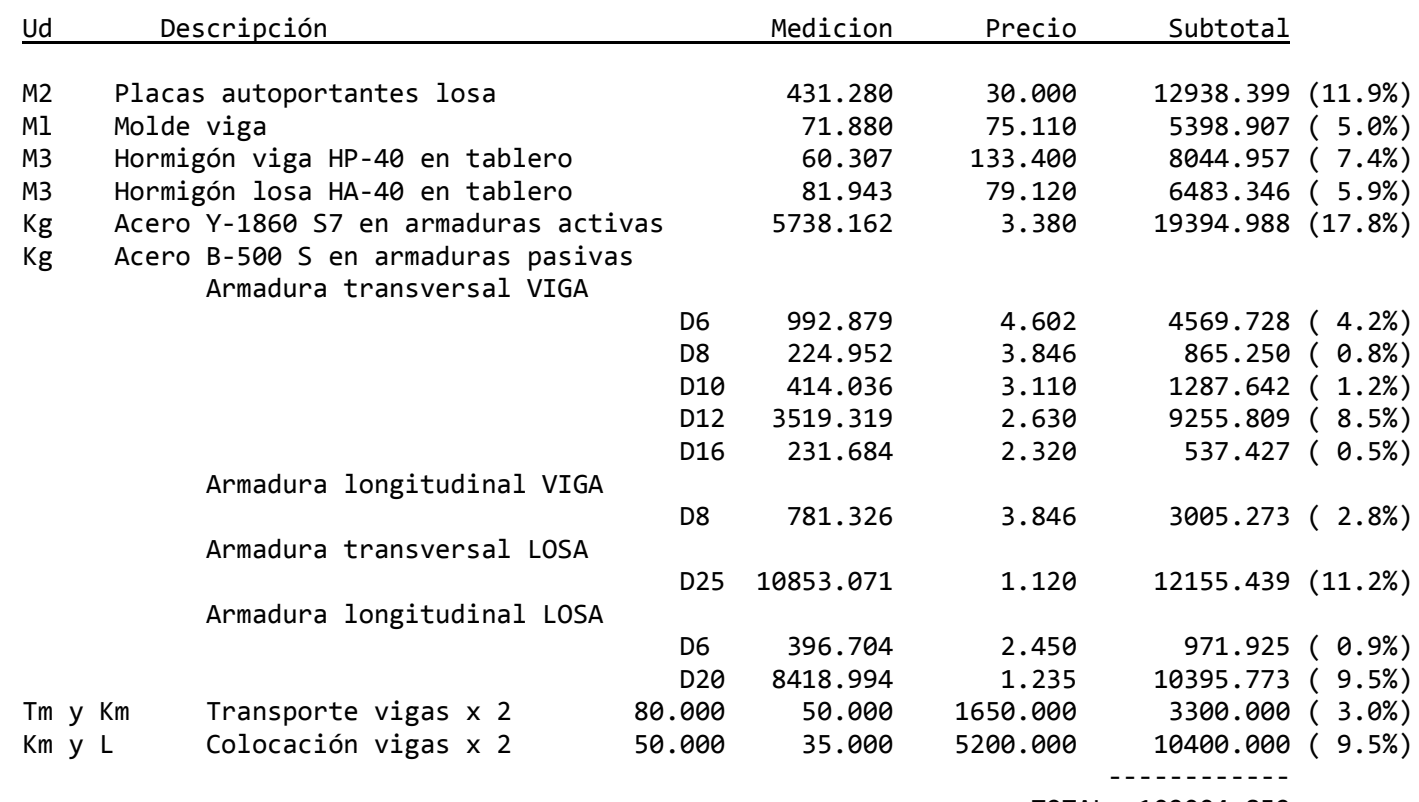

TOTAL: 109004.859 


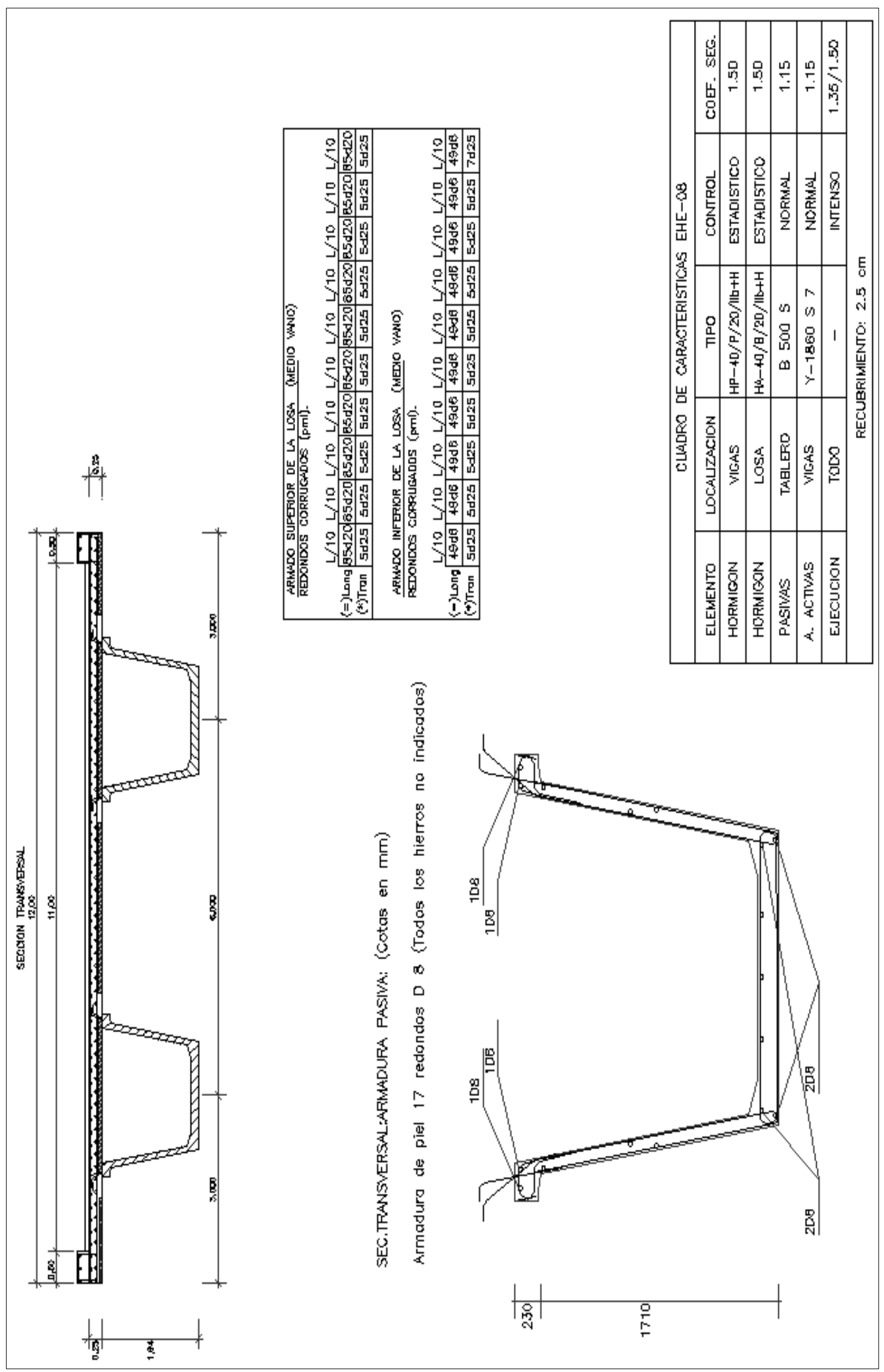




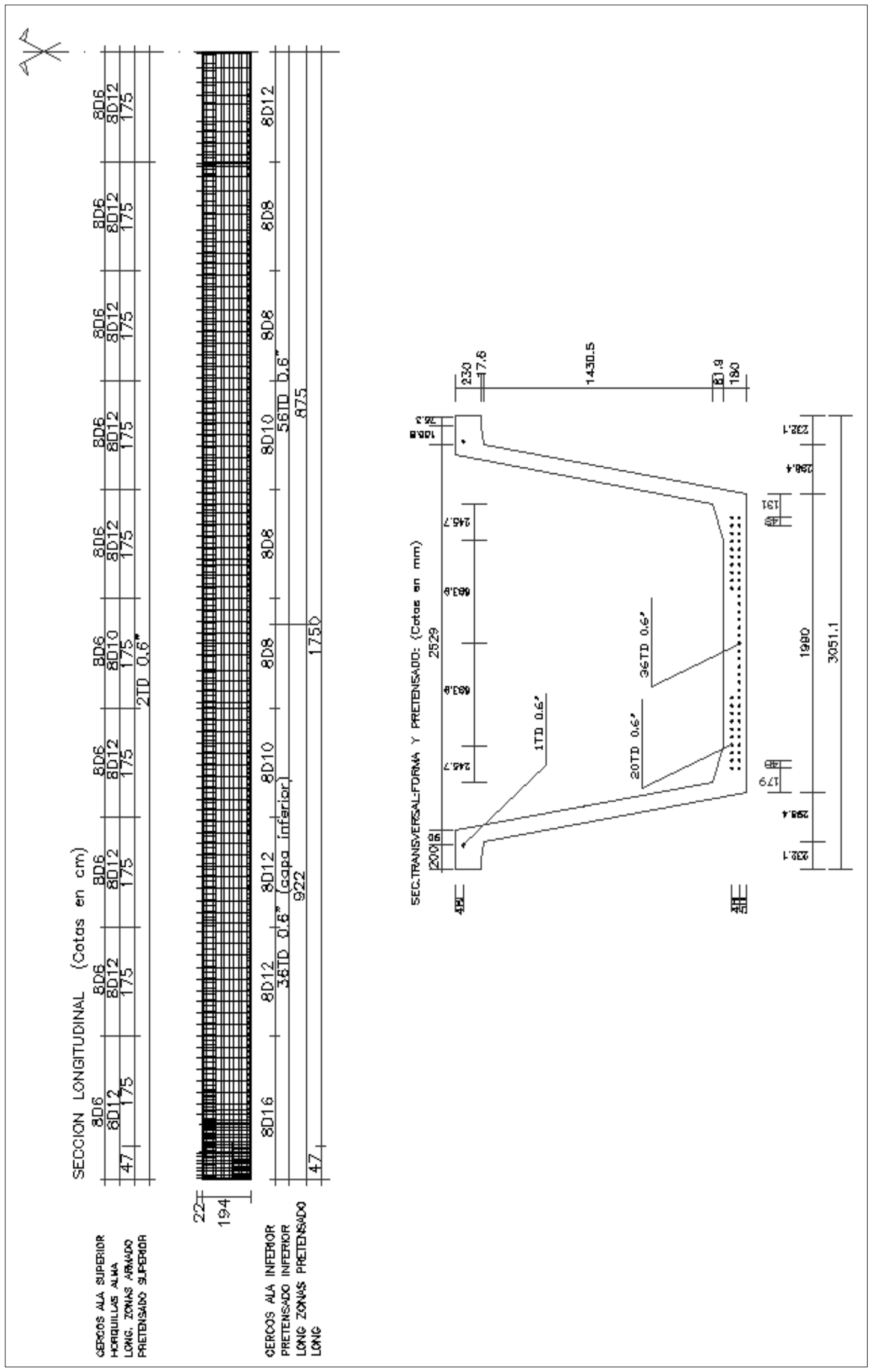


$\mathrm{L}=35 \mathrm{~m} . \mathrm{HPF}$

ARMADURA PASIVA

Armadura transversal

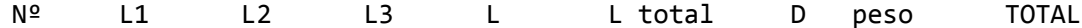

$\mathrm{sec}$ barras $<\ldots[\mathrm{mm}][\mathrm{kg} / \mathrm{m}] \quad[\mathrm{kg}]$

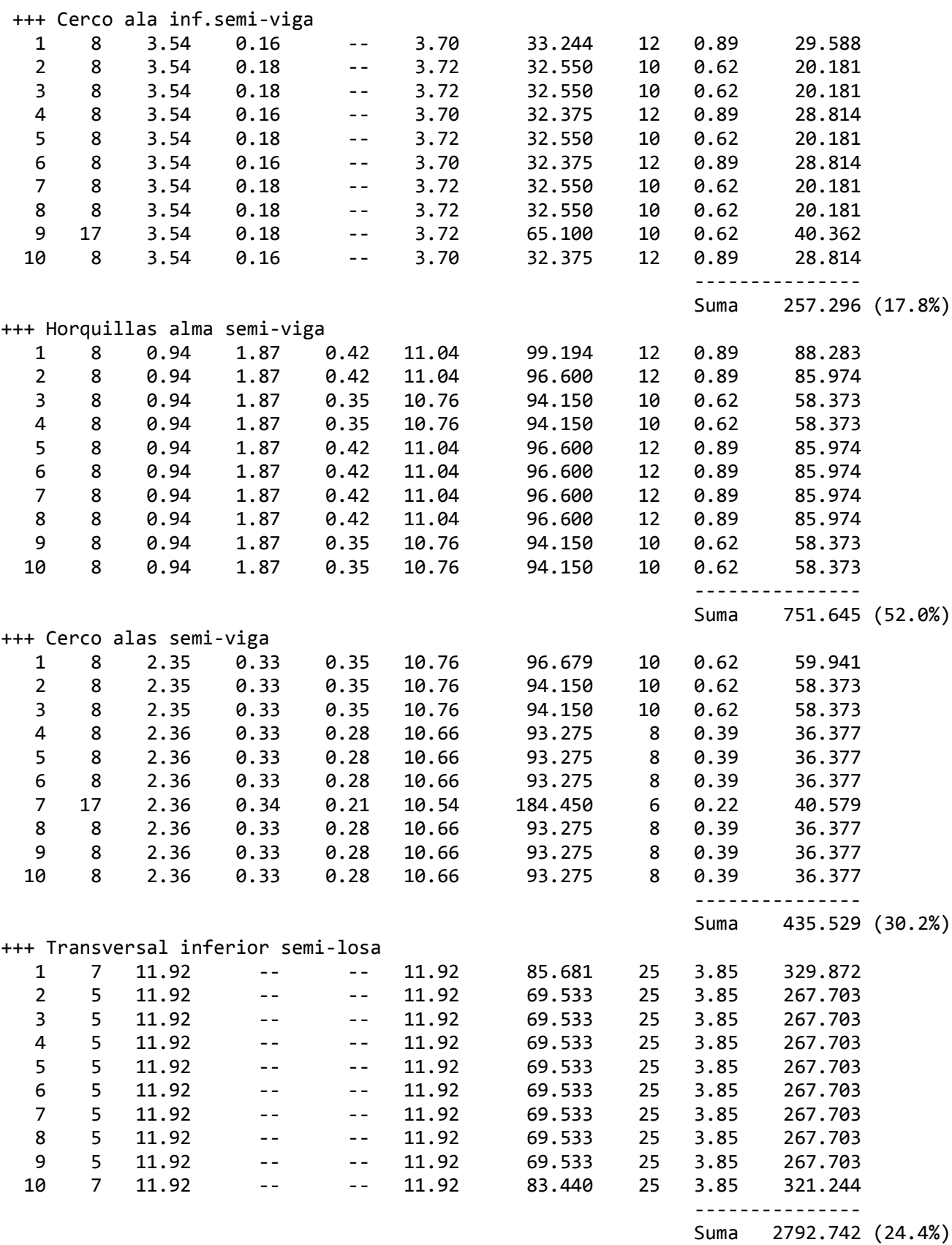




\begin{tabular}{|c|c|c|c|c|c|c|c|c|c|}
\hline 1 & 5 & 11.92 & -- & -- & 11.92 & 71.401 & 25 & 3.85 & 274.893 \\
\hline 2 & 5 & 11.92 & -- & -- & 11.92 & 69.533 & 25 & 3.85 & 267.703 \\
\hline 3 & 5 & 11.92 & -- & -- & 11.92 & 69.533 & 25 & 3.85 & 267.703 \\
\hline 4 & 5 & 11.92 & -- & - - & 11.92 & 69.533 & 25 & 3.85 & 267.703 \\
\hline 5 & 5 & 11.92 & -- & -- & 11.92 & 69.533 & 25 & 3.85 & 267.703 \\
\hline 6 & 5 & 11.92 & -- & -- & 11.92 & 69.533 & 25 & 3.85 & 267.703 \\
\hline 7 & 5 & 11.92 & -- & -- & 11.92 & 69.533 & 25 & 3.85 & 267.703 \\
\hline 8 & 5 & 11.92 & - & -- & 11.92 & 69.533 & 25 & 3.85 & 267.703 \\
\hline 9 & 5 & 11.92 & -- & -- & 11.92 & 69.533 & 25 & 3.85 & 267.703 \\
\hline \multirow[t]{2}{*}{10} & 5 & 11.92 & -- & -- & 11.92 & 69.533 & 25 & 3.85 & 267.703 \\
\hline & & & & & & & & Suma & 2684.223 \\
\hline
\end{tabular}

Armadura longitudinal total tablero

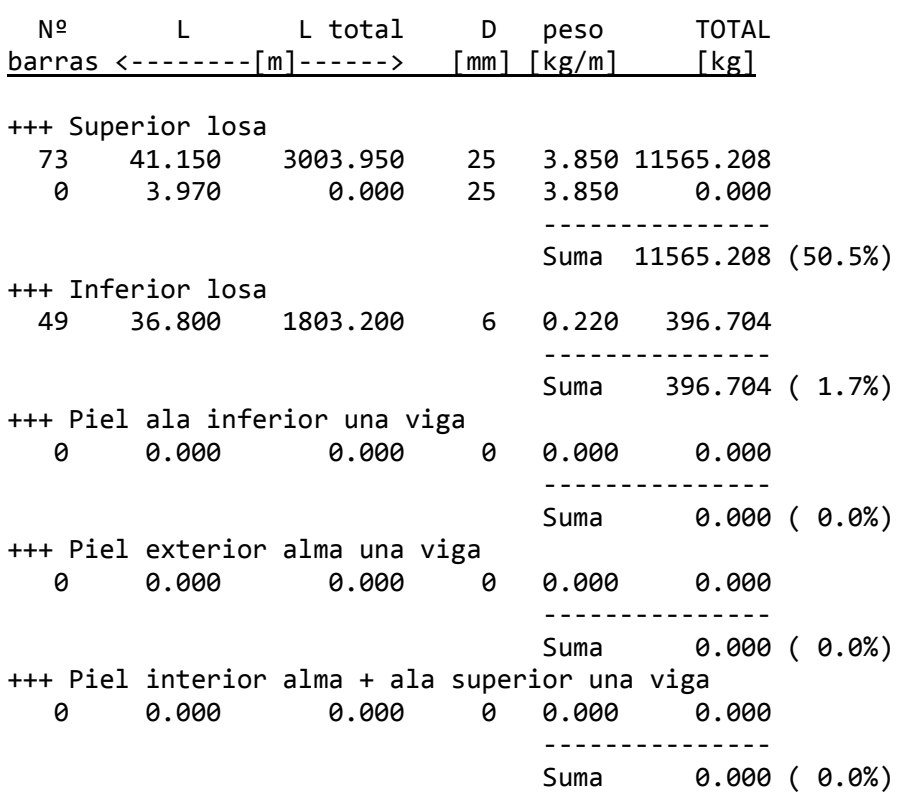

\section{ESTADO DE MEDICIONES}

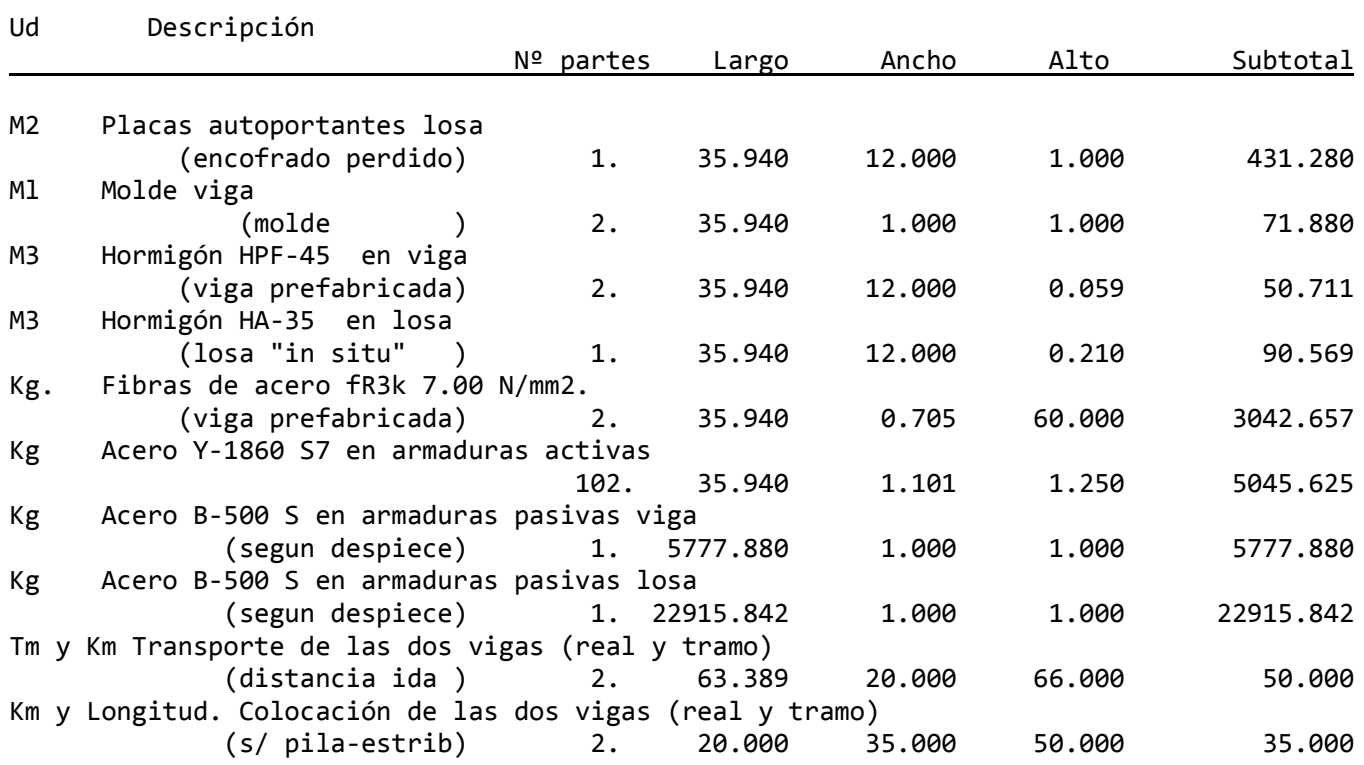




\section{CUANTIAS RESULTANTES}
$60.000 \mathrm{Kg} . / \mathrm{M3}$ hormigon
0.327 M3 hormigon / M2 tablero
$35.714 \mathrm{~kg}$ activas / M3 hormigón
$99.498 \mathrm{~kg}$ activas / M3 hormigón VIGA
$11.680 \mathrm{~kg}$ activas / M2 tablero
$203.099 \mathrm{~kg}$ pasivas / M3 hormigón
$113.938 \mathrm{~kg}$ pasivas / M3 hormigón VIGA
$66.421 \mathrm{~kg}$ pasivas / M2 tablero

\section{CALCULO DEL COSTE}

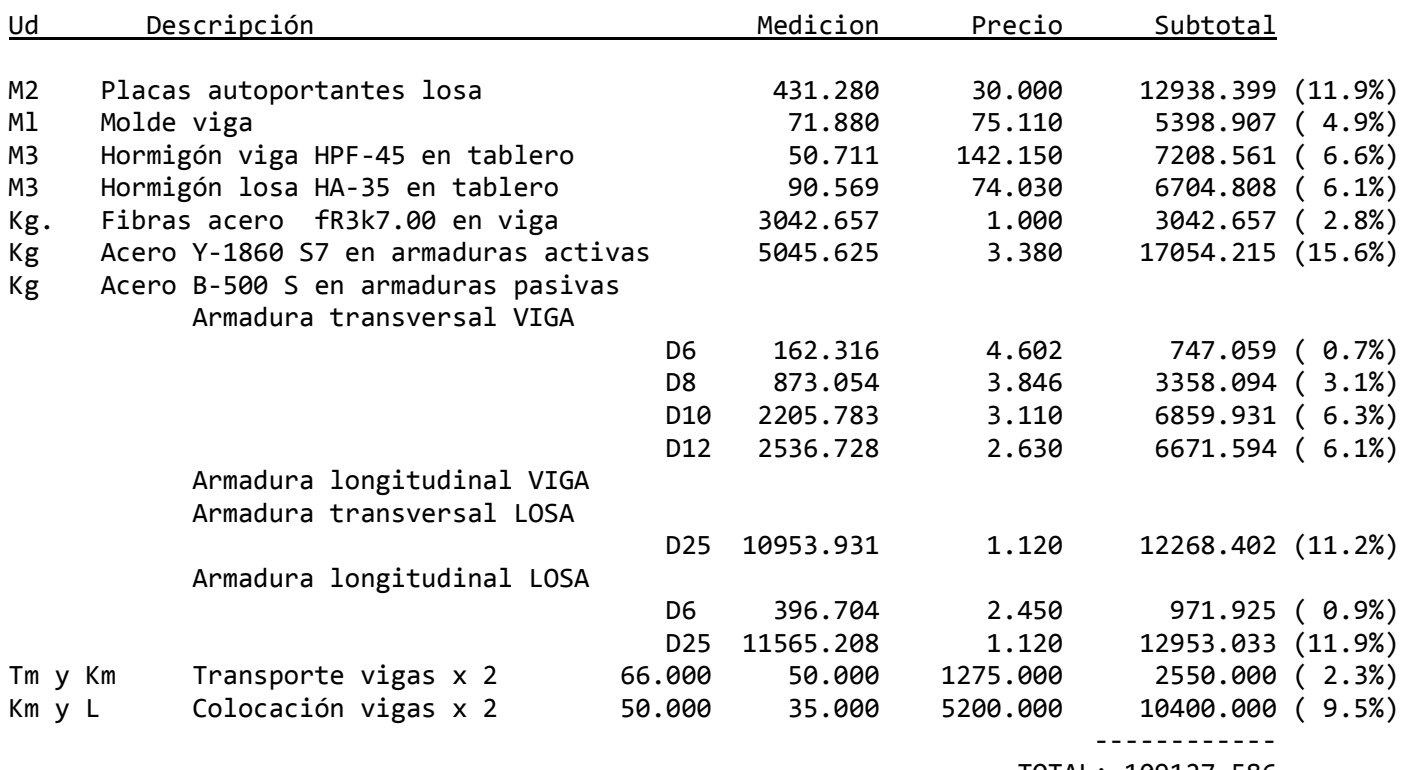

TOTAL: 109127.586 


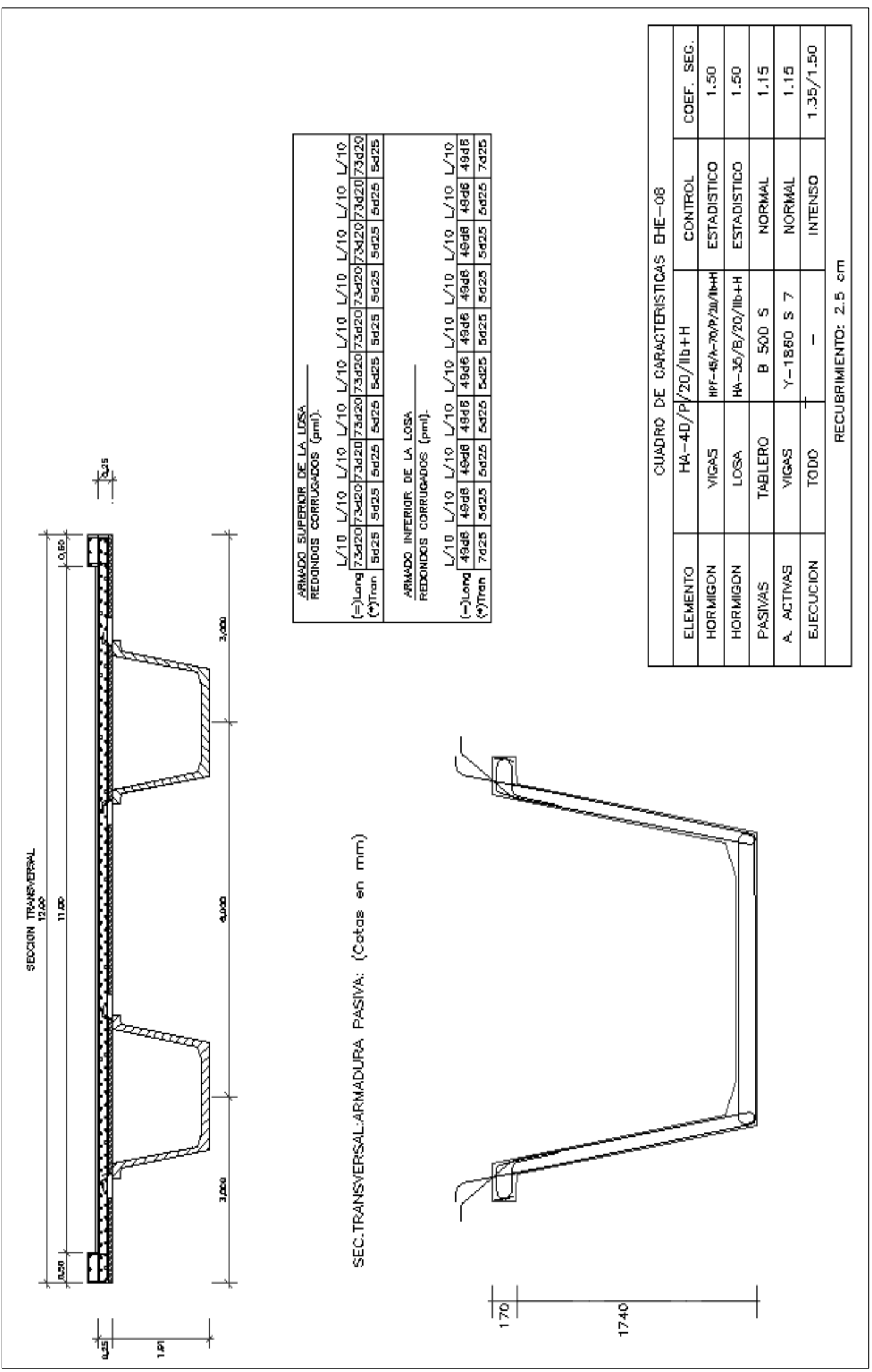




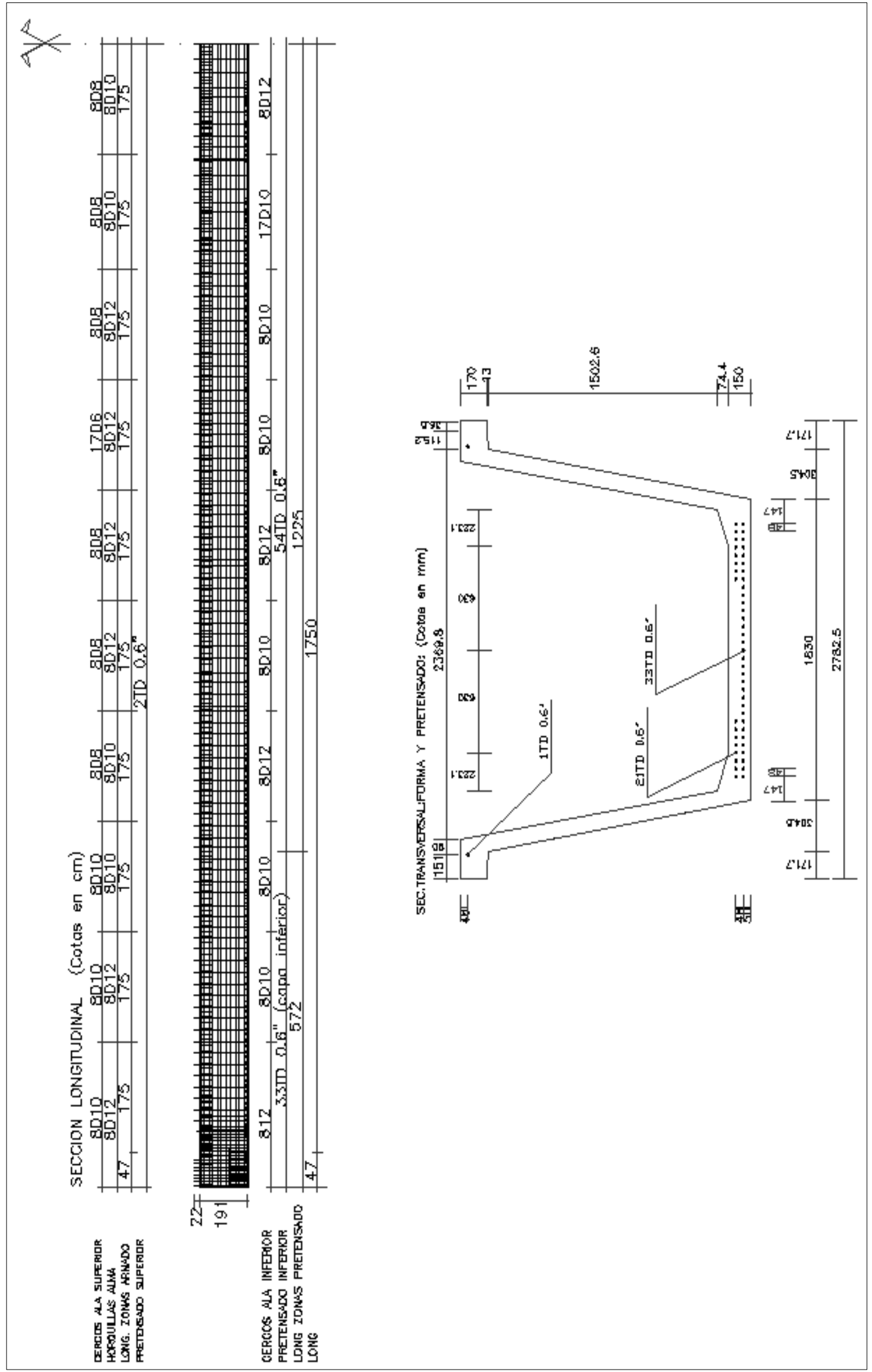


$\mathrm{L}=40 \mathrm{~m}$. HP

ARMADURA PASIVA

Armadura transversal

$\begin{array}{lllllllll}\text { No } & \mathrm{L} 1 & \mathrm{~L} 2 & \mathrm{~L} 3 & \mathrm{~L} & \mathrm{~L} \text { total } & \mathrm{D} & \text { peso } & \text { TOTAL }\end{array}$

$\mathrm{sec}$ barras $\langle\ldots \ldots[\mathrm{mm}][\mathrm{kg} / \mathrm{m}] \quad[\mathrm{kg}]$

\begin{tabular}{|c|c|c|c|c|c|c|c|c|c|c|}
\hline 1 & 10 & 3.86 & 0.28 & - - & 4,14 & 473 & 12 & 089 & 37.712 & \\
\hline 2 & 10 & 3.86 & 0.30 & - - & 4.16 & 41.600 & 10 & 0.62 & 25.792 & \\
\hline 3 & 10 & 3.86 & 0.28 & - - & 4.14 & 41.400 & 12 & 0.89 & 36.846 & \\
\hline 4 & 10 & 3.86 & 0.30 & - & 4.16 & 41.600 & 10 & 0.62 & 25.792 & \\
\hline 5 & 20 & 3.88 & 0.30 & -- & 4.18 & 83.600 & 6 & 0.22 & 18.392 & \\
\hline 6 & 10 & 3.86 & 0.28 & -- & 4.14 & 41.400 & 12 & 0.89 & 36.846 & \\
\hline 7 & 10 & 3.88 & 0.30 & -- & 4.18 & 41.800 & 8 & 0.39 & 16.302 & \\
\hline 8 & 10 & 3.86 & 0.28 & -- & 4.14 & 41.400 & 12 & 0.89 & 36.846 & \\
\hline 9 & 10 & 3.88 & 0.30 & -- & 4.18 & 41.800 & 8 & 0.39 & 16.302 & \\
\hline \multirow[t]{2}{*}{10} & 10 & 3.88 & 0.30 & -- & 4.18 & 41.800 & 8 & 0.39 & 16.302 & \\
\hline & & & & & & & & Suma & 267.132 & $(14.6 \%)$ \\
\hline \multicolumn{11}{|c|}{ +++ Horquillas alma semi-viga } \\
\hline 1 & 10 & 0.94 & 2.19 & 0.42 & 12.32 & 126.095 & 12 & 0.89 & 112.225 & \\
\hline 2 & 10 & 0.94 & 2.19 & 0.42 & 12.32 & 123.200 & 12 & 0.89 & 109.648 & \\
\hline 3 & 10 & 0.94 & 2.19 & 0.35 & 12.04 & 120.400 & 10 & 0.62 & 74.648 & \\
\hline 4 & 10 & 0.94 & 2.19 & 0.42 & 12.32 & 123.200 & 12 & 0.89 & 109.648 & \\
\hline 5 & 10 & 0.94 & 2.19 & 0.42 & 12.32 & 123.200 & 12 & 0.89 & 109.648 & \\
\hline 6 & 10 & 0.94 & 2.19 & 0.42 & 12.32 & 123.200 & 12 & 0.89 & 109.648 & \\
\hline 7 & 10 & 0.94 & 2.19 & 0.42 & 12.32 & 123.200 & 12 & 0.89 & 109.648 & \\
\hline 8 & 10 & 0.94 & 2.19 & 0.35 & 12.04 & 120.400 & 10 & 0.62 & 74.648 & \\
\hline 9 & 10 & 0.94 & 2.19 & 0.42 & 12.32 & 123.200 & 12 & 0.89 & 109.648 & \\
\hline \multirow[t]{3}{*}{10} & 10 & 0.94 & 2.19 & 0.42 & 12.32 & 123.200 & 12 & 0.89 & 109.648 & \\
\hline & & & & & & & & \multicolumn{2}{|c|}{-------------} & \\
\hline & & & & & & & & Suma & 1029.057 & $(56.4 \%)$ \\
\hline \multicolumn{11}{|c|}{ +++ Cerco alas semi-viga } \\
\hline 1 & 10 & 2.77 & 0.49 & 0.21 & 12.48 & 127.733 & 6 & 0.22 & 28.101 & \\
\hline 2 & 10 & 2.77 & 0.49 & 0.21 & 12.48 & 124.800 & 6 & 0.22 & 27.456 & \\
\hline 3 & 10 & 2.77 & 0.49 & 0.21 & 12.48 & 124.800 & 6 & 0.22 & 27.456 & \\
\hline 4 & 10 & 2.77 & 0.49 & 0.21 & 12.48 & 124.800 & 6 & 0.22 & 27.456 & \\
\hline 5 & 10 & 2.77 & 0.49 & 0.21 & 12.48 & 124.800 & 6 & 0.22 & 27.456 & \\
\hline 6 & 10 & 2.77 & 0.49 & 0.21 & 12.48 & 124.800 & 6 & 0.22 & 27.456 & \\
\hline 7 & 10 & 2.77 & 0.49 & 0.21 & 12.48 & 124.800 & 6 & 0.22 & 27.456 & \\
\hline 8 & 10 & 2.77 & 0.49 & 0.21 & 12.48 & 124.800 & 6 & 0.22 & 27.456 & \\
\hline 9 & 10 & 2.77 & 0.49 & 0.21 & 12.48 & 124.800 & 6 & 0.22 & 27.456 & \\
\hline \multirow[t]{3}{*}{10} & 10 & 2.77 & 0.49 & 0.21 & 12.48 & 124.800 & 6 & 0.22 & 27.456 & \\
\hline & & & & & & & & \multicolumn{2}{|c|}{-----------} & \\
\hline & \multicolumn{7}{|c|}{ +++ Transversal inferior semi-losa } & Suma & 275.205 & $(15.1 \%)$ \\
\hline 1 & 6 & 11.93 & - - & - - & 11.93 & 81.402 & 25 & 3.85 & 313.399 & \\
\hline 2 & 6 & 11.93 & - & - & 11.93 & 79.533 & 25 & 3.85 & 306.203 & \\
\hline 3 & 6 & 11.93 & -- & -- & 11.93 & 79.533 & 25 & 3.85 & 306.203 & \\
\hline 4 & 6 & 11.93 & -- & -- & 11.93 & 79.533 & 25 & 3.85 & 306.203 & \\
\hline 5 & 6 & 11.93 & -- & -- & 11.93 & 79.533 & 25 & 3.85 & 306.203 & \\
\hline 6 & 8 & 11.93 & -- & -- & 11.93 & 95.440 & 25 & 3.85 & 367.444 & \\
\hline 7 & 6 & 11.93 & -- & -- & 11.93 & 79.533 & 25 & 3.85 & 306.203 & \\
\hline 8 & 8 & 11.93 & -- & -- & 11.93 & 95.440 & 25 & 3.85 & 367.444 & \\
\hline 9 & 6 & 11.93 & -- & -- & 11.93 & 79.533 & 25 & 3.85 & 306.203 & \\
\hline \multirow[t]{3}{*}{10} & 6 & 11.93 & -- & - - & 11.93 & 79.533 & 25 & 3.85 & 306.203 & \\
\hline & & & & & & & & \multirow{2}{*}{\multicolumn{3}{|c|}{ Suma 3191711 (29 4\%) }} \\
\hline & & & & & & & & & & $(29.4 \%)$ \\
\hline
\end{tabular}




$\begin{array}{rrrrrrrrrr}+++ \\ 1 & 6 & 11.93 & -- & -- & 11.93 & 81.402 & 25 & 3.85 & 313.399 \\ 2 & 6 & 11.93 & -- & -- & 11.93 & 79.533 & 25 & 3.85 & 306.203 \\ 3 & 6 & 11.93 & -- & -- & 11.93 & 79.533 & 25 & 3.85 & 306.203 \\ 4 & 6 & 11.93 & -- & -- & 11.93 & 79.533 & 25 & 3.85 & 306.203 \\ 5 & 6 & 11.93 & -- & -- & 11.93 & 79.533 & 25 & 3.85 & 306.203 \\ 6 & 6 & 11.93 & -- & -- & 11.93 & 79.533 & 25 & 3.85 & 306.203 \\ 7 & 6 & 11.93 & -- & -- & 11.93 & 79.533 & 25 & 3.85 & 306.203 \\ 8 & 6 & 11.93 & -- & -- & 11.93 & 79.533 & 25 & 3.85 & 306.203 \\ 9 & 6 & 11.93 & -- & -- & 11.93 & 79.533 & 25 & 3.85 & 306.203 \\ 10 & 6 & 11.93 & -- & -- & 11.93 & 79.533 & 25 & 3.85 & 306.203 \\ & & & & & & & & & \\ \end{array}$

Armadura longitudinal total tablero

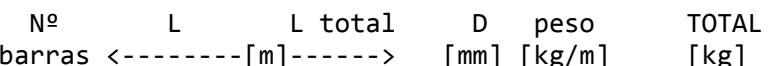

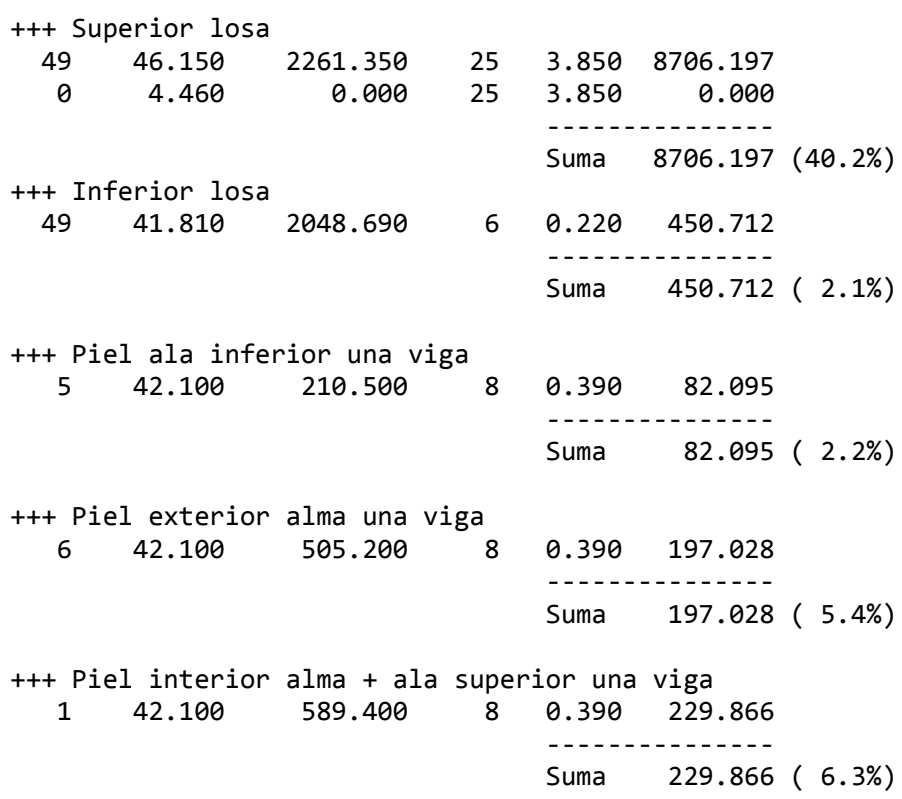

\section{ESTADO DE MEDICIONES}

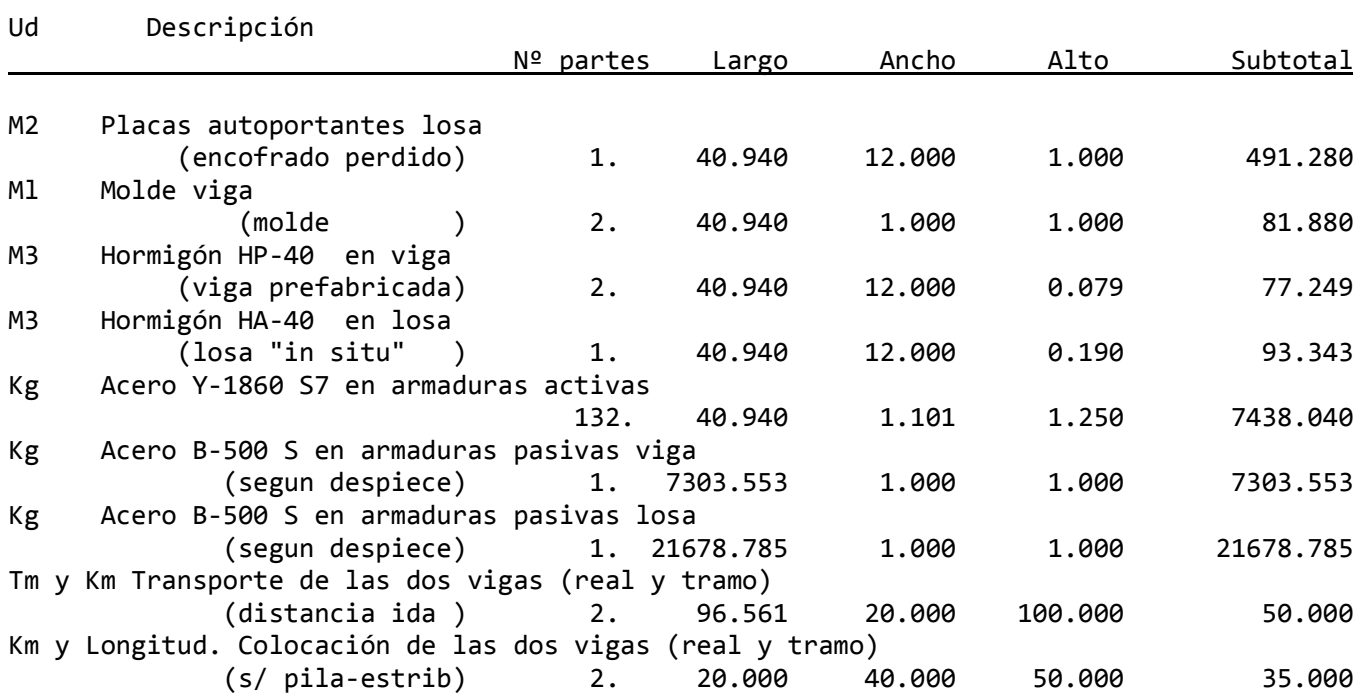




\section{CUANTIAS RESULTANTES}

0.347 M3 hormigon / M2 tablero

$43.601 \mathrm{~kg}$ activas / M3 hormigón

$96.287 \mathrm{~kg}$ activas / M3 hormigón VIGA

$15.118 \mathrm{~kg}$ activas / M2 tablero

$169.893 \mathrm{~kg}$ pasivas / M3 hormigón

$94.546 \mathrm{~kg}$ pasivas / M3 hormigón VIGA

$58.907 \mathrm{~kg}$ pasivas / M2 tablero

\section{CALCULO DEL COSTE}

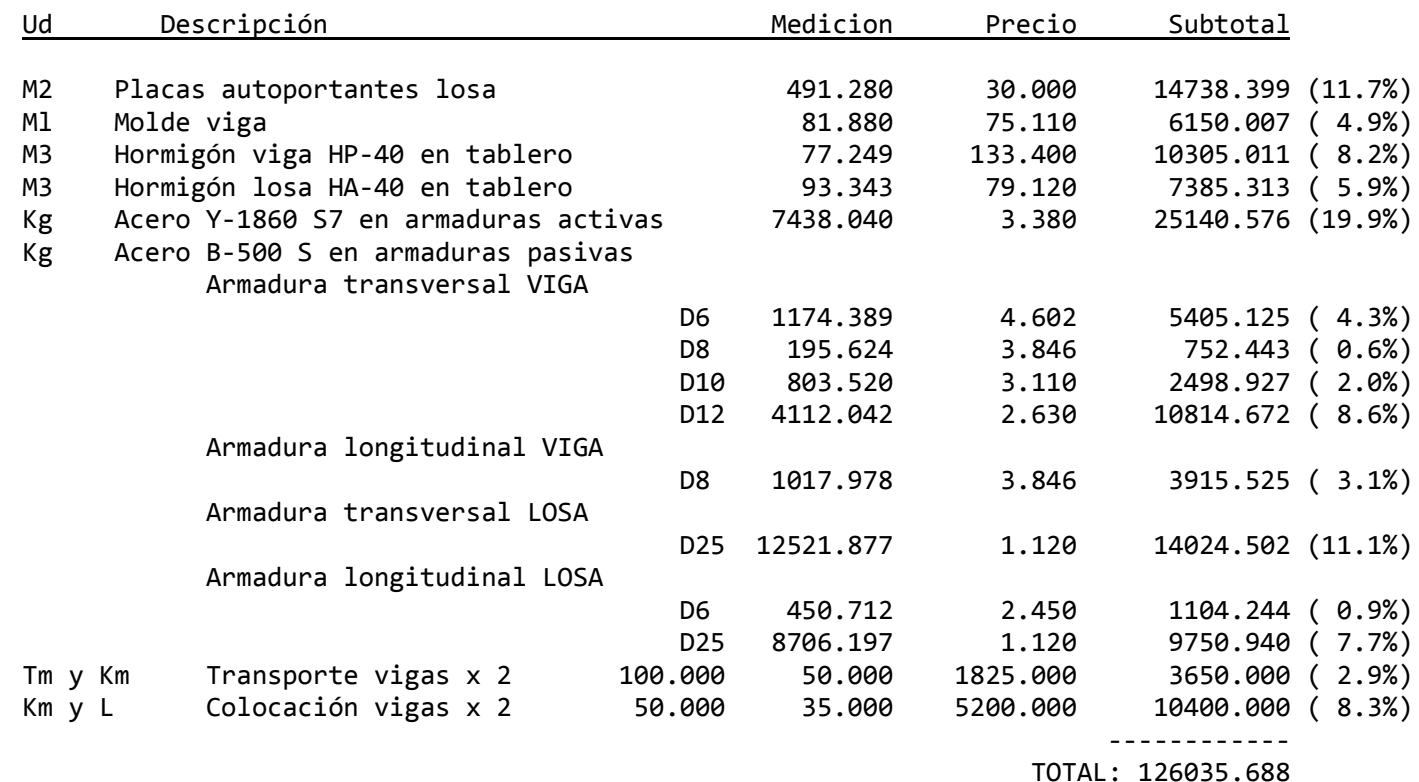

256.17 EUROS / M2 tablero 


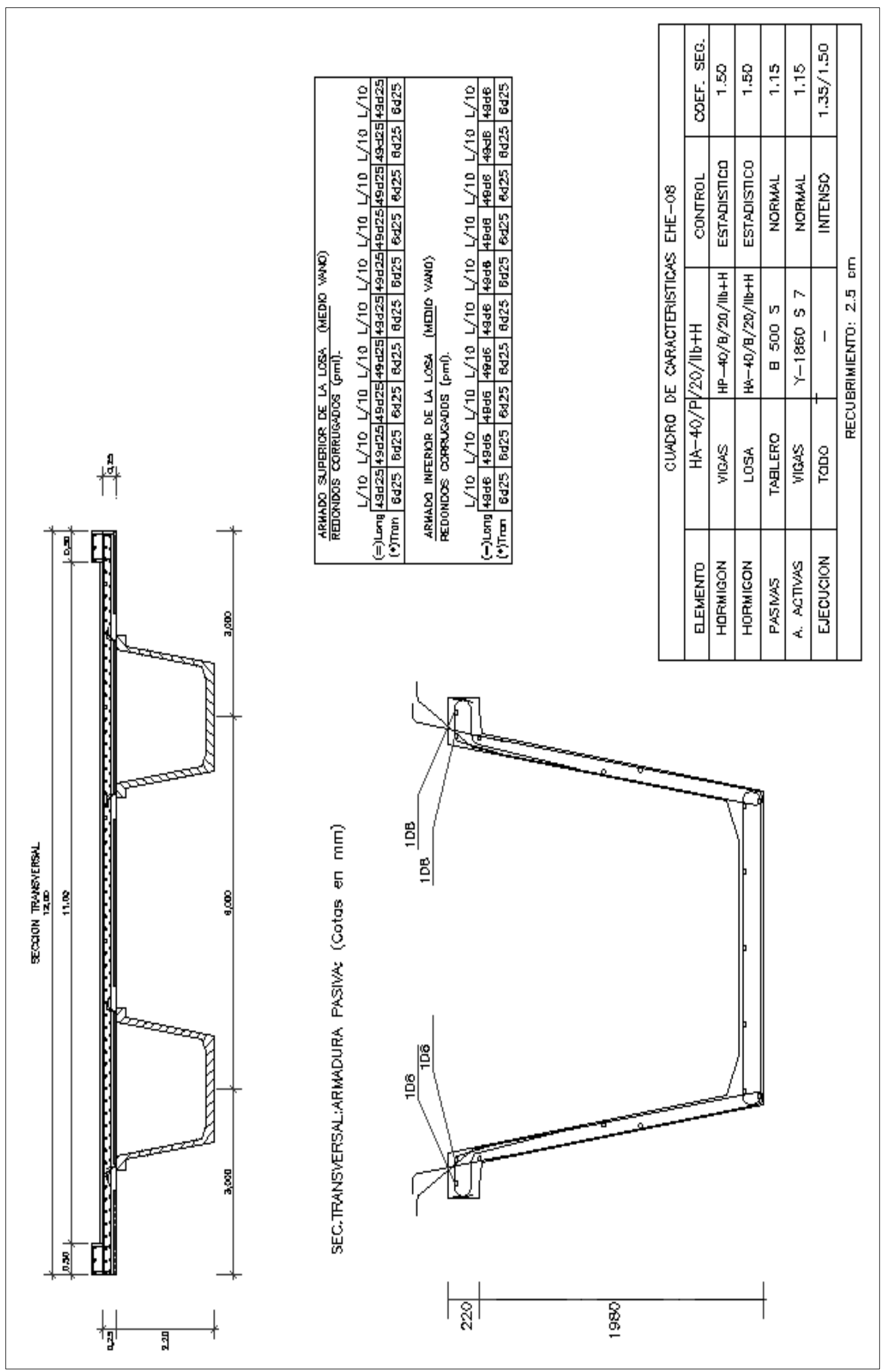




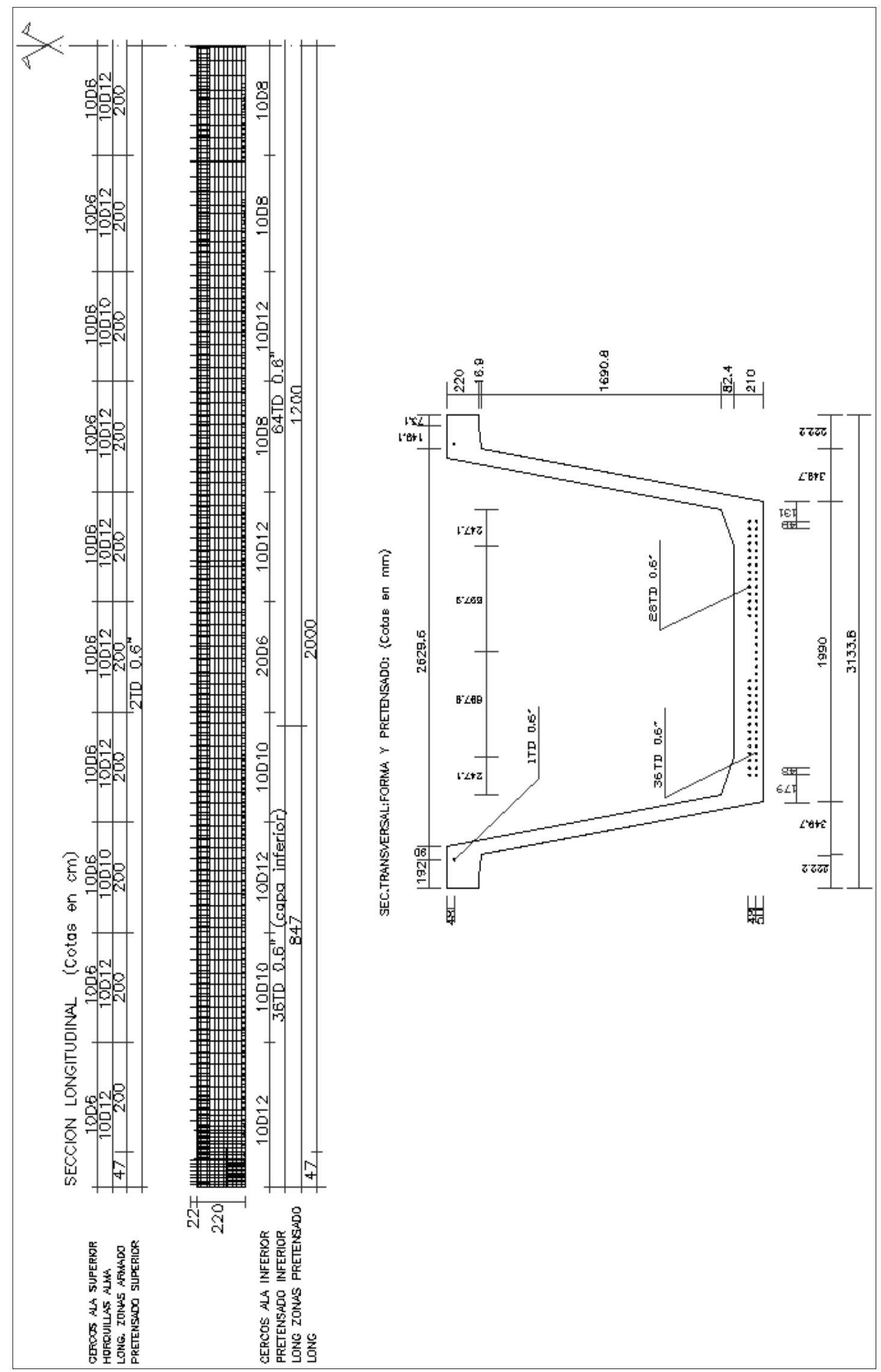


$\mathrm{L}=40 \mathrm{~m} . \mathrm{HPF}$

ARMADURA PASIVA

Armadura transversal

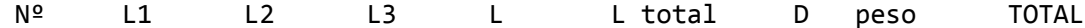

$\mathrm{sec}$ barras $<\ldots[\mathrm{mm}][\mathrm{kg} / \mathrm{m}] \quad[\mathrm{kg}]$

$\begin{array}{rccccc}+++ & \text { Cerco ala inf.semi-viga } & & \\ 1 & 20 & 3.88 & 0.20 & -- & 4.08 \\ 2 & 10 & 3.88 & 0.22 & -- & 4.10 \\ 3 & 20 & 3.90 & 0.22 & -- & 4.12 \\ 4 & 10 & 3.88 & 0.22 & -- & 4.10 \\ 5 & 20 & 3.90 & 0.22 & -- & 4.12 \\ 6 & 10 & 3.88 & 0.22 & -- & 4.10 \\ 7 & 10 & 3.88 & 0.20 & -- & 4.08 \\ 8 & 10 & 3.88 & 0.20 & -- & 4.08 \\ 9 & 10 & 3.88 & 0.20 & -- & 4.08 \\ 10 & 10 & 3.88 & 0.22 & -- & 4.10\end{array}$

+++ Horquillas alma semi-viga

$\begin{array}{rrrrrrrrrr}1 & 10 & 0.94 & 2.17 & 0.42 & 12.24 & 125.276 & 12 & 0.89 & 111.496 \\ 2 & 10 & 0.94 & 2.17 & 0.42 & 12.24 & 122.400 & 12 & 0.89 & 108.936 \\ 3 & 10 & 0.94 & 2.17 & 0.42 & 12.24 & 122.400 & 12 & 0.89 & 108.936 \\ 4 & 10 & 0.94 & 2.17 & 0.35 & 11.96 & 119.600 & 10 & 0.62 & 74.152 \\ 5 & 10 & 0.94 & 2.17 & 0.42 & 12.24 & 122.400 & 12 & 0.89 & 108.936 \\ 6 & 10 & 0.94 & 2.17 & 0.42 & 12.24 & 122.400 & 12 & 0.89 & 108.936 \\ 7 & 10 & 0.94 & 2.17 & 0.42 & 12.24 & 122.400 & 12 & 0.89 & 108.936 \\ 8 & 10 & 0.94 & 2.17 & 0.42 & 12.24 & 122.400 & 12 & 0.89 & 108.936 \\ 9 & 10 & 0.94 & 2.17 & 0.42 & 12.24 & 122.400 & 12 & 0.89 & 108.936 \\ 10 & 10 & 0.94 & 2.17 & 0.42 & 12.24 & 122.400 & 12 & 0.89 & 108.936 \\ & & & & & & & & & -.---.---.--\end{array}$

+++ Cerco alas semi-viga

\begin{tabular}{|c|c|c|c|c|c|c|c|c|c|c|}
\hline 1 & 10 & 2.77 & 0.49 & 0.21 & 12.48 & 127.733 & 6 & 0.22 & 28.101 & \\
\hline 2 & 10 & 2.77 & 0.49 & 0.21 & 12.48 & 124.800 & 6 & 0.22 & 27.456 & \\
\hline 3 & 10 & 2.77 & 0.49 & 0.21 & 12.48 & 124.800 & 6 & 0.22 & 27.456 & \\
\hline 4 & 10 & 2.77 & 0.49 & 0.21 & 12.48 & 124.800 & 6 & 0.22 & 27.456 & \\
\hline 5 & 10 & 2.77 & 0.49 & 0.21 & 12.48 & 124.800 & 6 & 0.22 & 27.456 & \\
\hline 6 & 10 & 2.77 & 0.49 & 0.21 & 12.48 & 124.800 & 6 & 0.22 & 27.456 & \\
\hline 7 & 10 & 2.77 & 0.49 & 0.21 & 12.48 & 124.800 & 6 & 0.22 & 27.456 & \\
\hline 8 & 10 & 2.77 & 0.49 & 0.21 & 12.48 & 124.800 & 6 & 0.22 & 27.456 & \\
\hline 9 & 10 & 2.77 & 0.49 & 0.21 & 12.48 & 124.800 & 6 & 0.22 & 27.456 & \\
\hline 10 & 10 & 2.77 & 0.49 & 0.21 & 12.48 & 124.800 & 6 & 0.22 & 27.456 & \\
\hline & & & & & & & & Suma & 275.205 & $(16.6 \%)$ \\
\hline + Transversal inferior semi-losa & 6 & 11.93 & - & - & 1193 & 81.402 & 25 & 3.85 & 313.399 & \\
\hline 2 & 6 & 11.93 & - & -- & 11.93 & 79.533 & 25 & 3.85 & 306.203 & \\
\hline 3 & 6 & 11.93 & -- & -- & 11.93 & 79.533 & 25 & 3.85 & 306.203 & \\
\hline 4 & 6 & 11.93 & -- & -- & 11.93 & 79.533 & 25 & 3.85 & 306.203 & \\
\hline 5 & 6 & 11.93 & -- & -- & 11.93 & 79.533 & 25 & 3.85 & 306.203 & \\
\hline 6 & 6 & 11.93 & -- & -- & 11.93 & 79.533 & 25 & 3.85 & 306.203 & \\
\hline 7 & 6 & 11.93 & -- & -- & 11.93 & 79.533 & 25 & 3.85 & 306.203 & \\
\hline 8 & 6 & 11.93 & -- & -- & 11.93 & 79.533 & 25 & 3.85 & 306.203 & \\
\hline 9 & 6 & 11.93 & -- & -- & 11.93 & 79.533 & 25 & 3.85 & 306.203 & \\
\hline 10 & 6 & 11.93 & -- & - - & 11.93 & 79.533 & 25 & 3.85 & 306.203 & \\
\hline
\end{tabular}




\begin{tabular}{rlllllllll}
+++ & \multicolumn{7}{c}{ Transversal superior semi-losa } \\
1 & 6 & 11.93 & -- & -- & 11.93 & 81.402 & 25 & 3.85 & 313.399 \\
2 & 6 & 11.93 & -- & -- & 11.93 & 79.533 & 25 & 3.85 & 306.203 \\
3 & 6 & 11.93 & -- & -- & 11.93 & 79.533 & 25 & 3.85 & 306.203 \\
4 & 6 & 11.93 & -- & -- & 11.93 & 79.533 & 25 & 3.85 & 306.203 \\
5 & 6 & 11.93 & -- & -- & 11.93 & 79.533 & 25 & 3.85 & 306.203 \\
6 & 6 & 11.93 & -- & -- & 11.93 & 79.533 & 25 & 3.85 & 306.203 \\
7 & 6 & 11.93 & -- & -- & 11.93 & 79.533 & 25 & 3.85 & 306.203 \\
8 & 6 & 11.93 & -- & -- & 11.93 & 79.533 & 25 & 3.85 & 306.203 \\
9 & 6 & 11.93 & -- & -- & 11.93 & 79.533 & 25 & 3.85 & 306.203 \\
10 & 6 & 11.93 & -- & -- & 11.93 & 79.533 & 25 & 3.85 & 306.203 \\
& & & & & & & & & \\
\end{tabular}

Armadura longitudinal total tablero

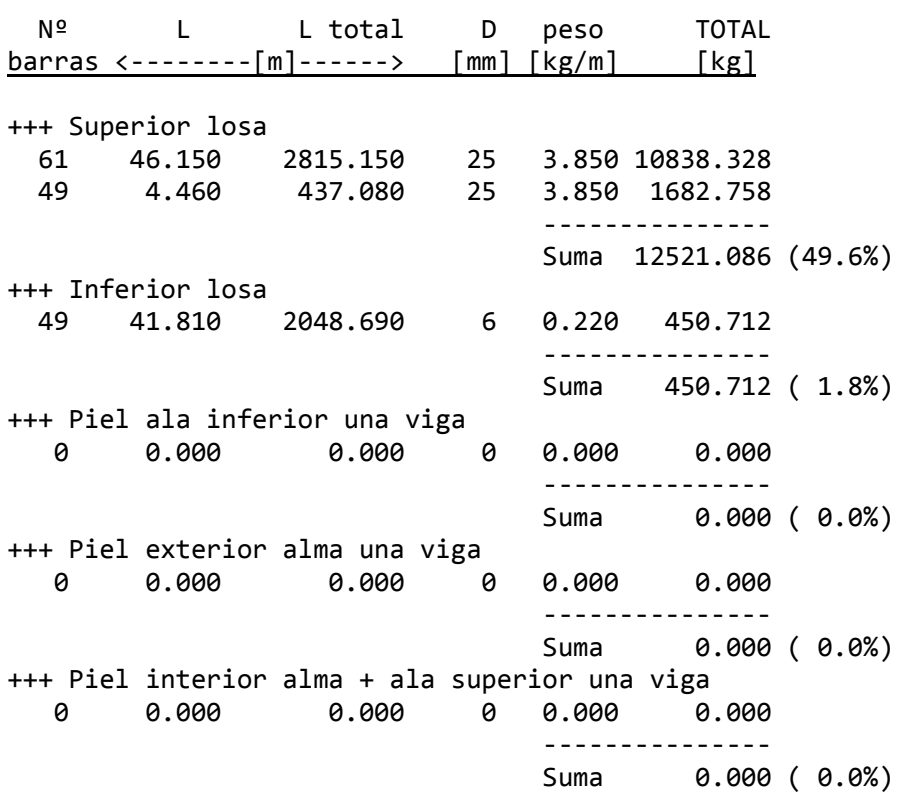

\section{ESTADO DE MEDICIONES}

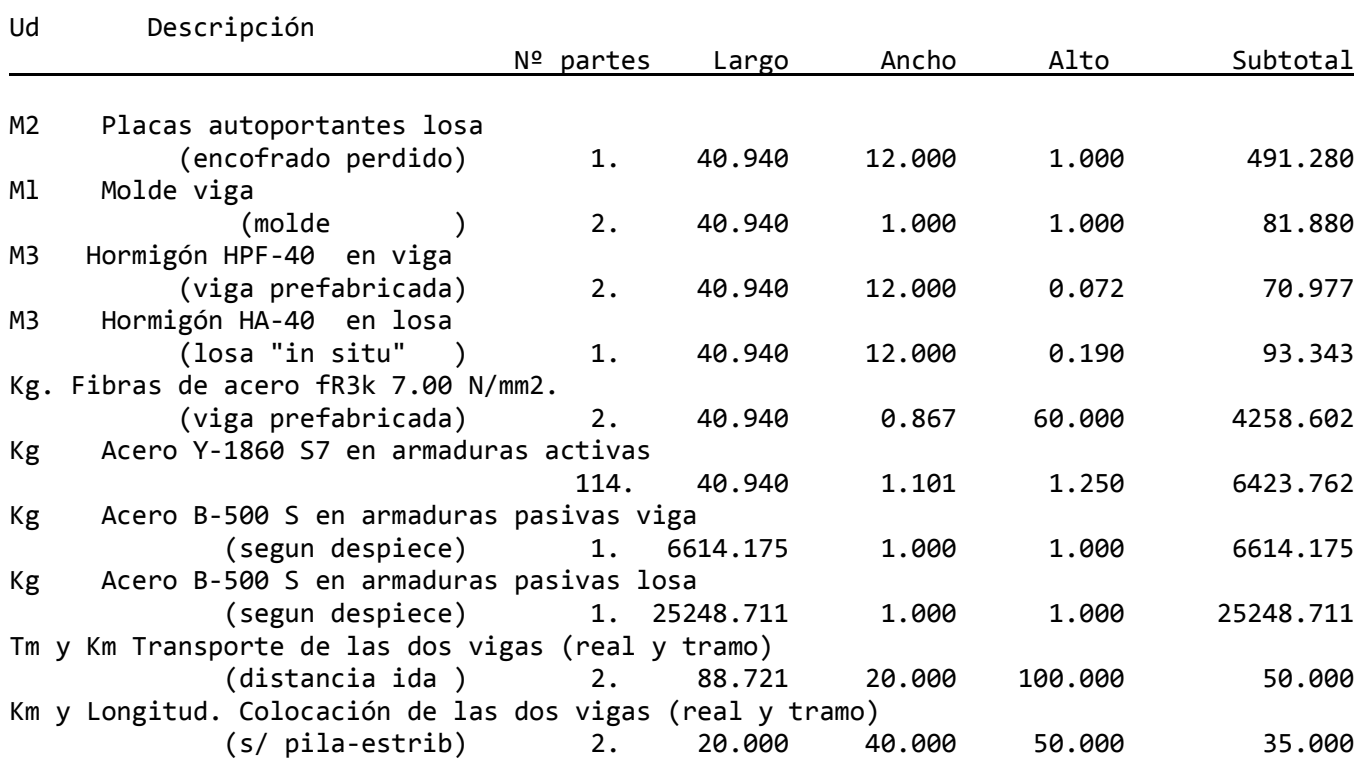




\section{CUANTIAS RESULTANTES}
$60.000 \mathrm{Kg} . / \mathrm{M3}$ hormigon
0.334 M3 hormigon / M2 tablero
$39.093 \mathrm{~kg}$ activas / M3 hormigón
$90.505 \mathrm{~kg}$ activas / M3 hormigón VIGA
$13.056 \mathrm{~kg}$ activas / M2 tablero
193.908 kg pasivas / M3 hormigón
$93.188 \mathrm{~kg}$ pasivas / M3 hormigón VIGA
$64.762 \mathrm{~kg}$ pasivas / M2 tablero

\section{CALCULO DEL COSTE}

\begin{tabular}{|c|c|c|c|c|c|c|}
\hline$\underline{U d}$ & Desc & cripción & Medicion & Precio & Subtotal & \\
\hline M2 & Placas & autoportantes losa & 491.280 & 30.000 & 14738.399 & $(11.6 \%)$ \\
\hline M1 & Molde & viga & 81.880 & 75.110 & 6150.007 & $(4.8 \%)$ \\
\hline M3 & Hormigc & ón viga HPF-40 en tablero & 70.977 & 133.400 & 9468.291 & $(7.5 \%)$ \\
\hline M3 & Hormigc & yón losa HA-40 en tablero & 93.343 & 79.120 & 7385.313 & $(5.8 \%)$ \\
\hline $\mathrm{Kg}$. & Fibras & acero fR3k7.00 en viga & 4258.602 & 1.000 & 4258.602 & $(3.4 \%)$ \\
\hline $\mathrm{Kg}$ & Acero & Y-1860 S7 en armaduras activas & 6423.762 & 3.380 & 21712.314 & $(17.1 \%)$ \\
\hline \multirow[t]{9}{*}{ Kg } & Acero & $\begin{array}{l}\text { B-500 S en armaduras pasivas } \\
\text { Armadura transversal VIGA }\end{array}$ & & & & \\
\hline & & 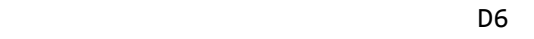 & 1245.845 & 4.602 & 5734.000 & $(4.5 \%)$ \\
\hline & & D10 & 703.328 & 3.110 & 2187.333 & $(1.7 \%)$ \\
\hline & & D12 & 4665.002 & 2.630 & 12268.957 & $(9.7 \%)$ \\
\hline & & $\begin{array}{l}\text { Armadura longitudinal VIGA } \\
\text { Armadura transversal LOSA }\end{array}$ & & & & \\
\hline & & D25 & 12276.914 & 1.120 & 13750.144 & $(10.8 \%)$ \\
\hline & & Armadura longitudinal LOSA & & & & \\
\hline & & 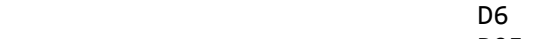 & 450.712 & 2.450 & 1104.244 & $(0.9 \%)$ \\
\hline & & D25 & 12521.086 & 1.120 & 14023.616 & $(11.1 \%)$ \\
\hline & & Transporte vigas $\times 2$ & 50.000 & 1825.000 & 3650.000 & $(2.9 \%)$ \\
\hline $\mathrm{Km}$ y & $\mathrm{L}$ & Colocación vigas $\times 2$ & 35.000 & 5200.000 & 10400.000 & $(8.2 \%)$ \\
\hline
\end{tabular}

257.79 EUROS / M2 tablero 


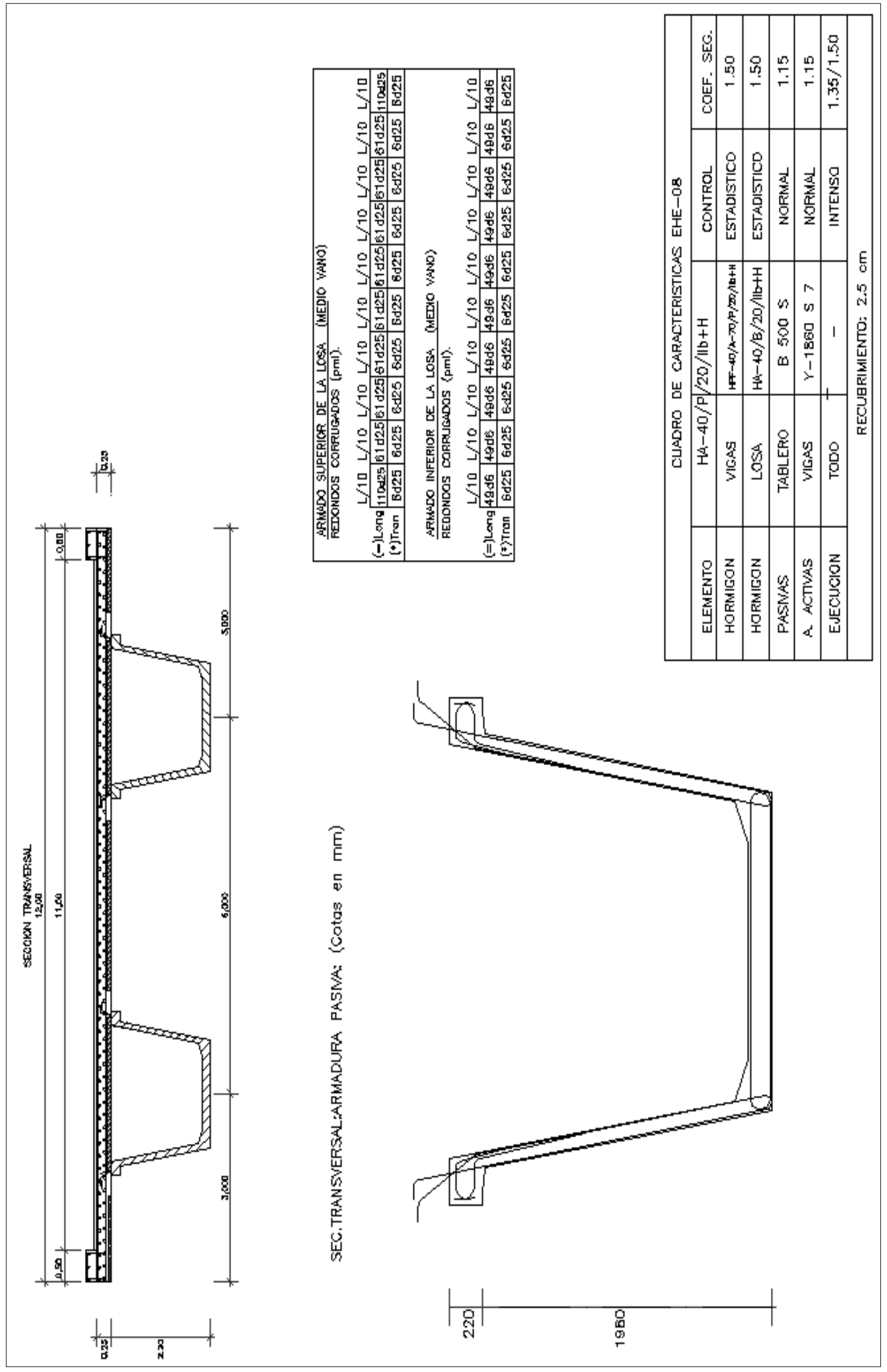




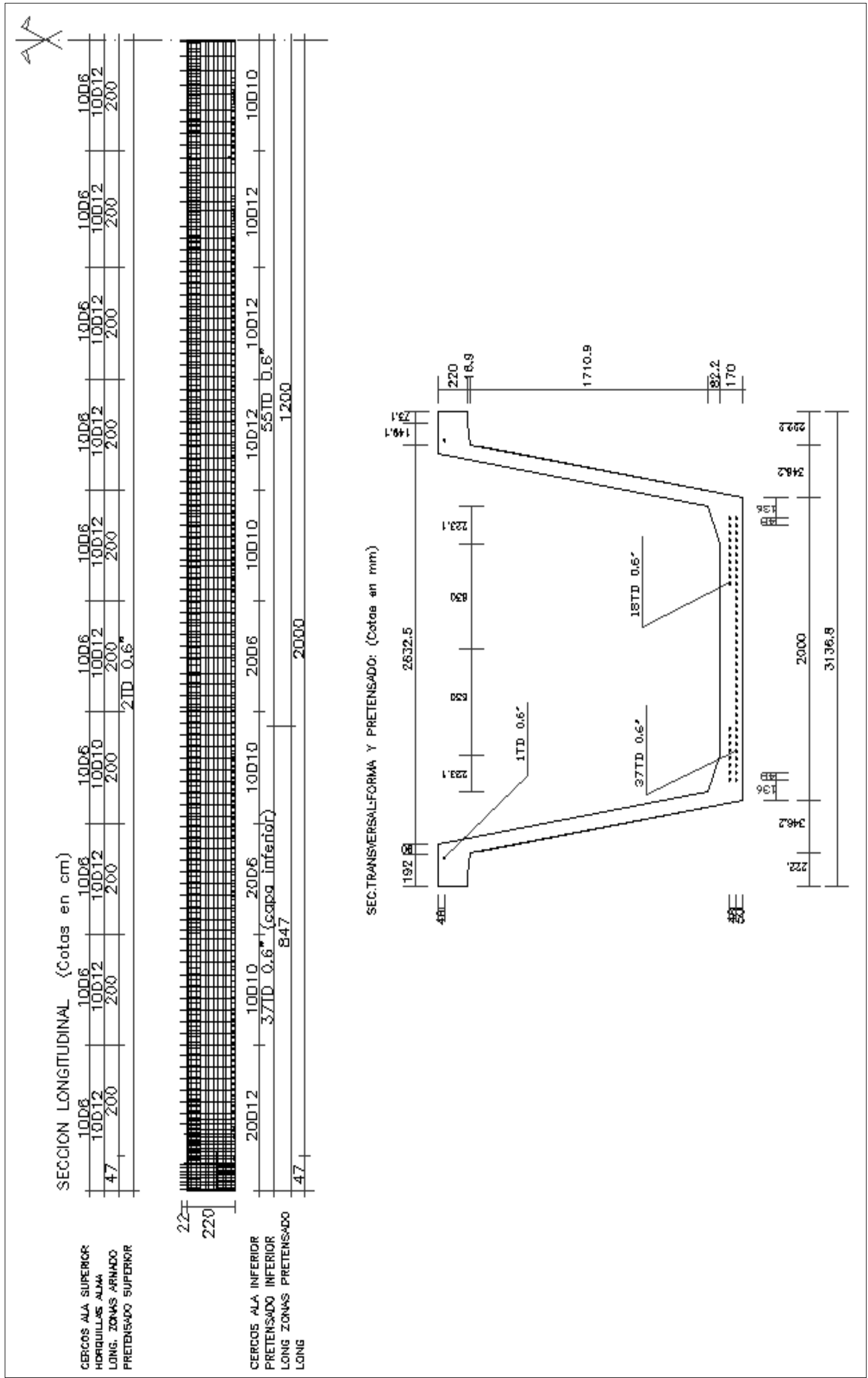


